\title{
Real-Time 3-D SAFT-UT System Evaluation and Validation
}

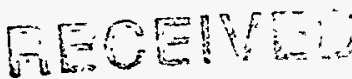

DEC 23 १९95

OSTI

Prepared by

S. R. Doctor, G. J. Schuster, L. D. Reid, T. E. Hall

Pacific Northwest National Laboratory

Prepared for

U.S. Nuclear Regulatory Commission 


\section{AVAILABILITY NOTICE}

Availability of Reference Materials Cited in NRC Publications

Most documents cited in NRC publications will be available from one of the following sources:

1. The NRC Public Document Room, 2120 L Street, NW., Lower Level, Washington, DC 20555-0001

2. The Superintendent of Documents, U.S. Government Printing Office. P. O. Box 37082 , Washington. DC 20402-9328

3. The National Technical Information Service, Springfield, VA 22161-0002

Although the listing that follows represents the majority of documents cited in NRC publications, it is not intended to be exhaustive.

Referenced documents available for inspection and copying for a fee from the NRC Public Document Room include NRC correspondence and internal NRC memoranda; NRC bulletins, circulars, information notices. inspection and investigation notices; licensee event reports; vendor reports and correspondence; Commission papers; and applicant and licensee documents and correspondence.

The following documents in the NUREG serles are available for purchase from the Government Printing Office: formal NRC staff and contractor reports, NRC-sponsored conference proceedings, international agreement reports, grantee reports, and NRC booklets and brochures. Also available are regulatory guides, NRC regulations in the Code of Federal Regulations, and Nuclear Regulatory Commission Issuances.

Documents avallable from the National Technical Information Service include NUREG-series reports and technical reports prepared by other Federal agencies and reports prepared by the Atomic Energy Commission, forerunner agency to the Nuclear Regulatory Commission.

Documents available from public and special technical libraries include all open literature items, such as books. Journal articles, and transactions. Federal Register notices. Federal and State legislation, and congressional reports can usually be obtained from these libraries.

Documents such as theses, dissertations, foreign reports and translations, and non-NRC conference proceedings are available for purchase from the organization sponsoring the publication cited.

Single copies of NRC draft reports are available free. to the extent of supply, upon written request to the Office of Administration, Distribution and Mail Services Section, U.S. Nuclear Regulatory Commission, Washington, DC 20555-0001.

Coples of industry codes and standards used in a substantive manner in the NRC regulatory process are maintalned at the NRC Library. Two White Flint North, 11545 Rockville Pike, Rockville. MD 20852-2738. for use by the public. Codes and standards are usually copyrighted and may be purchased from the originating organization or, if they are American National Standards. from the American National Standards Institute, 1430 Broadway, New York, NY 10018-3308.

\section{DISCLAIMER NOTICE}

This report was prepared as an account of work sponsored by an agency of the United States Government. Neither the United States Govemment norany agency thereof, norany of their employees, makes any warranty, expressed or implied, or assumes any legal liability or responsibility for any third party's use, or the results of such use, of any information, apparatus, product, or process disclosed in this report, or represents that its use by such third party would not infringe privately owned rights. 

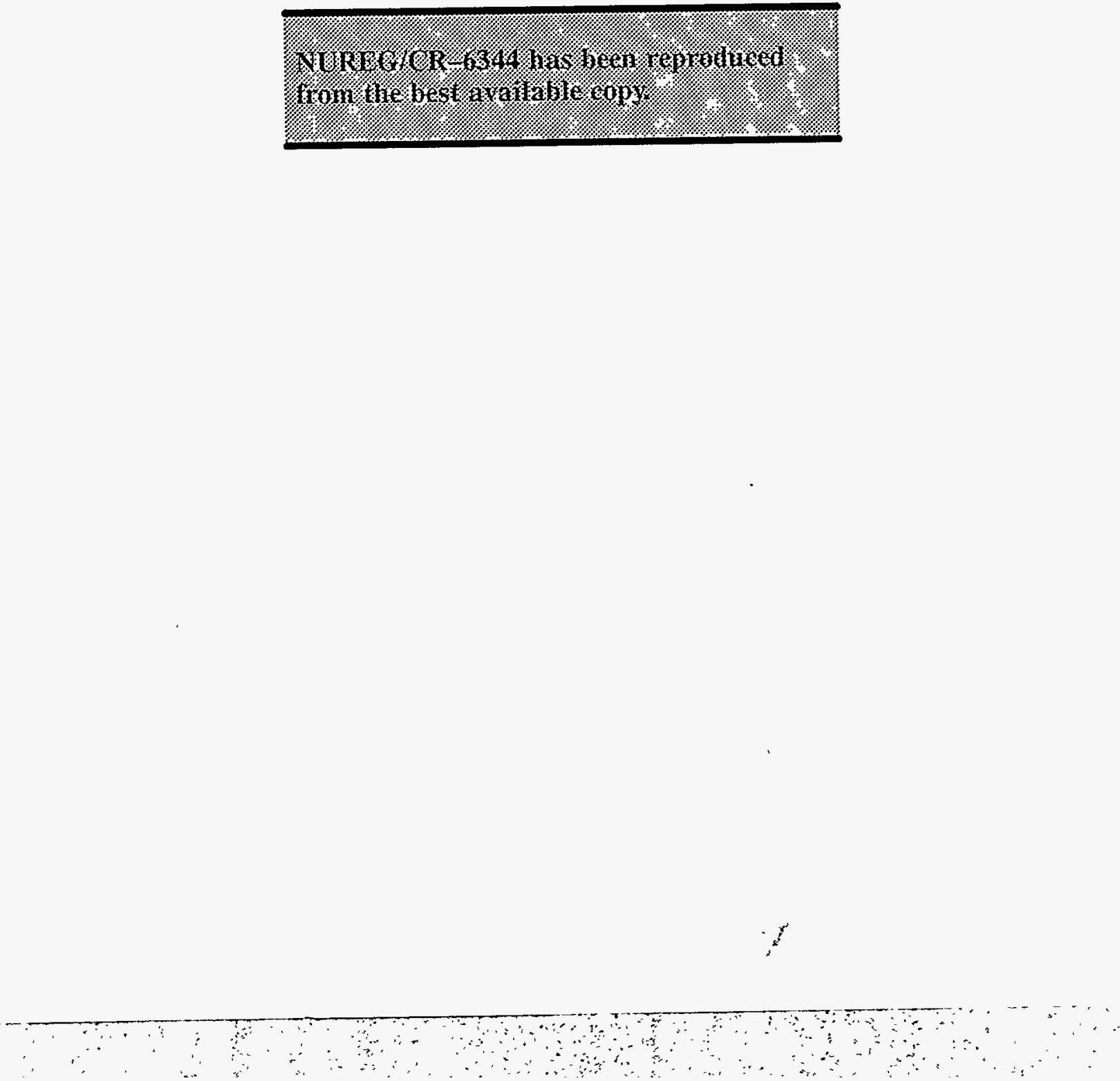
NUREG/CR-6344

PNNL-10571

\section{Real-Time 3-D SAFT-UT System Evaluation and Validation}

Manuscript Completed: March 1995

Dáte Published: September 1996

Prepared by

S. R. Doctor, G. J. Schuster, L. D. Reid, T. E. Hall

Pacific Northwest National Laboratory

Richland, WA 99352

J. Muscara, NRC Project Manager

Prepared for

Division of Engineering Technology

Office of Nuclear Regulatory Research

U.S. Nuclear Regulatory Commission

Washington, DC 20555-0001

NRC Job Codes B2467, B2913

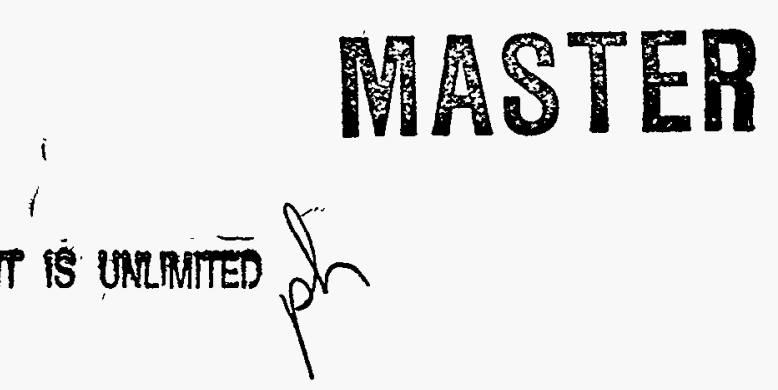

DASTRIBUTION OF THIS DOCUNENT IS UMLPMITED 
Abstract

\begin{abstract}
SAFT-UT technology is shown to provide significant enhancements to the inspection of materials used in U.S. nuclear power plants. This report provides guidelines for the implementation of SAFT-UT technology and shows the results from its application.

An overview of the development of SAFT-UT is provided so that the reader may become familiar with the technology. Then the basic fundamentals are presented with an extensive list of references. A comprehensive operating procedure, which is used in conjunction with the SAFT-UT field system developed by Pacific Northwest Laboratory (PNL), provides the recipe for both SAFT data acquisition and analysis.
\end{abstract}

The specification for the hardware implementation is provided for the SAFT-UT system along with a description of the subsequent developments and improvements. One development of technical interest is the SAFT real time processor. Performance of the real-time processor is impressive and comparison is made of this dedicated parallel processor to a conventional computer and to the newer high-speed computer architectures designed for image processing. Descriptions of other improvements, including a robotic scanner, are provided.

Laboratory parametric and application studies, performed by PNL and not previously reported, are discussed followed by a section on field application work in which SAFT was used during inservice inspections of operating reactors. 


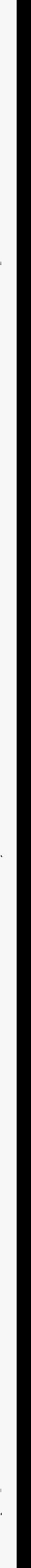




\section{DISCLAIMER}

Portions of this document may be illegible in electronic image products. Images are produced from the best available original document. 


\section{DISCLAIMER}

This report was prepared as an account of work sponsored by an agency of the United States Government. Neither the United States Government nor any agency thereof, nor any of their employees, makes any warranty, express or implied, or assumes any legal liability or responsibility for the accuracy, completeness, or usefulness of any information, apparatus, product, or process disclosed, or represents that its use would not infringe privately owned rights. Reference herein to any specific commercial product, process, or service by trade name, trademark, manufacturer, or otherwise does not necessarily constitute or imply its endorsement, recommendation, or favoring by the United States Government or any agency thereof. The views and opinions of authors expressed herein do not necessarily state or reflect those of the United States Government or any agency thereof. 


\section{Contents}

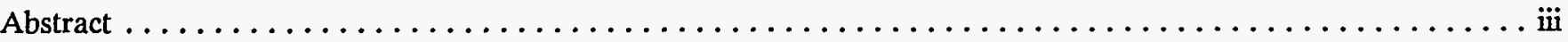

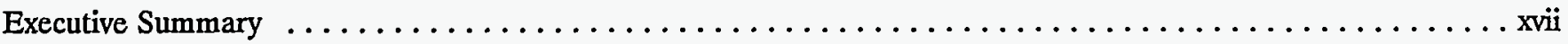

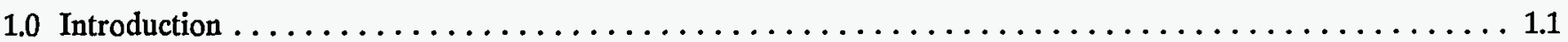

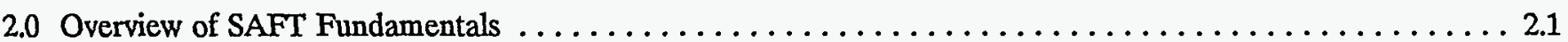

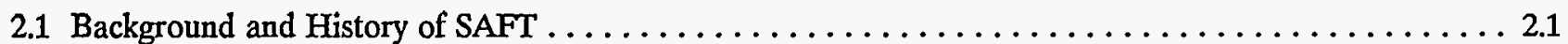

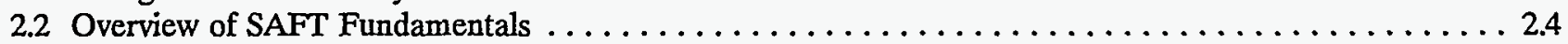

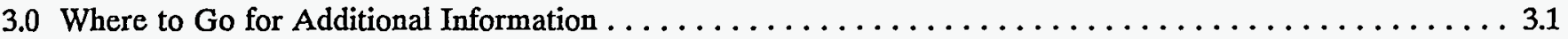

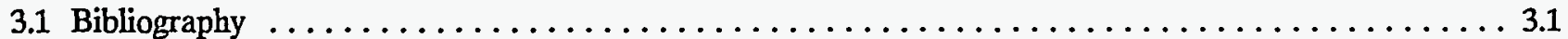

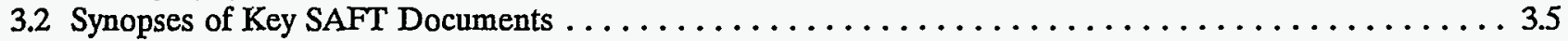

3.2.1 Review and Discussion of the Development of Synthetic Aperture Focusing Technique (SAFT-

UT) (Busse, Collins, and Doctor 1984) . . . . . . . . . . . . . . . . . 3.5

3.2.2 Development and Validation of a Real-Time SAFT-UT System for the Inspection of Light

Water Reactor Components, Vol. 1 (Doctor et al. 1986) . . . . . . . . . . . . . . . . 3.6

3.2.3 Development and Validation of a Real-Time SAFT-UT System for the Inspection of Light

Water Reactor Components, Vol. 2 (Doctor et al. 1987a) . . . . . . . . . . . . . . . . 3.7

3.2.4 Development and Validation of a Real-Time SAFT-UT System for the Inspection of Light

Water Reactor Components, Vol. 3 (Doctor et al. 1987b) . . . . . . . . . . . . . . 3.8

3.2.5 The SAFT-UT Real-Time Inspection System Operational Principles and Implementation (Hall,

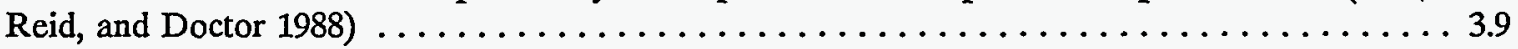

3.2.6 Program for Field Validation of the Synthetic Aperture Focusing Technique for Ultrasonic

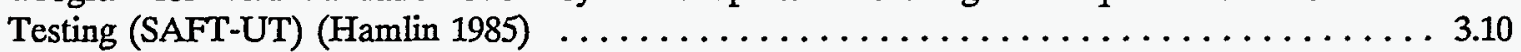

3.2.7 Development of a Real-Time Residue Number Processor for SAFT Inspection, Phase II Final Report, September 1984 through April 1986 (Polky 1986) . . . . . . . . . . . . . . . 3.10

3.2.8 An Ultra-High Speed Residue Processor for SAFT Inspection System Image Enhancement,

Final Report, October 1983 through March 1984 (Polky and Miller 1985) . . . . . . . . . 3.11

3.2.9 Design and Development of a Special Purpose SAFT System for Nondestructive Evaluation of

Nuclear Reactor Vessels and Piping Components (Ganapathy et al. 1985) . . . . . . . . 3.11

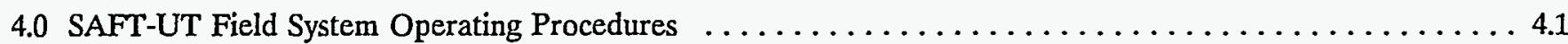

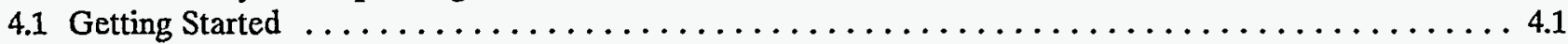

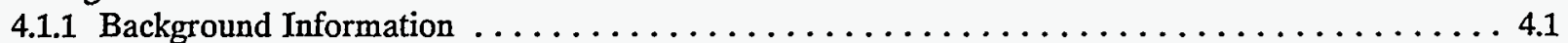

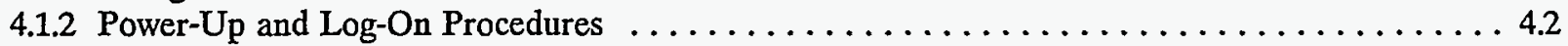

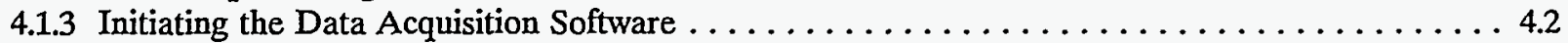

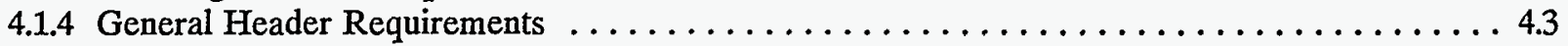

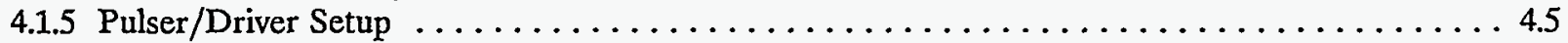

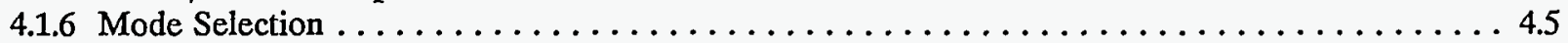

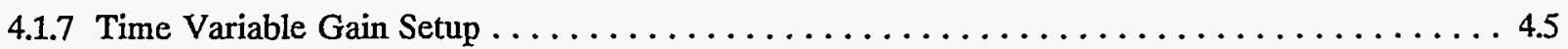

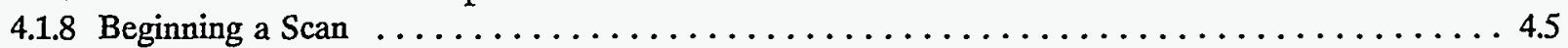

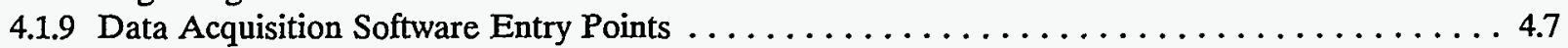

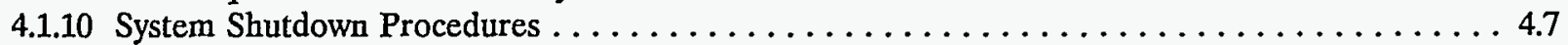

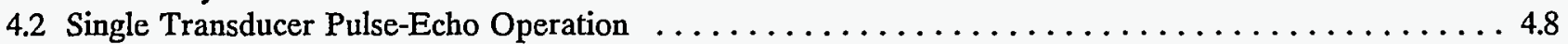

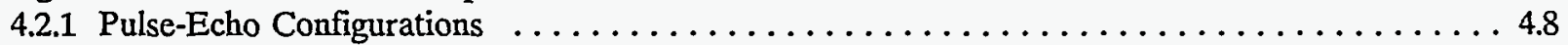

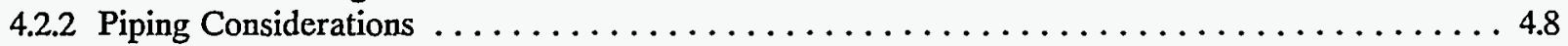

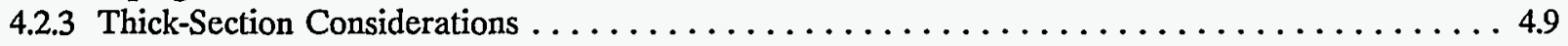




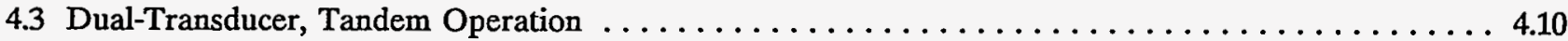

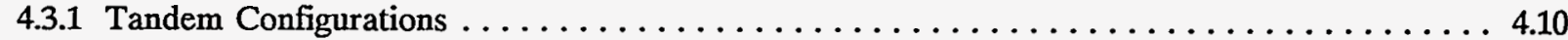

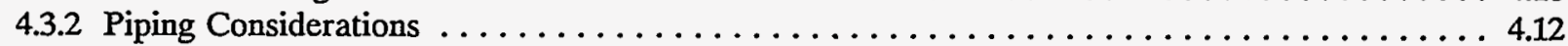

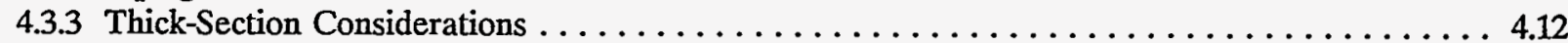

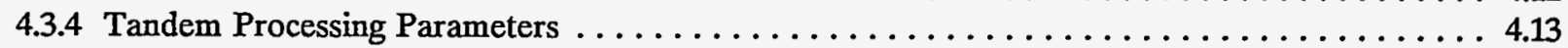

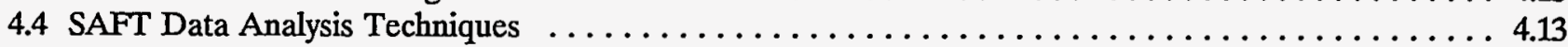

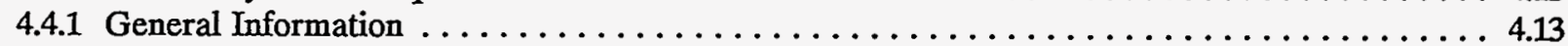

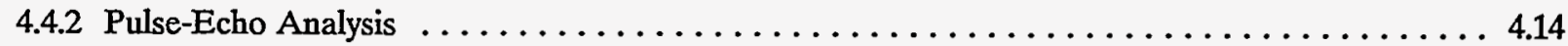

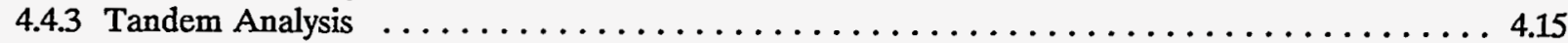

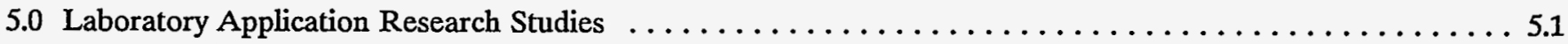

5.1 Investigation of SAFT Imaging in Thick Material $\ldots \ldots \ldots \ldots \ldots \ldots \ldots \ldots \ldots \ldots \ldots \ldots \ldots \ldots \ldots \ldots \ldots \ldots$

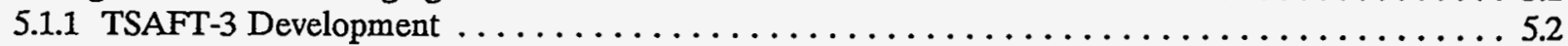

5.2 Application of Advanced Signal Processing Techniques to Improve SAFT-UT Performance on CCSS

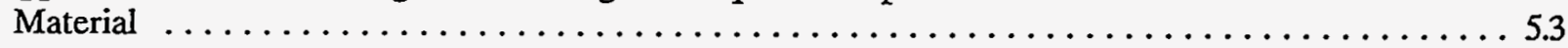

5.2.1 Applying the Spectral Analysis and Subsequent Filter Technique to a CCSSRRT Data File . . . 5.4

5.2.2 Software Tools Used to Define the Optimal Bandpass Filter Used to Enhance the SAFT-

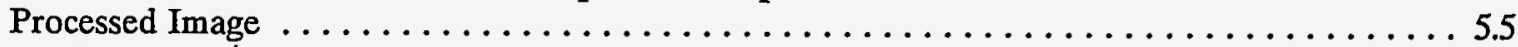

5.2.3 Statistical Analysis of Defect and NonDefect CCSS A-Scans $\ldots \ldots \ldots \ldots \ldots \ldots \ldots \ldots \ldots .6$

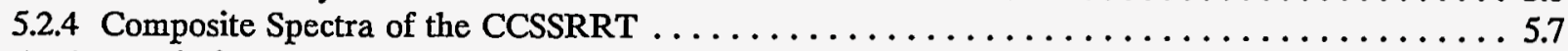

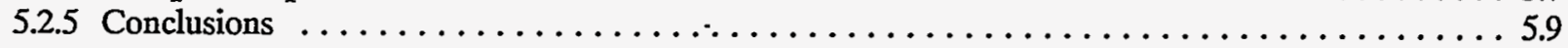

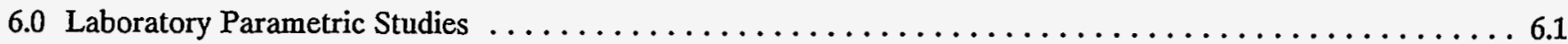

6.1 TSAFT-2 Configuration Velocity and Thickness Parametric Study $\ldots \ldots \ldots \ldots \ldots \ldots \ldots \ldots \ldots .1$

6.2 Bandwidth Compensation Study $\ldots \ldots \ldots \ldots \ldots \ldots \ldots \ldots \ldots \ldots \ldots \ldots \ldots \ldots \ldots \ldots \ldots \ldots \ldots \ldots \ldots \ldots \ldots \ldots \ldots .2$

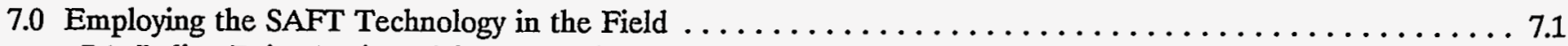

7.1 Indian Point Station Unit II Inservice Inspection $\ldots \ldots \ldots \ldots \ldots \ldots \ldots \ldots \ldots \ldots \ldots \ldots \ldots \ldots \ldots \ldots \ldots \ldots \ldots \ldots$

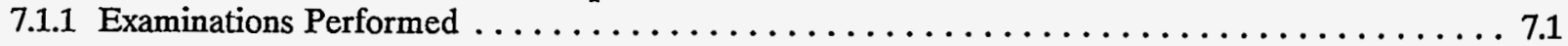

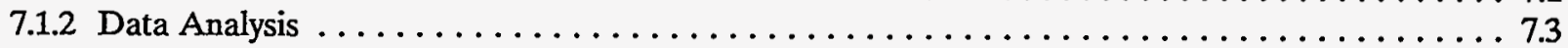

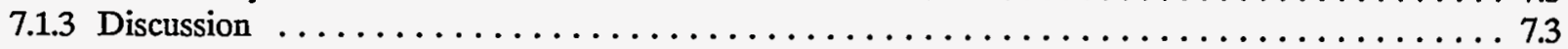

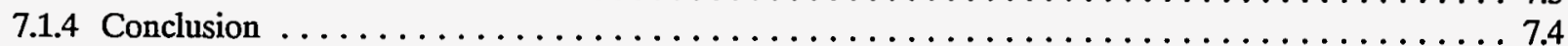

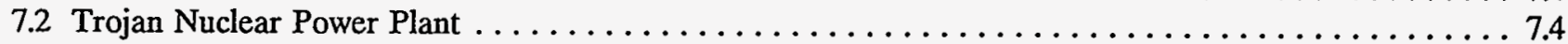

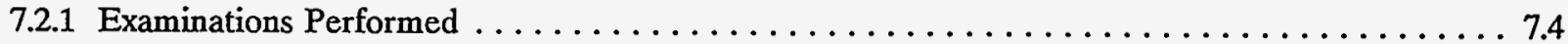

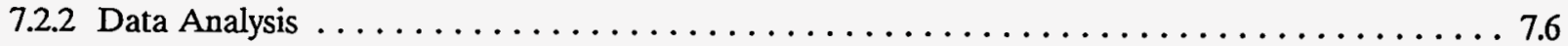

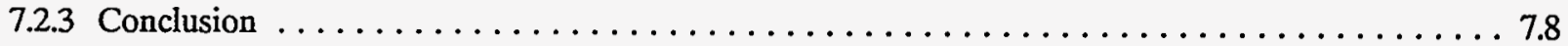

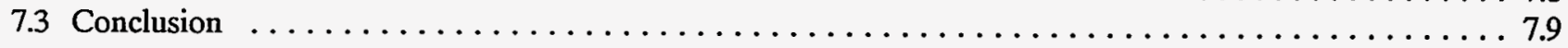

8.0 SAFT Real-Time Processor Performance Analysis and Overview $\ldots \ldots \ldots \ldots \ldots \ldots \ldots \ldots \ldots \ldots \ldots$

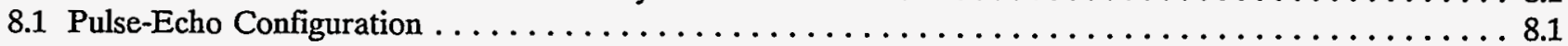

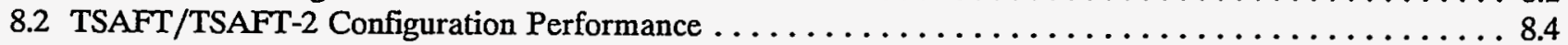

8.3 Overview of the Software Implementation of the SAFT-UT Real-Time Processor $\ldots \ldots \ldots \ldots \ldots \ldots .6$

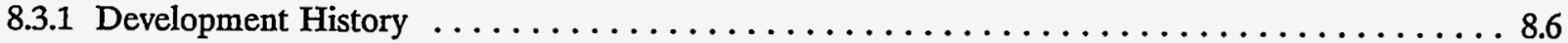

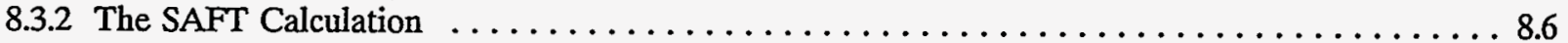

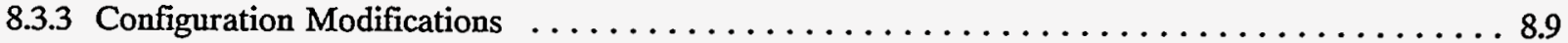

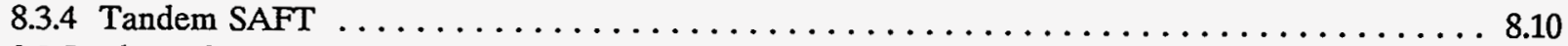

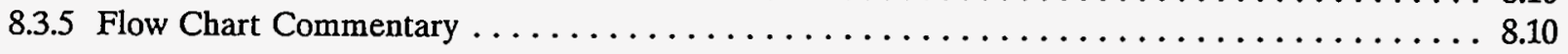




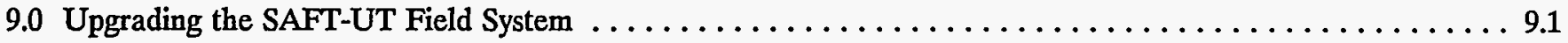

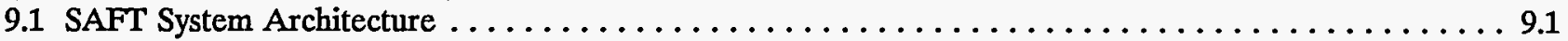

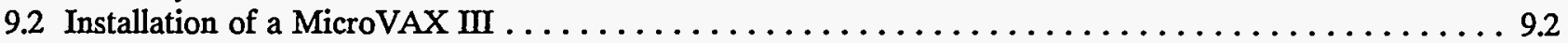

9.3 Other System Upgrades for the Inspection of Nuclear Reactor Pressure Vessels . . . . . . . . . 9.2

9.4 SAFT-UT Upgrades for NRC's NDE Mobile Laboratory $\ldots \ldots \ldots \ldots \ldots \ldots \ldots \ldots \ldots \ldots \ldots \ldots$

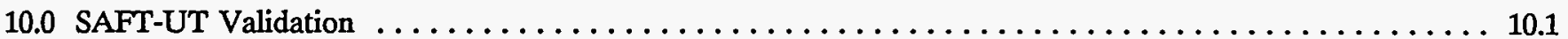

10.1 Validation of SAFT-UT in the Mini-Round Robin $\ldots \ldots \ldots \ldots \ldots \ldots \ldots \ldots \ldots \ldots \ldots \ldots \ldots \ldots$

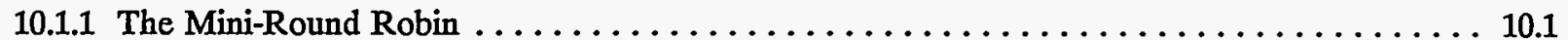

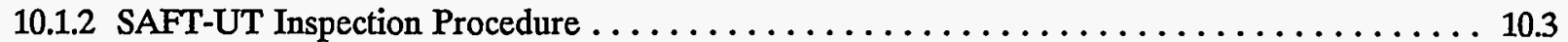

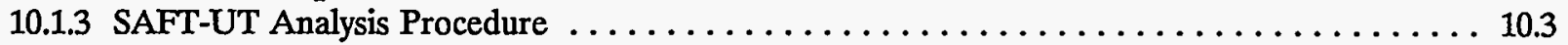

10.1.4 SAFT-UT Performance Results and Discussion $\ldots \ldots \ldots \ldots \ldots \ldots \ldots \ldots \ldots \ldots \ldots$

10.1.5 Conclusions of the SAFT-UT Performance on the MRR $\ldots \ldots \ldots \ldots \ldots \ldots \ldots \ldots \ldots$

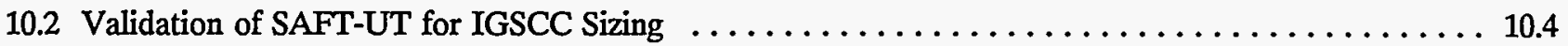

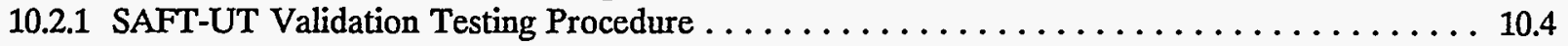

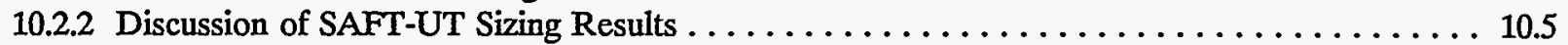

10.2.3 Conclusions of SAFT-UT from the IGSCC Sizing Test $\ldots \ldots \ldots \ldots \ldots \ldots \ldots \ldots \ldots \ldots$

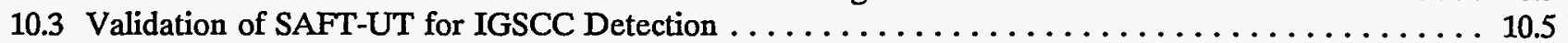

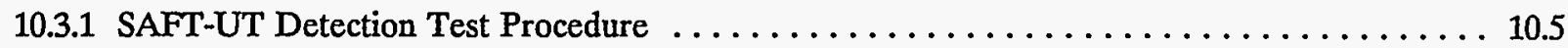

10.3.2 SAFT-UT Detection Test Results and Discussions $\ldots \ldots \ldots \ldots \ldots \ldots \ldots \ldots \ldots \ldots \ldots \ldots \ldots$

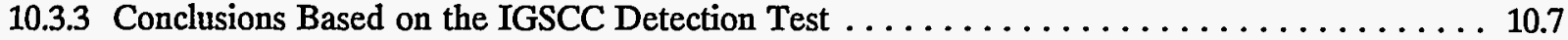

10.4 Validation of the SAFT-UT on the PISC-III Full-Scale Vessel Test $\ldots \ldots \ldots \ldots \ldots \ldots \ldots \ldots \ldots 10.7$

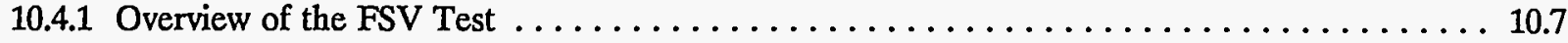

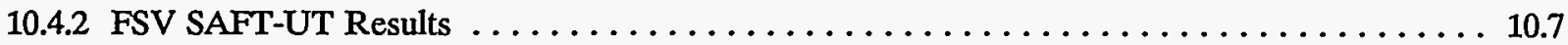

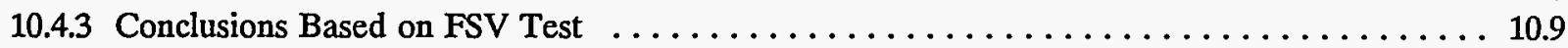

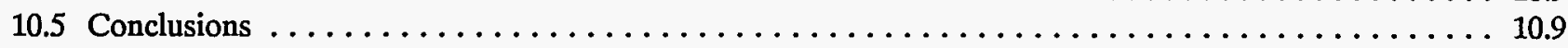

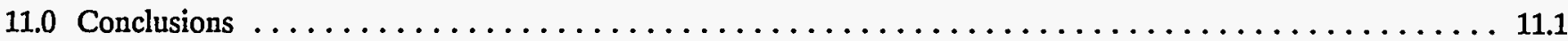

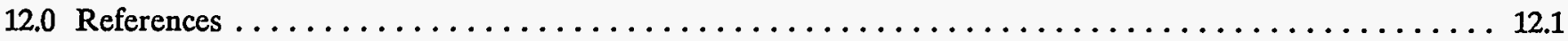

Appendix A: Detailed Description of the SAFT-UT Field Inspection System $\ldots \ldots \ldots \ldots \ldots \ldots \ldots$

Appendix B: Tutorial Procedure for Operating the SAFT-UT Robotic Scanner $\ldots \ldots \ldots \ldots \ldots \ldots \ldots$

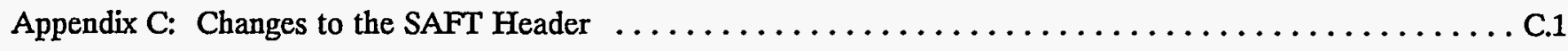

Appendix D: Definition of SAFT-UT ASCII Header $\ldots \ldots \ldots \ldots \ldots \ldots \ldots \ldots \ldots \ldots \ldots \ldots \ldots \ldots \ldots$ 


\section{Figures}

2.1. Schematic of the Results Produced by the Original SAFT-UT Experiments $\ldots \ldots \ldots \ldots \ldots \ldots \ldots$

2.2. Demonstration of Signal-to-Noise Improvement Gained by Subtraction of Front Surface Signal $\ldots \ldots \ldots 2.6$

2.3. Illustration of the Effect of Front Surface Signal Removed upon SAFT-UT Processed Images . . . . . . . 2.7

4.1. SAFT-UT Field System Identifying Switches, Knobs, and Modules $\ldots \ldots \ldots \ldots \ldots \ldots \ldots \ldots$

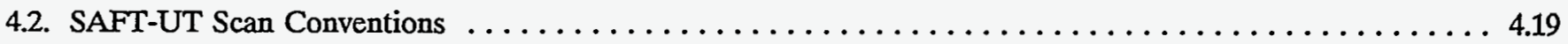

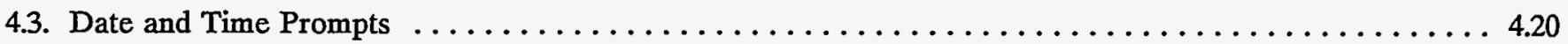

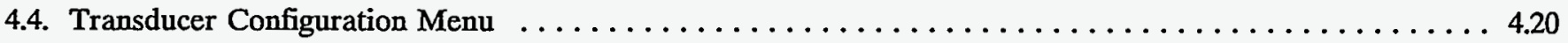

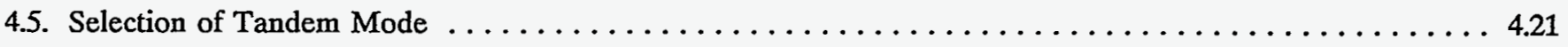

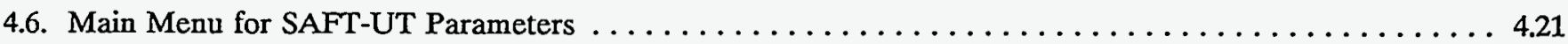

4.7. View of Current Parameters (Pulse-Echo) $\ldots \ldots \ldots \ldots \ldots \ldots \ldots \ldots \ldots \ldots \ldots \ldots \ldots \ldots \ldots \ldots \ldots \ldots \ldots \ldots \ldots \ldots .22$

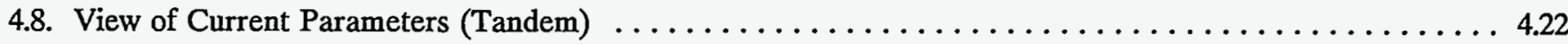

4.9. Transducer Header Information $\ldots \ldots \ldots \ldots \ldots \ldots \ldots \ldots \ldots \ldots \ldots \ldots \ldots \ldots \ldots \ldots \ldots \ldots \ldots \ldots \ldots \ldots \ldots$

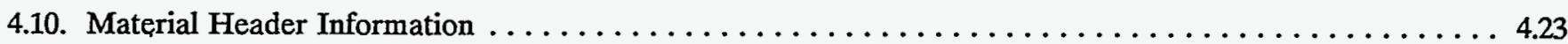

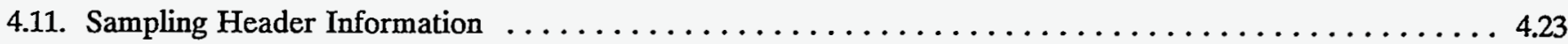

4.12. Scan Header Information (Pulse-Echo) $\ldots \ldots \ldots \ldots \ldots \ldots \ldots \ldots \ldots \ldots \ldots \ldots \ldots \ldots \ldots \ldots \ldots \ldots \ldots \ldots$

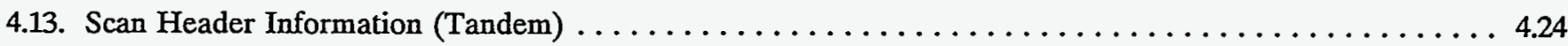

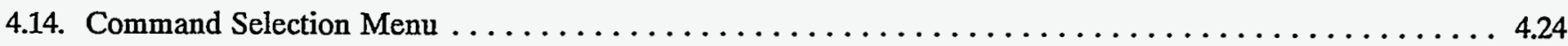

4.15. Prompt to Change the Initial Delay $\ldots \ldots \ldots \ldots \ldots \ldots \ldots \ldots \ldots \ldots \ldots \ldots \ldots \ldots \ldots \ldots \ldots \ldots \ldots \ldots \ldots \ldots \ldots .25$

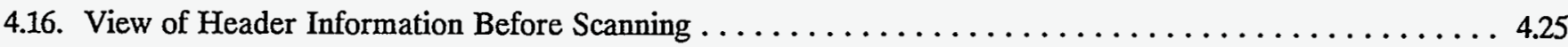

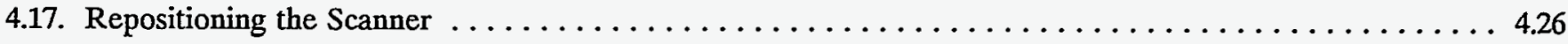

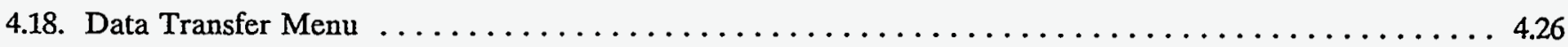

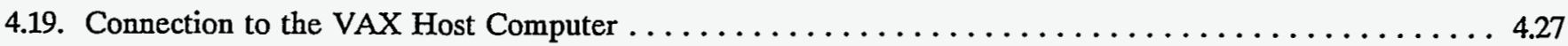

4.20. Starting Data Collection on the VAX Host Computer $\ldots \ldots \ldots \ldots \ldots \ldots \ldots \ldots \ldots \ldots \ldots \ldots \ldots$

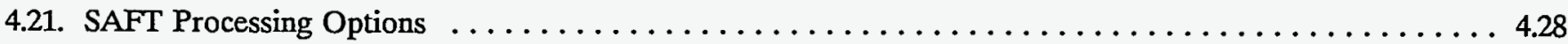

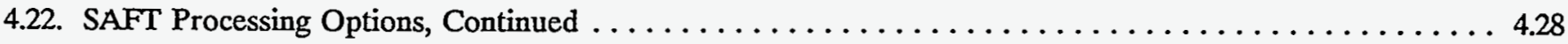




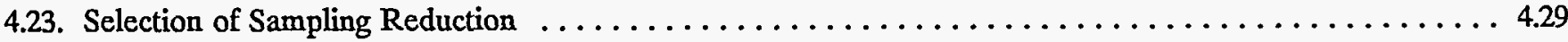

4.24. Entering Comments and Setting the Scanner Speed $\ldots \ldots \ldots \ldots \ldots \ldots \ldots \ldots \ldots \ldots \ldots \ldots \ldots \ldots \ldots \ldots \ldots \ldots \ldots .29$

4.25. Normal-Beam Pulse-Echo Inspection Using a Water Column $\ldots \ldots \ldots \ldots \ldots \ldots \ldots \ldots \ldots \ldots \ldots .30$

4.26. Angle-Beam Pulse-Echo Inspection Using a Lucite Wedge and Small-Diameter Transducer $\ldots \ldots \ldots 4.31$

4.27. Comparison Between Pulse-Echo (top) and Tandem Images of a 0.3-in.-deep (0.8-cm) Semicircular Sawcut in a 0.6 -in.-thick $(1.5-\mathrm{cm})$ Aluminum Coupon $\ldots \ldots \ldots \ldots \ldots \ldots \ldots \ldots \ldots \ldots \ldots \ldots \ldots \ldots \ldots \ldots \ldots \ldots . .32$

4.28. Angle-Beam Tandem Inspection Using a Lucite Wedge and Small-Diameter Transducers $\ldots \ldots \ldots . . .43$

4.29. Four Propagation Paths for Ultrasonic Energy Produced by the Tandem Transmission Technique . . .. 4.34

4.30. TSAFT-2 Configuration Using $1 / 2$-Vee Transmit Path $\ldots \ldots \ldots \ldots \ldots \ldots \ldots \ldots \ldots \ldots \ldots \ldots \ldots$

4.31. TSAFT-2 Image of 0.7-in.-deep (1.8-cm) Sawcut in 9-in.-thick (22.9-cm) Specimen, 54 Transmitter and

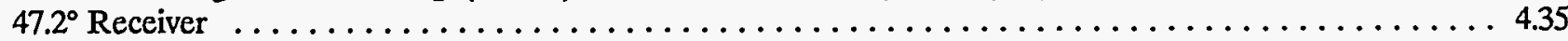

4.32. TSAFT-3 Initial (a) and Ending (b) Transducer Positions for a Single Sweep $\ldots \ldots \ldots \ldots \ldots \ldots . . \ldots$

4.33. TSAFT-3 Configuration Showing Paths Contributing to the Real (Path 2) and Conjugate (Path 1) Image . 4.36

4.34. Side Views of TSAFT-3 Setups Showing 9-in.-thick (22.9-cm) Steel Block with 0.7-in.-deep (1.8-in.) Sawcut and Fictitious Material Thickness with "Folded" Transmit/Receive Paths $\ldots \ldots \ldots \ldots \ldots \ldots \ldots \ldots .37$

4.35. TSAFT-3 Image of 0.7-in.-deep (1.8-cm) Sawcut in 9-in.-thick (22.9-cm) Specimen, $47.2^{\circ}$ Transmitter and

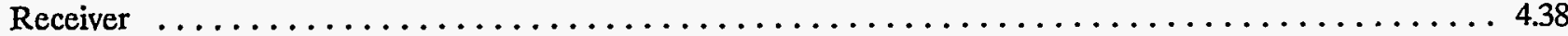

4.36. Additional Tandem Processing Parameters, Initial Screen Display $\ldots \ldots \ldots \ldots \ldots \ldots \ldots \ldots \ldots \ldots$

4.37. Additional Tandem Processing Parameters, Subsequent Screen Display . . . . . . . . . . . . . 4.39

4.38. Pulse-Echo Image of a 0.2-in.-diameter (0.5-cm), Side-Drilled Hole, $0.5 \mathrm{in} .(1.3 \mathrm{~cm})$ up from the Far

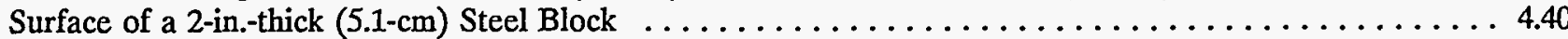

5.1. Pulse-Echo Image of 0.050 -in.-deep $(0.13-\mathrm{cm})$ Sawcut in a 9-in.-thick $(22.86-\mathrm{cm})$ Steel Block $\ldots \ldots \ldots .10$

5.2. Pulse-Echo Image of 0.700 -in.-deep $(1.8-\mathrm{cm})$ Sawcut in a 9-in.-thick $(22.86-\mathrm{cm})$ Steel Block $\ldots \ldots \ldots . . .10$

5.3. TSAFT-2 Image of 0.050 -in.-deep $(0.13-\mathrm{cm})$ Sawcut in a 9 -in.-thick $(22.86-\mathrm{cm})$ Steel Block $\ldots \ldots \ldots \ldots .11$

5.4. TSAFT-2 Image of 0.700 -in.-deep (1.8-cm) Sawcut in 9-in.-thick $(22.86-\mathrm{cm})$ Steel Block $\ldots \ldots \ldots \ldots . . .11$

5.5. Images of Specimen 5 of the CCSSRRT Data Set $\ldots \ldots \ldots \ldots \ldots \ldots \ldots \ldots \ldots \ldots \ldots \ldots \ldots \ldots \ldots \ldots \ldots \ldots \ldots .12$

5.6. Image of Specimen 5, Selected A-Scans, and Spectra Obtained After Application of Step-Wise Filter to

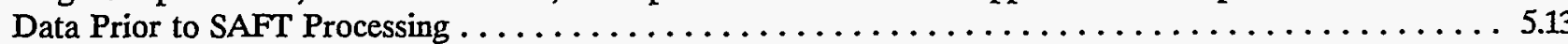


Figures

5.7. Image of Specimen 5, Selected A-Scans, and Spectra Obtained After Application of Time Derivative Filter

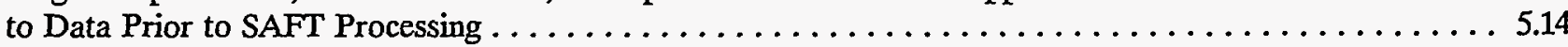

5.8. Image of Specimen 5, Selected A-Scans, and Spectra After Application of Time Derivative and Stepwise

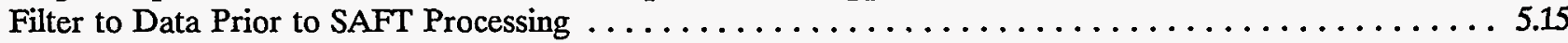

5.9. Maximum Correlation Values at Different Frequencies for Equiaxed Material $\ldots \ldots \ldots \ldots \ldots \ldots$

5.10. Maximum Correlation Values at Different Frequencies for Columnar Material $\ldots \ldots \ldots \ldots \ldots \ldots$

5.11. Composite Power Spectra for Sawcut Test File $\ldots \ldots \ldots \ldots \ldots \ldots \ldots \ldots \ldots \ldots \ldots \ldots \ldots \ldots \ldots \ldots \ldots \ldots$

5.12(a). Composite Power Spectra Plot for Equiaxed Shear-Wave Data Files $\ldots \ldots \ldots \ldots \ldots \ldots \ldots \ldots$

5.12(b). Specimen 3 Images Showing Before (top) and After (bottom) Processed Images (Filter parameters for After image were selected from the power spectra plot.) $\ldots \ldots \ldots \ldots \ldots \ldots \ldots \ldots \ldots \ldots \ldots .18$

5.13(a). Composite Power Spectra Plot for Columnar Shear-Wave Data Files $\ldots \ldots \ldots \ldots \ldots . \ldots \ldots$

5.13(b). Specimen 4 Images Showing Before (top) and After (bottom) Processed Images (Filter parameters for After image were selected from the power spectra plot.) $\ldots \ldots \ldots \ldots \ldots \ldots \ldots \ldots \ldots \ldots \ldots \ldots .19$

$5.13(\mathrm{c})$. Single Slice End View 0.5 in. $(1.3 \mathrm{~cm})$ from Weld Center $\ldots \ldots \ldots \ldots \ldots \ldots \ldots \ldots \ldots \ldots \ldots \ldots \ldots$

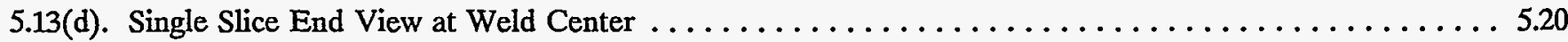

5.14. Images of the Equiaxed Side of Specimen 6 Showing the Before (top) and After (bottom) Processed Images (Filter Parameters for After Image were Selected From the Equiaxed Power Spectra Plot) . . . . 5.21

5.15. Images of the Columnar Side of Specimen 6 Showing the Before (top) and After (bottom) Processed Images (Filter Parameters for After Image were Selected From the Columnar Power Spectra Plot) . . . . 5.21

5.16. Plot Showing the Composite Power Spectra for Equiaxed Shear-Wave Specimens that had a High Probability of Detection $\ldots \ldots \ldots \ldots \ldots \ldots \ldots \ldots \ldots \ldots \ldots \ldots \ldots \ldots \ldots \ldots \ldots \ldots \ldots \ldots \ldots \ldots \ldots .22$

5.17. Composite Power Spectra for Columnar Shear-Wave Specimens Having High Probability of Detection ... 5.22

6.1. TSAFT-2 Images of a 0.3-in.-deep (0.8-cm) Sawcut in 0.750-in.-thick (1.9-cm) Aluminum Material with the

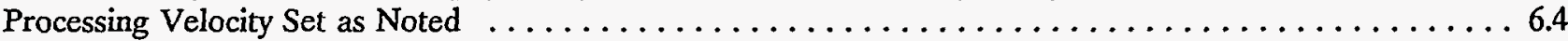

6.2. TSAFT-2 Images of a 0.3-in.-deep (0.8-cm) Sawcut in 0.750-in.-thick (1.9-cm) Aluminum Material with the

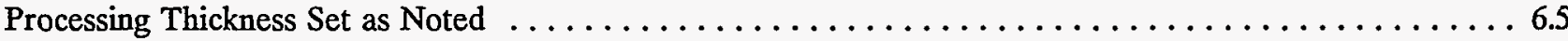

6.3. Image of Unprocessed Data (C-Scan View) from 0.5 -in.-diameter $(1.3-\mathrm{cm})$ Steel Sphere $\ldots \ldots \ldots . \ldots 6$

6.4. A-Scan Near Center of Unprocessed Data from 0.5 -in.-diameter $(1.3-\mathrm{cm})$ Steel Sphere $\ldots \ldots \ldots \ldots .6 .6$

6.5. A-Scan Showing Only Echo Trace from 0.5 -in.-diameter $(1.3-\mathrm{cm})$ Steel Sphere $\ldots \ldots \ldots \ldots \ldots \ldots . \ldots$

6.6. Fourier Transform Plot of Echo Trace from 0.5 -in.-diameter $(1.3-\mathrm{cm})$ Steel Sphere $\ldots \ldots \ldots \ldots . \ldots .6$. 
6.7. C-Scan View of SAFT Processed Echo Trace from 0.5 -in.-diameter $(1.3-\mathrm{cm})$ Steel Sphere $\ldots \ldots \ldots .6$

6.8. C-Scan View of Time Derivative Raw Data Echo Trace from 0.5 -in.-diameter $(1.3-\mathrm{cm})$ Steel Sphere $\ldots \ldots 6.8$

6.9. A-Scan Near Center of Unprocessed Time Derivative Data from 0.5 -in.-diameter $(1.3-\mathrm{cm})$ Steel Sphere $\ldots .6 .9$

6.10. Fourier Transform Plot of Time Derivative Data from 0.5 -in.-diameter $(1.3-\mathrm{cm})$ Steel Sphere $\ldots \ldots \ldots .9$

6.11. C-Scan View of SAFT Processed Time Derivative Data from 0.5-in.-diameter (1.3-cm) Steel Sphere . . . 6.10

6.12. C-Scan View of SAFT Processed Echo Trace from 0.5-in.-diameter (1.3-cm) Steel Sphere After Applying

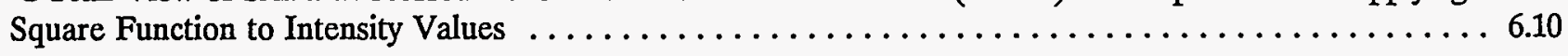

6.13. Pulse-Echo Image of Raw Data from 0.3-in.-deep (0.8-cm) Sawcut in 0.585-in.-thick (1.5-cm) Stainless

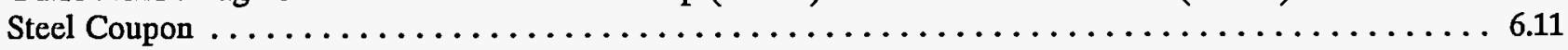

6.14. A-Scan Near Center of Raw Data from 0.3-in.-deep (0.8-cm) Sawcut in 0.585-in.-thick (1.5-cm) Stainless

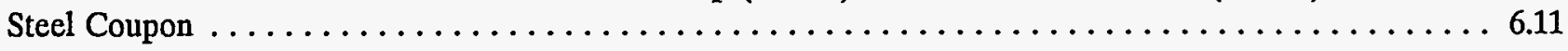

6.15. Time Derivative of A-Scan Near Center of Raw Data from 0.3-in.-deep (0.8-cm) Sawcut in 0.585-in.-thick $(1.5-\mathrm{cm})$ Stainless Steel Coupon $\ldots \ldots \ldots \ldots \ldots \ldots \ldots \ldots \ldots \ldots \ldots \ldots \ldots \ldots \ldots \ldots \ldots \ldots \ldots \ldots \ldots \ldots \ldots \ldots \ldots \ldots \ldots .12$

6.16. Pulse-Echo Image of Time-Derivative Raw Data from 0.3-in.-deep (0.8-cm) Sawcut in 0.585-in.-thick (1.5-

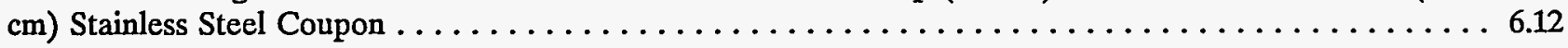

6.17. Pulse-Echo Image of SAFT-Processed Time Derivative Data from 0.3-in.-deep (0.8-cm) Sawcut in 0.585in.-thick $(1.5-\mathrm{cm})$ Stainless Steel Coupon $\ldots \ldots \ldots \ldots \ldots \ldots \ldots \ldots \ldots \ldots \ldots \ldots \ldots \ldots \ldots \ldots \ldots \ldots \ldots \ldots \ldots \ldots \ldots .13$

6.18. Pulse-Echo Image of SAFT-Processed Data from 0.3-in.-deep $(0.8-\mathrm{cm})$ Sawcut in 0.585 -in.-thick Stainless Steel Coupon After Applying Square Function to Intensity Values $\ldots \ldots \ldots \ldots \ldots \ldots \ldots \ldots \ldots \ldots$

7.1. $45^{\circ}$ Shear-Wave Image of Corner-Trap Echo of 0.3-in.-deep (0.8-cm) Notch in 9-in.-thick (22.9-cm) Cladded Vessel Calibration Block (Waltz Mill) $\ldots \ldots \ldots \ldots \ldots \ldots \ldots \ldots \ldots \ldots \ldots \ldots \ldots \ldots$

7.2. $45^{\circ}$ Shear-Wave Image of Corner-Trap Echo of 0.3-in.-deep (0.8-cm) Notch in 9-in.-thick (22.9-cm)

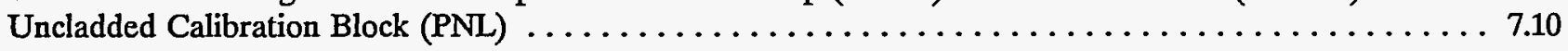

7.3. $45^{\circ}$ Shear-Wave Image (3-D Processed) of the Vessel Indication at Indian Point Unit II $\ldots \ldots \ldots . . . . .11$

7.4. $45^{\circ}$ Longitudinal-Wave Image (3-D Processed) of the Vessel Indication at Indian Point Unit II . . . . . 7.12

7.5. $45^{\circ}$ Shear-Wave Image (3-D Processed) of 0.2-in.-diameter $(0.5-\mathrm{cm})$ Side-Drilled Hole 0.5 in. $(1.3 \mathrm{~cm})$ from

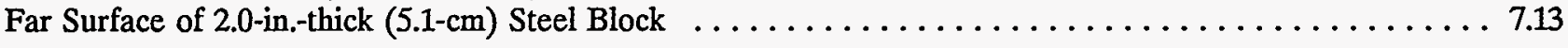

7.6. Cumposite Spectra Plot of Suspected Defect and Non-defect Areas of Data File TR9SH5D . . . . . . . 7.14

7.7(a). B-Scan Side View and C-Scan Top View of Data File TR9SH5A $\ldots \ldots \ldots \ldots \ldots \ldots \ldots \ldots$

7.7(b). Expanded View of Indication \#2, TR9SH5A $\ldots \ldots \ldots \ldots \ldots \ldots \ldots \ldots \ldots \ldots \ldots \ldots \ldots \ldots \ldots$ 
Figures

7.7(c). Expanded View of Indication \#3, TR9SH5A $\ldots \ldots \ldots \ldots \ldots \ldots \ldots \ldots \ldots \ldots \ldots \ldots \ldots \ldots$

7.7(d). Expanded View of Indication \#5, TR9SH5A $\ldots \ldots \ldots \ldots \ldots \ldots \ldots \ldots \ldots \ldots \ldots \ldots \ldots \ldots \ldots$

7.8(a). B-Scan Side View and C-Scan Top View of Data File TR9SH5C $\ldots \ldots \ldots \ldots \ldots \ldots \ldots \ldots \ldots 7.17$

7.8(b). Expanded View of Indication \#2, TR9SH5C $\ldots \ldots \ldots \ldots \ldots \ldots \ldots \ldots \ldots \ldots \ldots \ldots \ldots \ldots \ldots \ldots \ldots$

7.8(c). Expanded View of Indication \#3, TR9SH5C $\ldots \ldots \ldots \ldots \ldots \ldots \ldots \ldots \ldots \ldots \ldots \ldots \ldots \ldots \ldots \ldots \ldots \ldots \ldots \ldots .18$

7.8(d). Expanded View of Indication \#5, TR9SH5C $\ldots \ldots \ldots \ldots \ldots \ldots \ldots \ldots \ldots \ldots \ldots \ldots \ldots \ldots \ldots \ldots \ldots \ldots$

7.8(e). Expanded View of Indication A, TR9SH5C $\ldots \ldots \ldots \ldots \ldots \ldots \ldots \ldots \ldots \ldots \ldots \ldots \ldots \ldots$

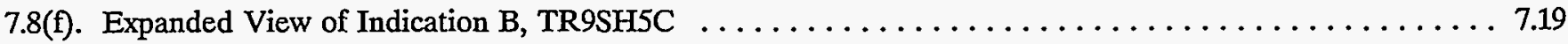

7.9(a). B-Scan Side View and C-Scan Top View of Data File TR9SHSD $\ldots \ldots \ldots \ldots \ldots \ldots \ldots \ldots \ldots$

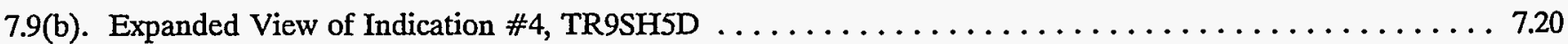

7.10. B-Scan Side View and C-Scan Top View of Data File TR9SH5G $\ldots \ldots \ldots \ldots \ldots \ldots \ldots \ldots \ldots \ldots .21$

8.1. SAFT Computational Device or Real-Time Processor $\ldots \ldots \ldots \ldots \ldots \ldots \ldots \ldots \ldots \ldots \ldots \ldots \ldots .12$

8.2. Real-Time Processor Performance on Pipe Data File with Selective Processing Disabled and Output

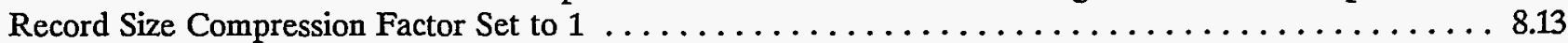

8.3. Real-Time Processor Performance on Pipe Data File with Selective Processing Disabled and Output

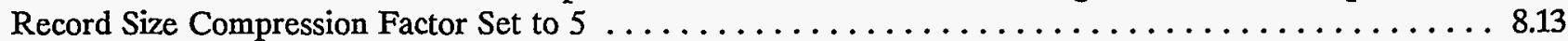

8.4. Real-Time Processor Performance on Pipe Data File with Selective Processing Set to $-20 \mathrm{~dB}$ and Output

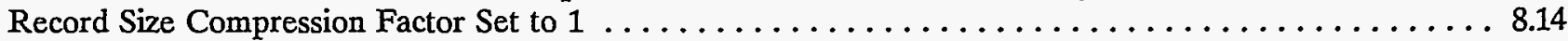

8.5. Real-Time Processor Performance on Pipe Data File with Selective Processing Set to $-20 \mathrm{~dB}$ and Output

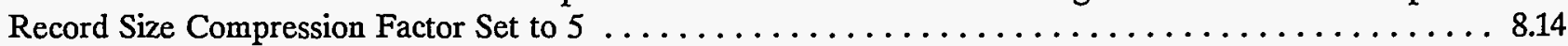

8.6. Real-Time Processor Performance on PISC II Data File with Selective Processing Disabled and Output Record Size Compression Factor Set to 1

8.7. Real-Time Processor Performance on PISC II Data File with Selective Processing Disabled and Output

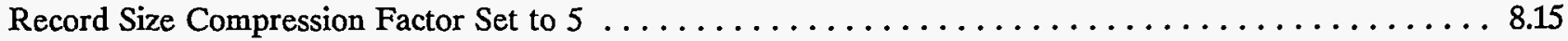

8.8. Real-Time Processor Performance on PISC II Data File with Selective Processing Set to $-20 \mathrm{~dB}$ and Output Record Size Compression Factor Set to $1 \ldots \ldots \ldots \ldots \ldots \ldots \ldots \ldots \ldots \ldots \ldots \ldots \ldots \ldots \ldots \ldots \ldots \ldots \ldots \ldots .16$

8.9. Real-Time Processor Performance on PISC II Data File with Selective Processing Set to $-20 \mathrm{~dB}$ and Output Record Size Compression Factor Set to 5

8.10. Real-Time Processor Performance, Tandem Configuration, on Thin-Pipe Data File (TSAFT; 0.585 in. thick [1.49 cm]) with Selective Processing Disabled and Output Record Size Compression Factor Set to 1 . 8.17 
8.11. Real-Time Processor Performance, Tandem Configuration, on Thick-Pipe Data File (TSAFT; 1.5 in. thick [3.8 cm]) with Selective Processing Disabled and Output Record Size Compression Factor Set to $1 \ldots \ldots 8.17$

8.12. Real-Time Processor Performance, Tandem Configuration, on Thick-Pipe Data File (TSAFT-2; 1.5 in. thick [3.8 cm]) with Selective Processing Disabled and Output Record Size Compression Factor Set to 1 . . 8.18

8.13. Real-Time Processor Performance, Tandem Configuration, on Thin-Pipe Data File (TSAFT; 0.585 in. thick [1.49 cm]) with Selective Processing Disabled and Output Record Size Compression Factor Set to 5 . 8.18

8.14. Real-Time Processor Performance, Tandem Configuration, on Thick-Pipe Data File (TSAFT; 1.5 in. thick [3.8 cm]) with Selective Processing Disabled and Output Record Size Compression Factor Set to 5 .. 8.19

8.15. Real-Time Processor Performance, Tandem Configuration, on Thick-Pipe Data File (TSAFT-2; 1.5 in. thick [3.8 cm]) with Selective Processing Disabled and Output Record Size Compression Factor Set to 5 . 8.19

8.16. Real-Time Processor Performance, Tandem Configuration, on Thin-Pipe Data File (TSAFT; 0.585 in. thick $[1.49 \mathrm{~cm}])$ with Selective Processing Set to $-20 \mathrm{~dB}$ and Output Record Size Compression Factor Set

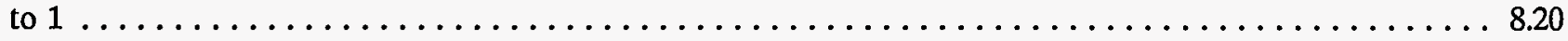

8.17. Real-Time Processor Performance, Tandem Configuration, on Thick-Pipe Data File (TSAFT; 1.5 in. thick [3.8 cm]) with Selective Processing Set to $-20 \mathrm{~dB}$ and Output Record Size Compression Factor Set to

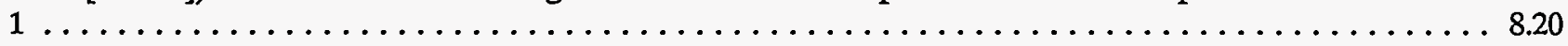

8.18. Real-Time Processor Performance, Tandem Configuration, on Thick-Pipe Data File.(TSAFT-2; 1.5 in. thick $[3.8 \mathrm{~cm}]$ ) with Selective Processing Set to $-20 \mathrm{~dB}$ and Output Record Size Compression Factor Set to

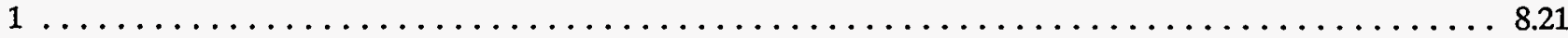

8.19. Real-Time Processor Performance, Tandem Configuration, on Thin-Pipe Data File (TSAFT; 0.585 in. thick [1.49 cm]) with Selective Processing Set to $-20 \mathrm{~dB}$ and Output Record Size Compression Factor Set to 5

8.20. Real-Time Processor Performance, Tandem Configuration, on Thick-Pipe Data File (TSAFT; 1.5 in. thick [3.8 cm]) with Selective Processing Set to $-20 \mathrm{~dB}$ and Output Record Size Compression Factor Set to

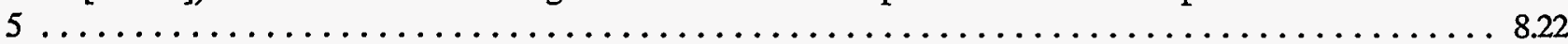

8.21. Real-Time Processor Performance, Tandem Configuration, on Thick-Pipe Data File (TSAFT-2; 1.5 in. thick [3.8 cm]) with Selective Processing Set to $-20 \mathrm{~dB}$ and Output Record Size Compression Factor Set to

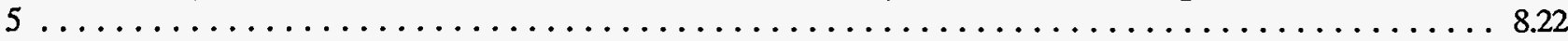

8.22. Example of How Transducer Locations Translate to Memory Locations $\ldots \ldots \ldots \ldots \ldots . \ldots \ldots$

8.23. Logical Flow of Events During SAFT Data Processing Using Real-Time Processor $\ldots \ldots \ldots \ldots .24$

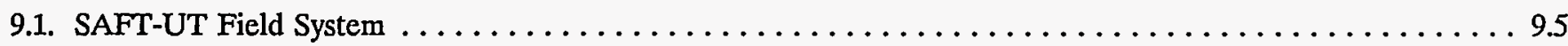

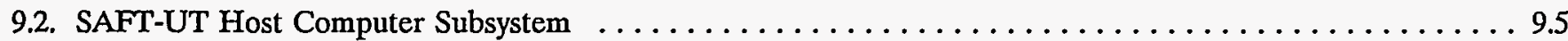

9.3. SAFT-UT PDP11 Data Acquisition Subsystem $\ldots \ldots \ldots \ldots \ldots \ldots \ldots \ldots \ldots \ldots \ldots \ldots \ldots \ldots \ldots \ldots \ldots \ldots \ldots \ldots$

9.4. SAFT-UT Field System Used at Pressure Vessel Research User Facility (PVRUF) $\ldots \ldots \ldots \ldots \ldots$ 
Figures

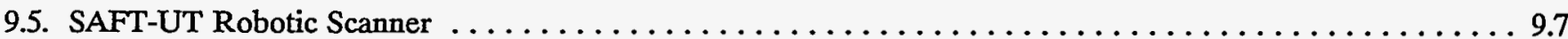

9.6. NDE Mobile Laboratory SAFT-UT Field System $\ldots \ldots \ldots \ldots \ldots \ldots \ldots \ldots \ldots \ldots \ldots \ldots \ldots \ldots \ldots$

9.7. Electronic Component Diagram for the NRC Mobile Laboratory SAFT-UT System $\ldots \ldots \ldots \ldots . . \ldots$

9.8. NDE Mobile Laboratory SAFT-UT Field System Scanner $\ldots \ldots \ldots \ldots \ldots \ldots \ldots \ldots \ldots \ldots \ldots \ldots$

9.9. First Deployment of NDE Mobile Laboratory SAFT-UT Field System Electronics at a Reactor Site--WNP-

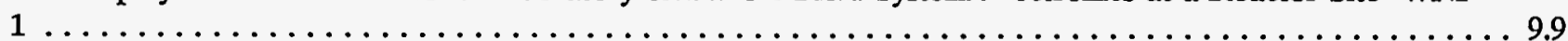

9.10. NDE Mobile Laboratory SAFT-UT PC-486 $\ldots \ldots \ldots \ldots \ldots \ldots \ldots \ldots \ldots \ldots \ldots \ldots \ldots \ldots \ldots \ldots$

9.11. NDE Mobile Laboratory SAFT-UT Host Computer $\ldots \ldots \ldots \ldots \ldots \ldots \ldots \ldots \ldots \ldots \ldots \ldots \ldots \ldots$

9.12. Typical Data Analysis Display from NRC Mobile Laboratory SAFT-UT System . . . . . . . . . 9.11

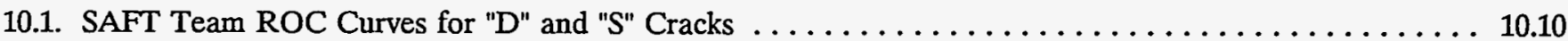

10.2. SAFT Team ROC Curves for "D" and "L" Cracks $\ldots \ldots \ldots \ldots \ldots \ldots \ldots \ldots \ldots \ldots \ldots \ldots \ldots \ldots 10.10$

10.3. Manual Teams' (Average) ROC Curves for "D" and "S" Cracks $\ldots \ldots \ldots \ldots \ldots \ldots \ldots \ldots \ldots \ldots \ldots .11$

10.4. Manual Teams' (Average) ROC Curves for " $D$ " and "L" Cracks $\ldots \ldots \ldots \ldots \ldots \ldots \ldots \ldots \ldots \ldots \ldots .11$

10.5. Advanced Teams' (Average) ROC Curves for " $\mathrm{D}$ " and "S" Cracks $\ldots \ldots \ldots \ldots \ldots \ldots \ldots \ldots \ldots \ldots .12$

10.6. Advanced Teams' (Average) ROC Curves for " $\mathrm{D}$ " and "L" Cracks $\ldots \ldots \ldots \ldots \ldots \ldots \ldots \ldots \ldots \ldots$

10.7. Results of SAFT-UT Technique on IGSCC Performance Demonstration Sizing Test Conducted at EPRI

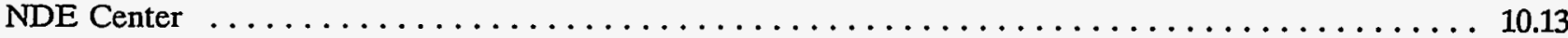




\section{Tables}

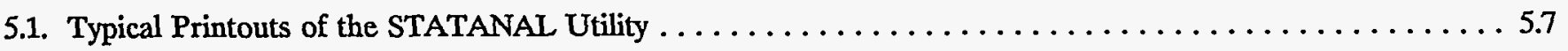

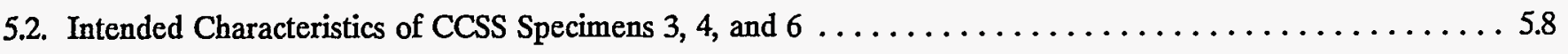

7.1. SAFT Sizing of the Static-Cast-Elbow Indications $\ldots \ldots \ldots \ldots \ldots \ldots \ldots \ldots \ldots \ldots \ldots \ldots \ldots$

8.1. VAX 11/780 TSAFT/TSAFT-2 Executive Time (-20-dB selective processing implemented) $\ldots \ldots \ldots .4$

8.2. SAFT-UT Real-Time Processor TSAFT/TSAFT-2 Executive Time (-20-dB selective processing implement-

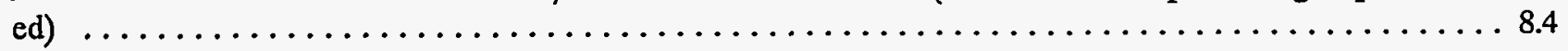

8.3. SAFT-UT Real-Time Processor TSAFT/TSAFT-2 Executive Time (selective processing disabled) $\ldots \ldots 8.5$

8.4. Tandem Mode Analysis (Total Number of Points Processed) $\ldots \ldots \ldots \ldots \ldots \ldots \ldots \ldots \ldots \ldots . \ldots \ldots$

9.1. Performance for Upgrades to SAFT-UT System $\ldots \ldots \ldots \ldots \ldots \ldots \ldots \ldots \ldots \ldots \ldots \ldots \ldots \ldots \ldots \ldots \ldots \ldots$

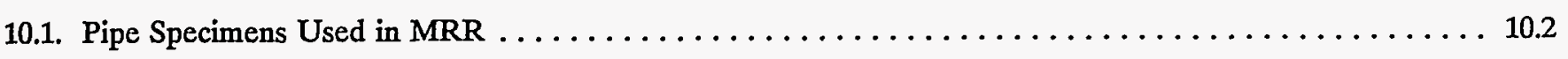

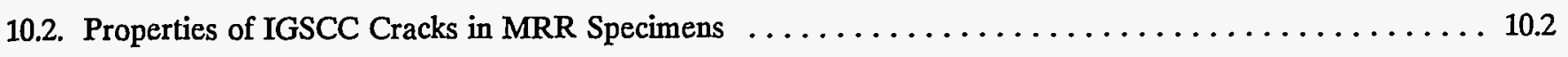

10.3. Crack/No-Crack POD and FCP, SAFT-UT $\ldots \ldots \ldots \ldots \ldots \ldots \ldots \ldots \ldots \ldots \ldots \ldots \ldots \ldots \ldots \ldots \ldots \ldots$

10.4. Crack/No-Crack POD and FCP, Average of Advanced Teams $\ldots \ldots \ldots \ldots \ldots \ldots \ldots \ldots \ldots \ldots$

10.5. Crack/No-Crack POD and FCP, Average of Manual Teams $\ldots \ldots \ldots \ldots \ldots \ldots \ldots \ldots \ldots \ldots$

10.6. PISC-III Action No. 2 Full-Scale Vessel Test - Phase $1 \ldots \ldots \ldots \ldots \ldots \ldots \ldots \ldots \ldots \ldots \ldots \ldots$

10.7. Statistical Values of SAFT-UT Performance on ALL FSV Flaws $\ldots \ldots \ldots \ldots \ldots \ldots \ldots \ldots \ldots$

10.8. Statistical Values of SAFT-UT Performance on All FSV Flaws Except No. $6 \ldots \ldots \ldots \ldots \ldots \ldots$ 


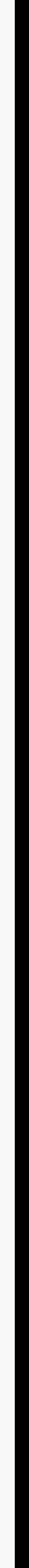




\section{Executive Summary}

A multi-year program for the Development and Validation of a Real-Time SAFT-UT System for Inservice Inspection of Light Water Reactors was established at the Pacific Northwest Laboratory (PNL) to develop the Synthetic Aperture Focusing Technique for Ultrasonic Testing (SAFT-UT) and demonstrate the technology in reactor inservice inspections.

The objectives of this program for the Nuclear Regulatory Commission (NRC) Office of Nuclear Regulatory Research include:

- Design, fabricate, and evaluate a real-time flaw detection and characterization system based on SAFT-UT for inservice inspection (ISI) of all required light-water reactor (LWR) components.

- Establish calibration and field operating procedures.

- Demonstrate and validate the SAFT-UT system through actual reactor ISIs.

- Generate an engineering data base to support the American Society of Mechanical Engineers

(ASME) Code acceptance of the real-time SAFTUT technology.

The scope of the program is defined by the following:

- Conduct laboratory tests to provide engineering data for defining SAFT-UT system performance.

- Complete the development of a special processor to make SAFT a real-time process for ISI applications.

- Fabricate and field test a real-time SAFT-UT system suitable for field inspection of nuclear reactor piping, nozzles, and pressure vessels.

The technology has been developed and put into a form for easy transfer to others. A substantial laboratory data base exists for showing the effectiveness of the SAFT technology for reliably detecting and characterizing defects. Some field validation work has occurred, but the extensive work for field validation can only be said to have begun. Several field trips occurred to Dresden Unit \#2 and to Vermont Yankee where data was gathered on-site but the data processing portion of the SAFT-UT system, which became the real-time SAFT processor, had not been developed at that time. Subsequently, data processing and analysis occurred in the PNL laboratory.

The objectives of the program, for the NRC, have been achieved with the end product being a transportable field system that can achieve real-time SAFT imaging. In conjunction with Westinghouse and Dynacon, it was shown that other commercial data acquisition equipment could be used to acquire suitable data, which was subsequently SAFT processed and analyzed. These data were acquired from the reactor pressure vessel at the Indian Point Unit II Nuclear Power Plant operated by Consolidated Edison of New York. Further demonstrations of this capability came when Combustion Engineering and Amdata, Inc. performed an ISI with an I-98 ultrasonic testing (UT) system of a static-cast elbow in the hot-leg of the Trojan Nuclear Power Plant operated by the Portland General Electric Company. Data was subsequently SAFT processed, analyzed, and used to further characterize the indications found.

With the evolution of the real-time SAFT processor, pre-processing techniques such as selective filtering were used on laboratory and field data to evaluate if improvement in the images would result. Slow processing times had previously prohibited utilizing different pre-processing techniques because feedback of the results was too slow in coming. Pre-processing techniques proved most useful on coarse grained inhomogeneous materials such as Centrifugally Cast Stainless Steel (CCSS). All the defects in round-robin studies on CCSS have generally been detected by SAFT, but the problem has been one of a high false call rate because of coherent scattering from the large grains. False calls were reduced significantly by the pre-processing filtering, leading to the application of the technique to staticcast data files from the Trojan Nuclear Power Plant. Results from the Trojan data files were not as dramatic as were observed in the large grained CCSS data set; however, the signal-to-noise ratios of the images did improve substantially, thereby making interpretation of the images more straightforward.

Subsequently, fast processing times have also opened the door for thick section materials such as pressure vessels. Previously, thick material data files were too large to process in a reasonable amount of time and would typically take days or weeks to process. The 
speed of the real-time processor performance is impressive. A typical thick-section pulse-echo data file that previously took 36 days with 260 hours of host CPU time was completed in 64.2 minutes and required only 6.8 minutes of host CPU time! Thus, a real-time SAFT-UT field system is a reality, meeting the primary objective of the NRC program.

The ability to SAFT process data files in real time has reduced the time necessary for conducting parametric studies that help predict the effects of errors in processing parameters and algorithm assumptions. Thickness and material velocity errors in TSAFT-2 data files were studied. The placement of the resulting image is dramatically affected by deviations of either thickness or velocity from the true values of the specimen under test. However, the general image integrity is retained. Another study focused on the narrow band approximation of the SAFT algorithm. By performing a time derivative prior to SAFT processing, it was found that the signal-to-noise ratio improved for wide-band data files.

Computer technology has greatly advanced since the SAFT-UT field system was first designed and developed by PNL. The original SAFT-UT field system consists of three equipment racks plus a RAMTEK image processor. The most recent system developed at PNL, for the NRC NDE Mobile Laboratory, fits on a single table top based on a 486 PC and a SUN workstation.
Beginning with an overview of SAFT history and basic fundamentals, this report provides a single source one can use to become familiar with the SAFT technology. A comprehensive list of references as well as summaries of key SAFT documents are provided. Laboratory and field application work not previously reported, a comprehensive operating procedure used in conjunction with the SAFT-UT field system developed by PNL, and new parametric studies will be of interest to both the novice and experienced SAFT investigator. The Conclusions section highlights the significant achievements of the SAFT program and discusses the future possibilities for the SAFT technology. The SAFT-UT field system was assembled from commercially available hardware; a complete description of the specifications of the hardware used, equipment model numbers, addresses of vendors that supplied the hardware, and pertinent drawings are included as an Appendix. 


\section{Acknowledgements}

The authors would like to thank the many people who over the years have worked on the evolution of the SAFT technology for the NRC. The software implemented on the SAFT Real-Time Field System was the result of this evolutionary process and the effort by these contributors should not be overlooked. Following is a list (not a complete list) of the researchers who have been in the forefront of the development at their institutes, although many of them have moved to other research activities: University of Michigan -- J. R. Frederick, S. N. Ganapathy, J. A. Seydel, B. L. Jackson, C. Vandenbroek, and M. R. Hamano; from Southwest Research Institute -- J. L. Jackson and D. R. Hamlin; and from PNL: A. J. Baldwin, R. E. Bowey, L. J. Busse, H. D. Collins, S. L. Crawford, J. D. Deffenbaugh, R. W. Gilbert, R. P. Gribble, R. J. Littlefield, G. A. Mart, P. D. Sperline, L. G. Van Fleet, and L. P. Van Houten.
We would like to thank K. E. Hass of PNL for preparing and editing this manuscript.

We would like to acknowledge the Combustion Engineering (now ABB Amdata) contribution for support and guidance on field applications; and, in particular, Jack LeReau and Mark Kirby.

We would like to thank Chuck Serpan of NRC RES for defending and supporting the SAFT program. We would also like to thank Joe Muscara of NRC RES for supporting and guiding the development of the SAFT technology over the years; and, in particular, for his leadership in this program. 


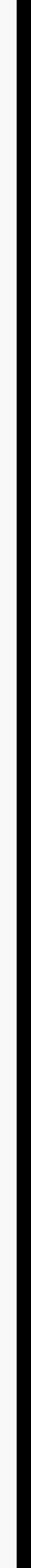




\subsection{Introduction}

Since the mid-1970s, the Nuclear Regulatory Commission (NRC) has supported development of the Synthetic Aperture Focusing Technique for Ultrasonic Testing (SAFT-UT). Specifically, the inspection of structurally important components in nuclear reactors has been the major application emphasis. Research began at the University of Michigan, which included laboratory adaptation of existing SAFT algorithms from radar applications to the ultrasonic realm. An extensive database and laboratory-oriented software library was developed on a Digital Equipment Corporation VAX-11/780 running the UNIX operating system.

The NRC also funded Southwest Research Institute in the late 1970s and early 1980s to take the SAFT technology being developed at the University of Michigan and demonstrate technology for some limited field applications.

In 1983, the research effort was transferred to the $\mathrm{Pa}$ cific Northwest Laboratory (PNL) to develop a fieldable system based on the technology developed at the University of Michigan. At PNL, the effort was focused on accelerating the computation process, streamlining the software package, and reducing the physical size of the hardware involved. "Portability" became the key word in terms of both the hardware and software.

A major objective was to offer a practical and fieldable technology to commercial companies. This process many times is referred to as "technology transfer." It was considered important to the success of technology transfer to provide a user-friendly software package and offer the ability of integrating the developed SAFT processing accelerator (Real-Time SAFT Processor) on the user's host computer.

Section 2 is intended to give the reader an overview of the SAFT technology, beginning with background information from a historical perspective and concluding with an overview of SAFT fundamentals. In the background and history section, one is led from the early radar applications of the SAFT technology, through the early ultrasonic applications at the University of Michigan, to the culmination of the SAFT technology at PNL. The SAFT fundamentals section provides a basic understanding of the logic employed to process data files that have been collected using the SAFT pulseecho configuration.
Section 3 provides the reader with a complete bibliographical listing of all NRC publications written about the SAFT technology, including all publications written by the University of Michigan and PNL. Further, synopses of key publications are included for readers whom may have only this SAFT final report at their disposal.

Section 4 provides a complete operating procedure for the SAFT-UT field inspection system developed at PNL. Included is an in-depth review of the analysis logic that has evolved from the many field and laboratory studies that have transpired over the years. Both the novice and experienced SAFT user will find this section extremely beneficial.

Section 5 reports on the outcome of a thick-section study; i.e., pressure vessels. Further, a discussion of the development of TSAFT- 3 is included. Centrifugally cast stainless steel (CCSS) has historically been a difficult medium in which to reliably detect and size cracks. Included in Section 5 is a section on advanced preprocessing techniques developed at PNL, which can be used prior to SAFT processing to enhance the subsequent images, thereby reducing the number of false calls and improving the inspectability of anisotropic materials such as CCSS.

Laboratory tests were performed to examine the effects of thickness and velocity parameters on TSAFT-2 images. Section 6 goes on to report a bandwidth compensation study performed by PNL to document the-limitations of the narrow-band approximation used in the SAFT algorithm. A utility developed to improve the SAFT images of wide-band data files is described and explained.

Twice SAFT was used to process data acquired by commercial equipment during inservice inspections of operating reactors. Section 7 begins with a report on the Indian Point Unit II pressure vessel inspection, where data was acquired by Westinghouse/Dynacon and subsequently processed and analyzed by PNL using the SAFT technology. A second report describes the Trojan Nuclear Power Plant inservice inspection, where data was acquired by Combustion Engineering/Amdata from a static-cast stainless steel hot-leg elbow. Once again, data was subsequently processed and analyzed, using the SAFT technology, at PNL. 
One of the most important contributions made by PNL to further the SAFT technology is the development of the Real-Time Processor (RTP). Section 8 covers in detail the performance benefits of the RTP for both pulse-echo and tandem SAFT applications under various conditions. Also included in Section 8 is an overview discussion of the implementation of the Real-Time Processor from a programmer's perspective.

Section 9 covers the upgrades to the SAFT-UT inspection system. The field host computer, described in Appendix A, was upgraded from a VAX 11-730 to a MicroVAX III. A robotic scanner was added to the SAFT-UT system. The robot permits the scanning of large objects with complex surface geometry. A new data acquisition system, based on a PC/486 computer, was assembled and installed. This upgrade increased the amount at data that could be acquired in a single scan. The SAFT code was ported to a Sun Microsystems, SparcStation computer. This permits SAFT data processing and analysis on inexpensive graphics workstations. A redesigned SAFT-UT system has been assembled for the NRC's NDE Mobil Laboratory. This new system is significantly smaller in overall size and significantly faster in operation.

Section 10 discusses the SAFT-UT technology that has been validated by participation in various blind tests including the Mini-Round Robin, the performance demonstration test for intergranular stress corrosion crack (IGSCC) detection and sizing and the PISC III Full Scale Vessel Test. These tests have shown that the SAFT-UT technology is a viable technique for detecting and characterizing defects in light water reactor materials.

In an effort to tie together all of the years of research, both at PNL and the University of Michigan, a conclusions section (Section 11) is included. In this section, the high points of the SAFT technology are reiterated pointing out the significant achievements that have brought the SAFT technology to where it is and an insight as to what the future of SAFT might be.

Section 12 provides a list of references used to write this final SAFT report.

The SAFT-UT field system was assembled from commercially available hardware. The specifications of the hardware used, equipment model numbers, addresses of vendors who supplied the hardware, and pertinent drawings are described completely in an appendix. 


\subsection{Overview of SAFT Fundamentals}

Section 2 is intended to give an overview of the SAFT technology, beginning with background information from a historical perspective and concluding with an overview of SAFT fundamentals. The background and history section leads the reader from the early radar applications of the SAFT technology, through the early ultrasonic applications at the University of Michigan, to the culmination of the SAFT technology at PNL. The SAFT fundamentals section provides a basic understanding of the logic employed to process data files that have been acquired employing the SAFT pulse-echo configuration.

\subsection{Background and History of SAFT}

The resolution of all imaging systems is limited by the effective aperture area, that is, the area over which data can be detected, collected, and processed. Before synthetic aperture techniques were developed, this maximum aperture size was limited by the ability to fabricate and control the physical aperture used for data collection; e.g., in optics and astronomy, large-aperture telescopes were limited by lens/mirror aberrations and atmospheric turbulence; in radar and radio astronomy, the physical size of antennae was limited by the need for portability. SAFT is an imaging method that was developed to overcome some of the limitations imposed by large physical apertures. SAFT has been successfully applied in a broad range of imaging contexts--radar, geophysical exploration, radio astronomy, ultrasonic testing, and others.

First applications of SAFT involved improving the lateral resolution of airborne radar mapping systems. For airborne data collection systems, antenna size is necessarily limited to some fraction of the size of the airplane being used to scan the antenna. By scanning a small antenna over a large area, a large effective aperture can be synthesized and resolution can consequently be improved.

Analogies between SAFT processing of radar data and SAFT processing of ultrasonic data are significant; however, there are some fundamental differences between microwave propagation and ultrasonic propagation. These differences have led to different implementations of SAFT processing. First, radar frequencies are many orders of magnitude higher than ultrasonic frequencies. This requires the use of special detection and recording techniques to maintain phase coherence in radar systems. In ultrasonic systems operating in the 1- to 10$\mathrm{MHz}$ frequency range, the propagating disturbance can be recorded directly using high-speed, analog-to-digital conversion techniques. Second, radar imaging is performed in a relatively uniform medium with a single index of refraction and a single mode of propagation. Ultrasonic imaging, on the other hand, uses media with variable indices of refraction. This fact leads to a number of complicating phenomena such as beam steering caused by refraction, multiple-mode propagation caused by mode conversion, and guided-wave phenomena caused by material anisotropy.

Original ultrasonic applications of SAFT followed directly from the radar experience (Prine 1972; Burckhardt, Grandchamp, and Hoffman 1974). Ultrasonic data were recorded on photographic film by means of a phase reference or coherent demodulator. Such a recording procedure is diagrammed in Burckhardt, Grandchamp, and Hoffman (1974). The received signals were amplified and mixed with a signal from a reference source to provide a coherently demodulated signal used to modulate the intensity on the oscilloscope. The output of the oscilloscope was recorded on film using a camera that was scanned in synchrony with the scanning ultrasonic transducer. The results of such a scanning procedure are illustrated conceptually in Figure 2.1. Figure 2.1(a) shows the plane to be imaged (including two point reflectors), the scanning ultrasonic transducer, and the divergent ultrasonic beam. Figure 2.1(b) shows the data that were recorded on film. In range $\mathrm{Z}$, the signals are highly confined. This range resolution, $\mathrm{DZ}$, is determined by the bandwidth of the ultrasonic pulse. In azimuth $\mathrm{X}$, the indications from the two point targets are smeared laterally because of the divergent beam used to collect the data; however, these smeared indications are modulated and appear as onedimensional Fresnel zone plates. The phase variation is slow near the center of the indication and fast near the edges. The spatial frequency content of these indications is directly related to the range of the point reflector. This recorded data was processed optically by a laser and a series of lenses that allows refocusing of this data in the $\mathrm{X}$ direction only. The processed image was then recorded photographically.

Even though this mode of ultrasonic synthetic aperture focusing has long since been outmoded, it does illustrate a number of points inherent with SAFT: 
- Imaging is a multiple-step process. Some kind of collection and storage scheme must be implemented to organize data for a subsequent reconstruction process.

- The object being imaged must remain stationary while it is scanned.

- Range resolution is controlled primarily by the bandwidth of the insonifying pulse.

- Lateral resolution is controlled by the effective aperture. The effective aperture is limited by the divergence of the insonifying ultrasonic beam, the directivity of the target, and the size of the scanned aperture.

- Lateral resolution can be independent of range because points located at larger distances have a larger "effective aperture."

The first digital implementations of one-dimensional SAFT processing in ultrasound soon followed these initial demonstrations. Digital SAFT processing was demonstrated in the area of nondestructive testing (NDT) by the University of Michigan (Frederick et al. 1976) and in medical imaging at the Mayo Clinic (Johnson et al. 1975) recording, and coherent summation to demonstrate the feasibility of digital SAFT. The major differences between the two demonstrations lay in the data collection procedure. The NDT system used a single, scanned, focused transducer to simulate an array of point sources and receivers. The medical system used multiple-channel recording and an acoustic array for data collection. This method was used because the medical system had to image moving objects; all the data had to be recorded before the objects moved an appreciable distance.

This need for high-speed data collection in medical imaging has subsequently caused the SAFT imaging approach to be abandoned in favor of phased-array and annular-array imaging techniques. In nondestructive evaluation (NDE), the goals of high resolution and imaging system flexibility have far outweighed the data acquisition speed requirements.

Research, sponsored primarily by the Nuclear Regulatory Commission, has been carried out at the University of Michigan (Frederick et al. 1976; Frederick et al.
1977; Frederick, VandenBroek, Elzinga et al. 1979; Frederick, VandenBroek, Ganapathy et al. 1979; Ganapathy et al. 1981; Ganapathy, Wu, and Schmult 1982; Ganapathy and Schmult 1982; Ganapathy et al. 1983). In addition, cooperative programs have been carried out at the University of Missouri at Columbia (Seydel 1978; Hamano 1980) and at Southwest Research Institute (Jackson 1978a, 1978b, 1981). The original goal of the work at the University of Michigan was to demonstrate the feasibility of SAFT to "obtain improved resolution with respect to other NDT methods, and in this way to obtain a better idea of the size, shape, and orientation of a discontinuity in the pressure vessel or piping." The initial results demonstrated improved lateral and axial resolution with planar SAFT-UT applied to a series of artificial defects (side-drilled holes) in aluminum test blocks. All of the initial work was performed with longitudinal ultrasonic waves (Frederick, Seydel, and Fairchild 1976).

The second year's work at the University of Michigan concentrated primarily on extending the SAFT processing algorithm to three-dimensional data sets (Frederick, Fairchild, and Anderson 1977). This extension involved far-reaching changes in data collection, processing, and display techniques.

During the third year of research (Frederick et al. 1978), three-dimensional longitudinal-wave (L-wave) SAFT processing was demonstrated with natural and artificial defects in several series of test blocks. At this time, the University of Michigan joined the University of Missouri at Columbia in a collaborative effort to develop signal processing procedures that would yield further improvements in axial flaw resolution and suppression of "front-surface ringdown."

Signal processing techniques studied intended to deconvolve the transducer impulse response for the reflected ultrasonic signal. Spectral division and linear predictive deconvolution were two techniques successfully applied to this problem. Linear predictive deconvolution was suggested as a means for performing "real-time" deconvolution because it can be implemented in the time domain as a recursive digital filter.

Also demonstrated was a digital subtraction technique for suppression of the large front-surface echo encountered in L-wave SAFT. Figure 2.2 shows the benefits of 
applying this technique. The raw A-scan shown in Figure 2.2(a) contains a large indication caused by the ultrasonic signal reflected from the front surface of the test block and from a small side-drilled hole located 4.6 $\mathrm{mm}$ below the front surface. By subtracting only the front-surface signal from Figure 2.2(a), it is possible to improve the signal-to-noise ratio of the side-drilled hole indication as shown in Figure 2.2(b). Figure 2.3 shows two SAFT-processed images derived from this type of data. Figure 2.3(a) shows the contour plot of a conventionally processed SAFT image. Figure 2.3(b) shows SAFT images produced from the same data; however, before processing, the front-surface echo was digitally subtracted. With this sort of preprocessing of the raw radio frequency $(\mathrm{rf})$ data, it appears possible to detect artificial defects within $2.0 \mathrm{~mm}$ of the top surface.

A significant effort during the final years of the program at the University of Michigan was the design and development of a special-purpose SAFT processor for real-time operation. A processor was built and demonstrated by GARD, Inc. (Ganapathy et al. 1985). This pioneering work in parallel processing established performance marks that proved that the SAFT processing could be performed at rates making field implementation a real-time process. However, the advances in computer equipment and performance resulted in the design and fabrication of the PNL special-purpose processing using off-the-shelf boards. The successful PNL SAFT real-time processor was a logical extension of the work at the University of Michigan and GARD, Inc.

Starting in 1977, a "Program for the Field Validation of Synthetic Aperture Focusing Technique for Ultrasonic Testing" was undertaken at Southwest Research Institute (SwRI) (Jackson 1978a and 1978b). The thrust of this program was to apply many of the techniques developed by the University of Michigan group and to produce a SAFT-UT system suitable for use during site examination of actual nuclear power reactors. The initial work performed by SwRI was aimed at improving the ultrasonics and electronics "front end" of the SAFTUT system. A final report on all of the work performed by SwRI was published (Hamlin 1985), and the reader is referred to that report for all of the details.

Over the past twelve years, work has continued to improve SAFT-UT inspection systems for both field and laboratory applications. The following list summarizes the highlights of this work:

- Software was developed and implemented for performing SAFT-UT on specimens with regular surface topography; e.g., cylinders, planes not parallel to the scan plane.

- Software was developed and implemented for performing SAFT-UT on specimens with arbitrary surface geometry.

- Software was developed to perform multi-depth focusing and expanding aperture SAFT-UT.

- The focusing properties of SAFT-UT were numerically analyzed. The point spread function of SAFT was studied as a function of aperture size, pulse attenuation, random position errors, pulse bandwidth, horizontal sample intervals, and frontsurface variations.

- Advanced data presentation techniques were analyzed. Perspective contour plotting, grey scale, color plotting, and interactive displays were considered. Finally, a RAMTEK high-resolution graphics imaging display system was selected and a graphics/analysis utility was written.

- $\quad$ Processing needs, associated with performing realtime SAFT-UT, were analyzed. Commercially available computer systems and array processors were analyzed and found to be either inadequate or too expensive for performing real-time SAFTUT. The concept of a special-purpose, hardware implementation, SAFT processor was proposed and developed. However, this also was inadequate (variations of the SAFT algorithm could not be easily implemented) and a second real-time processor was developed using a parallel processor architecture. This second real-time processor utilized commercially available hardware and the software could be easily modified to accommodate different SAFT algorithms (pulse-echo, TSAFT, TSAFT-2, TSAFT-3).

- Special-purpose scanning head hardware has been developed. Such hardware allows for high-speed scanning of arbitrary surfaces and allows for constant transducer offset. The scanning hardware 
was further modified to accommodate multiple transducer configurations such as TSAFT-2 and TSAFT-3.

- Tandem SAFT configurations were developed to overcome the ambiguities inherent in pulse-echo, single-transducer configurations. Further, a special tandem configuration (TSAFT-3) was developed for thick-section materials such as reactor pressure vessels.

- Parametric studies were performed to study the effects of errors in parameters used during SAFT processing. Included in these studies were effects from assumptions made within the SAFT algorithm itself.

- The SAFT-UT field data acquisition system was developed and employed in the field during inservice inspections of operating reactors (Dresden and Vermont Yankee) with subsequent data processing at PNL. Further, SAFT data was acquired using two different commercial data acquisition systems and subsequently processed and analyzed to characterize real defects in a reactor pressure vessel (Indian Point Unit II) and a static-cast stainless steel elbow in the hot-leg of a reactor (Trojan Nuclear Power Plant).

- Advanced preprocessing techniques were developed for anisotropic materials such as centrifugally cast stainless steels.

- Installation of the SAFT utilities on a VAX 11/750 owned by Combustion Engineering (CE) constituted the first step in educating $\mathrm{CE}$ personnel in the operation and inspection requirements of SAFT. This step paved the way for field validation testing.

- A real-time processor was built for Sandia National Laboratories and the SAFT utilities were installed on a Micro-VAX computer, which was designated as the host computer in the Sandia SAFT-UT system. This was the first attempt and trial for technology transfer.

To summarize, much developmental research work has been performed over the past twelve years to improve and implement SAFT-UT. Many of the basic questions regarding the ultimate resolution and computational complexity associated with performing SAFT-UT have been answered. Culmination of the SAFT program has resulted in a real-time SAFT-UT field system and handin-hand work with commercial companies performing special inservice inspections on operating reactors. Transfer of the SAFT technology to the commercial sector has been a goal of the NRC. This goal has not been fully met, but the process has started.

\subsection{Overview of SAFT Fundamentals}

The reader is referred to Busse, Collins, and Doctor (1984); Frederick and Fairchild (1976); Frederick, Fairchild, Anderson (1977); Frederick, VandenBroek, Elzinga, et al. (1979); and Frederick, VandenBroek, Ganapathy, et al. (1979) for extensive details on SAFT fundamentals. The following is a brief overview of the SAFT process and why it leads to significant improvement in full-volume imaging. SAFT relies on the physics of ultrasonic wave propagation and thus is a very robust technique.

Synthetic aperture focusing refers to a process in which the focal properties of a large-aperture focused transducer are synthetically generated from data collected over a large area using a small transducer with a divergent sound field. The processing required to focus this collection of data has been called beam-forming, coherent summation, or synthetic aperture processing.

Inherently, SAFT has an advantage over physical focusing techniques in that the resulting image is a full-volume focused characterization of the inspected area. Traditional physical focusing techniques provide focused data only at the depth of focus of the lens. For the typical pulse-echo data collection scheme used with SAFT-UT, a focused transducer is positioned with the focal point located at the surface of the part to be inspected. This configuration is used to produce a broad, divergent ultrasonic beam in the object under test. Alternatively, a small-diameter contact transducer may be used to generate a divergent beam in the specimen material. As the transducer is scanned over the surface of the object, the A-scan record (rf waveform) is digitized for each position of the transducer. Each reflector produces a collection of echoes in the A-scan records. If the reflector is an elementary single point 
reflector, then the collection of echoes will form a hyperbolic surface within the data-set volume. The shape of the hyperboloid is determined by the depth of the reflector in the test object and the velocity of sound in the material under test. This relationship between echo location in the series of A-scans and the actual location of the reflectors within the test object makes it possible to reconstruct a high-resolution and high signal-to-noise ratio image from the acquired signals.

If the scanning and surface geometries are well known, it is possible to accurately predict the shape of the locus of echoes for each point within the test object. The process of coherent summation for each image point involves shifting a locus of A-scans, within a regional aperture, by predicted time delays and summing the shifted A-scans. This process may also be viewed as performing a spatial matched filter operation for each point within the volume to be imaged. Each element is then averaged by the number of points that were summed to produce the final processed value. If the particular location correlates with the elementary point response hyperboloid, then the values summed will be in phase and produce a high-amplitude result. If the location does not correlate with the predicted response, then destructive interference will take place and the spatial average will result in a low amplitude value, reducing the noise level to a very small value.

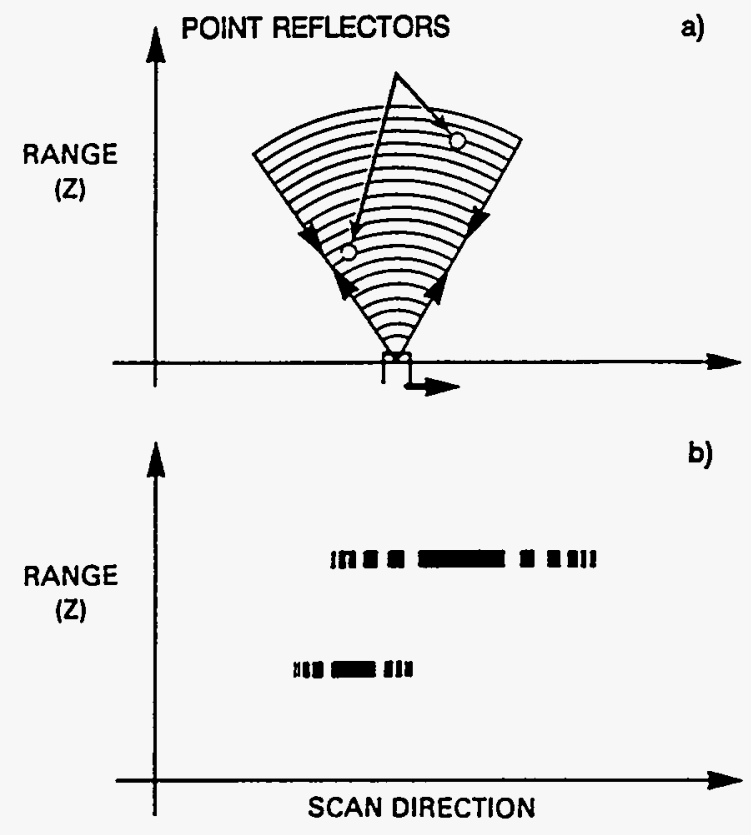

Figure 2.1. Schematic of the Results Produced by the Original SAFT-UT Experiments 
WITHOUT FRONT-SURFACE REMOVAL

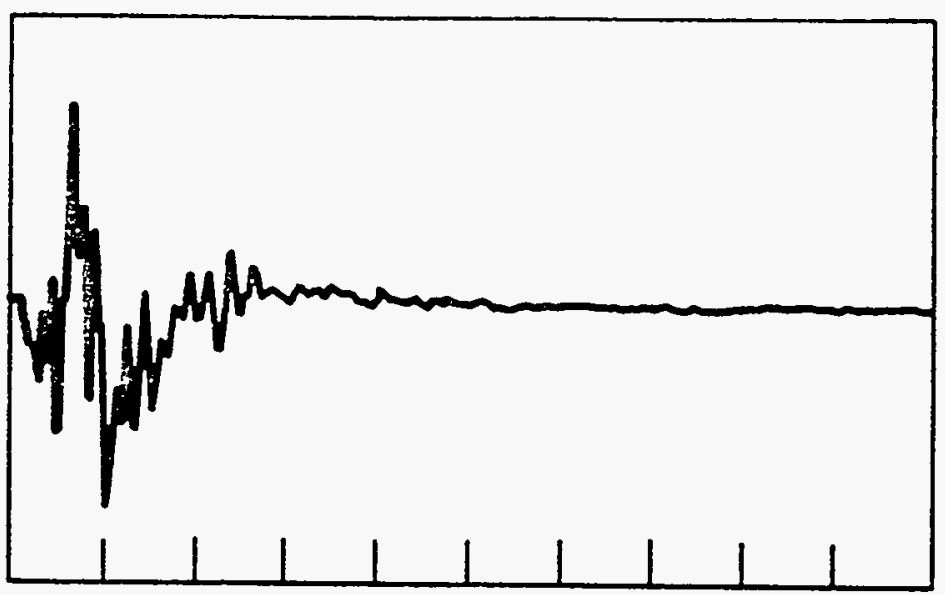

WITH FRONT-SURFACE REMOVAL

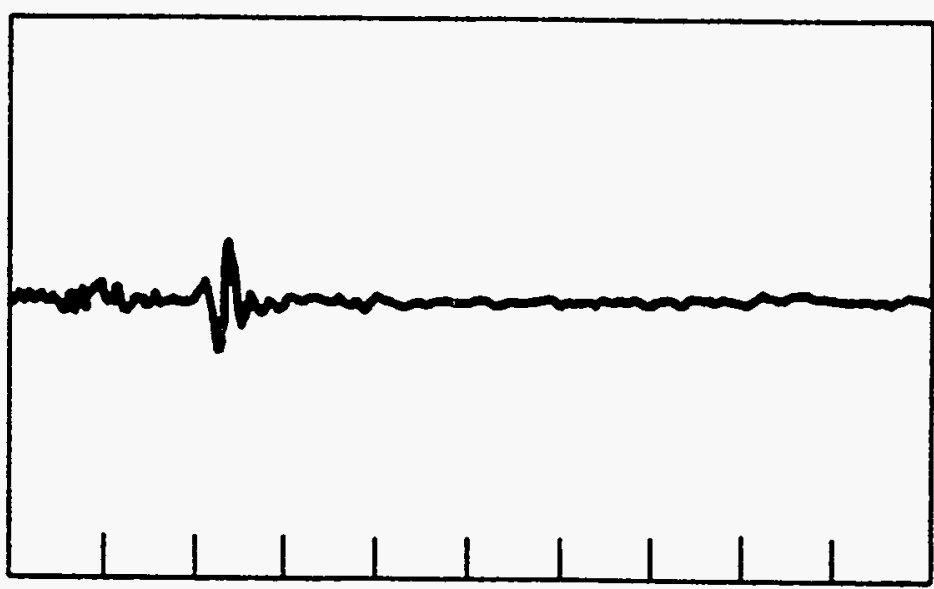

Figure 2.2. Demonstration of Signal-to-Noise Improvement Gained by Subtraction of Front Surface Signal 


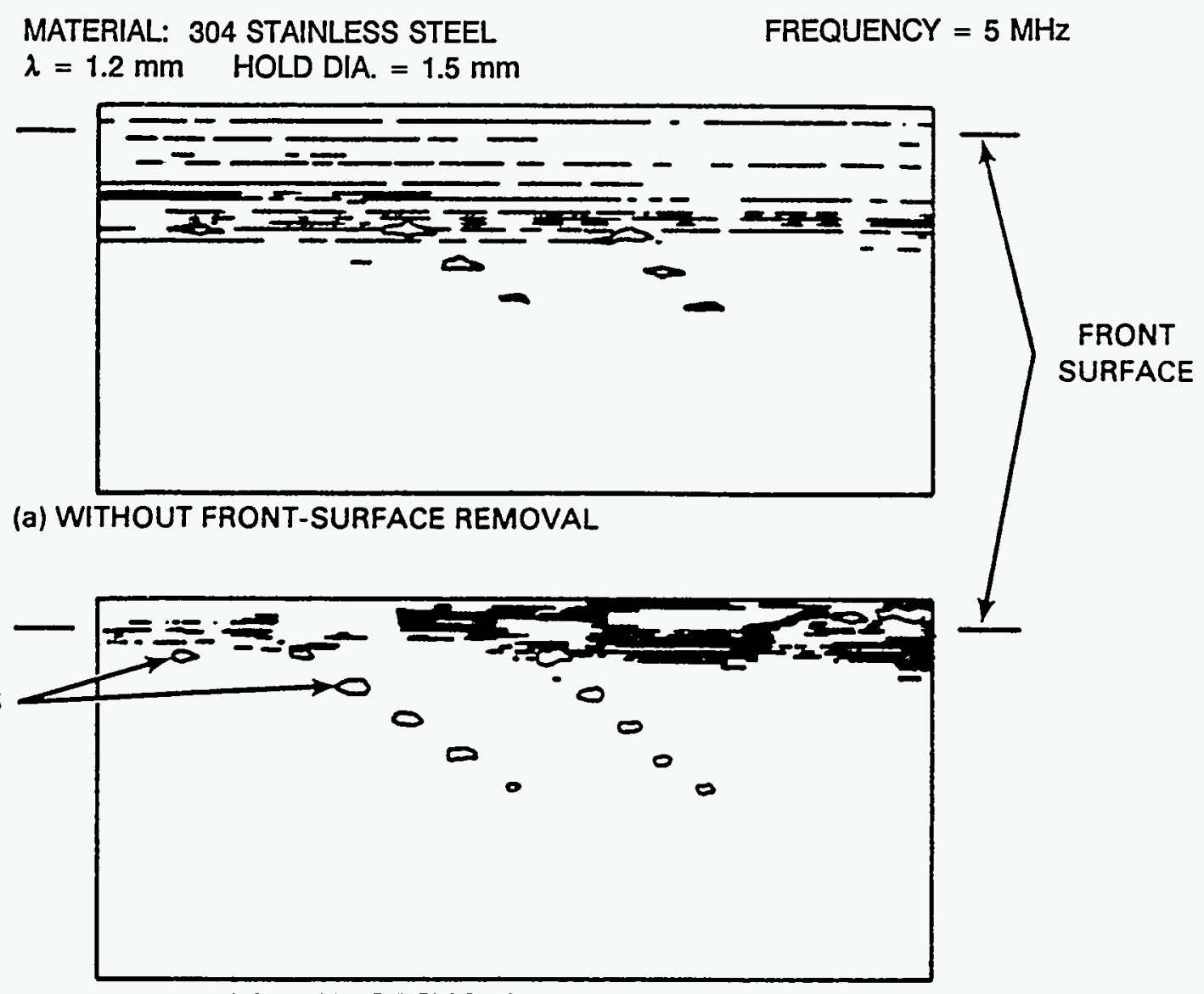

(b) WITH FRONT-SURFACE REMOVAL

Figure 2.3. Illustration of the Effect of Front Surface Signal Removed upon SAFT-UT Processed Images 


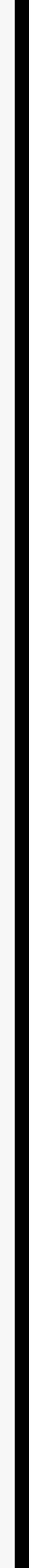




\subsection{Where to Go for Additional Information}

Section 3 provides the reader with a complete bibliographical listing of all NRC publications and selected other relevant publications concerning the SAFT technology. This listing includes all the documents written by the University of Michigan and PNL. This section also provides synopses of key publications for readers who may have only the SAFT final report at their disposal.

\subsection{Bibliography}

A comprehensive, but certainly not complete, bibliographical listing is provided to readers interested in further pursuing the SAFT technology.

Barna, B. A., and J. A. Johnson. 1981. "The Effects of Surface Mapping Corrections and Synthetic Aperture Focusing Techniques on Ultrasonic Images." EGG-FM-5314, EG\&G Idaho, Inc., Idaho Falls, ID.

Barna, B. A., and J. A. Johnson. 1982. "The Effects of Surface Mapping Corrections with Synthetic Aperture Focusing Techniques on Ultrasonic Imaging," in Review of Progress in Quantitative NDE, eds. D. O. Thompson and D. E. Chimenti, p. 753. Plenum Press, New York, NY.

Berkhout, A. V., J. Ridder, and F. L. V. d. Wal. 1980. "Acoustic Imaging by Wave Field Extrapolation, Part I: Theoretical Considerations," in Acoustical Imaging, Vol. 10, eds. P. Alais and A. F. Metherell. Plenum Press, New York, NY.

Bracewell, R. N. 1978. The Fourier Transform and Its Application. Second edition, Chapter 12. McGraw-Hill, New York, NY.

Burckhardt, C. B., P. A. Grandchamp, and H. Hoffmann. 1974. "Methods for Increasing the Lateral Resolution of B-Scan," in Acoustical Imaging, Vol. 5, ed. P. S. Green. Plenum Press, New York, NY.

Busse, L. J., H. D. Collins, and S. R. Doctor. 1984. "The Emerging Technology of Synthetic Aperture Focusing for Ultrasonic Testing," presented at 1984 Pressure Vessel and Piping Conference, San Antonio, Texas, 84-PUD-122, June 18-20, 1984.
Busse, L. J., H. D. Collins, and S. R. Doctor. 1984. Review and Discussion of the Development of Synthetic Aperture Focusing Technique for Ultrasonic Testing (SAFT-UT). NUREG/CR-3625, PNL4957. Pacific Northwest Laboratory, Richland, WA.

Collins, H. D., and B. P. Hildebrand. 1972. "The Effects of Scanning Position and Motion Errors on Hologram Resolution," in Acoustical Imaging, Vol. 4, ed. G. Wade. Plenum Press, New York, NY.

Collins, H. D., L. J. Busse, and D. K. Lemon. 1982. "Acoustic Emission Linear Pulse Holography." Ultrasonics Symposium Proceedings, Cat. \#82CH1823-4, Institute of Electrical and Electronics Engineers, Piscataway, NJ.

Corl, P. D., G. S. Kino, C. S. DeSilets, and P. M. Grant. 1980a. "A Digital Synthetic Focus Acoustic Imaging System," in Acoustical Imaging, Vol. 8, ed. A. F. Metherell. Plenum Press, New York, NY.

Corl, P. D., and G. S. Kino. 1980b. "A Real-Time Synthetic-Aperture Imaging System," in Acoustical Imaging, Vol. 9, ed. K. Y. Wang. Plenum Press, New York, NY.

Doctor, S. R., L. J. Busse, S. L. Crawford, T. E. Hall, R. P. Gribble, A. J. Baldwin, and L. P. Van Houten. 1986. Development and Validation of $a$ Real-Time SAFT-UT System for the Inspection of Light Water Reactor Components, Semi-annual Report, April 1984 to September 1984. NUREG/CR-4583, PNL-5822, Vol. 1. Pacific Northwest Laboratory, Richland, WA.

Doctor, S. R., T. E. Hall, L. D. Reid, S. L. Crawford, R. J. Littlefield, R. W. Gilbert. 1987. Development and Validation of a Real-Time SAFT-UT System for the Inspection of Light Water Reactor Components, Annual Report, October 1984 to September 1985. NUREG/CR-4583, PNL-5822, Vol. 2. Pacific Northwest Laboratory, Richland, WA.

Doctor, S. R., T. E. Hall, L. D. Reid, G. A. Mart. 1987. Development and Validation of a Real-Time SAFT-UT System for the Inspection of Light Water 
Reactor Components, Annual Report, October 1985 to September 1986. NUREG/CR-4583, PNL-5822, Vol. 3. Pacific Northwest Laboratory, Richland, WA.

Doctor, S. R., L. J. Busse, and H. D. Collins. 1983. "The SAFT-UT Technology Evolution," in 6th International Conference on NDE in the Nuclear Industry, pp. 145-151. Zurich, Switzerland, November 28-December 2, 1983.

Doctor, S. R., L. J. Busse, and H. D. Collins. 1984. "Development and Validation of a Real-Time SAFT-UT System for Inservice Inspection of LWRs," Proc. of the U.S. NRC Eleventh Water Reactor Safety Research Information Meeting, Vol. 4, NUREG/CP-0048, pp. 65-80. U.S. Nuclear Regulatory Commission, Washington, D.C.

Doctor, S. R., H. D. Collins, L. P. Van Houten, S. L. Crawford, T. E. Hall, A. J. Baldwin, R. E. Bowey, and R. P. Gribble. 1985. "Development and Validation of a Real-Time SAFT-UT System for Inservice Inspection of LWRs," Proc. of the U.S. NRC Twelfth Water Reactor Safety Research Information Meeting, Vol. 4, NUREG/CP-0058, pp. 319-341. U.S. Nuclear Regulatory Commission, Washington, D.C.

Doctor, S. R., A. J. Baldwin, L. J. Busse, H. D. Collins, S. L. Crawford, and L. P. Van Houten. 1985. "SAFT-UT for Stainless Steel Pipe Inspection," Proc. of the 7th International Conference on NDE in the Nuclear Industry, Grenoble, France, January 28-February 1, 1985.

Doctor, S. R., S. L. Crawford, T. E. Hall, and L. D. Reid. 1985. "SAFT Imaging in the Tandem Mode - TSAFT," ASNT Topical Meeting on Digital Signal Acquisition and Processing, Dallas, Texas, May 14-16, 1985.

Doctor, S. R., L. J. Busse, H. D. Collins, S. L. Crawford, and L. P. Van Houten. 1985. "Development and Validation of a Real-Time,SAFT-UT System for Inservice Inspection of LWŔ," Nuclear Engineering and Design, Vol. 86, pages 31-38, North Holland, Amsterdam.
Doctor, S. R., S. L. Crawford, and T. E. Hall. 1985. "SAFT-UT Field Experience," presented at 1985 Pressure Vessel and Piping Conference, New Orleans, Louisiana, PVP-Vol. 98-1, pp. 203-210, June 22-26, 1985.

Doctor, S. R., T. E. Hall, S. L. Crawford, and L. P. Van Houten. 1985. "Advanced Ultrasonic Imaging of Pipes in Dresden and Vermont Yankee Reactors," 8 th International Conference on Structural Mechanics in Reactor Technology, Vol. C-D, August 19-23, 1985.

Doctor, S. R., T. E. Hall, and L. D. Reid. June 1986. "SAFT -- The Evolution of a Signal Processing Technology for Ultrasonic Testing," NDT International, Vol. 19, No. 3, pp. 163-167.

Doctor, S. R., T. E. Hall, L. D. Reid, and G. A. Mart. 1986. "Application of Real-Time 3-D SAFT in the Tandem Mode," PNL-SA-14328, presented at the MPA Seminar, Stuttgart, West Germany, October 10, 1986.

Doctor, S. R., T. E. Hall, L. D. Reid, G. A. Mart, R. Littlefield, and R. Gilbert. 1986. "Development and Validation of a Real-Time SAFT-UT System for Inservice Inspection of LWRs," Proc. of Fourteenth Water Reactor Safety Information Meeting, Gaithersburg, Maryland, October 27-31, 1986, pp. 19-41.

Doctor, S. R., T. E. Hall, and L. D. Reid. 1986. "RealTime SAFT-UT for Nuclear Component Inspection," presented at the 8th International Conference on NDE in the Nuclear Industry, Orlando, Florida, November 17-20, 1986.

Doctor, S. R., T. E. Hall, L. D. Reid, and G. A. Mart. 1987. Development and Validation of a Real-Time SAFT-UT System for the Inspection of Light Water Reactor Components, Annual Report, October 1985-September 1986. NUREG/CR-4583, PNL5822, Vol. 3, Pacific Northwest Laboratory, Richland, WA.

Doctor, S. R., T. E. Hall, and L. D. Reid. 1988. The SAFT-UT Real-Time Inspection System Operational Principles and Implementation. NUREG/CR- 
5075, PNL-6413, Pacific Northwest Laboratory, Richland, WA.

Frederick, J. R., J. A. Seydel, and R. C. Fairchild. 1976. Improved Ultrasonic Non-Destructive Testing of Pressure Vessels. NUREG-0007-1, University of Michigan, Ann Arbor, MI.

Frederick, J. R., R. C. Fairchild, and B. H. Anderson. 1977. Improved Ultrasonic Nondestructive Testing of Pressure Vessels. NUREG-0007-2, University of Michigan, Ann Arbor, MI.

Frederick, J. R., C. VandenBroek, R. C. Fairchild, and M. B. Elzinga. 1978. Improved Ultrasonic Nondestructive Testing of Pressure Vessels.

NUREG/CR-0135, University of Michigan, Ann Arbor, MI.

Frederick, J. R., C. VandenBroek, M. Elzinga, M. Dixon, D. Papworth, N. Hamano, and K. Ganapathy. 1979. Improved Ultrasonic Nondestructive Testing of Pressure Vessels. NUREG/CR-0581, University of Michigan, Ann Arbor, MT.

Frederick, J. R., C. VandenBroek, S. Ganapathy, M. Elzinga, W. DeVries, D. Papworth, and N. Hamano. 1979. Improved Ultrasonic Nondestructive Testing of Pressure Vessels. NUREG/CR0909, University of Michigan, Ann Arbor, MI.

Ganapathy, S. N. Hamano, M. R. Bether, W. S. Wu, T. G. Dennehy, C. Purnaveja, M. M. Murray, M. B. Elzinga, and F. Raam. 1981. Ultrasonic Imaging Techniques for Real-time In-service Inspection of Nuclear Power Reactors. NUREG/CR-2154, University of Michigan, Ann Arbor, MI.

Ganapathy, S., W. S. Wu, and B. Schmult. 1982. "Analysis and Design Considerations for a RealTime System for Nondestructive Evaluation in the Nuclear Industry." Ultrasonics 20:249.

Ganapathy, S., and B. Schmult. 1982. "Design of a Real-time Inspection System for NDE of Reactor Vessels and Piping Components." Presented at I MECH E Conference, London, England. I MECH E 1982-9.
Ganapathy, S., B. Schmult, W. S. Wu, N. Hamano, and D. Bristor. 1983. Investigation of Special Purpose Processors for Real-Time Synthetic Aperture Focusing Techniques for Nondestructive Evaluation of Nuclear Reactor Vessels and Piping Components. NUREG/CR-2703, University of Michigan, Ann Arbor, MI.

Ganapathy, S., B. Schmult, W. S. Wu, T. G. Dennehy, N. Moayeri, and P. Kelly. 1985 Design and Development of a Special Purpose SAFT System for Nondestructive Evaluation of Nuclear Reactor Vessels and Piping Components, NUREG/CR-4365, U.S. Nuclear Regulatory Commission, Washington, D.C.

Gehlbach, S. M., and R. E. Alvarez. 1981. "Digital Ultrasound Imaging Techniques using Vector Sampling and Raster Line Reconstruction." Ultrasonic Imaging 3:83.

Hall, T. E., S. R. Doctor, and L. D. Reid. 1987. "A Real-Time SAFT System Applied to the Ultrasonic Inspection of Nuclear Reactor Components," Review of Progress in Quantitative Nondestructive Evaluation, Vol. 6A, pp. 509-517. Plenum Press, NY.

Hall, T. E., S. R. Doctor, L. D. Reid, R. J. Littlefield, and R. W. Gilbert. 1987. "Implementation of a Real-Time Ultrasonic SAFT System for Inspection of Nuclear Reactor Components," Acoustical Imaging, Vol. 15, pp. 253-266. Plenum Press, NY,

Hall, T. E., L. D. Reid, and S. R. Doctor. 1988. The SAFT-UT Real-Time Inspection System--Operational Principles and Implementation. NUREG/CR5075, PNL-6413, Pacific Northwest Laboratory, Richland, WA.

Hamano, N. 1980. "Deconvolution of Ultrasonic Nondestructive Testing Data." $\mathrm{PhD}$ Dissertation, Electrical Engineering Dept. University of Missouri, Columbia, MO.

Hamlin, D. R. 1985. Program for Field Validation of the Synthetic Aperture Focusing Technique for Ultrasonic Testing (SAFT-UT). NUREG/CR-4078, Southwest Research Institute. 
Herman, G. T., H. K. Tuy, K. J. Langenberg, and P. C. Sabatter. 1987. Basic Methods of Tomography and Inverse Problems. Melvern Physics Series. IOP Publishing Ltd. Bristol, England.

Highmore, P. J., A. J. Willets and P. Clough. 1982. "Circe - A Versatile Ultrasonic Digital Data Recording, Analysis, and Display System," in I MECH E Conference Proceedings, C154/82:209.

Hildebrand, B. P., and K. A. Haines. 1969. "Holography by Scanning." J. Opt. Soc. Am. 59(1):1.

Hildebrand, B. P., and B. B. Brenden. 1972. An Introduction to Acoustical Holography. Plenum Press, New York, NY.

Jackson, J. L. 1978a. Program for Field Validation of the Synthetic Aperture Focusing Technique for Ultrasonic Testing (SAFT-UT) - Analysis Before Test. NUREG/CR-0288, Southwest Research Institute.

Jackson, J. L. 1978b. Program for Field Validation of the Synthetic Aperture Focusing Technique for Ultrasonic Testing (SAFT-UT) Midyear Progress Report. NUREG/CR-0290, Southwest Research Institute.

Jackson, J. L. 1981. Program for Field Validation of the Synthetic Aperture Focusing Technique for Ultrasonic Testing (SAFT-UT). NUREG/CR-1885, Vol. 1, 2, and 3, Southwest Research Institute.

Johnson, S. A., J. F. Greenleaf, F. A. Duck, A. Chu, W. R. Samayou, and B. K. Gilbert. 1975. "Digital Computer Simulation Study of a Real-Time Collection, Post-Processing Synthetic Focusing Ultrasound Cardiac Camera," in Acoustical Holography, Vol. 6, ed. N. Booth. Plenum Press, New York, NY.

Johnson, S. A., and J. F. Greenleaf. 1979. "New Ultrasound and Related Imaging Techniques." Transactions on Nuclear Science NS-26:8212. IEEE.

Johnson, J. A. 1982. "Parameter Study of SyntheticAperture Focusing in Ultrasonics," in Review of Progress in Quantitative NDE, eds. D. O. Thomp- son and D. E. Chimenti. Plenum Press, New York, NY.

Kino, G. S., D. Corl, S. Bennett, and K. Peterson. 1980. "Real Time Synthetic Aperture Imaging System." Ultrasonics Symposium Proceedings, Cat. \#80CHJ1602-2SU, IEEE.

Kupperman, D. S., et al. 1982. "Light-Water-Reactor Safety Research Program: Quarterly Progress Report." Nuclear Regulatory Commission Report NUREG/CR-2970, Vol. 1; 2-15.

Moore, M. J., and F. J. Dodd. 1982. "Real-Time Signal Processing in an Ultrasonic Imaging System." Materials Evaluation 40:976.

Murgatroyd, R. A. 1982. "Automated Defect Location and Sizing by Advanced Ultrasonic Techniques," in Proceedings of Quantitative NDE in the Nuclear Industry, 82-74019:243.

Norton, S. J. 1976. "Theory of Acoustic Imaging." Stanford Technical Report No. 4956-2.

Peterson, D. K., S. D. Bennett, and G. S. Kino. 1981. "Real Time Digital Imaging." Ultrasonic Symposium Proceedings, Cat. \#81CH1689-9SU. IEEE.

Polky, J. N., and D. D. Miller. 1985. An Ultra-High Speed Residue Processor for SAFT Inspection System Image Enhancement. NUREG/CR-4170, Sigma Research Inc., Redmond, WA.

Polky, J. N. 1986. Development of a Real-Time Residue Number Processor for SAFT Inspection. NUREG/CR-4634, Sigma Research Inc., Redmond, WA.

Prine, D. W. 1972. "Synthetic Aperture Ultrasonic Imaging," in Proceedings of the Engineering Applications of Holography Symposium:287. Society of Photo-optical Instrumentation Engineers.

Ridder, J., A. J. Berkhout, and L. F. v.d. Wal. 1980. "Acoustic Imaging by Wave Field Extrapolation, Part II: Practical Considerations," in Acoustical Imaging, Vol. 10, eds. P. Alais and A. F. Metherell. Plenum Press, New York, NY. 
Schafer, G., V. Schmitz, and W. Muller. 1983. "Comparison of Different Signal Averaging Methods to Improve SNR for the NDT of Coarse Grained Materials." Nondestructive Testing Communications. Gordon \& Breach Science Publishers, New York, London, Paris.

Schmitz, V., W. Muller, and G. Schafer. 1982. "A New Ultrasonic Imaging System." Materials Evaluation 40:101.

Schmitz, V., W. Muller, and G. Schafer. 1983. "Classification and Reconstruction of Defects by Combined Acoustical Holography and Line-SAFT," in Proceedings of the Germany-United States Workshop on Research and Development of New Procedures in NDT. Springer Verlag.

Schmitz, V., O. A. Barbian, and W. Gebhardt. 1983. "Defect Assessment by Ultrasonic Testing," in Measurement and Characterization of Weld Defects - A Critical Appraisal of Nondestructive Testing by the British Welding Institute, Cambridge CB1 6AL England.

Seydel, J. A. 1978. (Signal Processing) Methods Development. NUREG/CR-0135, University of Missouri, Columbia.

VandenBroek, C., M. B. Elzinga, J. R. Frederick, and S. Ganapathy. November 1980. "Synthetic Aperture Ultrasonic Imaging in Metals." National Bureau of Standards Special Publication 596. Ultrasonic Materials Characterization: 249.

\subsection{Synopses of Key SAFT Documents}

For readers who may have only the "SAFT Final Report" at their disposal, synopses of key SAFT documents have been assembled. These synopses are intended to give an overview of past information published about the SAFT technology. For more detailed information, readers should acquire the particular documents desired.

\subsubsection{Review and Discussion of the Devel- opment of Synthetic Aperture Focusing Tech- nique (SAFT-UT) (Busse, Collins, and Doctor 1984)}

The synthetic aperture focusing technique for ultrasonic testing (SAFT-UT) is an ultrasonic imaging technique designed to enhance the performance of conventional ultrasonic, nondestructive testing procedures for performing two basic functions: 1) detection of flaws and defects within structural and functional components; and 2) classification and/or characterization of these detected defects in terms of size, shape, orientation, location, and composition. This enhanced performance is brought about by a high-resolution ultrasonic image, which is the output of the SAFT process. SAFT-UT is a particularly flexible imaging method that can be adapted to many different materials, specimen geometries, and propagation modes. SAFT-UT is accomplished by applying a signal processing algorithm to a collection of raw amplitude versus time data (A-scans). The algorithm allows each point within the inspected volume to be focused upon by mathematically simulating the action of a lens specifically formed for imaging that point in the volume. This method of image formation permits each of the points to be imaged at the maximum available resolution. The images produced are volumetric; i.e., they provide a three-dimensional map of any defect and the surrounding area. Such a map can be accurately interpreted.

Many aspects of SAFT-UT are treated in this report:

- Historical Background of SAFT-UT

- Theory of SAFT-UT

- Implementation of and Results Using SAFT.

From the results presented, it appears that SAFT-UT has been truly successful in providing a flexible highresolution imaging system for the laboratory environment. This method of imaging appears to be ideal for the inspection of critical industrial components, especially in the nuclear power industry.

The application of SAFT-UT imaging methods to fieldtesting of reactor piping, pressure vessels, and nozzles appears to be a feasible and highly desirable extension 
of the basic research carried out so far. With the development of a special-purpose, real-time SAFT processor, this transfer of defect detection/imaging technology from the laboratory to the field appears to be both practical and feasible.

\subsubsection{Development and Validation of a Real- Time SAFT-UT System for the Inspection of Light Water Reactor Components, Vol. 1 (Doctor et al. 1986)}

A three-year program for the Development and Validation of a Real-Time SAFT-UT System for Inservice Inspection of Light Water Reactors was established at PNL to develop SAFT and demonstrate the technology in reactor inservice inspections.

The objectives of this NRC program include these:

- Design, fabricate, and evaluate a real-time flaw detection and characterization system based on SAFT-UT for inservice inspection (ISI) of all required light water reactor (LWR) components.

- Establish calibration and field operating procedures.

- Demonstrate and validate the SAFT-UT system through actual reactor inservice inspections.

- Generate an engineering data base to support ASME Code acceptance of the realtime SAFT-UT technology.

The scope of the program is defined by the following:

- Conduct laboratory tests to provide engineering data for defining SAFT-UT system performance.

- Complete the development of a special processor to make SAFT a real-time process for ISI applications.

- $\quad$ Fabricate and field-test a real-time SAFTUT system suitable for field inspection of nưclear reactor piping, nozzles, and pressure vessels.

Ongoing work is advancing the evolution of the SAFTUT field system from concept to reality. The final system was operational by the end of fiscal year 1985 . Reported here are a system overview, optimization of front-end electronics, and procurement of hardware for the final system configuration.

In demonstration tests, a special-purpose SAFT processor being designed by General American Research and Development, Incorporated, met its speed requirement for real-time operation. However, because the hardwired system was intended to implement pulse-echo mode only, it could not be easily changed to accommodate other algorithms, e.g., Tandem SAFT. Also, redesign was needed to produce a reliable unit suitable for field work because the first version had been intended only for laboratory demonstration of the concept. PNL proposed a new design based on commercial hardware.

The theory of pulse-echo operation is described here and extended to cover the tandem mode of operation. The tandem mode was developed to image vertically oriented defects by overcoming the ambiguities of corner-trap pulse-echo data. Laboratory experiments were conducted to begin refinement of criteria to be used for sizing vertically oriented defects for both modes of operation.

SAFT data were obtained from several examinations of material samples and piping welds in the field. Samples included a specimen provided by Southwest Research Institute from the Duane Arnold reactor, a thick section of steel plate with induced defects examined by Risley Nuclear Laboratory, and plate 2 of the PISC II program. The field welds included four at the Dresden Unit 3 power plant and nine at the Vermont Yankee power plant. Analyses of these data are presented.

Several changes were made in the SAFT processing scheme to speed up the processing of ultrasonic data for SAFT analysis. The changes included optimization of the SAFT algorithm on the VAX 11/780 computer, selective processing, acceleration of the envelope detection algorithm, and conversion of SAFT software from the UNIX implementation of the $C$ language to VAX/VMS C. 


\subsubsection{Development and Validation of a Real- Time SAFT-UT System for the Inspection of Light Water Reactor Components, Vol. 2 (Doctor et al. 1987a)}

A multiyear program for the Development and Validation of a Real-Time SAFT-UT System for Inservice Inspection of Light Water Reactors was established at PNL to develop the synthetic aperture focusing technique (SAFT) and demonstrate the technology in reactor inservice inspections.

The objectives of this program for the NRC include these:

- Design, fabricate, and evaluate a real-time flaw detection and characterization system based on SAFT-UT for ISI of all required LWR components.

- Establish calibration and field operating procedures.

- Demonstrate and validate the SAFT-UT system through actual reactor inservice inspections.

- Generate an engineering data base to support ASME Code acceptance of the realtime SAFT-UT technology.

The scope of the program is defined by the following:

- Conduct laboratory tests to provide engineering data for defining SAFT-UT system performance.

- Complete the development of a special processor to make SAFT a real-time process for ISI applications.

- $\quad$ Fabricate and field test a real-time SAFTUT system suitable for field inspection of nuclear reactor piping, nozzles, and pressure vessels.

Ongoing work is advancing the evolution of the SAFTUT field system from concept to reality. The final system was operational by the end of the fiscal year 1985, and its features are described in terms of operatororiented software designed to minimize operator computer skill requirements. Features such as transducer view, compare, B-scan, C-scan, and enlarge are described.

The modes of signal processing with the emphasis on the implementation of the tandem SAFT mode are described. Examples, where image ambiguity is reduced, are presented. Processing speed-up routines, including selective processing and envelope detection, are described and performance is demonstrated.

The real-time SAFT processor box using the 68000 family of microprocessor is described and performance matrices presented for this design versus the VAX $11 / 780$ and VAX 11/730 for both thin material (pipe) and thick material (pressure vessel) files. This design approach clearly provides the processor speed necessary to achieve real-time SAFT operation.

The field experience consisted of taking the SAFT-UT system to Commonwealth Edison and demonstrating for the first time in-field processing of SAFT data on a narrow-gap pipe weldment containing fabrication flaws.

A detailed analysis was conducted on PISC II Plate 2 data and compared to other PISC II team performances. This block was the first out of laboratory testing on the SAFT-UT data acquisition system with subsequent PNL processing of the data. Many things were learned about how to develop procedures to reduce potential inspection errors. The SAFT results were very good under the circumstances and were better than the global average and average of the advanced teams. The data clearly shows that image sizing criteria must be applied as a systematic function of defect type (i.e., planar versus volumetric); otherwise, consistent over- or under-sizing will result. For the data presented, a lossof-signal criterion was applied resulting in an over-sizing trend.

Data was collected on a series of 15 centrifugally cast stainless steel weldments that are part of a round robin being conducted under PISC II. The data collection methods are described, but no analysis was performed on the data during this reporting period. 


\subsubsection{Development and Validation of a Real- Time SAFT-UT System for the Inspection of Light Water Reactor Components, Vol. 3 (Doctor et al. 1987b)}

A multiyear program for the Development and Validation of a Real-Time SAFT-UT System for Inservice Inspection of Light Water Reactors was established at the Pacific Northwest Laboratory (PNL) to develop the synthetic aperture focusing technique (SAFT) and demonstrate the technology in reactor inservice inspections.

The objectives of this program for the Nuclear Regulatory Commission (NRC) include these:

- Design, fabricate, and evaluate a real-time flaw detection and characterization system based on SAFT-UT for inservice inspection (ISI) of all required LWR components.

- Establish calibration and field operating procedures.

- Demonstrate and validate the SAFT-UT system through actual reactor inservice inspections.

- Generate an engineering data base to support ASME Code acceptance of the real-time SAFTUT technology.

The scope of the program is defined by the following:

- Conduct laboratory tests to provide engineering data for defining SAFT-UT system performance.

- Complete the development of a special processor to make SAFT a real-time process for ISI applications.

- Fabricate and field test a real-time SAFT-UT system suitable for field inspection of nuclear reactor piping, nozzles, and pressure vessels.

During this year, a formal agreement was put in place with Combustion Engineering in which they joined the program and began assisting in validating the SAFT technology on nuclear power plants.
Ongoing work is focused on advancing the evolution of the SAFT-UT field system from concept to reality. The final field system was operational by the end of fiscal year 1985; and the emphasis this past year has been in terms of parametric sensitivity studies on operational parameters, further development of the real-time SAFT processor box, and detailed analysis of a number of defected samples.

The tandem SAFT mode was developed in 1985 to overcome some of the serious shortcomings of the pulse-echo mode. This first implementation of the TSAFT mode was developed by using a fixed source and scanned receiver. During this year, the TSAFT-2 mode was developed, which uses a scanned source and scanned receiver. The performance of the TSAFT- 2 mode is clearly superior and allows defects with depth larger than the transducer beam diameter to be sized accurately and shows an improvement of the resolution performance.

Extensive efforts were expended to conduct parametric studies on the sensitivity of the SAFT-UT system to some of the processing variables. The variables studied during this reporting period included: pulse-echo sampling rate, 3-D SAFT mode versus the Line-SAFT mode, TSAFT velocity changes of up to $\pm 20 \%$, TSAFT thickness changes of up to $\pm 20 \%$, TSAFT resolution performance, imaging of nonvertical defects, and performance of TSAFT and TSAFT-2 for accurately sizing vertical defects. The SAFT-UT system performed as predicted based on theory. As the sampling rate increased, the imaging resolution improved until twice the Nyquist sampling rate was achieved. The resolution performance of the 3-D SAFT mode was shown to be very superior to the results for Line-SAFT for reflectors exhibiting high spatial frequencies. The surprising result for changes in velocity and thickness was to have a large shift in the defect location and a minor blurring or resolution loss in the image. The SAFT-UT system performance deteriorated the same as other acoustic methods because of signal loss when defects became non-vertical and scattered the acoustic energy outside the receiver aperture. The changes are documented and predictable from simple acoustic scattering theory. The superiority of the TSAFT-2 mode over the original TSAFT configuration was demonstrated through a series of sawcut sizing measurements showing the increase in resolution performance by a factor of 1.4. 
The evolution of the real-time SAFT processor box is continuing, and further studies of the performance is showing that the real-time rates of ten A-scans per second can be achieved when the new 68020 microprocessor boards are used.

Some very good studies were performed in the laboratory on blocks and pipe specimens from a number of sources. The SAFT performance was very good and was within predictions for the apertures that the specimens allowed. TSAFT-2 was shown to provide improved near-surface performance over pulse-echo on the NAVSEA block. The large-diameter pipes in the Materials Reliability Center at PNL were examined and much was learned about refining the SAFT procedure to minimize efforts during the data collection phase. Furthermore, it was found that if the electronics saturate, the resultant image will be blurred and result in systematic oversizing of the defect. Data were collected on a series of KAPL specimens, and the images were good although no feedback has been received on the SAFT result versus the destructive results or even intended defects. Results were obtained on a series of CCSS specimens and all thermal fatigue cracks in the specimens were detected; but at the same time, a number of false calls were made. The false calls result from the coherent backscattering that is produced by the large anisotropic grain structure. Data were also collected on a series of 45 quadrants of pipe specimens that are part of the Mini-Round Robin study in progress at PNL. These data were analyzed and has not been compared with true-state destructive results. This is planned for next fiscal year.

\subsubsection{The SAFT-UT Real-Time Inspection System Operational Principles and Imple- mentation (Hall, Reid, and Doctor 1988)}

A multiyear program for the Development and Validation of a Real-Time SAFT-UT System for Inspection of Light Water Reactors was established at the Pacific Northwest Laboratory (PNL) to develop the synthetic aperture focusing technique (SAFT) and demonstrate this advanced imaging technology in reactor inservice inspections. The developed system presents a visual image of ultrasonic indications from reflectors located in piping and pressure vessels of nuclear reactors. A significant portion of the effort at PNL was oriented toward building a system based on SAFT technology that is transportable to field environments, and provides on-the-spot (real-time) images for defect sizing and characterization. This includes accelerating the computation process, streamlining the software package, and reducing the physical size of the hardware involved.

In addition to field system design, it was considered necessary for the advancement of SAFT-UT technology to offer a software and hardware package that is easy to use and could readily be implemented by other organizations. "Technology transfer" became an important item in the scope of the project and, "Portability" became the key word in terms of both the hardware and software.

Development of a real-time SAFT processor was an important achievement in providing rapid SAFT-UT images. This device is a parallel architecture peripheral to the host computer and performs all of the computationally intensive SAFT computations. It significantly accelerates the SAFT computation and relieves the host computer so that it may more effectively perform the executive and graphics operations.

A variety of software utilities were developed to assist the user in processing the data collected and interpreting the results. These include an on-line editor for data files and their associated parameter blocks, an interactive interface to the real-time SAFT processor and SAFT processing software, and three graphic utilities that provide extensive defect sizing and characterization capability.

A major hardware design goal of the transportable realtime field system was to implement off-the-shelf components. The purpose of this was to facilitate technology transfer. While it is apparent that the overall size of the field system could be reduced through custom designs of certain modules, commercially available components were chosen wherever possible to make this technology more available to other organizations. The few exceptions to this objective are detailed in the final portion of this document.

This document was written as a basis for implementing the SAFT-UT software and hardware developed at PNL. A theoretical overview of the PNL implementation of SAFT-UT is given. Included is an architectural overview of the SAFT-UT field system including the real-time SAFT processor. A detailed operating de- 
scription is provided for each SAFT-UT software utility offered in the package. A precise description of the installation SAFT-UT software utilities is also given, along with an explanation of the technique for interfacing foreign data files to this program set. Finally, an itemized parts list and hardware description is given to complete the comprehensive documentation of the SAFT-UT real-time fieldable imaging system.

\subsubsection{Program for Field Validation of the Synthetic Aperture Focusing Technique for Ultrasonic Testing (SAFT-UT) (Hamlin 1985)}

An NDE system capable of using the SAFT-UT technique was developed and tested. The performance capability of this system was evaluated and is summarized below:

1) SAFT-UT is a viable NDE technique capable of performing accurate measurements of the spatial location and extent of acoustically reflective surfaces contained in objects similar to the structural components and weldments in nuclear power reactor systems.

2) SAFT-UT inspection equipment demonstrated effective operation at higher sensitivity levels than is practical using conventional UT inspection equipment.

3) Interpretation of the SAFT image displays is much more definitive and easier to perform than the evaluation of indications obtained from conventional UT equipment.

4) To provide a SAFT-UT system possessing the flexibility necessary to perform general field-rated flaw analysis functions, both 0-degree and anglebeam inspection capabilities must be incorporated.

5) In contrast to the use of an immersed focusedbeam inspection mode for SAFT-UT, the surface inspection mode can also be utilized. The surface contact inspection mode can be applied to component surfaces representative of actual field components. Use of this inspection mode offers engineering advantages over the focused-beam mode because of greater simplification (lower cost, shorter development time, higher reliability) of the mechanics and software control of the algorithms required to permit system operation.

6) The SAFT-UT technique can be used to generate images of both volumetric and crack-type defects.

7) The SAFT-UT technique can be used to generate images of defects located beneath cladding or located in the vicinity of strong geometrical reflectors.

8) SAFT-UT can be utilized to produce images of a variety of defect types and will be successful when the UT technique employed returns sufficient energy to the receiving transducer for image generation.

The single most important criterion limiting the ability of SAFT-UT to image defects is the ability to define the physics of the acoustic interactions that take place at the surface of the defect. The SAFT-UT technique depends on the acoustic energy returned from the defect and, if not present due to orientation or other problems, results in failure to image the defect. Further work in evolving this technique should concentrate on defining a better understanding of the limitations of the UT technique itself from an acoustic physics standpoint.

\subsubsection{Development of a Real-Time Residue Number Processor for SAFT Inspection, Phase II Final Report, September 1984 through April 1986 (Polky 1986)}

A high-speed SAFT-UT imaging system has been designed using residue number system (RNS) computational methods. The imaging system is based on a new frequency domain correlation process applied to conventional pulse-echo ultrasonic data, wherein the data is collected over a two-dimensional aperture. The resulting three-dimensional data set in $\mathrm{x}, \mathrm{y}$, and "time-offlight" may be processed by the frequency domain SAFT (FSAFT) system in either real-time or batch (post-test) modes. In the real-time mode, it is expected that true flaw recognition would be of primary interest and the resulting images would be competitive with current time-domain SAFT techniques. However, the greatest benefit of FSAFT is for detailed analysis of critical flaw types using the high-speed batch or fast 
inspection mode. The system's performance results from using custom RNS hardware to speed the correlation process, which for typical sub-volumes of $64 \times 128 \times$ 400 sample points would execute in 11 seconds. This rapid execution time includes all memory exchange overhead as well as forward and inverse number theoretic transforms (NTTs) and point spread function (PSF) multiplications.

The hardware design concentrated on a custom memory management processor and RNS computational modules. Although no hardware was fabricated, it is believed that a commercial system could be realized with conventional electronic components operating at a base clock frequency of $10 \mathrm{MHz}$.

A FORTRAN-coded software simulation was developed in conjunction with the analysis phase of this effort. Through the use of this code, it was determined that data volume slicing, overlap save, and frequency domain techniques could be used effectively in the real-time hardware implementation.

It was determined by analysis and simulation that the PSF is the critical element in the effective use of FSAFT for inspection. The PSF may be customized by the user to affect a variety of reconstruction results. Most important of these is the ability to mold the PSF to enhance unusually difficult flaw characterization problems. However, more research is required to better understand the nature of optimum PSFs and their limitations in the FSAFT algorithm. Not only can the PSF be customized for direct imaging, but it may also be hybridized to include image enhancement processing within the reconstruction process. The overhead incurred by forming a composite PSF is zero. Therefore, FSAFT imaging can give the user greater capability for characterizing flaws than other methods, and can do so without any additional computational burden.

\subsubsection{An Ultra-High Speed Residue Proces- sor for SAFT Inspection System Image En- hancement, Final Report, October 1983 through March 1984 (Polky and Miller 1985)}

The Phase-I feasibility study of residue number system (RNS) image processing for SAFT inspection has successfully determined that an advanced inspection system may be built using a correlation-reconstruction SAFT algorithm, implemented with RNS techniques and offthe-shelf electronic components. Images are reconstructed in a number of theoretic transform components. All computations can be made in a highly parallel computational architecture. These methods also allow image enhancement to be easily performed for improved flaw visualization, and with negligible speed reduction. It has been determined that high-resolution three-dimensional flaw images may be generated and that a commercially viable product could result through development of a prototype real-time RNS processor. The hardware is expected to be made up of 100 -nsec bit slice microprocessor components and large RAM storage units. Such a processor would provide the NDE community with a valuable new tool that could generate significant improvements in real-time flaw visualization of critical components encountered in nuclear power plant inspection. Special inspection conditions, such as multiple reflections and crack tip detection in the presence of corner reflecting effects, may be overcome by modifying the functional form of the PSF. It is also possible that semiautomatic flaw classifications could be included in the process without significantly adding to the computational burden. Based on the performance estimates of the Phase-I effort, this new image processing system has the potential to acquire and focus the equivalent of $145 \mathrm{~A}$-scans per second, which translates into an inspection rate of more than 1000 cubic inches per minute for typical pressure vessel specimens. A near-term prototype version can be fabricated, using some disk memory to reduce risk and cost, with a throughput of 40 focused A-scans per second. This prototype design may be easily upgraded to the higher throughput rate for a future production model.

\subsubsection{Design and Development of a Special Purpose SAFT System for Nondestructive Evaluation of Nuclear Reactor Vessels and Piping Components (Ganapathy et al. 1985)}

This technical report describes research work performed by the Ultrasonic Imaging Laboratory for the U.S. Nuclear Regulatory Commission (NRC) in fulfillment of NRC Contract No. NRC-04-75-182, "Ultrasonic Imaging Techniques for Real-Time In-service Inspection of Nuclear Power Reactors." 
The purpose of this multiyear contract (1980-1983) was to develop a field-implementable real-time system based on Synthetic Aperture Focusing Techniques (SAFT) developed previously at the University of Michigan. To accomplish this objective, the first year's effort was spent on implementing improved display techniques and analyzing potential bottlenecks in the development of a field-implementable real-time system. The second year's effort concentrated on the detailed analysis of processing requirements and initial design of a SAFT processor. The results of the first two years' efforts are detailed in the NRC reports NUREG/CR-2154 (October 1981) and NUREG/CR-2703 (January 1983), respectively.

The final year was devoted to the detailed design of the special purpose SAFT processor and an overall design of a field-implementable SAFT-UT system. The design specifications were subsequently handed over to General American Research and Development, Incorporated, as part of a subcontract to enable them to build a prototype. This prototype was successfully fabricated by late 1983 and met the overall objectives of the design. This report, the third and final annual report, discusses the design details and the results of the construction of the SAFT processor and includes recommendations on the other components of a field- implementable NDE system based on SAFT.
The work was performed in the Ultrasonic Imaging Laboratory, Department of Electrical and Computer Engineering, the University of Michigan, Ann Arbor, Michigan. The project was performed under the technical direction of Prof. S. Ganapathy. The project monitor was Dr. J. Muscara of the U.S. Nuclear Regulatory Commission.

This technical report covers work done primarily over the period January 1, 1982, to September 30, 1983. The details of this report are based heavily on the results reported in earlier reports, primarily NUREG/CR-2154 and NUREG/CR-2703. Details of previous work on all aspects of this research are available in one or more of the following NRC technical reports:

NUREG-0007-1: January 1976

NUREG-0007-2: September 1977

NUREG/CR-0135: May 1978

NUREG/CR-0581: January 1979

NUREG/CR-0909: September 1979

NUREG/CR-2154: October 1981

NUREG/CR-2703: January 1983. 


\subsection{SAFT-UT Field System Operating Procedures}

This section provides a general operating procedure for the U.S. Nuclear Regulatory Commission (NRC) fieldusable Synthetic Aperture Focusing Technique for Ultrasonic Testing (SAFT-UT) system.

The result of the PNL effort to develop a fieldable SAFT-UT system is housed in three transportable racks and consists of four functional parts: the host computer (a VAX-11/730), graphics processing and display, data acquisition, and real-time processor. A detailed hardware description may be found in Appendix A of this document. All of the SAFT software utilities referenced throughout this section are described in detail in The SAFT-UT Real-Time Inspection System - Operational Principles and Implementation (Hall, Reid, and Doctor 1988).

The focus of this section is on operating the SAFT-UT field system. Each section will discuss a different aspect of the SAFT data acquisition and/or analysis.

Section 4.1 covers turning on the power, interfacing with the data acquisition software, making preliminary adjustments, and beginning a scan.

Section 4.2 deals with pulse-echo data acquisition. It includes a discussion of things to consider when scanning piping or thick-section vessels.

Section 4.3 gives detailed information about the three available tandem configurations. Application of the tandem configuration on piping and thick-section vessels is also covered.

Finally, Section 4.4 provides an insight to both the strengths and limitations of the SAFT-UT field system as it pertains to the analysis of acquired SAFT data sets. The logic used to analyze both pulse-echo and tandem images has been developed over the years and is a result of reviewing the large data base that PNL has acquired. A description of the analysis logic is provided.

The integrity of the data base is important. No amount of sophisticated processing will help the resultant image if the data base is of a low quality. One must always pay close attention to details when using the SAFT-UT field system to ensure that the mechanical scanner positioning, front-end analog adjustments, and file header information are correct. It has been our experience at
PNL that the robust nature of the SAFT technique and algorithm provides very high-quality ultrasonic imaging, and that the majority of erroneous interpretations have been due to errors made during the data acquisition phase.

Consequently, this section will lay out a procedural format that can be followed by new SAFT-UT users to provide them with the information necessary to acquire and analyze SAFT-UT data.

\subsection{Getting Started}

This section provides the user with background information that should be read prior to actually operating the SAFT-UT field system. It then goes on to explain how to turn on the system, interface with the data acquisition software, and make preliminary adjustments to the SAFT-UT equipment. Everything necessary to set up a particular scan configuration and collect SAFT data is covered. Figure 4.1 provides a picture of the SAFT-UT field system identifying switches, knobs, and/or modules by a number. Each function is subsequently described in this section. As each switch, knob, and/or module is described, a number in brackets (e.g., $<1>$ ) corresponds to one of the numbers in Figure 4.1, so the reader can easily identify the physical location.

\subsubsection{Background Information}

To operate the SAFT-UT field system properly, one must have a basic understanding of the SAFT philosophy and scanning conventions that are used. Figure 4.2 graphically shows the scan conventions assumed throughout the SAFT software set. The $Z$ axis represents depth into the material with the axis origin located at the surface of the material; the deeper into the material, the larger the value of $Z$.

The $\mathrm{X}$ axis is the scan axis at the surface of the material and is normally perpendicular to the wall being scanned. The $\mathrm{Y}$ axis is the increment axis and is parallel to the weld being scanned. The scan mechanism is assumed to have

1. traveled in the positive $\mathrm{X}$ direction while collecting a plane of data

2. incremented in the $Y$ direction 
3. scanned back to the original position of $\mathrm{X}$ without collecting data

4. again traveled in the positive $\mathrm{X}$ direction collecting the next plane of data.

It is essential that the data file A-scan records be ordered in this manner. An A-scan record consists of a predetermined number of points describing the rf waveform along the $Z$ axis. SAFT utilities have been developed to transform data that have been acquired with different axes orientations.

For angle-beam applications, care must be taken to conform to the incident angle convention. Universally, the utilities expect the transducer to be tilted along the $X$ (scan) axis and in the negative direction, as shown in Figure 4.2. If the transducer is tilted so that the sound field precedes it, then this is considered a negative incident angle. In most applications, the incident angle will be negative. Again, SAFT utilities have been developed to transform data that has been acquired using a positive incident angle.

\subsubsection{Power-Up and Log-On Procedures}

Ensure that POWER SWITCHES $<1,2,3,4\rangle$ (no particular order) are in the ON position and that the DC $\underline{\text { PWR }}<5>$ and $\underline{\text { CLK }}<6>$ buttons are depressed. Two more power switches must be ON; the first is located at the rear of the DISK DRIVE $<7>$ unit, and the second is located at the rear of the DEC-11/23 $<8>$ microcomputer. Each of the aforementioned switches is normally left in the $\mathrm{ON}$ and/or depressed position.

Turn on the main power to the DATA ACOUISITION RACK $<9>$ by switching the MAIN POWER SWITCH $<10>$ to the ON position; observe that the $\underline{\text { RUN }}<11>$ light is lit, indicating that the DEC-11/23 microcomputer is on line. Turn on the power to the host VAX and the rest of the SAFT-UT field system by turning the MAIN POWER KEY SWITCH $<12>$ to the LOCAL position. Depress the RUN/STOP $<13>$ button to bring the disk drive up to speed (observe that the READY $<14>$ light is lit after the disk drive comes up to speed). The DEC-11/23 microcomputer is now ready for the user to log on and for the host VAX to be booted.
The terminal, connected to the data acquisition rack, should now be prompting the user for a date entry (Figure 4.3--remember to include any dashes or colons); enter the date using the prompted format (dd-mmmyy). Next, the user is prompted for the time (Figure 4.3); enter the time using the prompted format (hh:mm:ss). Once the user has entered the date and time, a period appears on the terminal; this is the DEC-11/23 microcomputer prompt symbol. The user is now in the RT-11 operating system environment and may perform any of the allowable RT-11 commands. SAFT data acquisition is controlled by the DEC-11/23 microcomputer, which performs the executive function; it monitors and supervises the scanner stepper motor controllers, data digitization, delay gate generator, and position encoder circuitry. Storage and interface functions, with respect to communication with the field host analysis computer (the VAX-11/730), are also controlled by the microcomputer. The data acquisition microcomputer software serves as the operator interface for the real-time mode of operation. A menu-driven format is used to ensure that the user will correctly enter, prior to the scan, SAFT parameters that are used to process the raw data file.

The host VAX needs to be booted. Press the return key on the terminal connected to the host VAX (console port); the prompt symbol ( $>>>$ ) should be seen. Type, $>>>$ B <return $>$ to start the booting process. Upon successful completion of the boot process, the terminal should be prompting the user, "PLEASE ENTER DATE AND TIME (DD-MMM-YYYY HH:MM)." Enter the date and time in the promoted format and press the return key. Some initial programs will run and then the final message "The PNL SAFT FIELD VAX VAX-11/730 system has been rebooted" will be presented to the user. The SAFT-UT field system is now ready to use. The user must invoke the data acquisition software that will prompt the user for information to begin collecting, processing, and displaying data.

\subsubsection{Initiating the Data Acquisition Soft- ware}

A command file named "SCAN," executed by typing @SCAN <return> (Figure 4.3), places the user in the SAFT data acquisition program. Figure 4.4 presents what the user will see next on the terminal. The user is 
first prompted to select the transducer configuration. A variety of common scanner configurations are presented to the user, each with various standoff options. The appropriate response is to type $>$ a number, 1 through 8) <return>. Next, the user is prompted to select the scan axis to be used (Figure 4.4; pulse-echo configurations only); type $>(1$ or 2$)<$ return $>$. Finally, the user is prompted to enter if this scan is to straddle (transducer scanning from the side opposite the scanner location) the object plane or not. This entry is necessary to ensure that the scan axis will be directed to travel in the correct direction to collect data in the standard SAFT format; type, $>(\mathrm{Y}$ or $\mathrm{N})<$ return $>$. Figure 4.5 presents what the user would see if a tandem configuration were selected.

The user is now presented the menu in Figure 4.6, which allows (1) viewing the header parameters, (2) editing the transducer characteristics, (3) editing the material characteristics, (4) modifying the sampling data, (5) modifying the scan parameters, $(0)$ continuing to the next menu, and (-1) modifying the configuration mode. Each option may be exercised by selecting the appropriate code, $>(1,2,3,4,5,0$, or -1$)<$ return $>$.

\subsubsection{General Header Requirements}

If the user types $>1<$ return $>$, the complete header information is viewed on one screen, as shown in Figure 4.7 (pulse-echo configuration) and 4.8 (tandem configuration).

The transducer header information (Figure 4.9) consists of items such as transducer frequency, standoff criteria, angle of incidence, full beam angle, and beam entry diameter. Parameters such as the refracted angle and $\underline{\mathrm{f}}$ number are calculated from the header information. Frequency, entered in megahertz, is used for reference only and has no effect on the outcome of the processed image. However, the standoff height and velocity affect the initial delay, which determines the depth along the sound path where digitization begins. The standoff height is entered in inches, and the standoff velocity is entered in inches per second. An error in the entry of these parameters will result in the initial delay (period of time from the initial pulse to where digitization of the rf waveform begins) being incorrect. An error in the initial delay may result in
- not digitizing the area of interest

- an error in the actual starting depth.

An error in the actual starting depth affects the correlation of the data when the SAFT algorithm is applied. The resulting processed image would most likely be of poor quality. The incident angle is the angle of the central ray (before entering the material) relative to an imaginary line perpendicular to the near surface of the material under test and is entered in degrees. If the sound field precedes the transducer as it is scanned, then the angle is negative. Errors in the incident angle will affect the initial delay and the resulting image in the same way as errors in the standoff parameters. The full beam angle, also entered in degrees, will affect only the processed image. It determines the size of the synthetic aperture to be used when SAFT processing is performed. A calculated value may be entered initially using Equation (4.1) for a small-diameter, flat piston radiator:

$$
\text { Full Beam Angle }=\sin ^{-1} \frac{0.5 * \lambda}{d}
$$

where: $\lambda=$ the wavelength in the material given in inches (of the transducer's center frequency)

$d=$ the diameter of the transducer element given in inches.

Most likely, the full beam angle will be altered later using the DEDIT (Hall, Reid, and Doctor 1988) utility and a new process file generated. Typically, small beam angles are used initially to reduce the processing time and larger angles are used later if a higher image quality is desired. Beam entry diameter, in inches, affects only the processed image and is normally zero. SAFT assumes a synthetic aperture with a point source at the beginning, expanding in the general shape of a cone. Since the aperture is small at the near surface, the number of off-center A-scans used during processing is also small. The front surface echo is often very large and degrades the near-surface image; one may take advantage of the spatial averaging inherent in the SAFT processing by entering a value for the beam entry diameter (a diameter normally equal to one-half the transducer element diameter is entered). The effect on the synthetic aperture used in processing is to create a 
cylinder with a diameter equal to the beam entry diameter parameter that would extend into the material until it intersects the normal aperture cone.

The material category (Figure 4.10) includes specimen velocity, thickness, and pipe radius. Material velocity will affect the initial delay. Therefore, the start of the digitization window will be incorrect if an error exists in the material velocity entry. As with the standoff and incident angle parameters, material velocity errors may result in not digitizing the area of interest and/or poor image quality. Material velocity is entered in inches per second.

In the tandem configuration, both the material thickness and the velocity will affect the position of the image. Experimental results at PNL show the tandem image integrity is not compromised when small errors (up to $\pm 20 \%$, assuming the area of interest was digitized) in thickness and/or velocity exist; however, the image will be mispositioned within the image space. For this reason, the pulse-echo images are relied on for confirmation of the defect location. In the pulse-echo configuration, the material thickness, in inches, is used as a reference only, indicating the near-to-far surface extent of the scanned object. The pipe radius entry is used as a reference value and is entered in inches. If the object is flat, then "flat" is entered.

Sampling (Figure 4.11) includes items related to the temporal sampling of the waveform; i.e., sample period (entered in nanoseconds) and start/stop of the sampling window (entered in inches along the sound path). Parameters such as the number of points and initial delay are calculated from the header information.

Temporal sampling (sample period) is a very important parameter in a SAFT-UT system and should always be a minimum of one-fourth the wave-length period (i.e., the wave-length period of $2.25 \mathrm{MHz}$ is $444 \mathrm{nsec}$; a temporal sampling of $100 \mathrm{nsec}$ will provide at least four samples per cycle). Generally, the image quality improves as the temporal sampling is increased. The sample period may range from 500,000 to 50 in a $5,2,1$ sequence. A sample period of $30 \mathrm{nsec}$ (primarily used for frequencies of $5 \mathrm{MHz}$ and greater) may be achieved by removing the cable from the LeCroy 8501 Programmable Clock $<15>$ clock 1 output jack (the other end of the cable is connected to the Programmable Pulse Divider $<16>$ ) and connecting it to the clock output jack of the Lecroy TR8837F Transient Recorder $<17>$. An entry of $30 \mathrm{nsec}$ is then entered for the header sample period. One must remember to switch the cable back to the external clock if sample periods other then $30 \mathrm{nsec}$ are desired. The start sampling point is entered in inches along the sound path in the material and marks the beginning of the digitization window. The stop sampling point is also entered in inches along the sound path in the material and marks the end of the digitization window. If the digitization window is not adequate, aperture limiting will occur and system resolution will degrade.

Finally, lateral scan parameters (Figure 4.12) are displayed. Default values are initially presented. All of the scan parameters are entered in inches. The initial receiver $X$ relative to the weld center entry is for reference only. This entry places an arrow (when the image is viewed with one of the graphics utilities) where the weld center or some other reference line should be relative to the image space. In the tandem configuration (Figure 4.13), one additional scan entry is the transmit $X$ relative to the initial receiver $X$ and is measured from the center of each beam entry point. This entry is critical in tandem configurations; an error will result in poor image quality. In the tandem configuration, the number of transmit $1 / 2$ vees to the object plane are also displayed for reference information. The scan increment: $X, Y$ axes, $X$ scan length, and $\underline{Y \text { scan }}$ length are straightforward entries. However, in a SAFT-UT system, one must remember that the $X$ and $Y$ increments are the spatial sampling parameters and generally should be no greater than one wave-length in the material under test. As the spatial sampling becomes more dense, the image quality typically improves. Physical aperture limiting may occur if the $X$ and $Y$ scan axes lengths are too small. Often, aperture limiting (physical or synthetic) results in the edges of the image being poorly defined.

In general, the header entries should be made with great care. A wrong entry could produce erroneous results; however, mistakes may be corrected later using the host computer DEDIT utility. Often the correct values are not available if much time has passed since the data was collected.

At this time, some preliminary hardware settings need to be verified before a " 0 " is selected to present the next menu. Specific setup considerations for pulse-echo and 
tandem configurations will be addressed in the sections reserved for each configuration. The remainder of this section will address the preliminary settings and/or adjustments of the Pulser/Driver, Mode Selection, and Time Variable Gain (TVG) modules. Beginning a scan, data acquisition software entry points, and system shutdown procedures will also be addressed.

\subsubsection{Pulser/Driver Setup}

Before selection "0" (Figure 4.4) is entered and the Command Selection Menu in Figure 4.14 presented, a manual repetition rate must be selected. When in the command selection menu, the digitizer is read each time the return key is pressed. If no repetition rate is selected, no sync pulse is provided to the digitizer, and the DEC-11/23 microcomputer is in an endless wait loop with nothing else happening. The Pulser/Driver $<21>$ provides four preset repetition rates that may be selected by pressing the button directly below the series of light-emitting diodes (LEDs) labeled "REP RATE." With each push of the button, a new repetition rate is selected. The upper LED indicates "REMOTE." In the remote position, the repetition rate is provided by the Programmable Pulse Divider $\langle 16\rangle$ module when each scan increment value is reached. To the right of the bottom LED is a screwdriver-adjustable potentiometer that allows the repetition rate to be set to a userspecified value.

A range of pulse widths is available and may be selected by pressing the button to the right of the repetition rate selection button. Each time the pulse-width button is pressed, the corresponding LED lights to indicate the range selected. The adjustment just above the pulsewidth selection button is for fine-tuning the pulse width to produce a peaked symmetrical echo when looking at the TVG amplifier output.

\subsubsection{Mode Selection}

Mode selection is accomplished by pressing one of four pushbuttons on the Mode Selection Module $<18>$. The upper button selects the front transducer for pulseecho mode. The next button selects the rear transducer for pulse-echo operation. Tandem configurations are selected by pressing the third button for a front-source and rear-receive transducer, or the fourth button if a rear-source and front-receive transducer is desired.
When power is first applied, the SAFT-UT system defaults to the tandem mode with the rear transducer being the source and the front transducer being the receiver.

\subsubsection{Time Variable Gain Setup}

Both the TVG amplifier "RF output" and "DC ramps" may be viewed on the Tektronix Oscilloscope Monitor $<20\rangle$. Channel 1 of the oscilloscope monitors the TVG amplifier output, and the sensitivity should be set to $0.1 \mathrm{~V}$ per division. The input to the digitizer accepts a $0.5-\mathrm{V}$ peak-to-peak signal. Channel 2 of the oscilloscope monitors the TVG ramp and the sensitivity should be set to $1 \mathrm{~V}$ per division. The TVG ramp varies from 0 to $5 \mathrm{~V}$. The Time Variable Gain Amplifier $<19>$ may be used to compensate for material attenuation and/or reduce the front-surface echo (pulse-echo configuration). Normally, a calibration block with defects at various depths is used to adjust the slope of the TVG amplifier to obtain equal amplitude echoes. The upper pair of adjustments control the slope starting time relative to the initial pulse and the initial gain. Each of the next three pairs of adjustments allows a complex TVG slope to be set. Each pair adjusts the slope segment starting time relative to the end of the previous slope segment and the gain of that segment. The final gain is controlled by the bottom adjustment. When a scan is being set up, a flat gain should be used to find the echoes of interest. Then, a TVG slope may be set using calibration block echoes to determine the gain slope of the TVG amplifier.

\subsubsection{Beginning a Scan}

To proceed to the command selection menu, ensure that a manual repetition rate is selected and type $>\underline{0}$ $<$ return $>$. The initial delay is shown (Figure 4.15), and the user is given the opportunity to enter a different value. CAUTION: This value determines where along the sound path (usually the standoff time plus the desired depth in the material) digitization will begin, and erroneous results may occur if this value is incorrect. If the user does not wish to change the initial delay, the return key should be pressed. The header is presented (Figure 4.16) one last time so it may be checked. Pressing the return key again presents the Command Selection Menu shown in Figure 4.14. This menu is intended primarily to allow the user to reset encoders, 
position the scanner, set gains, and verify signal integrity. After placing the scanner in a reference location, the user may then select "RS" to reset the encoder counters and establish a "home" position. Precise movement of the scanner may then be performed by selecting "X1," "X2," or "Y" (Figure 4.17) depending on which axis the user wishes to reposition. Each time a selection is made, the information at the top of the screen (Figure 4.14) is updated. This information is related to the actual digitized waveform and scanner position. Pressing the return key will digitize a new waveform at the current location of the scanner. At this time, the user may view the oscilloscope that displays the digitized signal to verify expected echo timing and signal gains.

The user may select a simple "help" facility from this menu by entering "HE." Also, the header parameters may be viewed by selecting "VI," or control may be passed to the previous menu by selecting " $\mathrm{CM}$ " (change menu) or "CS" (change to scan parameters).

When the operator is convinced that the parameters are correct and the scanner is positioned to begin a scan, the "SC" (scan) option is selected and control is passed to the menu shown in Figure 4.18. The user must now decide if the data acquisition system is to transfer the collected data to the host VAX-11/730 computer directly or store it on the local disk drive. Normally, the data is transferred directly to the host VAX. However, if the data acquisition system is being operated in a standalone mode, then local storage would be selected. If transfer to the host VAX is selected, a virtual terminal program is invoked, making the DEC-11/23 microcomputer emulate a terminal and connecting the user to the VAX host computer. Figure 4.19 shows what the user would see on the terminal screen. It provides the user with some cues about what to do next. If the user has not previously invoked the virtual terminal program (it may be invoked stand-alone by typing . Run VT < re-

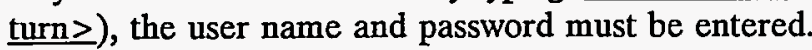
The "\$" is the VAX VMS prompt symbol. Once it appears, the user is in the VMS operating system environment and may invoke any of the VMS commands or utilities. To begin a scan, the user must first set the default directory in which data will be stored in using the VMS change directory format; i.e., type $\$$ set default [directory tree] <return>. If a new directory is to be created, type $\$$ create/directory [directory tree] $\leq$ return $>$. The Scanrd program (Figure 4.20) may then be started by typing $\$$ SCANRD < return>. This command invokes the SAFT data collection utility on the host VAX. It prompts the user to determine if an existing data file will be used as the input or the data acquisition system will provide the input; it also asks if concurrent processing and graphics will be used. Processing and analysis of the images may take place after the data is collected. If concurrent processing is desired, further parameter input is necessary.

Figures 4.21 and 4.22 are presented on the terminal when concurrent processing is selected. The user is prompted to enter a number of processing parameters. Processing parameters determine

- if envelope detection of the processed file will occur (smoothing of the processed data by removing the high frequencies)

- what threshold value will be used (only data values equal to or greater than -[selected decibel level] of the maximum value will be processed)

- the normalization mode - If a value falls below the threshold value, either 0 or the raw value will be used; and if the point is processed, it will be divided by one of the following: a) number of offcenter values that were summed, b) a constant, or c) the square root of the number of off-center values that were summed. Each normalization option also provides the choice of substituting zero or the raw data value for data not processed.

- data sampling adjustment: no, automatic, or manual sampling adjustment - The automatic sampling option adjusts the $Z$ axis increment of the output file to be equal to the $X$ and $Y$ axes. Therefore, a smaller processed file will be created. Manual sampling provides the user with a method of setting the $X, Y$, and $Z$ sampling reduction value; i.e., after selecting option 2 (Figure 4.23 for manual sampling adjustment), an entry of 2 for the $Z$ and the $X ; Y$ axes would reduce the data used during processing by a factor of 2 . The $Z$ axis of the processed file may be reduced; an entry of 2 would reduce the $Z$ axis of the output file by a factor of 2 . 
- Finally, the user may select to process beyond the far surface; if "Y" is entered, processing will occur to data that lies beyond the path time to the far surface.

At this time, if a tandem configuration is chosen, some additional processing parameter queries will appear. Refer to Section 4.3.4 (tandem processing parameters) before continuing.

To invoke concurrent graphics, one simply types $\mathrm{Y}<\mathrm{re}-$ turn $>$ in answer to the prompt shown in Figure 4.22. At this time, control is transferred back to the DEC$11 / 23$ microcomputer.

Figure 4.24 shows the final menu in the sequence. Two lines of comments may be entered by the operator related to the particular scan being performed. These comments will become a permanent record in the data file. The desired scanner speed is queried next--a 0 will invoke the default speed value or the user may specify a new value in inches per second. With each stroke of the scanner, the speed is automatically adjusted until the desired scanner speed or the maximum speed relative to the data acquisition loop is achieved.

Finally, the data file name is entered; i.e., $x \times x x x x . x x x$ if the data file is to be created on the data acquisition system or $x x x x x x x x . x x x$ if the data file is to be created on the host VAX. Automatic scanning begins.

At this time, the scanner is told to travel a specified scan-axis distance. At specified increment intervals, the remote pulser is triggered. After the initial delay period, the transient recorder digitizes the analog signal and this waveform is stored in memory. At the end of a single scan or stroke, the full scan length of data is transferred to the host computer while the scanner is returning (data is taken in only one direction). If concurrent processing and data display are selected, the host computer will begin processing each scan line of data and present an image on the graphics display when the number of scan lines of data collected equal half of the synthetic aperture width. The user may view the image while scanning is in progress.

The preceding is a description of the menu sequence used to initiate a typical SAFT scanning process. The menus have been designed to provide a logical sequence to help the user define all the necessary
SAFT-UT parameters. Collecting data can be a timeconsuming and difficult task. Therefore, a little extra time spent ensuring header parameters are correct may preclude repeating data collection.

\subsubsection{Data Acquisition Software Entry Points}

The data acquisition software is actually a chain of programs, and program entry may be at a different link in the chain than the beginning. The sequence of events will occur normally from the point of entry. There are five main program entry points:

- SCANMN (to execute, type .run scanmn $\leq$ return $>$ )

- QUERY (to execute, type run query < return>)

- SCANMU (to execute, type .run scanmu $\leq$ return $>$ )

- $\quad$ SCANVT (to execute, type .run scanvt <return>)

- $\quad$ SCANPT (to execute, type run scanpt <return >).

A virtual terminal program called VT may be run (type .run VT <return>) to communicate with the host VAX; however, do not attempt to run SCANRD on the host VAX because the serial interface does not get initialized properly. The user must run the SCANVT program to initialize the serial interface; SCANRD may then be invoked on the host VAX.

The user must remember that when the data acquisition program is entered at other than the beginning entry point, previously specified parameter values will be used for that part of the software that is by-passed. This attribute is desirable if the user is doing multiple scans that have identical parameters.

\subsubsection{System Shutdown Procedures}

If the field system is to be powered down after use, type CNTL $\mathrm{P}<$ return > on the terminal connected to the host VAX. The user will see the system prompt symbol $(>>>$ ); type $>>>\mathrm{H}<$ return $>$ to halt the host VAX. Press the RUN/STOP $<13>$ button to spin down the disk. When the READY $<14>$ lamp goes off, the SAFT-UT field system power may be shut 
off using the MAIN POWER SWITCH $<10>$ and the MAIN POWER KEY SWITCH $<12>$.

Sections 4.2 and 4.3 will address configuration-specific details, and Section 4.4 will discuss image analysis.

\subsection{Single Transducer Pulse-Echo Op- eration}

A single transducer to transmit and receive the sound wave is the fundamental configuration used with the SAFT-UT field system; i.e., the same transducer used to generate the sound field in the material under test is also used to receive the return echo. A rectilinear pattern is obtained by incrementing the scanner one increment at the completion of each stroke of the scanner.

This section will discuss the various uses of the pulseecho configuration and how to apply that configuration to piping and/or thick-section vessels.

\subsubsection{Pulse-Echo Configurations}

When the central ray of the transducer's sound beam is perpendicular to the material under test, the inspection is referred to as a normal-beam inspection. If the central ray of the transducer is not perpendicular to the material under test, then it is referred to as an anglebeam inspection.

A normal-beam inspection may be performed by using a focused transducer, housed in a water column (Figure 4.25 ), with the focal point of the transducer placed at the near surface of the object. This placement produces a diverging field of sound within the material under test. Volumetric defects, geometrical conditions, and even weld location may be imaged using the normalbeam configuration. Application of the SAFT processing to the raw data set focuses the entire volume, not just a single plane as with conventional methods. Therefore, a single normal-beam inspection may reveal a multitude of information about the material under test.

An angle-beam inspection is performed if the defect presents a small cross-sectional area when a normalbeam inspection is performed (such as cracks). Often, many different refracted angles are tried (if the actual orientation of the defect is not known) before the optimum inspection angle is found. The desired scenario is for the central ray of the transducer to be normal to the defect, producing a higher-amplitude echo and yielding the best signal-to-noise ratio. If a focused transducer is to be used, the focal point is placed at the near surface. A water column is used to provide the proper standoff and the coupling of the sound wave into the material. The water column is tilted at an angle to provide the desired refracted angle of the sound field within the material. A small-diameter, contact transducer may also be used for angle-beam inspections by using a Lucite wedge to provide the proper incident angle (Figure 4.26) and an ultrasonic gel to provide the coupling of the sound wave into the material. Typically, shear waves are generated in the material, although longitudinal waves are sometimes used when investigating cracks to enhance the tip-diffracted echo. Tip-diffracted echoes are very weak when compared to the high-amplitude, corner-trap echo (the corner-trap echo is from the intersection of the crack and the far surface of the material) and are difficult to distinguish from acoustic noise. Longitudinal waves produce a loweramplitude echo from the corner-trap, making the tipdiffracted echo more distinguishable because it has similar amplitude. Because of the nature of the pulseecho algorithm (direct path to the defect), thickness and velocity errors have a minimum effect on the location of the defect within the image space. Therefore, the pulse-echo configuration is relied on for defect detection and location.

One may use any of several pulse-echo configurations to characterize a material under test, profiting from the full-volume focusing and spatial averaging that a SAFTUT system provides. One of the most common applications of SAFT-UT has been imaging cracks associated with welds that join two sections of pipe together. The next section describes the procedures commonly used by PNL staff to collect data on pipe sections with the SAFT-UT field system.

\subsubsection{Piping Considerations}

Piping comes in many different diameters and thicknesses. The primary problem one encounters with any piping is the counterbore cut normally done to pipe when preparing to weld two sections together. Other problems include coupling the sound into the material, 
physical alignment of the scanner, and physical dimensions of the pipe.

Often, echoes from the geometry of the counterbore are interpreted as cracks. One may use the normalbeam image to profile the far surface of the pipe, identifying possible geometrical conditions created by the counterbore or other weld preparation procedures. SAFT is particularly affected by counterbore problems due to the large physical aperture scanned and the divergent nature of the sound within the material. Return echoes from the far surface, to the defect, and back to the receiver will not be the path length expected if the counterbore is very severe. Image quality may degrade because SAFT assumes that the near and far surfaces will be parallel. Normal-beam scans are a must when scanning pipes to determine the severity of the counterbore.

Near-surface shrinkage in the area of the weld is common when pipe sections are joined by a weld. Shrinkage of the near surface may add to the already difficult problem of coupling the sound into the material. Coupling is always a problem but even more so when scanning a pipe, because often one must scan the pipe in a position other than horizontal. PNL has favored mineral oil as a couplant/lubricant. Ultrasonic gel adheres better when the pipe is vertical or overhead. Therefore, the gel is the usual choice in these situations. The pressure applied to the wedge or water column also plays a part in how well sound is coupled into the material; too much pressure and the wedge or water column tends to act as a squeegee, reducing, or in some cases totally eliminating, coupling of the sound into the material. Plastic wedges should be arced to conform to the pipe curvature, and grooves may be filed into the front, back, and sides to improve the flow of the couplant under the wedge, reducing the chances of the wedge acting like a squeegee.

Accurate location of a defect is dependent on the care taken to enter valid header information and the physical set up of the scanner. Typically, the types of defects one looks for in piping are cracks that lay along circumferential welds. Defect location is relative to a circumferential scribe reference line, usually describing the center of the weld (the normal-beam image helps certify this assumption). Care must be taken when making measurements from the transducer to the scribe line; all measurements should be taken from the central ray, where it enters the material under test, to the scribe line. Access to each side of the weld may be limited. Often, data is taken on the far side of the weld with the scanner track sitting on the near side of the weld. In this case, the transducer "straddles" the weld and is looking back toward the scanner. Straddling the weld is more convenient because the scanner and the track need not be moved. Only the transducer holder is rotated $180^{\circ}$, which is a simple process. One must always check that there is enough "throw" in the scan axis to allow a sufficient physical aperture to be scanned; if not, the scanner track may need to be moved closer to the weld center line.

The size of the piping is also important when considering SAFT-UT. A small radius pipe presents two problems. First, the radius of the pipe creates an error between the desired distance to be moved and the actual distance moved along the circumferential axis. The mechanical scanner must always be calibrated along the circumferential axis by moving the scanner along the $\mathrm{Y}$ axis, under program control, a predetermined distance (usually 6 in. $[15.2 \mathrm{~cm}]$ ). Verify the actual distance moved and edit the AMAPS.SCN file to reflect the correct counts per inch needed for both the $\mathrm{Y}$-axis steeper motor and encoder. Equation (4.2) may be used to determine the corrected counts-per-inch value.

$$
\frac{\text { corrected counts-per-inch }}{\text { existing counts-per-inch }}=\frac{\text { predetermined distance }}{\text { actual distance } \text { moved }}
$$

Second, the pipe thickness may be a problem. SAFT uses a divergent sound field, as previously mentioned, and the aperture is very small at the near surface. Therefore, not much focussing takes place near the top of the aperture cone. Thin materials inherently limit the aperture size so focusing is limited. One may still take advantage of the spatial averaging inherent in the SAFT processing algorithm when a thin-section material is scanned. SAFT resolution performance improves as the thicknesses of the material increase, permitting a larger physical aperture.

\subsubsection{Thick-Section Considerations}

Thick-section materials such as steel pressure vessels are well suited for a SAFT-UT system. The physical 
size of the divergent sound field is more than adequate, plus usually fewer obstacles prevent scanning of a large spatial aperture. One does need to be mindful of attenuation problems and large data files.

Attenuation problems may be overcome somewhat by setting the TVG amplifier ramp (see Section 4.1.6). The "F number" (transducer diameter to focal length ratio) of the transducer selected should be reasonable. Too low an "F number" may result in a poor signal-tonoise ratio, and too high an "F number" will limit the physical aperture. Experience at PNL has shown that an F4 transducer is a good compromise for most thicksection work.

Large data files become very cumbersome to handle when applying any of the SAFT utilities; for this reason, data files should be kept to a manageable size. When a scan is first set up, being conscientious of the spatial aperture dimensions, digitization window, and sampling density will minimize the file size. Once a defect is localized, a finer grid scan may be performed. Typically, data files greater than 10,000 blocks ( 1 block = 512 bytes) should be avoided. The data file size is calculated and displayed (Figure 4.24) prior to beginning a scan. $A$ control $C$ at this point will abort the scan if the user wants to change some of the parameters to obtain a smaller file size. Equation (4.3) may be used to calculate the data file size.

$$
\text { File size in blocks }=\frac{(x) *(y) *(z+32)}{512}
$$

$$
\text { where: } \begin{aligned}
\mathrm{x}= & \begin{array}{l}
\text { number of points in the } \mathrm{X} \text { dimension } \\
\text { (X length } / \mathrm{X} \text { increment) }
\end{array} \\
\mathrm{y}= & \begin{array}{l}
\text { number of points in the } \mathrm{Y} \text { dimension } \\
\text { (Y length } / \mathrm{Y} \text { increment) }
\end{array} \\
\mathrm{z}= & \begin{array}{l}
\text { number of points in the } \mathrm{Z} \text { dimension } \\
\text { (refer to Section } 4.1 .3 \text { under sampling). }
\end{array} \\
& \begin{array}{l}
\text { The extra } 32 \text { bytes per } \mathrm{A} \text {-scan are for } \\
\text { header records. }
\end{array}
\end{aligned}
$$

Although the pulse-echo configuration is reliable, ambiguous results may be obtained; i.e., a volumetric defect above a surface imperfection may look like the tip-diffracted echo from a deep crack. Figure 4.27 presents a comparison between a pulse-echo image and a tandem image of a 0.3-in.-deep $(0.8-\mathrm{cm})$ semi-circular sawcut in a 0.6 -in.-thick $(1.5-\mathrm{cm})$ aluminum coupon.
The tandem image is clearly easier to interpret. Accurate pulse-echo vertical measurements are difficult because of the elusive nature of the tip-diffracted echo. The tandem configuration was implemented to eliminate the ambiguities present in the pulse-echo configuration and to improve the sizing of vertically oriented planar defects.

\subsection{Dual-Transducer, Tandem Opera- tion}

A single-transducer, pulse-echo configuration works well for location and detection of a defect but may provide ambiguous results. The tandem configuration reduces the ambiguities and improves sizing of vertical defects. Three tandem configurations have evolved (TSAFT, TSAFT-2, and TSAFT-3), and each new configuration has improved the capabilities of the SAFT-UT field system.

\subsubsection{Tandem Configurations}

Fundamentally, tandem SAFT, or TSAFT, consists of a fixed transmitter placed in line with, or in tandem with, a scanned receiving transducer (Figure 4.28). The transmitter is placed such that the divergent sound beam illuminates the primary object area. The receiver is translated to receive the direct energy reflected from the defect area. At the completion of each pass of the receiver, the scanner is incremented so that a rectilinear pattern is obtained. Physically the transmitter must be positioned far enough behind the receiver to not aperture-limit the receive transducer (receiver physically running into the transmitter). Typically, the transmitter is placed $3 / 2$ vees back from the vertical object plane (the central ray strikes the far surface, the near surface, and the far surface once more before illuminating the object plane [path 2 of Figure 4.29]), providing enough rQom for the receiver to scan an adequate spatial aperture. In the case of surface-connected defects (cracks), the receiver is scanned over a larger spatial aperture so that echoes from path 2 and path 3 (Figure 4.29) may be received. When both paths are collected and processing occurs beyond the far surface, the result is the real and the conjugate image (Collins and Gribble 1986). Because multiple bounces off the surfaces of the material are used in the TSAFT configuration, errors in the material velocity and thickness affect the location of the defect relative to the far surface and the illuminated 
object plane. As with pulse-echo, severe counterbores or other irregularities in the far surface will degrade the image quality through distortion of the sound fields. Because of these variables, errors in vertical sizing would occur if the far surface were used as a reference to size the vertical extent of a surface-connected defect. For this reason, sizing is accomplished by measuring the extent of the real and conjugate images and dividing the resultant value by two.

The TSAFT implementation does not uniformly illuminate the vertical object plane, because the transmitter is stationary with a limited sound field aperture (due to transducer size and refraction effects) and the intensity of each ray within the aperture is not constant. The amplitude of the received echoes vary because of not only the characteristics of the defect but also the intensity of the sound field illuminating the defect. Depending on the thickness, some of the vertical image plane might not even be illuminated. If a defect exists in an area not illuminated, it would go undetected. Vertical sizing of defects was also influenced by the intensity distribution of the sound field; deeper cracks were not uniformly or fully illuminated, and the amplitude of the upper structure would drop-off rapidly, causing one to undersize the vertical extent.

A second implementation of tandem, called TSAFT-2, provides a uniform illumination of the vertical object plane. The central ray of the transmitter's divergent beam is always centered on the receive transducer by scanning the transmitter synchronous but in the opposite direction with the receiver. Since a more uniform illumination of the vertical object plane is possible, near-surface defects will be detected, and the vertical extent of a defect will be accurately measured.

Thick-section materials inherently prohibit the use of multiple bounce paths normally associated with TSAFT modes; attenuation of the sound field becomes intolerable. A practical solution is to limit the number of bounces to one (before the sound strikes the vertical object plane). Figure 4.30 shows the center ray paths to the vertical object plane for a 1/2-vee TSAFT-2 configuration. The difficulty using TSAFT- 2 in this configuration is the physical limitations; i.e., the transmitter can scan forward only until it hits the receiver. Unfortunately, a portion of the real image (path 2, Figure 4.29) and most, if not all, of the conjugate image (path 1, Figure 4.29) is lost. Figure 4.31 shows the
TSAFT-2 image (using $54^{\circ}$ transmit and $47.2^{\circ}$ receive inspection angles) of a 0.7 -in.-deep $(1.8-\mathrm{cm})$ sawcut that has been placed in the far surface of a 9-in.-thick (22.9$\mathrm{cm})$ carbon steel block. The B-scan end view reveals two weaknesses of this configuration. First, a portion of the real image is missing; second, the conjugate image is highly degraded.

A third tandem configuration, a special case of TSAFT2 called TSAFT-3, was implemented for thick-section vessels where it was not practical for the transmit transducer to be 3/2-vees from the vertical image plane due to the long material paths attenuating the sound field. TSAFT-3 overcomes the physical problems of TSAFT2 , allowing the transmit transducer to be $1 / 2$ vee from the vertical object plane. The transmit transducer is offset [when small Lucite wedges are used, the offset is typically $0.75 \mathrm{in} .(1.9 \mathrm{~cm})$ ] such that it passes by the receive transducer in a side-by-side fashion as shown in Figure 4.32. There are no provisions to compensate for the offset distance between the transmit and receive transducers; the long material paths from the transducers to the vertical object plane make the effect of the offset negligible when small transducers are used. The transmitter would initially start in front of the receive transducer as shown in Figure 4.32(a); both transducers are scanned equal distances but, as is typical of the TSAFT configurations, in opposite directions as shown in Figure 4.32(b). TSAFT-3 provides all of the advantages of TSAFT-2. In addition, it allows a shorter material path $(1 / 2 \mathrm{vee})$ to be used, greatly improving the signal-to-noise ratio in thick-section materials.

Figure 4.33 shows the positions of the transmit and receive transducers when they are toward the extremes of the scanned aperture. Path 1 provides data for the conjugate image, and path 2 data is used for the real image. The real and conjugate portions of the defect image may be observed simultaneously by choosing to process beyond the far surface during TSAFT2/TSAFT-3 processing. The vertical extent of a surface-connected defect may be accurately determined by measuring the full vertical extent of the image and dividing the result by two. This technique reduces the sensitivity of the vertical sizing measurements to variations in material velocity and material thickness, and the location of the far surface relative to the image need not be known accurately. 
Processing TSAFT-3 data requires manipulating three data file header parameters; i.e., material thickness, the number of transmit bounces, and the number of receive bounces. If the correct material thickness is used, the image space for TSAFT becomes too large to process. The TSAFT algorithm processes the entire material thickness; when processing beyond the far surface is chosen, twice the material thickness is processed.

When dealing with thick-section specimens and the TSAFT modes, it is necessary to limit the depth range that is processed and subsequently displayed. Figure 4.34(a) presents a typical TSAFT-3 setup, on a 9-in.thick $(22.9 \mathrm{~cm})$ steel block that has a 0.7-in.-deep (1.8$\mathrm{cm}$ )sawcut placed into the far surface. This setup will be used as an example. In our example, the region of interest is only the lower fifth of the material thickness. Processing is selected to be beyond the far surface, but limited in range as shown by the shaded area in Figure 4.34(a). To accomplish this, a fictitious material thickness value, equal to the original thickness divided by five $(1.8 \mathrm{in}$. [ $4.6 \mathrm{~cm}])$, is entered into the file header parameters using the DEDIT utility. If a different region of interest were desired, the material thickness could be divided by any odd number. The transmit and receive paths are then "folded" as shown in Figure 4.34(b), to give equivalent material path lengths. The correct number of transmit and receive bounces are counted and entered into the file header parameters. For our example, the number of transmit bounces would be 5 and the number of receive bounces would be 4. An easy rule-of-thumb is as follows:

- The number of transmit bounces will always be an odd value.

- The number of receive bounces will always be one less and an even value.

- The material thickness value to be entered is the true material thickness divided by the number of transmit bounces.

Once the necessary header modifications have been made, the TSAFT- 3 data file may be processed normally. Figure 4.35 shows the corresponding TSAFT-3 image of the identical sawcut shown in Figure 4.30. Notice that the conjugate image is much improved and that the full information is now available for operator interpretation. This technique greatly improves imaging of defects in thick-section materials.

\subsubsection{Piping Considerations}

Thin-wall piping does not present a long enough material path; hence, the receive transducer's aperture is small when compared to the receive aperture in thicker materials. If the transmit transducer is placed $5 / 2$ vees back from the object plane and the receiver is scanned $3 / 2$ vees back, a larger aperture may be realized. This technique is not always easy to implement if access to the pipe is a problem or the weld has been overlaid. As with the pulse-echo configuration, consistent coupling of the sound field into the material is of primary concern. Both the aperture size and the coupling consistency will affect the image quality. TSAFT-2 is the best configuration to use on piping if space permits. TSAFT may be used if space is a problem but should not be relied on for sizing information. The TSAFT configuration should be used only to resolve questions about pulse-echo results that may be ambiguous.

A different technique must be used for thick-section vessels. The long material paths encountered in thicksection vessels may help the image quality by allowing a large aperture to develop within the material; at the same time, the longer material paths may attenuate the sound field, decreasing the signal-to-noise ratio.

\subsubsection{Thick-Section Considerations}

Because of the large aperture present in thick-section vessels, an improvement in the image quality should be seen; however, a poorer signal-to-noise ratio is also common when longer material paths are encountered. Thick-section vessels do not lend themselves well to the normal tandem configurations. Both TSAFT and TSAFT-2 require the transmit transducer to be at least $3 / 2$ vees back from the object plane so the receive transducer will not collide with the transmitter when scanning the spatial aperture required. The material path would be much too long in a thick-section vessel if the transmitter were placed $3 / 2$ vees back from the object plane (i.e., a 9-in.-thick [22.7-cm] vessel would present a 51-in. [1.3-m] material path if a $45^{\circ}$ refracted angle were used). TSAFT-3 allows the transducers to pass each other in a side-by-side fashion; thus, a 1/2vee material path may be used to reduce the effect of 
material thickness and velocity errors normally associated with multiple-bounce TSAFT configurations.

Because the transmit and receive transducers are scanned simultaneously (TSAFT-2 or TSAFT-3 configurations), the path length to the defect remains constant. For this reason, if the area of interest is known, the digitization window does not need to be very large. The total data file size may be greatly reduced by keeping the digitization window just large enough to encompass the received echo over the entire aperture.

Some additional parameters need to be known before processing tandem data files. The user is queried for the processing mode and the number of transmit and receive bounces before the object plane.

\subsubsection{Tandem Processing Parameters}

Additional processing parameters are needed when processing tandem data files. Figure 4.36 presents the queried format for the processing mode. The user must enter a selection by typing $>(A, B, C$, or $D)<$ return $>$. Typically, mode $B$ is selected. If processing occurs beyond the far surface, provided a large enough spatial aperture is scanned, a real and conjugate image will be seen when the processed file is viewed. Processing modes other than B are intended for experimental use, and the resultant processed files may contain erroneous results.

Except in the case of TSAFT-3, tandem data acquisition usually requires that the energy field bounce off the far- and near-surface interfaces one or more times before striking the object plane. After striking the object plane, the energy may again bounce off the nearand far-surface interfaces one or more times before being received. The number of bounces for both the transmit and receive transducers are parameters that must be entered prior to processing a tandem data file.

Figure 4.37 graphically presents examples of $3 / 2$ - and 5/2-vee transmit paths. The user enters the number of transmit bounces prior to the object plane by typing $>$ (NUMBER OF TRANSMIT BOUNCES) < return>. Figure 4.37 also presents examples of $0 / 2$ - and 2/2-vee receive transducer paths. As with the transmit transducer, the user enters the number of bounces prior to the object plane; i.e., >(NUMBER OF RECEIVE BOUNCES) <return $>$.

Collecting tandem data is similar to collecting pulseecho data, except tandem data should not saturate. The edges of a processed image will be bloomed if the signal is allowed to saturate, and sizing errors will result. Tandem data is more sensitive to material thickness and velocity errors than pulse-echo data (see Section 4.1.3) because of the multiple vee paths normally used. Accurate values 'should be used for these header parameters.

Both the pulse-echo and tandem processed data files of an area of interest need to be analyzed prior to making any decisions about the character and size of possible defect indications.

\subsection{SAFT Data Analysis Techniques}

SAFT data analysis techniques have evolved over the course of several years as a direct result of reviewing the large, diverse data base that has been acquired on an extensive range of specimens and defects.

\subsubsection{General Information}

An understanding of the material properties, geometries, and defect characteristics is a must before one can properly analyze a processed data file. The material properties include the material thickness and sound velocity, while the geometries include the shape and nearand far-surface profiles. SAFT processing assumes flat parallel surfaces and homogeneous material. Deviations from these conditions may affect the position of indications within the processed image or the image quality. Studies have been completed showing the effects of velocity and thickness errors on TSAFT-2 images. A 0.3-in.-deep $(0.8-\mathrm{cm})$ vertical sawcut in a flat aluminum coupon was used as the target. Figures showing the results are referenced and described in Section 4.4.3.

In general, the procedure used to size an indication (pulse-echo or tandem) is as follows:

1. Enter the APLOT utility (Hall, Reid and Doctor 1988) by typing APLOT [FILENAME] <RE- 
TURN $>$. The prompt symbol will change to $\leq$ APLOT $>$.

2. Box the indication in both the $\mathrm{XZ}$ and $\mathrm{YZ}$ views using the following command sequence:

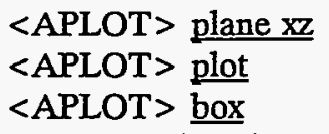

(At this point, select corner \#1 by placing the curser in the desired location and pressing the enter switch located on the remote curser control. Repeat the process for corner \#2.)

$<$ APLOT $>$ plot

$<$ APLOT $>$ plane yz

$<$ APLOT $>$ box

(Repeat the aforementioned procedure.) $<$ APLOT > plot

This allows the image to be normalized by the maximum value of a single indication. Because a $-6-\mathrm{dB}$ sizing criterion is used, this step is extremely important. If there is a larger indication nearby that is not discriminated against by the boxing procedure, the image will be normalized by the larger indication and -6- $\mathrm{dB}$ sizing of the smaller indication would be invalid (the indication would be undersized).

3. To enter the curser sizing mode, type <APLOT> curs <return $>$. Press the enter switch twice to establish the reference point, then move the curser to the point of measurement and press the enter switch again. The $\mathrm{XZ}$ or $\mathrm{YZ}$ (depending on the view used to take the measurement--measurement can be taken only on the last view plotted) values will be given on the terminal screen in inches.

4. If the far-surface distance is accurately known, the cursor can be referenced to the far surface (indicated by a dashed line). Vertical measurements can be made using the $-6-\mathrm{dB}$ criterion relative to the far surface. If the far-surface distance is suspect, one must use the $-6-\mathrm{dB}$ beginning of the corner-trap envelope (pulse-echo mode) and the 6-dB beginning of the tip-diffracted echo that is directly vertical for sizing references. For tandem files, one must measure the extent of the entire image and divide by two. Special techniques are used if the indication is not surface- connected and will be detailed later.

Section 4.4.2 will give a detailed description of techniques used when analyzing pulse-echo files, and Section 4.4.3 will go on to describe the techniques used when analyzing tandem files.

\subsubsection{Pulse-Echo Analysis}

When analyzing a SAFT-processed file, it is important to know as much as possible about the material, welding techniques used (if applicable), and characteristic defects that may be encountered. Often one may need to view many files. The files may have been collected from different sides of the defect or perhaps the files made use of different insonification angles. Whatever the case, it often requires viewing many files to ultimately determine what category of defect is being viewed.

Defects may be classified into three primary categories:

1. Category 1 consists of VOLUMETRIC indications. Volumetric indications include inclusions such as slag and/or porosity with a high degree of cylindrical or spherical size. Most often, volumetric indications are not surface-connected and may present several echoes. Figure 4.38 presents the image of a 0.2 -in.-diameter side-drilled hole that was placed 0.5 in. $(1.3 \mathrm{~cm})$ up from the far surface of a 2 -in.-thick steel block. The side-drilled hole emulates a volumetric defect that is not surface connected. Three echoes are evident. The uppermost echo is the direct reflection, while the echo located at the far surface (represented by a dashed line) is the corner-trap echo, and the echo that plots below the far-surface line is the mirror image.

2. Category 2 consists of PLANAR indications. Planar indications include lack of fusion or inclusions. A planar indication may be surface connected and provides a strong direct echo that is sensitive to the angle of insonification and may be the only echo received. 
3. Category 3 consists of $\mathrm{CRACK}$ indications. Crack indications are normally surface-connected, diffuse reflectors that provide a strong corner-trap echo and a weaker tip-diffracted echo. The sawcut emulates a crack providing a strong corner-trap echo and a weaker tip-diffracted echo. If the crack is tilted, the tip-diffracted echo will not be directly vertical from the corner-trap echo but will be displaced in the direction of the tilt. As the crack is tilted away from the direction of insonification, the tip-diffracted echo becomes stronger and will merge with the corner-trap echo as the crack becomes perpendicular to the angle of insonification.

One must first consider the processing parameters that will be used to process the data before analysis may begin. Of extreme importance is the full beam angle that will be used. Generally, the larger one can make the beam angle, the better the focussing will be, provided that information was gathered at the extremes of the defined aperture. A corner-trap echo will ideally focus to a line, centered around the far surface. If a larger beam angle than that over which data was actually acquired, the resultant image dynamic range may be greatly reduced as zero values will be averaged in where data does not exist.

The material velocity, couplant velocity, and incident angle will affect how well the data correlates. Errors in the values entered into the header will often result in an image with a reduced dynamic range and/or poor focusing. Care should be taken to ensure data integrity and accuracy of the values used in the SAFT data header.

To properly analyze an image, one should have a feel for what category of defect is expected. If the category of defect is not clear, invoke APLOT and plot the full $\mathrm{XZ}$ view. The $\mathrm{XZ}$ view can be sequenced plane by plane by typing APLOT $>$ SEQ $11 \leq$ return $>$. Sequencing through the $\mathrm{XZ}$ view by single planes often "reveals" the category into which the defect will best fit. One looks for the mirror echo (category 1) or the weaker tip-diffracted echo and strong corner-trap (category 3). Category 2 defects may require viewing several images, although the presence of a single echo above the far surface provides a strong case. Each category of defect realistically requires more than one image to confirm the exact category. The shape of the indication is very important; a pulse-echo image of a real echo will show a slanted appearance because of the different time-offlight paths. A globular indication may be noise or some other incoherent source. A real defect will present an indication that is present in more than one plane, and sequencing through the image gives one a feel for the consistency of the indication as well as its shape.

Volumetric and planar defects are measured at the -6$\mathrm{dB}$ levels, and it is extremely important to use the maximum beam angle possible (within the physical aperture constraints) to ensure optimum focusing and sizing.

In conclusion, the pulse-echo defect types can be divided into three categories: volumetric, planar, or crack. Sizing of a defect is normally performed by measuring the vertical extent (cracks) or the cross-sectional distance (volumetric/planar) at the $-6-\mathrm{dB}$ levels, once the defect has been isolated and the image normalized by the maximum value of the defect to be sized. Often multiple images may be required to classify the defect into a particular category and to further confirm the shape of the defect.

Tandem sizing and analysis use techniques similar to pulse-echo but provide images that are often easier to interpret. The next section provides an insight into analyzing tandem images.

\subsubsection{Tandem Analysis}

Tandem image analysis uses techniques similar to those of pulse-echo analysis. Defects may be categorized as volumetric, planar, or crack. The primary difference between the tandem and pulse-echo image is that the tandem image of a crack presents the entire cross section of the crack and not just the corner-trap and tipdiffracted echoes. Often, the tip-diffracted echo is very illusive because of the weak nature of the tip-diffracted echo compared to the very strong corner-trap echo; and without a tip-diffracted echo, the vertical extent of a crack is difficult to estimate. The pulse-echo image may be ambiguous as in Figure 4.38 where a cornertrap echo and direct echo are seen much the same as if they were cracks. If the mirror echo were not present, it would be hard to classify this image as a volumetric defect. A tandem image of a volumetric defect that is not surface-connected presents the real and conjugate 
(mirror) images separated by the distance of the defect from the far surface (Collins and Gribble 1986). If the defect is surface-connected and vertical, the real and conjugate images would be symmetrical; but if the defect is tilted, the image would be asymmetrical about the far surface (Collins and Gribble 1986).

The signal-to-noise ratio of a tandem image is often much superior to that of a pulse-echo image, because a separate receiver eliminates noise caused by the initial pulse, the near-surface interface. and the specular backscatter from the given structure. The criteria developed for sizing the SAFT images were based on the empirical results that have evolved over many years of testing and analysis. Other work (Hildebrand 1986; Thome 1985) has shown that one can determine a sizing criterion by mapping the pixel numbers versus the pixel intensity. Interestingly, the values those researchers have generated by this procedure are in agreement with the values that were derived empirically by the SAFT research. The nice thing about the pixel procedure is that it can be accomplished automatically; no operator skill is required.

Tandem image indications are vertical in appearance, as opposed to the slanted appearance of a pulse-echo image (Figure 4.27). The location of the indication within the image space is influenced by the material thickness, velocity, and refracted angle. Care should be taken to ensure accuracy of the tandem header entries. Errors normally do not change the appearance of the indication but do influence the location. Two performance evaluation studies were focused on the TSAFT-2 configuration to determine the sensitivity of this mode to velocity errors and thickness measurement errors. It was also a goal of these studies to determine the degradation that occurs due to these variations.

The SAFT algorithm implemented at PNL assumes isotropic and homogeneous material with rèspect to velocity; that is, the calculations performed by the SAFT processing make the approximation that the velocity is constant throughout the material. This, of course, is not exactly true in most specimens, and the variance depends greatly on the type of material under test. Also, the algorithm requires that the velocity be known to some degree of accuracy. This may seem to be a trivial requirement, but in fact in many cases it is not. For example, an extreme case is CCSS material, which varies in velocity as a function of angle in the material.

As stated previously, the placement of the resulting image is dramatically affected by deviations of either thickness or velocity from the true values of the specimen under test. However, the encouraging result is that the general image integrity is retained. Thus, with the complement of both pulse-echo and tandem scans of the same region, a judgement of size and location may be made. The pulse-echo corner-trap image may be used to position the defect laterally, and the TSAFT2 image may be used to determine the horizontal and vertical extent of the defect. The characteristics of both files may be used to verify the integrity of the other. A surface-connected crack sized by using the total vertical extent of the real and conjugate images and then dividing the resultant value by two would provide an accurate value of the vertical extent of the crack, even if there were minor errors in the header entries that caused the indication to not be located symmetrically about the far surface. Raw data should always be viewed to verify the integrity of the data and to ensure that the data was not aperture-limited.

The normal SAFT data analysis procedure involves a number of sequential steps that must be followed to perform the analysis correctly. These steps are summarized and include

1. $0^{\circ}$ SAFT scan with display in B- and C-scan to identify volumetric defects, length size volumetric defects, and all geometric conditions

2. $45^{\circ}$ shear mode pulse-echo scan with display in Band C-scan to identify defects and to determine defect length

3. $45^{\circ}$ shear mode pulse-echo scan from the opposite direction of Step 2 to see what the defect looks like from the other direction and to confirm defect length

4. $45^{\circ}$ TSAFT in the shear mode from the two directions in Steps 2 and 3 to be used for confirming the presence of a defect, sizing the length of the defect, and sizing the thickness dimension of the defect 
5. If the data is not consistent between views of the images created by the steps listed above, then other images taken at different inspection frequencies and inspection angles can aid in clarifying the interpretation.

6. For the inspection of pressure vessels from the inside, it is very useful to conduct an inspection with a $70^{\circ}$ longitudinal probe for the near-surface zone since this yields a larger aperture for the near-surface zone during SAFT processing.

7. Based on the calibration blocks, the transducer frequency is selected with normally $2.25 \mathrm{MHz}$ being the best except in the case of coarse-grained material in which a $1-\mathrm{MHz}$ longitudinal or a 500 $\mathrm{kHz}$ shear works better.

8. The sizing of defects is contingent on the type of defect. Empirical relationships that have evolved are to use a $-6 \mathrm{~dB}$-criterion for volumetric and defects that can be viewed by the specular reflection. In other cases, the defects are best sized by using a loss-of-signal criterion, and the actual decibel drop value will vary based on the noise level of the material. If the tip signal from planar defects can be identified, then it should be used to size the defect.

9. The defect type can be determined by the images in which the defect can be seen and the shape of the response in the images. A volumetric defect should be seen by all the inspections. If the defect has planar qualities, then it will be seen in only those images (insonification) in which the scattered energy returns to the receiver. 


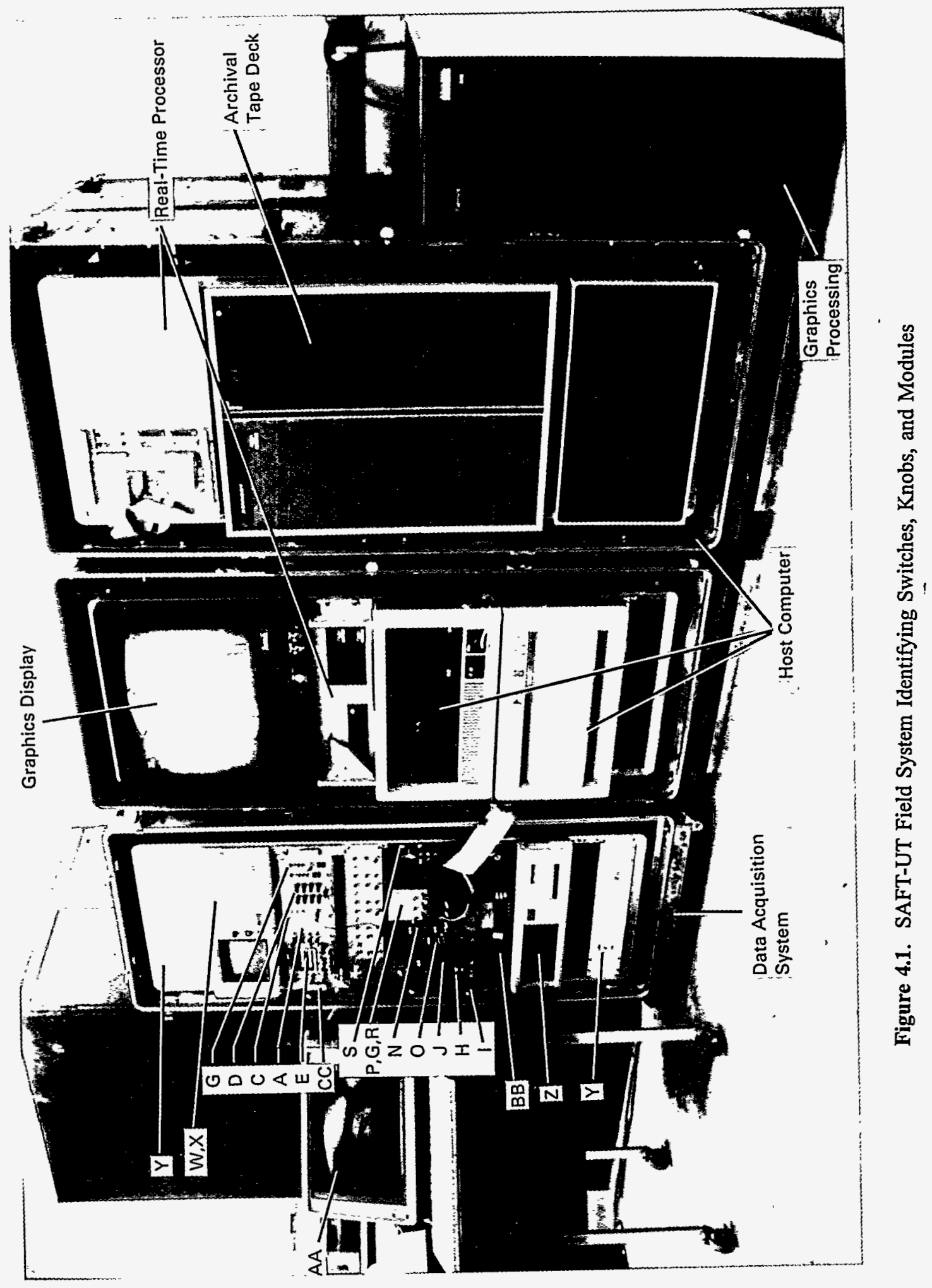




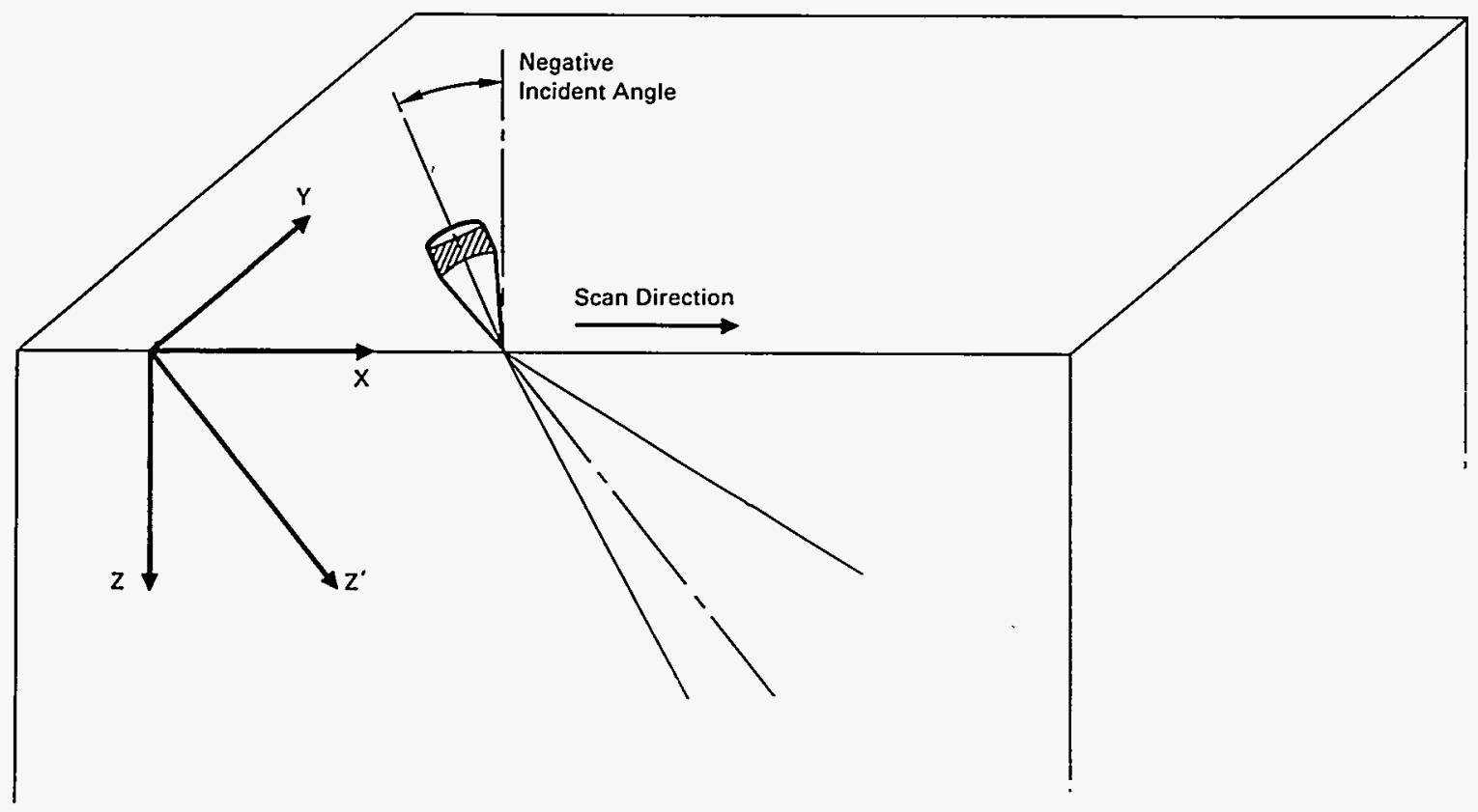

Figure 4.2. SAFT-UT Scan Conventions 
NEW VERSION OF DATA COLLECTION FOR AMAPS SCANNER ONLY, BUT SELECTABLE

FOR TANDEM OR PULSE-ECHO ....

TYPE 'Escan' or 7) 'run scanner'

Before proceeding, please enter the time and date.

Date [dd-mmm-yy]? 14-AUG-87

Time [hh:mm:ss] ? 11:19

Figure 4.3. Date and Time Prompts

$$
\text { MODE }
$$

1. SINGLE TRANSDUCER - PULSE ECHO

2. SINGLE TRANSDUCER - PULSE ECHO

3. SINGLE TRANSDUCER - PULSE ECHO

4. SAFT TANDEM CONFIGURATION

5. SAFT TANDEM CONFIGURATION

6. SAFT TANDEM CONFIGURATION

7. SAFT TANDEM2 CONFIGURATION

8. SAFT TANDEM2 CONFIGURATION

9. SAFT TANDEM2 CONFIGURATION

ENTER SELECTION ---> 2

Select desired scan axis: $1=X 1$

$$
2=\times 2
$$$$
----->1
$$

\section{STANDOFF}

water path

plastic wedge

(user specified standoff velocity)

xmit wedge, recv water xmit wedge, recv wedge

(user specified standoff velocity)

xmit wedge, recv water xmit wedge, recv wedge

(user specified standoff velocity)

Are you set up so that the transducer(s) are strattling the weld $(y / n /$ unsure $)$ ? 
(QUERY)

Select scanner configuration ...

MODE

1. SINGLE TRANSDUCER - PULSE ECHO

2. SINGLE TRANSDUCER - PULSE ECHO

3. SINGLE TRANSDUCER - PULSE ECHO

4. SAFT TANDEM CONFIGURATION

5. SAFT TANDEM CONFIGURATION

6. SAFT TANDEM CONFIGURATION

7. SAFT TANDEM2 CONFIGURATION

8. SAFT TANDEM2 CONFIGURATION

9. SAFT TANDEM2 CONFIGURATION

ENTER SELECTION --> 5

Select desired scan axis: $1=X 1$

$2=X 2$

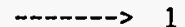

Are you set up so that the transducer(s) are strattling the weld $(y / n /$ unsure $)$ ?

\section{STANDOFF}

water path

plastic wedge

(user specified standoff velocity)

xmit wedge, recv water xmit wedge, recv wedge

(user specified standoff velocity)

xmit wedge, recv water

xmit wedge, recv wedge

(user specified standoff velocity)

Figure 4.5. Selection of Tandem Mode

(QUERY)

Select options:

1. VIEW current parameters

2. modify TRANSDUCER characteristic data

3. modify MATERIAL characteristic data

4. modify SAMPLING data

5. modify SCAN parameters

0 . continue to next menu

-1 . modify configuration mode

ENTER SELECTION --->

Figure 4.6. Main Menu for SAFT-UT Parameters 


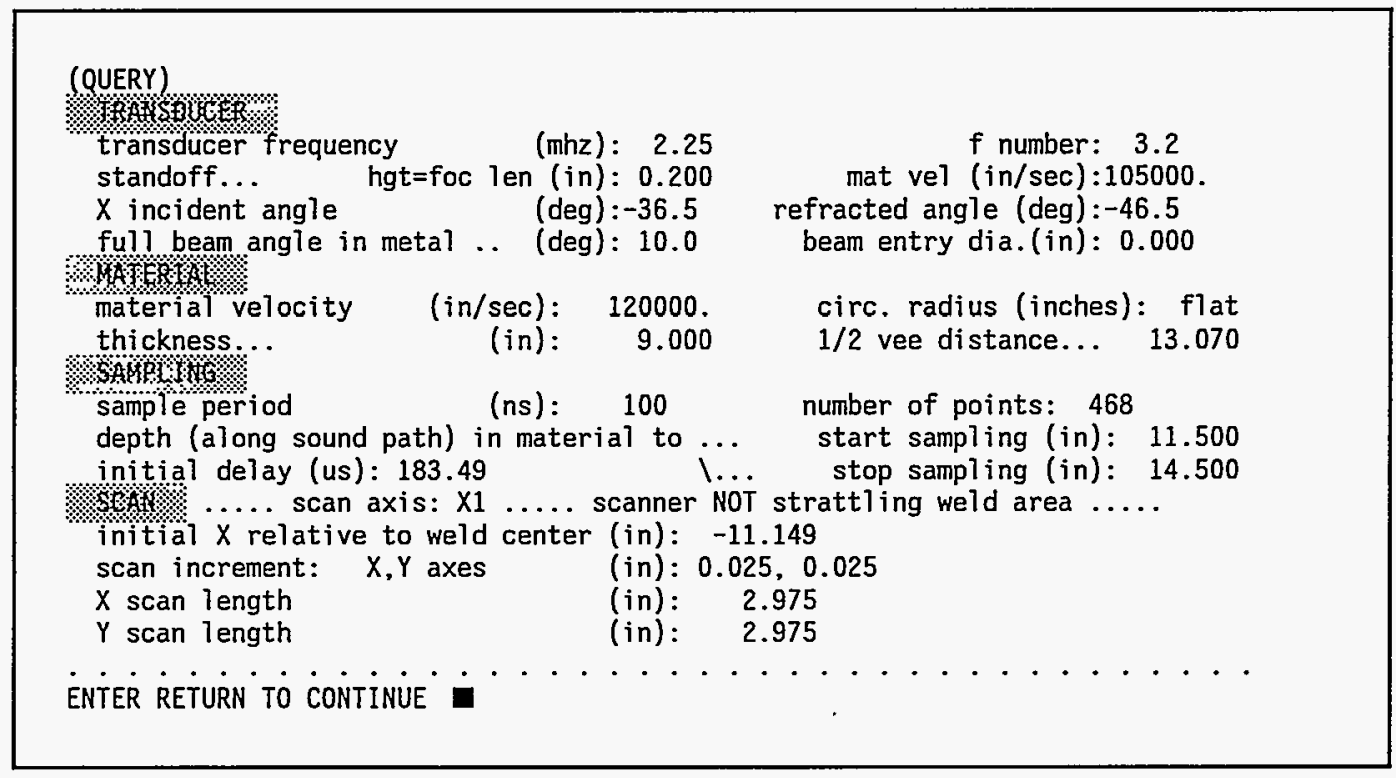

Figure 4.7. View of Current Parameters (Pulse-Echo)

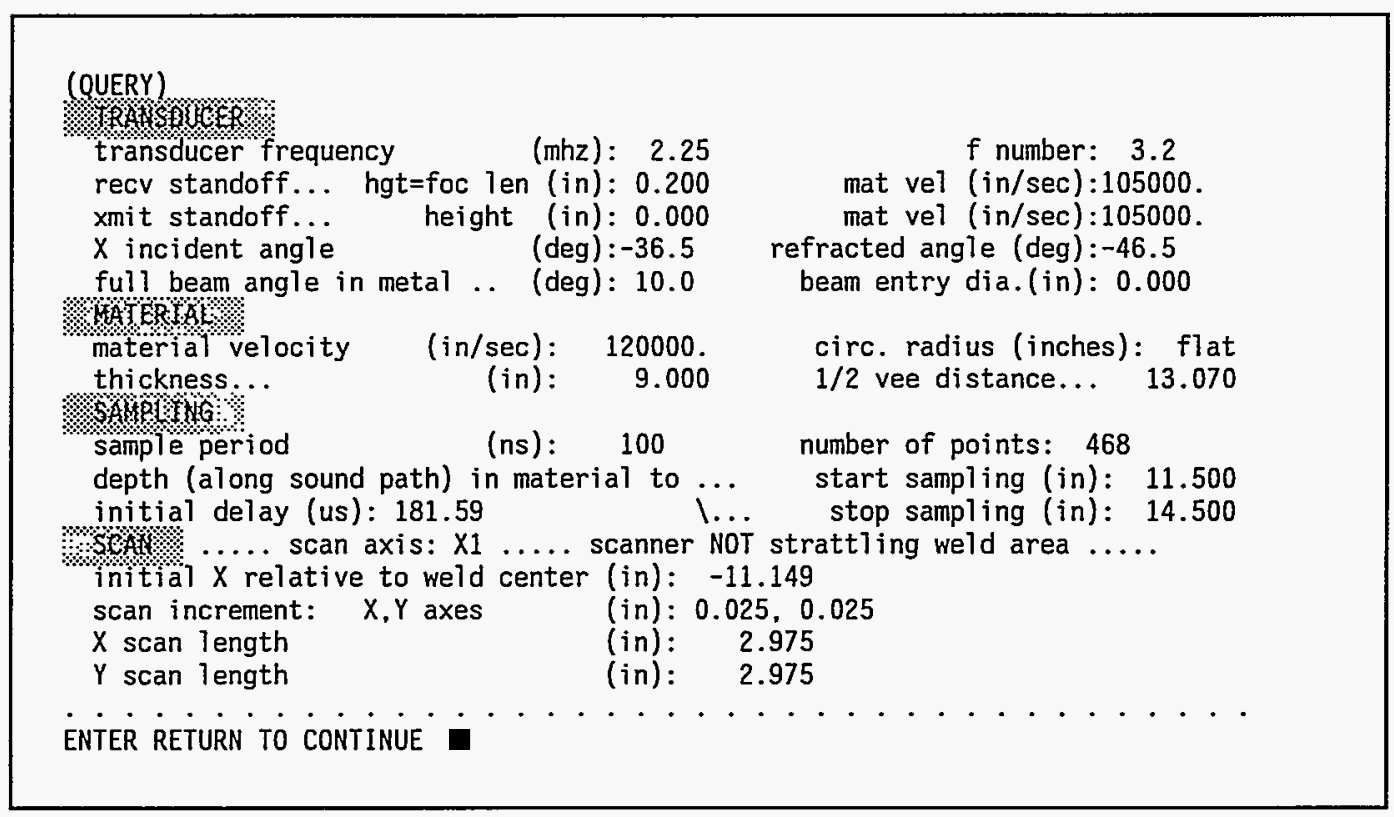

Figure 4.8. View of Current Parameters (Tandem) 
$X$ incident angle

hgt=foc len (in): 0.200

(deg): -36.5

full beam angle in metal .. (deg): 10.0 f number: 3.2

mat ve] (in/sec): 105000 .

refracted angle (deg): -46.5

beam entry dia.(in): 0.000

Figure 4.9. Transducer Header Information
(QUERY)
WHEYAO
material velocity
thickness...
(in/sec): 120000 .
circ. radius (inches): flat
(in): $\quad 9.000$
$1 / 2$ vee distance...
13.070

Figure 4.10. Material Header Information

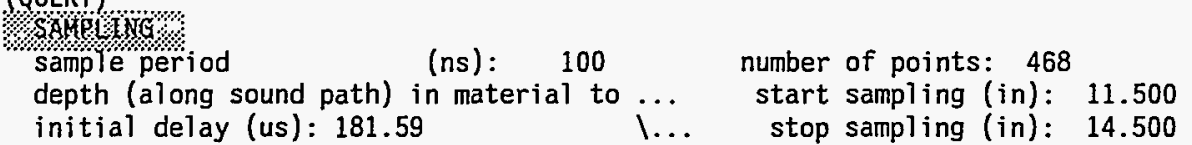

Figure 4.11. Sampling Header Information

Figure 4.12. Scan Header Information (Pulse-Echo)

Scal .... scan axis: $X 1 \ldots$.... scanner NOT strattling weld area .....

initial $X$ relative to weld center (in): -11.149

scan increment: $X, Y$ axes $\quad$ (in): $0.025,0.025$

$X$ scan length $\quad$ (in): 2.975

$Y$ scan length (in): 2.975 
(QUERY)

\$SOXI.... scan axis: XI .... scanner NOT strattling weld area .....

initial rov $X$ rel to weld center (in): $-11.149 \quad$ xmit $1 / 2 \mathrm{~V}$ 's to vel: $x$ mit $X$ rel to init rev $X$ (in): -0.600

1.2

scan increment: $X, Y$ axes

$X$ scan length

(in): $0.025,0.025$

$Y$ scan length

(in): 2.975

(in): $\quad 2.975$

Figure 4.13. Scan Header Information (Tandem)

<SCANPT $>$

Number of Points $=468 \quad$ Delay (usec) $=183.49$

Minimum" 189 Maximum" 142 AVerage $=125.92$

Present Scanner Position: $X_{1}=0.000 \quad X_{2}=0.000 \quad Y=0.000$ inches

\%o

' $H E^{\prime}$ - print help file

' $\mathrm{CM}$ ' - change setup parameters

'CS' - change scan parameters

' $V I$ ' - view current parameters

' $X 1$ ' - move scanner axis X1 (normally rcv xducer in tandem mode)

' $X 2$ ' - move scanner axis $X 2$

' $Y$ ' - move scanner in the $Y$ direction

'RS' - reset the scanner \& counters

'return' - digitize the signal \& display scanner position

'SC' - proceed to scanning module

'EN' - end this program and exit

ENTER COMMAND -->

Figure 4.14. Command Selection Menu 
<QUERY >

Select options:

1. VIEW current parameters

2. modify TRANSDUCER characteristic data

3. modify MATERIAL characteristic data

4. modify SAMPLING data

5. modify SCAN parameters

0 . continue to next menu

-1. modify configuration mode

ENTER SELECTION --->

The delay is 103.49 usec.

Press 'RETURN' if this is correct or if you wish to change it enter number. (WARNING: alternation of delay may result in erroneous processing) $\rightarrow$

Figure 4.15. Prompt to Change the Initial Delay

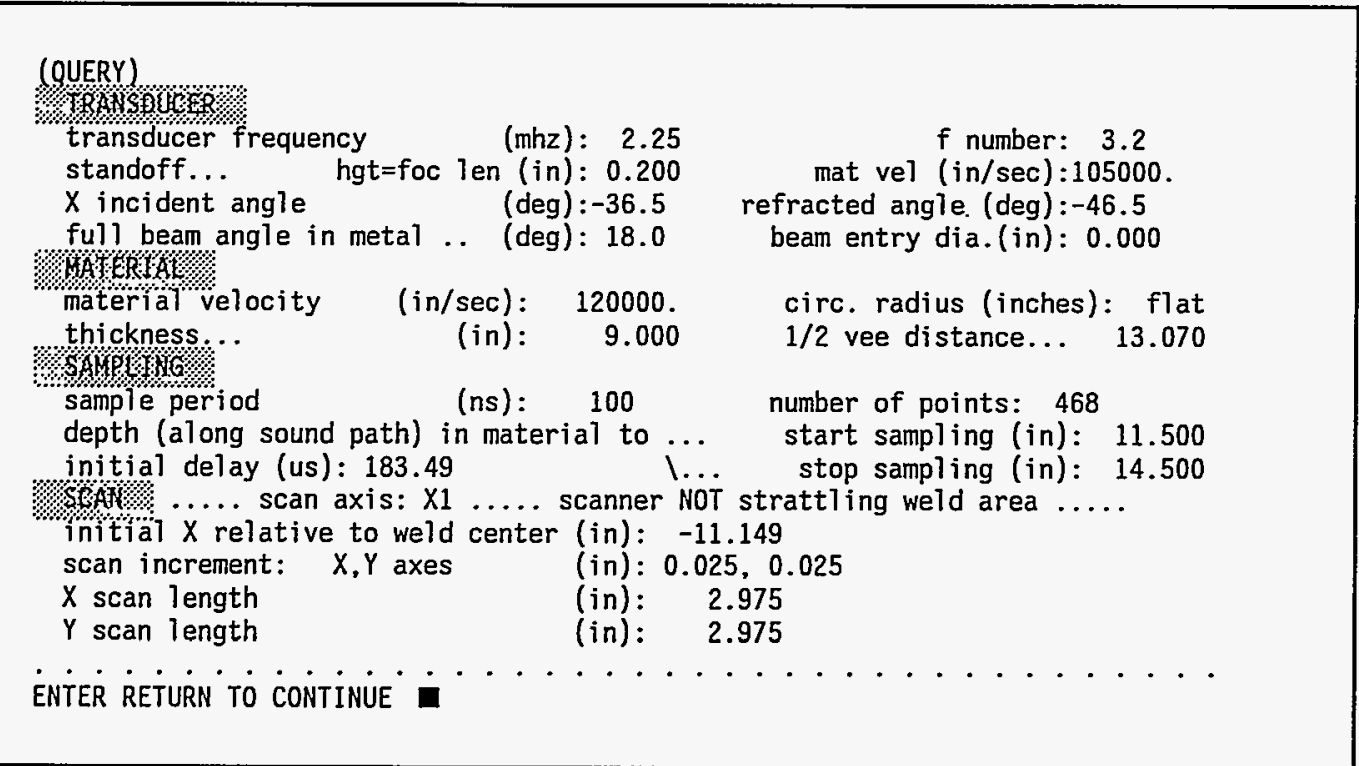

Figure 4.16. View of Header Information Before Scanning 
Present Scanner Position: $X 1=0.000 \quad X_{2}=0.000 \quad Y=0.000$ inches

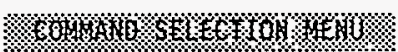

'HE' - print help file

' $\mathrm{CM}$ ' - change setup parameters

'CS' - change scan parameters

'VI' - view current parameters

' $X 1$ ' - move scanner axis $X 1$ (normally rov xducer in tandem mode)

' $X 2$ ' - move scanner axis $X 2$

' $Y$ ' - move scanner in the $Y$ direction

'RS' - reset the scanner \& counters

'return' - digitize the signal \& display scanner position

'SC' - proceed to scanning module

' $E N '$ ' - end this program and exit

ENTER COMMAND $-\rightarrow \quad X 1$

Present $X 1$ position is 0.0 inches

Enter $X 1$ Location (in): 1

Scanning speed $=0.30$ inches $/$ sec.

(30)

(1)

Figure 4.17. Repositioning the Scanner

<SCANPT>

Select storage/transfer mode:

1) transfer directly to VAX over PRONET.

2) store only on local PDP11/23 disk drive.

3) both (store locally and transfer over PRONET).

ENTER SELECTION >

Figure 4.18. Data Transfer Menu 
<SCANPT>

IF NOT LOGGED ON TO VAX THEN:

1. enter user name.

2. enter password. Then wait for prompt.

3. Set the default directory on the VAX to the directory in which you wish the data file to reside (e.g. 'SB. PARKS').

4. Then enter "SCANRD'. and follow subsequent instructions.

IF ALREADY LOGGED ON TO VAX THEN:

1. Type 'SD' to make sure in right directory which you wish the data file to reside

2. Then enter 'SCANRD' , and follow subsequent instructions.

Welcome to PNL $\$$ VAX-11/700 VMS Version V3.6

Username:

Figure 4.19. Connection to the VAX Host Computer

<SAFTDEFS> The current VMS version is ...3.6

<SAFTDESF> Today is ...... August 14, 1987 The time is ... 11:30 am

<SAFTDEFS> The SAFT-UT [VERSION 1.1] environment has been set.

<SAFTDEFS> Type HELP QSAFT for assistance with the SAFT utilities.

$\$$
$\$$
$\$$
$\$$
$\$$
$\$$
$\$$
$\$$
$\$$
$\$$
$\$$
$\$$

scanrd

Figure 4.20. Starting Data Collection on the VAX Host Computer 
<SAFTMAIN VMS.1> initiate SAFT collection, processing \& graphics

Do you want to COLLECT DATA FROM DATA ACQUISITION SYSTEM $(y / n)$ ? y Do you want to PROCESS data while scanning $(y / n)$ ? y

SAFT pulse-echo configuration

Envelope detect this data? (detection WILL be performed)

Enter data clipping threshold relative to max in $\mathrm{db}$.

Typically: -40 ( $1 \%$ of peak)

$-20(10 \%$ of peak $)$

$-10(30 \%$ of peak $)$

$-6(50 \%$ of peak)

$>-20$

NORMALIZATION MODE SELECTION:

1) average the data summed $(1 / N)$. raw data on skip.

2) average the data summed $(1 / N)$. zero on skip.

$3)$ constant normalization (1/C). raw data on skip.

4) constant normalization (1/C). zero on skip.

5) $1 / \mathrm{SQRT}(N)$ data normalization. raw data on skip.

6) $1 / S Q R T(N)$ data normalization. zero on skip. ENTER SELECTION >

Figure 4.21. SAFT Processing Options

$$
\begin{aligned}
& -20(10 \% \text { of peak }) \\
& -10(30 \% \text { of peak }) \\
& -6(50 \% \text { of peak }) \quad>-20
\end{aligned}
$$

NORMALIZATION MODE SELECTION:

1) average the data summed $(1 / N)$. raw data on skip.

2) average the data summed $(1 / N)$. zero on skip.

$3)$ constant normalization (1/C). raw data on skip.

4) constant normalization (1/C). zero on skip.

5) $1 /$ SQRT(N) data normalization. raw data on skip.

6) $1 / \mathrm{SQRT}(\mathrm{N})$ data normalization. zero on skip. ENTER SELECTION > 1

Data Sampling Adjustment Options:

0) no sampling adjustment to be performed.

1) perform automatic adjustment.

2) perform manual adjustment.

Enter data reduction selection $>1$

Do you wish to process beyond the back surface $(y / n) ?>n$ Do you want to DISPLAY data while scanning $(y / n)$ ?

Figure 4.22. SAFT Processing Options, Continued 


\subsection{Operating Procedures}

MANUAL DATA REDUCTION OPTION:

If data has been oversampled in the $x ; y$ or $z$ direction, sometimes it is desirable to create the output file with reduced sampling in this direction to minimize the size of the data.

If you do not wish to alter the resultant data enter 0 or 1 to the following inquiry. Otherwise enter the integer reduction factor you desire.

Reductions to data during processing:

$$
\begin{aligned}
& \text { Enter Z-axis reduction factor }> \\
& \text { Enter } X, Y \text { axis reduction factor }>
\end{aligned}
$$

Enter $X, Y$ axis reduction factor tion):

Reductions to data after processing (during envelope detec-

Enter Z-axis reduction factor >

Figure 4.23. Selection of Sampling Reduction

$<$ SCANPT $>$

ENTER 2 LINES OF COMMENTS ( 40 CHARACTERS EACH):

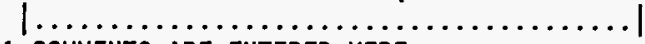

$1>$ COMMENTS ARE ENTERED HERE

2>

Enter desired scanner speed (inches/sec) ( $<0=$ default $>$ : 1

Number of 512 byte blocks $=14064$

Number of A-scans $=14400$

Number of points/a-scan $=468$

Enter name of data file to be created > FILENAME.DAT

Total points ......X $=120 \ldots . Y=120$

Scan Increment .... $0.025 \quad \ldots . . \quad 0.025$

53

Figure 4.24. Entering Comments and Setting the Scanner Speed 


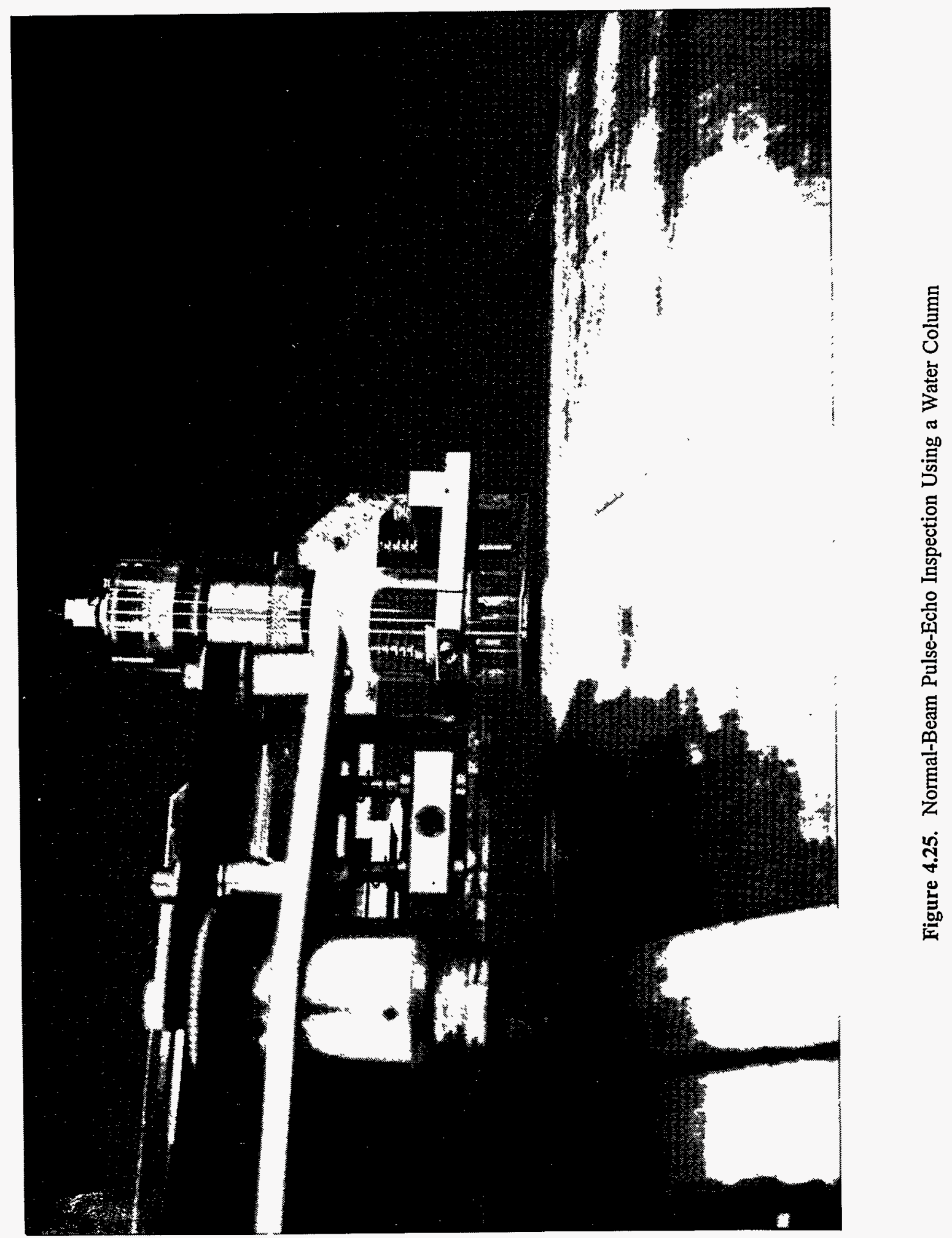




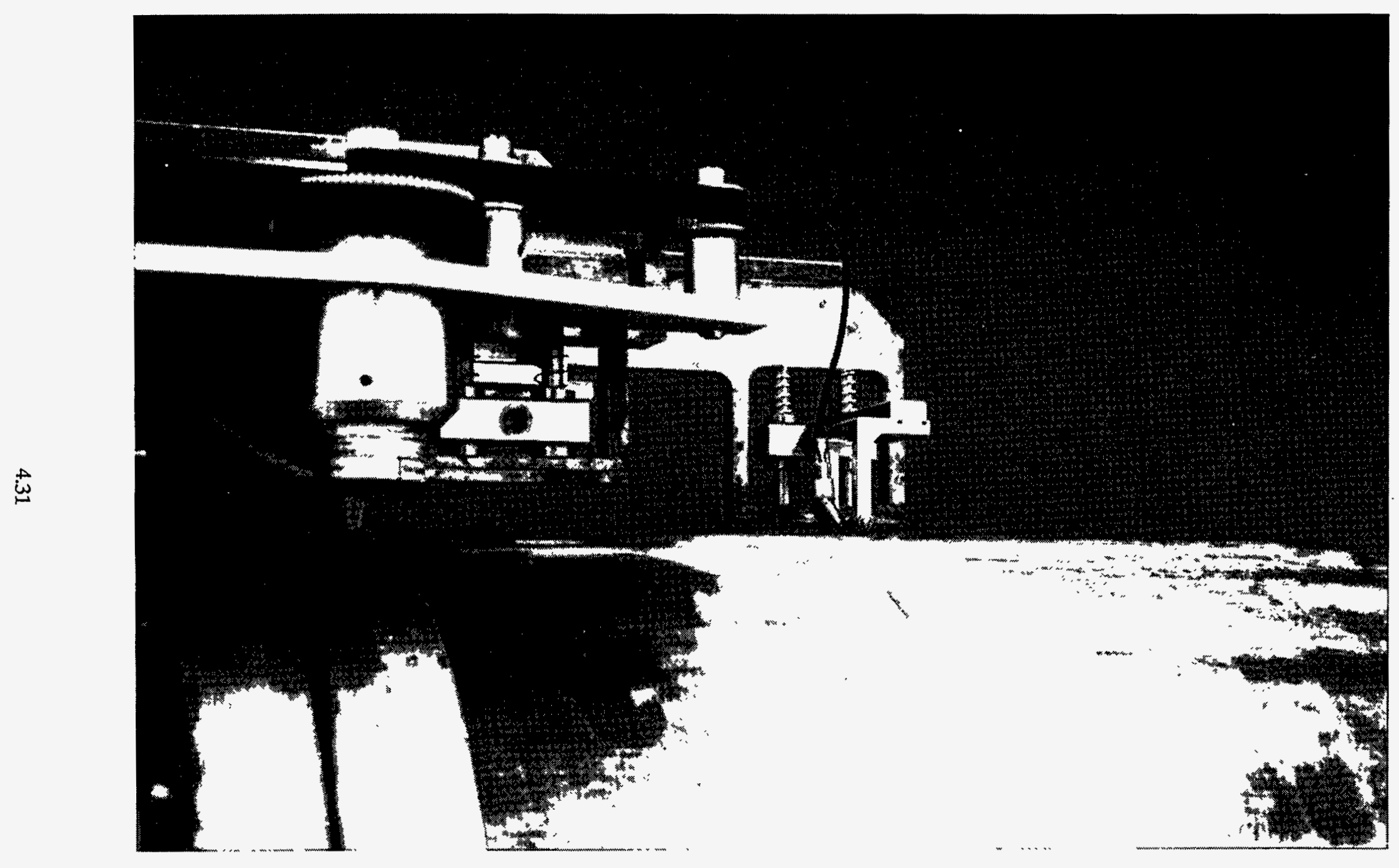

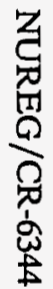

Figure 4.26. Angle-Beam Pulse-Echo Inspection Using a Lucite Wedge and Small-Diameter Transducer 


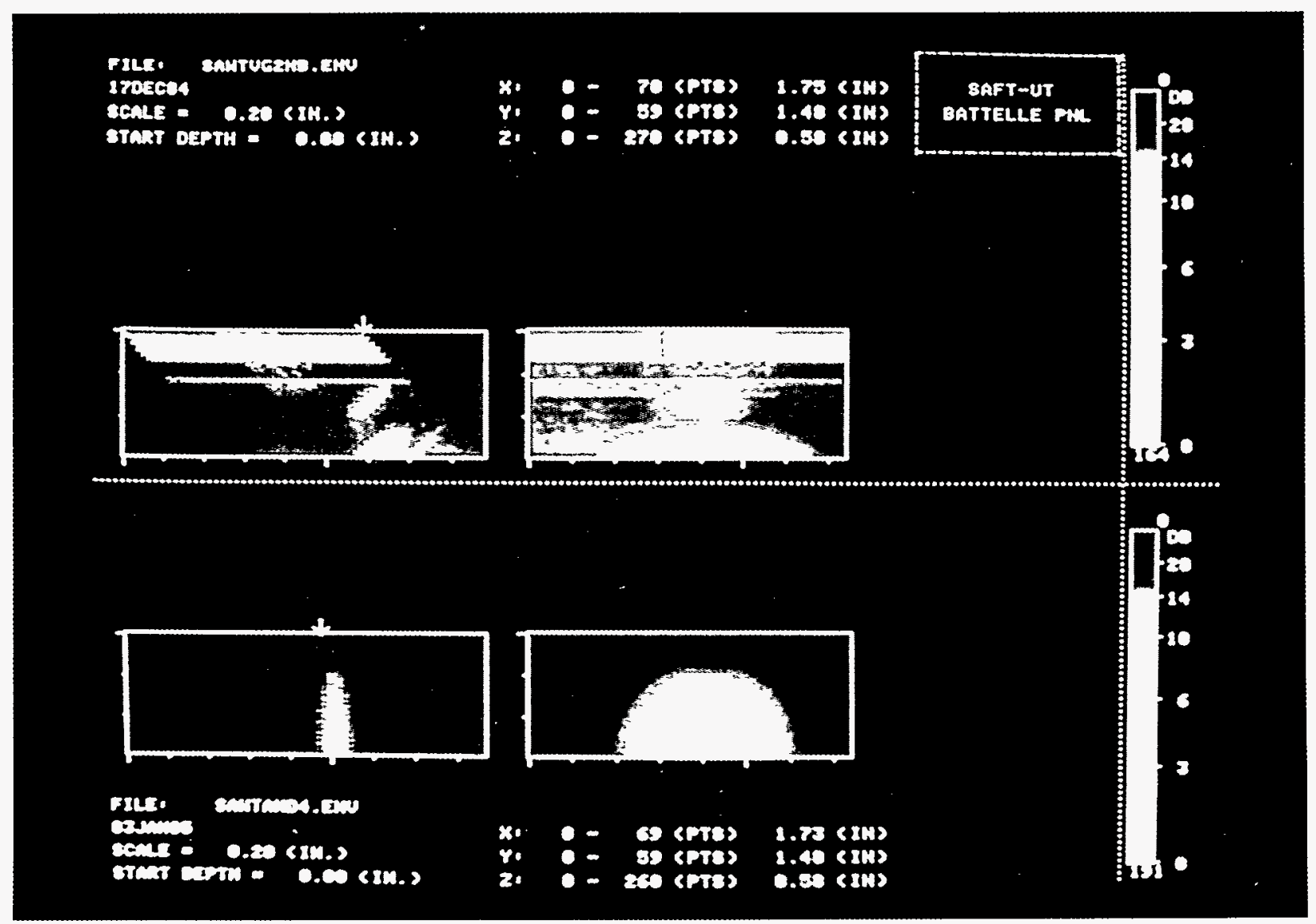

Figure 4.27. Comparison Between Pulse-Echo (top) and Tandem Images of a 0.3-in.-deep (0.8-cm) Semicircular Sawcut in a 0.6-in.-thick $(1.5-\mathrm{cm})$ Aluminum Coupon 


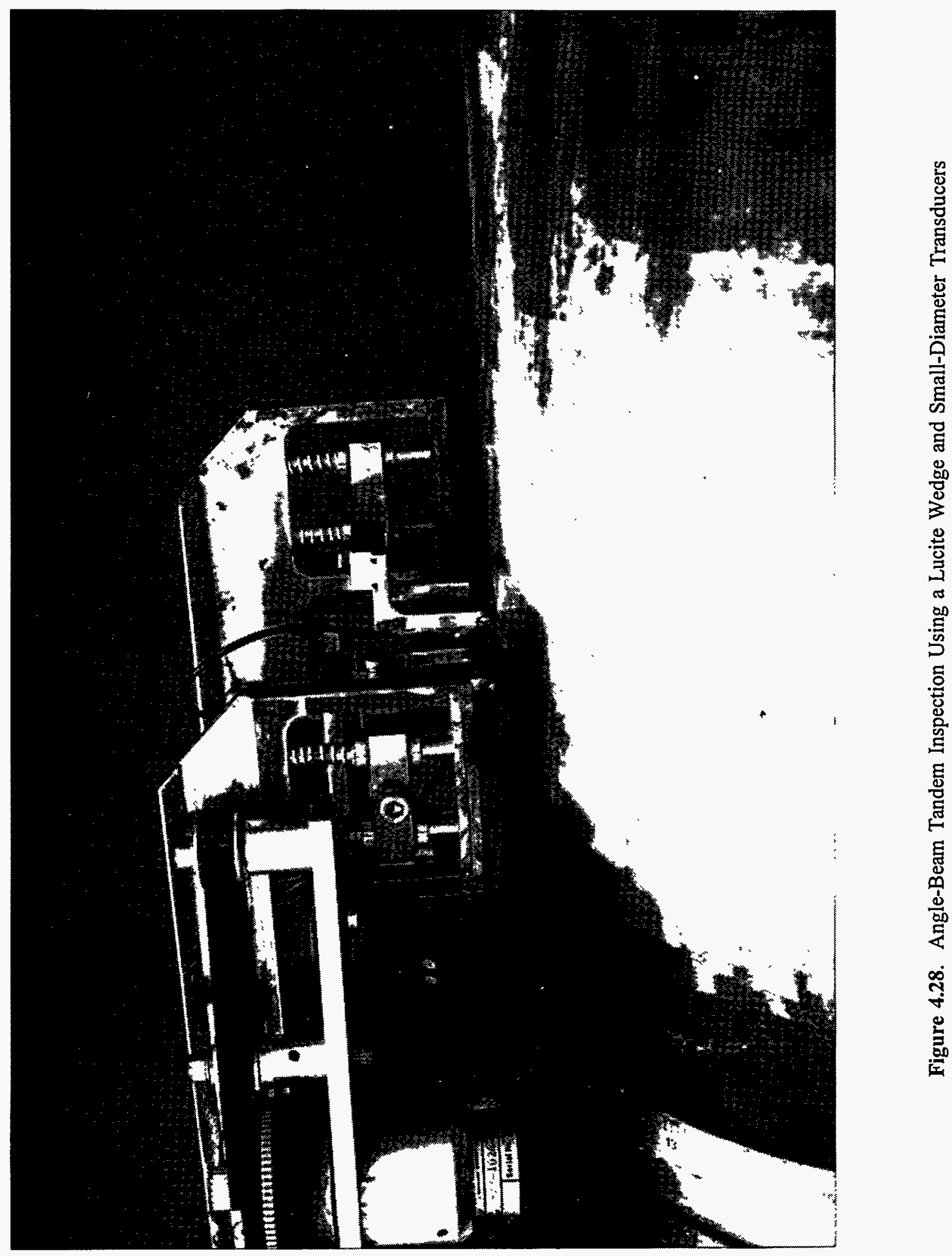



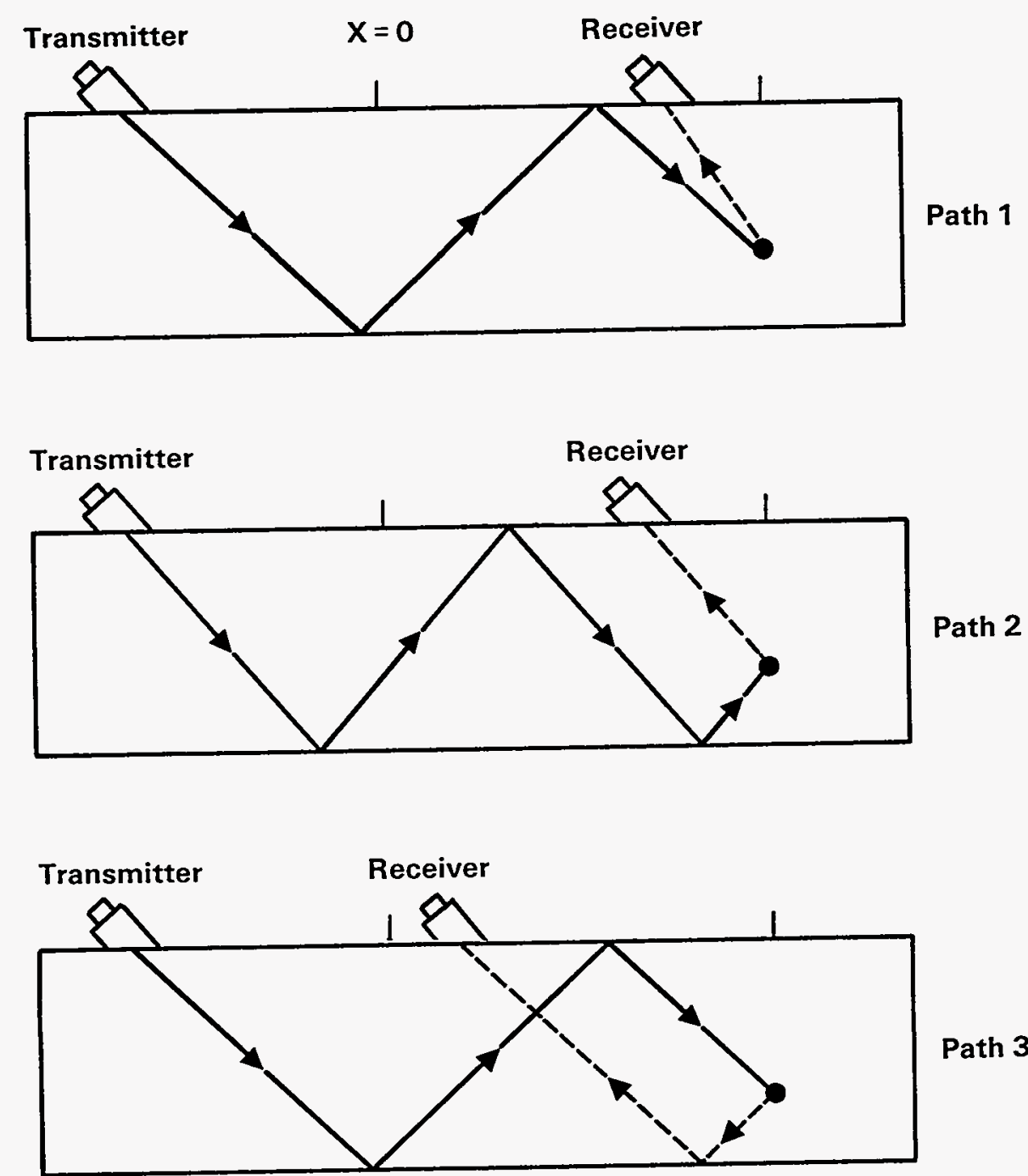

Path 3

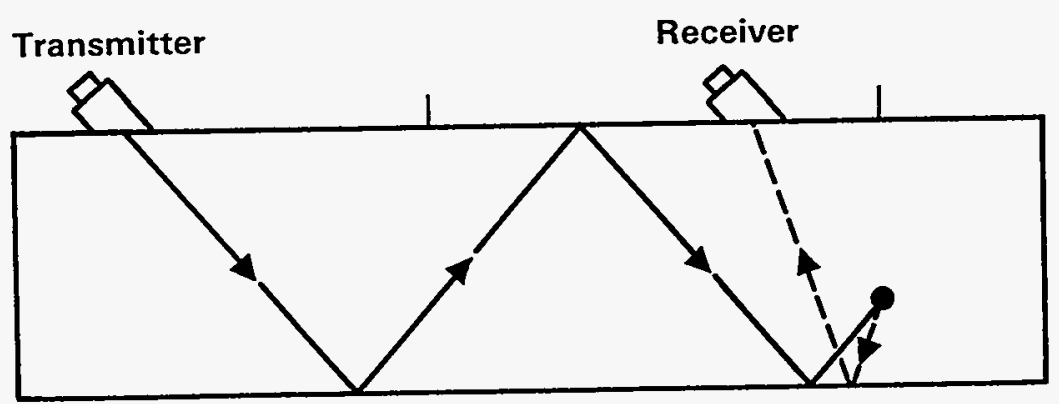

Path 4

Figure 4.29. Four Propagation Paths for Ultrasonic Energy Produced by the Tandem Transmission Technique 


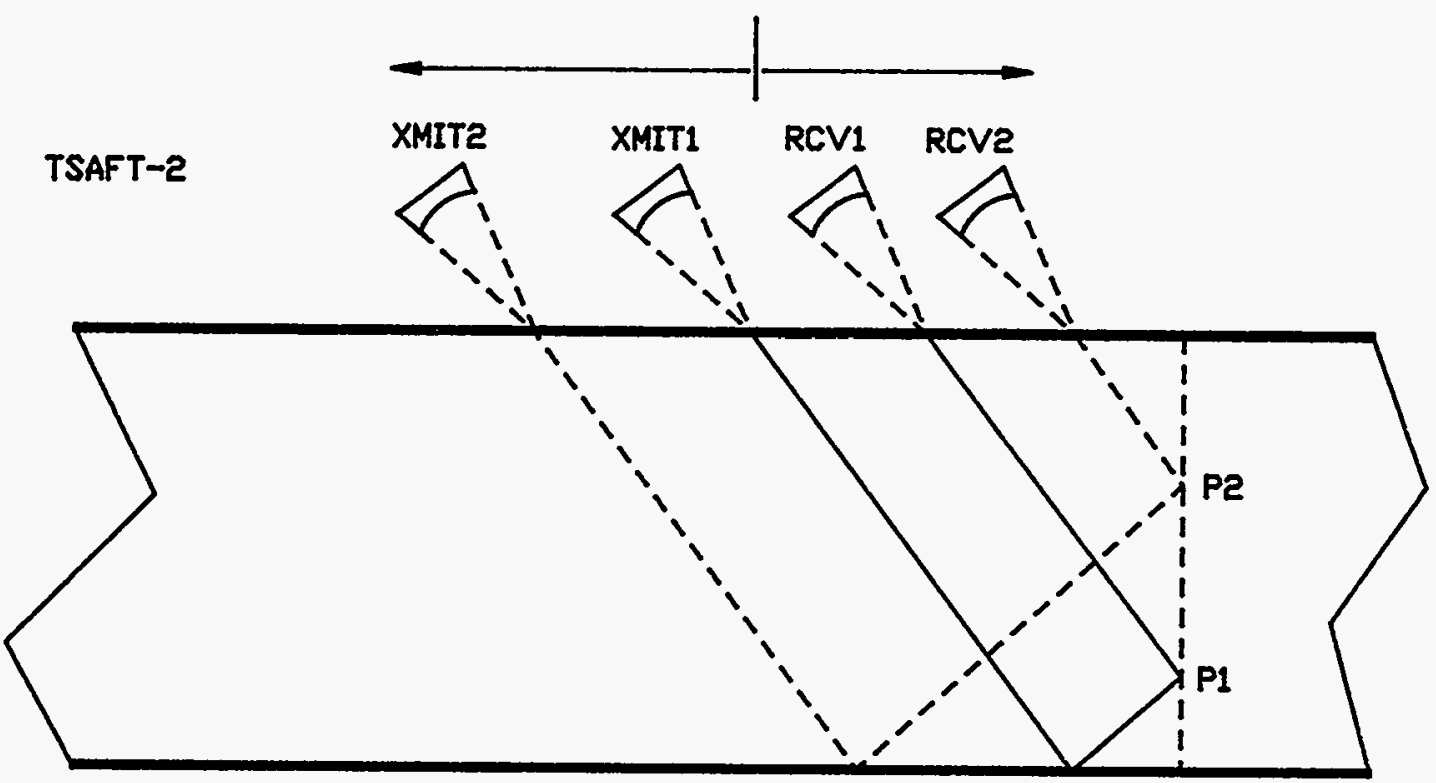

Figure 4.30. TSAFT-2 Configuration Using $1 / 2$-Vee Transmit Path

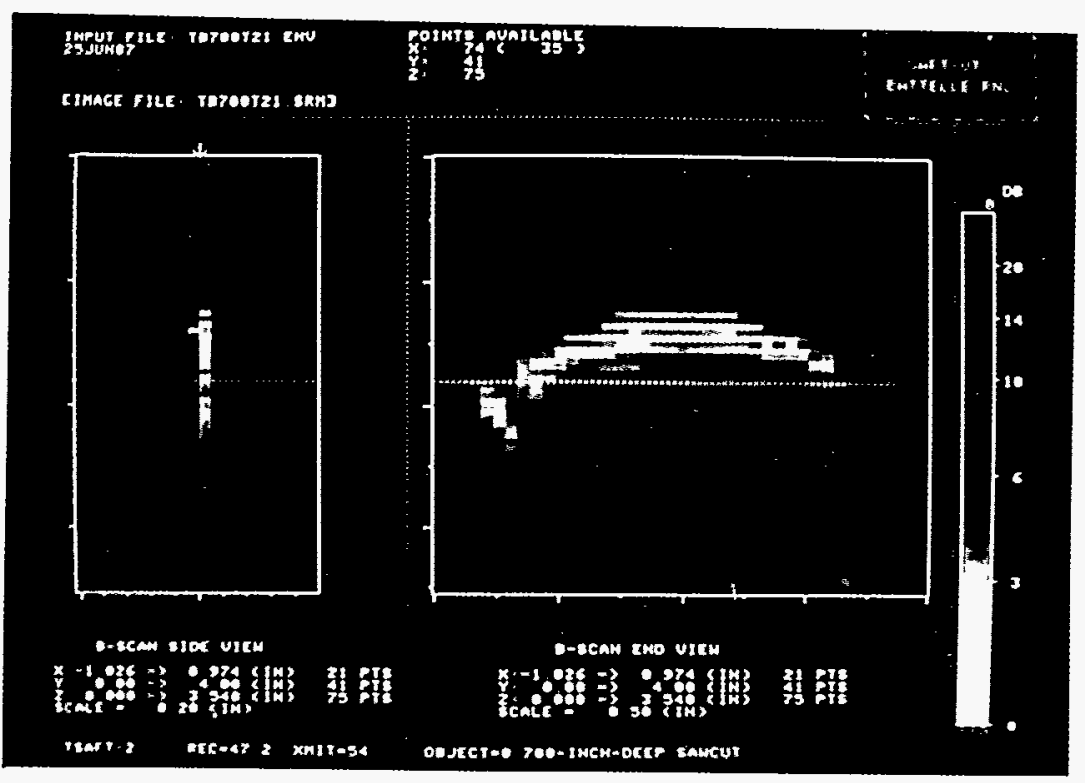

Figure 4.31. TSAFT-2 Image of 0.7-in.-deep (1.8-cm) Sawcut in 9-in.-thick (22.9-cm) Specimen, $54^{\circ}$ Transmitter and $47.2^{\circ}$ Receiver 


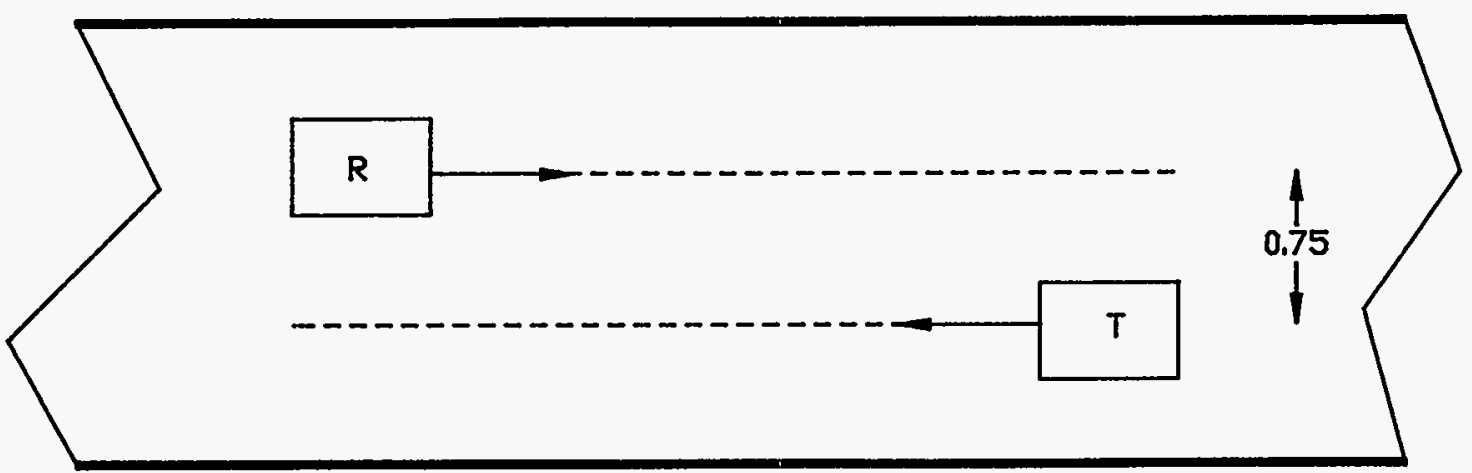

(a) TSAFT-3 Initial Position for a Single Sweep

TOP VIEW

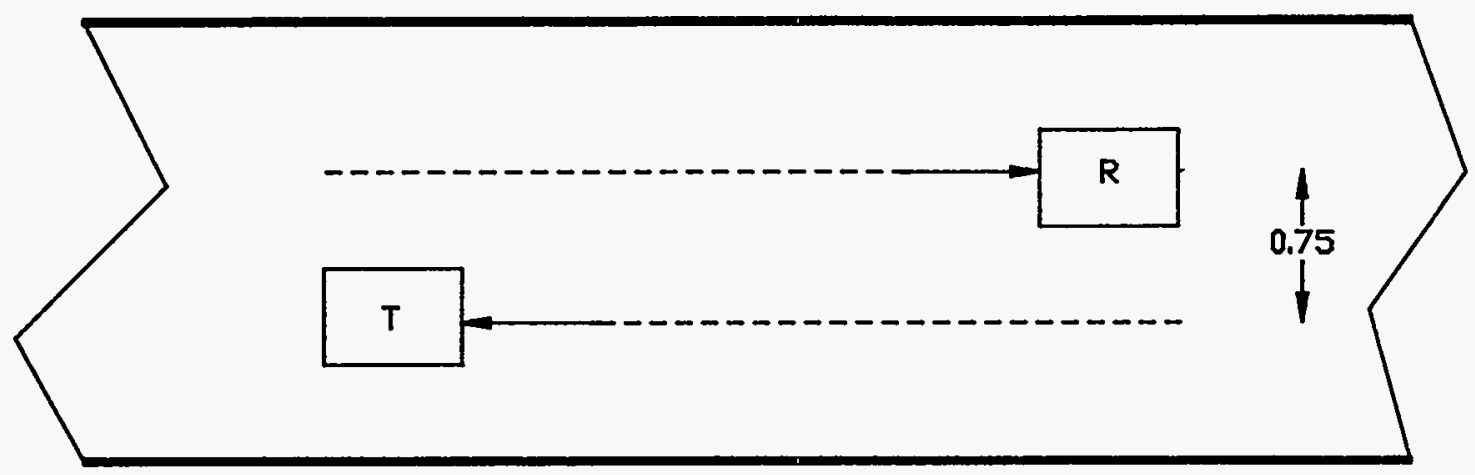

(b) TSAFT-3 Ending Position of a Single Sweep

Figure 4.32. TSAFT-3 Initial (a) and Ending (b) Transducer Positions for a Single Sweep

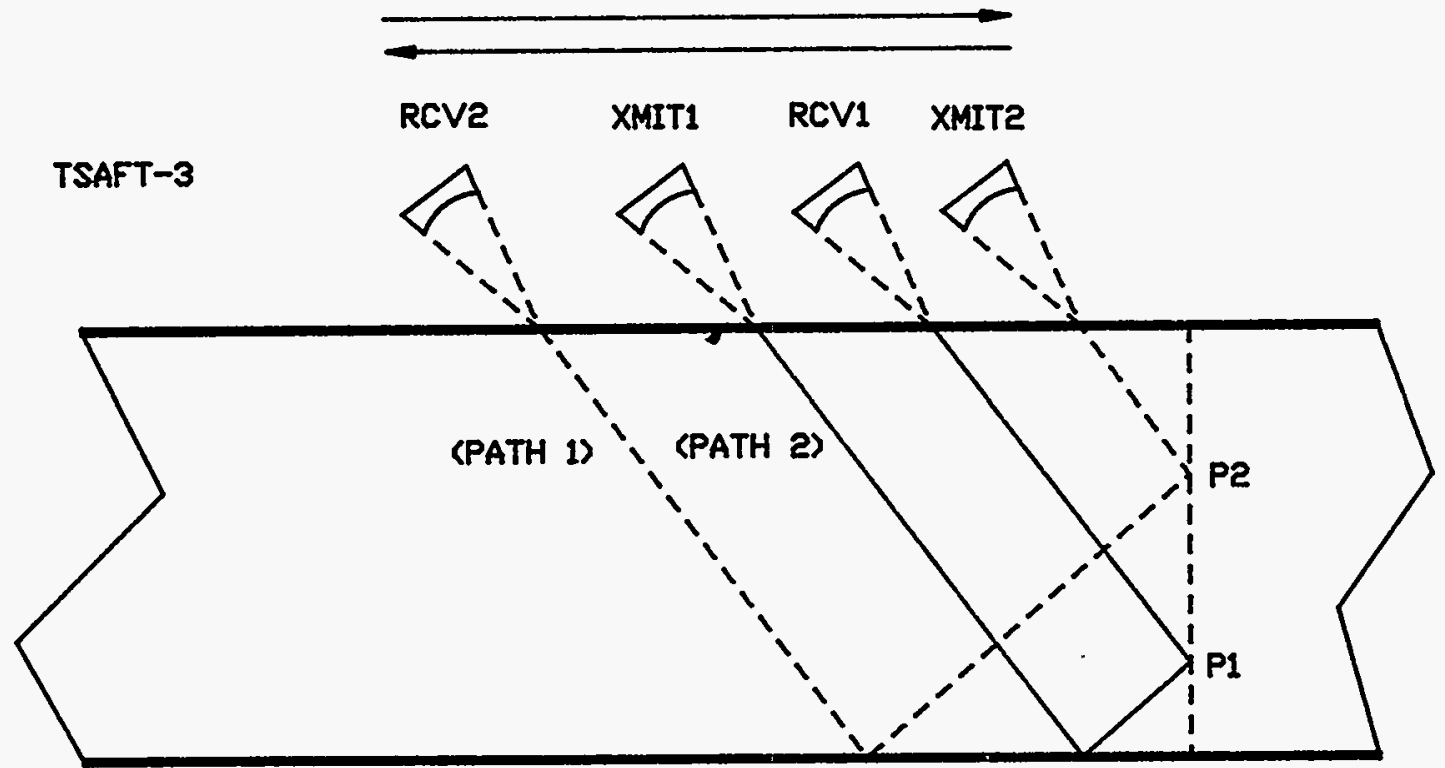

Figure 4.33. TSAFT-3 Configuration Showing Paths Contributing to the Real (Path 2) and Conjugate (Path 1) Image 

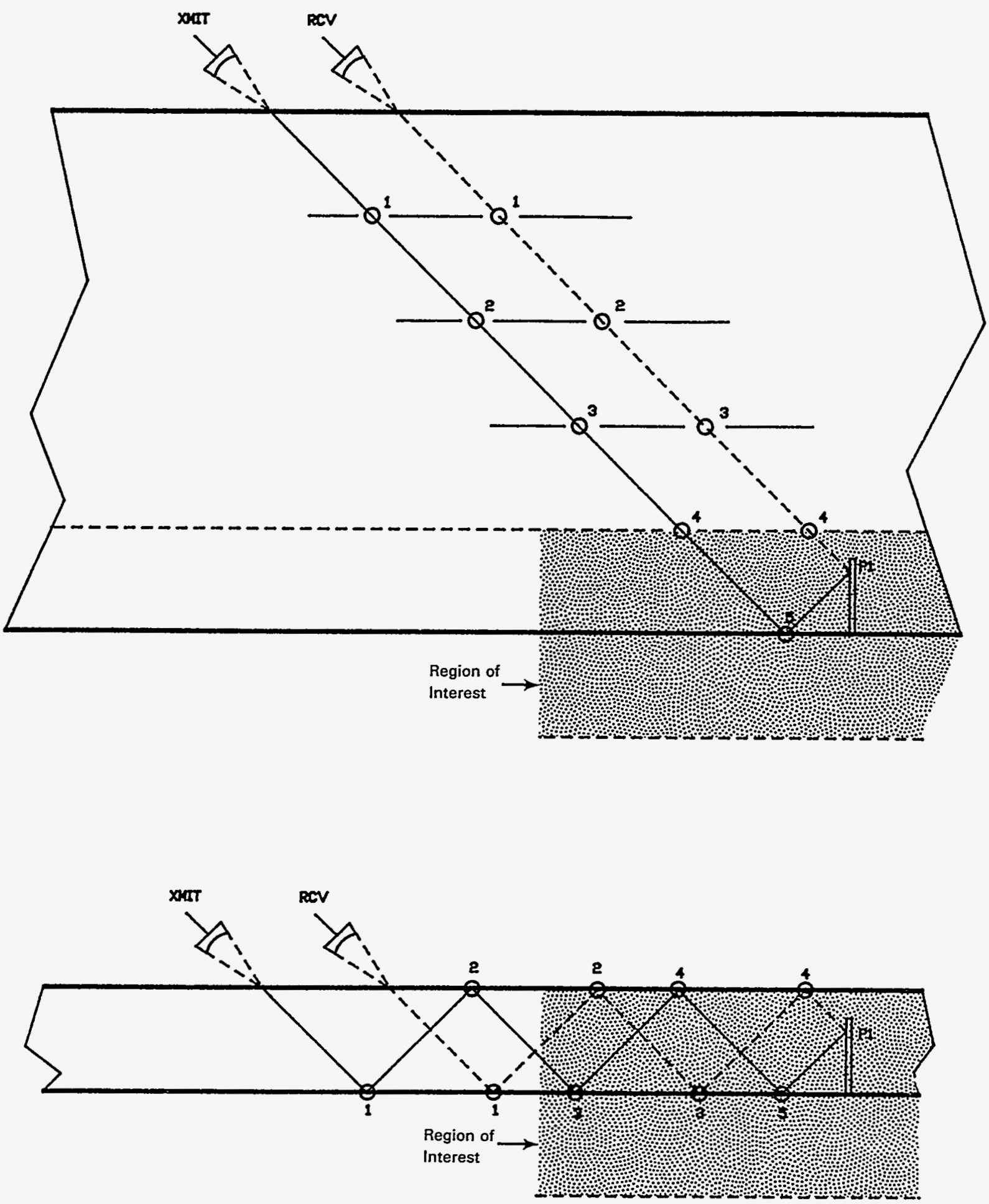

Figure 4.34. Side Views of TSAFT-3 Setups Showing 9-in.-thick (22.9-cm) Steel Block with 0.7-in.-deep (1.8-in.) Sawcut and Fictitious Material Thickness with "Folded" Transmit/Receive Paths 


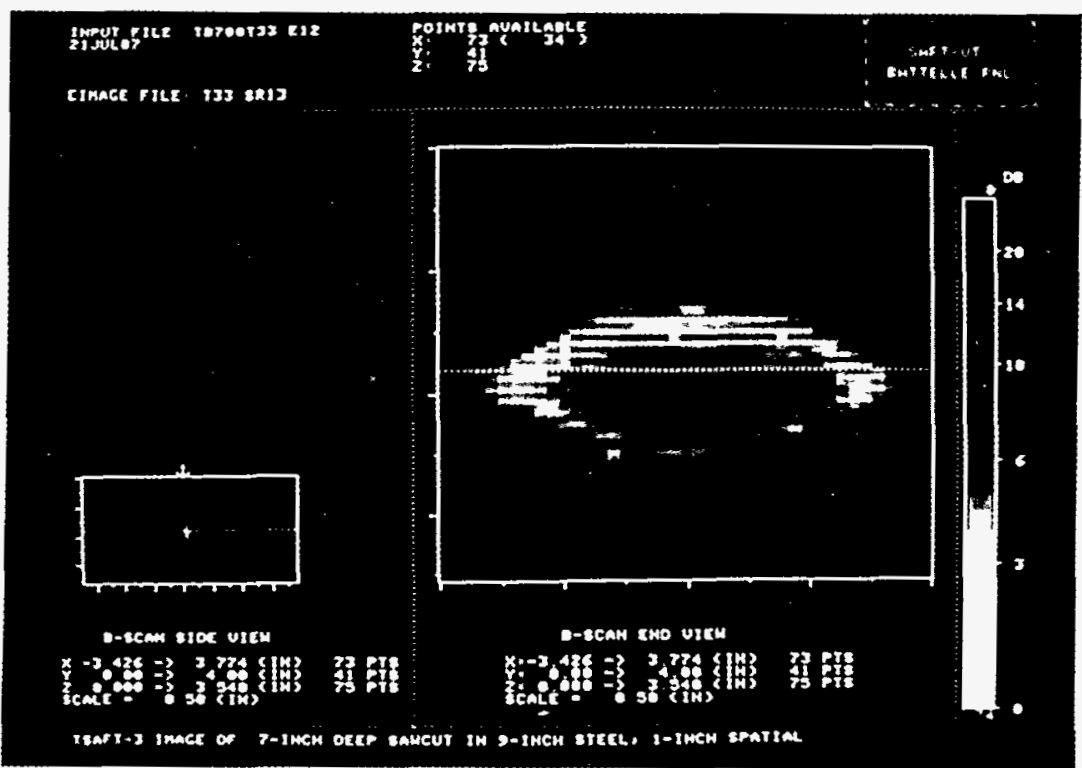

Figure 4.35. TSAFT-3 Image of 0.7 -in.-deep (1.8-cm) Sawcut in 9-in.-thick (22.9-cm) Specimen, $47.2^{\circ}$ Transmitter and Receiver

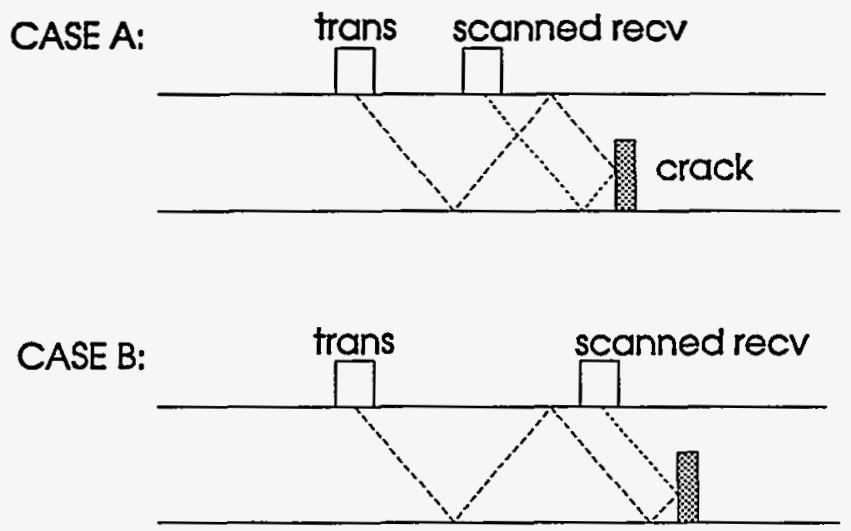

CASE C: both xmlt \& recV are direct paths to crack.

CASE D: both xmlt \& recV paths bounce off back surf to crack.

Select processing mode by entering case letters desired.

(If select more than one case

do not space between letters)

Please enter selection-

Figure 4.36. Additional Tandem Processing Parameters, Initial Screen Display 
Bounce $=3$

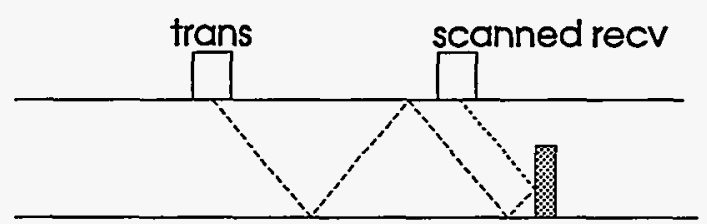

Bounce $=5$

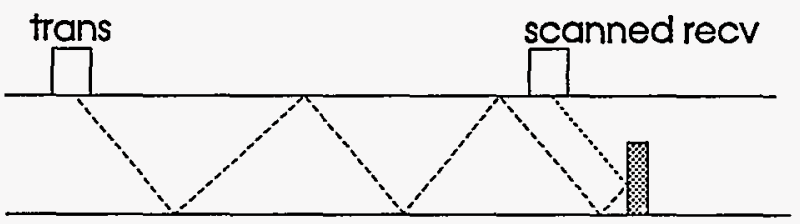

Enter number of bounces
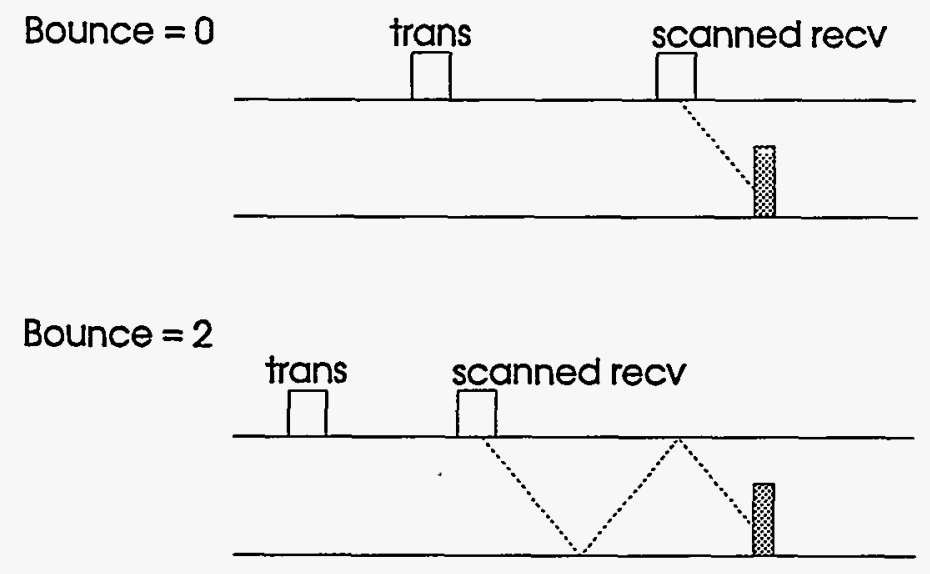

Enter number of bounces

Figure 4.37. Additional Tandem Processing Parameters, Subsequent Screen Display 


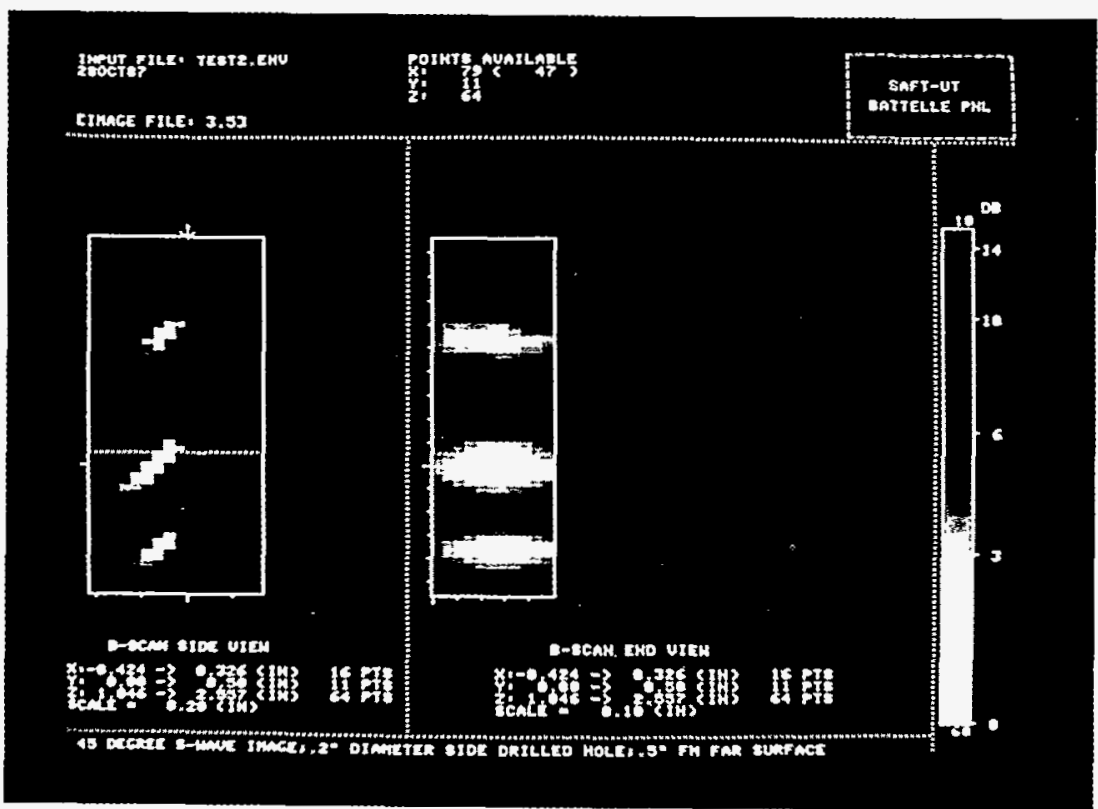

Figure 4.38. Pulse-Echo Image of a 0.2-in.-diameter $(0.5-\mathrm{cm})$, Side-Drilled Hole, 0.5 in. $(1.3 \mathrm{~cm})$ up from the Far Surface of a 2-in.-thick $(5.1-\mathrm{cm})$ Steel Block 


\subsection{Laboratory Application Research Studies}

This section describes the final extensive laboratory testing conducted on the SAFT technology. A review of how well SAFT-UT performed compared to other teams, employing both advanced and manual scanning techniques, who participated in a mini-round robin exercise, begins Section 5. The mini-round robin exercise utilized piping specimens with IGSCC to evaluate advanced and manual techniques, quantifying the results. Although these were laboratory tests, they have been included as part of the validation work discussed in Section 10. Section 5 reports on the outcome of a thick-section study i.e., pressure vessels. Further, a discussion of the development of TSAFT-3 is included. Also included is a section on advanced preprocessing techniques developed at PNL, which can be used prior to SAFT processing to enhance the subsequent images. Such image enhancement reduces the number of false calls, improving the inspectability of anisotropic materials such as CCSS, historically a difficult medium in which to reliably detect and size cracks.

For completeness, important earlier laboratory application research studies are cited as follows:

- inspection of a saft-end from the Duane Arnold nuclear reactor, provided to PNL by Southwest Research Institute (SwRI) (Doctor et al. 1986)

- inspection of a thick-section cladded plate with fabricated defects - data was acquired by Risley Nuclear Laboratories and sent to PNL for SAFT processing and analysis (Doctor et al. 1986).

- inspection of centrifugally cast stainless steel samples provided by the PISC II SSRRT - Data collected from these samples provided an extremely valuable database that was later used to develop preprocessing techniques that could be applied to anisotropic materials and is discussed in detail in Section 5.3 of this report (Doctor et al. 1987a).

- inspection of a submarine hull weld test specimen; Amdata, Inc. supplied the specimen. This was the first application where the TSAFT-2 configuration was employed and proved invaluable for detecting some near surface defects (Doctor et al. 1987b)

- inspection of three welds on two large-diameter (24-in. [60.96-cm] Schedule 80) 304 stainless steel test pipes; the pipes were located at the Materials
Reliability Center and were part of an Electric Power Research Institute Program to study IGSCC. The entire SAFT-UT field system was relocated on site and much of the processing and analysis took place on site as well (Doctor 1987b)

inspection of four stainless steel overlay specimens; the specimens were supplied by Knoll Atomic Power Laboratory and contained a variety of unknown defects (Doctor 1987b).

\subsection{Investigation of SAFT Imaging in Thick Material}

A series of tests was performed using a 9-in.-thick $(22.9-\mathrm{cm})$ carbon steel specimen with a number of machined defects placed in the far surface of the part. This specimen represents the first step in analyzing the performance of the SAFT-UT configurations in thick vessel material. Ten vertically oriented semicircular sawcuts were placed in this specimen, varying in depth from 0.050 in. to 0.700 in. $(0.13 \mathrm{~cm}$ to $1.9 \mathrm{~cm})$.

Figure 5.1 shows the B-scan side view and B-scan end view of a SAFT-UT image of the 0.050-in.-deep (0.13$\mathrm{cm})$ sawcut. The horizontal dotted line in the photograph represents the position of the far surface. This scan used a 0.25 -in.-diameter $(0.6-\mathrm{cm})$ transducer with a center frequency of $2.25 \mathrm{MHz}$. A Lucite wedge was used to cause a $47.2^{\circ}$ center-beam angle in the carbon steel material. Notice that the corner-trap reflection of this small defect is easily detected. However, the tip signal does not appear in the image presented. This is to be expected because the system resolution is on the order of two wave lengths $(0.100$ in. $[0.25 \mathrm{~cm}])$.

Figure 5.2 shows the corresponding SAFT-UT pulseecho configuration image of the 0.700 -in.-deep $(1.8-\mathrm{cm})$ sawcut. The color scale normalization has been adjusted in this photograph to enhance the display of the to image of the tip of the sawcut. Notice that the tip signal is now imaged very well, and that accurate through-wall sizing can now be performed from this image. However, because this is a single transducer configuration, the ambiguity limitations inherent with this illumination are still present. There is not a full characterization of the defect, as only the corner and the tip of the vertically oriented reflector are imaged. Therefore, it is important to implement the TSAFT-2 configuration for thick vessel material. 
It is impractical to implement a multiple-bounce configuration in thick materials, because the attenuation and geometries become intolerable. The thick material implementation of TSAFT-2, then, must involve using a $1 / 2$-vee transmitter and a $1 / 2$-vee receiver. This configuration illuminates the object plane by bouncing off the far surface only once, as opposed to the multiplebounce thin material configuration. The initial configuration that will be described here utilizes a $54^{\circ} \mathrm{S}$-wave transmitting probe and a $47.2^{\circ}$ receiving probe. Each transducer used in this configuration is 0.25 in. $(0.6 \mathrm{~cm})$ in diameter and has a center frequency of $2.25 \mathrm{MHz}$.

Figure 5.3 shows the TSAFT-2 B-scan side view and Bscan end view image of the 0.050 -in.-deep $(0.13-\mathrm{cm})$ sawcut. The horizontal dotted line represents the position of the far surface, and, although one can see the real and conjugate images of the sawcut, they are aperture-limited in this image. A vertical extent of approximately 0.18 in. $(0.46 \mathrm{~cm})$ would be measured from this image using a 6-db criterion. The size of this sawcut is smaller than the system resolution; consequently, oversizing occurs. This image becomes a measure of the vertical resolution of the system.

Figure 5.4 shows a TSAFT-2 image of the 0.700 -in.deep (1.8-cm) sawcut. The B-scan end-view presentation reveals two weaknesses of this configuration. The first apparent characteristic is that the conjugate image is highly degraded. This is due to limited scan in the $\mathrm{X}$ direction, because the two transducers obstruct each other in this direction. The conjugate image is needed to reduce the sensitivity of the vertical sizing measurements to variations in material velocity and material thickness.

Close observation of the B-scan end view of Figure 5.4 also shows that this configuration does not image the center part of the reflector. The zero order (low spatial frequencies) are lost because the wedges of the two transducers were selected such that the center angles are not equal. Thus only the top edge of the sawcut was imaged.

An alternate TSAFT-2 configuration (TSAFT-3) was conceived that eliminates the deficiencies shown in Figure 5.4. The small diameter probes were staggered slightly $(0.75$ in. $(1.9 \mathrm{~cm})$ center-to-center) in the $\mathrm{Y}$ direction so they pass by each other. Wedges were chosen to provide identical center beam angles and, as is characteristic of the TSAFT-2 configuration, they were scanned in opposing directions.

The corresponding image of the identical defect using the TSAFT-3 configuration is shown in Figure 4.36. Notice that the conjugate image is much improved and that the full information is now available for operator interpretation. This technique is expected to greatly improve imaging of defects in deep section material.

\subsubsection{TSAFT-3 Development}

Thick-section materials inherently prohibit the use of multiple-bounce paths normally associated with TSAFT modes; attenuation of the sound field becomes intolerable. A practical solution is to limit the number of bounces to one (before the sound strikes the vertical object plane). Figure 4.31 shows the center ray paths to the vertical object plane for a 1/2-vee TSAFT-2 configuration. The difficulty using TSAFT-2 in this configuration is the physical limitations; i.e., the transmitter can only scan forward until it hits the receiver. Unfortunately, a portion of the real image and most, if not all, of the conjugate image is lost. Figure 4.32 shows the TSAFT-2 image (using $54^{\circ}$ transmit and $47.2^{\circ}$ receive inspection angles) of a 0.7 -in.-deep $(1.9 \mathrm{~cm})$ sawcut that has been placed in the far surface of a 9-in.thick $(22.7-\mathrm{cm})$ steel block. The B-scan end-view reveals two weaknesses of this configuration. First, a portion of the real image is missing. Second, the conjugate image is highly degraded.

A third tandem configuration, a special case of TSAFT2 called TSAFT-3, was implemented for thick-section vessels where it was not practical for the transmit transducer to be $3 / 2$-vees from the vertical image plane due to the long material paths attenuating the sound field. TSAFT-3 overcomes the physical problems of TSAFT2 , allowing the transmit transducer to be $1 / 2$-vee from the vertical object plane. The transmit transducer is offset (when small Lucite wedges are used, the offset is typically $0.75 \mathrm{in} .[1.9 \mathrm{~cm}$ ) such that it passes by the receive transducer in a side-by-side fashion as shown in Figures 4.33(a) and 4.33(b). There are no provisions to compensate for the offset distance between the transmit and receive transducers; the long material paths from the transducers to the vertical object plane make the effect of the offset negligible when small transducers are used. The transmitter would initially start in front 
of the receive transducer as shown in Figure 4.33(a); both transducers are scanned equal distances but, as is typical of the TSAFT configurations, in opposite directions as shown in Figure 4.33(b). TSAFT-3 provides all of the advantages of TSAFT-2; plus, it allows a shorter material path (1/2-vee) to be used, improving the signal-to-noise ratio in thick-section materials.

Figure 4.34 shows the positions the transmit and receive transducers are in when they are toward the extremes of the scanned aperture. Path 1 provides data for the conjugate image, and path 2 data is used for the real image. The real and conjugate portions of the defect image may be observed simultaneously by choosing to process beyond the far surface during TSAFT-

2/TSAFT-3 processing. The vertical extent of a surface-connected defect may be accurately determined by measuring the full vertical extent of the image and dividing the result by two. This technique reduces the sensitivity of the vertical sizing measurements to variations in material velocity and material thickness, and the location of the far surface relative to the image need not be known accurately.

Processing TSAFT-3 data requires manipulating three data file header parameters; i.e., material thickness, the number of transmit bounces, and the number of receive bounces. If the correct material thickness is used, the image space for TSAFT becomes too large to process. The TSAFT algorithm processes the entire material thickness, and when processing beyond the far surface is chosen, twice the material thickness is processed.

When dealing with thick-section specimens and the TSAFT modes, it is necessary to limit the depth range that is processed and subsequently displayed. Figure 4.35(a) presents a typical TSAFT-3 setup, on a 9-in.thick $(22.9-\mathrm{cm})$ steel block with a $0.7-$ in.-deep $(1.8-\mathrm{cm})$ sawcut placed into the far surface. This setup will be used as an example. In our example, the region of interest is only the lower fifth of the material thickness. Processing is selected to be beyond the far surface, but limited in range as shown by the shaded area in Figure 4.35(a). To accomplish this, a fictitious material thickness value, equal to the original thickness divided by five $(1.8 \mathrm{in} .[4.6 \mathrm{~cm}])$, is entered into the file header parameters using the DEDIT utility. If a different region of interest were desired, the material thickness could be divided by any odd number. The transmit and receive paths are then "folded", Figure 4.35(b), to give equivalent material path lengths. The correct number of transmit and receive bounces are counted and entered into the file header parameters. For our example, the number of transmit bounces would be 5 and the number of receive bounces would be 4 . An easy ruleof-thumb is as follows:

1. The number of transmit bounces will always be an odd value,

2. the number of receive bounces will always be one less and an even value, and

3. the material thickness value to be entered is the true material thickness divided by the number of transmit bounces.

Once the necessary header modifications have been made, the TSAFT-3 data file may be processed normally.

\subsection{Application of Advanced Signal Processing Techniques to Improve SAFT-UT Performance on CCSS Mate- rial}

The initial results of the SAFT-UT inspection of the CCSSRRT samples were somewhat disappointing, because although all the cracks were detected, the false call rate was unacceptable. The samples consisted of laboratory-induced cracks in 2.4-in.-thick $(6.1-\mathrm{cm})$ CCSS material, with both columnar and equiaxed grain structures utilized. In general, SAFT performed as well as any of the teams who inspected the blocks; however, the algorithm implemented tended to generate a significant amount of false calls. Crack detection was not a problem, but distinguishing between cracks and coherent scattering from the microstructure was difficult. In the interest of improving SAFT performance on this type of specimen, further analysis was undertaken. Specifically, the emphasis of this analysis was focused on the frequency characteristics of this data base.

A distinct advantage of the SAFT-UT system is that the raw (unprocessed) data is archived and available for further analysis. With the SAFT-UT system, if one ensures that the integrity of the data files are of high quality, then this becomes a data base from which 
future analysis may draw. The CCSSRRT data falls into this category, and as such, the complete data set is fully retrievable.

It has been known for some time that the coherent summation algorithm incorporated in the SAFT-UT operation is a narrow-band approximation. This was reviewed by Busse, Collins, and Doctor (1984) in this program and was discussed earlier by Norton (1976) in his thesis paper at Stanford University. The exact method incorporates a time derivative of the raw data file prior to SAFT processing. When analyzing the spectral responses of data files collected on CCSS material with a $500 \mathrm{kHz}$ transducer, significant components as low as $150 \mathrm{kHz}$ and as high as $600 \mathrm{kHz}$ may be present. This suggests that perhaps the narrow-band approximation may not be totally valid in this case.

Also, as will be discussed later, one sees a difference in spectrum from a cracked area and an uncracked area. This suggests that a bandpass filter incorporated in the algorithm may enhance the image quality of the SAFTUT system. The purpose of the experiments reported herein is to define a bandpass filter that can be applied to a SAFT data file, prior to processing, that would reduce the ambiguities that exist between the coherent scattering from microstructures and cracks.

To assist in spectral analysis of the SAFT waveforms, the APLOT (SAFT-UT analysis graphics) utility was enhanced to provide a power spectrum plot on the RAMTEK of any selected A-scan of a given data file. Also, three additional SAFT utilities were added: one to perform a bandpass filter operation, one to perform a Fourier transform operation, and another to perform the time derivative of a given data file. The bandpass filter routine simply rejects all frequencies below a specified value and above a second specified value. Both of these values are user-selectable. No attempt was made to weight this in a Gaussian form or any more elaborate shape. The time derivative function simply performs the transform using the following equation shown in Bracewell's (1965) text:

$$
F^{\prime}(s)=i 2 \pi s \quad F(s)
$$

where

$$
s=\text { frequency, }
$$

$F(s)=$ the Fourier Transform of the original data set

$F^{\prime}(s)=$ the Fourier Transform of the desired time derivative, and

$$
\mathbf{i}=\sqrt{-1}
$$

\subsubsection{Applying the Spectral Analysis and Subsequent Filter Technique to a CCSSRRT Data File}

Specimen 5 of the CCSSRRT data set (see Bates, Doctor and Heasler 1987) was used for an initial analysis. The parent material of this block is $2.4 \mathrm{in} .(6.1 \mathrm{~cm})$ thick and consists of columnar material on one side of the weld and equiaxed material on the opposite side of the weld. The weld is approximately 2.2 in. $(5.6 \mathrm{~cm})$ thick and 7.6 in. $(19.3 \mathrm{~cm})$ long, with a crack introduced (estimated to be about $28 \%$ of wall thickness) on the columnar side of the weld. The crack in this specimen begins 4.2 in. $(10.7 \mathrm{~cm})$ and extends to 5.9 in. $(15 \mathrm{~cm})$ along the weld. SAFT data was collected from this specimen using a $0.5-\mathrm{MHz}$ transducer in $45^{\circ}$ shear mode. The primary data file of interest was collected on the columnar side of the weld area (the same side as the crack).

Figure 5.5(a) shows the SAFT-UT image of the area scanned. On the left of the photograph is the B-scan side view; while at the right, the B-scan end view is shown. The top of the image displayed corresponds to a 1-in. $(2.5-\mathrm{cm})$ depth in the material; and the dotted line, visible in the B-scan end view, represents the expected position of the far surface $(2.3 \mathrm{in}$. [5.8 cm] from the near surface). Some indications appear beyond the far surface because of velocity variations and beam skewing. The image is apparently very noisy; and in fact, from the B-scan end-view image, one would conclude that there is a crack the full length of the scan. In fact, the crack occurs only in the last 2 to 3 in. (5.1 to $7.6 \mathrm{~cm}$ ) of the scan. The indication that is apparent in the first 2 in. $(5.08 \mathrm{~cm}$ ) (the left side of the B-scan end view) is not from a crack structure but from the microstructures.

Figure 5.5(b) graphically shows the A-scan of an area that was believed to be cracked, while Figure 5.5(c) 
corresponds to an area known to be uncracked. Each A-scan presented here and in subsequent figures represents the full digitized return signal from this material. The digitized window includes from the front surface through an equivalent material path length of 4.5 in. $(11.4 \mathrm{~cm})$. These images, most obviously, are very noisy signals and, consequently, are difficult to interpret.

Figures 5.5(d) and 5.5(e) show the power spectra that correspond to the adjacent $A$-scans. If one closely reviews these figures, it can be seen that the general spectra are wide band, in that they contain much more low-frequency components than would be expected for a $0.5-\mathrm{MHz}$ center-frequency system. Also, the largest component in the uncracked region is shifted down in frequency by $70 \mathrm{kHz}$ with respect to the peak value in the cracked region. From these general observations, a series of tests was performed, including SAFT processing on the time derivative of the data set, on a bandpass filtered data set, and on a combination of both.

First, the time derivative was generated from the original data set. The results of this may be seen in Figure 5.6. An improvement in signal-to-noise level can be observed in the image shown in Figure 5.6(a). The indication in the region where no defect is located is reduced (in the B-scan end-view, this is at the 1-in. [2.5$\mathrm{cm}$ ] location). Also, a notable reduction in background noise is realized. When one of the spectra in this figure is compared with those shown in Figure 5.5 it can be seen that the effect of the time derivative on the power spectrum is to de-emphasize the low-frequency components.

A second test consisted of a more direct approach to altering the frequency spectrum of the waveforms. A step-wise filter was applied to the data set prior to SAFT processing. This filter rejected frequencies below $0.38 \mathrm{MHz}$ and above $0.68 \mathrm{MHz}$. The results of this test are shown in Figure 5.7. The final image presented in Figure 5.7(a) shows a substantial improvement in signal-to-noise level. The background noise is nearly eliminated, and the false indication at the $1-$ in. $(2.5-\mathrm{cm})$ location of the B-scan end view has vanished.

The final test consisted of a combination of the previous operations. The time derivative of the original data set was generated. This result was then passed through the bandpass filter previously described. Finally, the SAFT processing was performed on this result. The results of this are shown in Figure 5.8. The final image shown in this figure is comparable to that generated with only a filter operator (Figure 5.7). This can be expected because the narrow-band approximation becomes valid subsequent to this filter operation, thus reducing the effectiveness of the time-derivative operator.

\subsubsection{Software Tools Used to Define the Op- timal Bandpass Filter Used to Enhance the SAFT-Processed Image}

Initial results indicate that if an optimum bandpass filter is defined and applied prior to SAFT processing, the resulting image is greatly enhanced and the false call probability is decreased. The purpose of this section is to describe the software used to accomplish the task of defining the optimum bandpass filter limits.

From the initial results, one begins to believe that there may be a frequency range at which defect and nondefect areas may be discriminated. Proof of this assumption would be a high correlation to the premise that large components of the power spectral response from A-scans containing defects will correspond to small components of the power spectral response of $A$ scans containing nondefects. Using the CCSSRRT data base, a statistical analysis of the spectrum data files was conducted.

The CCSSRRT specimens provided a data base in which the defect and nondefect areas are well defined. The DEDIT utility was modified to allow a flag to be set in each A-scan recording in a given data file. This flag represents three conditions:

1. A "0" indicates an "unsure region." These A-scans may lay near the edge of a defect or the edge of the specimen itself and will not be used in the subsequent statistical analysis process.

2. A "1" indicates a "defect region." These A-scans are in line with a defect.

3. A "-1" indicates a "nondefect region." These Ascans are not in line with a defect.

To assist in the statistical analysis, a new utility, STATANAL, was implemented. The STATANAL 
utility calculates a correlation coefficient for selected frequency and transducer axial position ranges. Only records with the defect flag set to a nonzero value are used for input. Data files used as input to the STATANAL utility are first converted to spectrum data files using the Fourier transform utility, SPECTRUM, and each A-scan record defect flag is set to reflect the nature of the A-scan using the DEDIT utility. The statistical results reflect how well the data in the selected ranges fit the ideal (i.e., corresponding spectral values are of a low magnitude for nondefect areas and a high magnitude for defect areas). To better understand the correlation coefficient value, additional information is provided for each selected frequency and transducer position range, as shown below:

1. the total number of defect and nondefect A-scans used in the statistical analysis

2. the arithmetic mean of the spectral magnitudes

3. the standard deviation.

It is important to ensure that the transducer position entries are relative to the known defect location, except in the case of blank specimens where the transducer position entries are relative to the weld center line.

The following is a typical scenario used when employing the STATANAL utility:

1. Run the SPECTRUM utility, supplying the input and output filenames.

2. Use the DEDIT utility to appropriately set the defect flag in each A-scan record of a selected spectrum data file. Edit the initial transducer position to reflect the position relative to the known defect location for a specimen containing a defect, or relative to the weld center line for a specimen containing no defect.

3. Run the STATANAL utility. The user is prompted for the following input:
a. selected spectrum data file names
b. selected $X$ ranges (in inches)
c. selected frequency ranges (in $\mathrm{MHz}$ )
d. selected output filename.

Table 5.1 presents a typical output of the STATANAL utility for a sawcut data file, using a single frequency range of 2.1 to $2.4 \mathrm{MHz}$ and a single $\mathrm{X}$ position range of -0.7 to -0.5 in. $(-1.8$ to $-1.3 \mathrm{~cm}$ ) (normally, many small frequency and $X$ position ranges are used). The sawcut data file serves to check the validity of the STATANAL utility. The sawcut data file is a $45^{\circ}, 2.25-\mathrm{MHz}$ shear-wave file of a 0.3 -in.-deep $(0.8-\mathrm{cm})$ sawcut, placed in a $0.6-$ in. $(1.5-\mathrm{cm})$ stainless steel coupon. A perfect correlation would be $\mathrm{a}+1$ and an inverse correlation would be a -1 . Table 5.1 presents the maximum correlation coefficient for the selected frequency range. There is a near perfect correlation coefficient $(+0.907)$ for the $\mathrm{X}$ position and frequency range used. Attention is drawn to this fact by placing an asterisk next to any correlation coefficient values exceeding +0.9 . Finally, the last portion of the STATANAL printout lists the composite spectra values for defect and nondefect Ascans at each frequency increment. The difference value presented indicates what frequencies provide the largest magnitude difference between defect and nondefect A-scans.

\subsubsection{Statistical Analysis of Defect and Non- Defect CCSS A-Scans}

The CCSSRRT data set was split into two subsets: 1) data acquired from specimens having an equiaxed grain structure and 2) data from specimens having a columnar grain structure. Each data subset was evaluated at specific frequency and transducer position ranges to determine which ranges yielded the highest correlation to the hypothesis $A$ frequency range and transducer position exists where defect spectral response components will be large and corresponding nondefect spectral response components will be small.

Each equiaxed and columnar spectrum data file A-scan defect flag was set to reflect the nature of the A-scan. A-scans within 0.5 in. $(1.3 \mathrm{~cm}$ or one-half the transducer diameter) of the edge of the specimen or outside this tolerance about the defect were considered unsure, and the defect flag was set appropriately. The remainder of the A-scan records were either defect or nondefect in nature.

Figures 5.9 and 5.10 show the graphical results attained for equiaxed and columnar shear-wave spectrum data files. The maximum correlation value is plotted for 
Table 5.1. Typical Printouts of the STATANAL Utility <STATANAL> There are 1 files in the stack. <STATANAL> 1 SAWTVG2.SPM

Summary of $\mathrm{X}$-ranges:

$1-0.700$ to -0.500 inches

.. Region 1 frequency range (2.100000 to 2.400000 $\mathrm{MHz})$..

$\begin{array}{lr}\text { X-range } & 1 \\ \text { DEFECT } & 2448 \\ \text { NODEFECT } & 1872 \\ \text { AR MEAN } & 94.990 \\ \text { STD ERROR } & 0.209 \\ \text { COR COEF } & 0.907^{*}\end{array}$

NoTE:
$\begin{aligned} & \text { The following data was computed for } \\ & \text { the first specified } x \text { range only }(-0.700 \\ & \text { to }-0.500 \text { inches })\end{aligned}$

$\begin{array}{ll}\text { defect normalization factor } & =153 \\ \text { non-defect normalization factor } & =117\end{array}$

\begin{tabular}{|c|c|c|c|}
\hline $\mathrm{MHz}$ & Defect & NONdefect & Def-NON \\
\hline 2.109 & 139.059 & 42.590 & 96.469 \\
\hline 2.129 & 141.216 & 48.624 & 92.592 \\
\hline 2.148 & 148.693 & 46.846 & 101.847 \\
\hline 2.168 & 156.425 & 36.171 & 120.254 \\
\hline 2.187 & 157.013 & 20.393 & 136.620 \\
\hline 2.207 & 153.118 & 9.607 & 143.511 \\
\hline 2.226 & 155.268 & 14.718 & 140.550 \\
\hline 2.246 & 157.928 & 23.265 & 134.663 \\
\hline 2.265 & 153.791 & 30.145 & 123.646 \\
\hline 2.285 & 149.758 & 33.462 & 116.297 \\
\hline 2.305 & 152.000 & 30.205 & 121.795 \\
\hline 2.324 & 152.458 & 20.855 & 131.603 \\
\hline 2.344 & 146.549 & 9.769 & 136.780 \\
\hline 2.363 & 142.353 & 4.761 & 137.592 \\
\hline 2.383 & 141.980 & 7.949 & 134.032 \\
\hline 2.402 & 137.484 & 9.000 & 128.484 \\
\hline
\end{tabular}

each frequency range. The peak positive correlation values occur between 0.375 and $0.4 \mathrm{MHz}$ in Figure 5.9, and at 0.375 to $0.525 \mathrm{MHz}$ in Figure 5.10. One would expect the resulting images to be enhanced if the equiaxed and columnar data files were first processed using the filter utility, with the lower and upper frequency limits set to correspond to the lower and upper frequency limits of the peak correlation values.

\subsubsection{Composite Spectra of the CCSSRRT}

The composite spectra result is attained by summing the spectrum data for defect records in a single selected axial position, and also summing the spectrum data for nondefect records in the same axial position. This result is then normalized for comparison purposes. Figure 5.11 represents the composite spectra results of the SAWCUT test file. The composite spectra is plotted for the defect and nondefect records. Included in Figure 5.11 is a difference plot (composite spectrum defect values minus the composite spectrum nondefect values). The peak spectral component occurs at 2.25 $\mathrm{MHz}$, the transducer center frequency. From the difference plot in Figure 5.11, one may choose the lower and upper frequency limits that would be used in the FILTER utility, which would then be applied to the data file prior to SAFT processing. The FILTER utility would be expected to enhance the subsequent image.

Specimens 3, 4, and 6 of the CCSSRRT set were chosen to analyze the results of applying a filter, based on the composite spectra results, to the data files prior to SAFT processing. The parent material of each block is approximately 2.4 in. thick $(6.1 \mathrm{~cm})$ and consists of a columnar microstructure on one side of the weld and an equiaxed microstructure on the opposite side. Table 5.2 shows the intended characteristics of CCSS specimens 3,4 , and 6 .

Crack detection and length were of primary concern when dealing with the CCSSRRT data set. To this end, improvement was sought by discriminating against microstructure echoes based on spectra content. Indications at or near the far-surface location most likely would be echoes produced by the corner-trap or where the crack intersects the far surface. Relative location of indications is one discriminatory criterion that is applied when analyzing images. Therefore, indications located well above the far surface or at the weld centerline are dismissed when analyzing images for cracks. 
Table 5.2. Intended Characteristics of CCSS Specimens 3, 4, and 6

\begin{tabular}{|c|c|c|c|c|c|}
\hline Spectuen & Weld Length & Crack Offset & Crack Start & . Crack End & Depth \\
\hline 3 & 7.5 & .15 & 4.3 & 5.8 & $23 \%$ \\
\hline 4 & 7.0 & 0.5 & 5.0 & 6.0 & $21 \%$ \\
\hline 6 & 7.0 & N/A & N/A & N/A & N/A \\
\hline
\end{tabular}

NOTE: Weld Length, Crack Offset, Crack Start, and Crack End are all expressed in OD inches.

Crack Offset is the lateral distance of the crack from weld center.

The weld of specimen 3 is approximately $2.2 \mathrm{in.} \mathrm{thick}$ $(5.6 \mathrm{~cm})$ and $7.5 \mathrm{in}$. long $(19.1 \mathrm{~cm})(\mathrm{OD})$; a crack was introduced (estimated to be about $23 \%$ of wall thickness) along the equiaxed side of the weld. In this specimen, the crack begins at $4.3 \mathrm{in} .(10.9 \mathrm{~cm})$ and extends to $5.24 \mathrm{in} .(13.7 \mathrm{~cm})$ along the weld. SAFT data was collected from this specimen using a $0.5-\mathrm{MHz}$ transducer in $45^{\circ}$ shear mode. The primary data file of interest was collected from the equiaxed side of the weld area (the same side as the crack).

Figure 5.12(a) presents the resulting composite spectra plots (defect, nondefect, and difference) for shear-wave data files in equiaxed material. All of the equiaxed shear-wave data files from the CCSSRRT data set were used to arrive at the composite spectra plot shown in Figure 5.12(a). From the difference plot, one would choose a lower frequency limit of $0.375 \mathrm{MHz}$ and an upper frequency limit of $0.425 \mathrm{MHz}$ to be used in the FILTER utility. The filtered data file is then SAFT processed. Figure 5.12(b) shows the before (top) and after (bottom) results of applying the filter to specimen 3. Specimen 3 contains a crack that is 1.5 in. $(2.8 \mathrm{~cm})$ long. Figure 5.12(b) (top image) shows the crack to extend from 1.9 to 5.8 in. ( 4.8 to $14.7 \mathrm{~cm}$ ) along the weld, or 3.9 in. $(9.9 \mathrm{~cm})$ in length, when measured at the $-6-\mathrm{dB}$ points (green/amber transition). The bottom image of Figure 5.12(b) shows the crack to extend from 4.4 to 5.8 in. (3.6 to $14.7 \mathrm{~cm}$ ) along the weld, a length of $1.4 \mathrm{in}$. $(3.6 \mathrm{~cm})$ using the $-6-\mathrm{dB}$ measurement criterion (green/amber transition)--clearly an improvement.
The weld of specimen 4 is approximately 2.2 in. thick $(5.6 \mathrm{~cm})$ and 7.0 in. (OD) long $(17.8 \mathrm{~cm})$; a crack was introduced (estimated to be about $21 \%$ of wall thickness) on the columnar side of the weld. In this specimen, the crack began at $5.0 \mathrm{in} .(12.8 \mathrm{~cm})$ and extended to $6.0 \mathrm{in} .(15.2 \mathrm{~cm})$ along the weld. SAFT data was collected from this specimen using a $0.5-\mathrm{MHz}$ transducer in $45^{\circ}$ shear mode. The primary data file of interest was collected on the columnar side of the weld area (the same side as the crack).

Figure 5.13(a) presents the resulting composite spectra plots (defect, nondefect, and difference) for shear-wave data files in columnar material. From the difference plot, one would choose a lower frequency limit of 0.475 $\mathrm{MHz}$ and an upper frequency limit of $0.525 \mathrm{MHz}$ to be used in the FILTER utility. The filtered data file would then be SAFT-processed, and an enhancement of the subsequent image would be expected. Figure 5.13(b) shows the before (top) and after (bottom) results of applying the filter to specimen 4 . Specimen 4 contains a crack that is 1.0 in. long $(2.5 \mathrm{~cm})$. Figure 5.13(b) (top image) shows the crack to extend from 1.5 to 5.7 in. $(3.8 \mathrm{~cm}$ to $14.5 \mathrm{~cm})$ along the weld when measured at the $-6-\mathrm{dB}$ points (green/amber transition), or $4.2 \mathrm{in}$. $(10.7 \mathrm{~cm})$ in length. The bottom image of Figure 5.13 (b) shows the crack to extend from 3.7 to 5.4 in. (9.4 to $13.7 \mathrm{~cm}$ ) along the weld--a length of $1.7 \mathrm{in} .(4.3 \mathrm{~cm})$ using the $-6-\mathrm{dB}$ measurement criterion (green/amber transition)--clearly an improvement.

Further investigation revealed that the indications comprising the bottom B-scan end view in Figure 5.13(b) 
were actually displaced laterally as evident in the B-scan side view. The actual lateral location of the crack relative to the weld center is 0.5 in. $(1.3 \mathrm{~cm})$. Figures 5.13(c) and 5.13(d) show single-slice B-scan end views of specimen 4. Figure $5.13(\mathrm{c})$ is taken $0.5 \mathrm{in} .(1.3 \mathrm{~cm})$ from weld center, and Figure 5.13(d) is taken exactly on weld center. Most likely, the indication shown in Figure 5.13(d) is associated with the weld metal and not a crack. Therefore, Figure 5.13(c) probably better represents the crack and, in fact, does correlate better to the actual crack location; i.e., Figure 5.13(c) shows the crack starting at 4.9 and ending at 5.3 in. $(13.5 \mathrm{~cm})$.

Specimen 6 is a blank specimen; i.e., no cracks were intentionally introduced and no cracks were detected using penetrant techniques. Specimen 6 consisted of columnar and equiaxed material joined by a weld. Figure 5.14 (top image) shows the processed data file of the equiaxed side of specimen 6; no filtering was applied to the raw data file prior to SAFT processing. Indications are seen the entire length of the image, and initially no crack call was made because of the location of the indications relative to the weld center and the far surface. A bandpass filter using the equiaxed filter parameters ( 0.375 to $0.425 \mathrm{MHz}$ ) was applied to the equiaxed data file prior to SAFT processing. In Figure 5.14 (bottom image), the indications are greatly reduced; it is even easier to conclude that this side of specimen 6 is not cracked. Figure 5.15 (top image) shows the unfiltered SAFT-processed results of the columnar side of specimen 6 . Once again, indications are seen. It would be difficult in this case not to call a crack nearly the entire length of the image and, in fact, initially a crack was called. Figure 5.15 (bottom image) shows the processed filtered image of specimen 6 . A $0.475-$ to $0.525-\mathrm{MHz}$ filter was applied to the data prior to SAFT processing. The image is a vast improvement over the unfiltered image and, again, it would be easy not to call a crack. The indication seen is above the far surface (identified by the dashed line) and beyond the weld center (indicated by the arrow), which may allow one to hypothesize that the indications are associated with the weld material and not from cracks.

Finally, the results of the CCSSRRT indicated that cracks were easier to detect in some specimens than others. These "easy crack" specimens were used to generate a composite spectrum to see if there are characteristics about these specimens that indeed resulted in the higher detection and sizing scores. High probability data files are defined as those specimens that had a high percentage of correct calls, as shown in Appendix B of Stainless Steel Round Robin Test, Centrifugally Cast Stainless Steel Screening Phase (Bates, Doctor and Heasler 1987).

Figures 5.16 and 5.17 show the results of composite spectra for shear-wave defect and nondefect A-scans in equiaxed (Figure 5.16) and columnar (Figure 5.17) material for spectrum data files that had a high probability of defect detection. Figures 5.16 and 5.17 indicate that the defect spectra magnitudes are larger than average and near the center frequency of the transducer. The nondefect spectra show smaller than average magnitudes, suggesting these specimens have a spectral response closer to homogeneous materials, which could account for the higher detection rates.

\subsubsection{Conclusions}

Specific data files of the CCSSRRT data set seem to benefit from applying a bandpass filter to the data file before the SAFT processing. A statistical and composite spectra analyses of the spectrum content of the CCSSRRT data set indicates that applying a generic filter to all CCSS specimens to discriminate defect and nondefect areas can improve the subsequent SAFT images. More improvement in the image can be seen if one set of filter parameters is used for equiaxed microstructures and a different set of filter parameters is used for columnar microstructures. The particular filter parameters chosen are selected by first plotting the defect and nondefect composite spectra for each material by using samples with known defect locations. Filter parameters are then selected that encompass defect spectra and discriminate against nondefect spectra.

Some CCSS materials are closer in acoustic response to homogeneous materials than others. Therefore, detection of cracks may be easier and applying a filter to the data unnecessary. However, for the vast majority of CCSSRRT samples, application of a filter based on the spectra response of known defects did improve the image signal-to-noise levels of the processed CCSS data files, thereby significantly reducing the false call rate. 
5.0 Research Studies

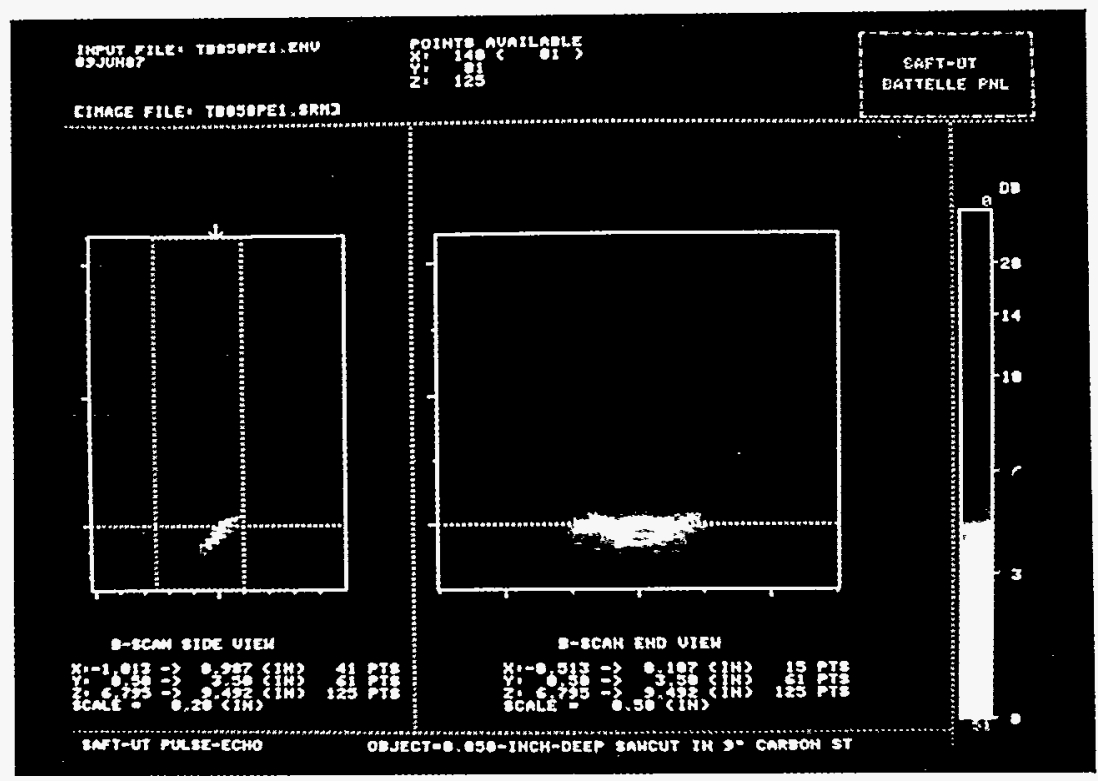

Figure 5.1. Pulse-Echo Image of 0.050 -in.-deep $(0.13-\mathrm{cm})$ Sawcut in a 9-in.-thick $(22.86-\mathrm{cm})$ Steel Block

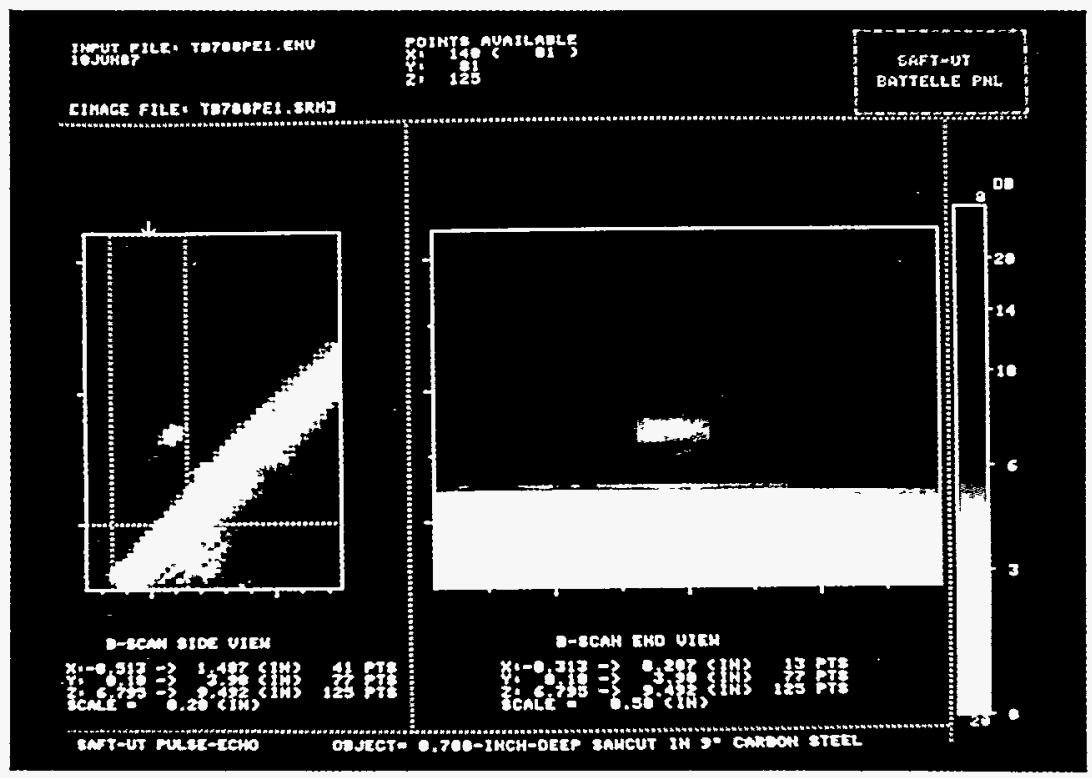

Figure 5.2. Pulse-Echo Image of 0.700-in.-deep (1.8-cm) Sawcut in a 9-in.-thick (22.86-cm) Steel Block 


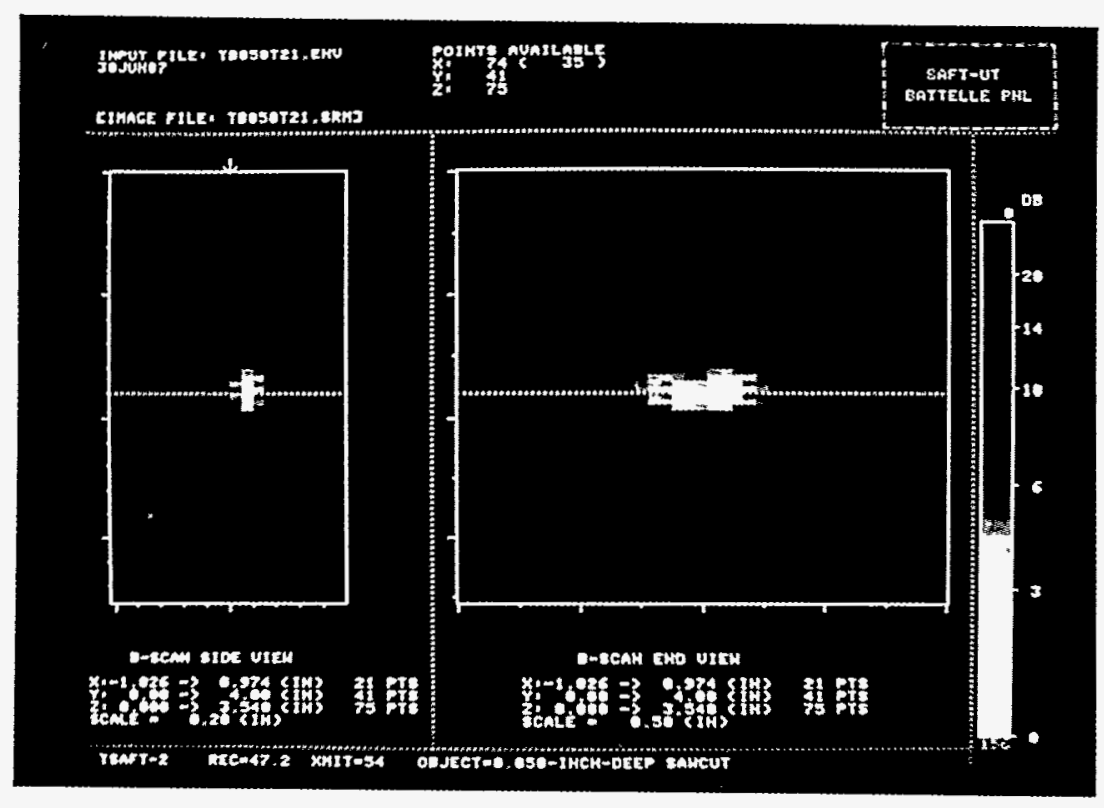

Figure 5.3. TSAFT-2 Image of 0.050 -in.-deep $(0.13-\mathrm{cm})$ Sawcut in a 9-in.-thick $(22.86-\mathrm{cm})$ Steel Block

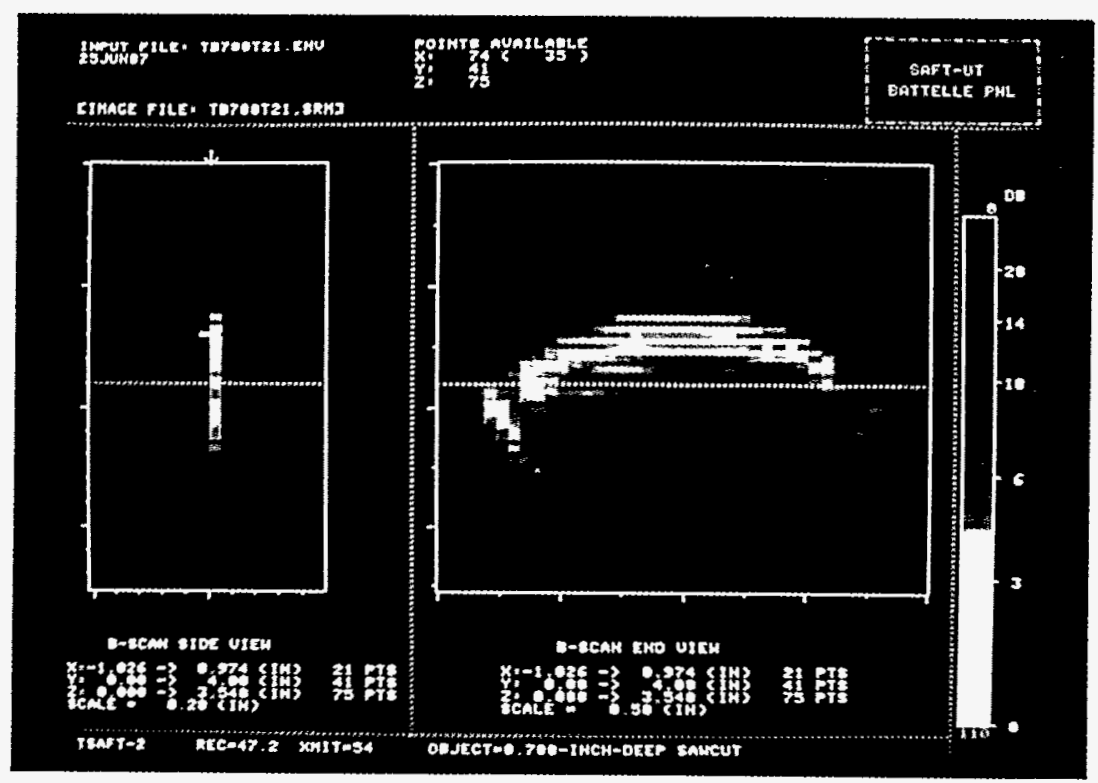

Figure 5.4. TSAFT-2 Image of 0.700 -in.-deep (1.8-cm) Sawcut in 9-in.-thick (22.86-cm) Steel Block 


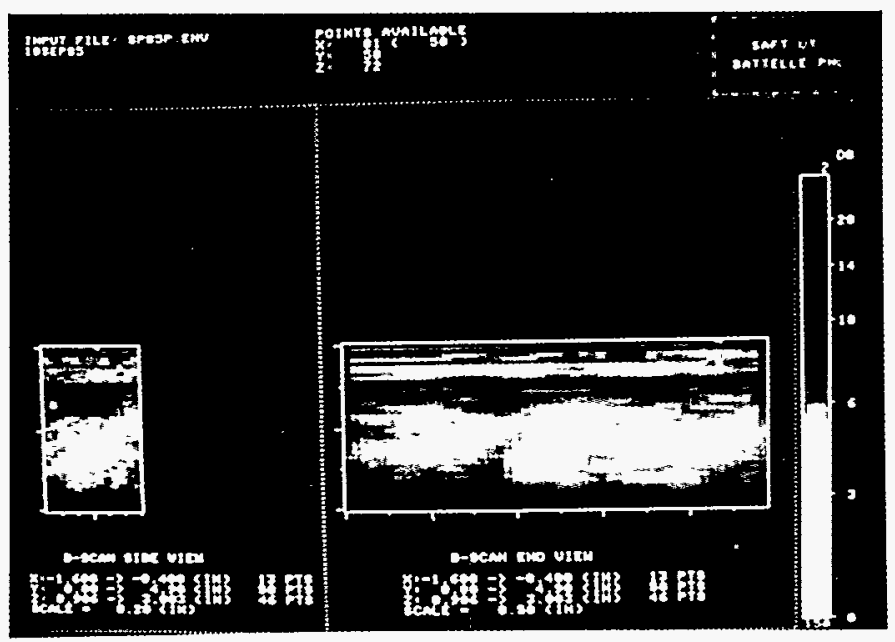

(a) SAFT processed image

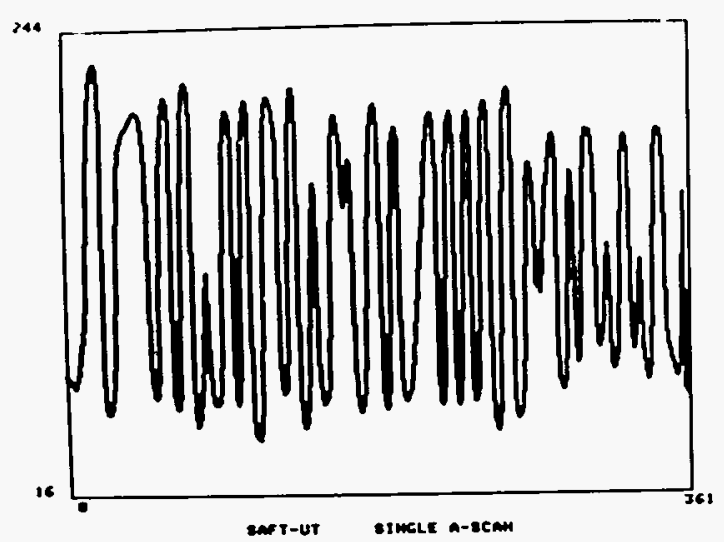

(b) A-scan of area believed to be cracked

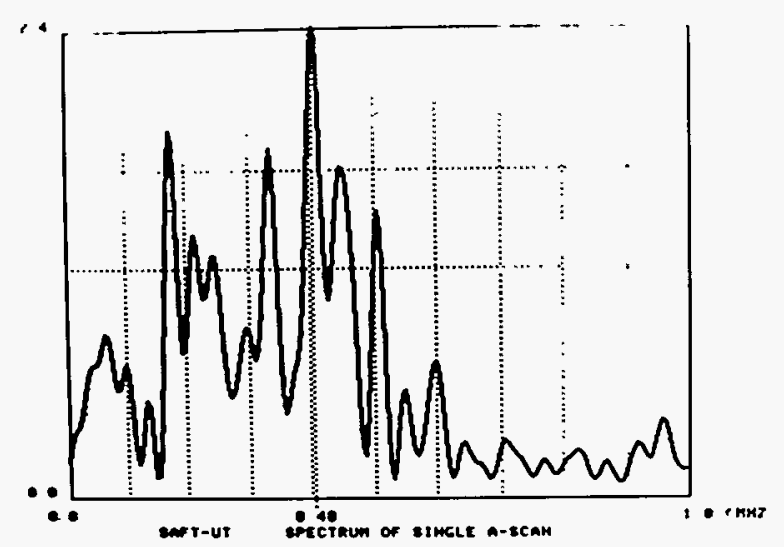

(d) power spectrum of area believed to be cracked

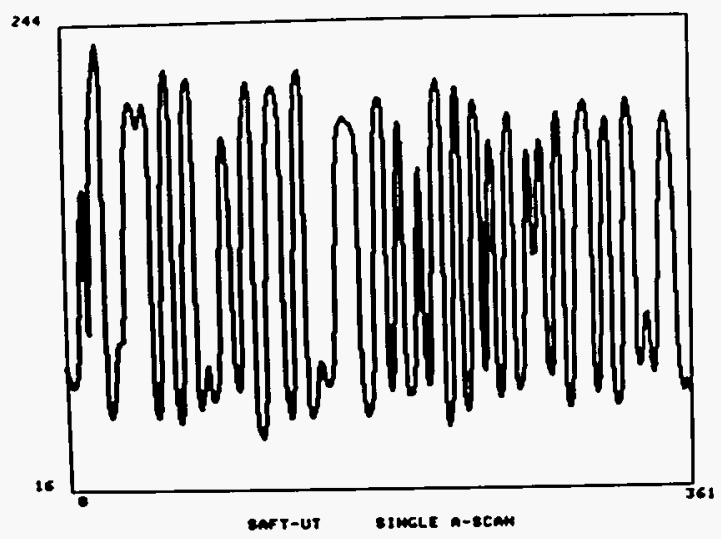

(c) A-scan of area believed not to be cracked

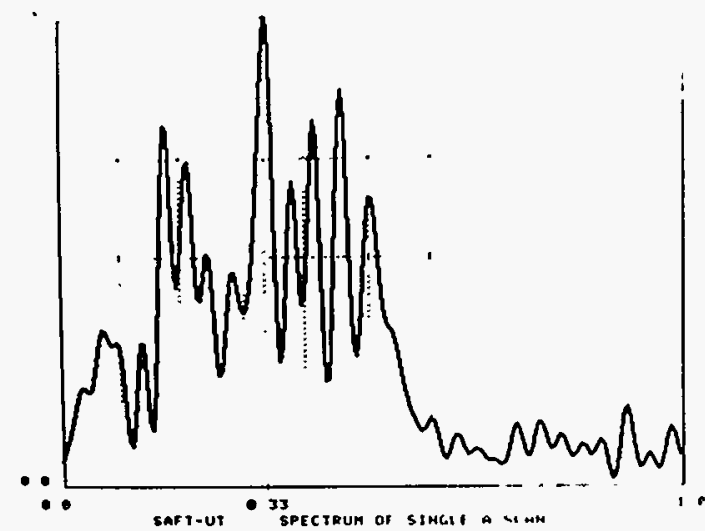

(e) power spectrum of area believed not to be cracked

Figure 5.5. Images of Specimen 5 of the CCSSRRT Data Set 


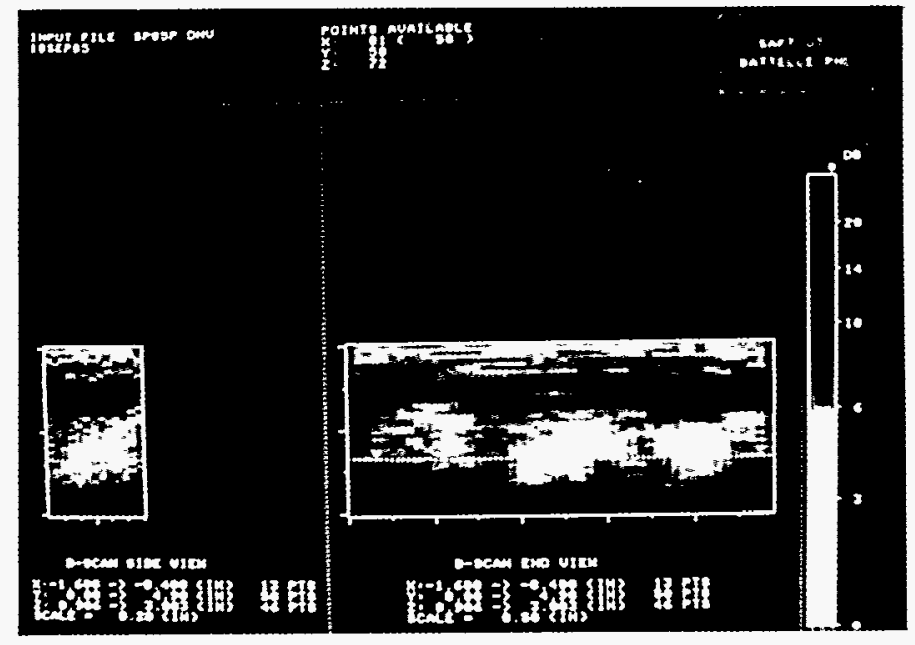

5.0 Research Studies

(a) SAFT Image

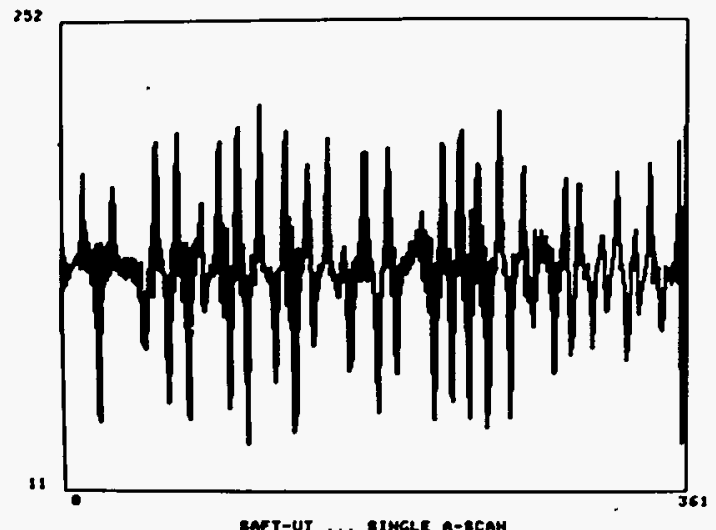

(b) A-scan of Cracked Region

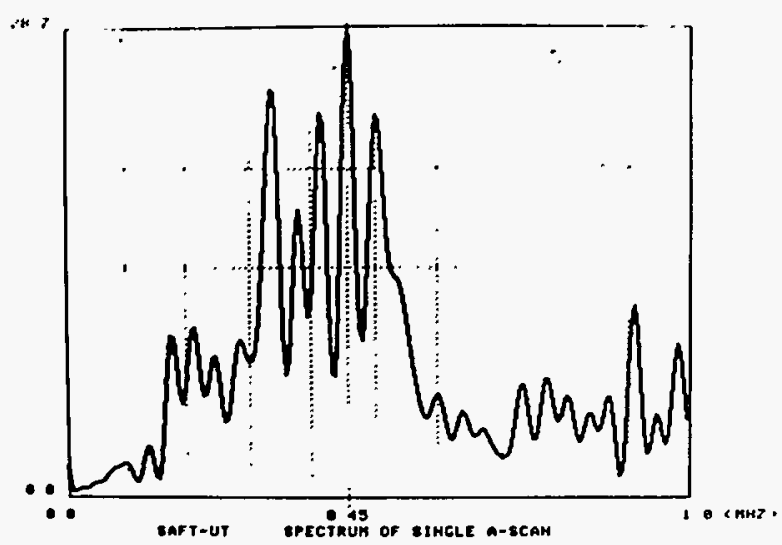

(d) Spectrum of Cracked Region

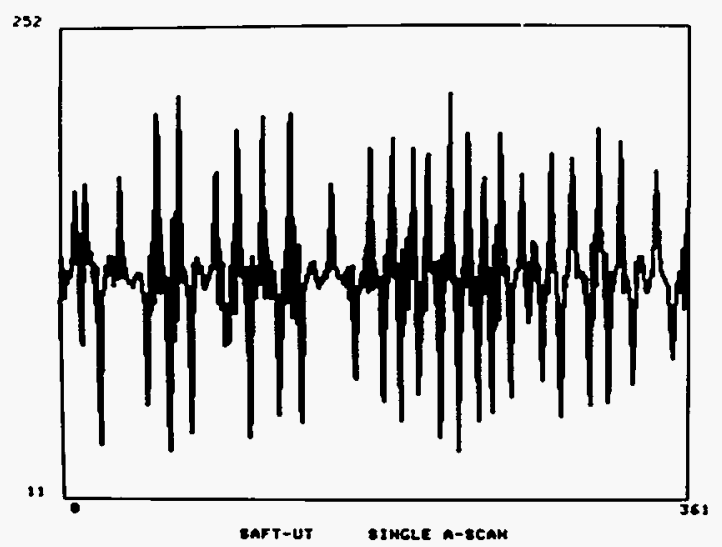

(c) A-scan of Uncracked Region

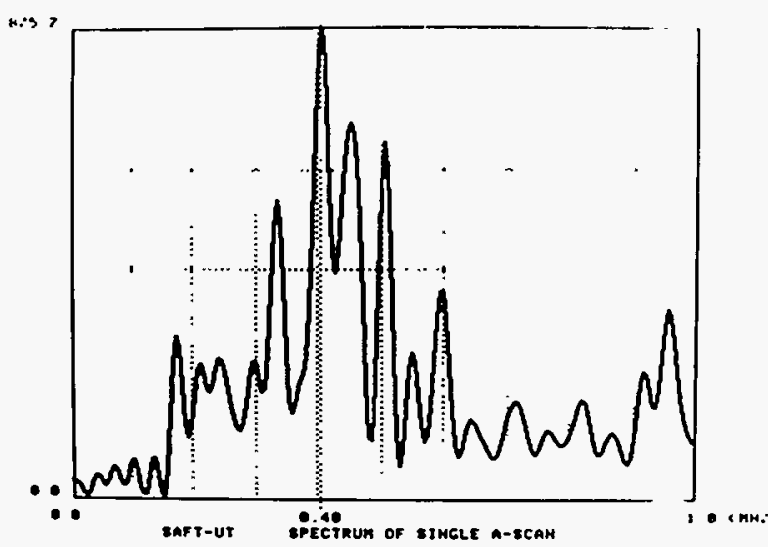

(e) Spectrum of Uncracked Region

Figure 5.6. Image of Specimen 5, Selected A-Scans, and Spectra Obtained After Application of Step-Wise Filter to Data Prior to SAFT Processing 


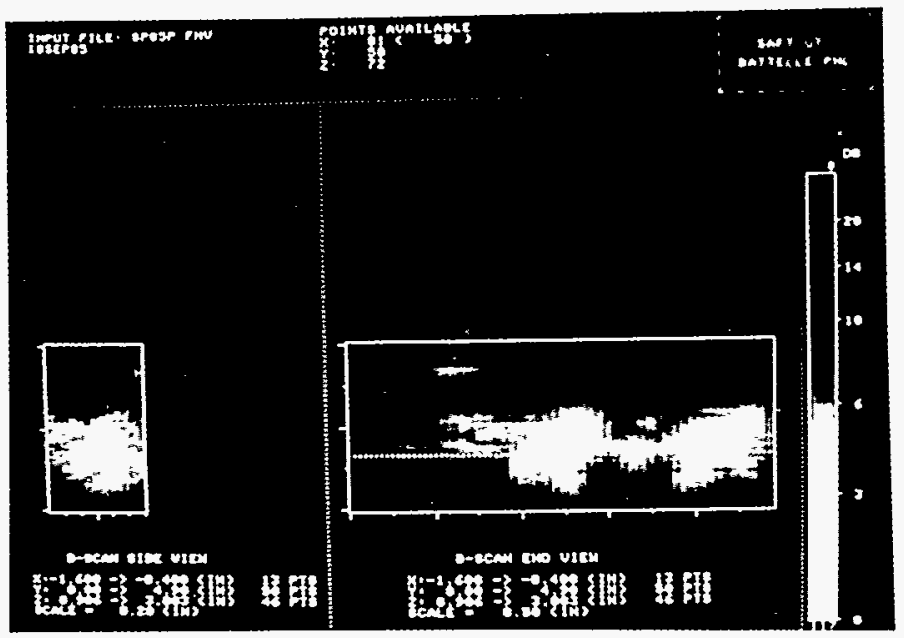

(a) SAFT Image

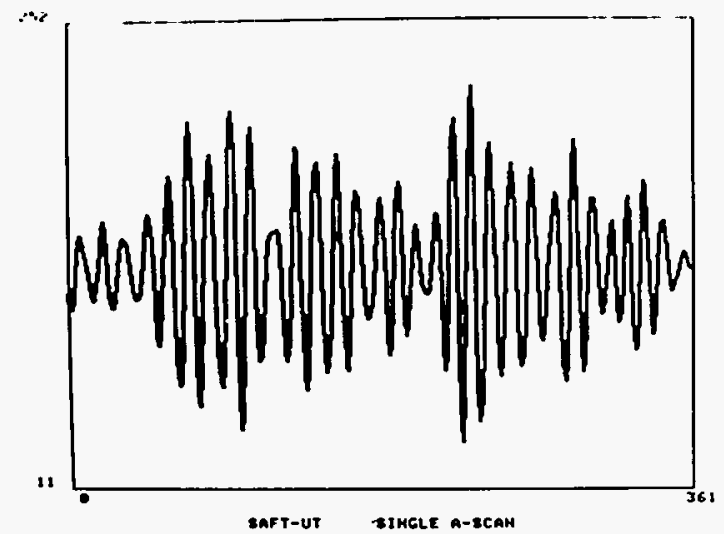

(b) A-scan of Cracked Region

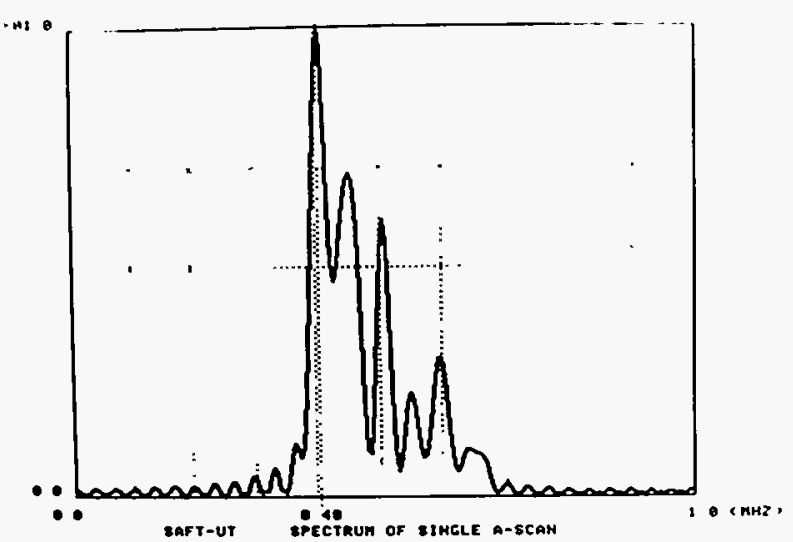

(d) Spectrum of Cracked Region

Figure 5.7. Image of Specimen 5, Selected A-Scans, and Spectra Obtained After Application of Time Derivative Filter to Data Prior to SAFT Processing 


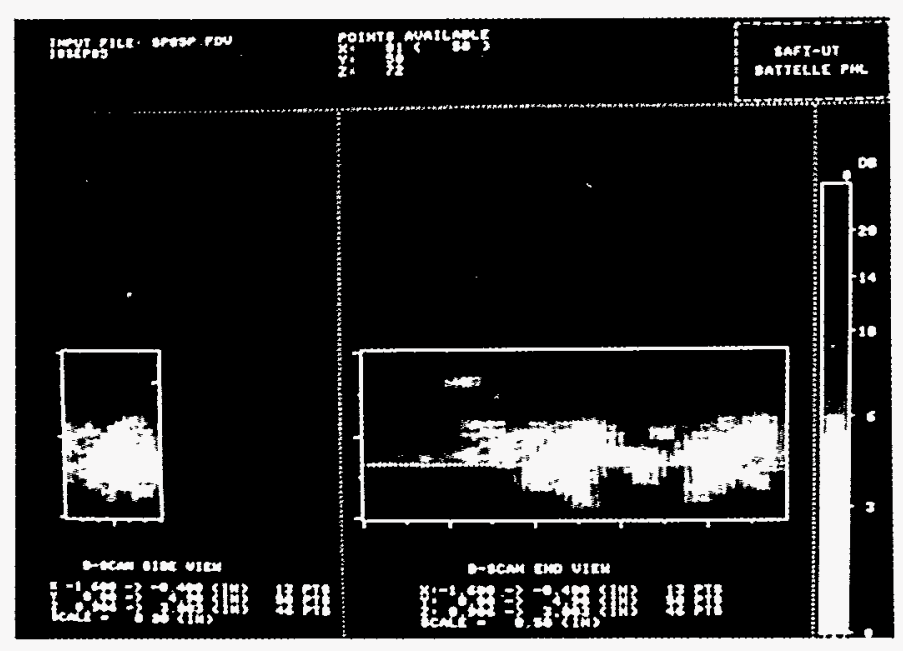

5.0 Research Studies

(a) SAFT Image

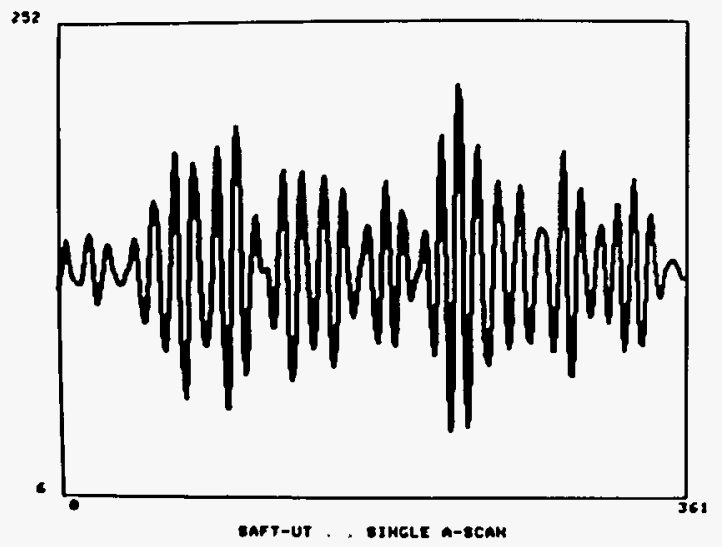

(b) A-scan of Cracked Region

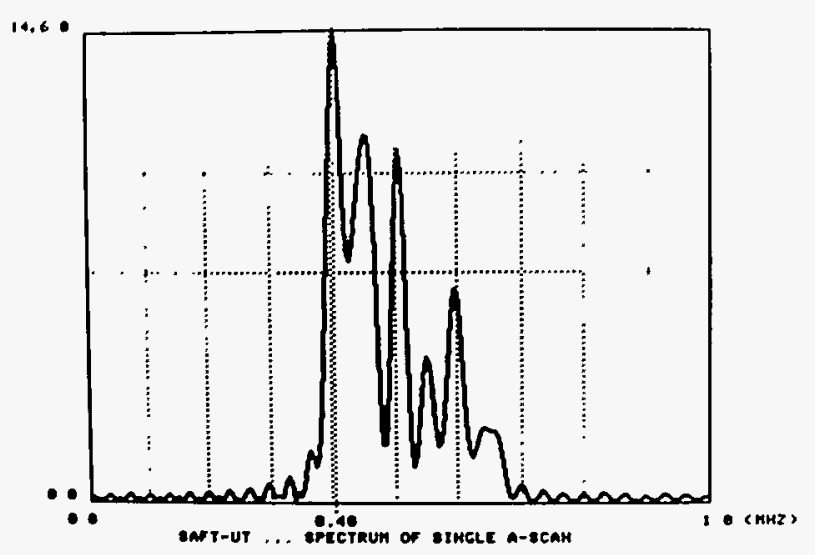

(d) Spectrum of Cracked Region

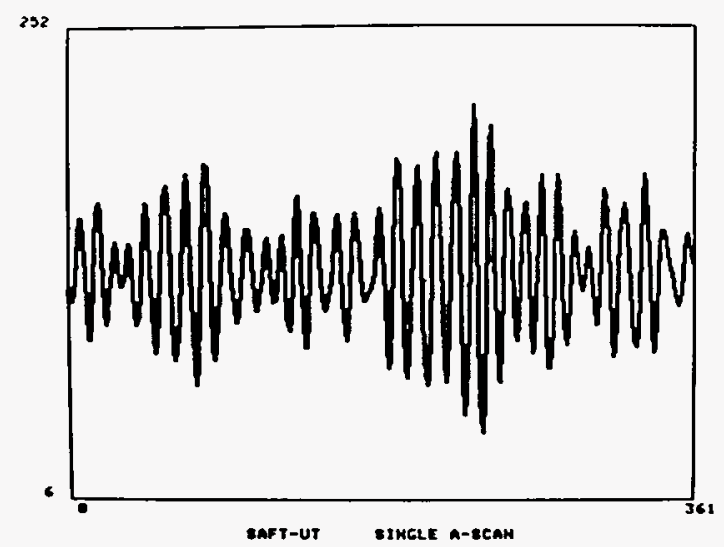

(c) A-scan of Uncracked Region

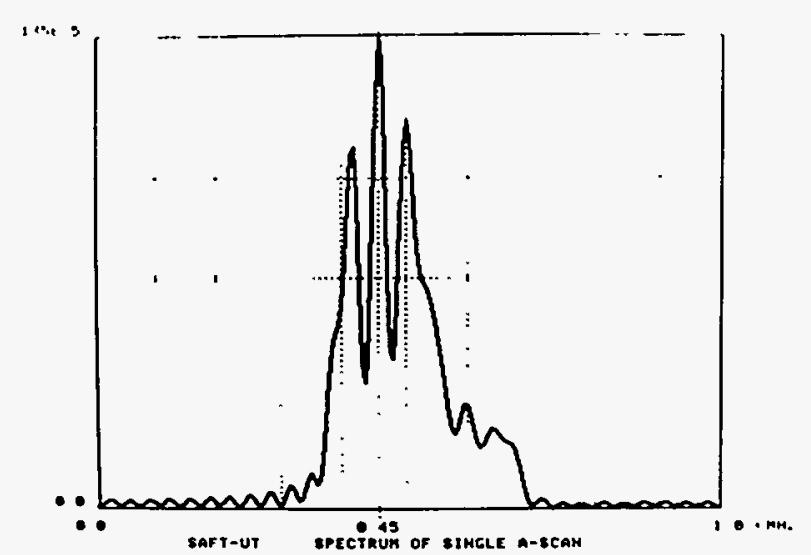

(e) Spectrum of Uncracked Regior.

Figure 5.8. Image of Specimen 5, Selected A-Scans, and Spectra After Application of Time Derivative and Stepwise Filter to Data Prior to SAFT Processing 


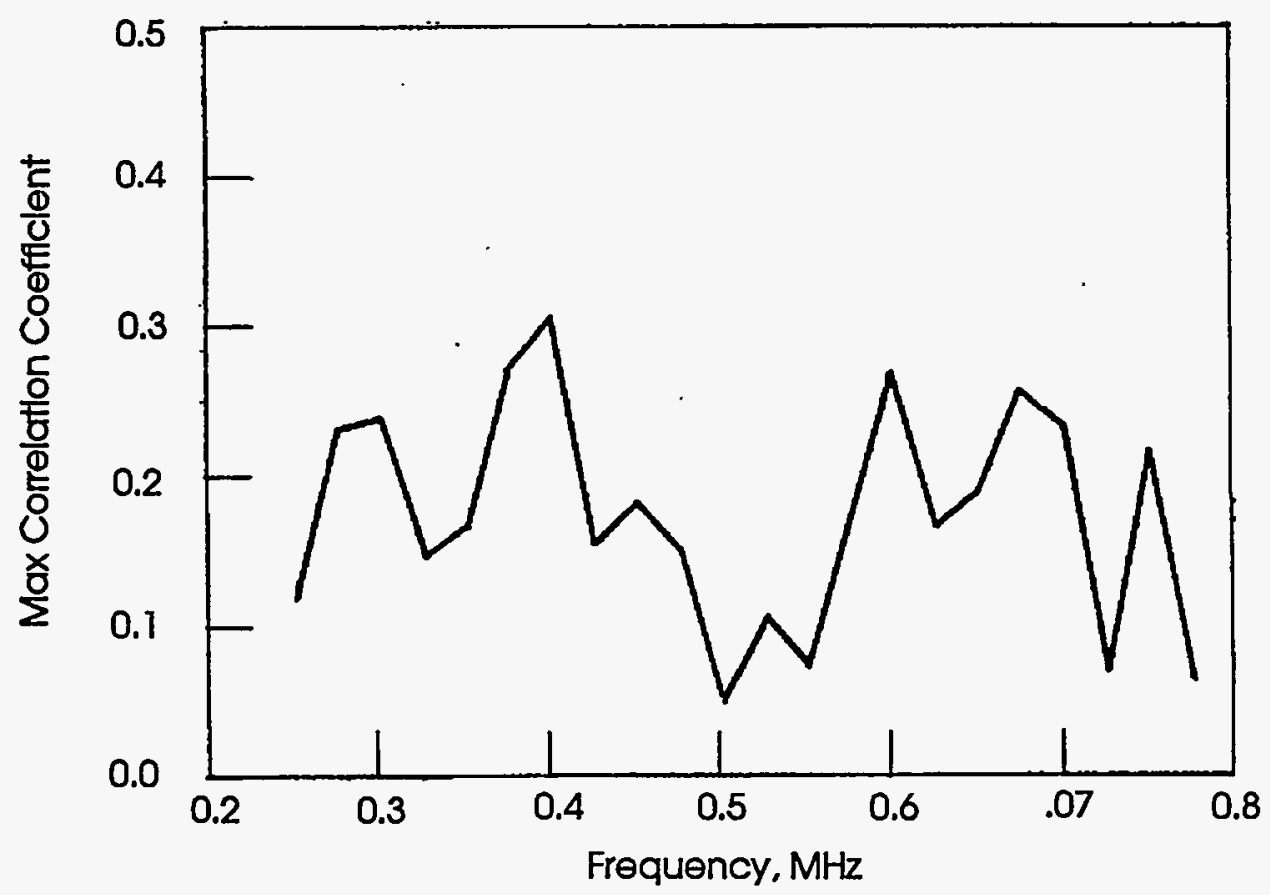

Figure 5.9. Maximum Correlation Values at Different Frequencies for Equiaxed Material

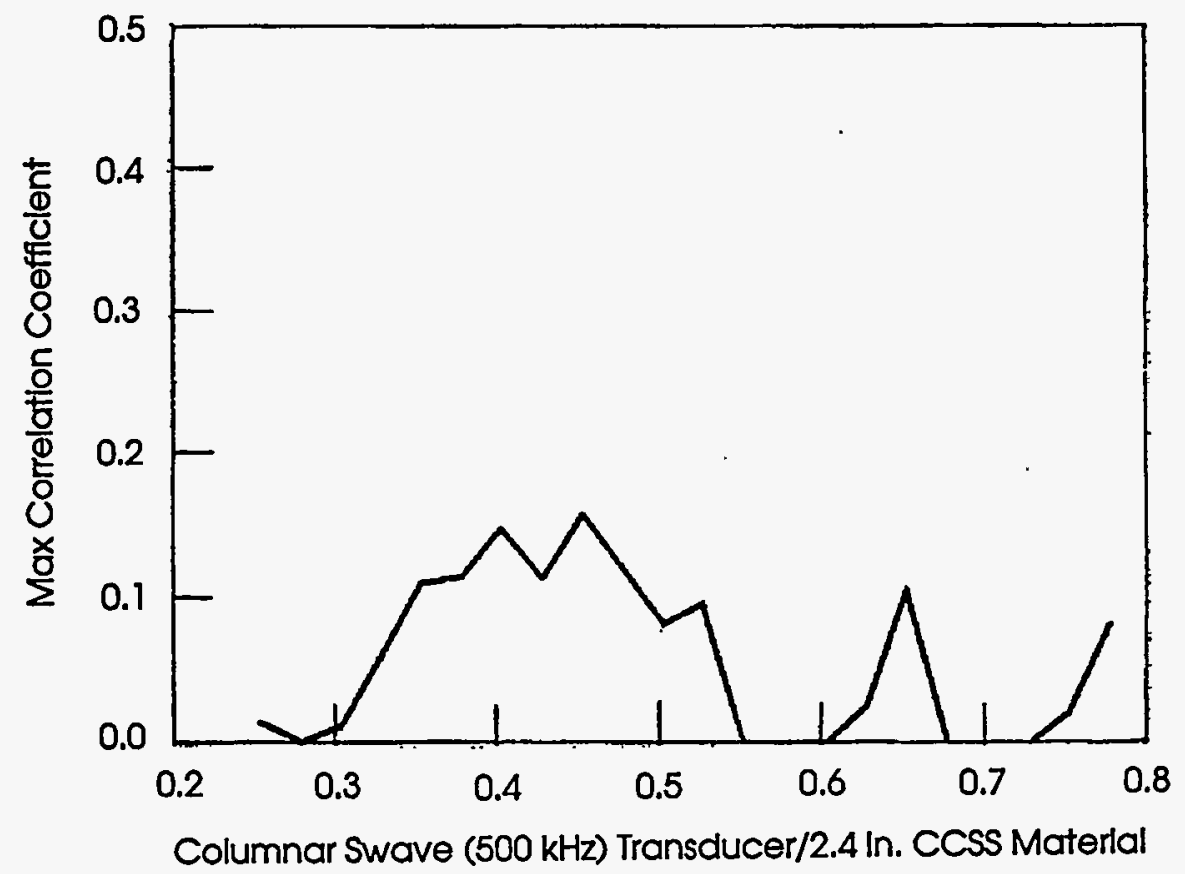

Figure 5.10. Maximum Correlation Values at Different Frequencies for Columnar Material 


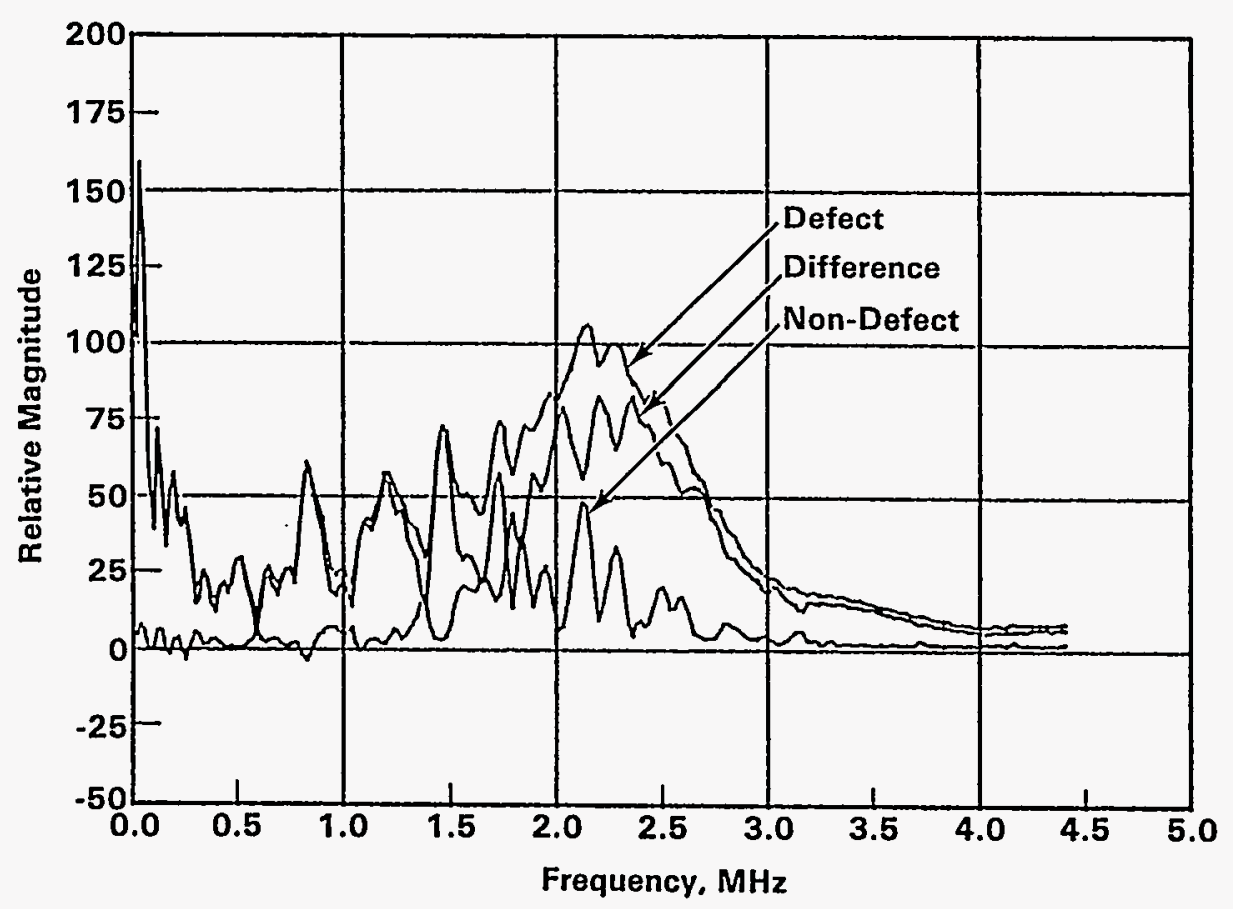

Figure 5.11. Composite Power Spectra for Sawcut Test File 


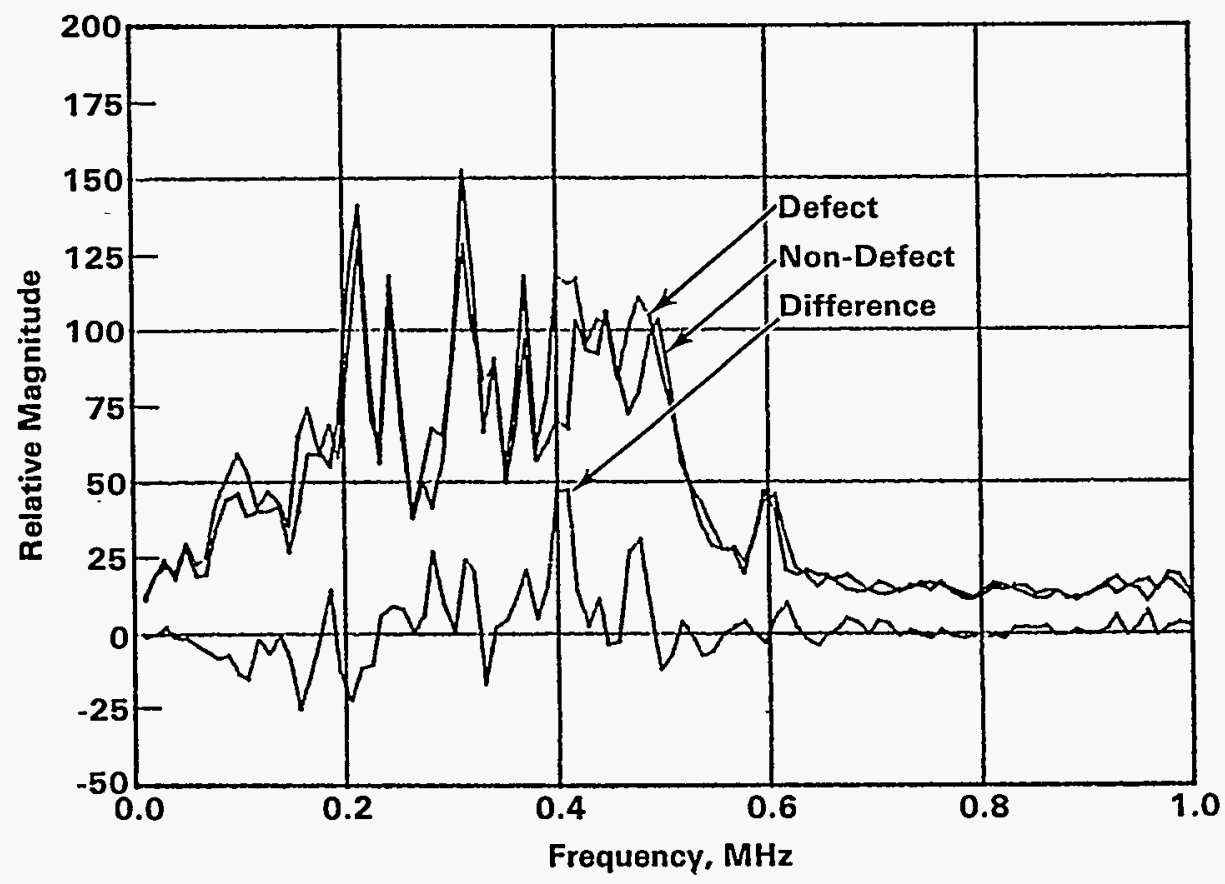

Figure 5.12(a). Composite Power Spectra Plot for Equiaxed Shear-Wave Data Files

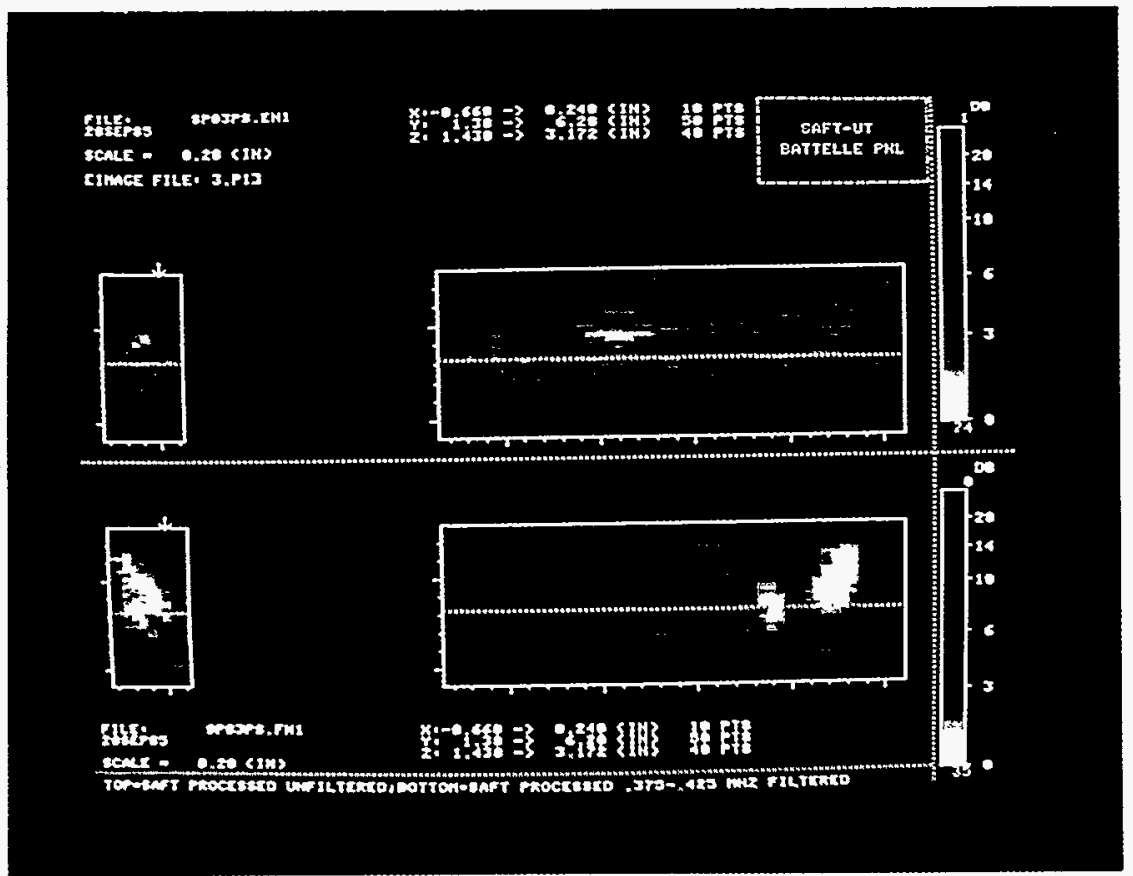

Figure 5.12(b). Specimen 3 Images Showing Before (top) and After (bottom) Processed Images (Filter parameters for After image were selected from the power spectra plot.) 


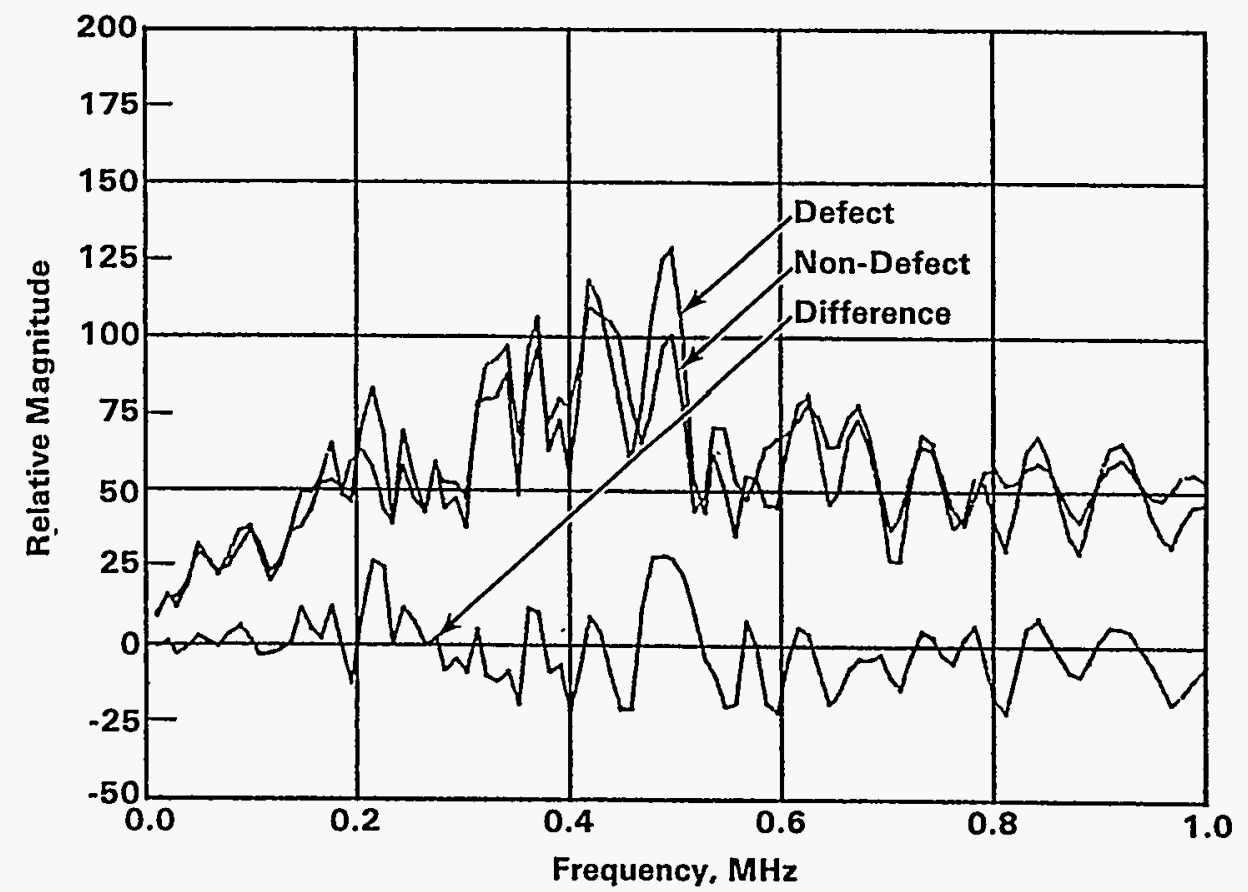

Figure 5.13(a). Composite Power Spectra Plot for Columnar Shear-Wave Data Files

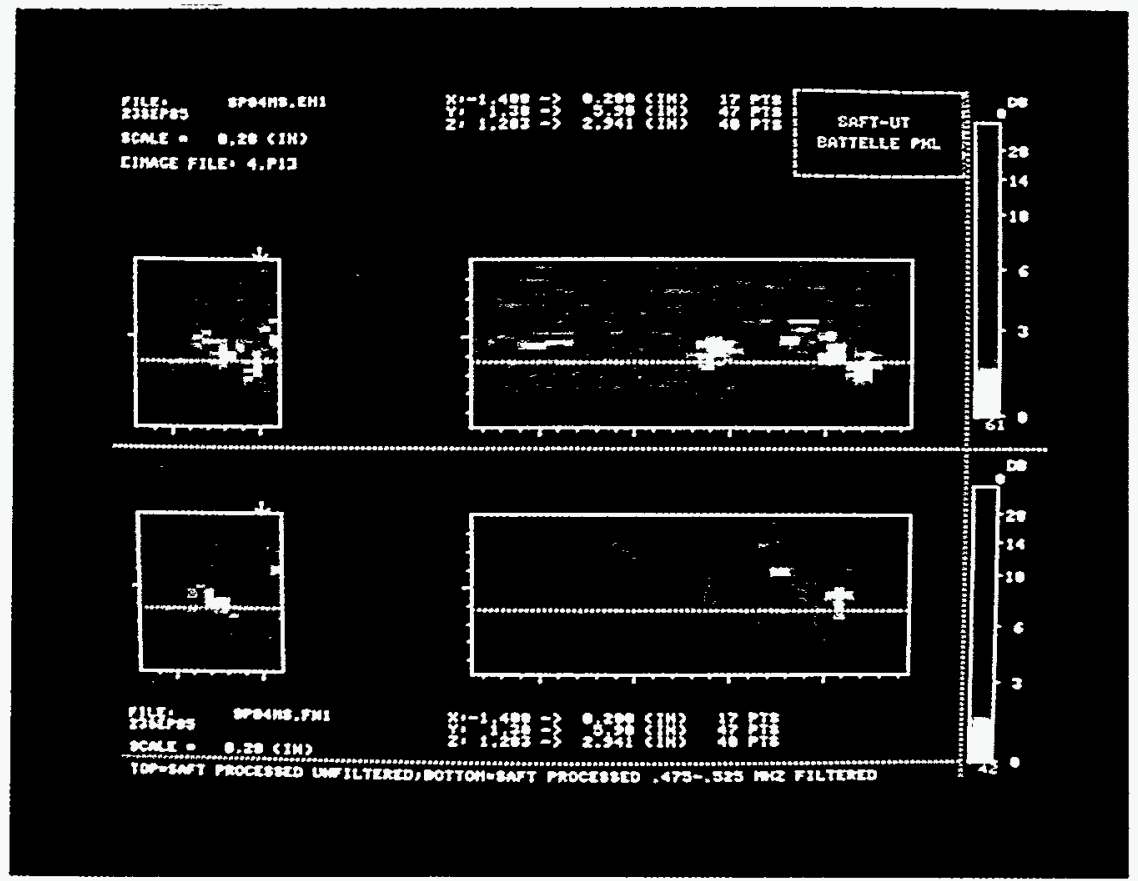

Figure 5.13(b). Specimen 4 Images Showing Before (top) and After (bottom) Processed Images (Filter parameters for After image were selected from the power spectra plot.) 
5.0 Research Studies

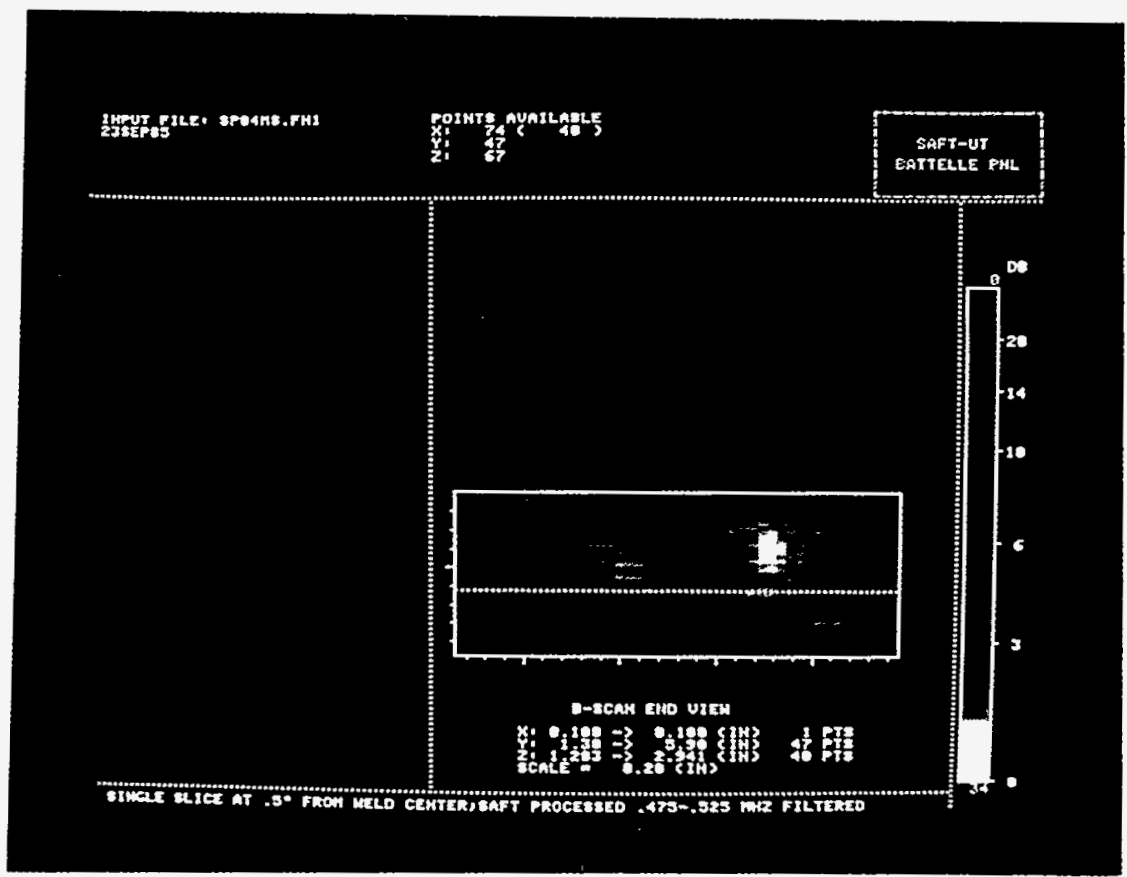

Figure 5.13(c). Single Slice End View $0.5 \mathrm{in} .(1.3 \mathrm{~cm})$ from Weld Center

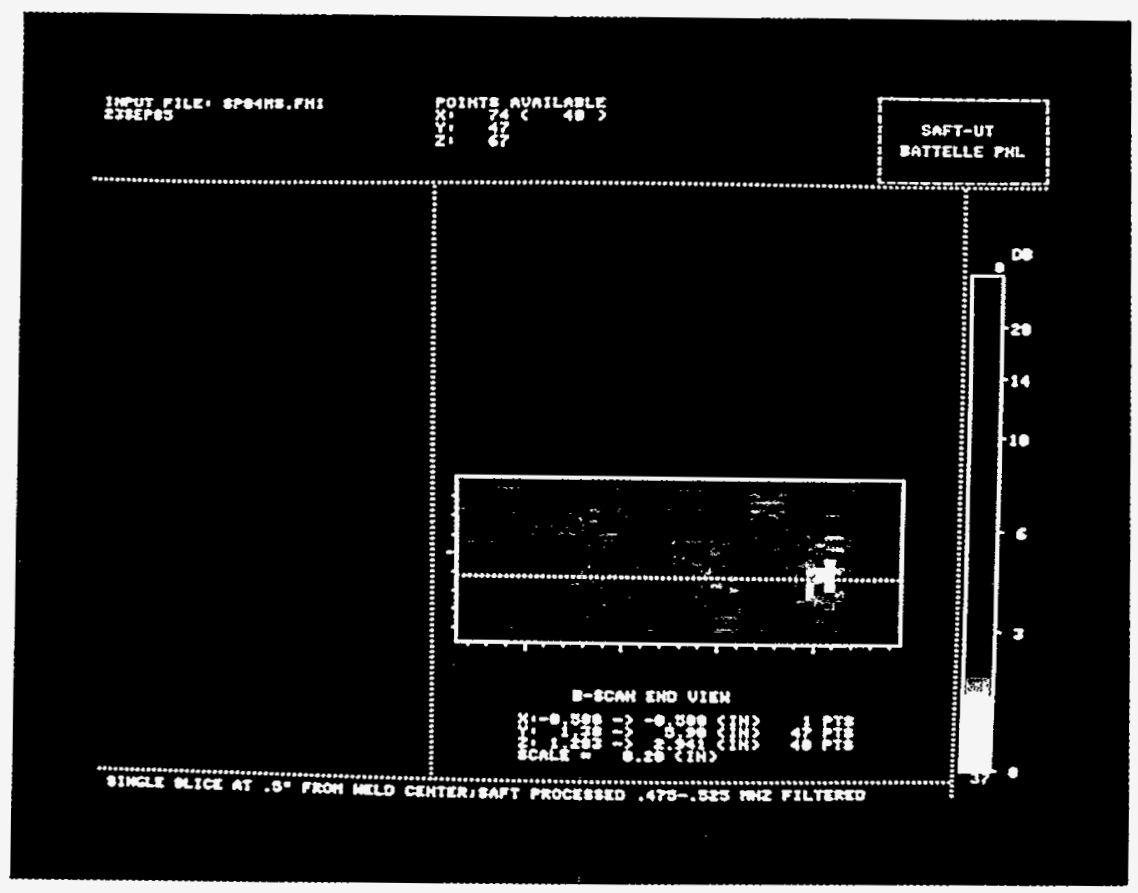

Figure 5.13(d). Single Slice End View at Weld Center 


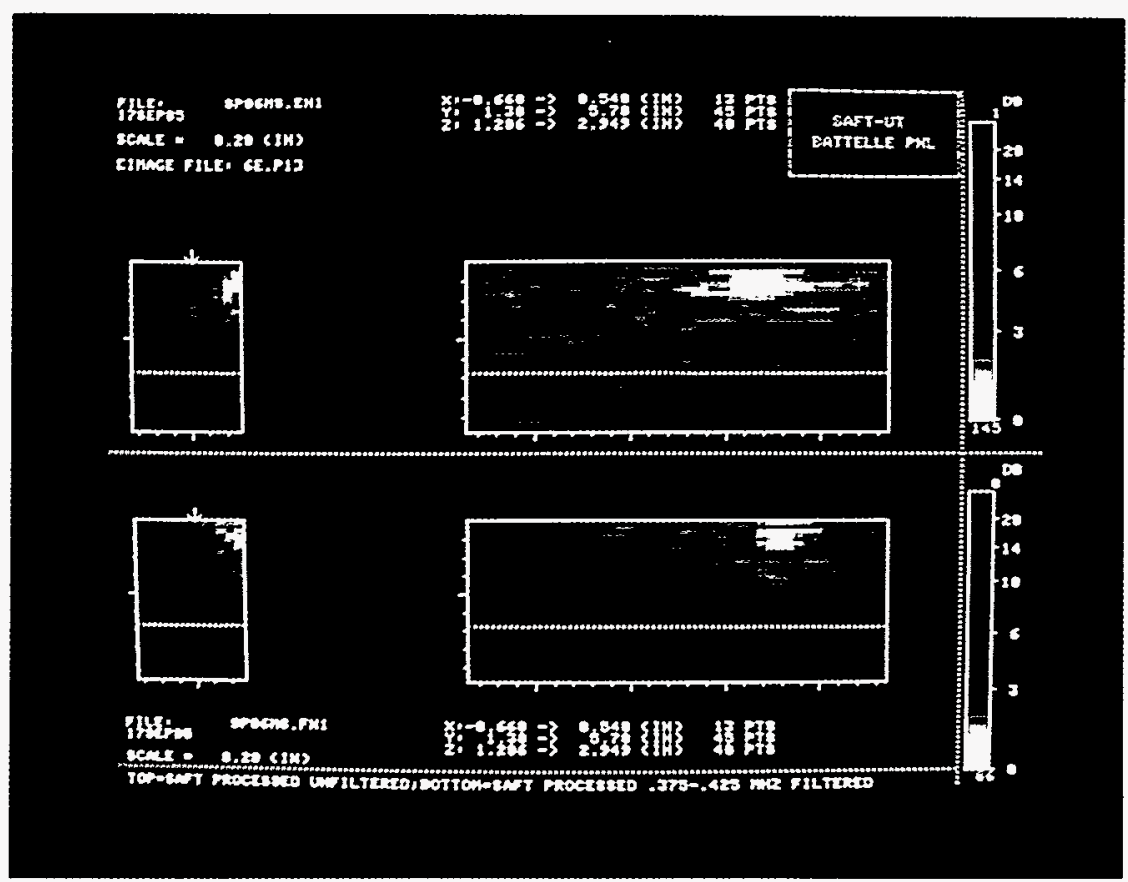

Figure 5.14. Images of the Equiaxed Side of Specimen 6 Showing the Before (top) and After (bottom) Processed Images (Filter Parameters for After Image were Selected From the Equiaxed Power Spectra Plot)

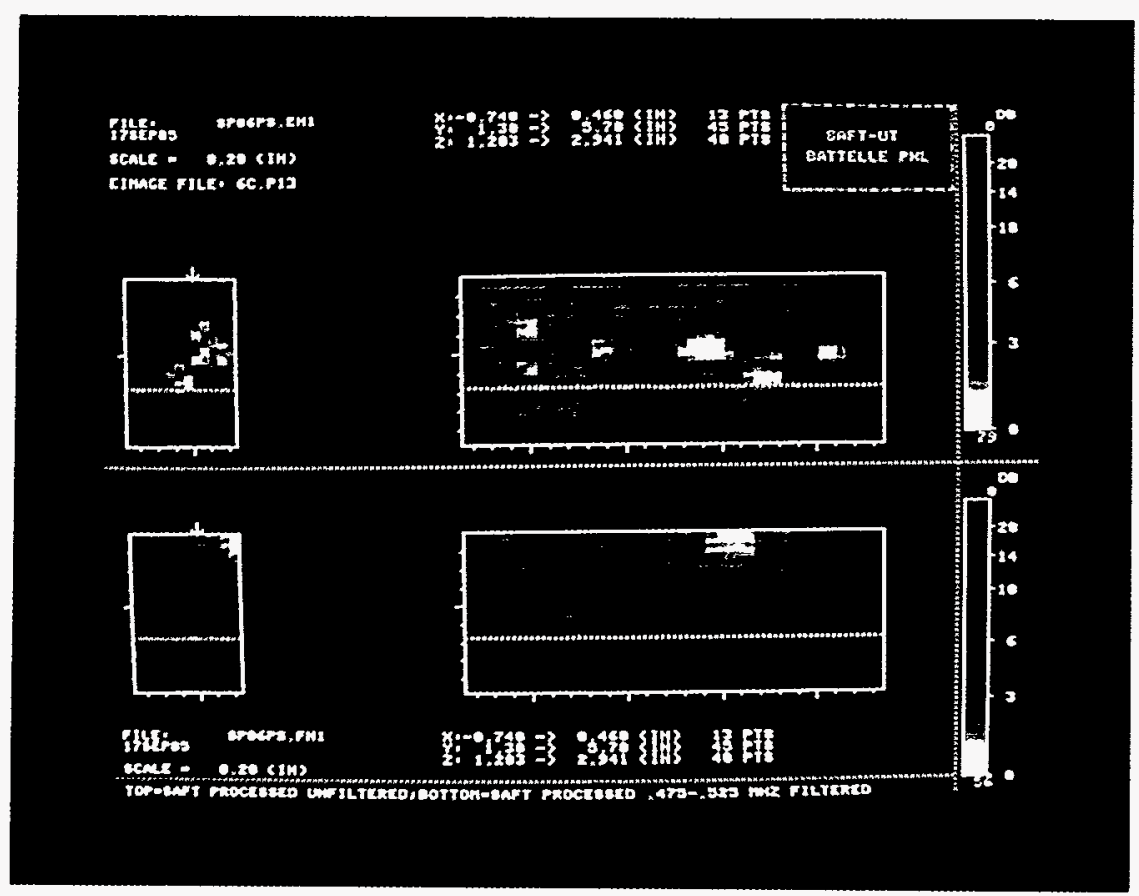

Figure 5.15. Images of the Columnar Side of Specimen 6 Showing the Before (top) and After (bottom) Processed Images (Filter Parameters for After Image were Selected From the Columnar Power Spectra Plot) 


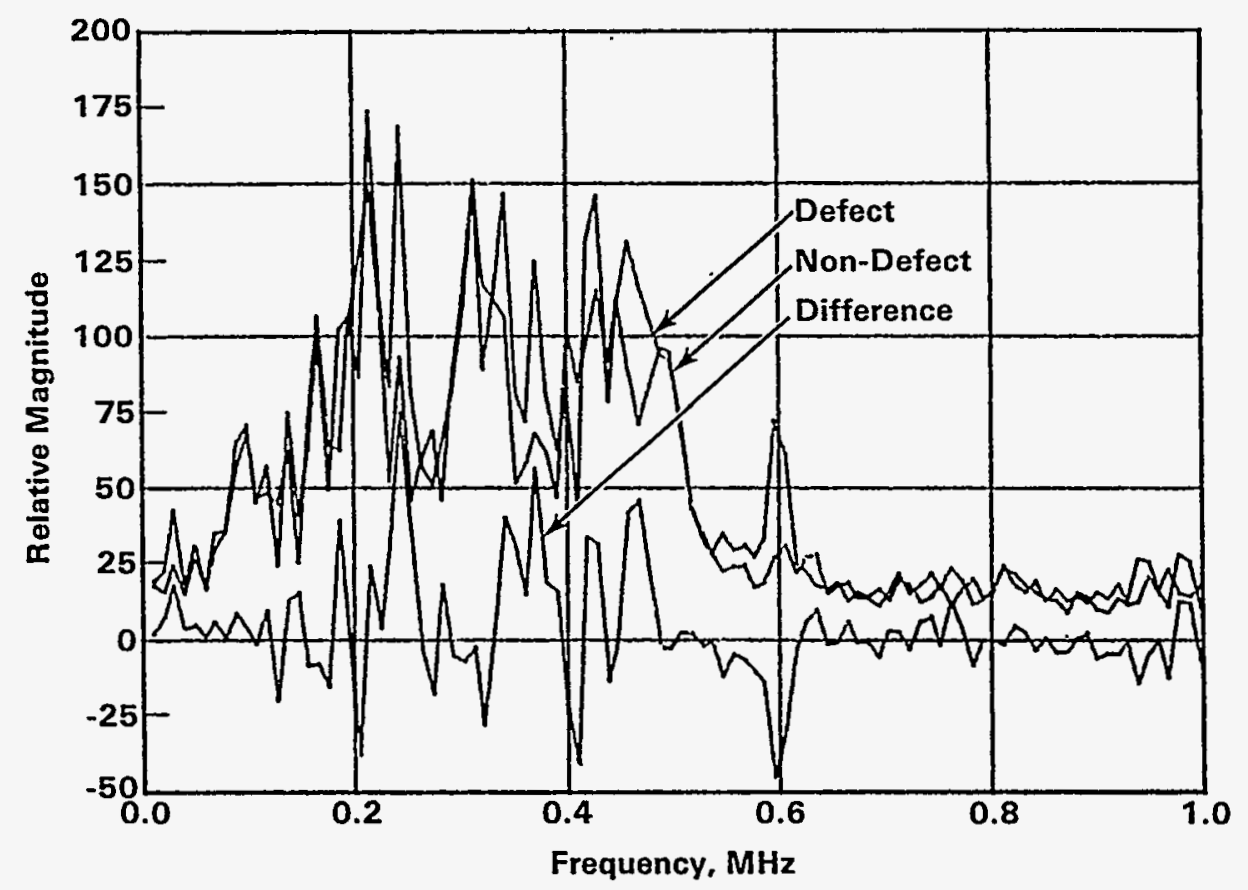

Figure 5.16. Plot Showing the Composite Power Spectra for Equiaxed Shear-Wave Specimens that had a High Probability of Detection

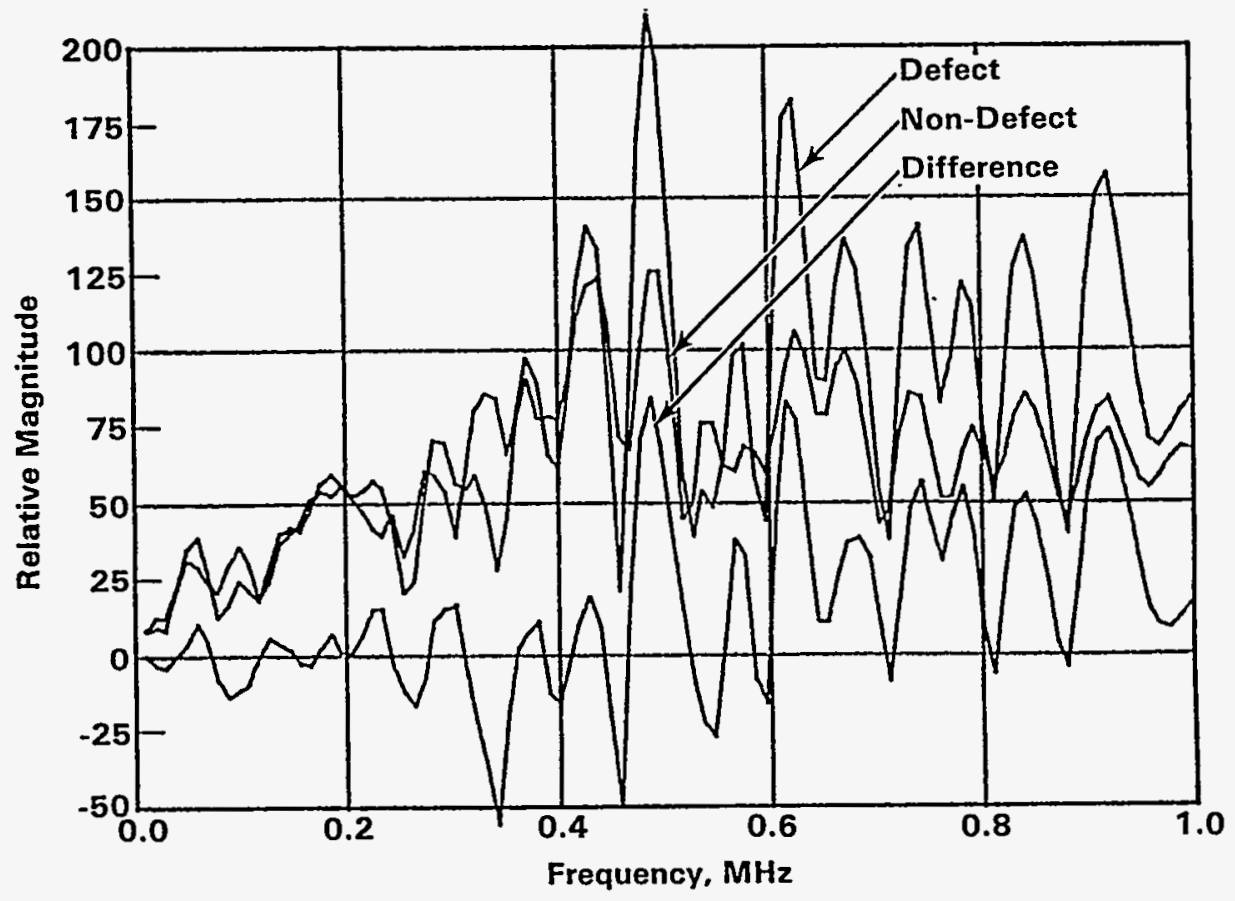

Figure 5.17. Composite Power Spectra for Columnar Shear-Wave Specimens Having High Probability of Detection 


\subsection{Laboratory Parametric Studies}

Laboratory tests were performed to examine the effects of thickness and velocity parameters on TSAFT-2 images. The tests were a natural follow-on to similar studies conducted on TSAFT images. Section 6 goes on to report a bandwidth compensation study performed by PNL to document the limitations of the narrow-band approximation used in the SAFT algorithm. A utility was developed to improve the SAFT images of wideband data files.

\subsection{TSAFT-2 Configuration Velocity and Thickness Parametric Study}

Velocity and thickness variation studies were performed on the TSAFT-2 configuration to augment a similar study performed on the TSAFT configuration in 1986. It was felt that, to be complete, a parallel investigation needed to be performed on the simultaneous sourcereceiver scanning configuration of TSAFT-2.

Two performance evaluation studies were focussed on the TSAFT-2 configuration to determine the sensitivity of this mode to velocity errors and thickness measurement errors. Another goal of these studies was to quantify the degradation that occurs due to these variations.

The SAFT algorithm implemented at PNL assumes isotropic and homogeneous material with respect to velocity; that is, the calculations performed by the SAFT processing make the approximation that the velocity is constant throughout the material. This, of course, is not exactly true in most specimens, and the variance depends greatly on the type of material under test. Also, the algorithm requires that the velocity be known to some degree of accuracy. This may seem to be a trivial requirement but, in many cases, is not. For example, an extreme case is CCSS material, which varies in velocity as a function of angle in the material. The following, then, is an experimental study to determine the image effects of erroneous velocity measurements on TSAFT-2 data files.

The data file chosen for this experiment is a TSAFT-2 scan of a 0.3 -in.-deep $(0.8-\mathrm{cm})$ sawcut in 0.750-in.-thick $(1.9-\mathrm{cm})$ aluminum material. The material is a very flat coupon with actual characteristics that are known very accurately. Each element in the chosen sequence utilizes the same data file but simply varies the velocity parameter in the data file header block. This simulates acquiring the data at these velocities and should produce identical results.

Figure 6.1 shows the series of images obtained by sequencing the velocity of the material. The known velocity is $122000 \mathrm{in} . / \mathrm{s}(309880 \mathrm{~cm} / \mathrm{s})$ since the illumination was in shear mode. Each element in the sequence represents approximately a $10 \%$ change in velocity. Figure 6.1(a) was processed at 97000 in./s (246380 $\mathrm{cm} / \mathrm{s}),(\mathrm{b})$ at $109000 \mathrm{in} . / \mathrm{s}(27686 \mathrm{~cm} / \mathrm{s}),(\mathrm{c})$ is the actual velocity of $122000 \mathrm{in} . / \mathrm{s}(309880 \mathrm{~cm} / \mathrm{s}),(\mathrm{d})$ is $134000 \mathrm{in} . / \mathrm{s}(340360 \mathrm{~cm} / \mathrm{s})$, and (e) is $146000 \mathrm{in} . / \mathrm{s}$ $(370840 \mathrm{~cm} / \mathrm{s})$.

It should be noted that each file was processed to include the complete data; therefore, both the true and conjugate images are visible. Since the object is a semicircular shaped sawcut, then one would expect the fully illuminated TSAFT-2 image to be elliptical with its centerline located at the back surface line. When one observes the image using the actual velocity, Figure 6.1(c), this is indeed the case.

The images in the sequence may now be analyzed to determine the major differences that have occurred. The most pronounced effect of velocity differences is shown to be a vertical and lateral displacement error. As the velocity increases, it is apparent that the image is mapped deeper into the material and translates further away from the scanning device. When observing the vertical movement of these images, it can be noted that, in general, for every $10 \%$ increase in material velocity, a vertical movement of about $1 / 4 \mathrm{~T}$ is realized. This observation is important. It is apparent that TSAFT- 2 is significantly sensitive to velocity errors and results primarily in positional errors. This response is very similar to that observed for the TSAFT (fixed source) configuration.

As with TSAFT, the general image quality and characteristics deviate much less than one might expect. This is important to consider. Even with dramatic velocity errors, the image integrity is retained with respect to general shape and size features.

Another parameter of the TSAFT-2 that is interesting to study is the measured material thickness. Just as the material is assumed to be homogeneous with respect to velocity, it is also currently assumed to have a constant 
thickness along the region of illumination or, in other words, it assumes that the front and back surfaces are uniform and parallel. This is obviously an approximation, since in many pipe materials shrinkage may occur near the weld area or a large counterbore may be present on the ID surface. For TSAFT-2 especially, one may wonder what effects this will have on the image quality, since TSAFT-2 relies on multiple bounce paths to illuminate the object zone. These paths are assumed to be well known in the image calculation but, in fact, may not be that well known.

Again, the data file chosen for this experiment is the TSAFT-2 scan of a 0.3-in.-deep (0.8-cm) sawcut in 0.750-in.-thick $(1.9-\mathrm{cm})$ aluminum material. The same data file is used to create the thickness variation sequence, with just the thickness parameter in the data file header adjusted to the appropriate value. This should accurately simulate separate scans that were acquired using these various thickness values.

The resultant images are shown in Figure 6.2. The sequence contains five images, incrementing the thickness by $10 \%$ each. Figure 6.2 (a) shows the image representing a thickness of $0.600 \mathrm{in} .(1.52 \mathrm{~cm})$, (b) $0.675 \mathrm{in}$. (1.7 $\mathrm{cm})$, (c) the correct thickness value of 0.750 in. (1.9 $\mathrm{cm})$, (d) 0.825 in. $(2.1 \mathrm{~cm})$, and (e) 0.900 in. $(2.29 \mathrm{~cm})$.

As the assumed thickness is increased, one can observe from these images that the algorithm maps the result more shallow in the material. The characteristics of thickness deviation are very similar to velocity deviation. This should not be too unexpected, since both parameters directly affect where the algorithm predicts time of flight of a given signal.

As stated previously, the placement of the resulting image is dramatically affected by deviations of either thickness or velocity from the true values of the specimen under test. However, the encouraging result is that the general image integrity is retained. Thus, with the complement of both pulse-echo and tandem scans of the same region, a judgement of size and location may be made. The pulse-echo corner trap image may be used to position the defect laterally, and the TSAFT2 image may be used to determine the horizontal and vertical extent of the defect. The characteristics of both files may be used to verify the integrity of the other.

\subsection{Bandwidth Compensation Study}

The SAFT algorithm currently being implemented assumes a homogeneous, isotropic medium. It also assumes that the system being implemented is of moderate bandwidth. It has been known for some time that the coherent summation algorithm incorporated in the SAFT-UT operation is a narrow-band approximation. This was reviewed by Busse, Collins, and Doctor (1984) in this program and was discussed earlier by Norton (1976) in his thesis paper at Stanford University. The exact method incorporates a time derivative of the raw data file prior to SAFT processing. When analyzing the spectral responses of certain data files, one may determine that the narrow-band approximation may be invalid.

A software utility was written to perform the time derivative on a SAFT-UT data file. This utility uses the identity (Bracewell 1965)

$$
q^{\prime}(t)=F^{-1}(i 2 \pi f F(q(t)))
$$

$$
\begin{aligned}
\text { where: } & \\
\mathbf{t} & =\text { time } \\
\mathbf{f} & =\text { frequency } \\
\mathbf{i} & =\sqrt{-1} \\
\mathbf{F} & =\text { Fourier transform } \\
\mathrm{F}^{-1} & =\text { Inverse Fourier transform operator } \\
\pi & =\mathbf{p i}
\end{aligned}
$$

The time derivative algorithm consists of performing the Fourier transform of each A-scan, multiplying the transformed value by the quantity ( $(2 \pi f)$, and performing the inverse transform.

Figure 6.3 shows the C-scan projection of unprocessed data collected in a water tank using a 0.5 -in.-diameter $(1.3-\mathrm{cm})$ steel sphere as an object. This data file was collected at $2.25 \mathrm{MHz}$ with a 1.0 -in.-diameter $(2.5-\mathrm{cm})$ 4.0-in. $(10.2-\mathrm{cm})$ focal point probe. The sphere was placed $3.0 \mathrm{in} .(7.6 \mathrm{~cm})$ from the focal point. Figure 6.4 plots an A-scan near the center of this data set. The echo shown near the beginning of the trace is from the sphere object. Figure 6.5 shows the plot of the echo trace from the specimen only. Figure 6.6 is the Fourier 
transform plot of the information shown in Figure 6.5. The spectrum shows a peak at $2.27 \mathrm{MHz}$ and a $6-\mathrm{dB}$ bandwidth of approximately $1.5 \mathrm{MHz}$ or $66 \%$. Figure 6.7 shows the $\mathrm{C}$-scan view of the resulting image subsequent to SAFT processing.

The time derivative operation was performed on this raw data file. A display of this is shown in Figure 6.8. The plot of an A-scan near the center of the file is shown in Figure 6.9. This corresponds to the plot shown in Figure 6.4 of the unprocessed data. Figure 6.10 shows the spectrum of the echo region of this Ascan. Notice that the low-frequency components are much diminished as compared to the raw data version shown in Figure 6.6. This makes sense based on Equation (6.1). The derivative action in the frequency domain consists of a linear filter; the $0-\mathrm{Hz}$ component is null, and the weighting function is simply a linear ramp. The resulting image subsequent to SAFT processing of this data is shown in Figure 6.11.

A substantial image improvement is apparent when comparing Figure 6.11 with Figure 6.7. However, through close observation, the scaling range appears to be modified. If one simply performs a square function (intensity plot) of the direct SAFT processed file, the identical results occur. Figure 6.12 shows this image.

A more complex object was implemented in the test. Figure 6.13 shows the B-scan side view and B-scan end view of a SAFT-UT pulse-echo data set. This was collected with a $2.25-\mathrm{MHz}, 0.25$-in.-diameter $(0.6-\mathrm{cm})$ contact probe configured to provide $45^{\circ}$ shear mode illumination. The object was a 0.3 -in.-deep $(0.8-\mathrm{cm})$ sawcut in a 0.585 -in.-thick $(1.5-\mathrm{cm})$ stainless steel coupon. This image shows the unfocused raw data set. Noise is present from the front surface signal, and the tip signal is much subdued. Figure 6.14 shows a time series plot of an A-scan that is located near the center of the scan, and contains tip signal information. Figure 6.15 shows a plot of the derivative of this A-scan. Notice that the low-frequency components located near the front surface are removed.
Figure 6.16 shows the SAFT-UT image of the raw data file. The tip signal is apparent in this photo, but there is a great deal of background noise due to the amplifier ring-down of the surface signal. Figure 6.17 shows the SAFT processed image of the time derivative data file. Notice that the background noise is much suppressed; however, the tip signal is also reduced. This latter phenomenon is due to the intensity (square of the data) distribution nature of this calculation process as stated previously. We can observe this by squaring the data set displayed in Figure 6.16. This result is shown in Figure 6.18. Note that the intensity range of this image looks very much like that of Figure 6.17. However, the noise level of the time derivative image file is much reduced.

In conclusion, it can be stated that the addition of the time derivative in the SAFT calculation would considerably improve the signal-to-noise ratio. The output of this operation should pass through a square-root function to scale the data in a manner similar to the direct SAFT-UT results. Also, a hardware array processor would be necessary to accelerate the FFT operations inherent in this function to make it practical to do in real-time in a reasonable time for application during the data analysis phase. Currently, the VAX 11/730 host-executed FFT performance is unacceptable and inhibits the use of this tool. 


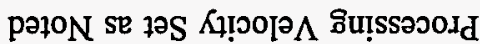

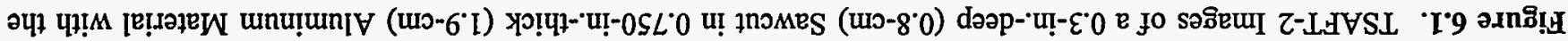

Jəs/u! 0009tI $=\Lambda$

(ә)

วəS/U! 000tEI $=\Lambda$

วəs/u! 000ZZI $=\Lambda$

(0)

O2S/U! 00060I $=\Lambda$

(q)

วəs $/$ u! $000 \angle 6=\Lambda$

(e)
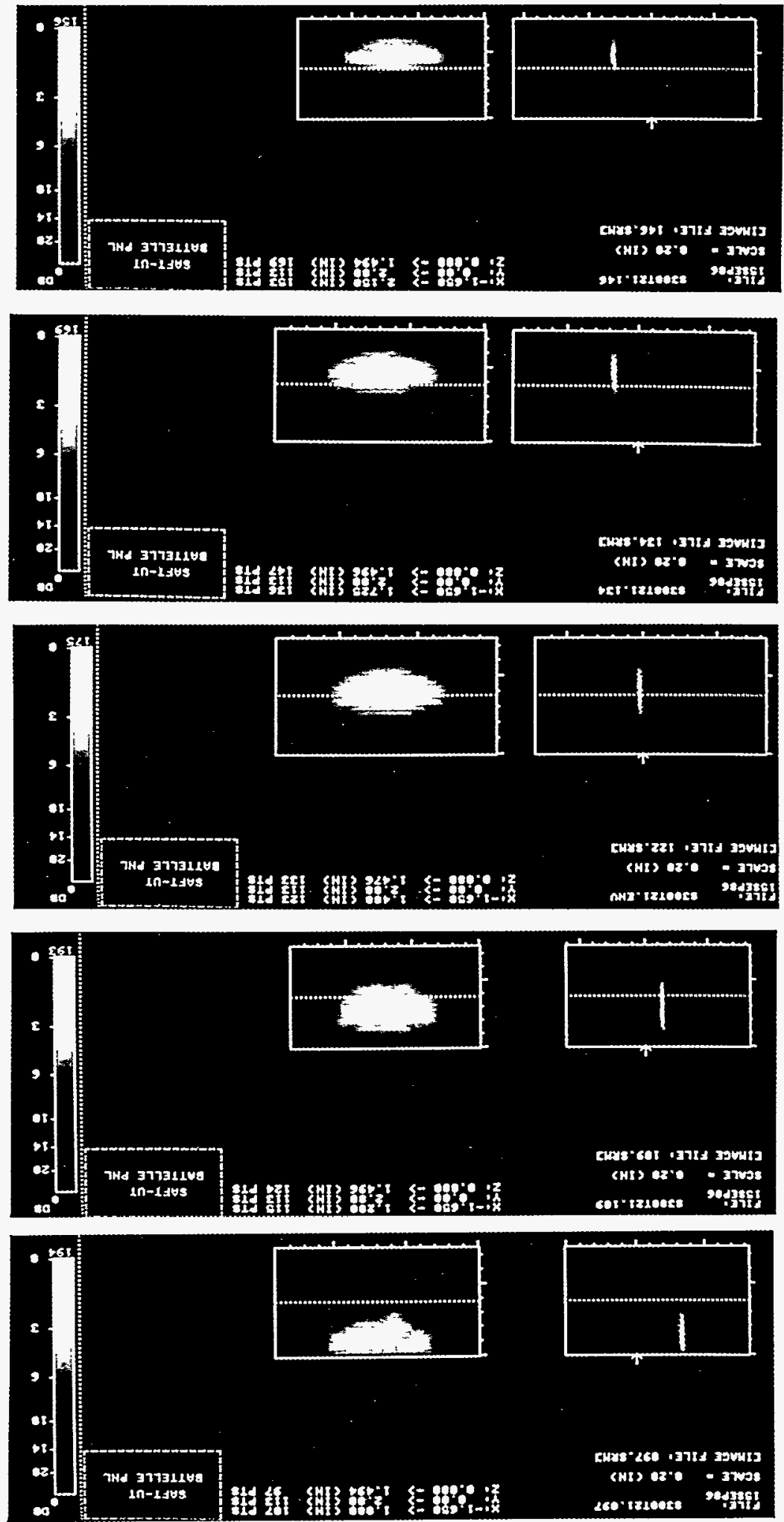

sәrpms olnourered Kroje.roqeT 0.9 


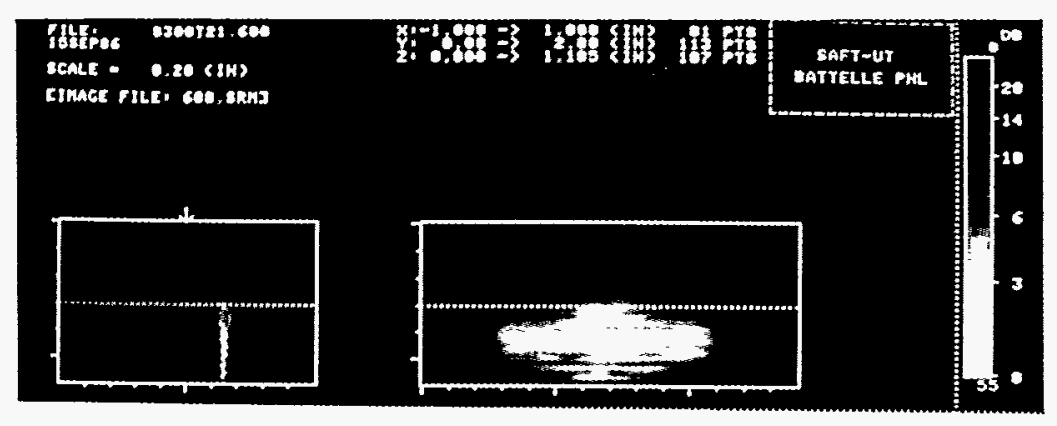

6.0 Laboratory Parametric Studies

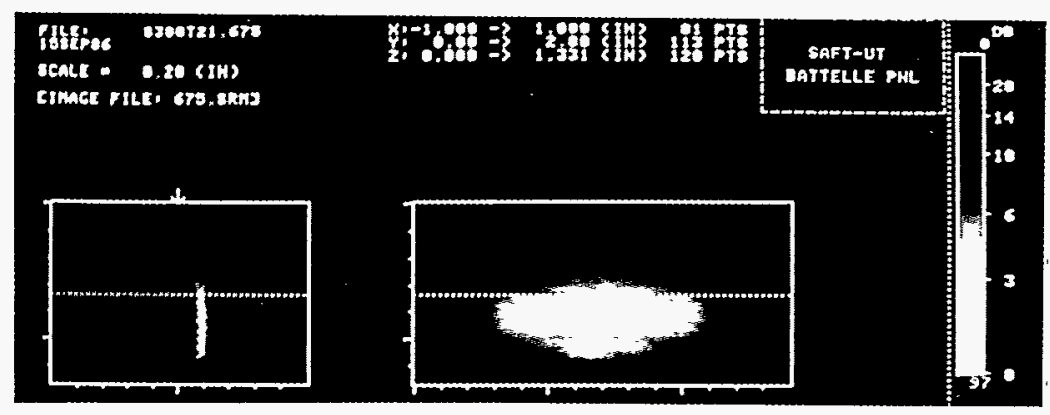

(a) $T=0.600$

(b) $T=0.675$

(c) $T=0.750$

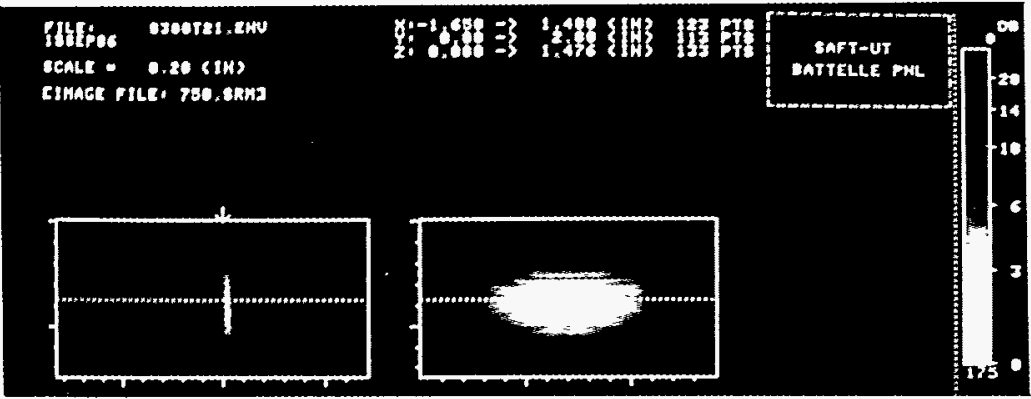

$$
\text { (d) } T=0.825
$$
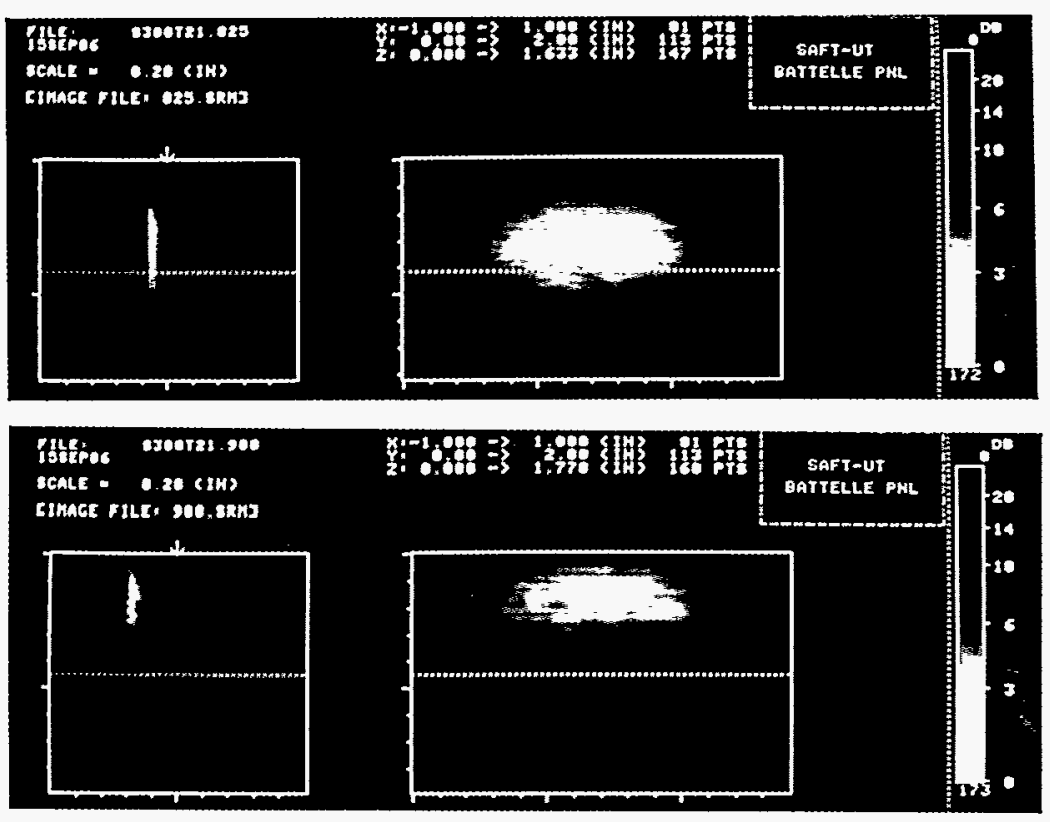

(e) $T=0.900$

Figure 6.2. TSAFT-2 Images of a 0.3-in.-deep (0.8-cm) Sawcut in 0.750-in.-thick (1.9-cm) Aluminum Material with the Processing Thickness Set as Noted 


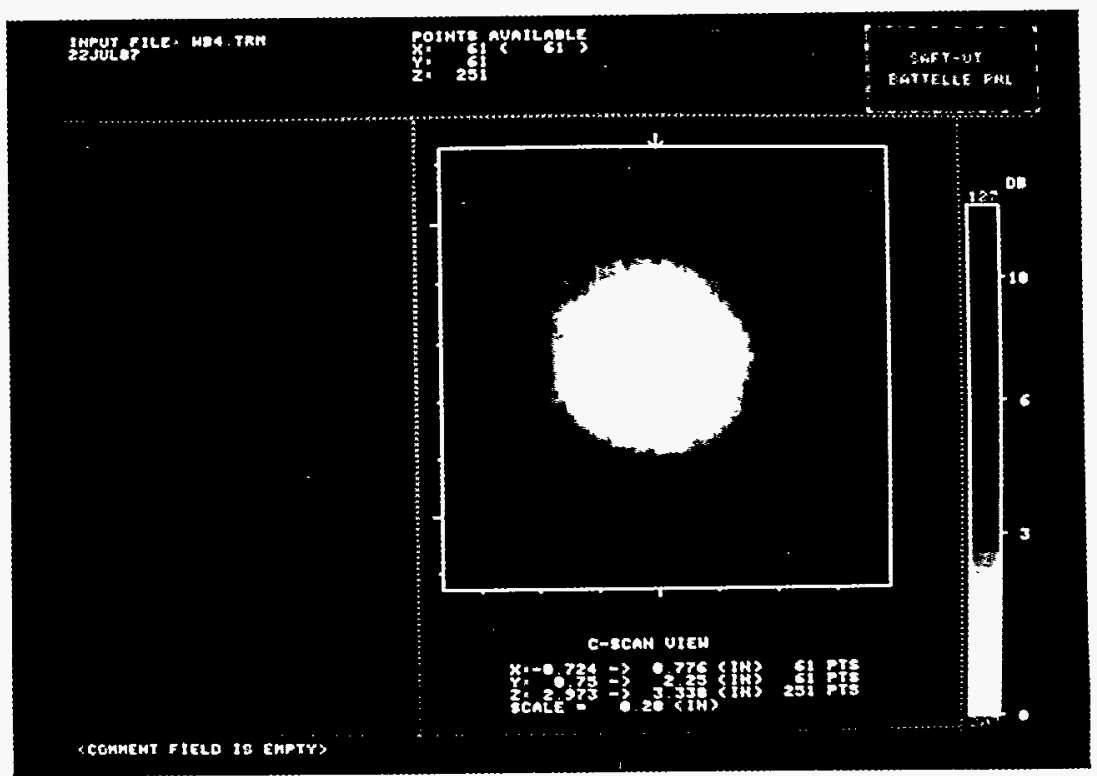

Figure 6.3. Image of Unprocessed Data (C-Scan View) from 0.5-in.-diameter (1.3-cm) Steel Sphere

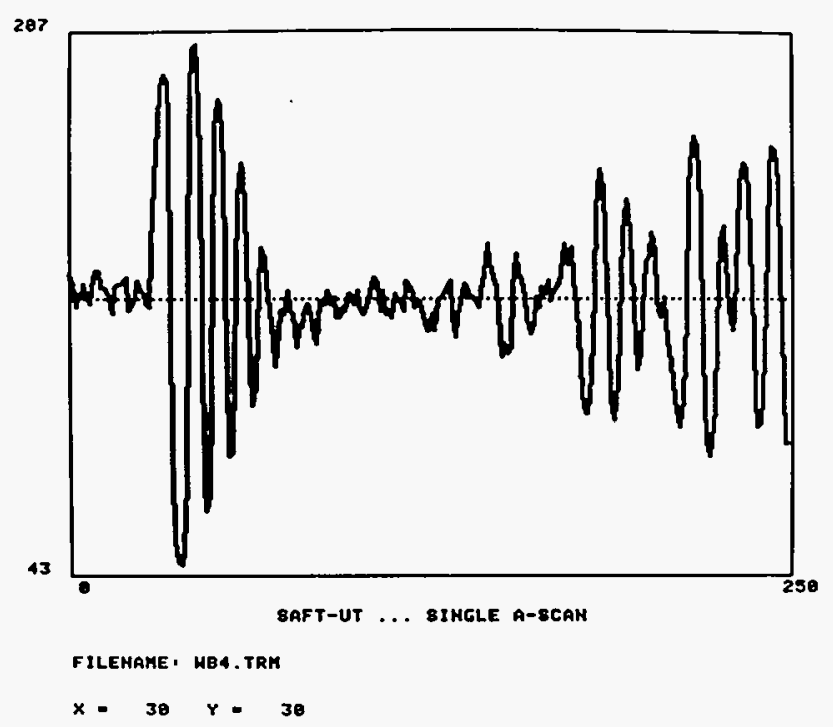

Figure 6.4. A-Scan Near Center of Unprocessed Data from 0.5-in.-diameter $(1.3-\mathrm{cm})$ Steel Sphere 


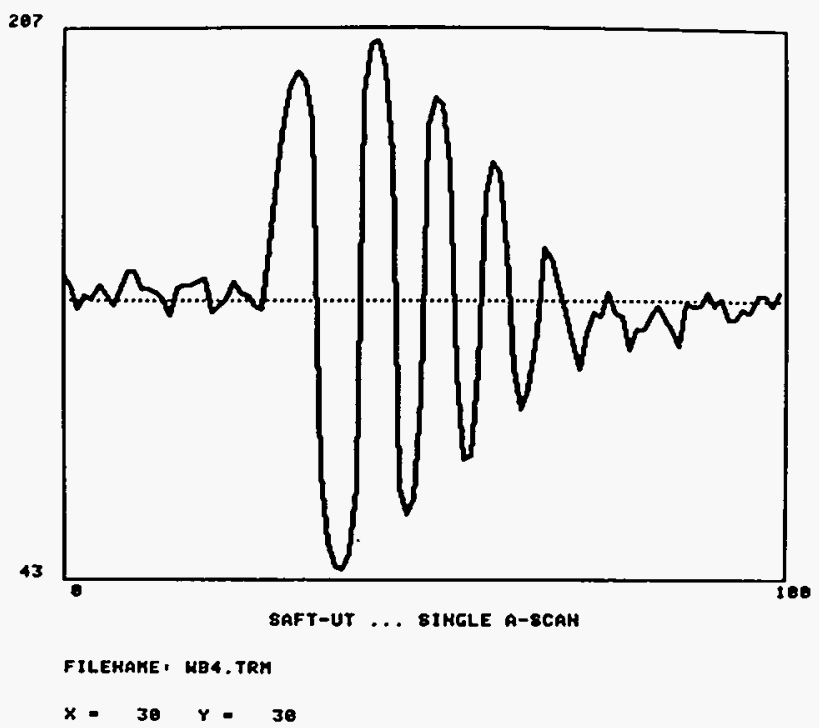

Figure 6.5. A-Scan Showing Only Echo Trace from 0.5-in.-diameter (1.3-cm) Steel Sphere

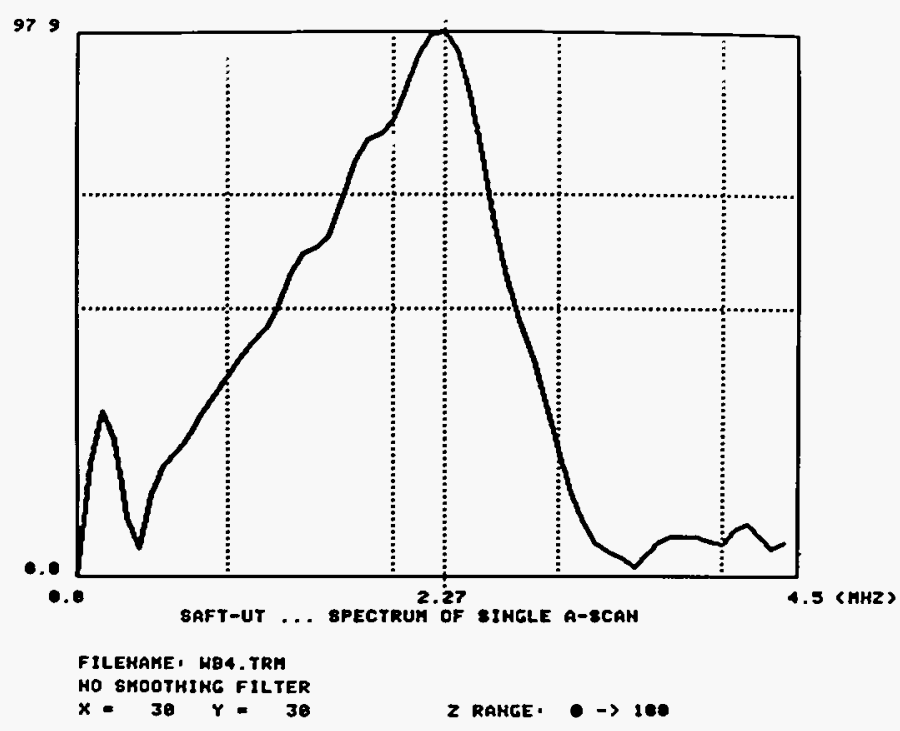

Figure 6.6. Fourier Transform Plot of Echo Trace from 0.5-in.-diameter (1.3-cm) Steel Sphere 


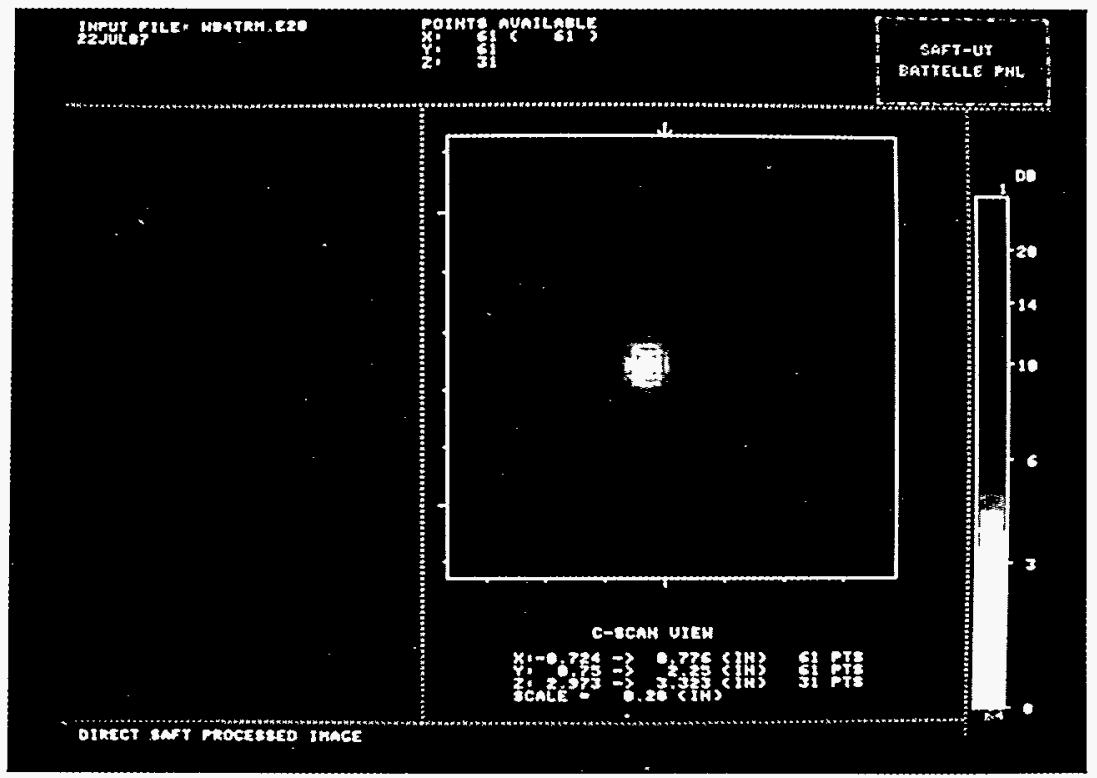

Figure 6.7. C-Scan View of SAFT Processed Echo Trace from 0.5-in.-diameter (1.3-cm) Steel Sphere

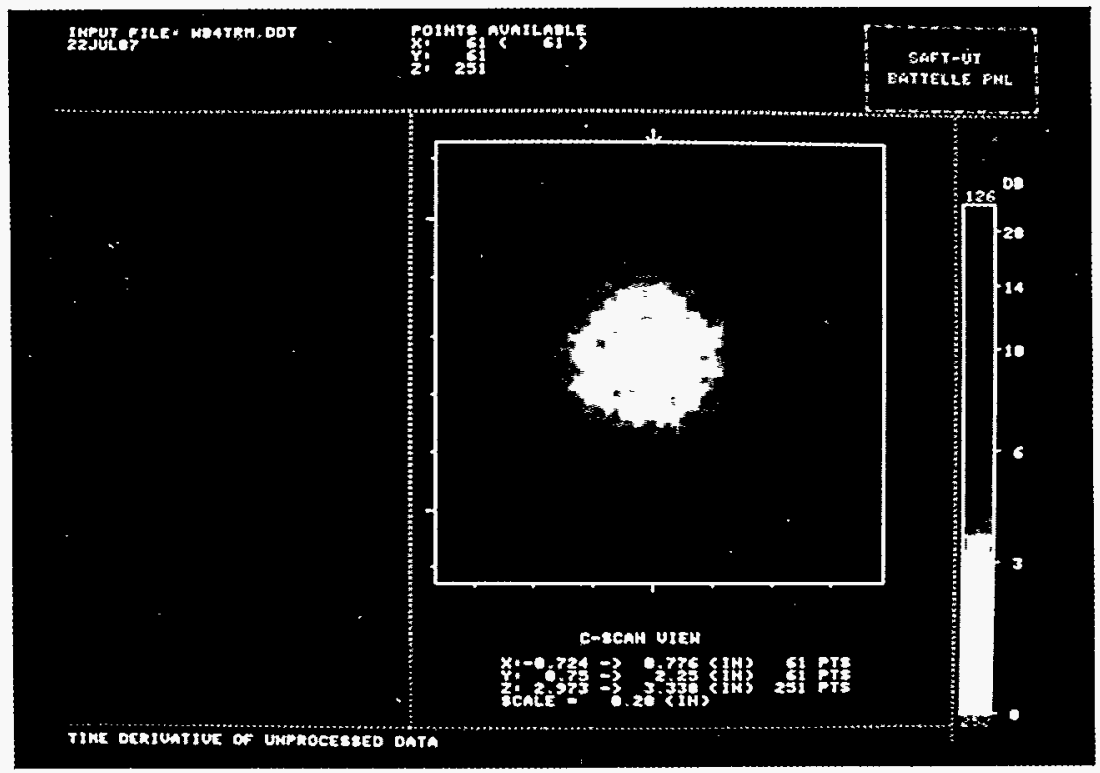

Figure 6.8. C-Scan View of Time Derivative Raw Data Echo Trace from 0.5-in.-diameter $(1.3-\mathrm{cm})$ Steel Sphere 


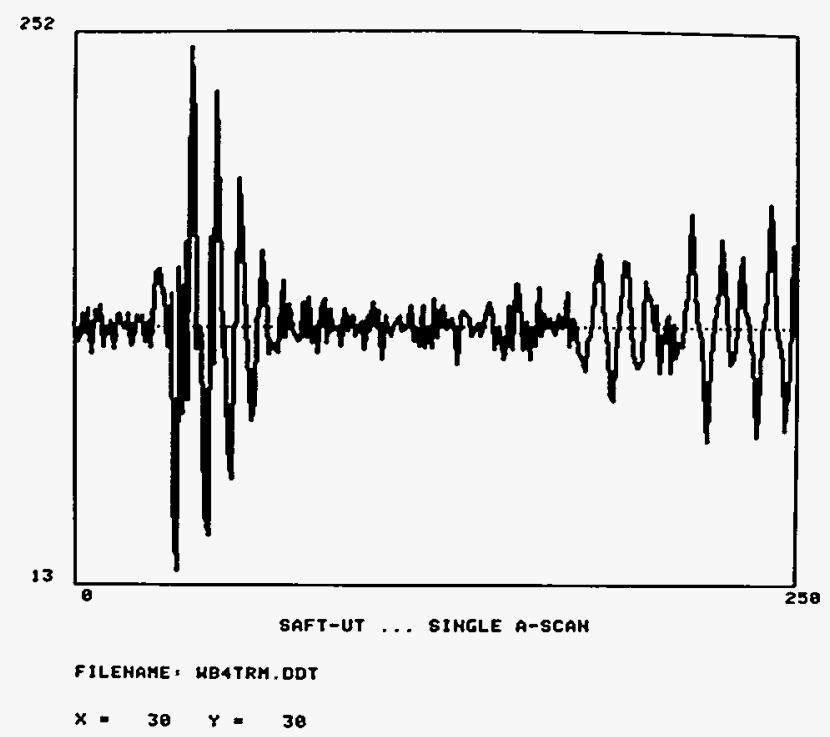

Figure 6.9. A-Scan Near Center of Unprocessed Time Derivative Data from 0.5 -in.-diameter $(1.3-\mathrm{cm})$ Steel Sphere

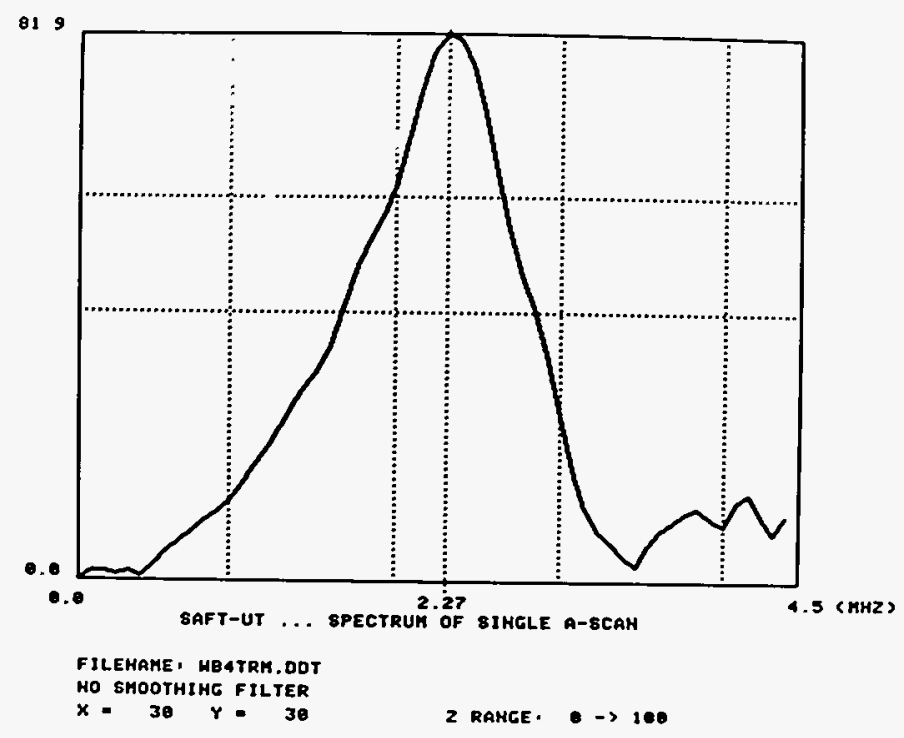

Figure 6.10. Fourier Transform Plot of Time Derivative Data from 0.5-in.-diameter (1.3-cm) Steel Sphere 


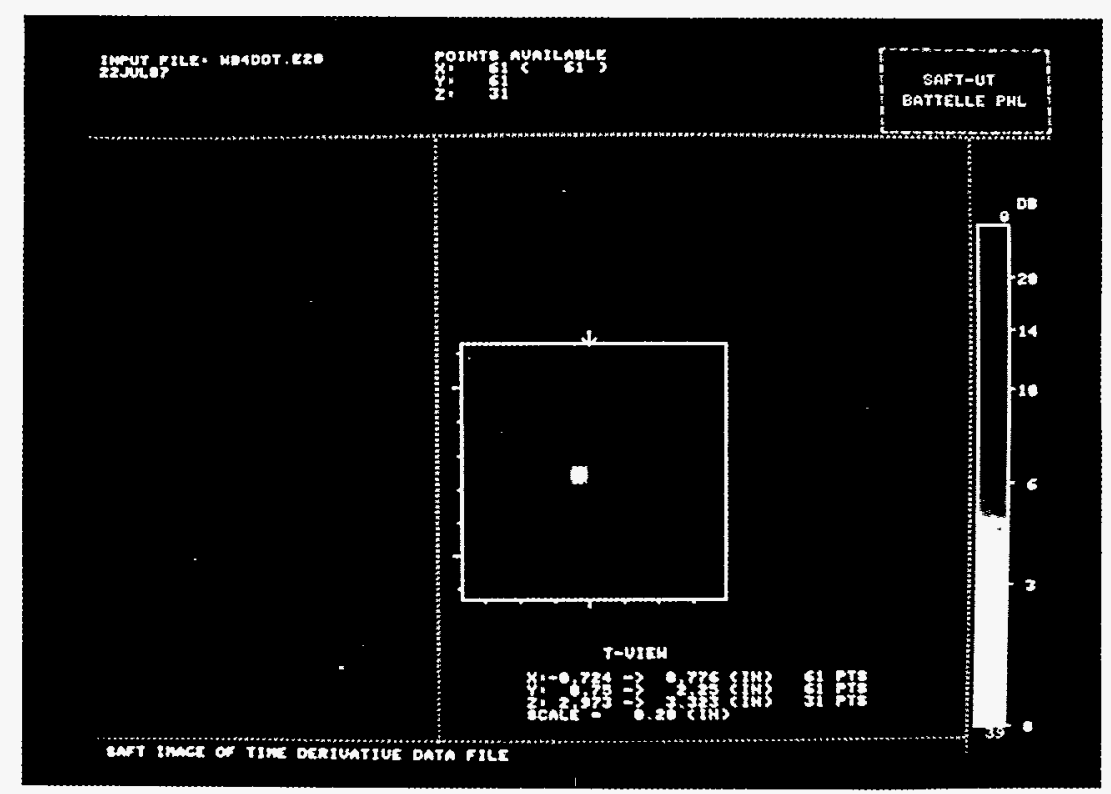

Figure 6.11. C-Scan View of SAFT Processed Time Derivative Data from 0.5-in.-diameter $(1.3-\mathrm{cm})$ Steel Sphere

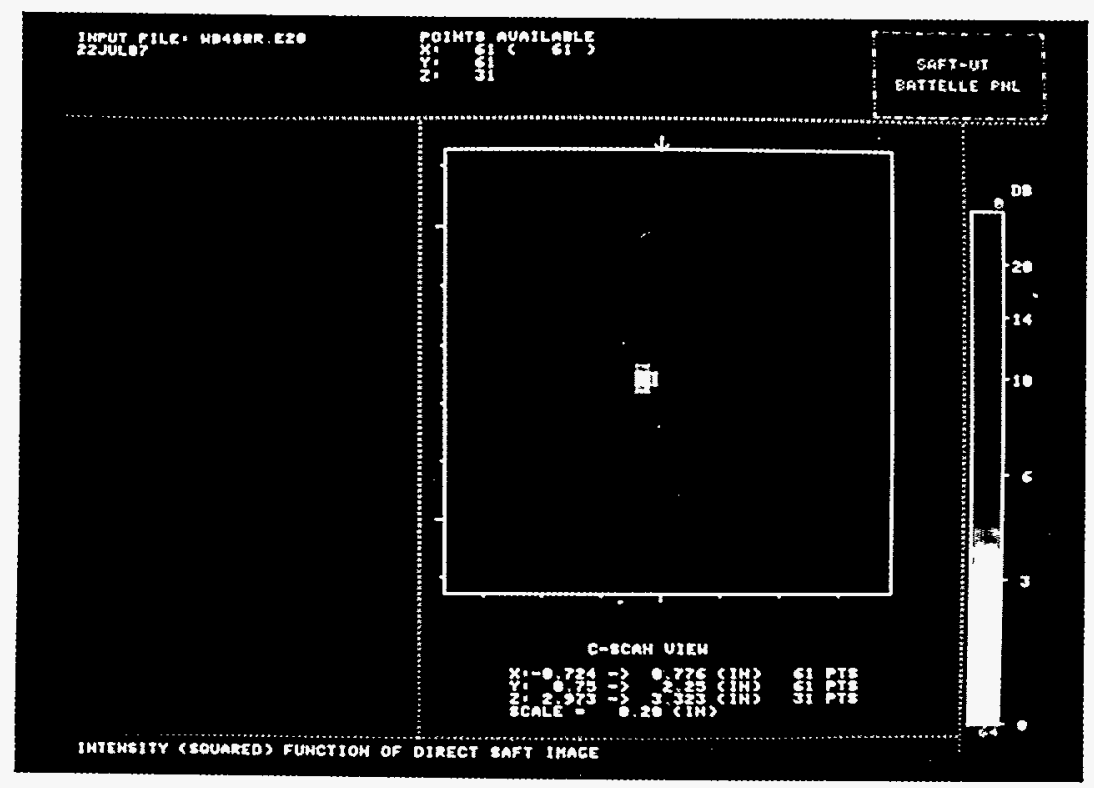

Figure 6.12. C-Scan View of SAFT Processed Echo Trace from 0.5-in.-diameter (1.3-cm) Steel Sphere After Applying Square Function to Intensity Values 


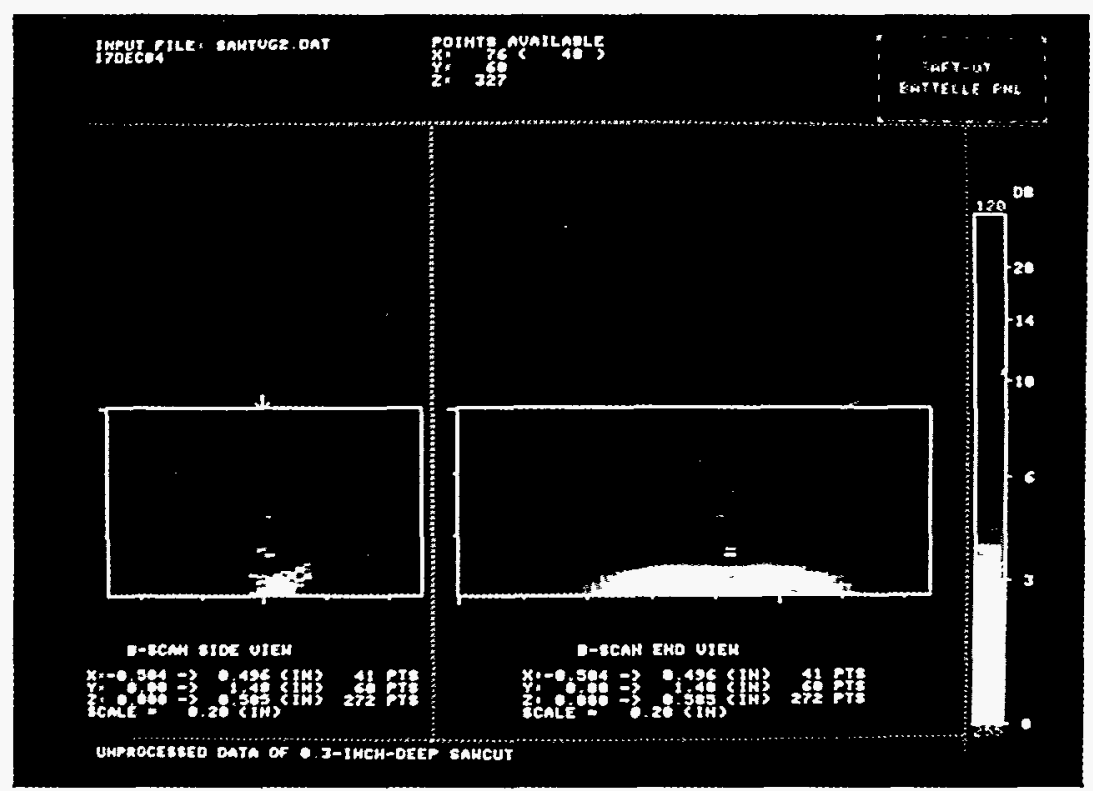

Figure 6.13. Pulse-Echo Image of Raw Data from 0.3-in.-deep (0.8-cm) Sawcut in 0.585-in.-thick (1.5-cm) Stainless Steel Coupon

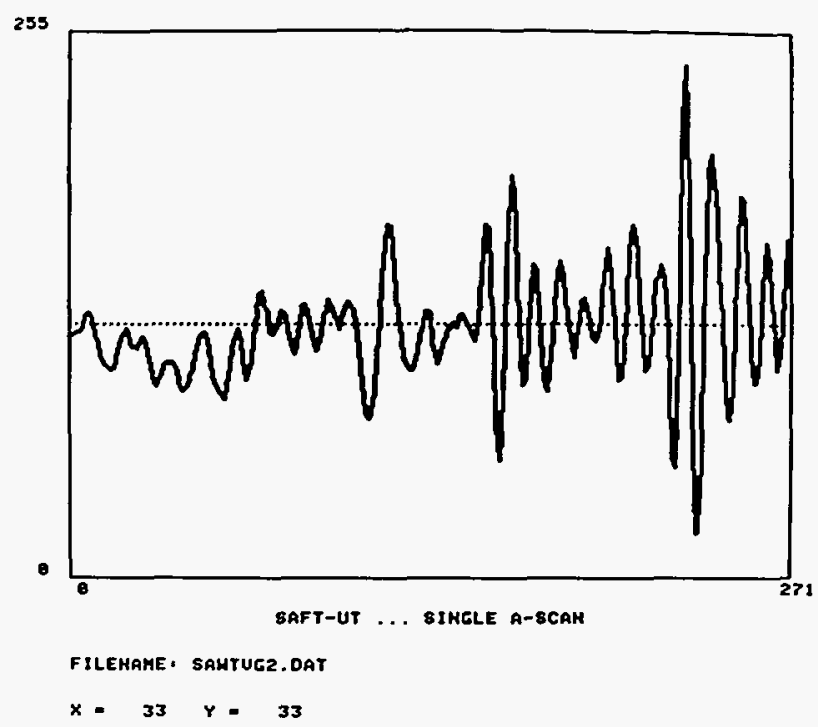

Figure 6.14. A-Scan Near Center of Raw Data from 0.3-in.-deep (0.8-cm) Sawcut in 0.585-in.-thick (1.5-cm) Stainless Steel Coupon 


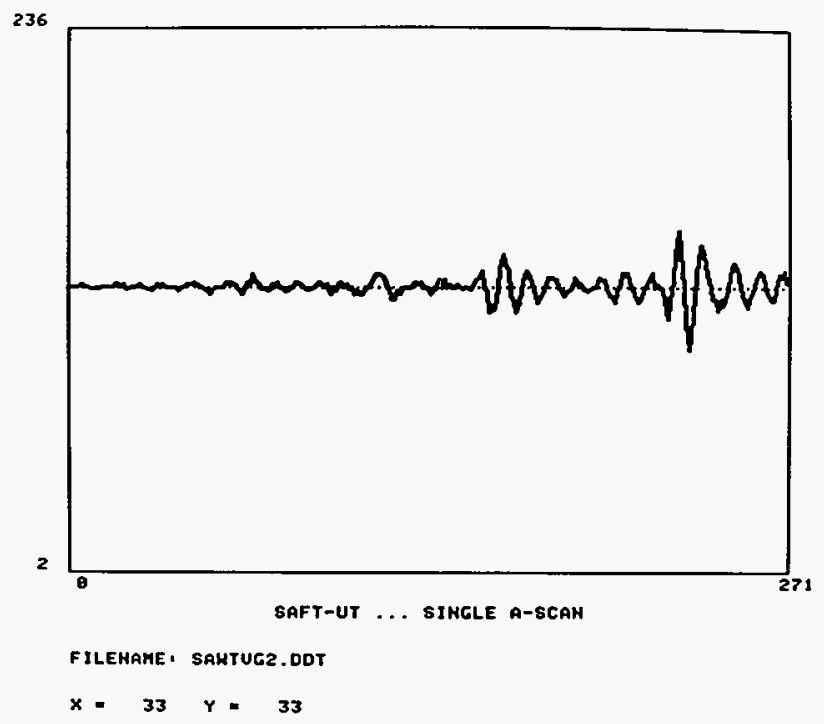

Figure 6.15. Time Derivative of A-Scan Near Center of Raw Data from 0.3-in.-deep (0.8-cm) Sawcut in 0.585-in.-thick $(1.5-\mathrm{cm})$ Stainless Steel Coupon

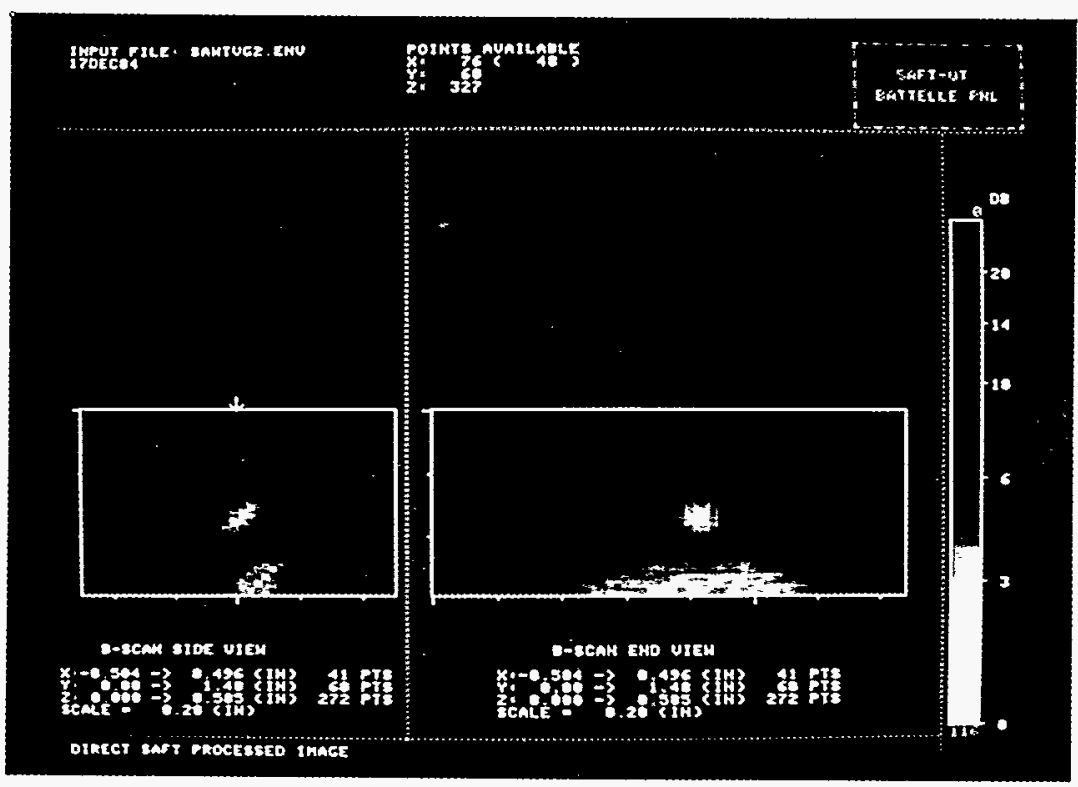

Figure 6.16. Pulse-Echo Image of Time Derivative Raw Data from 0.3-in.-deep (0.8-cm) Sawcut in 0.585-in.-thick (1.5$\mathrm{cm})$ Stainless Steel Coupon 


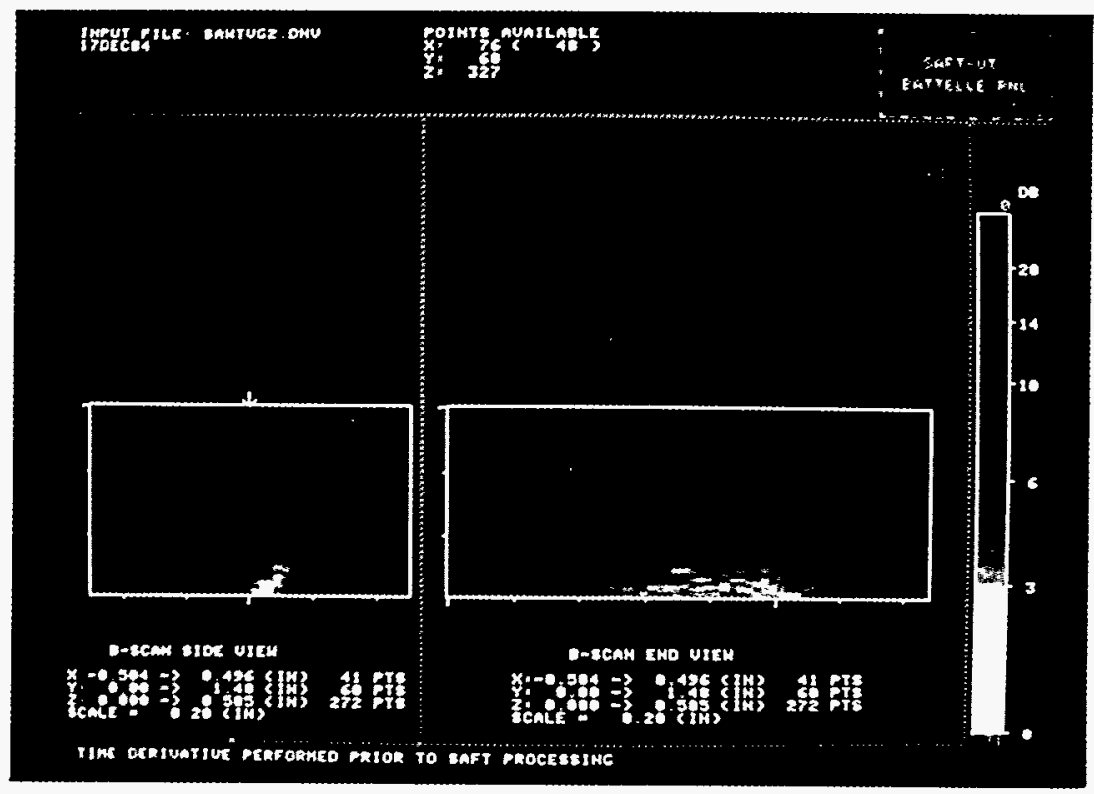

Figure 6.17. Pulse-Echo Image of SAFT-Processed Time Derivative Data from 0.3 -in.-deep (0.8-cm) Sawcut in 0.585in.-thick (1.5-cm) Stainless Steel Coupon

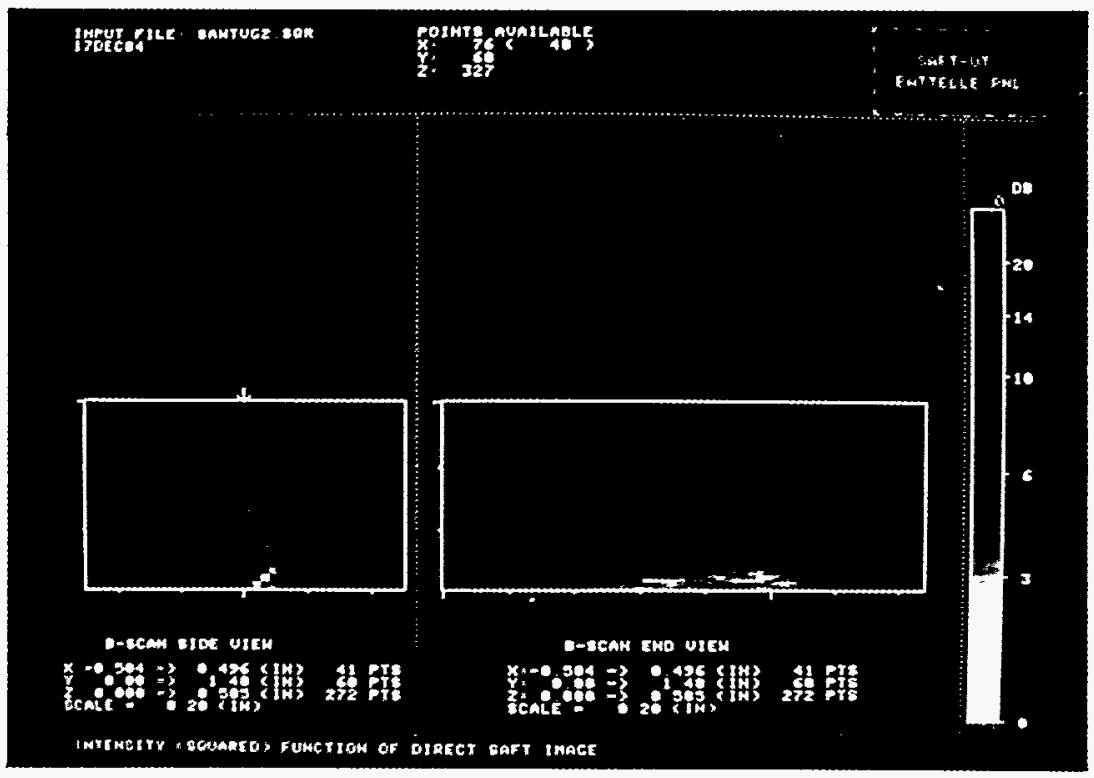

Figure 6.18. Pulse-Echo Image of SAFT-Processed Data from 0.3-in.-deep (0.8-cm) Sawcut in 0.585 -in.-thick Stainless Steel Coupon After Applying Square Function to Intensity Values 


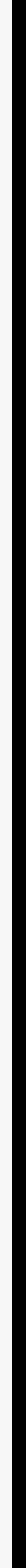




\subsection{Employing the SAFT Technology in the Field}

SAFT has been used at two reactors for detecting and sizing IGSCC in boiling water reactor (BWR) recirculation piping. These efforts have been documented in previous reports of this series. Two new field trials will be reported here. These were, in some ways, difficult because the data was acquired by commercial equipment during inservice inspections of operating reactors and not by the PNL SAFT-UT field system. Section 7 reports on the Indian Point Station Unit II pressure vessel inspection, where data was acquired by Westinghouse/Dynacon and subsequently processed and analyzed by PNL using the SAFT technology. Included in Section 7 is a report on the Trojan Nuclear Power Plant inservice inspection, where data was acquired by Combustion Engineering/Amdata using an Introspect98 UT system from a static-cast stainless steel hot-leg elbow. Once again data was subsequently processed and analyzed, using the SAFT technology, at PNL.

The three previous field trips--the first to General Electric (PISC II), a second to Dresden Unit III, and a third to Vermont Yankee BWR nuclear power plant (Doctor et al. 1986, 1987a, 1987b)--set the stage for PNL using the SAFT technology for independent evaluation. Inspection of the PISC II Plate 2 provided the first field trip for the SAFT-UT data acquisition system and tested the transportability of the system. During one week, 56 files were collected and stored on magnetic tape for subsequent processing and analysis at PNL. Subsequent field trips helped develop analysis techniques described in Section 4 (operating procedures); most of the hardware and software improvements made to the SAFT-UT field system are rooted in these field trips. Shipping-related equipment problems were nonexistent, and the SAFT-UT field system proved, throughout the program, to be very reliable. Although the SAFT-UT field system was not shipped to either the Indian Point Unit II or Trojan nuclear power plants, the processing and analysis portion of the SAFTUT field system was used at PNL. The ability to interface easily to other commercial data acquisition systems is definitely a plus; technology transfer depends on acceptance by the commercial sector, and certainly one criterion of acceptance will be the ease with which SAFT-UT can be implemented into existing systems.

\subsection{Indian Point Station Unit $\mathbf{I}$ Inservice Inspection}

The 1984 ultrasonic examination, conducted during a scheduled outage of the Consolidated Edison pressurized water reactor (PWR) Indian Point Station Unit II in Buchanan, New York, revealed an indication in the vicinity of $345^{\circ}$ vessel azimuth. Further examination using ASME Code-required and advanced ultrasonic techniques was scheduled during a planned 1987 refueling outage to try to further characterize the indication first noted in 1984. One such advanced ultrasonic technique is SAFT. SAFT data was acquired using equipment supplied by Westinghouse and Dynacon, the principal contractors performing the ultrasonic inservice examination. This data was collected using an ultrasonic transducer specified by PNL; the data was subsequently processed and analyzed at PNL. This section describes the SAFT scanning performed, the data processing performed, and the data analysis conducted by PNL. Conclusions are offered on the type, size, and location of the ultrasonic indication. The NRC-funded research program at PNL funded the initial procedure development and the Waltz Mill evaluation. Consolidated Edison funded PNL for the actual vessel examination and data analysis.

\subsubsection{Examinations Performed}

SAFT was first evaluated at the Westinghouse Waltz Mill site (September 1987) on a demonstration block (IPP-2T) that contained a number of artificial notches with varying depths. A number of test scans and transducer configurations were performed to evaluate the quality of the data, vertical resolution, and the overall SAFT performance. Analysis of the Waltz Mill data revealed the following factors:

1. Processing data along both the sweep axis and the increment axis did not produce a highly correlated resultant image. Primarily, adjacent data points did not align well from one sweep to the next. Processing along the sweep axis only produced the more favorable results. Because of this, the data processing proposed for the Indian Point Unit II data was line SAFT and aperture-limited threedimensional (3-D) SAFT. 
2. In an effort to limit the number of different configurations to be used and maintain a high degree of resolution, a $2.25-\mathrm{MHz}$ transducer was used in the $45^{\circ}$ shear-wave mode. Analysis of the Waltz Mill data indicated that the cladded surface was severely impairing the weaker tip-diffracted echo used to measure the vertical extent of the notches. However, the higher amplitude corner-reflected echo did correlate well and produced a corner trap image showing only minor degradation.

3. Laboratory tests on a 3-in.-thick (7.6-cm) multiwire cladded block containing a sawcut performed at PNL during the Waltz Mill data analysis revealed that the same transducer using the $45^{\circ}$ longitudinal mode would generate a higher amplitude and more coherent tip-diffracted echo. The tip-diffracted echo is very important in determining the vertical extent of planar reflectors with sharp radiused edges such as cracks. It was felt that the longitudinal mode scans would be very valuable, although the resolution would be onehalf that of the shear-wave scan.

The SAFT transducer selected and used at Waltz Mill was also used at Indian Point Unit II. An immersion transducer analysis form and data acquisition procedures were provided to Westinghouse. The SAFT transducer used was a Panametrics A305S, Serial \#69838, and has the following characteristics:

1. center frequency of $2.25 \mathrm{MHz}$

2. diameter of $0.75 \mathrm{in} .(1.91 \mathrm{~cm})$

3. focal length of 3 in. $(7.6 \mathrm{~cm})$

4. F number of 4

5. bandwidth approaching $50 \%$

6. The theoretical resolution of the transducer is 0.17 in. $(0.43 \mathrm{~cm})$ in water. The theoretical SAFT resolution in steel (for a limited SAFT aperture with a $6^{\circ}$ full-beam angle) is 0.19 in. $(0.48 \mathrm{~cm})$ for the shear mode and 0.35 in. $(0.9 \mathrm{~cm})$ for the longitudinal mode. The actual resolution obtained is expected to be degraded from the theoretical resolution because of cladding effects and system effects such as positional scanning tolerances.
Acquisition, of data to be processed using the SAFT algorithm requires digitizing the rf data and sampling at least twice per wavelength in the scanned axis direction and at least once per wavelength in the incremental axis. Temporal sampling must be at least four times per wavelength and encompass the full physical aperture (defined as the loss of signal in both the positive and negative directions of the scanned and incremental axes). This high density is required to produce the high resolution and high signal-to-noise ratio imaging. Resultant data files from thick-section materials, such as vessels, are inherently large and require more time to acquire, archive, and analyze.

During SAFT data collection on the Indian Point Unit II vessel, it was decided to acquire data using three scan configurations. The SAFT transducer was mounted in slot 29 of the Westinghouse ten-year plate. It was determined that when the plate was normal to the vessel wall, the SAFT transducer would produce a $45^{\circ}$ refracted shear wave in the vessel. Westinghouse could rotate the plate $10.8^{\circ}$, producing a $45^{\circ}$ refracted longitudinal wave in the vessel. Hence, with one transducer and little effort, SAFT data could be acquired in both the shear and longitudinal modes. The shear-wave scan would yield a higher-resolution image, and the longitudinal scan would enhance the tip-diffracted echo if it existed.

The following describes the three scans in which data were collected:

1. Scan \#1 was a $45^{\circ}$ longitudinal scan with the transducer pointing in the clockwise direction (this clockwise direction produced a lower-amplitude response than did the counterclockwise direction). The physical aperture was determined by signal loss in each direction ( \pm scan axis and \pm increment axis).

2. Scans \#2 and \#3 were with the transducer pointing in the counterclockwise direction (this side produced the largest amplitude echoes). Scan \#2 was a $45^{\circ}$ longitudinal insonification, and scan \#3 was a $45^{\circ}$ shear-wave scan. The physical aperture was again determined by signal loss but encompassed both transducer configurations to avoid programming the scanner twice (the programming task took an hour to perform because of the dense sampling parameters required). 
Quality of the three data files was fair to good. During scan \#1, a synchronization problem caused an erratic sampling from sweep to sweep. A retake of scan \#1 was not accomplished because it was considered a low priority. Scan \#1 was processed but proved to be of little value. Scans \#2 and \#3 were adequate, with scan \#3 providing the best signal-to-noise ratio and ultimately the most information.

\subsubsection{Data Analysis}

SAFT data acquisition setup began around 1900 hours on October 20, 1987, and was completed at 1100 hours on October 21, 1987. The data was collected with the intent of evaluating the use of SAFT on thick-walled vessels and to further characterize the ultrasonic vessel indication. Three cases or classes of indications could exist in the area where SAFT data was acquired:

- $\quad$ Case 1-Volumetric - slag inclusion or porosity with a high degree of spherical or cylindrical size

- $\quad$ Case 2 - Flat - lack of fusion or inclusion (the width dimension being small relative to the height or length dimension)

- $\quad$ Case 3-Crack - diffuse reflector, probably surface-connected.

All of the images analyzed conveyed a single message-the indication seemed to be above the far surface and not surface-connected. Figure 7.1 presents the $45^{\circ}$ shear-wave image of the corner-trap echo of a 0.3-in.deep $(0.76-\mathrm{cm})$ notch (data acquired at Waltz Mill). The indication center is around the far surface, indicated by a dashed line that has been adjusted to compensate for the block curvature. Furthermore, note the effect of the cladding by observing the periodic signal loss present in the B-scan end view. Figure 7.2 presents the $45^{\circ}$ image of the corner trap of a 0.3 -in.-deep (0.8$\mathrm{cm})$ sawcut in a 9-in.-thick $(22.9-\mathrm{cm})$ unclad steel block (data acquired at PNL). The images pictured in Figures 7.1 and 7.2 are in substantial agreement, although the system hardware used and the ease with which the materials can be ultrasonically inspected differ. Figure 7.3 presents the 3-D SAFT processed $45^{\circ}$ shear-wave data acquired on the Indian Point Unit II reactor pressure vessel. The indication is clearly off the far surface. (NOTE: The dashed line in Figure 7.3 represents the vessel far surface and has not been compensated for vessel curvature. The actual vessel far surface is located $0.25 \mathrm{in} .(0.6 \mathrm{~cm})$ above the dashed line, and this is one-half a minor division on the vertical axis.) If the indication was a crack (Case 3), it would most likely be surface-connected, presenting a corner-trap echo that would be oriented similar to that shown in Figure 7.1. If it was a crack and not surface-connected, the tipdiffracted echo should appear if it is within the system resolution. The 3-D SAFT-processed $45^{\circ}$ longitudinal wave image (Figure 7.4) did not contain any tip indications. (The dashed line in Figure 7.4 represents the vessel far surface and has been adjusted to compensate for the vessel curvature.)

If the indication was volumetric with a significant acoustic cross section (Case 1), the normal beam scans performed by Westinghouse should show an indication. Finally, if the indication was flat and of a proper orientation (Case 2), a mirror image should be seen projected below the far surface and possibly a corner-trap echo projected on the far surface. Figure 7.5 presents the image of a side-drilled hole that is 0.5 in. $(1.3 \mathrm{~cm})$ from the far surface of a 2.0 -in.-thick $(5.1-\mathrm{cm})$ steel block showing the direct, corner-trap echo, and mirror echoes one would expect for a volumetric defect with good acoustic scattering properties.

The SAFT $45^{\circ}$ shear-wave data file did not sample far enough to see the mirror echo, if it exists, and a cornertrap echo is not evident. Perhaps the geometry of the indication is such that the corner echo is severely reduced and therefore may not be distinguishable from the background noise. The $45^{\circ}$ longitudinal scans support the shear-wave scans in that the indication seems to be above the far surface. The data collected by Westinghouse sampled beyond the far surface and should corroborate the SAFT data. Westinghouse used a much larger variety of scan configurations (normal beam, pitch/catch, delta, $45^{\circ}$ shear, $60^{\circ}$ shear, and $45^{\circ}$ longitudinal), which should help confirm the analysis of the SAFT data.

\subsubsection{Discussion}

A review of the SAFT data shows that this indication does not present a geometry exhibiting tip signals or a strong corner-trap echo. This indication appears to be above the back surface although the remaining ligament 
and through-wall height cannot be established by the SAFT data alone; it is based almost entirely on the one aperture-limited scan of $45^{\circ}$ shear in the pulse-echo mode. The SAFT performance was based on notches (vertically oriented planar reflectors), and clearly this indication does not image like a notch. The $45^{\circ}$ shear image shows that the defect does not have uniform through-wall height over its length. The indication has an image center that is located on average about $0.3 \mathrm{in}$. $(0.8 \mathrm{~cm})$ above the far surface. The indication has a continuous length of 1.9 in. $(4.8 \mathrm{~cm}$ ) (based on a $-6 \mathrm{~dB}$ drop criterion) and an intermittent length of 2.4 in. (6.1 $\mathrm{cm})$. (The exact termination of the indication may be beyond $2.4 \mathrm{in} .(6.1 \mathrm{~cm})$, but this area was beyond the aperture limits chosen for the examination. The longitudinal scan does support the length measurement, and it was not aperture limited.) The images show that there are acoustic hot spots, but it is not possible to conclude if this reflector is a collection of small reflectors or a larger reflector with some acoustical preferential facets.

\subsubsection{Conclusion}

The main purpose of this work was to provide useful information for characterizing the indications in the Indian Point Unit II reactor pressure vessel. The SAFT scans did provide useful information for locating and sizing the indication length. The SAFT data analysis clearly shows that the defect is volumetric and is not surface-connected.

In addition, another purpose of using the SAFT technology for this examination was to assess whether the technology could be deployed in the field with conventional reactor pressure vessel inspection hardware. The conclusion derived from this work shows that some limitations exist with conventional hardware in achieving the accuracies needed for positioning the transducer in the required grid pattern. As a consequence, a limited processing aperture and the line SAFT mode was used. However, some very useful data and results were obtained. If SAFT were to be fully utilized in similar future applications, it will be necessary to modify the SAFT inspection protocol to include other SAFT scans to more accurately characterize indications.

\subsection{Trojan Nuclear Power Plant}

In May 1988, PNL personnel traveled to the Trojan Nuclear Power Plant to advise Amdata, Inc. personnel concerning the conduct of a special ultrasonic examination on a static-cast stainless steel hot-leg elbow. The purpose was to acquire data from regions already known to have ultrasonic indications and subsequently apply SAFT to the data. PNL was to process and analyze the SAFT data at its laboratory.

The SAFT technology was selected because it could be used to apply various signal processing techniques to the data. The inspection of cast materials is difficult because the material is coarse-grained, anisotropic, and inhomogeneous. As a consequence, one of the major problems is the identification of true defects; another is accurate characterization of the defects. Generally, typical approaches have included zone-focused probes coupled with some signal processing such as spatial or temporal averaging. The approach of SAFT is to acquire broad-band if signals with no filtering. Then the data can be processed by a number of techniques to select those most effective in removing innocuous reflectors and, at the same time, provide high resolution and high signal-to-noise images of the defects.

Laboratory work at PNL has shown that band-limited signals, based on measurements from the material, could be used to improve the inspectability of the cast material. Thus, the approach was to gather sufficient data to allow the development of a special filter based on the cast elbow material. The filter would then be used on the data prior to SAFT processing.

\subsubsection{Examinations Performed}

Amdata personnel calibrated the I-98 data collection system on a calibration standard provided by Trojan (reference Amdata drawing C-UTC-AL-244-004). Unfortunately, the $0.5-\mathrm{MHz}$ high-pass filter was used when calibration data was acquired. The calibration data was not useful to select filter parameters based on composite spectra results of known defect and nondefect areas.

A total of four data files were acquired on the 9 o'clock side of the static-cast elbow in the short time allotted for gathering SAFT data: 
1. TR9SH5A.D1 - Data file number one included three inside surface indications that Amdata personnel identified as \#2, \#3, and \#5. An $0.5-\mathrm{MHz}$ high-pass filter was used during data acquisition, receiver gain was set at $+12 \mathrm{~dB}$, and the transducer view was skewed $180^{\circ}$.

2. TR9SH5C.D1 - Data file number two was set up identically to data file TR9SH5A.D1 except no filters were used during data acquisition.

3. TR9SH5D.D1 - Data file number three included one inside surface indication that Amdata personnel identified as \#4, data was acquired with the transducer skewed $0^{\circ}$, and the receiver gain was set to $+12 \mathrm{~dB}$.

4. TR9SH5G.D1 - Finally, data file number four included one midwall indication identified as \#6, the transducer was skewed $270^{\circ}$, and, once again, the gain was set to $+12 \mathrm{~dB}$.

A Panametrics 500-kHz transducer (serial \#B32180E) was used with a $\mathrm{KB}$ Aerotech $45^{\circ}$ shear-wave wedge. Spatial increments of 0.05 in. $(0.13 \mathrm{~cm})$ were chosen for both the incremental and scanned axes in conjunction with a temporal sampling of $100 \mathrm{~ns}$.

The I-98 data acquisition system had not been used extensively with the SAFT utilities; therefore, some empirical manipulation of the data order was necessary to place the data in the proper SAFT format. Particular attention was given to the skew angle and the bidirectional acquisition of the data file.

Each data file was first converted to the SAFT data format using a software utility called VIPD. A shuffle or reordering of every other sweep was required to compensate for bidirectional data acquisition. Data files, where the transducer was skewed $180^{\circ}$, required using the SAFT utility EVENSHUFX to reorder the even sweeps and using the SAFT utility DEDIT to change the incident angle sign to minus (the SAFT processing utilities always expect the incident angle direction to be $\mathrm{X}$ and the sign to be minus). The $0^{\circ}$ skew data file required using the SAFT utility ODDSHUFX to reorder the odd sweeps, and the $270^{\circ}$ skew data file required using the SAFT utility EVENSHUFX to reorder the even sweeps. The I-98 conversion utility VIPD automatically makes the sweep or scan axis (no matter which axis it is, $X$ or $Y$ ) the $X$ axis. We found that when the sweep axis was circumferential $\left(270^{\circ} \mathrm{skew}\right)$, the header said the incident angle was in the $Y$ axis and positive; this was not correct because of the previously mentioned axis transformation that the VIPD utility performs. Therefore, we used the SAFT utility DEDIT to change the direction and sign of the incident angle of the $270^{\circ}$ skew data file.

Filter parameters were chosen by looking at the composite spectra analyses of suspected defect and nondefect areas of data file TR9SH5D (Figure 7.6). Unfortunately, the composite spectra were not very revealing, except to point out that a large $500-\mathrm{kHz}$ component was present. Based on the composite spectra, a 0.4- to 0.6$\mathrm{MHz}$ band-pass filter was used, primarily to keep the file with about a $50 \%$ bandwidth limit desired for the SAFT processing. Each data file was band-pass filtered using a $40 \%$ bandwidth filter (although with some of the data files, we experimented with different filter parameters) and then processed using the 3-D SAFT algorithm, which coherently sums the data over a predefined aperture. Data files were then processed using a $12^{\circ}$ and $6^{\circ}$ full-beam angle; a beam angle of $6^{\circ}$ was subsequently chosen for analysis because the $12^{\circ}$ images were being "washed out" due to the summing of null data. From the thickness measurements provided by Amdata personnel, a nominal thickness of 3.7 in. (9.4 $\mathrm{cm}$ ) was entered into the SAFT data file header for each data file. Thickness measurements ranged from 3.5 in. $(8.9 \mathrm{~cm})$ to 4.0 in. $(10.2 \mathrm{~cm})$ in the approximate area of the $180^{\circ}$ and $0^{\circ}$ skew scans, and the nominal value used is a truncated average of these values. Typically, the following scenario was followed:

1. Filter the raw data file based on composite spectrum results. A $0.4-$ to $0.6-\mathrm{MHz}$ filter was used on the unfiltered $180^{\circ}$ and $270^{\circ}$ skew data files; the $0^{\circ}$ skew data required no filtering.

2. SAFT process the resultant filtered file. Each data file was 3-D SAFT processed using a $6^{\circ}$ fullbeam angle.

3. Use the APLOT utility to analyze the processed results. A full B-scan side view and C-scan top view were initially analyzed. Each indication was boxed, and an expanded B-scan side view and Bscan end view were used for the final analysis and sizing. 


\subsubsection{Data Analysis}

A $0.5-\mathrm{MHz}$ high-pass filter was used during data acquisition of the $180^{\circ}$ skew data file TR9SH5A. Figure 7.7(a) shows the B-scan side and C-scan top view of data file TR9SH5A; three groups of indications are apparent. Each indication has been boxed on the Cscan view and labeled 2,3, and 5 on the accompanying $\mathrm{C}$-scan graphic. An expanded view of each indication is shown in Figures 7.7(b), 7.7(c), and 7.7(d). A sizing attempt has been made (Table 7.7), and the defects have been correlated to the indications identified by Amdata personnel.

Figure 7.7(a) shows indication 2 to be a dog-leg shaped indication; accurate sizing of the spot-like indications along the axial portion of the dog leg cannot be made and, therefore, have not been included in Table 7.1. The image of indication 2 is very difficult to interpret. Most of the indications seen in Figure 7.7(b), the Bscan side view, are at the same depth and may be corner-trap echoes from a surface-connected planar defect. Indication 3, Figure 7.7(c), is composed of a number of indications that have been considered as one because of the proximity of one to another. The depth of indication 3 varies; the strongest echo appears above the nominal thickness value, leading one to believe that this indication may not be surface-connected. Indication 5, Figure 7.7(d), presents a very interesting B-scan side view; the three major components of this indication are at different depths and uniform intensity. Characteristics of a surface-connected planar defect usually include a strong corner-trap indication and a reduced tip-diffracted echo. Indication 5 exhibits volumetric characteristics and does not image like one would expect if it were a surface-connected planar defect.

Figure 7.8(a) presents the B-scan side view and C-scan view of the $180^{\circ}$ skew data file TR9SH5C; a total of five indications have been identified and are labeled 2, 3, 5, $A$, and $B$ in the accompanying C-scan graphics. A 0.4to $0.6-\mathrm{MHz}$ band-pass filter was applied to this data file prior to SAFT processing primarily because this data file was very noisy and difficult to interpret. Figures 7.8(b), 7.8(c), 7.8(d), 7.8(e), and 7.8(f) show boxed views of indications $2,3,5, \mathrm{~A}$, and $\mathrm{B}$. Each indication size is listed in the comments section of the indication photograph and is repeated in Table 7.1.
Only a part of the axial portion of indication 2, which is the same area as the dog-leg indication 2 seen in data file TR9SH5A, Figure 7.7(a), is visible in Figure 7.8(a). The circumferential part of indication 2, B-scan side view, Figure 7.8(b), varies in depth as do indications 3 and 5 in Figures 7.8(c) and 7.8(d), suggesting either volumetric reflectors or a varying far surface. Indications 2,3, and 5 of data file TR9SH5C compare favorably with the same indications seen in data file TR9SH5A, although different filters were used prior to SAFT processing the data. Indication A, Figure 7.8(e), is an additional indication that has been noted; it is at least $-6 \mathrm{~dB}$ less intense than the other indications noted in data file TR9SH5C. The signal-to-noise ratio seen in Figure 7.8(e) makes characterization of this indication difficult. The most intense portion of indication $\mathrm{A}$ appears near the nominal thickness value line; therefore, this indication may be surface-connected. Finally, indication $B$, Figure $7.8(\mathrm{f})$, presents one high-intensity indication surrounded by shallower low-level indications. Once again, indication B appears near the nominal thickness value line and, for this reason, may be surface-connected.

Sizing of all the indications in files TR9SH5A and TR9SH5C included the surrounding low-level indications. Each boxed area was normalized by the most intense indication within the box. Sizing extended to the $-6-\mathrm{dB}$ points relative to the normalized value.

The best data file, by virtue of signal-to-noise ratio and a single indication, is TR9SH5D. Figure 7.9(a) shows a B-scan side view and C-scan top view of indication 4. Only one indication is seen, and the location of the satellite indications lead one to believe that the main indication may not be surface-connected and may actually be volumetric. Filtering of this data file did not produce significant changes in the size of the indication but did help resolve the satellite indications. Since the far surface location is not accurately known, it is difficult to estimate how far off the ID surface this indication might be in Figure 7.9(b), B-scan side view; however, based on the nominal thickness value, one would estimate $0.3 \mathrm{in} .(0.8 \mathrm{~cm})$. The temporal aperture should have been expanded in the near and far directions to include a possible direct echo, if the main indication seen is the corner-trap echo from a volumetric defect and/or mirror echo expected from a volumetric defect. Presence of these particular echoes 
Table 7.1. SAFT Sizing of the Static-Cast-Elbow Indications

\begin{tabular}{|c|c|c|c|c|}
\hline Data File & Indiction Nomber & Average Depth In; & lengthin & $\begin{array}{c}\text { X/X Location } \\
\text { in }\end{array}$ \\
\hline TR9SH5A & $\begin{array}{l}2 \\
3 \\
5 \\
\end{array}$ & $\begin{array}{l}3.4 \\
3.5 \\
3.4 \\
\end{array}$ & $\begin{array}{l}2.1 \\
2.3 \\
1.5 \\
\end{array}$ & $\begin{array}{l}0.8 / 5.3 \\
4.8 / 9.2 \\
0.8 / 9.5\end{array}$ \\
\hline TR9SH5C & $\begin{array}{l}2 \\
3 \\
5 \\
\text { A } \\
\text { B }\end{array}$ & $\begin{array}{l}3.4 \\
3.5 \\
3.5 \\
3.4 \\
3.6\end{array}$ & $\begin{array}{l}3.2 \\
1.2 \\
1.3 \\
1.9 \\
1.2\end{array}$ & $\begin{array}{l}4.0 / 6.7 \\
4.9 / 9.4 \\
1.2 / 9.5 \\
2.7 / 2.0 \\
5.0 / 1.0\end{array}$ \\
\hline TR9SH5D & 4 & 3.3 & 1.4 & $4.0 / 6.7$ \\
\hline TR9SH5G & 6 & \multicolumn{3}{|c|}{ Unable to size.*** } \\
\hline
\end{tabular}

Note: The lateral resolution limit imposed by the transducer is 0.5 inch ( $1 / 2$ the transducer diameter), which is about 2 wavelengths. A -6 $\mathrm{dB}$ (green/yellow transition) criteria was used to size the indications after each indication was boxed and normalized by the maximum value within the boxed area. Data was acquired with the gain increased $12 \mathrm{~dB}$ from that used when taking the calibration scans.

* Average depth was determined by averaging the depth of the indications (measured to the mid-point of the indication) seen in the B-scan side view image and truncating the value to one position to the right of the decimal point.

** The SAFT X location is the sweep axis and the SAFT $Y$ location is the incremental axis; $X / Y$ coordinates given in the summary of indications are relative to the scanned aperture $\mathrm{OD}$ dimensions and are the $\mathrm{X} / \mathrm{Y}$ coordinate of the maximum response for each indication.

*** Indication \#6 was not sized. The processed file revealed only noise, possibly do to 1) poor acoustic coupling, 2) incorrect area scanned, or 3) no defect.

would have certainly made a case for a volumetric defect.

A SAFT-processed normal-beam scan would have been helpful in resolving whether indications seen in data files TR9SH5A, TR9SH5C, and TR9SH5D are indeed planar or volumetric.

Finally, the last data file analyzed was the midwall indication (\#6) TR9SH5G. Figure 7.10 shows the B-scan side view and $C$-scan top view. There was some concern from Amdata personnel when we finished this scan that the transducer may not couple well in the $270^{\circ}$ skew position, which may account for the poor quality of the data. One cannot select a single indication from
Figure 7.10. One of the following conclusions may be made:

1. Poor acoustic coupling resulted in the signal-tonoise ratio being such that the indication was lost in the noise.

2. Reflector response to the $500-\mathrm{kHz}$ shear wave was nonexistent.

3. An incorrect area was scanned.

Most likely the coupling of the sound into the static-cast elbow was the problem. This theory is further supported by line-SAFT processing (focusing occurs only along 
the sweep direction of the scan) the midwall data file. Line-SAFT processing of the midwall data file revealed a near total drop-out of the data in the reverse direction of the scanner, which suggests coupling deficiencies. However, a videotape of the scanning process would help answer scanner positioning questions and would also help in the analysis process by giving the data analyst a better feel for the physical setup.

\subsubsection{Conclusion}

Data files TR9SH5A and TR9SH5C were acquired from the same scan aperture. The only difference was that an $0.5-\mathrm{MHz}$ filter was used during data acquisition for data file TR9SH5A, and data file TR9SH5C was acquired using no filters. A $40 \%$ band-pass filter was applied to the unfiltered data file prior to SAFT processing to reduce the noise. The composite spectrum of the unfiltered data file did not reveal unique features between spectra of suspected defect and nondefect areas; therefore, only a band-pass filter centered around the center frequency of the transducer $(500 \mathrm{kHz})$ was used. Both data files revealed similar indications (\#2, \#3, and \#5) that correlated positionally to the Amdata results (Table 5.2-1 of the 1988 Amdata Trojan report), although data file TR9SH5C did show two additional indications not reported by Amdata. Analysis by Amdata personnel may not have revealed the additional indications because of the low amplitude (greater than $6 \mathrm{~dB}$ down) relative to surrounding indications and/or spectral response of these indications.

The best data file was TR9SH5D. There was little change in the size or appearance of the single indication when different filters were applied prior to SAFT processing; however, the resolution of satellite indications did improve. This file was also used to see if there were unique features between spectra of suspected nondefect areas and suspected defect areas. There was a difference in the relative amplitudes of the spectra but no unique features.

The midwall indication did not image well, perhaps due to transducer coupling problems. Initially, we thought this would be the best data file because Amdata results pointed to the midwall indication as having the best response in terms of decibels. The pipe curvature in the $270^{\circ}$ skew direction may have been too severe for the flat wedge that was used, resulting in poor coupling of the sound field into the cast elbow. Line-SAFT processing of the midwall data file further substantiated that poor coupling was the problem; sweeps in the reverse scan direction exhibited a near total signal loss.

A SAFT-processed normal-beam scan would have been helpful in resolving whether indications seen in data files TR9SH5A, TR9SH5C, and TR9SH5D are indeed planar or volumetric.

PNL's analyses of the processed data files revealed the following:

1. Two of the inside surface indications in the $180^{\circ}$ skew data file (TR9SH5C), numbers 2 and 3 , seemed to be tied together, rather than being isolated indications. Lateral separation of these indications is approximately $0.5 \mathrm{in} .(1.3 \mathrm{~cm})$ or right at the lateral resolution imposed by the transducer diameter with low-level indications $(-6$ to $-10 \mathrm{~dB}$ ) between.

2. Two more indications were identified in the $180^{\circ}$ skew data file (TR9SH5C), indications A and B, not previously noted by Amdata. Analysis by Amdata personnel may not have revealed the additional indications because of the low amplitude (greater than $6 \mathrm{~dB}$ down) relative to surrounding indications and/or spectral response of these indications.

3. Four inside surface indications [number 4 (seen in the $0^{\circ}$ skew data file, TR9SH5D); numbers 3 and 5 (seen in the $180^{\circ}$ skew data file, TR9SH5A); and numbers 2,3 , and 5 (seen in the $180^{\circ}$ skew data file, TR9SH5C)] seem to have characteristics closer to volumetric indications and, therefore, may not be surface-connected.

4. The midwall indication $\left(270^{\circ}\right.$ skew data file, TR9SH5G) did not image well, possibly due to transducer coupling problems $\left(270^{\circ}\right.$ transducer skew position did not conform well to the Lucite wedge that was used); therefore, detection, confirmation, and/or sizing of this indication could not be accomplished.

Finally, it would be useful to conduct additional testing on these indications to provide an adequate technical basis to fully characterize the indications. Some fea- 
tures of the indications are salient, but other features necessary for confidence in classification and dimensional properties were not determined. Based on the tests already conducted, it is easy to lay out a testing procedure that generates the needed information.

\subsection{Conclusion}

The field trials have shown that SAFT can be successfully coupled with commercial UT equipment and scanner systems. The SAFT technology can be incorporated into commercial equipment in an on- or off-line mode. The SAFT results are easy to interpret and provide images that others can readily understand.

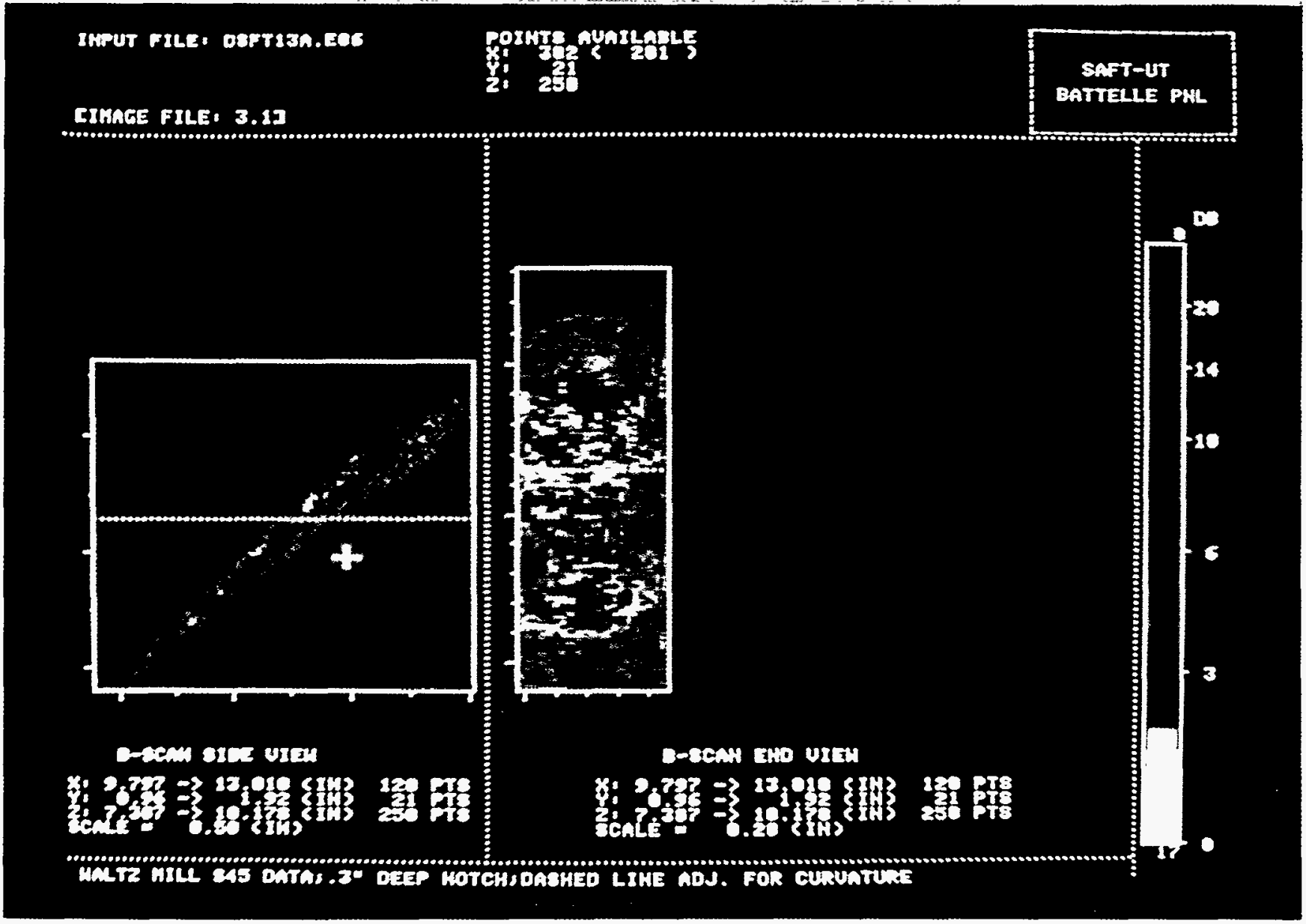

Figure 7.1. $45^{\circ}$ Shear-Wave Image of Corner-Trap Echo of 0.3-in.-deep (0.8-cm) Notch in 9in.-thick (22.9-cm) Cladded Vessel Calibration Block (Waltz Mill) 


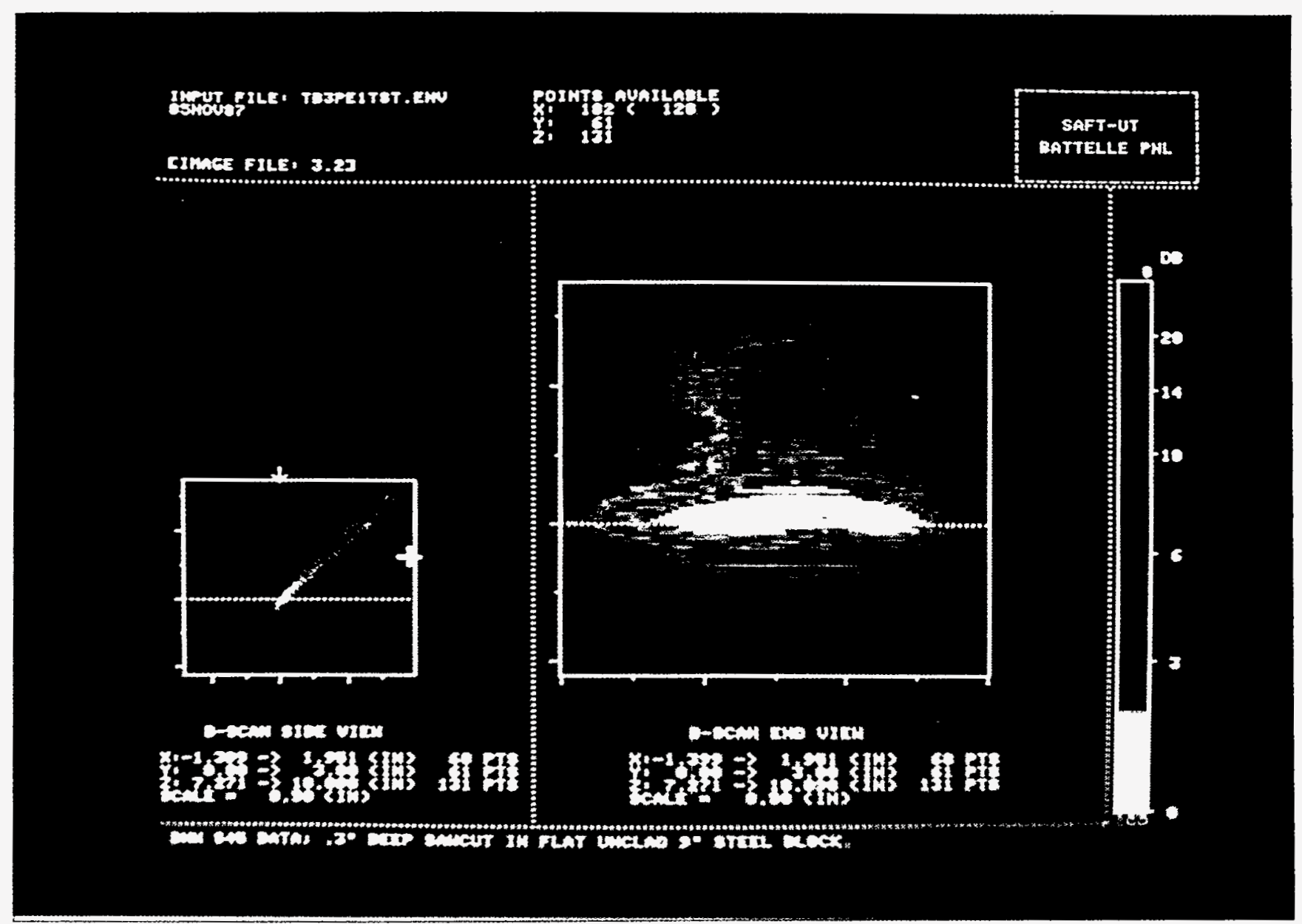

Figure 7.2. $45^{\circ}$ Shear-Wave Image of Corner-Trap Echo of 0.3-in.-deep (0.8-cm) Notch in 9-in.thick $(22.9-\mathrm{cm})$ Uncladded Calibration Block (PNL) 


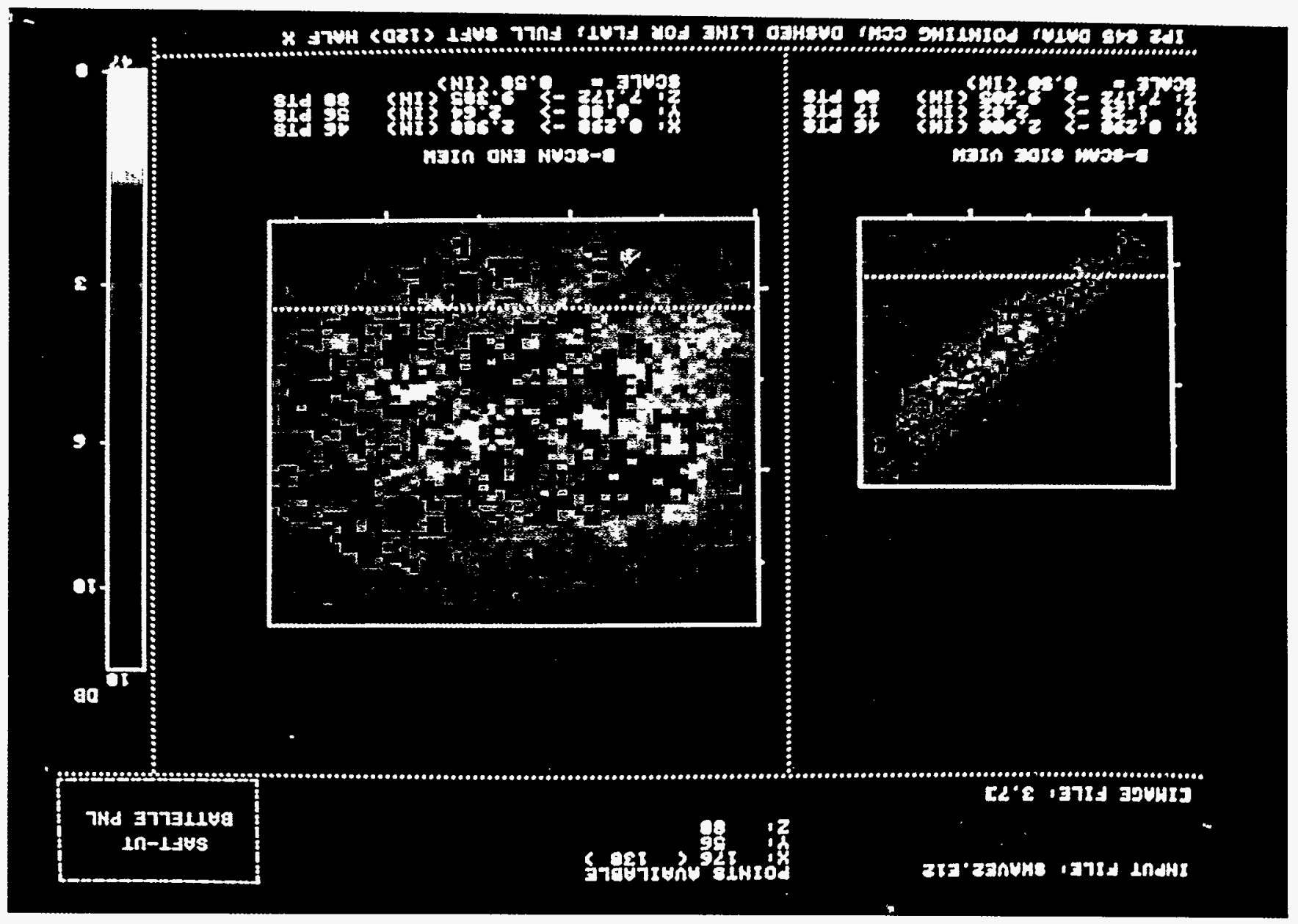




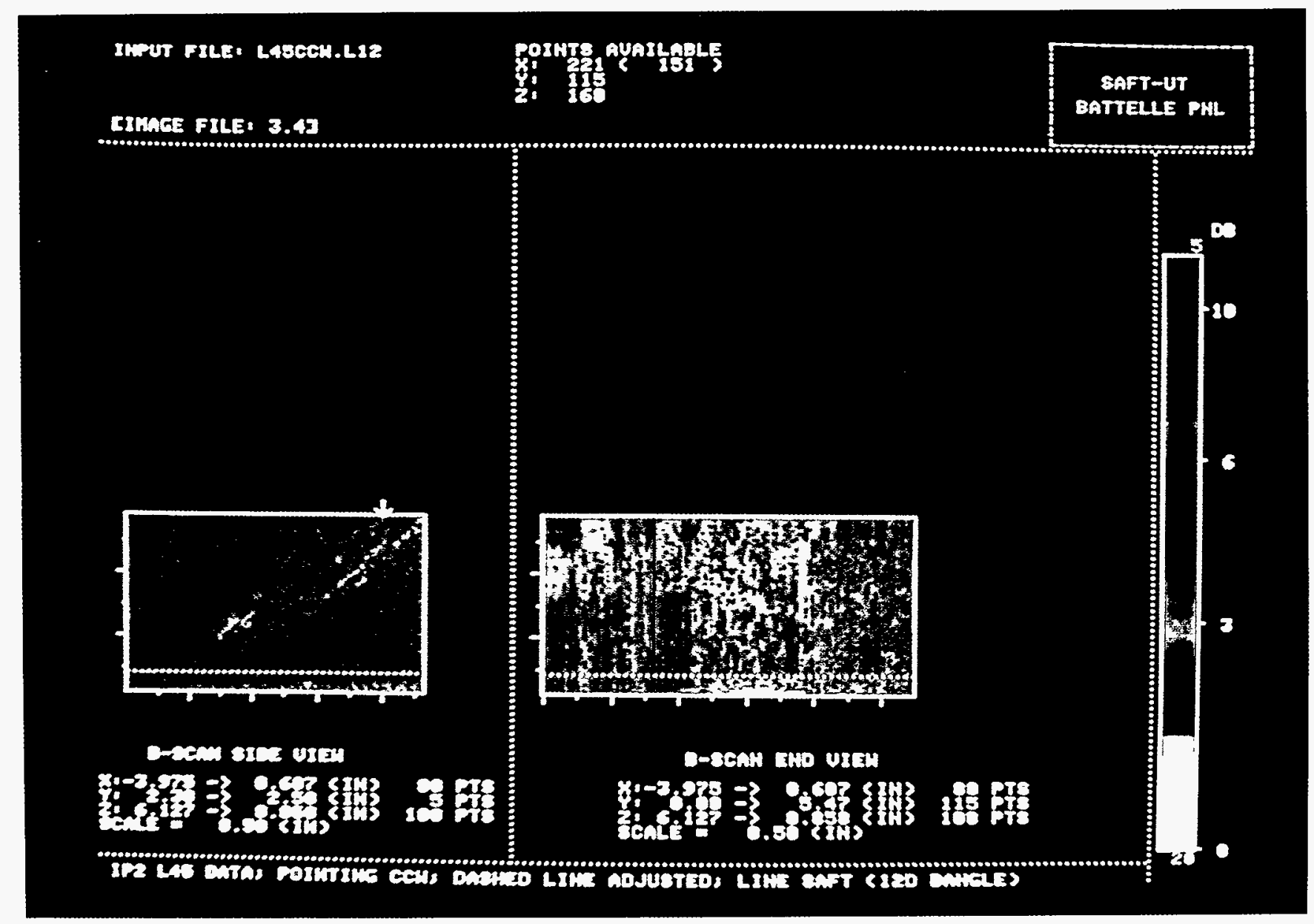

Figure 7.4. $45^{\circ}$ Longitudinal-Wave Image (3-D Processed) of the Vessel Indication at Indian Point Unit II 


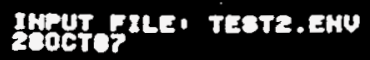

\section{CIMAGE FILE, 3.57}

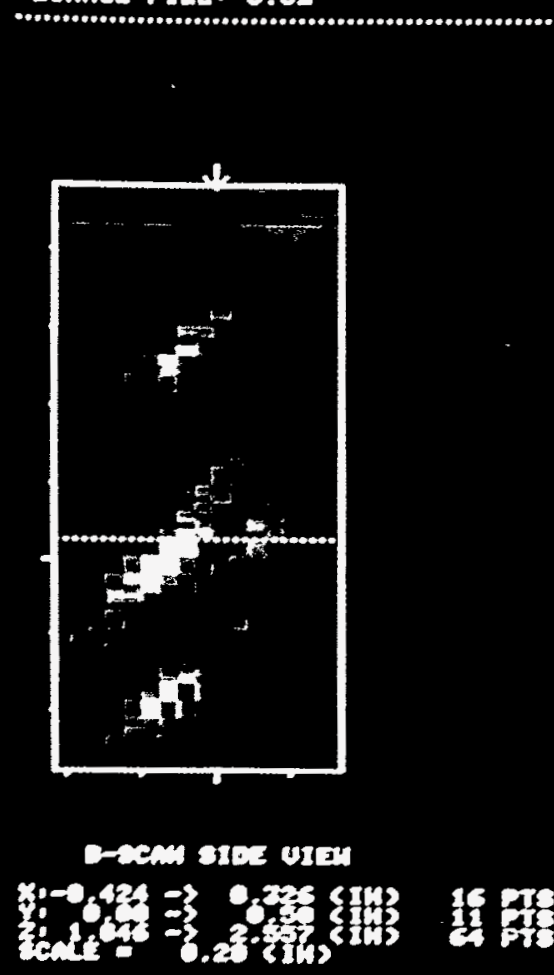

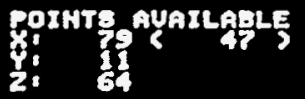

$$
28.24
$$

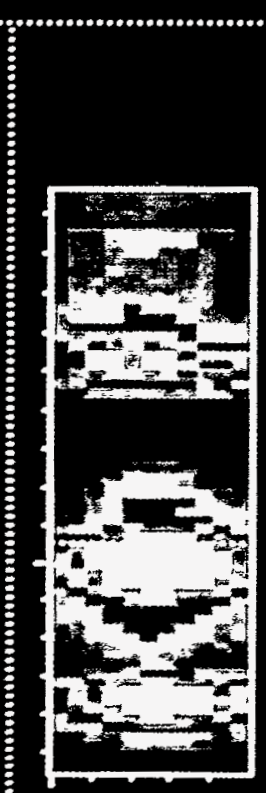

D-con ans uran

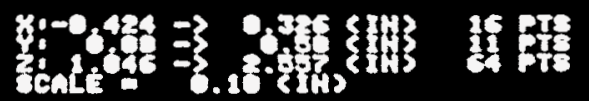

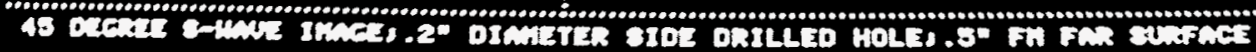

SAFT-UT

BATTELLE PHL

Figure 7.5. $45^{\circ}$ Shear-Wave Image (3-D Processed) of 0.2-in.-diameter $(0.5-\mathrm{cm})$ Side-Drilled Hole $0.5 \mathrm{in} .(1.3 \mathrm{~cm})$ from Far Surface of 2.0-in.-thick $(5.1-\mathrm{cm})$ Steel Block 
7.0 SAFT in the Field

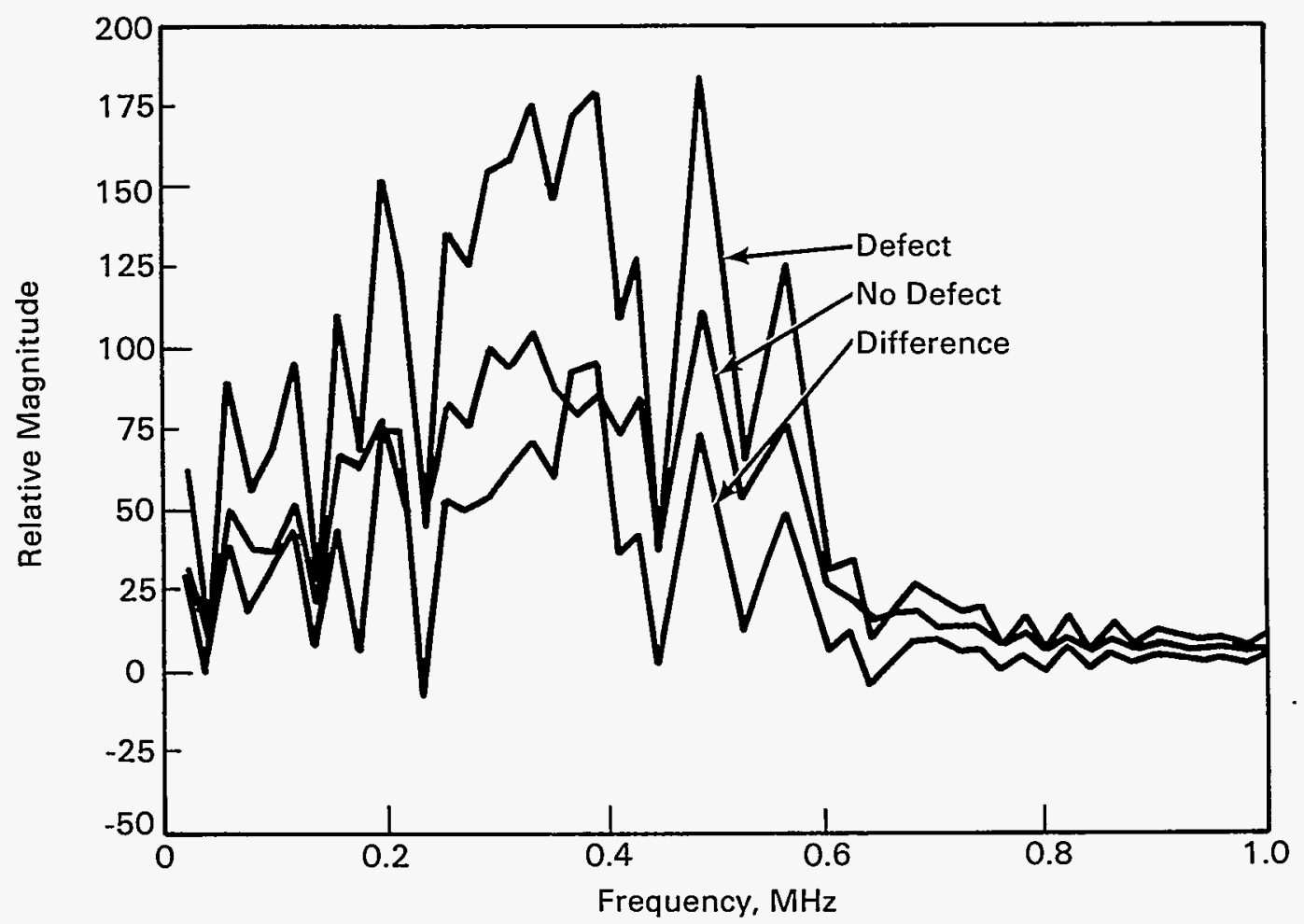

Figure 7.6. Composite Spectra Plot of Suspected Defect and Non-defect Areas of Data File TR9SH5D 

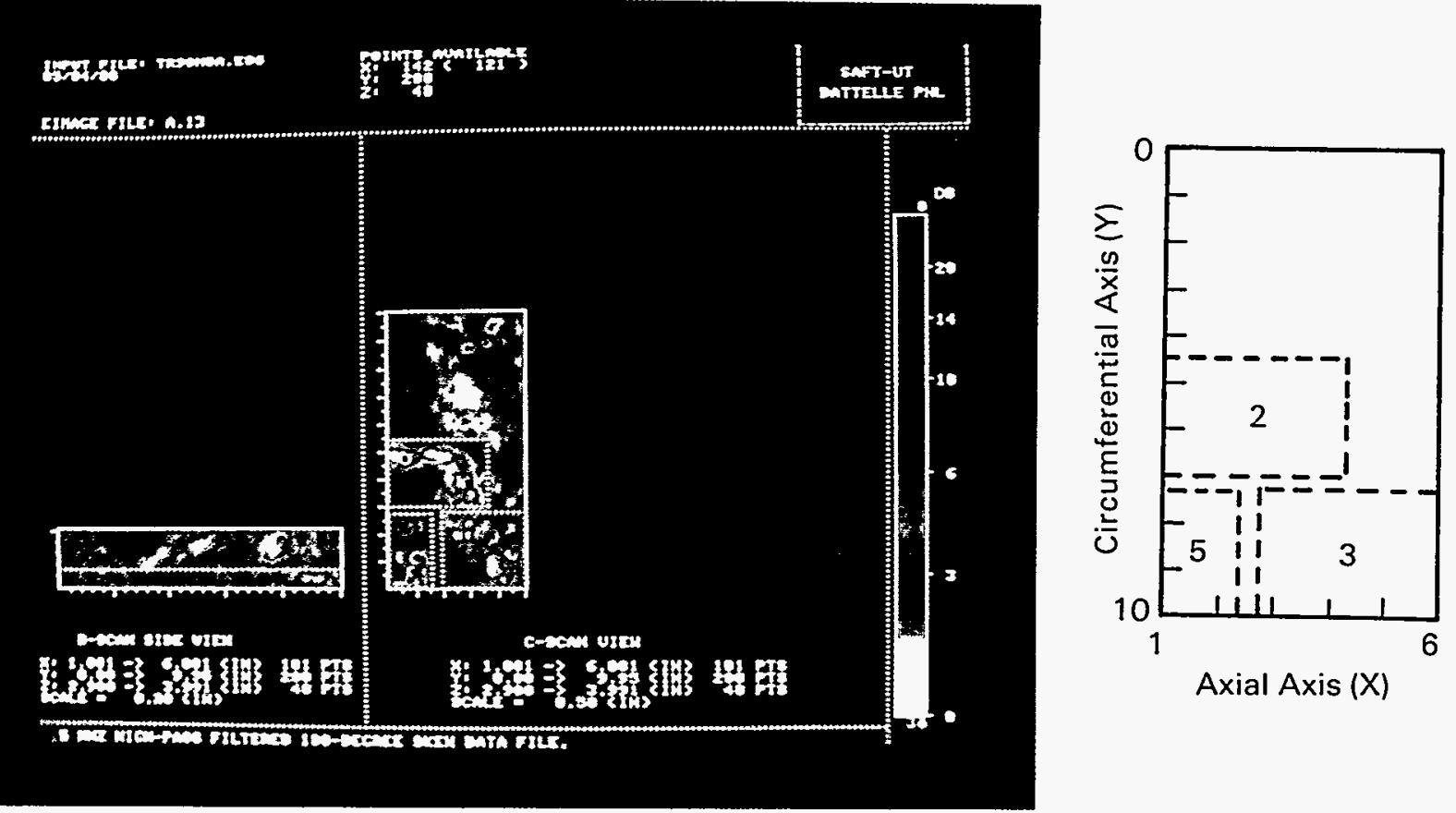

Figure 7.7(a). B-Scan Side View and C-Scan Top View of Data File TR9SH5A

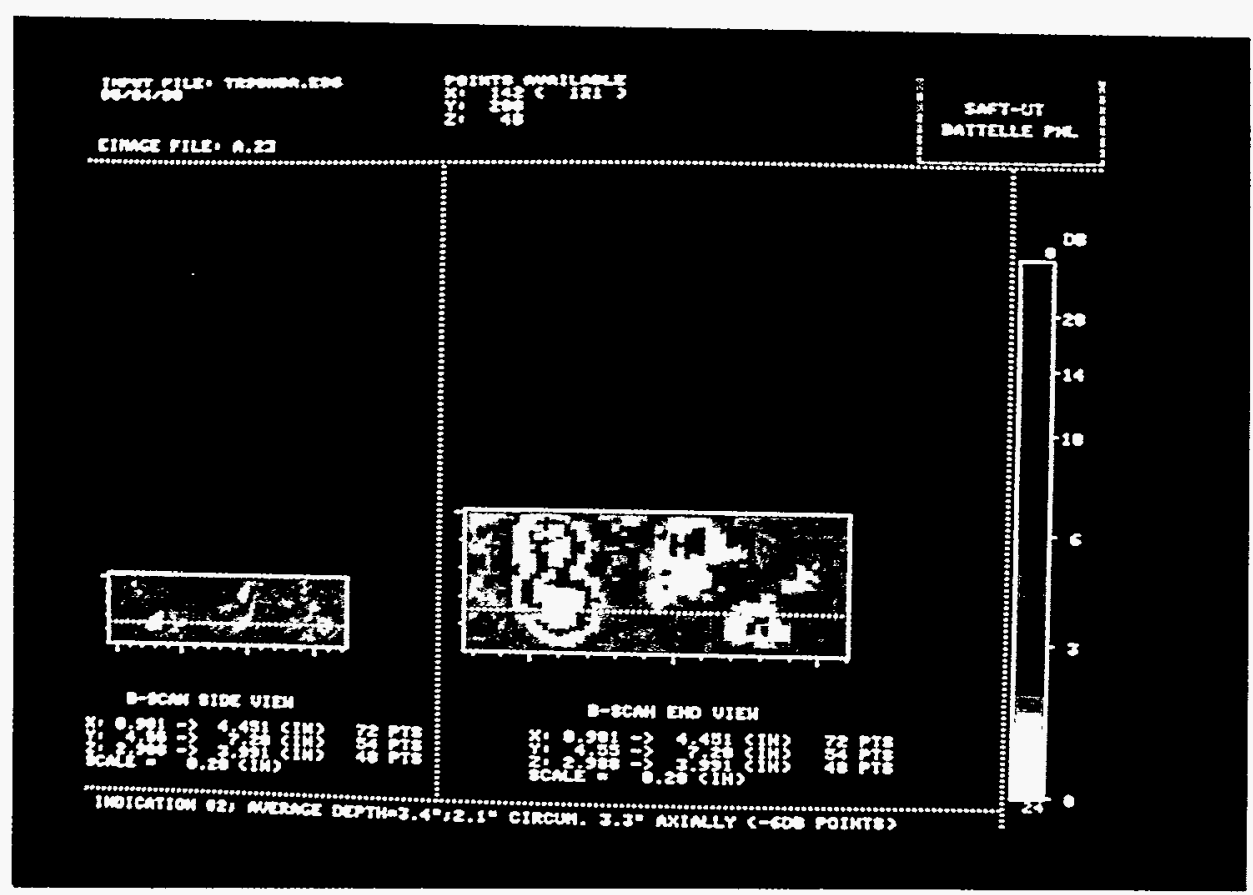

Figure 7.7(b). Expanded View of Indication \#2, TR9SH5A 


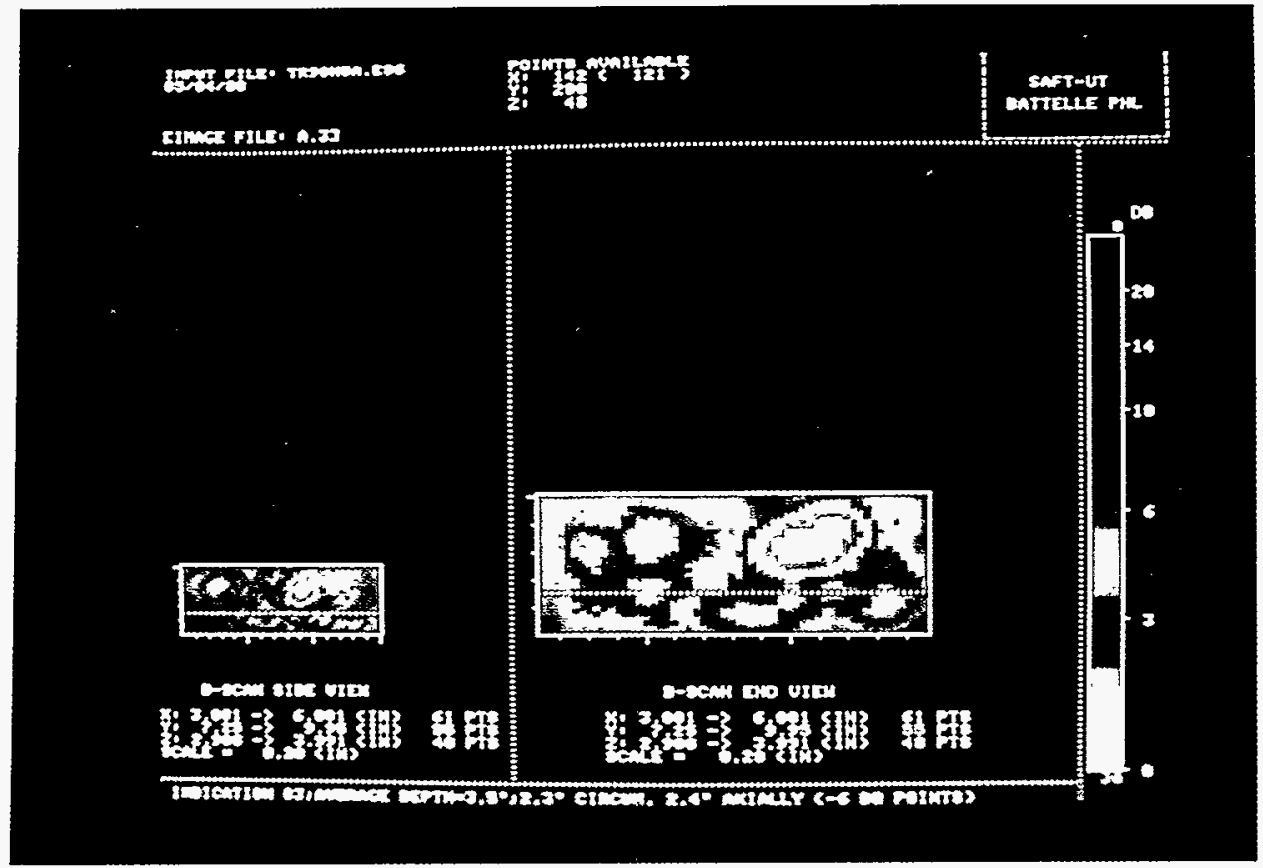

Figure 7.7(c). Expanded View of Indication \#3, TR9SH5A

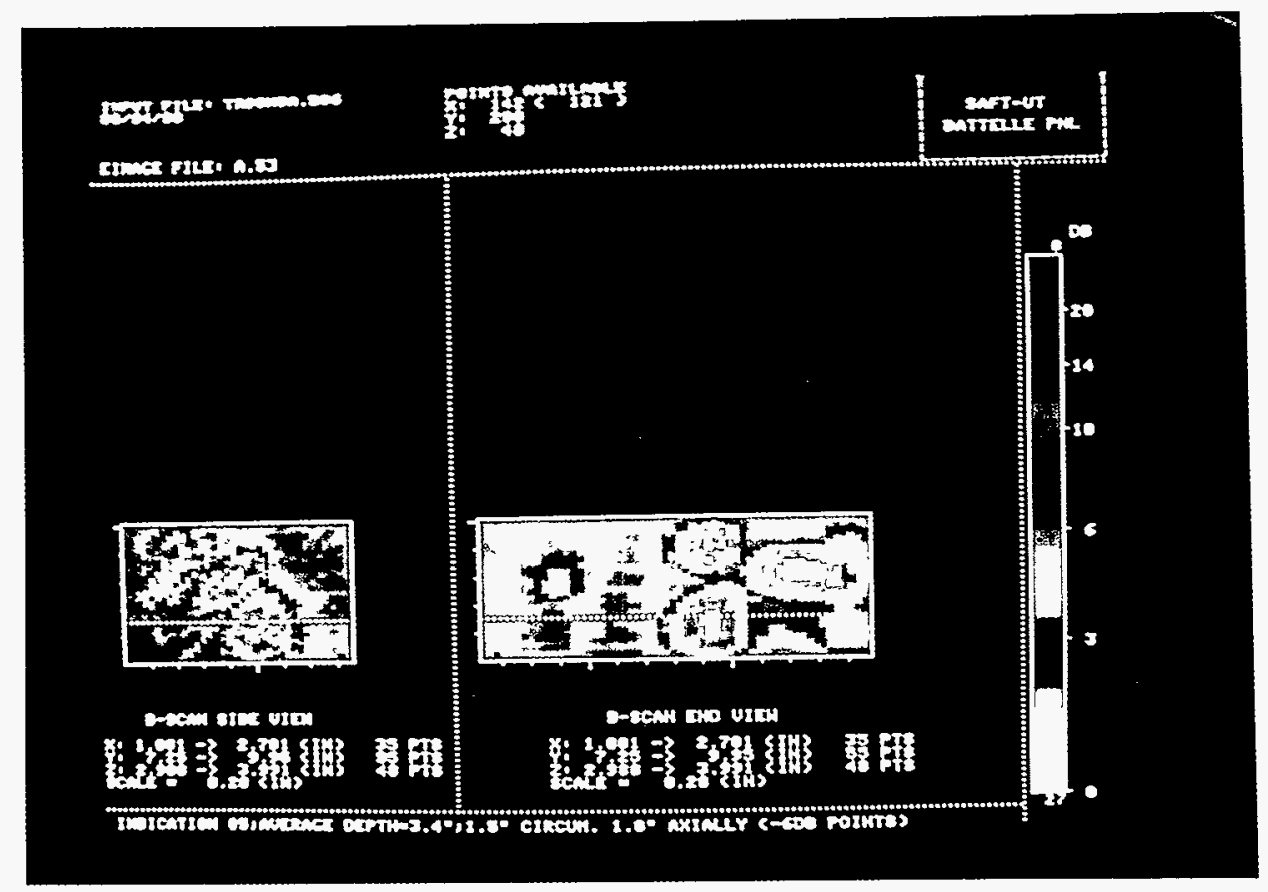

Figure 7.7(d). Expanded View of Indication \#5, TR9SH5A 

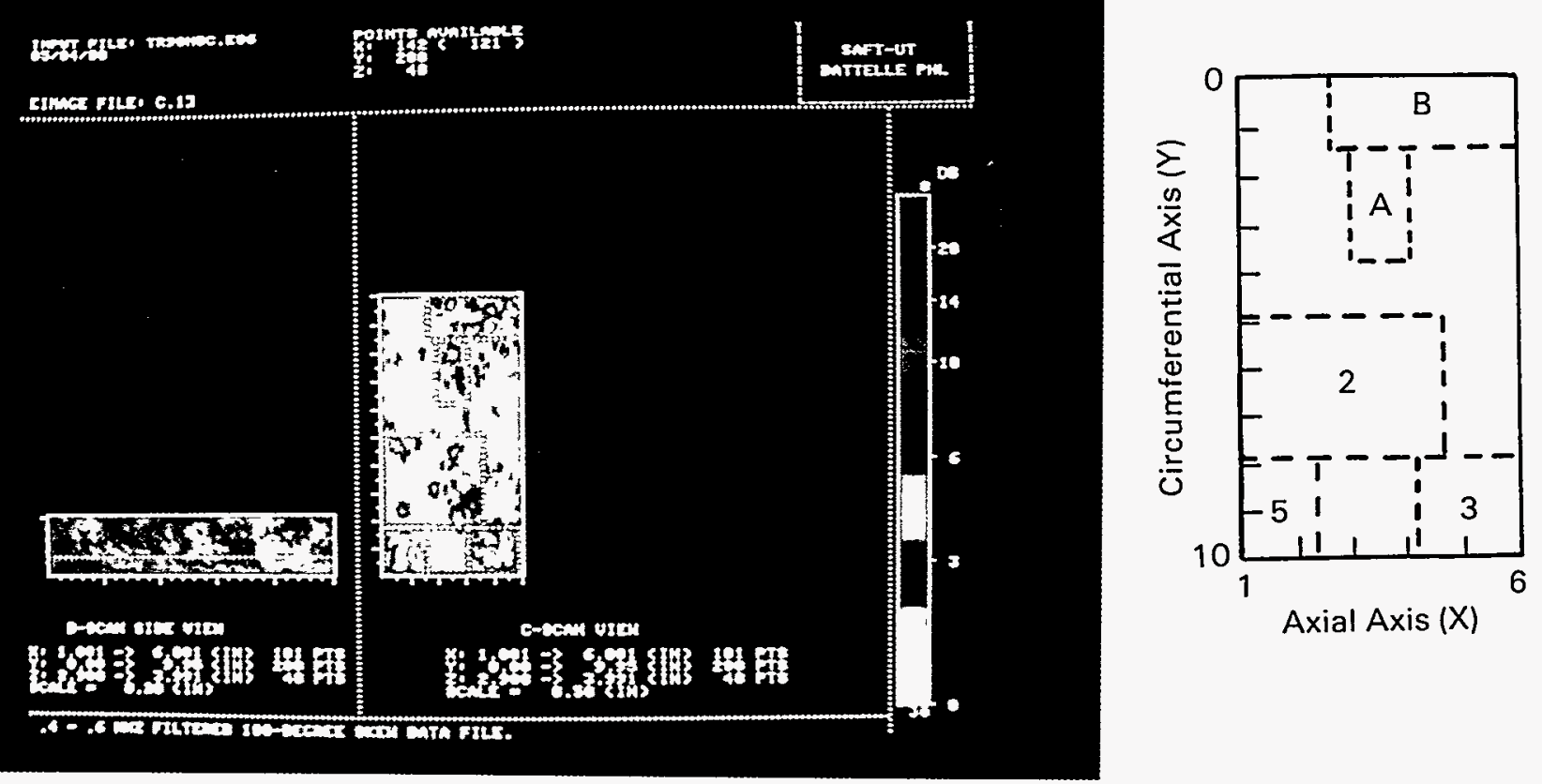

Figure 7.8(a). B-Scan Side View and C-Scan Top View of Data File TR9SH5C

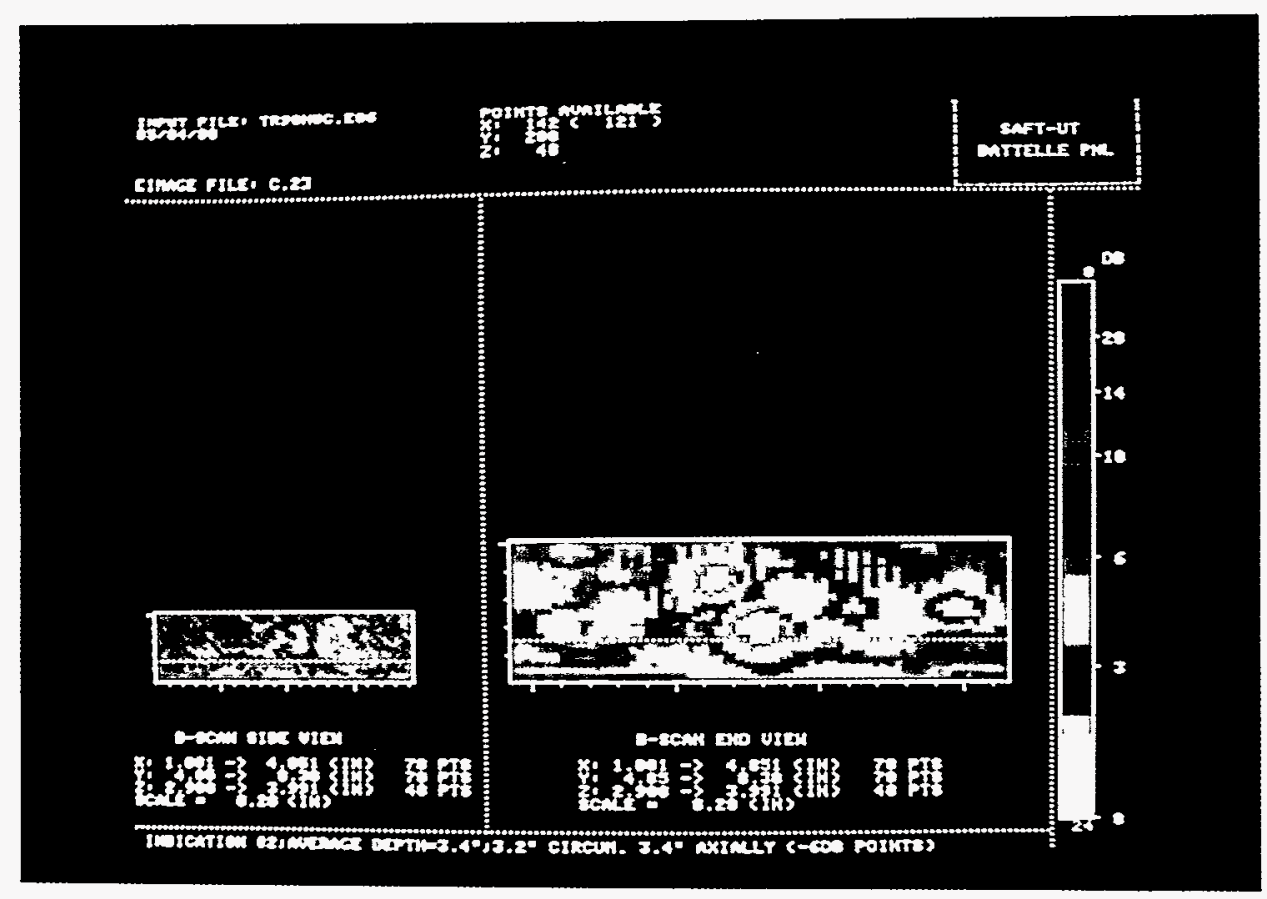

Figure 7.8(b). Expanded View of Indication \#2, TR9SH5C 


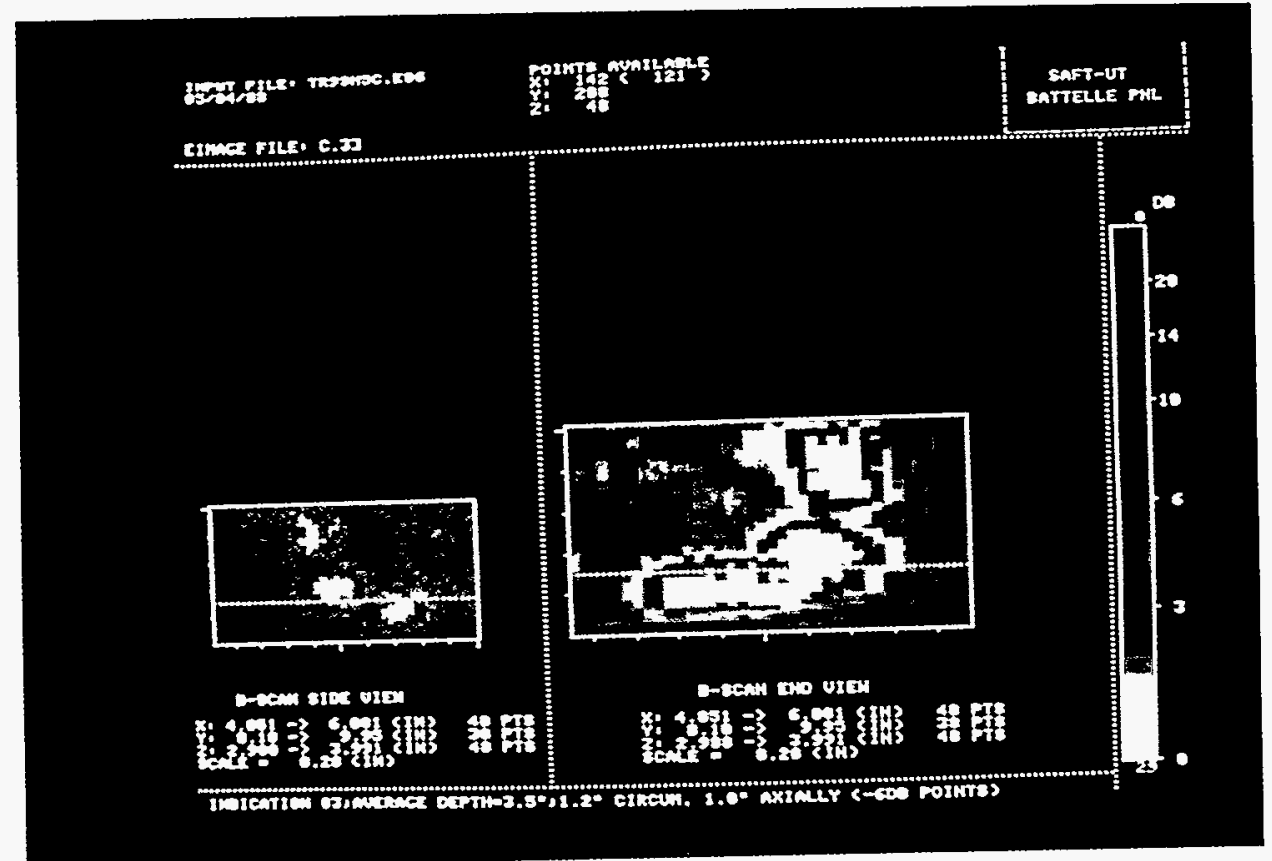

Figure 7.8(c). Expanded View of Indication \#3, TR9SH5C

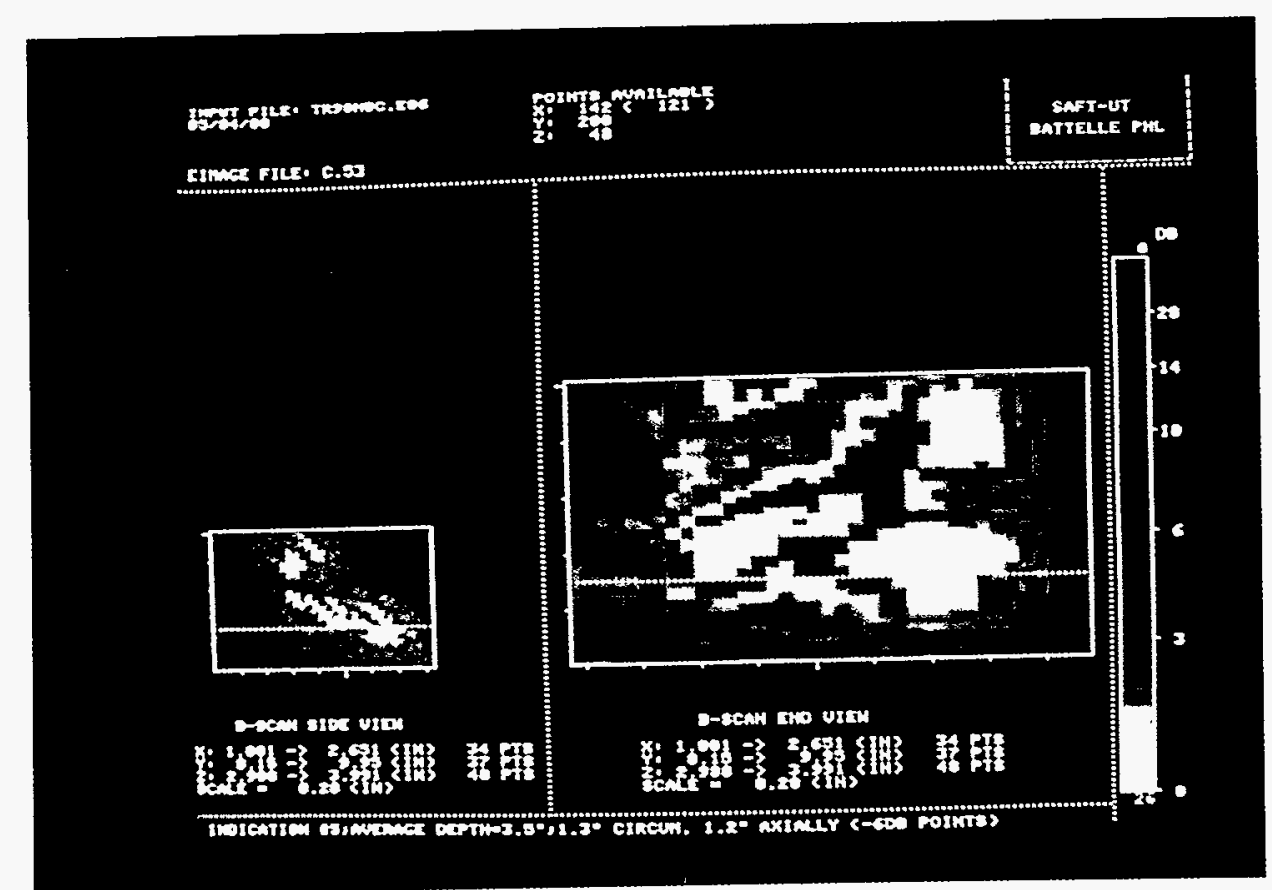

Figure 7.8(d). Expanded View of Indication \#5, TR9SH5C 


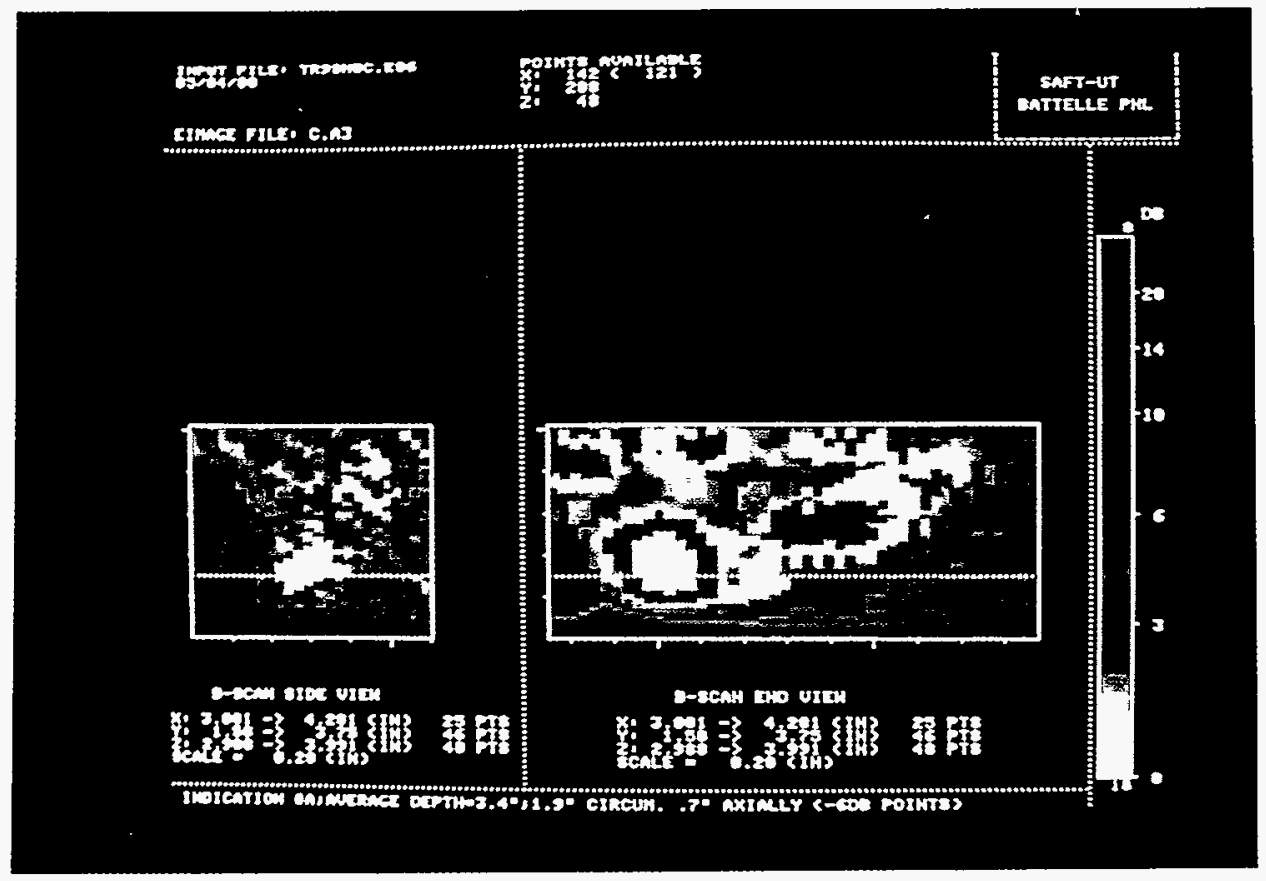

Figure 7.8(e). Expanded View of Indication A, TR9SH5C

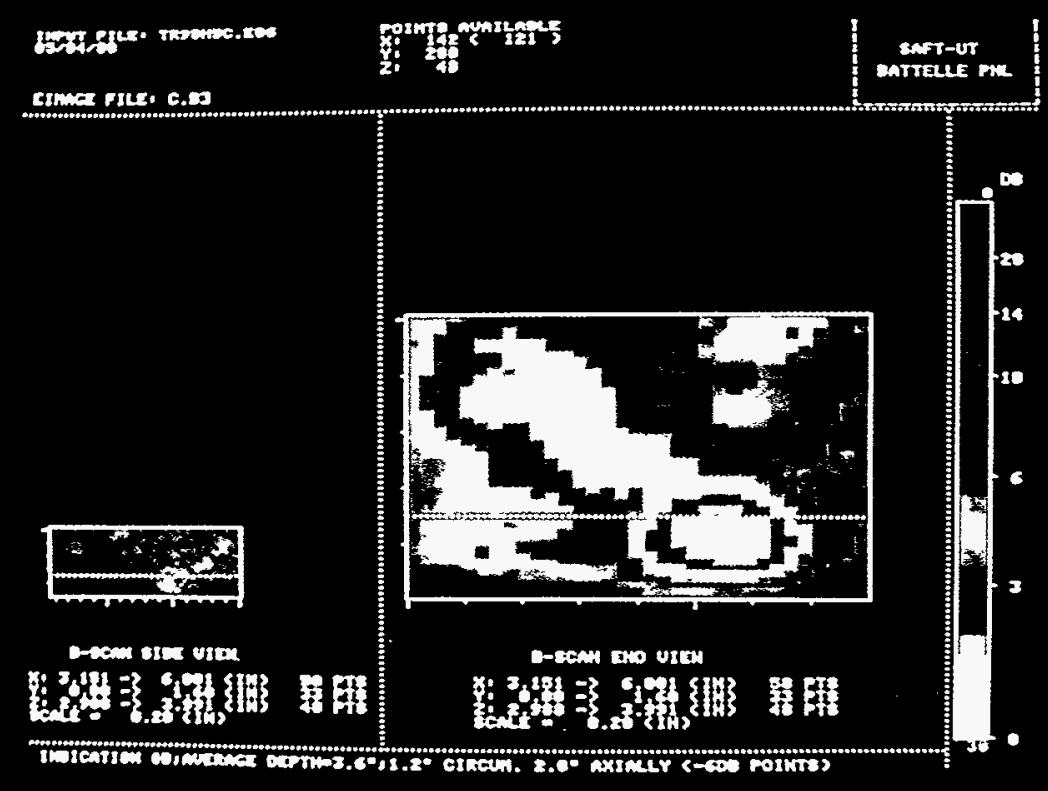

Figure 7.8(f). Expanded View of Indication B, TR9SH5C 

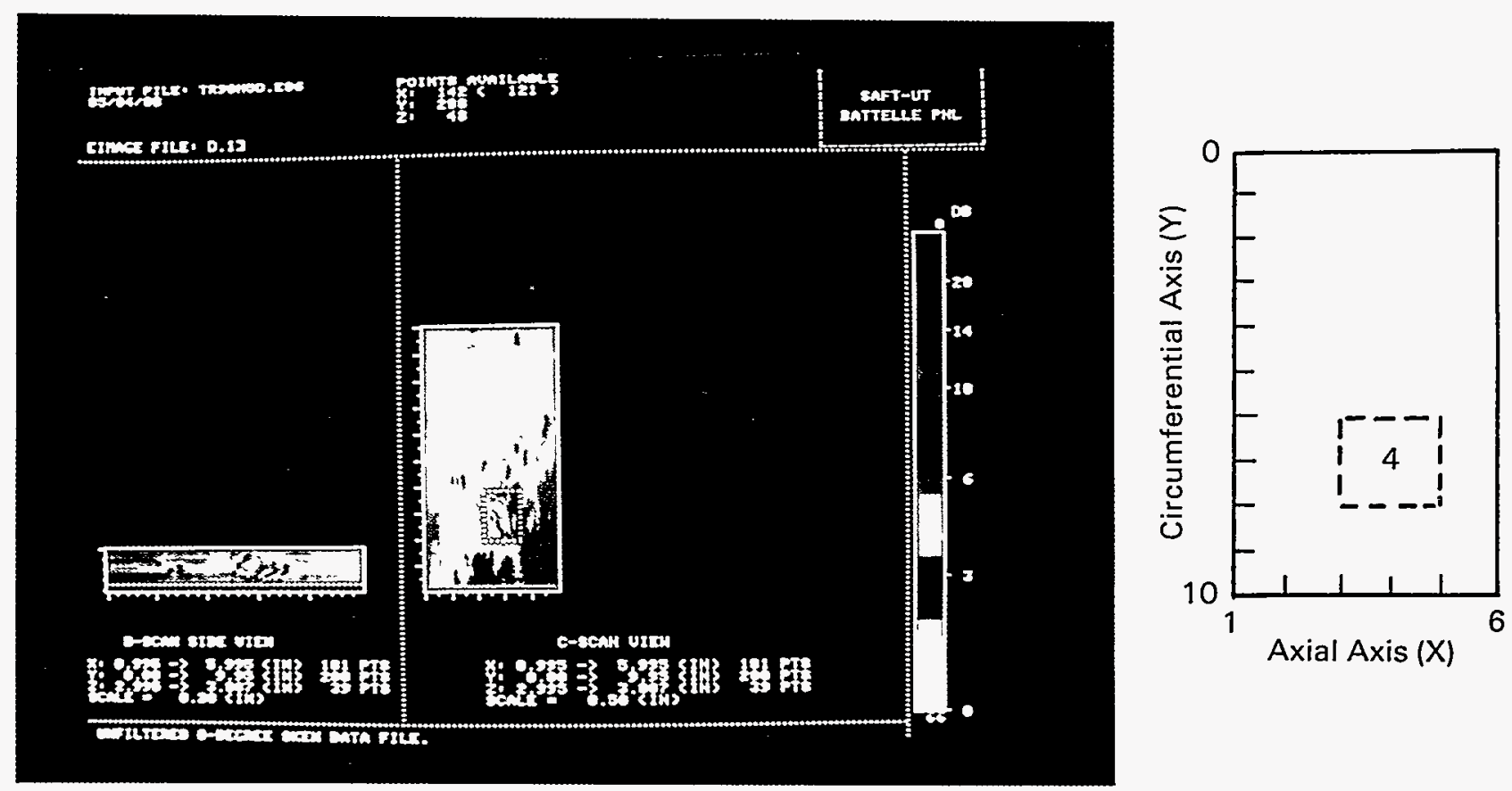

Figure 7.9(a). B-Scan Side View and C-Scan Top View of Data File TR9SH5D

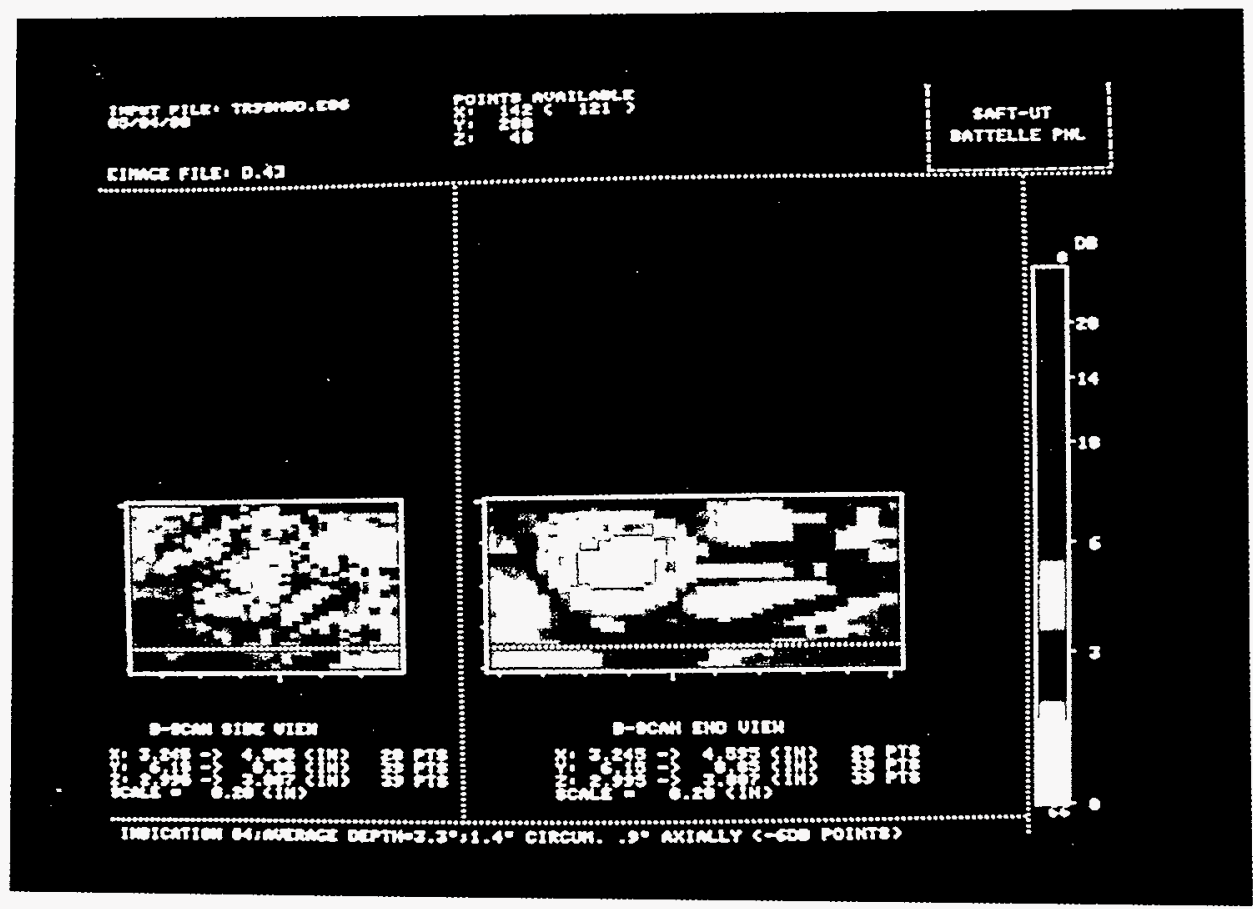

Figure 7.9(b). Expanded View of Indication \#4, TR9SH5D 


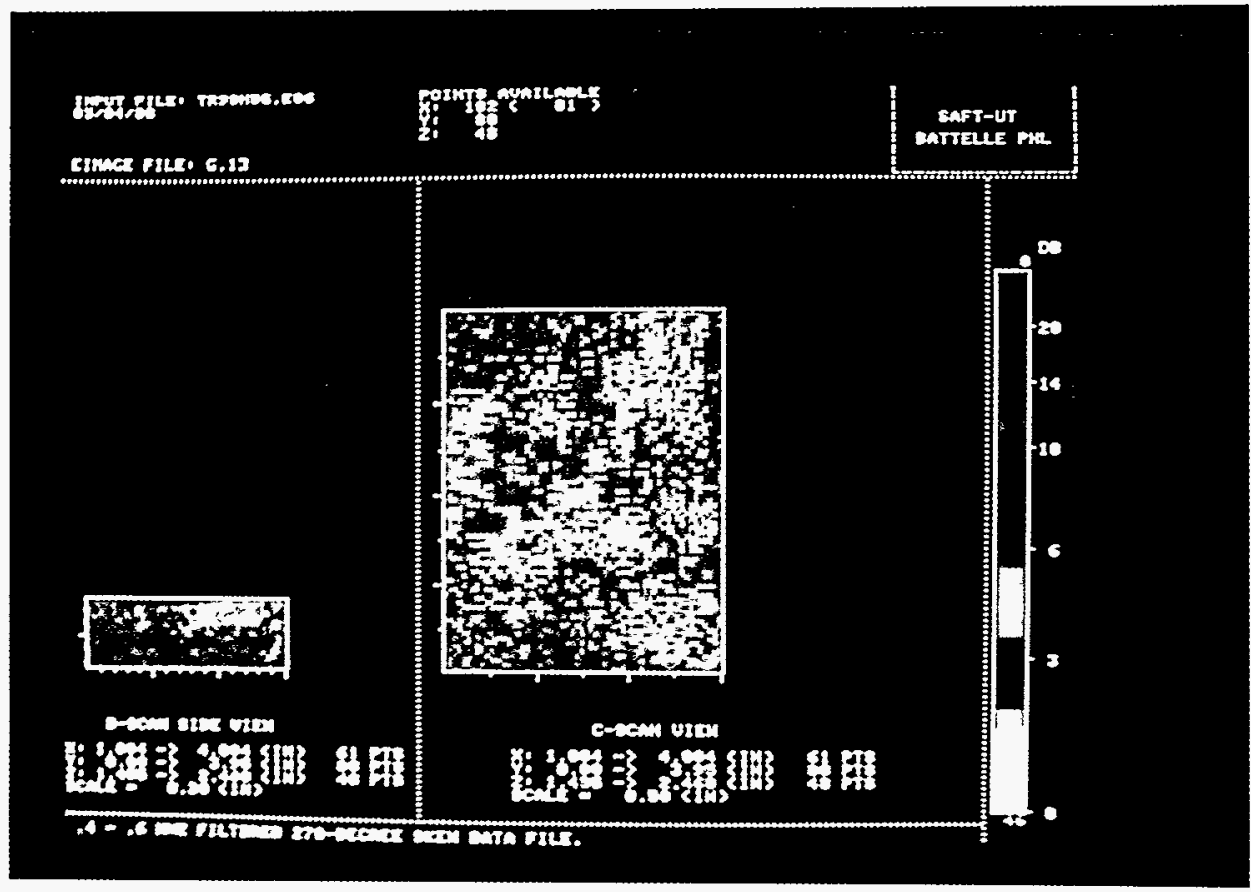

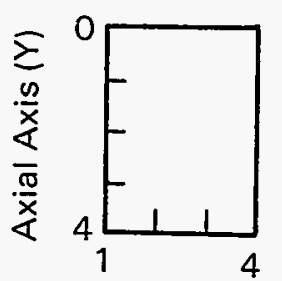

Circumferential Axis $(X)$

Figure 7.10. B-Scan Side View and C-Scan Top View of Data File TR9SH5G 


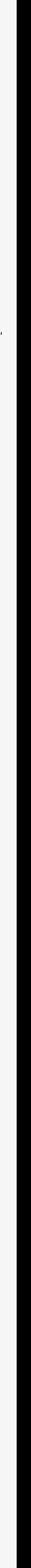




\subsection{SAFT Real-Time Processor Performance Analysis and Overview}

This section investigates the pulse-echo and tandem SAFT-UT configurations operating on the SAFT RealTime Processor (RTP). Performance analysis is focussed on determining the optimum hardware configuration with respect to the number of slave cards necessary to effectively operate the SAFT processor. Also, it is important to know which design component or group of components becomes the limiting factor with respect to performance. Also included in this section is an overview of the software implementation of the RTP.

Figure 8.1 shows a block diagram of the SAFT computational devices. The SAFT RTP acts as a peripheral to the Digital Equipment Corporation (DEC) VAX host computer and is linked to the host via a bidirectional, 16-bit parallel interface. The VAX computer retrieves the data file from disk storage and transfers this data over the parallel interface directly to the SAFT processor global memory. The input data is distributed to the slave cards, which then perform the partial summations and export the partial sums back to the global memory. The final result is then transported back to the host VAX, which stores it in an output disk file.

Four basic areas could potentially limit SAFT processing system performance. First, the host disk transfer rate is important. Although not truly a component in the measurement of the SAFT processor performance as will be shown later, it can and does affect the overall SAFT processing rate.

The second system performance component to consider is the parallel interface data transfer rate. A DR11W type of interface is used with a transfer rate of about $100 \mathrm{~kb} / \mathrm{s}$. This should not prove to be a bottleneck.

A third, and probably most significant, component in the processing performance is the VME bus contention. All system components have their focus on the global system memory, which resides on the VME bus. All input data comes in and is distributed from the global memory. All of the slave processors acquire their input data from the global memory and place the resultant partial sums back into the global memory. The resultant data array is then sent back to the host computer from the global memory. Each of these operations and a variety of other overhead functions require access to the global memory via the VME bus.
The final system performance component to be considered is the speed of the slave processors themselves. This becomes significant in larger data files where many values are summed for each resulting element.

\subsection{Pulse-Echo Configuration}

Two data files were used to analyze the performance of the SAFT RTP on the SAFT-UT field system in the single-transducer pulse-echo mode of operation. The first is a pulse-echo data file collected on a 26 -in.-diameter $(66-\mathrm{cm})$ Schedule 80 pipe at the PNL Materials Reliability Center. This is a 1.25 -in.-thick (3.2-cm) stainless steel pipe. The data was sampled in 0.050-in. $(0.13-\mathrm{cm})$ increments in the $\mathrm{X}$ and $\mathrm{Y}$ lateral directions. The temporal sampling was $100 \mathrm{~ns}$, from the near surface to $2.0 \mathrm{in}$. $(5.1 \mathrm{~cm})$ of metal path (326 sample points). A $2.25-\mathrm{MHz}, 0.25$-in.-diameter $(0.64-\mathrm{cm})$ transducer was implemented in the pulse-echo contact mode. A wedge was selected for a shear-mode center ray material refraction angle of $40^{\circ}$. Processing was performed with a $12.5^{\circ}$ beam angle established in the synthetic aperture. The defined aperture, then, required that a single point be summed at the top of the aperture cone and 83 points be summed to form the result at the bottom of the cone.

The second data file is a deep-section data set collected on the PISC-II carbon steel block Number 2. This block was 10.4 in. thick $(26.4 \mathrm{~cm})$. The data was sampled in 0.10-in. (0.25-cm) increments in the $X$ and $Y$ directions. The temporal sampling was 200 ns between data points, starting 5.5 in. $(14 \mathrm{~cm})$ along the sound path and ending 16 in. $(48.6 \mathrm{~cm})$ along the sound path in the material (854 sample points). The transducer used had a center frequency of $2.25 \mathrm{MHz}$ with a diameter of $0.75 \mathrm{in} .(1.9 \mathrm{~cm})$ and a focal distance of $3.0 \mathrm{in}$. $(7.6 \mathrm{~cm})$. The transducer was placed in a water column such that a $40.6^{\circ}$ shear-mode refraction angle was achieved for the center ray path. Processing was performed with a $12.5^{\circ}$ beam angle for the synthetic aperture, which established that there are 145 points to be summed at the top of the aperture cone and 1285 points to be summed to compute the result at the bottom of the cone.

A series of performance tests was run using these two data files as inputs. The SAFT-UT field system VAX $11 / 730$ was used for the host computer, which includes 
a DEC R80 disk drive for data storage. The focus of the tests was to determine the effects of adding slave processors, to determine the benefit of specific processing options, and to observe the effects of the host computer's disk output on the processing performance. The graphs shown in Figures 8.2 through 8.5 relate to the pipe data file described earlier. Each figure shows the processing speed of the overall system including the VAX 11/730 disk write (dashed line) and the performance when the disk input/output (I/O) is completely removed from the software during processing time.

Figure 8.2 shows the performance with selective processing disabled and with the output record size exactly the same length as the input record size. With disk writes, one can see that the performance is completely saturated and that the rate of processing is totally determined by the host disk system. More interesting is the solid line graph showing the response when the host is free from the disk duties. One can see that parallel processing saturation occurs after installing more than five slaves in the system. Notice that a speed improvement of a factor of 2.1 is achieved by increasing the number of slaves from one to five. After five slave cards, there is no benefit in performance for this data set.

Figure 8.3 shows the curves relating to selective processing disabled and an output record size compression factor of 5. Thus, the record being transmitted back to the host computer and stored in the disk file is five times smaller than the input data record length. This compression is performed after envelope detection, when the frequency components of the signal are much reduced; consequently, the sampling requirements of the signal are relaxed. This relates to about one data point per wavelength on the theoretical resolution limits. Furthermore, the data files are almost never displayed one-to-one because there are many more points than there are display pixels. Notice the level at which performance saturation of the dashed line has increased as compared to Figure 8.2. This increase is directly attributable to the fact that the amount of data-handling is reduced and therefore the disk I/O. becomes less significant. As expected, the solid line graph (no host disk activity) is identical to that shown in Figure 8.2.

Figure 8.4 shows the graphs related to a $-20-\mathrm{dB}$ threshold level in the selective processing option and no output record size compression selected. The chosen threshold level resulted in $58 \%$ of the data points being fully processed for this file. Remember that the other $42 \%$ of the data points are retained but just not processed. Notice that the dashed curve is identical to that shown in Figure 8.2. This is as expected, because the disk output rate dominates the performance once again. The solid line saturates much quicker, however, than that shown in Figure 8.2. This is from the large reduction in summations due to the selective processing algorithm. Performance saturation occurs at three slave processors for this file.

Figure 8.5 show the results of combining the options of Figures 8.3 and 8.4. In this case, -20-dB selective processing was chosen and an output data record compression factor of 5 was selected. As expected, the solid line (no host disk overhead) is identical to that shown in Figure 8.4, and the dashed line (disk output included) matches that shown in Figure 8.3.

The results for performing these tests on the larger PISC-II data set are shown in Figures 8.6 through 8.9. Because of the aperture size of this data set, the processing requires that at least three slaves be incorporated in this test (adequate slave memory to store the entire aperture). Figure 8.6 represents the performance observed with selective processing disabled and no output record compression chosen. The dashed line (host disk activity) saturates at about the 5.3-A-scans/s level, which corresponds to six slave processors. The solid line (no host disk activity) does not experience saturation; rather, a generally linear shape occurs. If one extrapolates the line back to a fictitious value for a single slave processor (1.7 A-scans/s), then the addition of the number of slave cards to ten achieves a speed increase of a factor of 3.9.

Figure 8.7 shows the graphs that result in choosing no selective processing and selecting an output record size compression factor of 5 . The results are identical to those in Figure 8.6, except the dashed line (with host disk activity) shows no saturation. In this case, the disk activity has no limiting effect on the processing performance and is expected for large A-scans that require extensive processing.

The results of choosing $-20-\mathrm{dB}$ selective processing with no output compression are shown in Figure 8.8. The selective processing option in this data set dramatically affects the amount of computations, because only $1 \%$ of 
the data points are fully processed. This is due to the high signal-to-noise level of the data set and is typical of pressure vessel steels. Both curves immediately achieve saturation levels and remain flat. The performance of both curves is dependent totally on the rate at which the data is transferred from device to device. The limit for the dashed line is the disk transfer rate of the host computer. The limit for the solid line is the throughput of the SAFT processor.

Figure 8.9 exhibits characteristics similar to those of Figure 8.8. The output record size has been compressed by a factor of 5 so the amount of data to be transferred is correspondingly reduced.

This performance analysis has been helpful in determining the benefit of adding slave processors and isolating bottlenecks with respect to data throughput. It is apparent, for most applications, that five slaves are probably sufficient. This is especially true if selective processing is used. Another important factor for system performance is the host disk I/O speed. It is important to select a fast disk system when initially specifying the SAFT host computer for real-time analysis.

A very large data set was processed on the SAFT RTP to observe the corresponding execution performance. This file is a finely sampled scan of a resolution pattern in an aluminum block. A $2.25-\mathrm{MHz}$ transducer was used with a diameter of 0.25 in. $(0.6 \mathrm{~cm})$. A wedge was selected to provide shear mode illumination with a refraction angle of $43.7^{\circ}$. The $X$ and $Y$ lateral sample spacing was 0.025 in. $(0.6 \mathrm{~cm})(\lambda / 2)$, and the temporal sampling was $20 \mathrm{MHz}(0.05 \mu \mathrm{sec}$ or about $\lambda / 10)$. Digitization began 2 in. $(5.1 \mathrm{~cm})$ into the material along the center ray sound path and continued until 4.5 in. $(11.4 \mathrm{~cm})$. There were 806 data points in each A-scan record, and there was a total of $14,400 \mathrm{~A}$-scan records in the data set.

Processing was performed on this data set with a synthetic aperture beam angle of $18.0^{\circ}$. With this angle selected, the number of off-center A-scans to sum at the top of the aperture $(2.0 \mathrm{in}$. [5.1 cm] into the material) is 707; the number to be summed at the deepest part of the defined aperture $(4.5 \mathrm{in} .[11.4 \mathrm{~cm}]$ into the material) is 3617 .
This data set required at least nine slave processors because of the immensity of the aperture size. The global memory had to be expanded to 3 Mbytes to perform this processing. Selective processing was not chosen, and an output record compression factor of 5 was implemented for this test. For this case, the host computer was the SAFT VAX/780.

The SAFT processor with ten slave processors successfully processed this data set with statistics that are quite impressive. The total elapsed processing time was 64.2 minutes, but the total central processing unit (CPU) time -- the time required for the host computer to do work - was 6.8 minutes. This means that the bulk of the processing is exported to the SAFT processor, and that comparatively very little is required of the host computer. This is the key to the success of the SAFT processor.

This file has not previously been processed without choosing selective processing to reduce the number of - summations required. Processing of this data file with these parameters was initiated on the VAX 11/780. However, practical completion of processing was not possible. The program was submitted into a special high-priority batch queue. After a connect time of 2.6 days and an elapsed CPU time of $18.5 \mathrm{~h}$, the processing was only $7.1 \%$ complete so it was terminated. It would have taken approximately 36 days and 260 CPU hours to complete. This is a reduction in processing time by a factor of 250 ! The primary reason for the long time in execution is the number of page faults that occurred during processing. There were $3 \times 10^{6}$ page faults at the point of termination, with approximately $42.2 \times 10^{6}$ page faults required to complete the processing. The VAX $11 / 780$ has only $4.75 \mathrm{Mb}$ of memory; thus, with a file this large and with an aperture of this size, it was spending most of the time in a VMS page fault waitstate rather than actually computing the result.

As stated previously, an attractive feature of the SAFT RTP architecture is that it exports the CPU-intensive labor required to execute the SAFT algorithm, thus dramatically increasing the speed of execution. This is especially true for the very large data files as experienced here. 


\subsection{TSAFT/TSAFT-2 Configuration Performance}

Three test files were used to evaluate speed improvements expected to be achieved utilizing the SAFT RTP for TSAFT and TSAFT-2 configuration files. The first data set was collected in the TSAFT configuration on a 0.585 -in.-thick $(1.5-\mathrm{cm})$ stainless steel coupon with a 0.3 in.-deep $(0.8-\mathrm{cm})$ vertical sawcut in the far surface.

This file is a familiar example that has commonly been used to evaluate the software performance. The second file is a TSAFT data set collected on a 1.0-in.-thick (2.5$\mathrm{cm}$ ) aluminum coupon with two opposing co-planar vertical sawcuts placed in it. File three is a TSAFT-2 data set based on the same aluminum coupon as the second file.

Table 8.1 shows the execution time utilizing the VAX $11 / 780$ with $-20-\mathrm{dB}$ selective processing implemented. Table 8.2 shows the performance of the SAFT processor with $-20-\mathrm{dB}$ selective processing implemented during the calculation. Performance improvement factors of 9.0, 12.0, and 9.6, respectively, are realized by implementing the SAFT processor on these TSAFT and TSAFT-2 files.

Table 8.3 shows the respective performance using the SAFT processor without implementing selective processing. This means that every point is processed regardless of the contents of the data elements. Notice the extended processing times and the total number of points that are summed. The processing time for File 2 is more than $20 \mathrm{~h}$, for nearly $2 \times 10^{9}$ points. Although this is a considerable amount of time, only three CPU minutes were required of the VAX host computer throughout this processing. In other words, the total workload was transported to the peripheral SAFT processor. Executing this solely on the VAX 11/780 would be impractical since it would take approximately 8.6 days to complete the processing.

Table 8.1. VAX 11/780 TSAFT/TSAFT-2 Executive Time (-20-dB selective processing implemented)

\begin{tabular}{|c|c|c|c|c|}
\hline 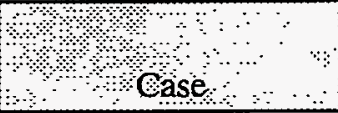 & Total trme & Noof A-Scans & No of Points & $\begin{array}{c}\text { Rate } \\
\text { A-Scans/sec. }\end{array}$ \\
\hline FLLE 1 & 51.5 & 2880 & $6.0 \times 10^{6}$ & 0.932 \\
\hline FLEE 2 & 365.3 & 12800 & $36.2 \times 10^{6}$ & 0.584 \\
\hline FULE 3 & 227.4 & 8640 & $30.5 \times 10^{6}$ & 0.633 \\
\hline
\end{tabular}

Table 8.2. SAFT-UT Real-Time Processor TSAFT/TSAFT-2 Executive Time (-20-dB selective processing implemented)

\begin{tabular}{|c|c|c|c|c|}
\hline Case & $\begin{array}{l}\text { Totäl Time, } \\
\text { munutes }\end{array}$ & No of A-Scans & $\begin{array}{l}\text { No of Points } \\
\text { Processed }\end{array}$ & A-Scans/sec. \\
\hline FILE 1 & 5.7 & 2880 & $6.0 \times 10^{6}$ & 8.4 \\
\hline FILE 2 & 30.4 & 12800 & $155.5 \times 10^{6}$ & 7.0 \\
\hline FILE 3 & 23.5 & 8640 & $27.2 \times 10^{6}$ & 6.1 \\
\hline
\end{tabular}


Table 8.3. SAFT-UT Real-Time Processor TSAFT/TSAFT-2 Executive Time

(selective processing disabled)

\begin{tabular}{|c|c|c|c|c|}
\hline Case & $\begin{array}{c}\text { Totall Time, } \\
\text { munutes } \\
\end{array}$ & $\begin{array}{c}0 . \\
\text { No. of } A-S c a n s\end{array}$ & $\begin{array}{l}\text { No. of Points } \\
\text { Processed }\end{array}$ & $\begin{array}{c}\text { Rate } \\
\text { A-Scans } / \mathrm{sec}\end{array}$ \\
\hline FILE 1 & 71.7 & 2880 & $120.1 \times 10^{6}$ & 0.669 \\
\hline FILE 2 & 1242.1 & 12800 & $1955.6 \times 10^{6}$ & 0.172 \\
\hline FILE 3 & 379.8 & 8640 & $2498.7 \times 10^{6}$ & 0.379 \\
\hline
\end{tabular}

Three variables are considered to be interesting with respect to further investigation of the system's performance. The first is the number of slave processors enabled during processing. This would affect the cost of implementing the SAFT processor. The second is the effect of implementing selective processing to reduce the total number of summations necessary to produce the final image. The third variable is output record size compression. This takes advantage of the low-frequency content of the envelope-detected result to reduce the size of the resulting A-scan, consequently reducing the amount of data-handling.

In the first set of tests run, $100 \%$ of the data files were processed. The output record size was not compressed. Figures $8.10,8.11$, and 8.12 show the graphs relating to these tests. In each graph, performance (A-scans/s) is plotted as a function of the number of slave processors enabled. Notice that each plot is nearly linear. Thus, adding the full complement of slave processors provides significant benefit because of the computationally intensive algorithm of the TSAFT/TSAFT-2 implementation. A high percentage of time is spent computing in the SAFT inner loop, as opposed to performing overhead functions.

Figures $8.13,8.14$, and 8.15 show the results of implementing a factor of 5 output record size compression. When these plots are compared with the previous set, they appear nearly identical. Again, this is due to the computationally intensive nature of the inner loop.

Figures 8.16, 8.17, and 8.18 show the performance curves under conditions of no output compression but a $-20-\mathrm{dB}$ selective processing threshold. Notice that a significant speed increase is realized with selective processing. This is due to two factors. The tandem mode data sets tend to have a high signal-to-noise ratio because the dual-transducer configuration has reduced backscatter. In addition, the computationally intensive nature of the algorithm implemented would contribute to this. Table 8.4 shows the benefit of using selective processing by tabulating the approximate number of summations that occurred when each complete data set was processed. As shown, the number of inner loop operations is dramatically reduced by using a threshold technique.

For completeness, Figures $8.19,8.20$, and 8.21 show the curves obtained with $-20-\mathrm{dB}$ selective processing and a factor of 5 output record compression. As expected, the processing performance is nearly identical to that shown in the preceding graphs.

In contrast to the results obtained in the pulse-echo mode (described in Section 8.1), the performance of the tandem implementation is highly sensitive to the number of slave processors installed and to the selective processing method implemented. The overhead functions, such as data transfer and VME buss contention problems, shown to be important factors in the pulseecho mode, are not significant in the tandem mode.

The implementation of TSAFT-2 on the SAFT processor has opened up new avenues of processing that were not practically available in the past. For example, full processing of File 3 took 6 hours and 20 minutes with 10 slaves enabled but would take weeks on the VAX $11 / 780$. Incidentally, although the elapsed time was more than 6 hours, the total CPU time (the time slice 
Table 8.4. Tandem Mode Analysis (Total Number of Points Processed)

\begin{tabular}{|c|c|c|c|}
\hline Data File & 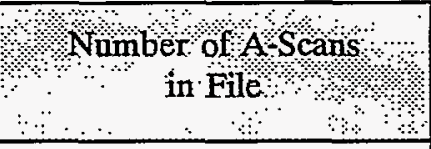 & $\frac{\text { Total Summations }}{400 \% \text { Processing }}$ & During Processing \\
\hline CASE 1 & 2880 & $0.114 \times 10^{9}$ & $0.069 \times 10^{9}$ \\
\hline CASE 2 & 12800 & $2.029 \times 10^{9}$ & $0.104 \times 10^{9}$ \\
\hline CASE 3 & 8640 & $2.568 \times 10^{9}$ & $0.131 \times 10^{9}$ \\
\hline
\end{tabular}

required by the host computer) was 5 minutes and 7 seconds. So the inclusion of a SAFT processor on the VAX host computer essentially makes the SAFT computation transparent to the load on the computer.

The TSAFT-2 tandem implementation processes TSAFT-3 data because these configurations are essentially the same on thicker materials. Actually, implementation of TSAFT-2 on the SAFT processor has made it possible to investigate TSAFT-3 on thick materials. Practical implementation of this configuration on the host VAX 11/780 has proven to be impossible.

\subsection{Overview of the Software Imple- mentation of the SAFT-UT Real-Time Processor}

This section provides an overview of the RTP from a programmer's perspective. A short history of the evolution of the SAFT software is provided followed by an explanation of the method of calculation. The various shortcuts used to both simplify implementation and improve the computational efficiency are reviewed. Finally, a flow chart provides a graphical representation of the logical flow of data processing events.

\subsubsection{Development History}

Initially, the SAFT calculations were coded on a VAX $11 / 780$ computer at the University of Michigan and some lookup tables were used to increase the speed of the calculation process. At PNL, all of the lookup tables, the sequence of calculations, and the organiza- tion of the data in memory were restructured to use the capabilities of the VAX computer to the best advantage. Even with all these improvements, the calculations could not be performed fast enough to provide inspection personnel with "real-time" (at least $10 \mathrm{~A}$ scans/s) images, and the decision was made to use several parallel processors to attain the necessary speed. When the SAFT algorithm was rewritten to use several processors, software was included that would allow execution in a simulation mode. In the simulation mode, all of the software is actually executed by the host computer. In places where several slave processes would be running at the same time, the software is actually executed sequentially. The simulation mode allowed the use of the better debugging facilities available on the VAX host computer. Consequently, the debugging time needed for the software was greatly reduced. Once the SAFT parallel-processor software was debugged and running properly in the simulation mode, final debugging was performed with the software running on the actual slave processors.

\subsubsection{The SAFT Calculation}

Consider the rectangular physical volume of material to be inspected and the accompanying "volume" of data. Data is acquired as a transducer is scanned over one surface (the data-collection surface) of the physical volume. For each spatial position $(X, Y, 0)$ at which the transducer records a waveform, a depth dimension $\left(Z_{p}\right)$ exists in the physical volume, corresponding to a timeof-flight dimension (TOF) within the data volume. If transducers were very directional and received echoes from a single direction only, then an echo in the data volume (X,Y,TOF) could result from only an acoustic 
reflector located in the physical volume at $\left(X, Y, Z_{\mathrm{p}}\right)$, where $Z_{p}=T O F / 2 V$ and $V$ is the material velocity. However, this is not the case.

Transducers "see" everything within some cone-shaped aperture; and, the best that can be said, when considering a single waveform, is that an echo located at $(\mathrm{X}, \mathrm{Y}, \mathrm{TOF})$ in the data volume may have resulted from an acoustic reflector at a distance $\mathrm{d}=\mathrm{TOF} / 2 \mathrm{~V}$ (or $\mathrm{Z}_{\mathrm{p}}$ ) from the transducer location $(X, Y, 0)$ and within the cone-shaped aperture. Of course, this is less than one would like to know about the location and size of reflectors within the physical volume. Clearly, some sort of focusing technique is needed.

Better results are achieved by reversing the scenario; i.e., consider a given point in the physical volume and determine where, within the data volume, the digitized amplitude values would be stored if an acoustic reflector were located at the point being considered. Several benefits arise from this approach. First, this approach would enable one to average over a locus of echoes, determining the strength of the reflector at the point being processed. All waveform data acquired at spatial locations from which the transducer cannot "see" the point in question may be eliminated because the point is outside the beam aperture. This leaves a set of transducer locations on the data-collection surface that correspond to a cone drawn from the point in the physical volume, toward the surface, and with the same fullbeam angle as the transducer aperture. For each spatial transducer location $(X, Y, 0)$ within the cone-shaped aperture, only the time dimension $(t)$ need be determined to locate the digitized amplitude value (within the data volume) corresponding to the echo produced by the point being processed. Since we know the distance from the transducer $(X, Y, 0)$ to the point in question $\left(X, Y, Z_{p}\right)$ and the sound velocity, we may calculate the TOF as twice the distance (sound path down and back) divided by the acoustic velocity; i.e.,

$$
T O F=\frac{2 * d}{V}
$$

where: $d=\sqrt{\left.\left(X-X_{p}\right)^{2}+\left(Y-Y_{p}\right)^{2}+\left(Z-Z_{p}\right)^{2}\right]}$
Therefore, from an elementary perspective, for every point in the physical volume

1. Determine which spatial transducer locations $(X, Y, 0)$ should receive an echo from the point being processed.

2. Calculate the TOF between the point being processed and the transducer for each spatial transducer location determined in step 1.

3. Sum the digitized values from the data volume for all (X,Y,TOF) locations determined in steps 1 and 2.

The final summations are stored in a second data volume at $(X, Y, Z)$, and their amplitude should directly relate to the strength of the acoustic reflector at $\left(\mathrm{X}, \mathrm{Y}, \mathrm{Z}_{\mathrm{p}}\right)$ in the physical volume. If the acoustic reflector at $\left(\mathrm{X}, \mathrm{Y}, \mathrm{Z}_{\mathrm{p}}\right)$ is strong and correlates with a locus of A-scan echoes, then the summation of the amplitude values by the SAFT calculation will produce a welldefined signal or high value. If the reflector is weak or nonexistent and does not correlate well with a locus of A-scan echoes, the amplitude values digitized would most likely be from random noise and the summation process would produce a spatial average of nearly zero. The SAFT algorithm is analogous to a spatial-temporal matched filter in that the raw A-scans are time-shifted by a predicted time delay and then averaged over a number of positions. Constructive interference is produced by coherent signals, and destructive interference is produced by incoherent signals.

The SAFT algorithm works well. However, when implemented in a straightforward or brute-force manner, there can be millions upon millions of calculations that can take hours, days, or even weeks to complete. Hence, the complexity of implementing the SAFT algorithm arises from measures taken to reduce the computational time required.

A solution was twofold:

First, many alterations in the data collection and computational procedures were made to eliminate redundant calculations and take advantage of the computer's abilities. Second, we divided the computational load among several processors. 
Redundant calculations were eliminated in the determination of which spatial transducer locations contain (within their beam aperture) the point in question. If the data is acquired over an ordered grid pattern, then the qualifying transducer locations will always have the same relative positions for all of the points (to be processed) at a given level $\left(Z_{p}\right)$ in the physical volume. The problem is simplified from a series of complex geometric calculations to one of constructing and keeping track of an orderly mapping in the computer memory. However, this does impose a relatively minor constraint on the data acquisition process: it must maintain a constant $\mathrm{X}$ and $\mathrm{Y}$ increment throughout the data acquisition scan.

Another, more important, benefit of the ordered grid data acquisition pattern is that the distance calculations between each point in the physical volume and each of the associated transducer locations are the same for all points (to be processed) at the same depth $\left(Z_{p}\right)$ level in the physical volume. Thus, the distance calculations need be computed only once for each depth $\left(Z_{p}\right)$ level in the volume and then subsequently stored in lookup tables to be used over and over again. Here again, the problem is reduced from a series of quadratic equation calculations to one of constructing and efficiently using lookup tables. Still, this imposes yet another constraint on the data acquisition process: the data collection surface must be relatively flat over an area described by the reverse projected cone. A study by Ganapathy et al. (1981) at the University of Michigan indicates that errors from surface variations are of minor significance for most geometries because of the small aperture angles normally used ( $12^{\circ}$ or less) and the inherent spatial averaging that occurs when the SAFT algorithm is employed. However, a complex and rapid change in the surface curvature (such as sometimes observed when inspecting pressure vessel nozzles) may produce a significant error, prompting the use of a correction algorithm.

The SAFT calculations are reduced to a series of memory accesses into the lookup tables and data storage. The final computational modifications consisted of organizing the lookup tables and data storage within the computer's RAM to further facilitate memory accesses.

A simplified example may illustrate how the distance and time calculations, which describe the physical data volume and transducer locations, are converted into a memory offset lookup table that can subsequently be used to find the data stored in the computer's memory. Assume the transducer collects a waveform, consisting of 100 data points in time, at each location in an $X, Y$ grid consisting of 10 increments in $\mathrm{Y}$ and 10 increments in $X$. These data points are stored sequentially in memory as follows:

1. the waveform taken at location $(1,1,0)$; points $(1,1,1) \ldots(1,1,100)$

2. followed by the waveforms taken at $(1,2,0)$.... $(1,10,0)$

3. followed by waveforms taken at $(2,1,0)$.... $(2,10,0)$, and so on.

Suppose that we are currently performing the SAFT calculation for a point in the physical volume at $(5,5,25)$. The center of the cone projected back from this point (in the data volume stored in that computer's memory) is at storage location number 4401 . That is 4 complete rows of data with 1000 points each $(10 \times 100)$, plus four waveforms in the fifth row at 100 points each, plus one for the first data point, in the fifth waveform, in the fifth row. (In practice, the memory location of this data point is not recalculated each time but rather incrementally changed as the point in the physical volume is moved; e.g., if the physical point is changed to $(5,6,25)$, then 100 is added to 4401 to get the correct position of 4501). In addition to the position of this waveform in the computer memory, positions of waveforms taken at adjacent transducer locations need to be known. These may be located as follows:

1. For each increment in the $\mathrm{X}$ direction in the physical volume, add (or subtract) 1000 storage locations.

2. For each increment in the $Y$ direction in the physical volume, add or subtract 100 storage locations (of course, the software also needs to keep track of when the transducer location is near the edge of the physical volume and preclude summing points that would actually be off the edge).

Thus, the location in the computer memory of each waveform taken within the cone, projected backwards from the point in the physical volume, may be found. 
Having previously calculated and stored in a lookup table the relative locations of the data from all of the spatial transducer locations in the backward projected cone, one can easily locate in memory all waveforms that should contain an echo from an acoustic reflector located at any given point in the physical volume. For example, if five transducer locations are within the backward projected cone and the memory address of the data from the point at the center of the backward projected cone is 4401 , the data from the other four points will be located at 4501, 4301, 5401, and 3401

(Figure 8.22).

The relationship between the time, depth, and data storage location works in a similar manner. Because each waveform is digitized at a constant rate, moving one storage location is equivalent to moving the reciprocal of the digitization rate in time; i.e., a $10-\mathrm{MHz}$ digitization rate would mean each storage location would be equivalent to $100 \mathrm{~ns}$. The precalculated TOF between a point in the physical volume and each transducer location, in its backward projected cone, is subsequently stored in a lookup table as a memory offset equal to the TOF/(time per data storage location).

Thus, the final SAFT algorithm consists of mathematical calculations no more time-consuming than additions and subtractions. A typical calculation would proceed as follows:

1. For every $Z$ level in the physical volume, precalculate the positions of the points in the backward projected cone in terms of $\mathrm{Y}$ and $\mathrm{Z}$ offsets relative to the center of the cone and the distance to each of those points.

2. Convert the calculated relative positions and distances to memory offsets and store them in two sets of lookup tables.

3. Now, for every point in the physical data volume, use the first lookup table to locate, in the computer memory, the position of the waveforms that should contain echoes from the point in question.

4. Use the second lookup table to locate the proper data point within each waveform and sum them all together.

\subsubsection{Configuration Modifications}

The SAFT algorithm lends itself to parallel processing because subsequent calculations do not depend upon the results of previous calculations. However, because the SAFT algorithm is primarily a series of memory accesses (into the lookup tables and the data storage area), parallel processors should not share the same memory for the lookup tables or data storage. If they did, they would frequently be competing for access to the same memory and performance would suffer. Each of the parallel processors has a copy of the lookup tables in its on-board memory. The handling of the data storage memory is a bit more complicated because the basic SAFT algorithm requires access to all of the data storage memory further, the massive size of the data makes it prohibitive to have a copy for each of the parallel processors. The solution to this problem is to divide the data among the parallel processors, with each processor storing every $\mathrm{N}^{\text {th }} \mathrm{Y}$ plane (where $\mathrm{N}$ is the total number of parallel processors) of the data in onboard memory. Consequently, all of the lookup tables and the SAFT algorithm were modified so that in performing the summation process, each slave would automatically skip over the data planes it did not process but still produce partial sums over the data it did have in its on-board memory, for every point in the total physical volume. Partial sums from each slave are then summed into a common storage area. Finally, the rf component of the processed data is removed using a procedure called envelope detection.

The sequence of operations is as follows:

1. The host computer inputs raw data from disk or the real-time data acquisition system.

2. The host calculates all of the lookup tables that will be used by the parallel processors.

3. One of the parallel processors is designated to be the supervisor. It handles all of the communications with the host and directs the operations of the other parallel processors, called slaves. Each slave runs the exact same software.

4. The host downloads the lookup tables and other parameters to the supervisor and starts to download the actual 'data. 
5. Upon a system reset, each slave executes a short startup sequence stored in its ROM and then waits for the supervisor to give the "go" signal.

6. The supervisor places into each slave's memory the code that the slave will execute, in performing its part of the SAFT algorithm, along with other parameters and each slave's number. The slave number causes each slave to execute the slave code in a slightly different sequence, compensating for the different sequence of data planes each slave processes.

7. When the supervisor has received enough data from the host for processing to start, it tells each slave in turn to begin executing the downloaded code.

8. Each slave copies every $\mathrm{N}^{\text {th }} \mathrm{Y}$ plane of data from the supervisor's shared memory into its private memory.

9. Each slave commences to compute the SAFT summations one $\mathrm{Y}$ plane at a time.

10. When a slave finishes a partial summation of each $Y$ plane, it transfers its results to the supervisor where they are combined with the partial summations from all the other slaves for the same $Y$ plane.

11. The supervisor transfers back to the host the fully summed $Y$ plane.

\subsubsection{Tandem SAFT}

In all respects commented on thus far, tandem SAFT is the same as pulse-echo SAFT. The exception is that when the TOF is computed, the distance used is the distance from the sending transducer to the point in the physical volume plus the distance from the point in the volume to the receiving transducer, rather than twice the distance from the point in the physical volume to the single pulse-echo transducer.

\subsubsection{Flow Chart Commentary}

Figure 8.23 shows the logical flow of events that occur during SAFT processing of data; from the host comput- er, to the real-time processor supervisor, to the slave processors, and back. Each box has a number that corresponds to numbers listed below commenting on the events that take place.

Host computer:

$<1>\quad$ Set up and receive data acquisition parameters.

$<2>\quad$ The VAX calculates all of the lookup tables that will be used by the slave processors.

<3> Initialize the supervisor. One of the parallel processors is designated to be the supervisor. It handles all of the communications with the VAX and directs the operations of the other parallel processors, called slaves. Each of the slaves runs the exact same software.

$<4>\quad$ The VAX downloads all of the lookup tables to the supervisor.

$<5>\quad$ Is the raw data ready for input?

$<6>\quad$ Is supervisor ready for more raw data?

$<7>\quad$ Is the supervisor ready with processed data?

$<8>\quad$ Is SAFT processing finished?

<9> Enter data from data acquisition system or disk file.

$<10>$ Download one plane of raw data to supervisor.

<11 Upload processed data from the supervisor and write to disk file.

$<12>\quad$ Close current files and exit.

Real-time processor supervisor:

$<13>$ Wait for the VAX to initialize, download lookup tables, and so forth.

$<14>$ Download slave software code and parameters to each slave. Give each slave the start- 
up signal in turn. The supervisor places into each slave's memory the code that the slave will execute in performing its part of the SAFT algorithm, along with other parameters, and each slave's number. The slave number causes each slave to execute in a slightly different sequence, compensating for the different sequence of data planes each slave processes.

$<15>\quad$ Need more raw data. Is the VAX ready with more raw data?

$<16>\quad$ Are all slave processors finished executing envelope detection processing?

$<17>\quad$ Are all slave processors finished writing processed data back to supervisor memory?

$<18>\quad$ Is the next (Y) plane of raw data available in supervisor's memory and are all the slaves ready? (All slaves must be initialized or have completed slave step 7.)

<19> Upload a (Y) plane of processed data to VAX. Exit if processing of data is complete.

$<20>\quad$ Download a (Y) plane of raw data from VAX.

$<21>\quad$ Give go-ahead for slaves to start envelope detection of data.

<22> Give go-ahead for slaves to start processing data.
Real-time processor slaves:

<23> On startup signal from supervisor, initialize, read lookup tables, and so on from supervisor memory. Upon any system reset, every slave executes a short startup routine stored in on-board ROM and then waits for the supervisor to give it the startup signal.

<24> Wait for go-ahead signal from supervisor. (All slaves must be initialized or have completed step 29.)

<25> Read raw data from supervisor memory. Each slave copies every $\mathrm{N}^{\text {th }} \mathrm{Y}$ plane (where $\mathrm{N}$ is the total number of slaves) of raw data from the supervisor's shared memory into on-board memory.

$<26>\quad$ Process one $(\mathrm{Y})$ plane of raw data.

$<27>\quad$ Write one (Y) plane of raw data back to supervisor memory. Processed data is written back to the supervisor's memory with an ADD instruction so that the partial sums from each slave are combined.

$<28>\quad$ Wait for go-ahead from supervisor. (All slaves must have completed step 27.)

$<29>\quad$ Envelope detect completed ( $Y$ ) plane in supervisor memory. 


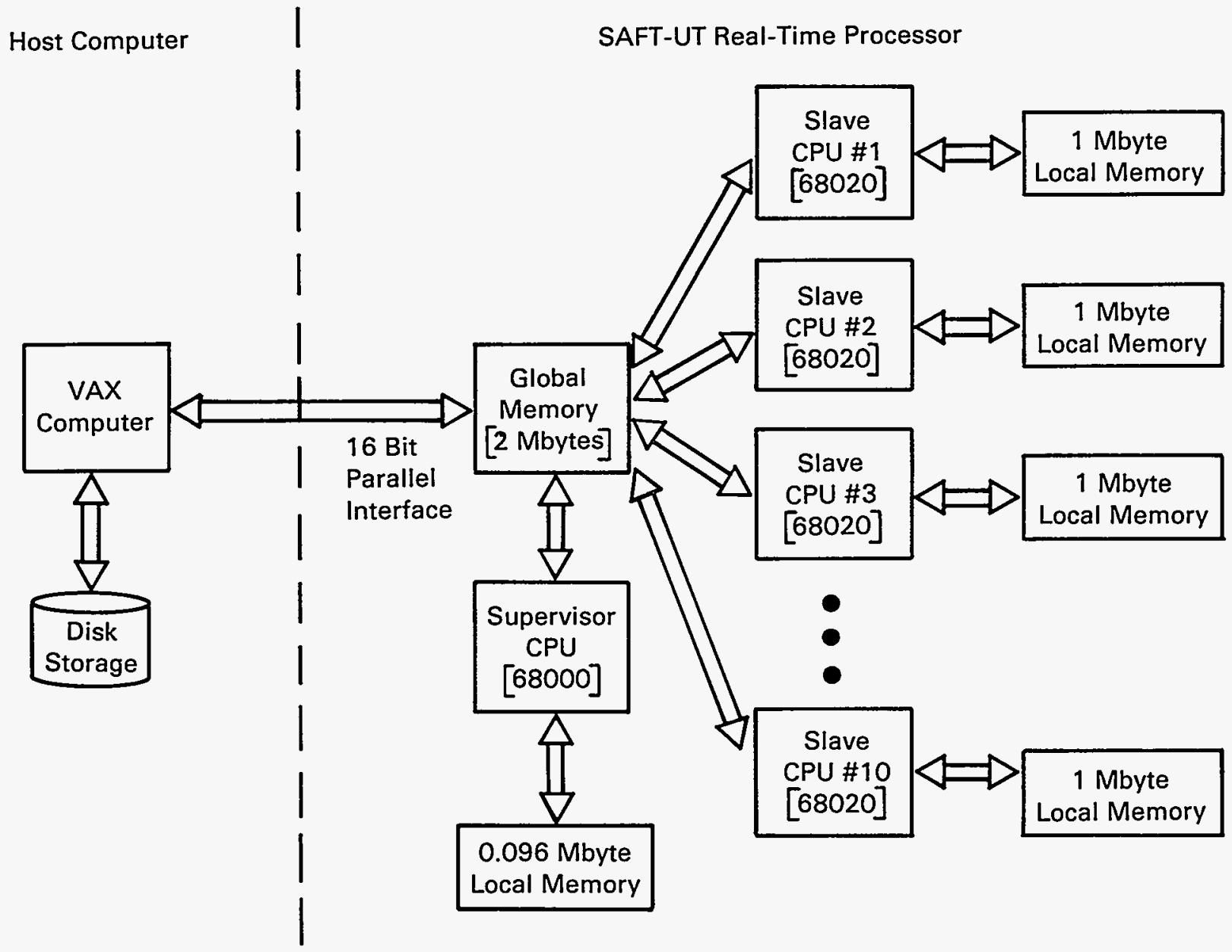

Figure 8.1. SAFT Computational Device or Real-Time Processor 
8.0 Processor Performance

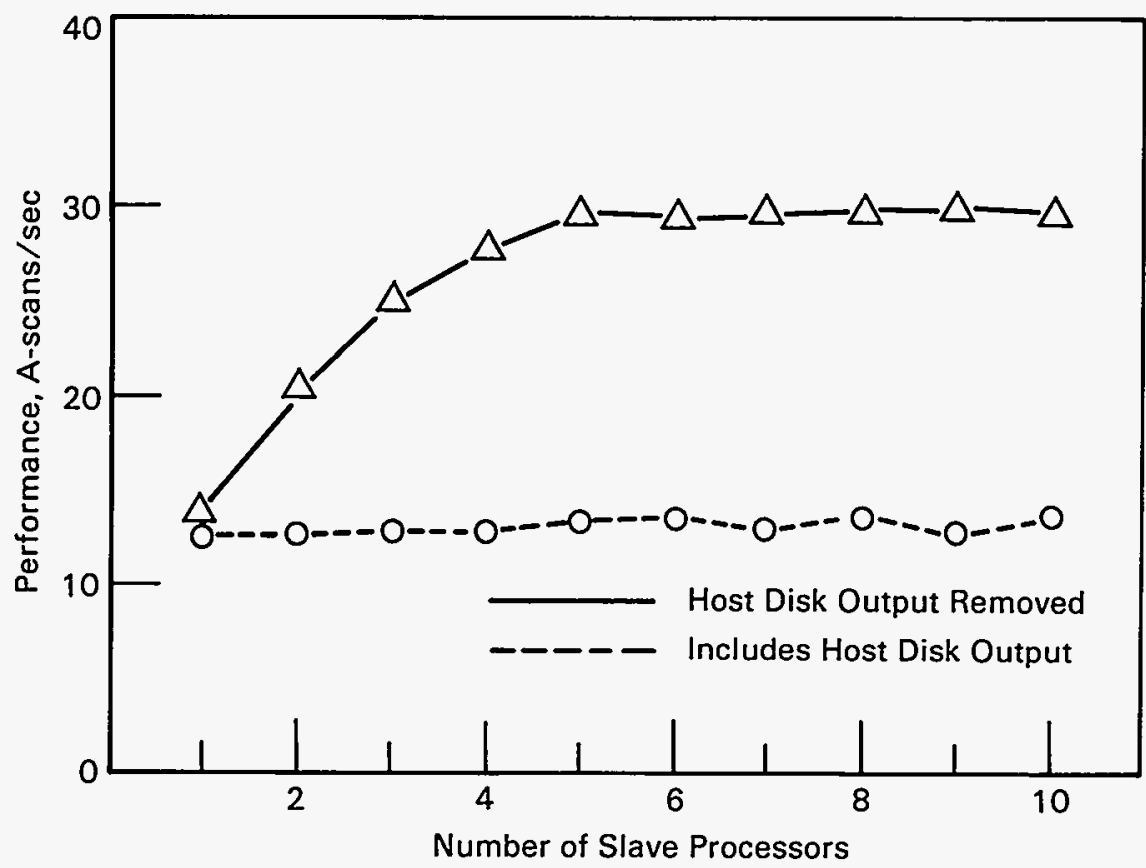

Figure 8.2. Real-Time Processor Performance on Pipe Data File with Selective Processing Disabled and Output Record Size Compression Factor Set to 1

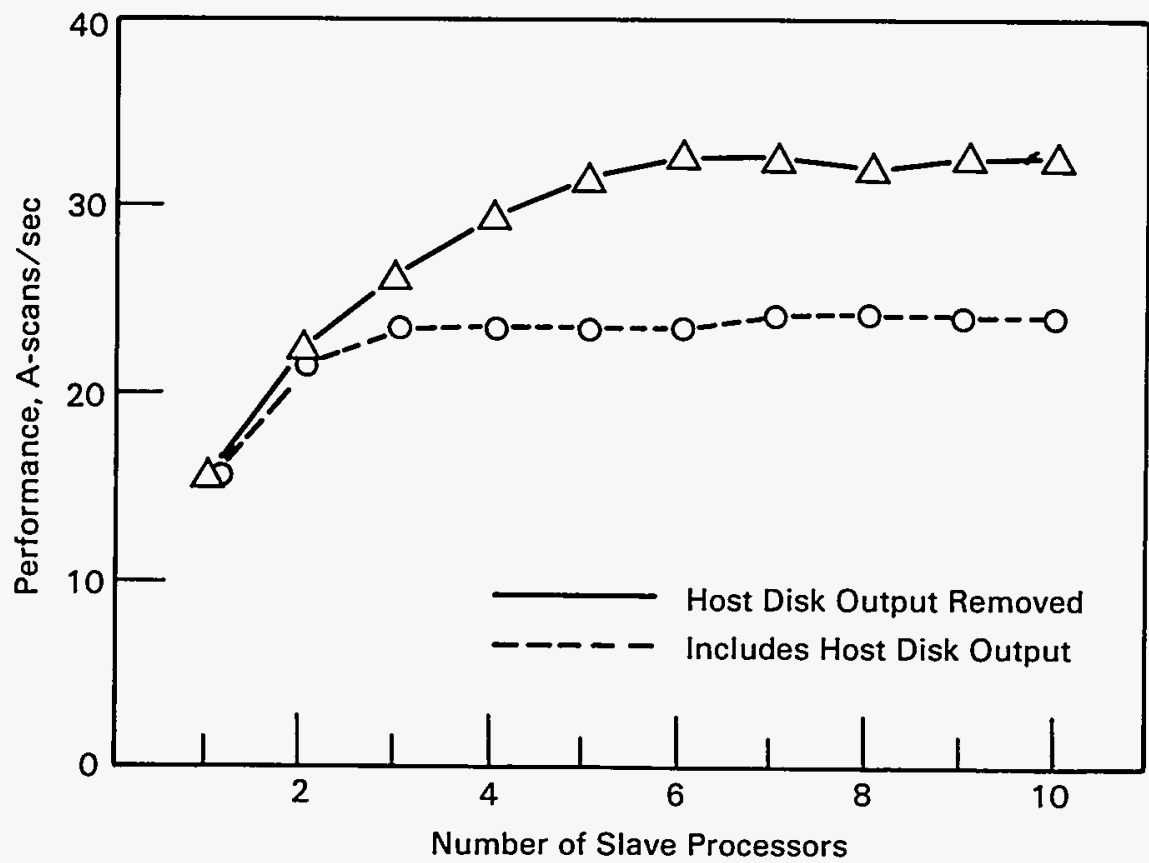

Figure 8.3. Real-Time Processor Performance on Pipe Data File with Selective Processing Disabled and Output Record Size Compression Factor Set to 5 


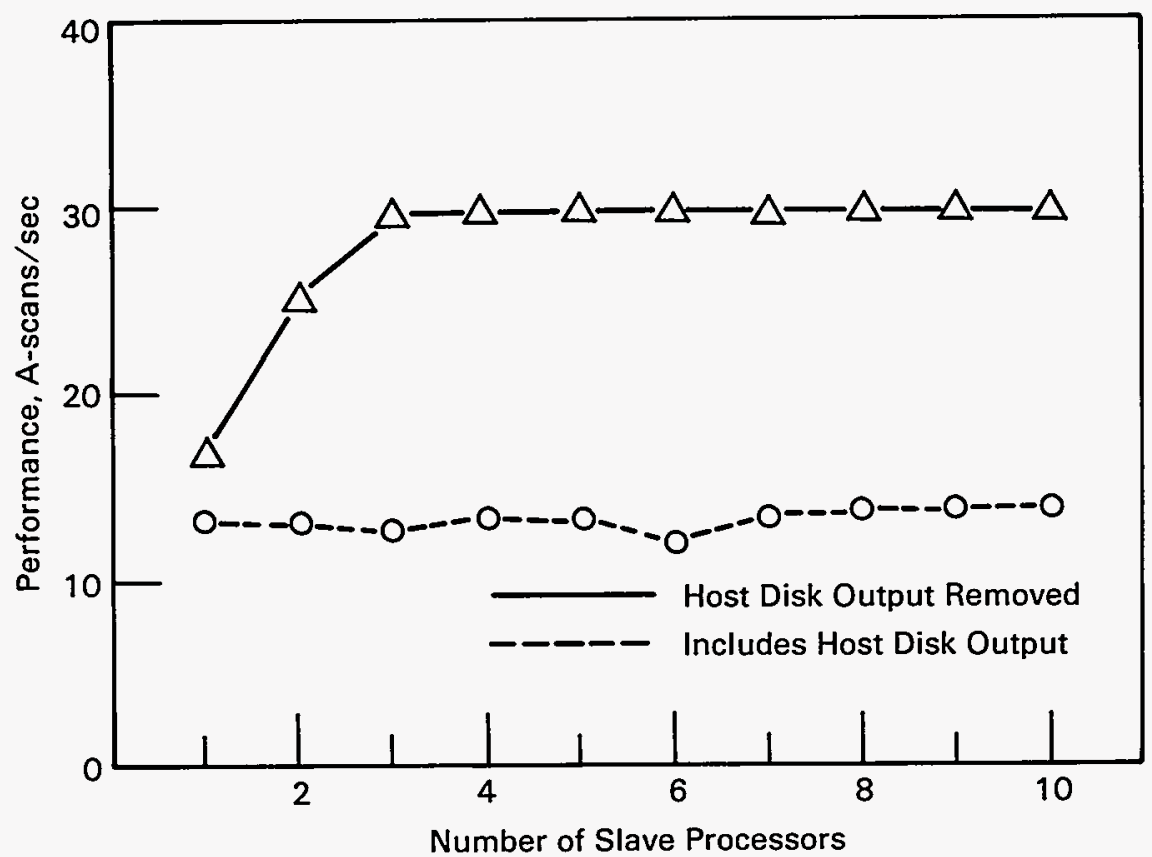

Figure 8.4. Real-Time Processor Performance on Pipe Data File with Selective Processing Set to $-20 \mathrm{~dB}$ and Output Record Size Compression Factor Set to 1

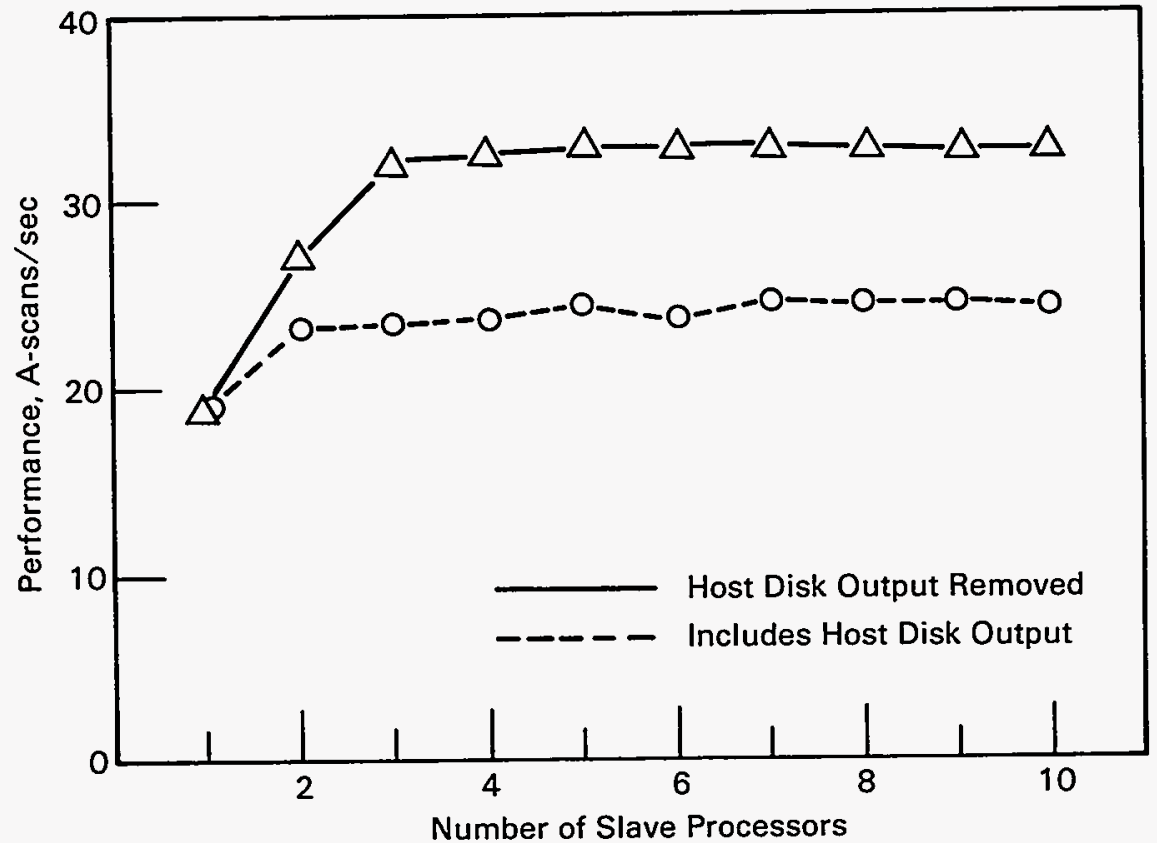

Figure 8.5. Real-Time Processor Performance on Pipe Data File with Selective Processing Set to $-20 \mathrm{~dB}$ and Output Record Size Compression Factor Set to 5 


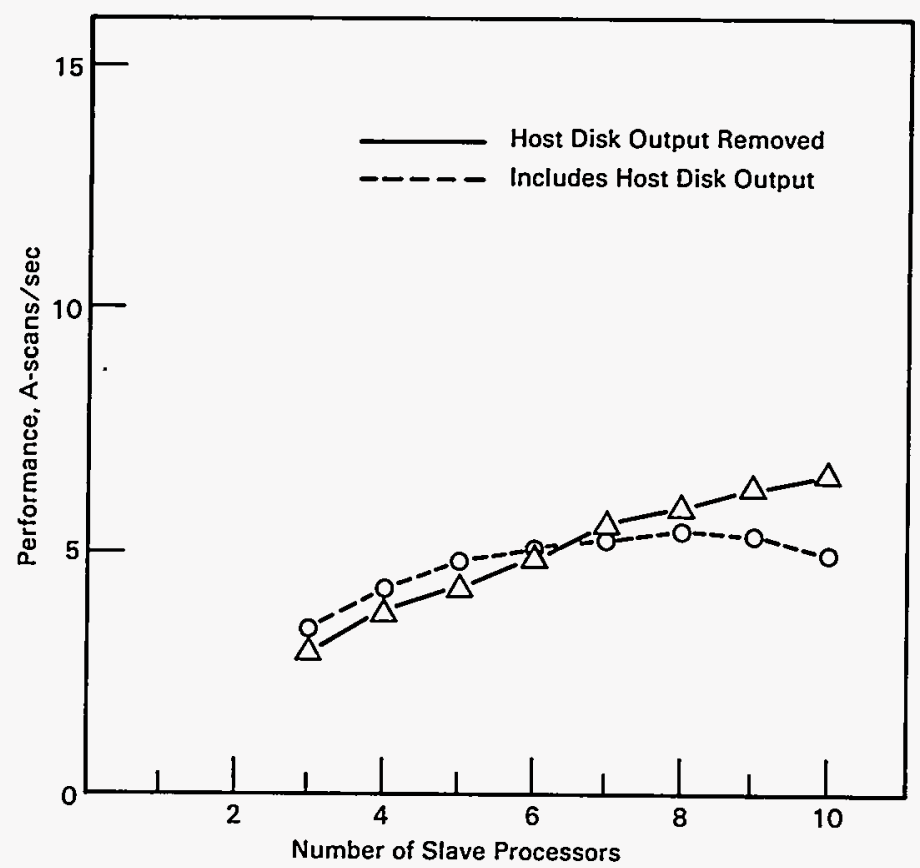

Figure 8.6. Real-Time Processor Performance on PISC II Data File with Selective Processing Disabled and Output Record Size Compression Factor Set to 1

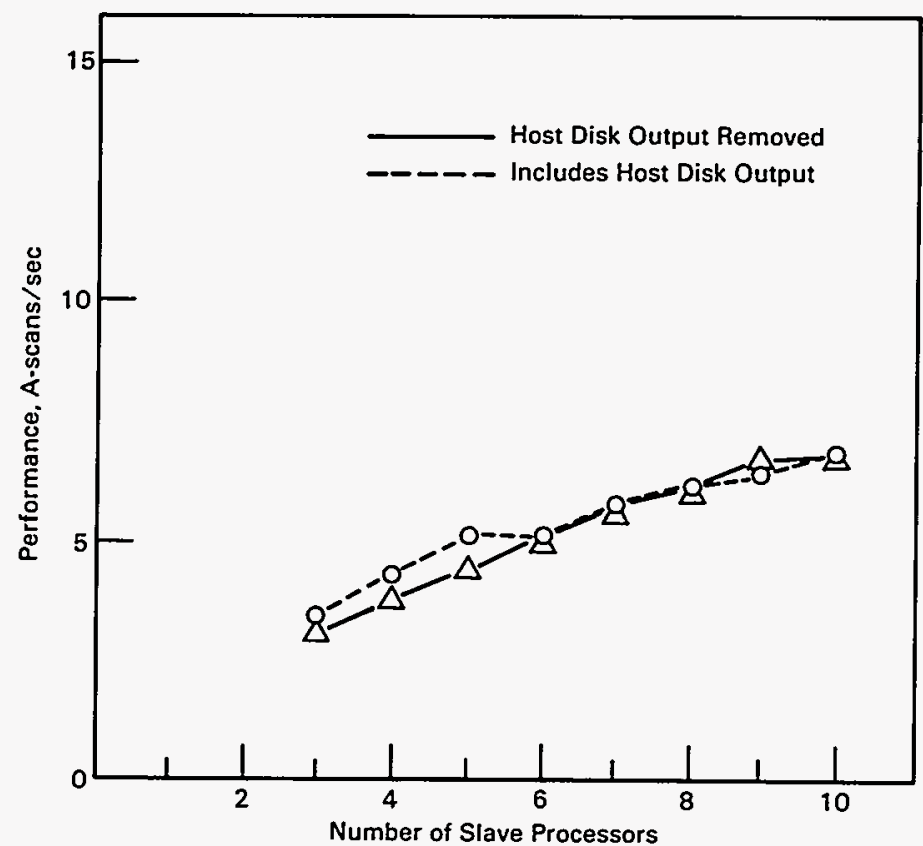

Figure 8.7. Real-Time Processor Performance on PISC II Data File with Selective Processing Disabled and Output Record Size Compression Factor Set to 5 


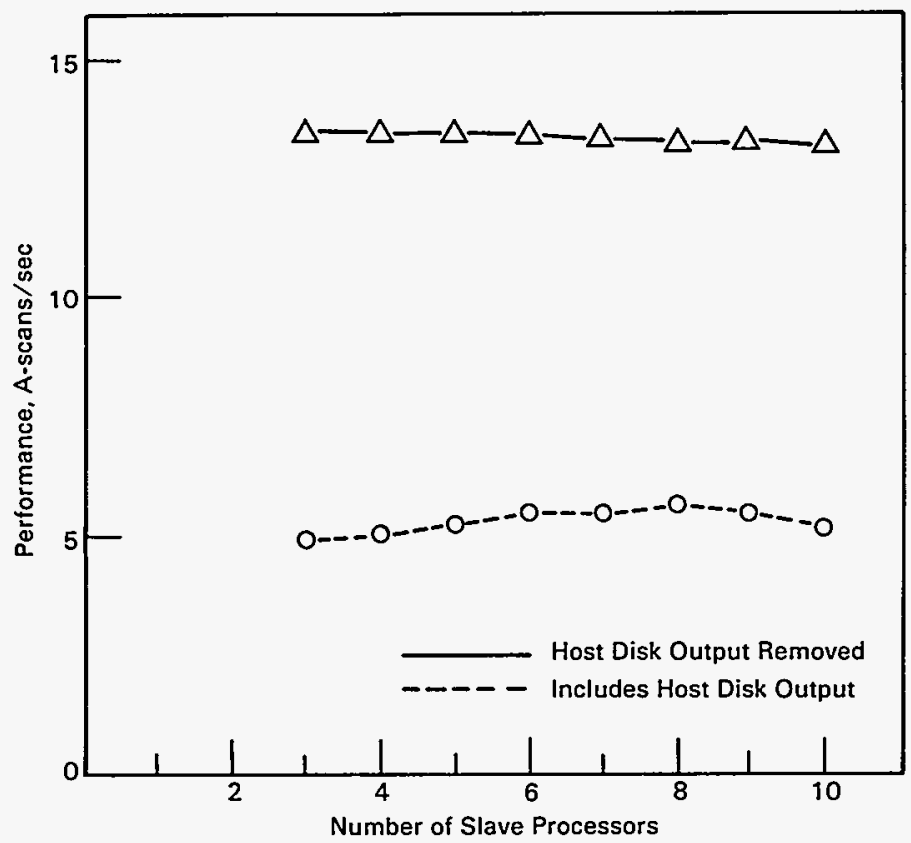

Figure 8.8. Real-Time Processor Performance on PISC II Data File with Selective Processing Set to $-20 \mathrm{~dB}$ and Output Record Size Compression Factor Set to 1

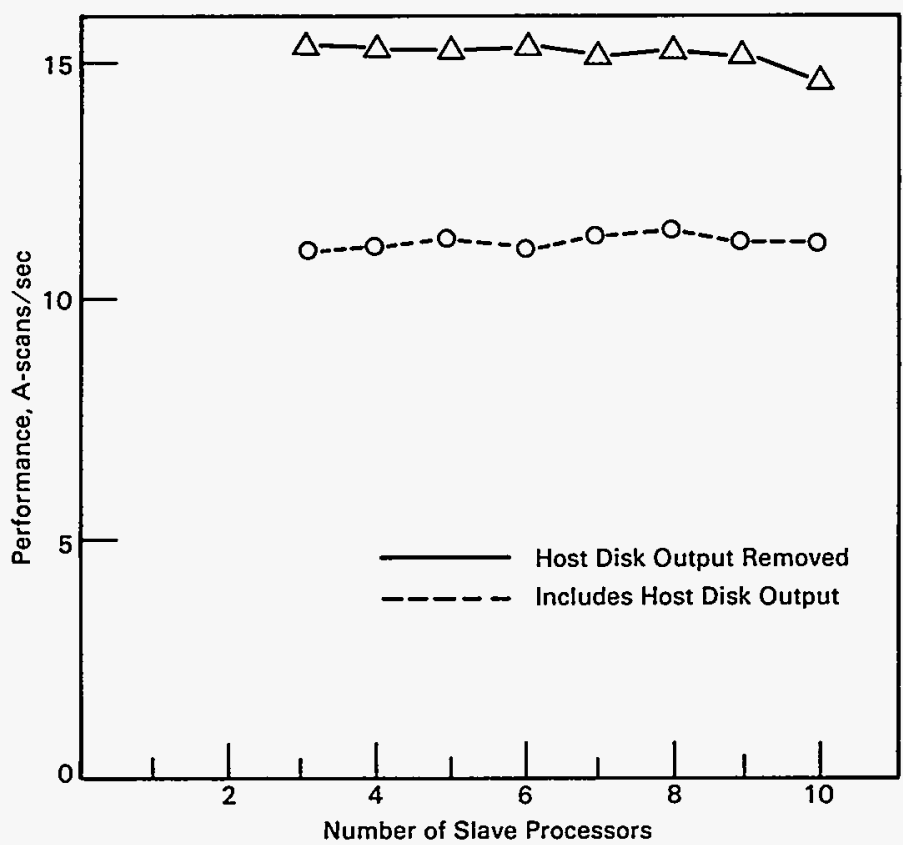

Figure 8.9. Real-Time Processor Performance on PISC II Data File with Selective Processing Set to $-20 \mathrm{~dB}$ and Output Record Size Compression Factor Set to 5 


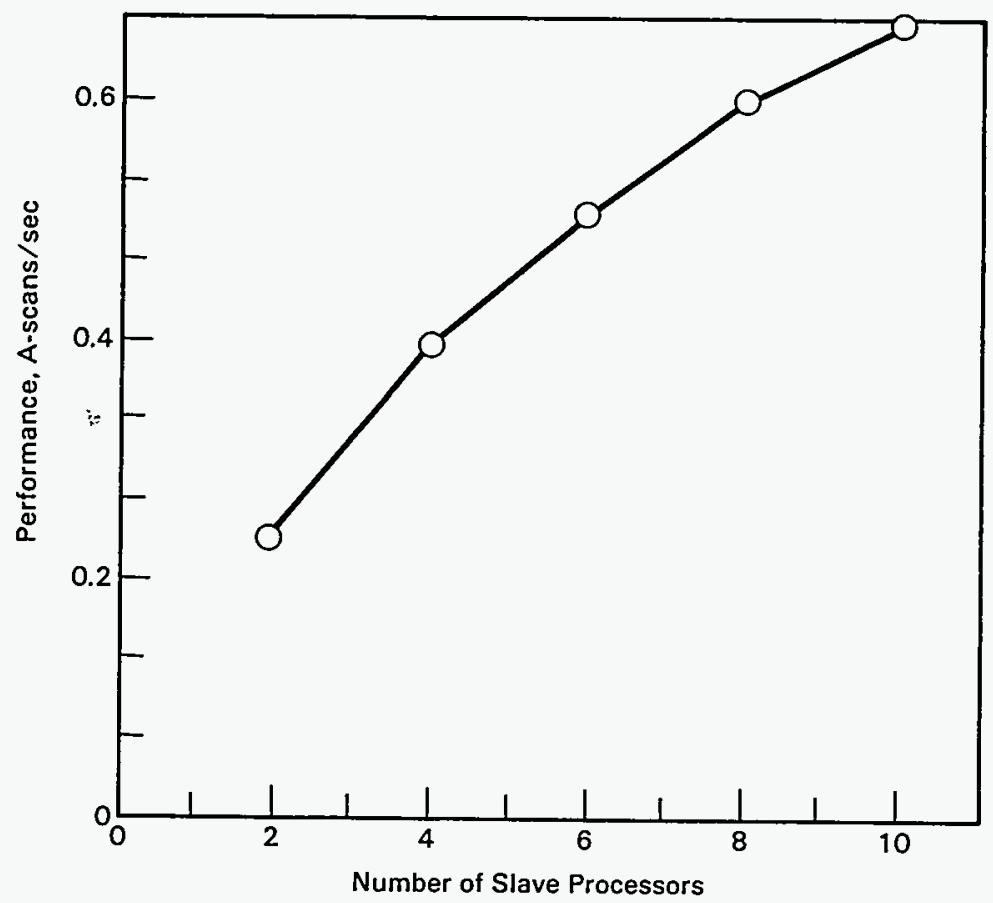

Figure 8.10. Real-Time Processor Performance, Tandem Configuration, on Thin-Pipe Data File (TSAFT; 0.585 in. thick [1.49 cm]) with Selective Processing Disabled and Output Record Size Compression Factor Set to 1

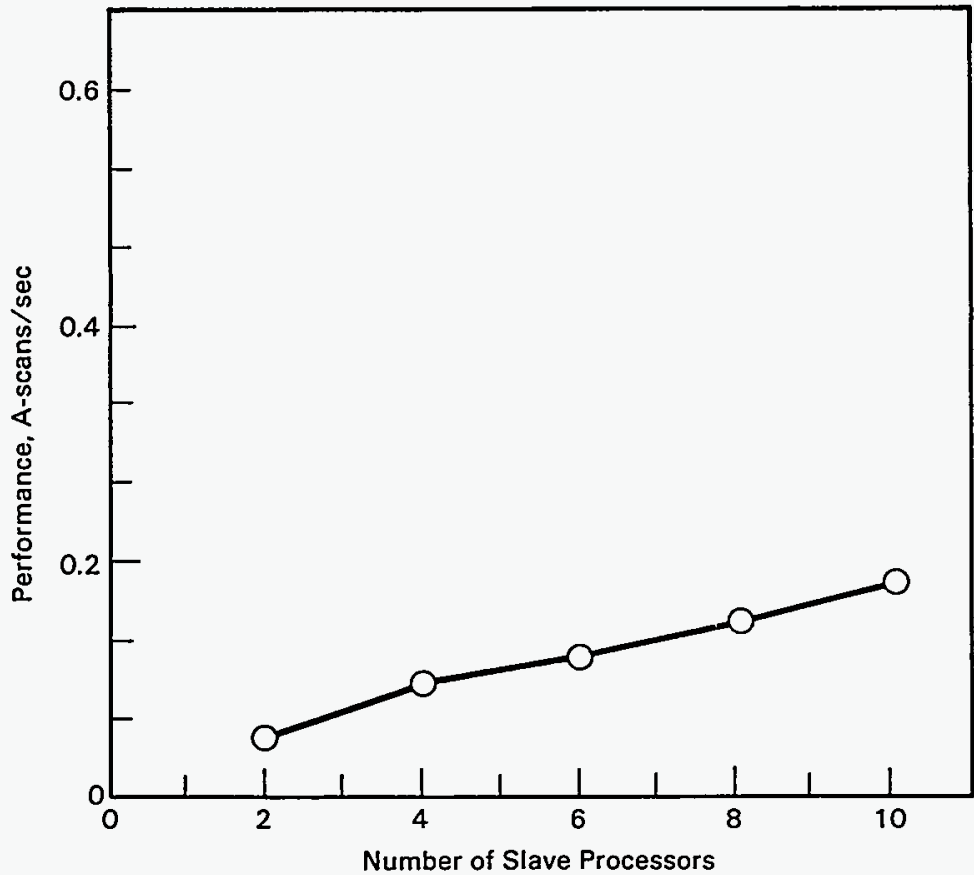

Figure 8.11. Real-Time Processor Performance, Tandem Configuration, on Thick-Pipe Data File (TSAFT; 1.5 in. thick [3.8 cm]) with Selective Processing Disabled and Output Record Size Compression Factor Set to 1 


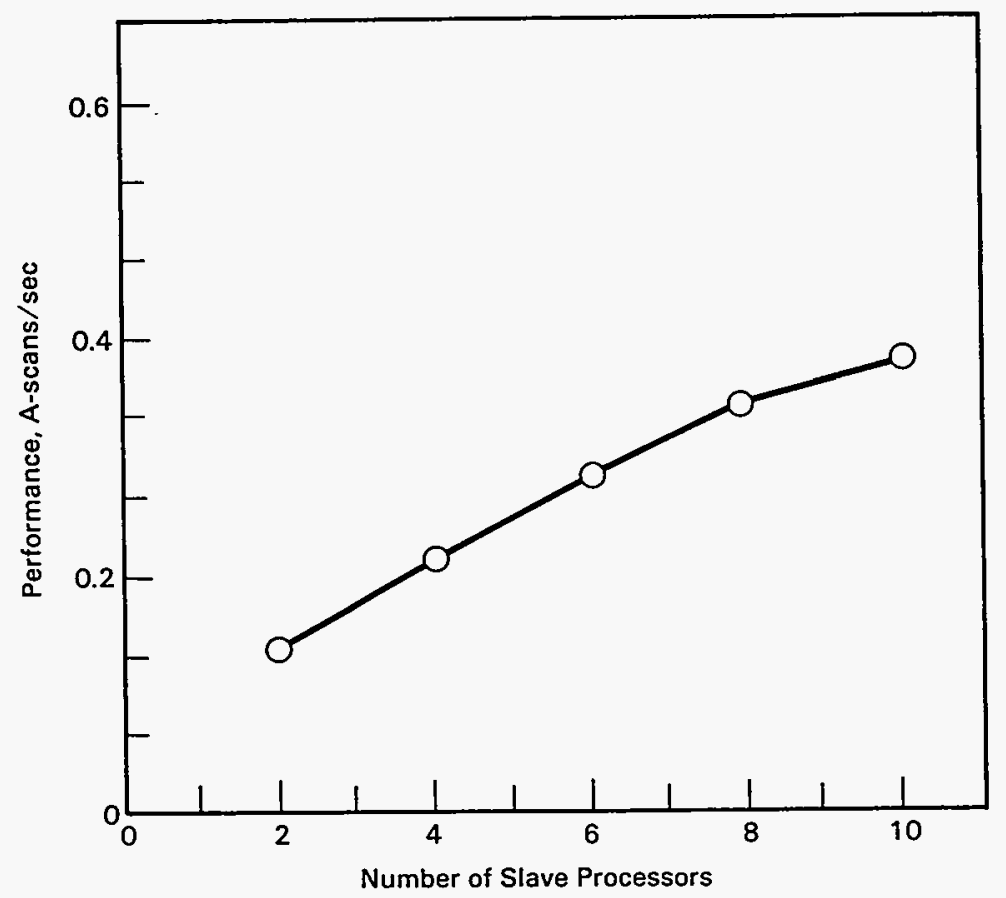

Figure 8.12. Real-Time Processor Performance, Tandem Configuration, on Thick-Pipe Data File (TSAFT-2; 1.5 in. thick $[3.8 \mathrm{~cm}])$ with Selective Processing Disabled and Output Record Size Compression Factor Set to 1

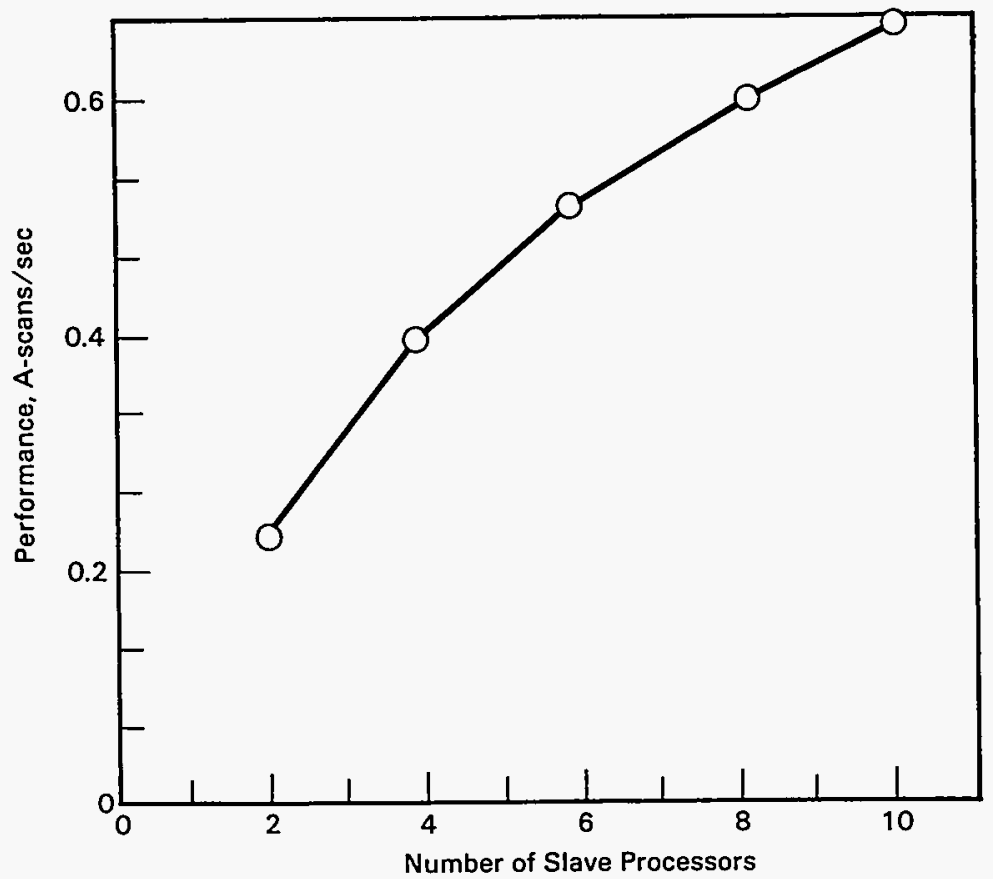

Figure 8.13. Real-Time Processor Performance, Tandem Configuration, on Thin-Pipe Data File (TSAFT; 0.585 in. thick [1.49 cm]) with Selective Processing Disabled and Output Record Size Compression Factor Set to 5 


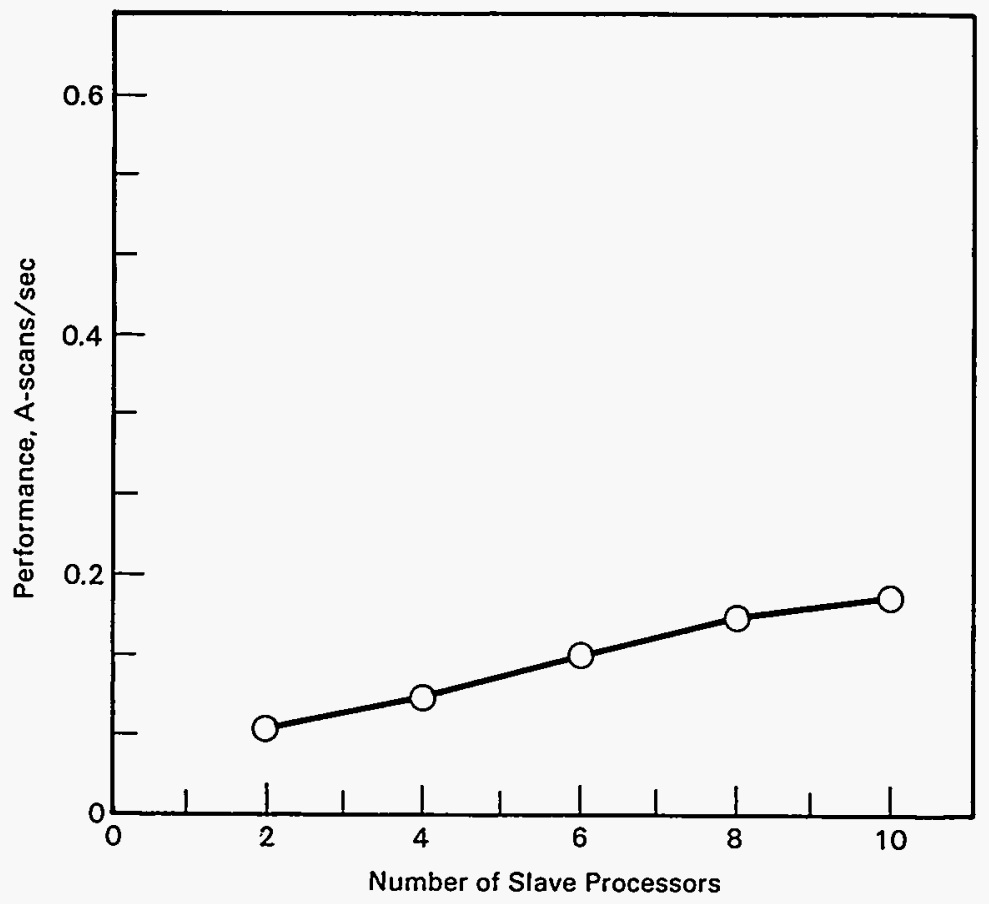

Figure 8.14. Real-Time Processor Performance, Tandem Configuration, on Thick-Pipe Data File (TSAFT; 1.5 in. thick [3.8 cm]) with Selective Processing Disabled and Output Record Size Compression Factor Set to 5

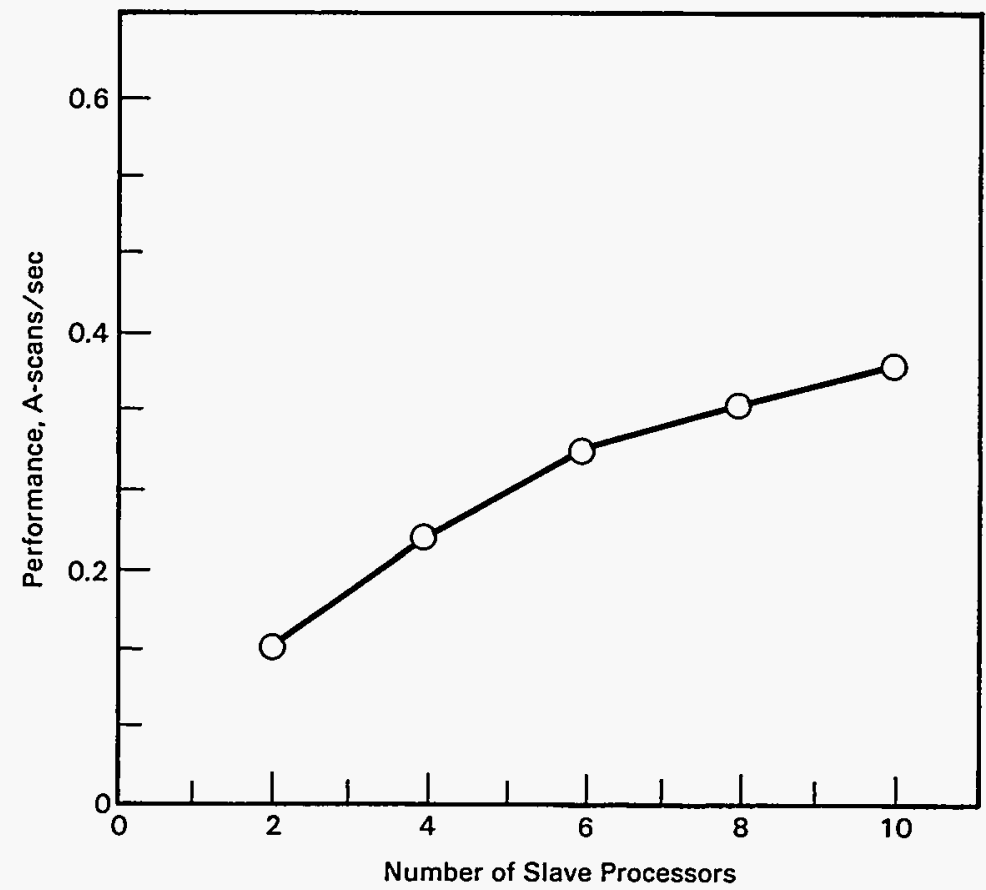

Figure 8.15. Real-Time Processor Performance, Tandem Configuration, on Thick-Pipe Data File (TSAFT-2; 1.5 in. thick [3.8 cm]) with Selective Processing Disabled and Output Record Size Compression Factor Set to 5 


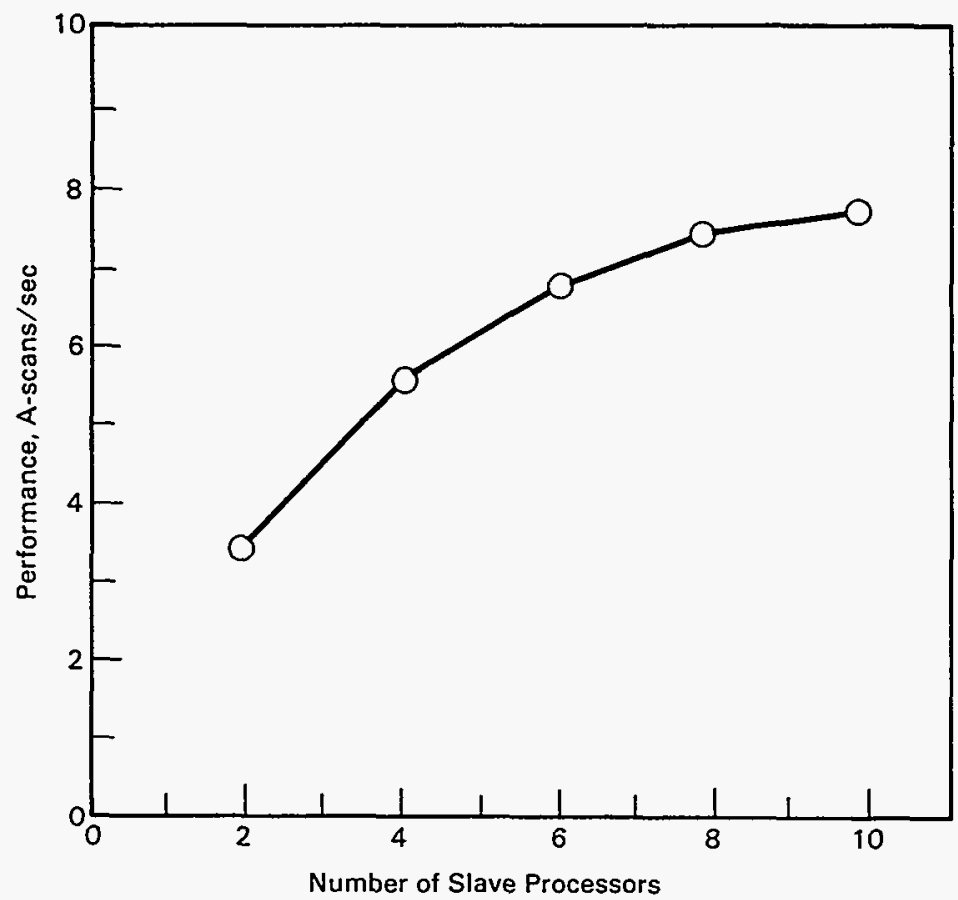

Figure 8.16. Real-Time Processor Performance, Tandem Configuration, on Thin-Pipe Data File (TSAFT; 0.585 in. thick [1.49 cm]) with Selective Processing Set to $-20 \mathrm{~dB}$ and Output Record Size Compression Factor Set to 1

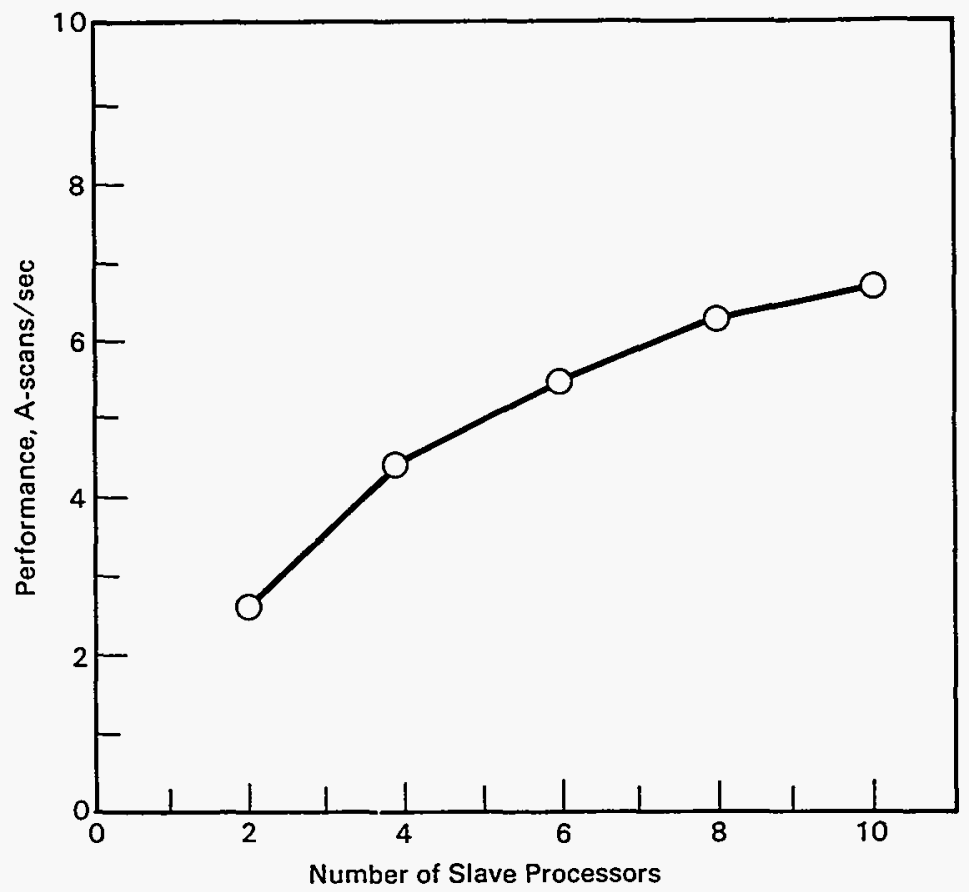

Figure 8.17. Real-Time Processor Performance, Tandem Configuration, on Thick-Pipe Data File (TSAFT; 1.5 in. thick $[3.8 \mathrm{~cm}])$ with Selective Processing Set to $-20 \mathrm{~dB}$ and Output Record Size Compression Factor Set to 1 
8.0 Processor Performance

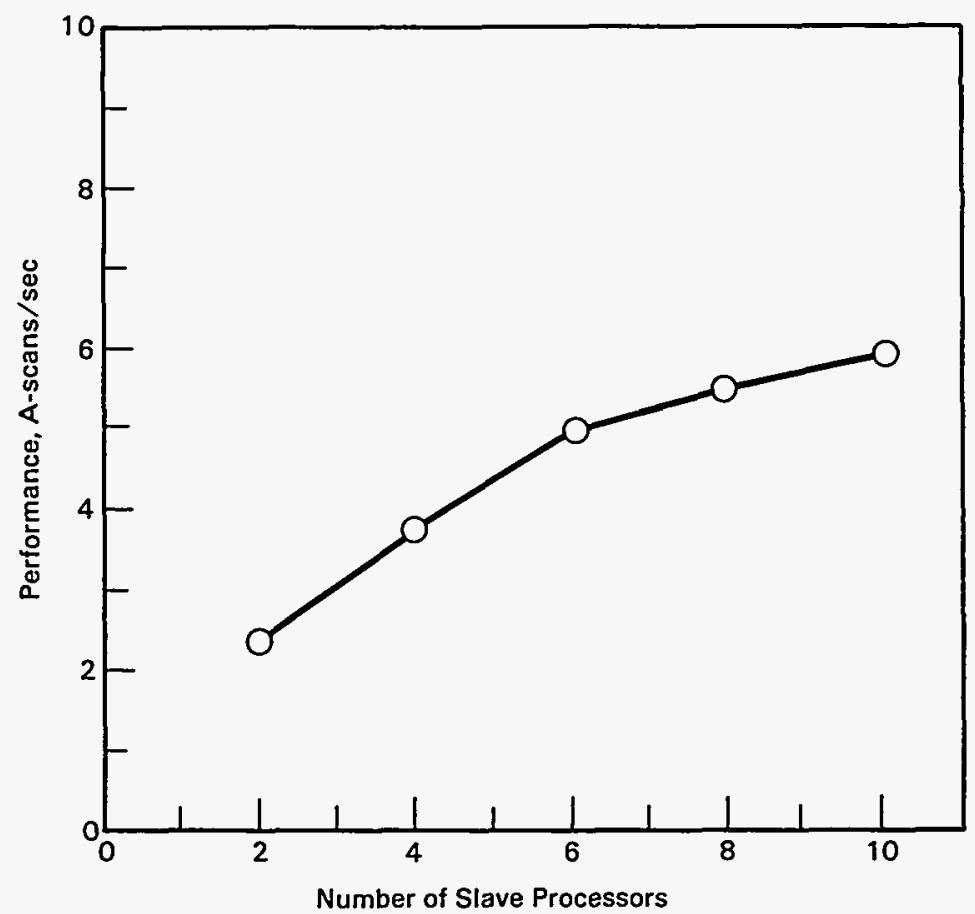

Figure 8.18. Real-Time Processor Performance, Tandem Configuration, on Thick-Pipe Data File (TSAFT-2; 1.5 in. thick [3.8 cm]) with Selective Processing Set to $-20 \mathrm{~dB}$ and Output Record Size Compression Factor Set to 1

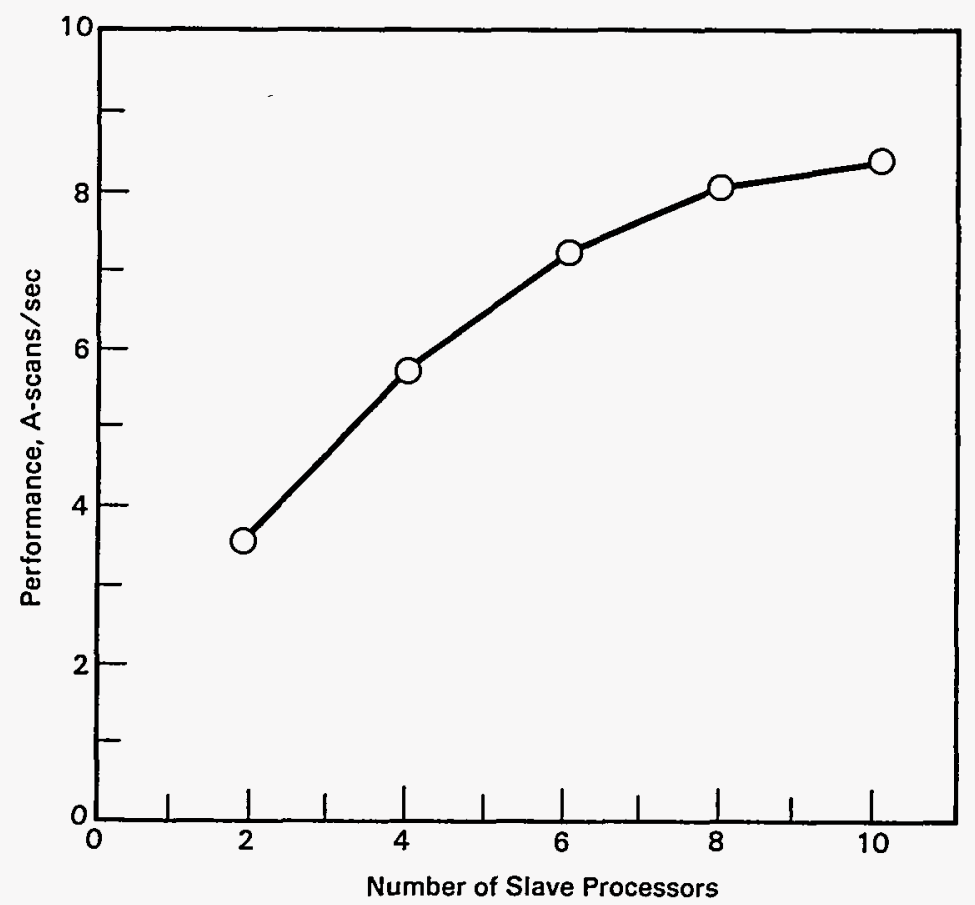

Figure 8.19. Real-Time Processor Performance, Tandem Configuration, on Thin-Pipe Data File (TSAFT; 0.585 in. thick [1.49 cm]) with Selective Processing Set to $-20 \mathrm{~dB}$ and Output Record Size Compression Factor Set to 5 


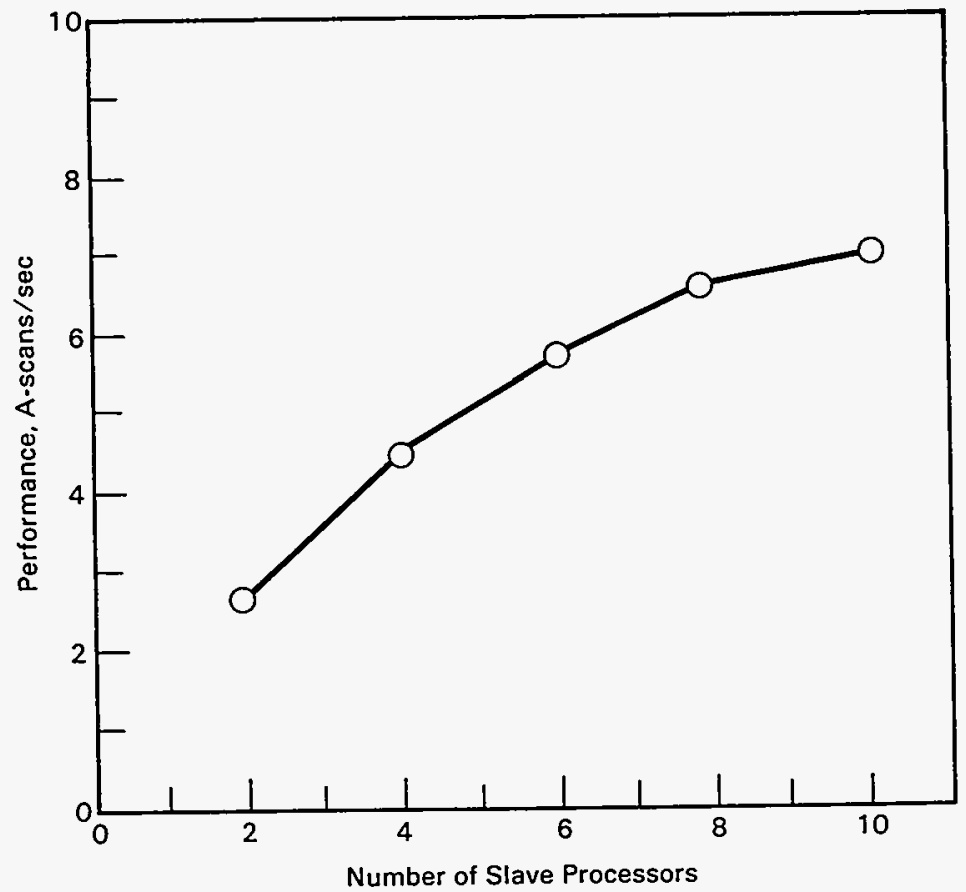

Figure 8.20. Real-Time Processor Performance, Tandem Configuration, on Thick-Pipe Data File (TSAFT; 1.5 in. thick [3.8 cm]) with Selective Processing Set to $-20 \mathrm{~dB}$ and Output Record Size Compression Factor Set to 5

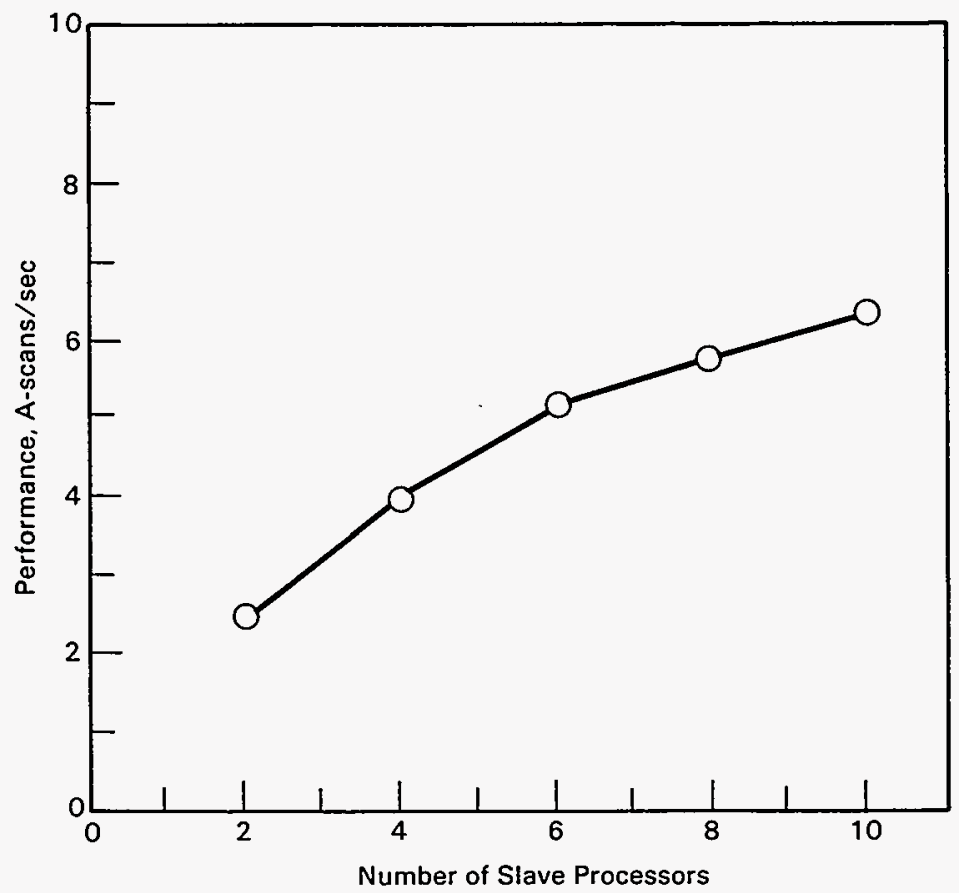

Figure 8.21. Real-Time Processor Performance, Tandem Configuration, on Thick-Pipe Data File (TSAFT-2; 1.5 in. thick [3.8 cm]) with Selective Processing Set to $-20 \mathrm{~dB}$ and Output Record Size Compression Factor Set to 5 


\section{CENTER GF BACKWARD}

PRDJECTED CONE

MEMIRY LICATION 4401
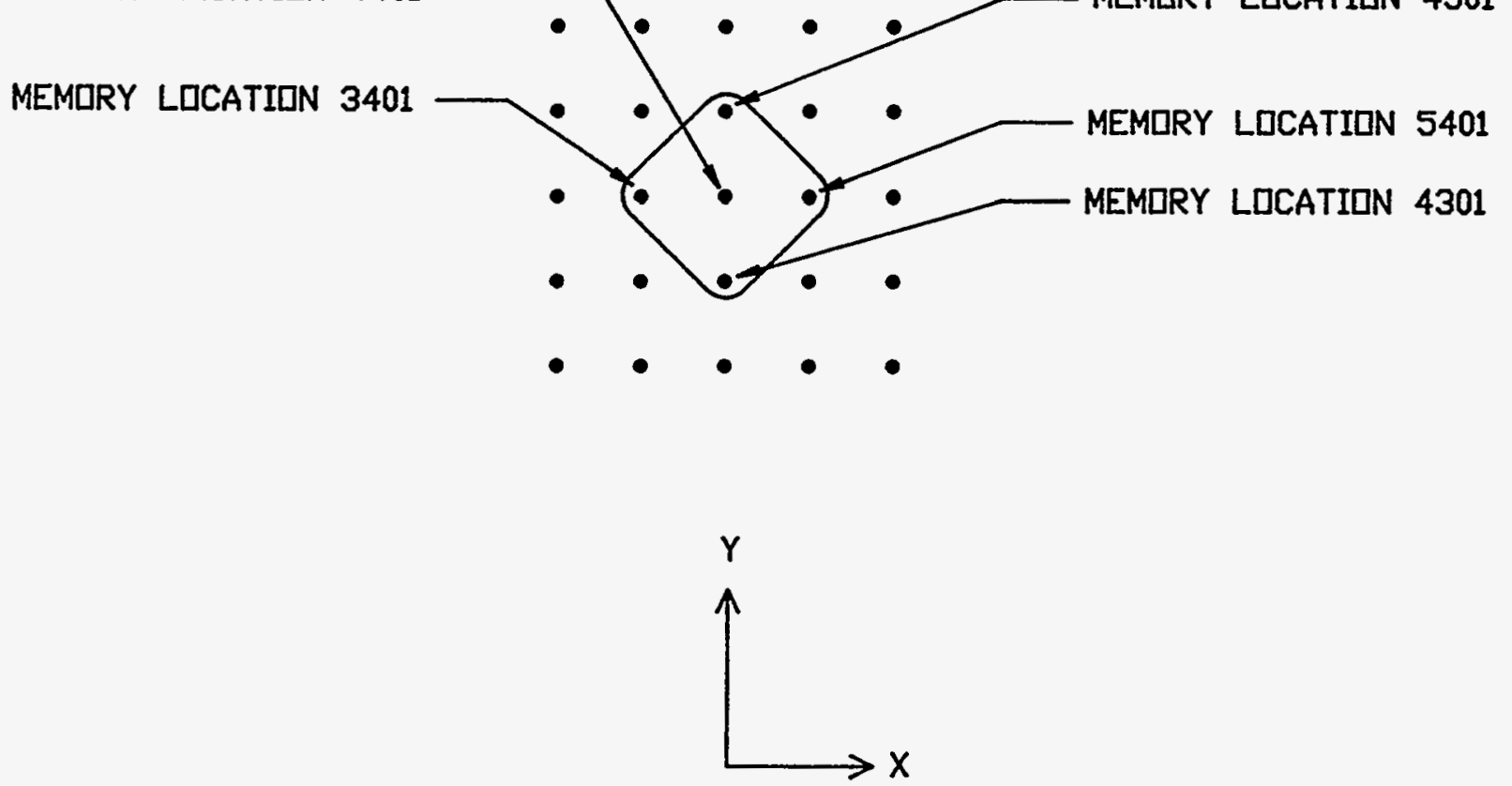

Figure 8.22. Example of How Transducer Locations Translate to Memory Locations 


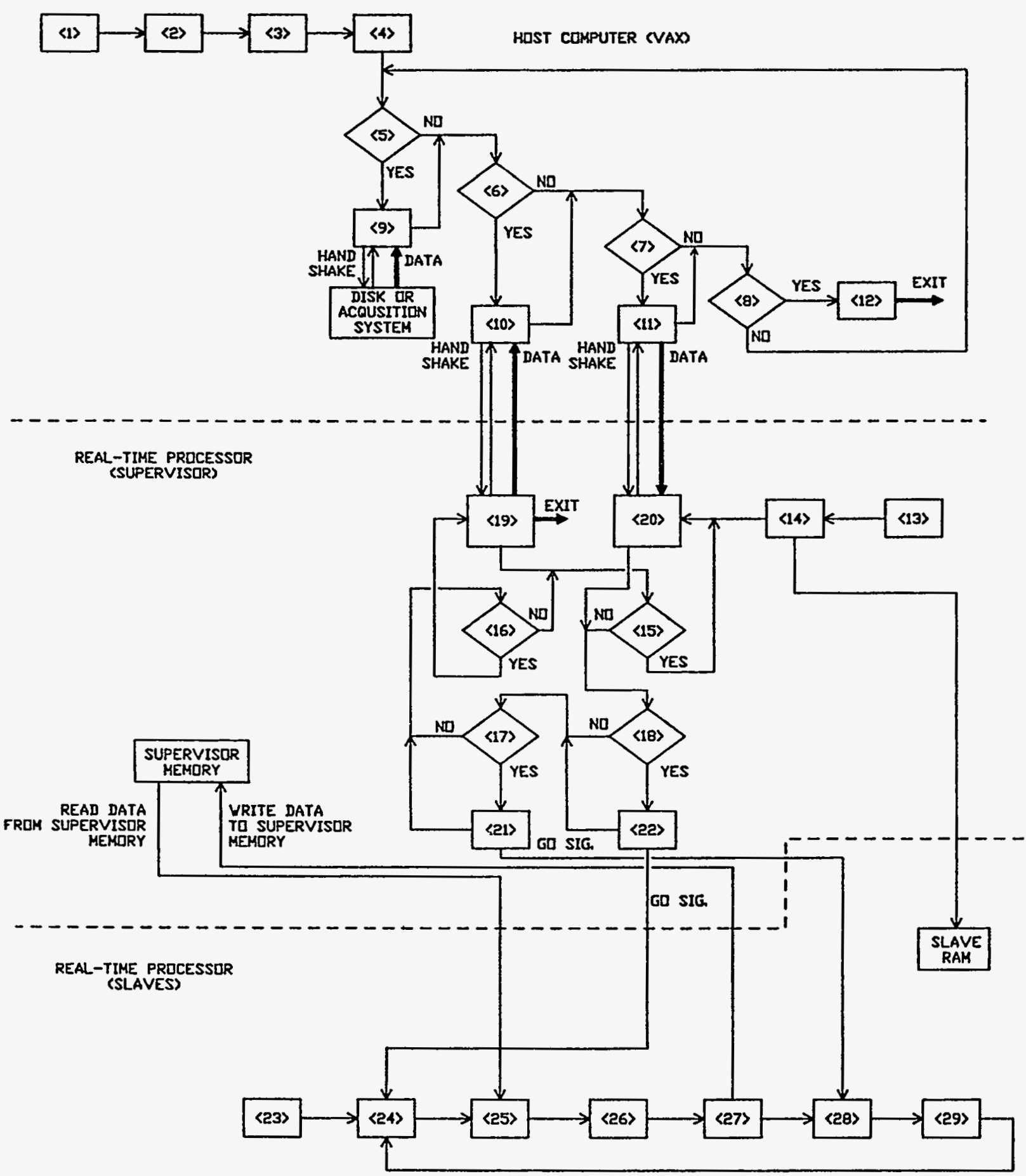

Figure 8.23. Logical Flow of Events During SAFT Data Processing Using Real-Time Processor 


\subsection{Upgrading the SAFT-UT Field System}

In the development and evaluation of the SAFT-UT technology, one of the important goals has been to improve the reliability of the inspection of reactor pressure vessels. In addition, another NRC program (JCN L1099) has as a goal to develop a data base on the distribution of fabrication flaws (i.e., flaws that existed at the time of vessel fabrication) that exist in U.S. nuclear reactor pressure vessels. SAFT-UT field inspections of unused pressure vessel material are planned for this project. The planned inspections require ultrasonic tests with equipment best suited to providing fast and accurate scans of the selected reactor pressure vessel areas. The SAFT-UT field data acquisition system has been upgraded to better cope with the large volume of data that will result from this kind of inspection.

In this section, we discuss the hardware and software methods used to achieve various rates of data collection, processing, and display. This text is useful for duplicating the system and for planning the implementation of SAFT-UT on other UT/ISI systems. Performance of the SAFT-UT system is measured using met- rics: sustained data acquisition rate, SAFT processing rate, and the data display rate. Table 9.1 shows the changes in these three rates as the SAFT-UT system was improved.

\subsection{SAFT System Architecture}

The SAFT-UT system is composed of four functional subsystems as shown in Figure 9.1. The data acquisition subsystem controls the scan of the part and collects the data for transfer to the host computer. The host computer receives the data from the data acquisition subsystem, files the data, and transfers the data to the other subsystems for processing and display. The SAFT real-time processor performs the computationally intensive SAFT calculations. Finally, the data display module provides the image display to the operator for interpretation.

Table 9.1. Performance for Upgrades to SAFT-UT System

Sustained Data Acquisition Rate

(kBytes/sec)

\begin{tabular}{|c|c|c|c|}
\hline PDP11 and VAX-11/730 & PDP11 and Micro Vax & PC and MroroVax & PCand Sun \\
\hline 5 & 20 & 200 & 500 \\
\hline
\end{tabular}

Approximate Relative SAFT Processing Rate

(for thick material)

\begin{tabular}{|c|c|c|c|c|}
\hline VAX-11/780 & $\because$ SAFTRTP* & Sun Sparc 10, Model 30 \\
\hline 1 & 25 & 3 & MicrovAX LII & Sul \\
\hline
\end{tabular}

Data Display Rate

\begin{tabular}{|c|c|c|}
\hline Vax 11/730 with Ramtek & MicroVari with Ramtek & Sun Spar 10, Model 30 \\
\hline 1 & 25 & 250 \\
\hline
\end{tabular}

*RTP = real-time processor 


\subsection{Installation of a MicroVAX III}

The host computer subsystem was upgraded from a VAX 11/730 to a MicroVAX 3400 computer. This upgrade improved the three functions of the host computer subsystem: 1) transfer of data from the data acquisition module, 2) archive the data to a removable disk platter instead of 9-track tape, and 3) transfer of data to the display module. A functional diagram of the subsystem is shown in Figure 9.2. The hardware specifications are the following:

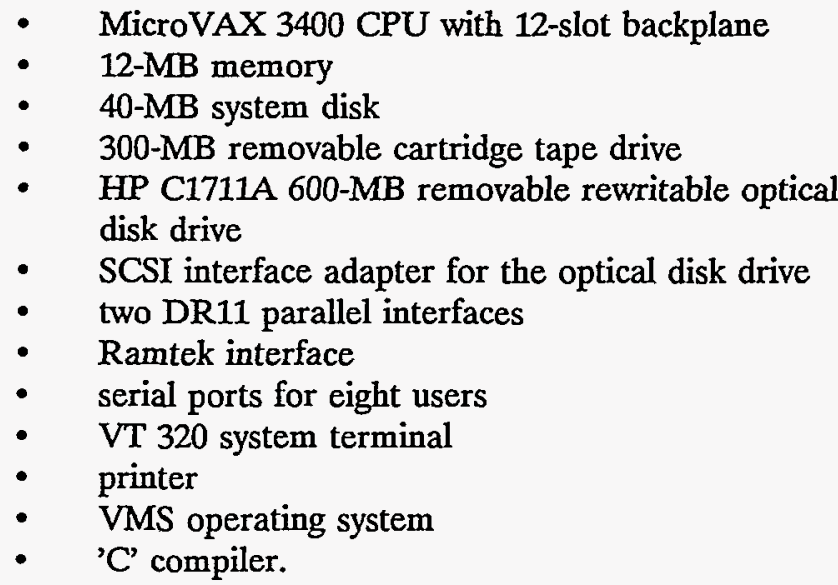

In addition, a few changes were made to the SAFT-UT data acquisition subsystem. The upgraded subsystem, shown in Figure 9.3, is divided into five components: mechanical scanner, acoustic subsystem, computer, disk drive, and interface to the host computer subsystem. The upgrades included

- a new 100-MB disk drive - This permits the data acquisition subsystem to operate better in the stand-alone mode.

- a joy-stick added to the scanner component to more easily position the scanner before beginning the inspection

- a DRV11-WA parallel interface added to more quickly transfer data to the host computer - A software device driver was written for this interface because this software was not available from the manufacturer.
Using this upgraded system, the time required to perform an inspection was decreased by a factor of 4 over the previous system. This was accomplished by improving the data transfer rate to the host computer from 5 $\mathrm{kBytes} / \mathrm{s}(\mathrm{kB} / \mathrm{s})$ to $20 \mathrm{kB} / \mathrm{s}$. The data acquisition rate to PDP11 memory was unchanged at $50 \mathrm{kB} / \mathrm{s}$ and limited to a total of $128 \mathrm{kB}$ per scan line with each A-scan not more than 1024 bytes in length.

The most dramatic improvement was made in the performance of data display calculations that routinely occur on the host CPU. The time required to perform axis transformations and image projections was decreased by a factor of 25 over the time required when using the VAX 11-730.

\subsection{Other System Upgrades for the Inspection of Nuclear Reactor Pres- sure Vessels}

Additional system upgrades included a robotic ultrasonic scanner, improvements to the SAFT-UT data file header, and a new data acquisition subsystem. A block diagram of the upgraded system is shown in Figure 9.4. The robotic scanner shown in Figure 9.5 uses a PUMA 562 industrial robotic arm, an RTD probe holder, and the existing SAFT-UT transducer gimbals. The PUMA 562 is a six-axis robotic arm with a reach of $914 \mathrm{~mm}$ (36 in.) and a repeatability of better than $0.10 \mathrm{~mm}$ (0.004 in.).

The robot is blind in the sense that it must be taught the inspection surface. However, the robot permits the systematic scanning of large complex objects, such as reactor pressure vessel nozzles, without moving a scanner track along the surface of the vessel. The SAFTUT robotic scanner assumes that the contact method will be used. The transducers are attached to the robot's end effector and moved along the surface of the material, permitting data acquisition at appropriately spaced intervals. The robotic scanner queries the operator about the location of the test material and asks the operator to teach it how to find the test material if it has no memory of the material's location in its work space. Once this is done, the robot can position itself anywhere on the surface of the test material that is within the robot's reach. The operator can move the robot's transducer along the surface of the test material 
by using the arrow keys on the data acquisition computer or by entering a material coordinate. Once the robot has been taught the location of the test material, it can perform the transformation from its own coordinates, which are fixed with respect to the base of its pedestal, to the coordinates of the test material. Of course, the robot can also perform the inverse of this transformation, that is, from material coordinates to its own coordinate system. If the robot is taught the location of the opening of a nozzle, it can move along and across the nozzle's blend zone. It can move along the inside of the nozzle as well. This can all be done by using the coordinates of the test material (reactor vessel). This feature of the robotic scanner gives it a significant advantage over a conventional (pipe) scanner. Scans of the test material can be controlled by the specification of material coordinates. This information, saved in the SAFT-UT data file header, permits the unambiguous identification of the location of ultrasonic indications. A complete description of the operation of the SAFT-UT robotic scanner is given in example form in Appendix B, "Tutorial Procedure for Operating the SAFT-UT Robotic Scanner."

To make the SAFT-UT data files portable to any computer platform, the header was upgraded to use an ASCII encoded format. For example, 'vel in material' was a floating point number in a 4- byte format that only a DEC computer could interpret. This header field is now an ASCII encoded string representation of the floating point number and, as such, requires 17 bytes. A complete list of changes to the SAFT-UT header is given in Appendix C. Some header fields were added to provide better documentation of the data. The unused header fields were deleted. The SAFT software was modified to recognize the new ASCII header as well as the old binary header. A software program was written to convert between the two header types. A listing of the ASCII header definitions is given in Appendix D.

The robotic data acquisition system controls the data acquisition process. It also provides local data storage, a data interface to the host computer, and the operator interface for the real-time mode of operation. The software is menu-driven to assist in ensuring that the SAFT parameters are correctly entered prior to data acquisition. An overview of the menu sequence is given in Appendix B.
If data are to be transferred to the SAFT subsystem concurrent with the scanning operation, then the connection is made to the host computer. A command from the robotic data acquisition module invokes the data transfer utility, and the user is prompted to determine if concurrent SAFT processing and/or real-time data display are desired.

The performance of the robotic data acquisition subsystem is as follows:

- The sustained data acquisition rate to MicroVAX 3400 memory is $200 \mathrm{kB} / \mathrm{s}$.

- The maximum amount of data in a scan line is limited only by the amount of host memory that can be allocated to it. Because the SAFT-UT MicroVAX has $12 \mathrm{MB}$, approximately $1 \mathrm{MB}$ is available for one scan line of data.

- The maximum length of the A-scans is $4 \mathrm{kB}$ in this system.

\subsection{SAFT-UT Upgrades for NRC's NDE Mobile Laboratory}

A redesigned SAFT-UT system has been assembled for the NRC's NDE Mobile Laboratory. This new system is significantly smaller in overall size and is referred to as the NDE Mobile Laboratory SAFT-UT field system. A block diagram of this new system is shown in Figure 9.6. The PC-486 computer performs the data acquisition function and the Graphics Workstation performs the SAFT-UT processing and data display functions. An electronic component diagram is shown in Figure 9.7.

The pipe scanner, shown in Figure 9.8, for this system is equipped with dual $\mathrm{X}$ axes where each axis is capable of $305 \mathrm{~mm}(12 \mathrm{in}$.) of movement. The scanner uses the style of pegged track that the NRC most commonly finds in inservice inspections of U.S. nuclear power plants. A 61-m (200-ft) cable connects the motor drivers with the pipe scanner. The field system electronics are shown in Figure 9.9. The motor drivers are kept out of the radiation zone and free of contamination. The controller (indexer) for the motor drivers is a host 
adapter for the PC ISA bus and is installed in the PC486 computer.

The UT electronics modules function as follows: The remote pulser/preamp is located on the pipe scanner. The preamp portion of this module provides a fixed 20 $\mathrm{dB}$ of gain and a 3-dB bandwidth of $7 \mathrm{MHz}$. This approach significantly increases signal-to-noise ratio by amplifying the echo signal before transmission on the $61-\mathrm{m}$ (200-ft) UT cable. Thus, any noise introduced during the transmission will be significantly less with respect to the echo signal. The pulser portion of the remote pulser/preamp produces the $400-\mathrm{V}$ excitation for the transducer. This excitation is a negative-going $400-\mathrm{V}$ square wave of programmable width. This width is set to produce the best excitation of the transducer; the setting usually matches one-half the wavelength of the transducer center frequency. The pulser obtains low-current $400-\mathrm{V}$ dc power via the $61-\mathrm{m}$ (200-ft) UT cable along with trigger and pulse width logic. This approach, used in the original SAFT-UT system, prevents distortion of the high-voltage transducer excitation by the $61-\mathrm{m}(200-\mathrm{ft})$ cable and prevents coupling with other signals.

A time-variable-gain (TVG) amplifier was supplied with the SAFT-UT system for the NDE Mobile Laboratory. The TVG amplifier adjusts the gain during the reception of the ultrasonic echo in such a way as to compensate for attenuation, compensate for beam divergence, and increase the dynamic range of the system. The TVG amplifier supplies the input for the STR 8100 digitizer where the signal is sampled and converted to digital form.

The PC-486 computer, shown in Figure 9.10, transfers the ultrasonic data to the SAFT-UT host computer through its 488 interface. A 5.25 -in. (13.3 cm), writeonce (WORM) laser disk drive is attached to the PC486 to provide local data storage if necessary.

The SAFT-UT host computer, shown in Figure 9.11, consists of a SUN Sparc 10, Model 30 computer configured as follows:
- SUN Sparc 10 Model 30 computer with color monitor

- $\quad 32-\mathrm{MB}$ memory

- $\quad 400-\mathrm{MB}$ internal system disk

- 400-MB internal disk for UT data

- write-once laser disk drive for 5.25 -in. $(13.3 \mathrm{~cm})$ 800-MB removable platters

- IEEE 488 interface.

The programs available on the MicroVAX III VMS system described in Hall, Reid, and Doctor (1988) were ported to the SUN Sparc 10 computer running UNIX $\mathrm{X}$-Windows operating system. Only the graphics (data presentation) tool required significant modification. Figure 9.12 shows the $X$-windows displays typical for the Mobile Laboratory SAFT-UT system. The other code required minor changes to remove VMS-specific calls to the disk I/O routines and nothing more.

The NDE Mobile Laboratory SAFT-UT system performs as follows:

- $\quad$ sustained data rate to host memory: $500 \mathrm{kB} / \mathrm{s}$

- maximum amount of data per scan line: $4 \mathrm{MB}$

- maximum length of the A-scans: $16 \mathrm{kB}$

- SAFT processing rate equal to SAFT-UT RealTime Processor

- maximum data file size: $300 \mathrm{MB}$.

In summary, the advances in electronics and computer methods are making SAFT-UT more portable (smaller system), less expensive, and much more powerful. The applicability of SAFT-UT to the inspection of LWR components will continue to become more attractive from a cost and performance standpoint as advances occur in digital electronics and data visualization software. 


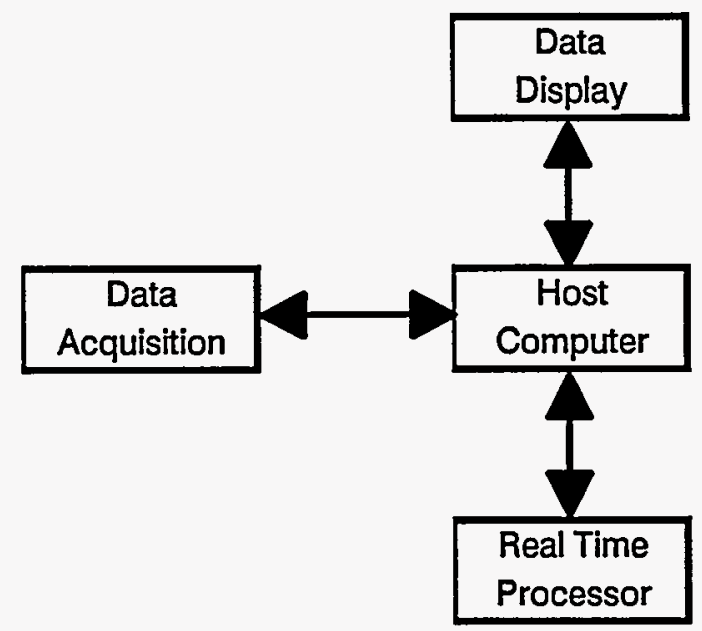

Figure 9.1. SAFT-UT Field System

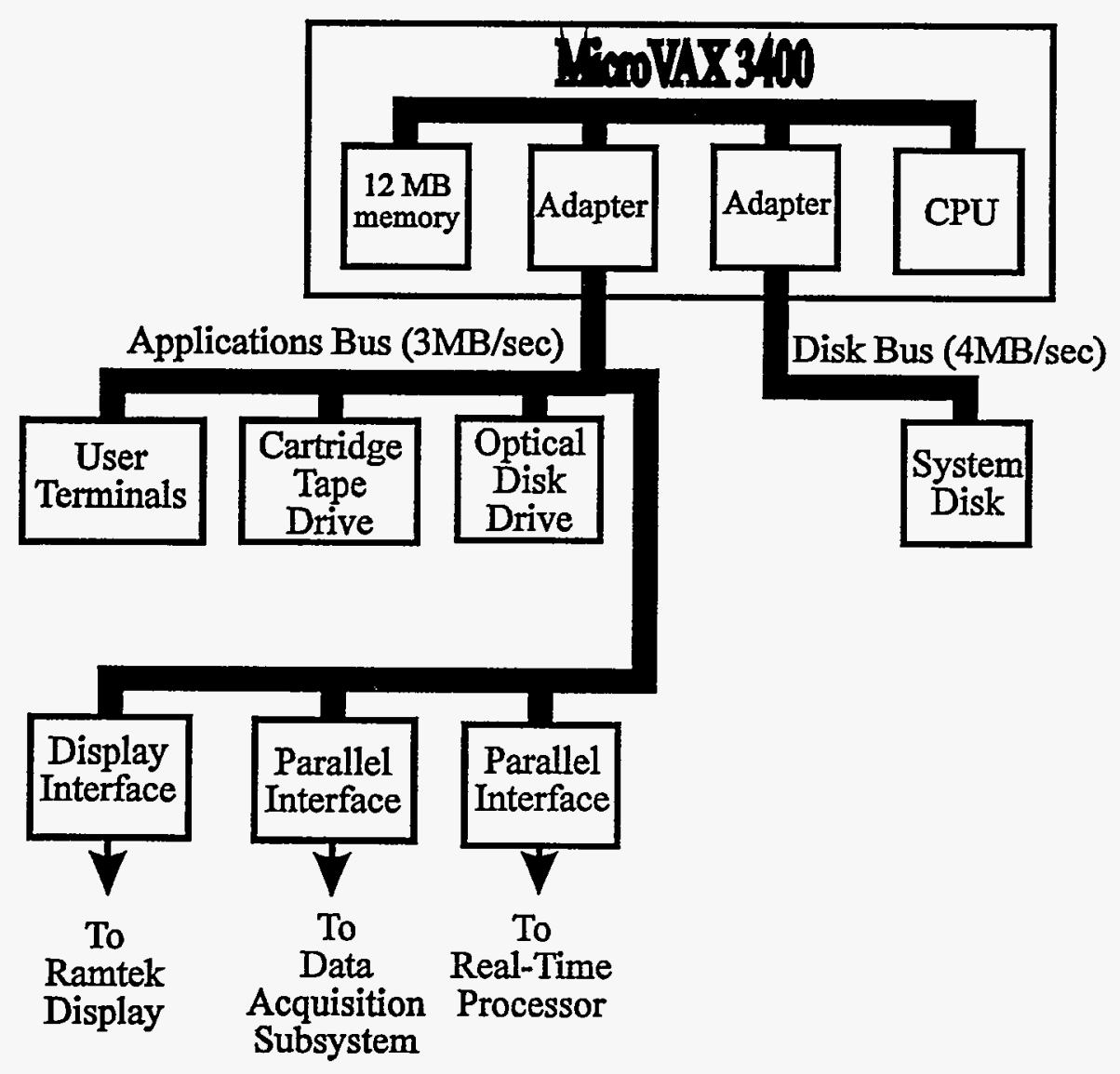

Figure 9.2. SAFT-UT Host Computer Subsystem 


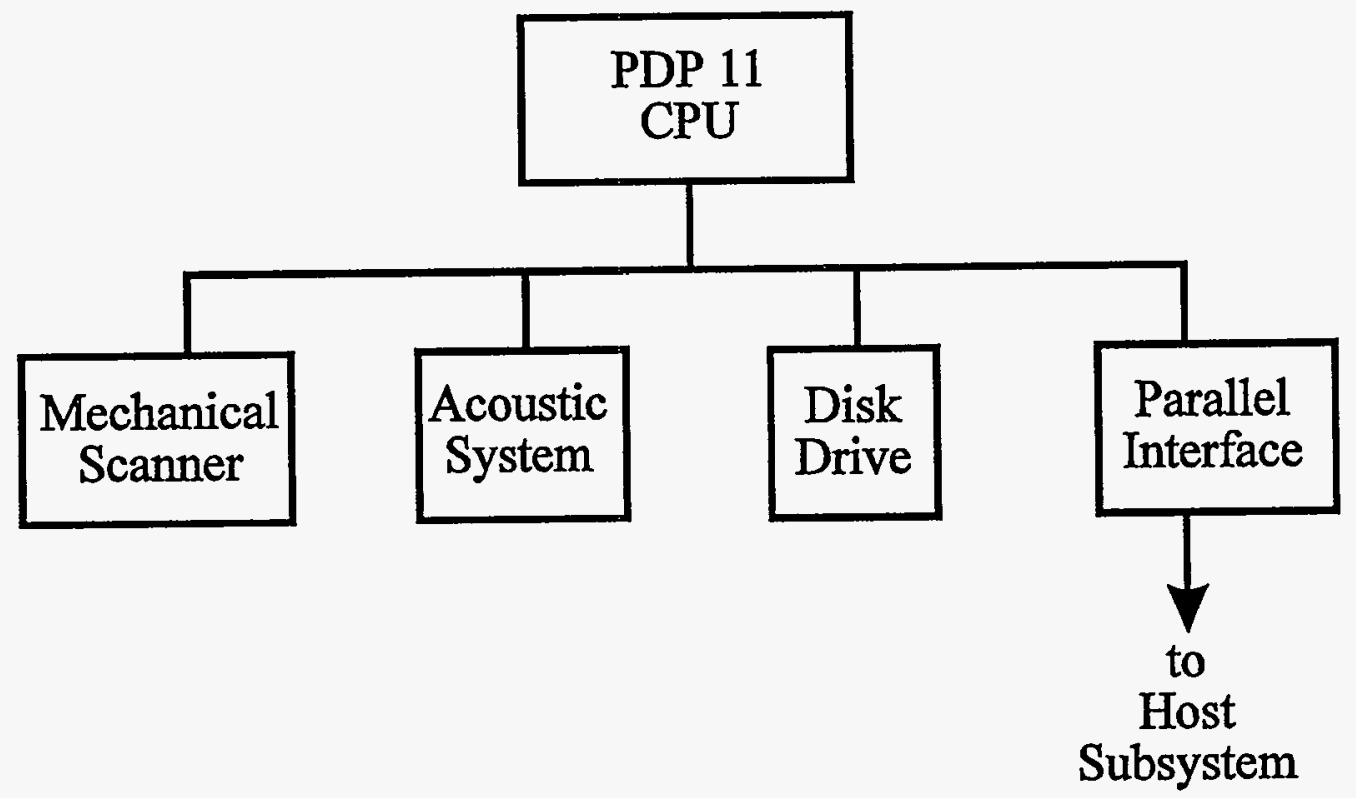

Figure 9.3. SAFT-UT PDP11 Data Acquisition Subsystem

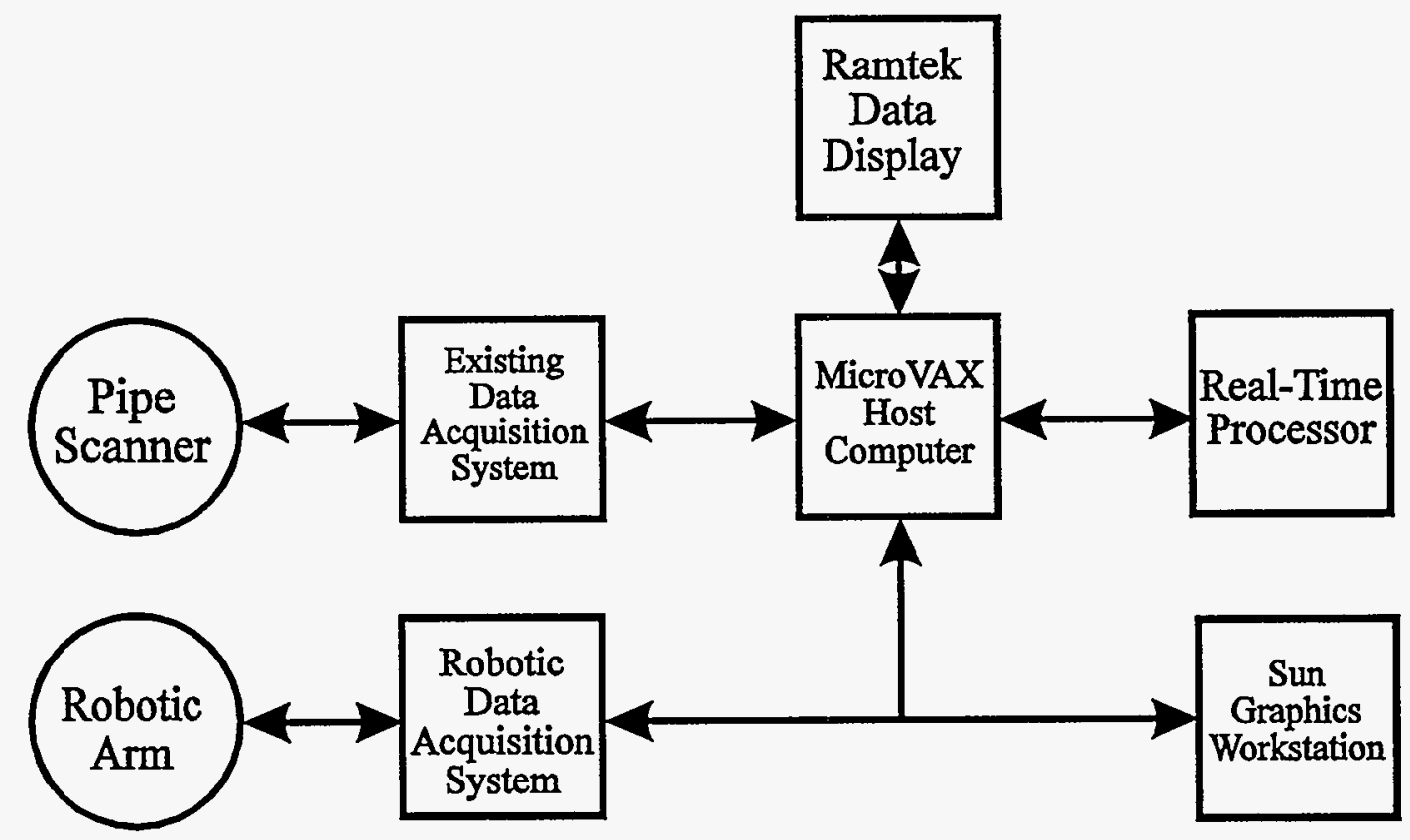

Figure 9.4. SAFT-UT Field System Used at Pressure Vessel Research User Facility (PVRUF) 


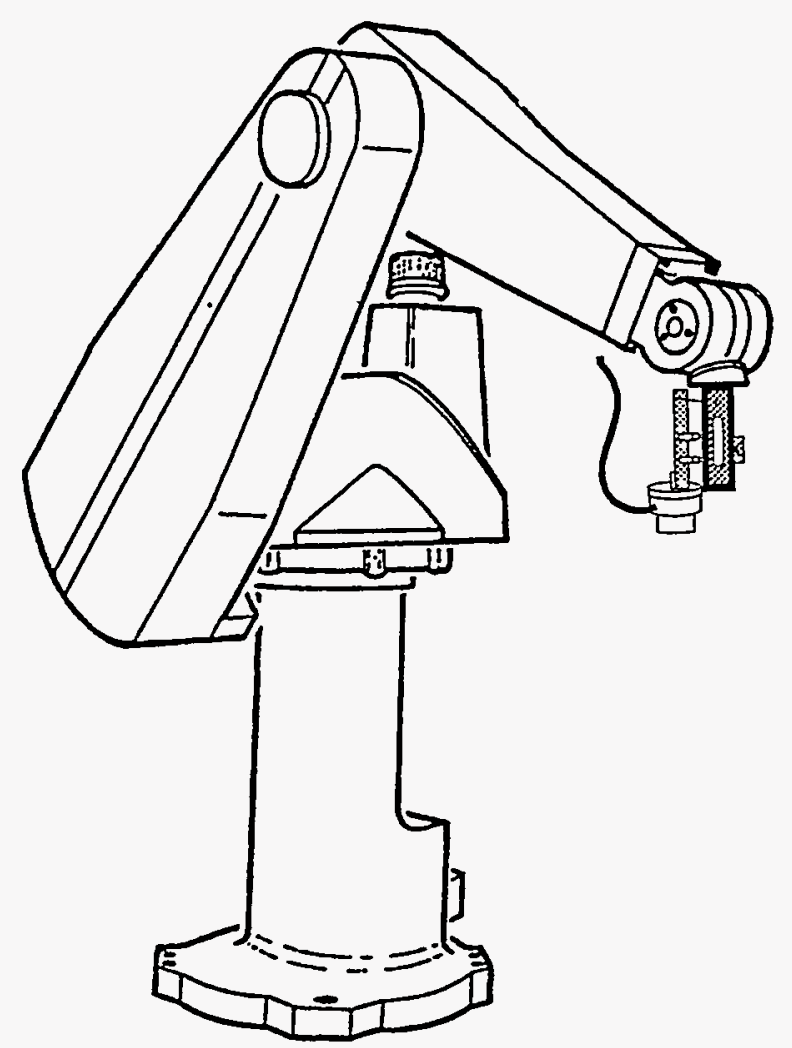

Figure 9.5. SAFT-UT Robotic Scanner

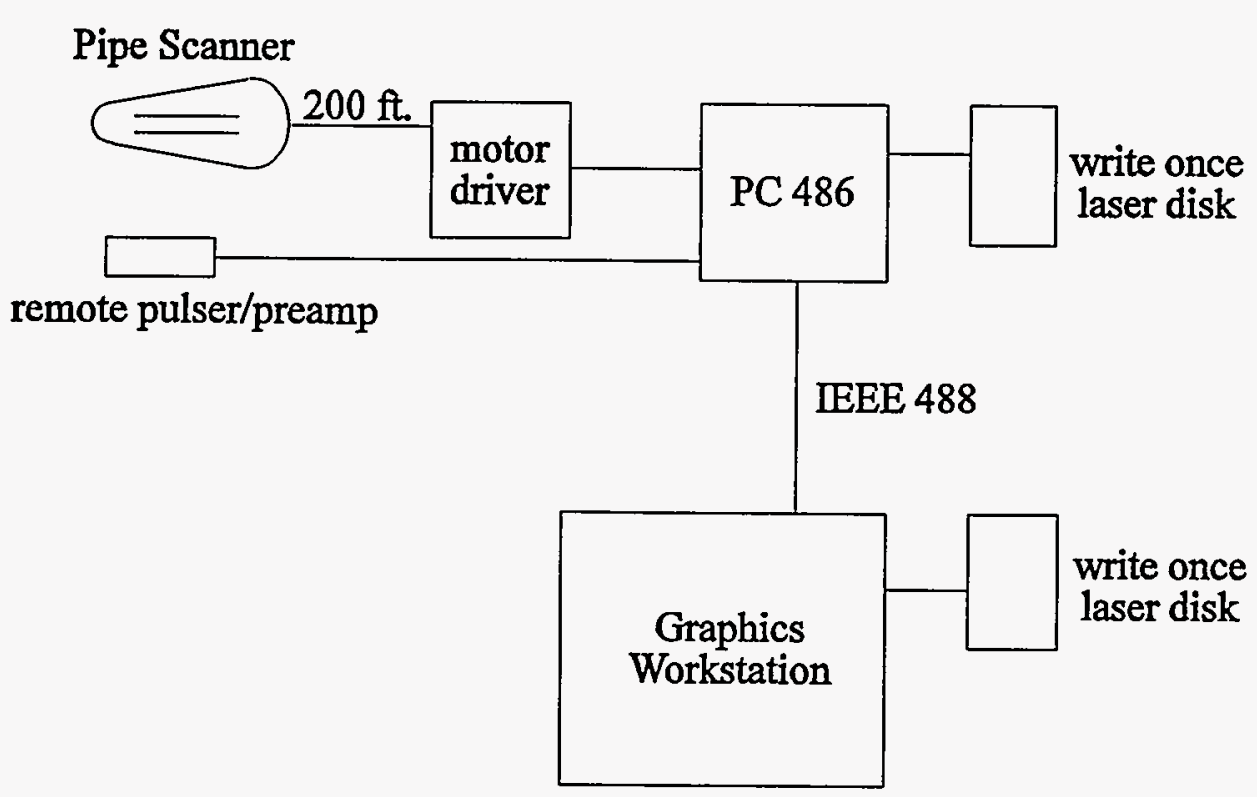

Figure 9.6. NDE Mobile Laboratory SAFT-UT Field System 


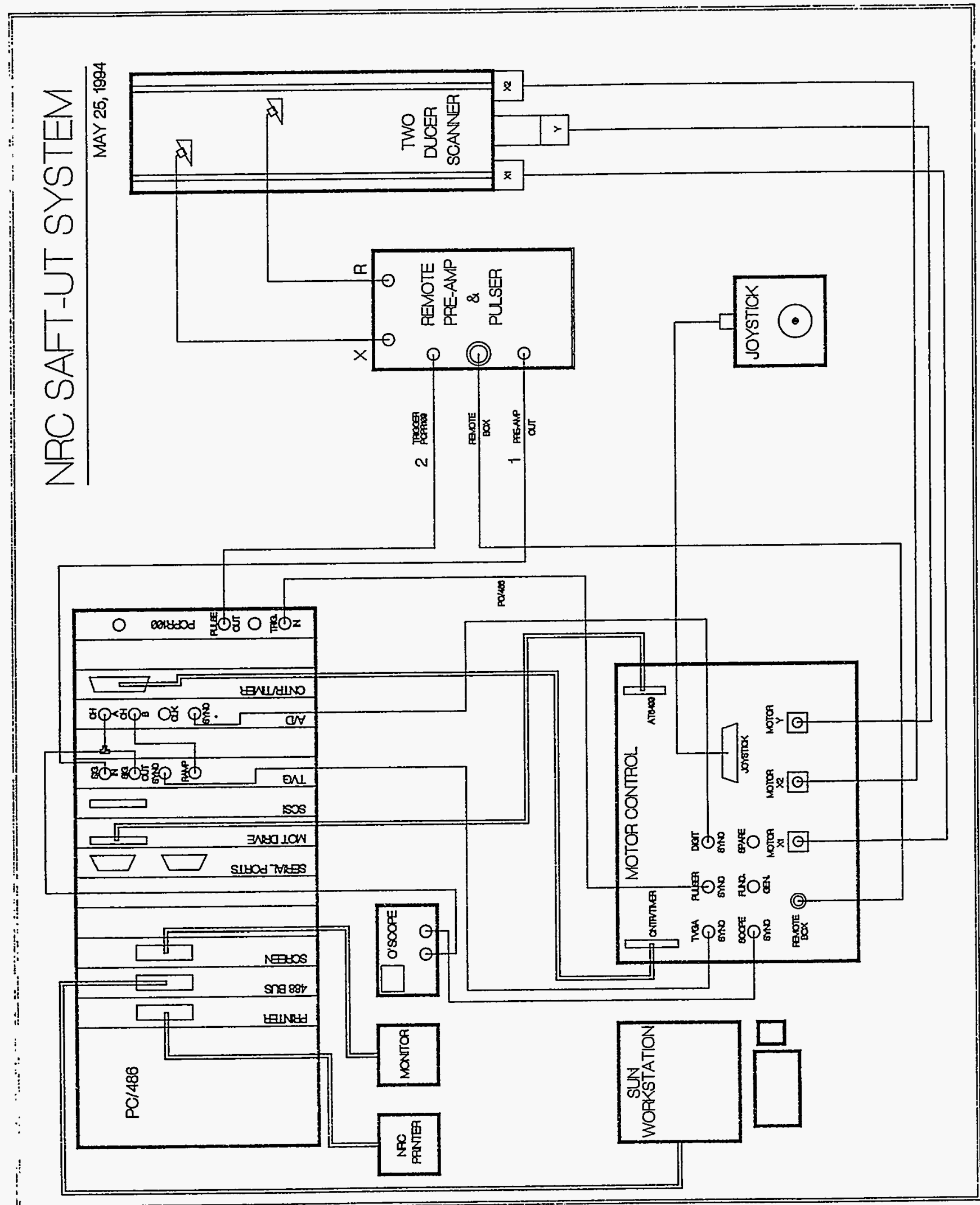




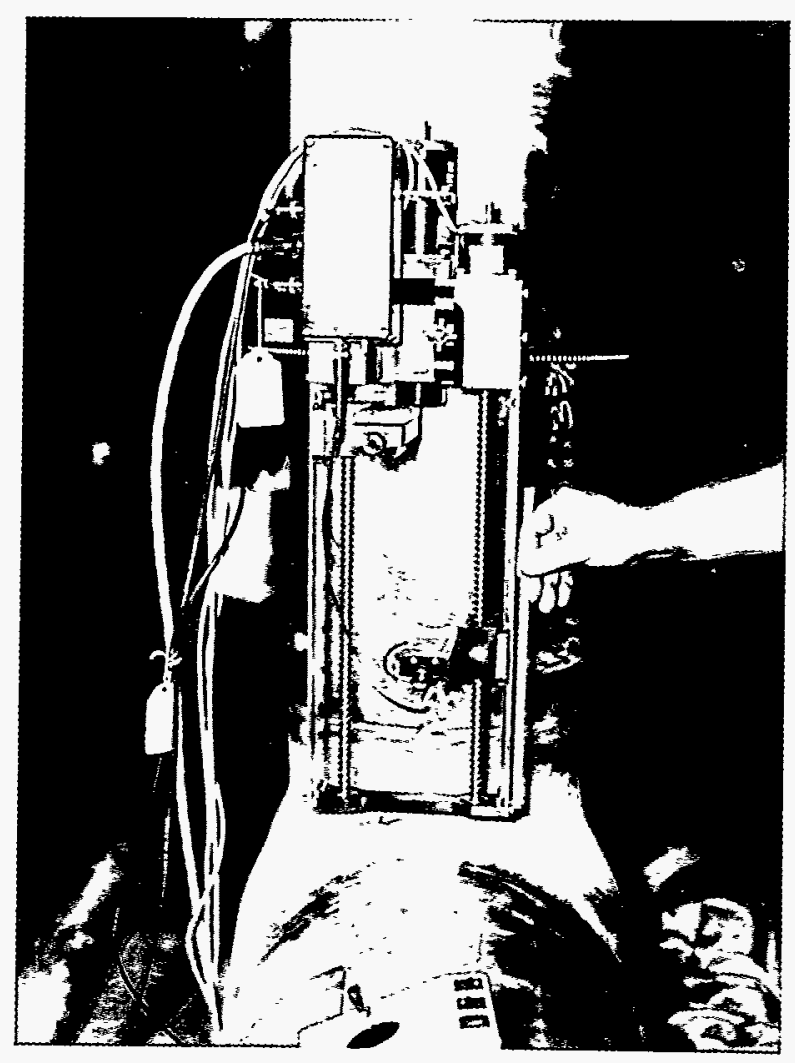

Figure 9.8. NDE Mobile Laboratory SAFT-UT Field System Scanner

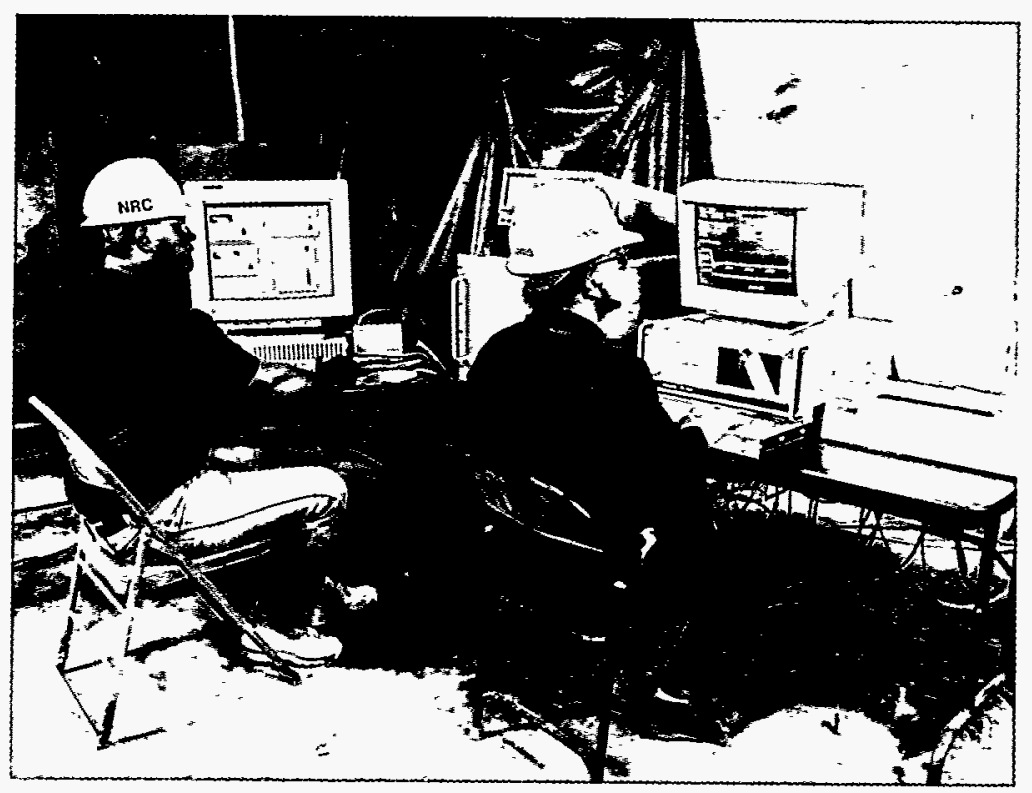

Figure 9.9. First Deployment of NDE Mobile Laboratory SAFT-UT Field System Electronics at a Reactor Site--WNP-1 


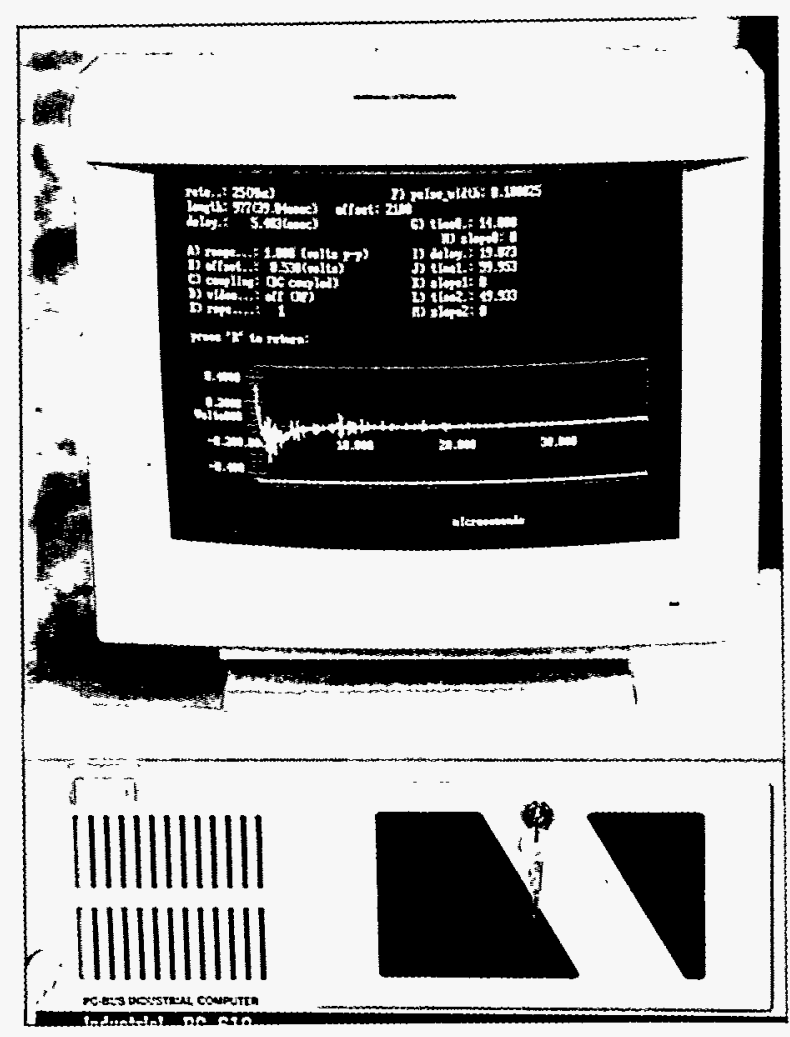

Figure 9.10. NDE Mobile Laboratory SAFT-UT PC-486

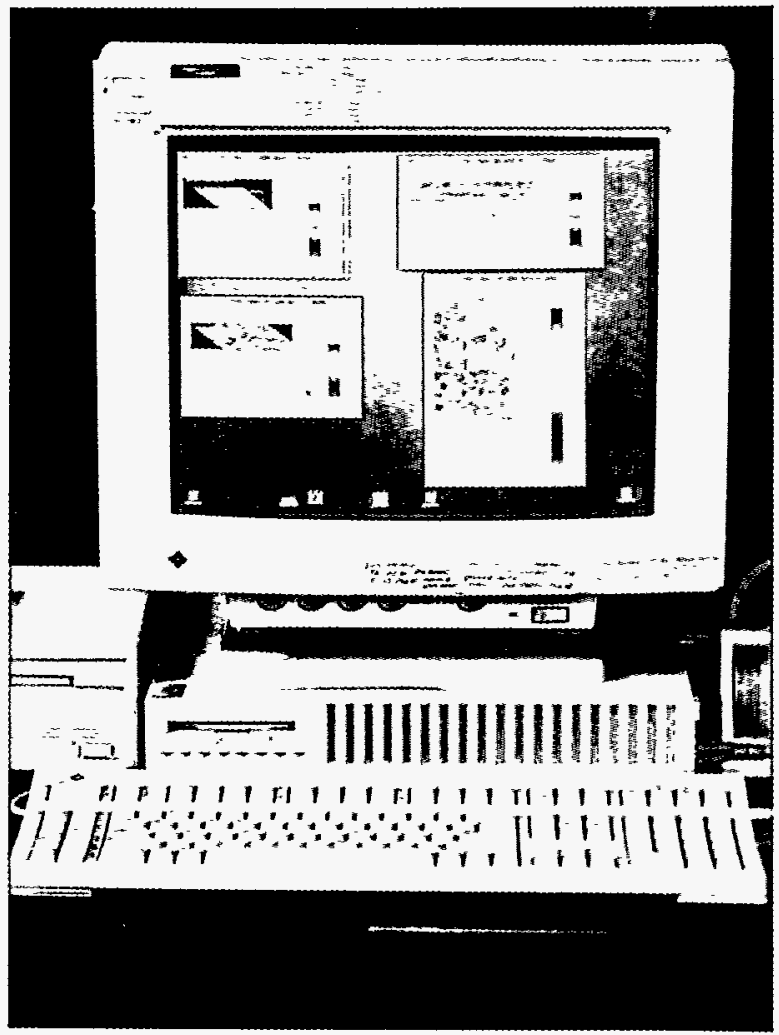

Figure 9.11. NDE Mobile Laboratory SAFT-UT Host Computer 


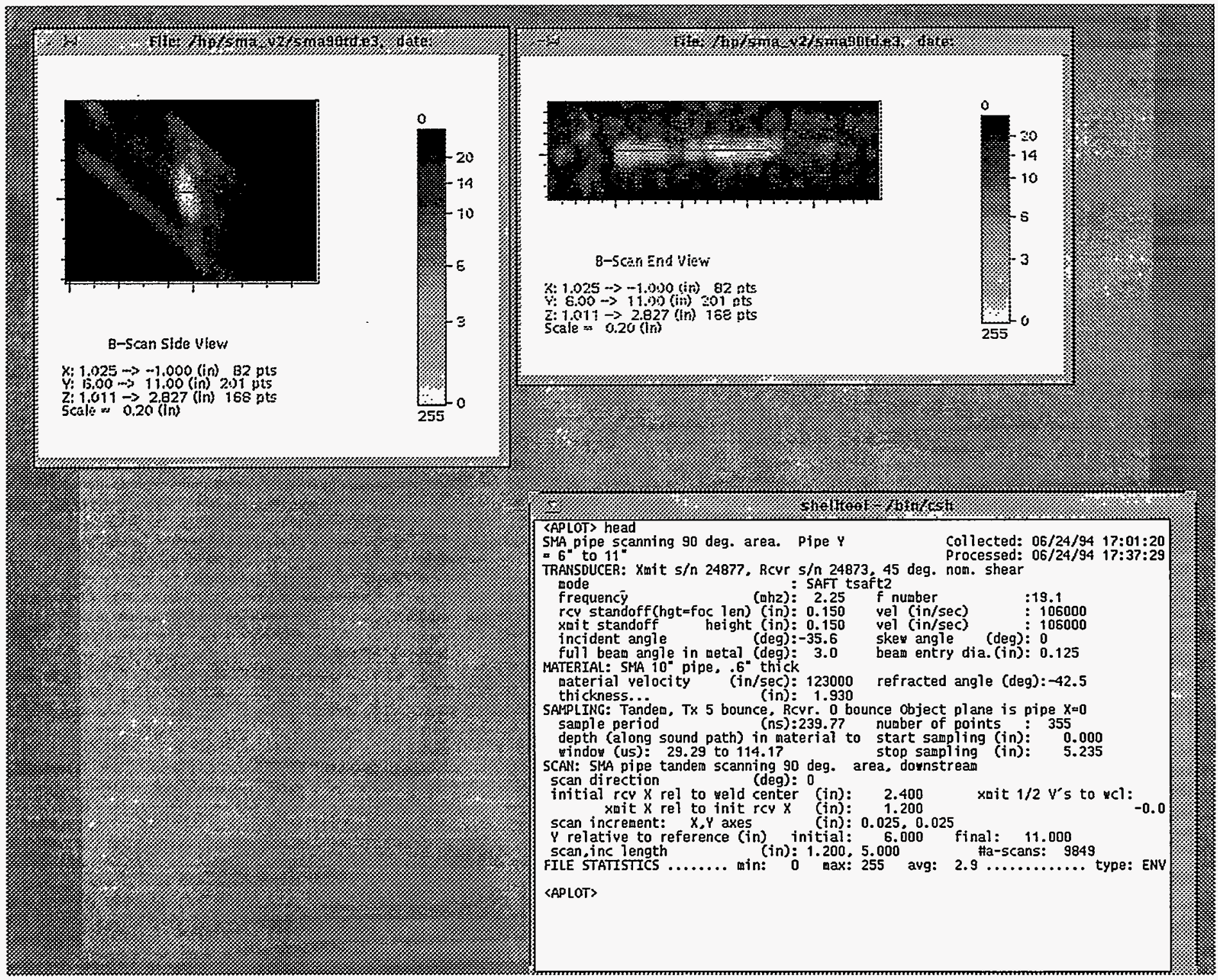

Figure 9.12. Typical Data Analysis Display from NRC Mobile Laboratory SAFT-UT System 


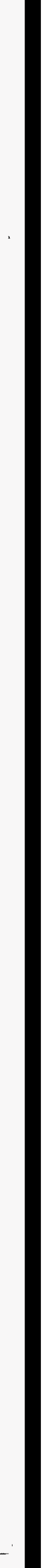




\subsection{SAFT-UT Validation}

The SAFT-UT technology has been under evaluation since the program started a number of years ago. However, many of these evaluations have been conducted on small sample sets without objective and blind testing conditions. In other cases, the defects detected and characterized were not destructively validated and, thus, do not provide hard evidence of the technology's effectiveness.

This section describes several such tests conducted to more fully quantify SAFT-UT system performance. The testing included SAFT-UT participation in the Mini-Round Robin (MRR) conducted at PNL, testing at the Electric Power Research Institute (EPRI) NDE Center, and testing conducted as part of the Programme for the Inspection of Steel Components Phase 3 (PISC-III).

\subsection{Validation of SAFT-UT in the Mini-Round Robin}

\subsubsection{The Mini-Round Robin}

The round robin study, conducted at PNL is completely described in NUREG/CR-4908 (Heasler et al. 1990). The study was conducted to provide an engineering data base for UT/ISI that would help

- quantify the effect of training and performance demonstration testing that resulted from IEB 8302

- quantify the differences in capability between detecting long (greater than $76 \mathrm{~mm}$ [3 in.]) cracks versus short (less than $51 \mathrm{~mm}$ [ 2 in.]) cracks

- quantify the capability of UT/ISI inspectors to determine the length and depth of intergranular stress corrosion cracks (IGSCCs).

The round robin study involved six two-person manual inspector teams and three automated inspection teams. SAFT-UT was an add-on after the original testing had been completed and before destructive testing. It was decided not to include the SAFT-UT results in NUREG/CR- 4908 because its inclusion as part of the original study had not been planned. It needs to be noted that the SAFT-UT inspections were conducted by a PNL staff member who was totally ignorant of the
MRR study and had no connection with the specimens in any way. The specimens used in this study contained laboratory-produced IGSCC. Key features of the specimens used in the MRR are listed in Tables 10.1 and 10.2.

The results were evaluated using the concept of grading units to define two performance parameters-probability of detection (POD) and false call probability (FCP). Each inspector's detection capabilities are estimated using these two statistics, which are defined by

$$
\begin{aligned}
P O D & =\frac{\text { Number of Correct Detections }}{\text { Number of Cracked Grading Units Inspected }} \\
F C P & =\frac{\text { Number of Blank Grading Units Called Cracked }}{\text { Number of Blank Grading Units Inspected }}
\end{aligned}
$$

The round robin was constructed so these two statistics could be estimated efficiently for different inspection conditions. These statistics are employed in this section to examine inspection performance.

The POD and FCP statistics always refer to a unit of material of particular size, called a grading unit. Therefore, individual detection and false call statistics are always associated with a unique grading unit. The detection statistic for a particular grading unit is 1 if a crack was called within the unit and 0 if no crack was called. Individual grading units were separated by a minimum of $25 \mathrm{~mm}$ ( $1 \mathrm{in}$.) of blank weld material in the circumferential direction with no separation axially for grading units on opposite sides of the weld; all the grading units have the same circumferential length. Grading units are randomly located within the test specimens but are not actually marked on the outside of the specimen, so the inspectors do not know their location. See Appendix E of Heasler et al. (1990), which shows all the grading units on each MRR specimen.

Each grading unit was classified as defective if it contained a crack or as blank if it did not contain a crack. A true-state data base describing defective and blank grading units was developed for each test specimen used in the MRR.

The 69 grading units were large enough to encompass the cracks in the specimens. Because the cracks were 
Table 10.1. Pipe Specimens Used in MRR

\begin{tabular}{|c|c|c|c|c|c|}
\hline \multirow{2}{*}{ Nupesamples } & \multirow[b]{2}{*}{ Oiameter, $\mathrm{n}$} & \multirow{2}{*}{$\begin{array}{l}\text { Nominal wall } \\
\text { Thicknesss, } \\
\text { in /Schedule }\end{array}$} & \multirow{2}{*}{ Wlawtsength, in } & \multicolumn{2}{|c|}{$\begin{array}{l}\text { Number of Grading } \\
\text { Units }\end{array}$} \\
\hline & & & & cracked & olank \\
\hline 5 & $\begin{array}{c}10 \\
(254 \mathrm{~mm})\end{array}$ & $\begin{array}{c}0.593 / \mathrm{Sch} .80 \\
(15 \mathrm{~mm})\end{array}$ & $\begin{array}{c}\text { Short }(<1-1.5) \\
(<25 \mathrm{~mm}-60 \mathrm{~mm})\end{array}$ & 13 & 31 \\
\hline 4 & $\begin{array}{c}12 \\
(305 \mathrm{~mm})\end{array}$ & $\begin{array}{c}0.688 / \mathrm{Sch} .80 \\
(17.5 \mathrm{~mm})\end{array}$ & $\begin{array}{c}\text { Long }(3-40) \\
(76 \mathrm{~mm}-1016 \mathrm{~mm}) \\
\end{array}$ & 12 & 6 \\
\hline 1 & $\begin{array}{c}12 \\
(305 \mathrm{~mm})\end{array}$ & $\begin{array}{c}0.844 / \text { Sch. } 100 \\
(21.4 \mathrm{~mm})\end{array}$ & $\begin{array}{c}\text { Long }(3-9) \\
(76 \mathrm{~mm}-229 \mathrm{~mm})\end{array}$ & 3 & 4 \\
\hline \multicolumn{4}{|c|}{ Total } & 28 & 41 \\
\hline
\end{tabular}

of two disparate lengths, two different lengths were used to calculate the statistical information. Some grading units were $76 \mathrm{~mm}$ (3 in.) in length and were classified with respect to through-wall penetration by designating them "S" or "D." If the cracks were particularly long, then they were labeled with an "L" designator, and the grading units were expanded to be $203 \mathrm{~mm}$ (8 in.) in length. " $S$ " cracks had an average maximum depth of $4 \%$ through-wall, while "D" cracks had an average maximum depth of $18 \%$. The "L" cracks had an average length of $152 \mathrm{~mm}$ (6 in.), while the "S" cracks had an average length of $7.6 \mathrm{~mm}(0.3 \mathrm{in}$.), and the "D" cracks had an average length of $22.9 \mathrm{~mm}(0.9$ in.).

Table 10.2. Properties of IGSCC Cracks in MRR Specimens

\begin{tabular}{|c|c|c|c|c|c|}
\hline \multirow{2}{*}{$\begin{array}{l}\text { erack } \\
\text { Oategory }\end{array}$} & \multirow{2}{*}{$\begin{array}{l}\text { Noo of } \\
\text { Cracks }\end{array}$} & \multicolumn{2}{|c|}{ Average } & \multicolumn{2}{|c|}{ अ $:$ Range $\because$} \\
\hline & & $\begin{array}{l}\text { Tength, } \\
\text { in. }\end{array}$ & $\begin{array}{l}\text { Depth, } \\
\text { in } / \% \text { of wall }\end{array}$ & Kength; in. & 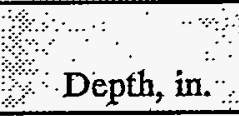 \\
\hline$S$ & 5 & $0.29(7.4 \mathrm{~mm})$ & $0.042(1.1 \mathrm{~mm}) / 4$ & $\begin{array}{c}0.1-0.67 \\
\left(\begin{array}{c}2.5 \mathrm{~mm}-17 \\
\mathrm{~mm})\end{array}\right.\end{array}$ & $\begin{array}{c}0.011-0.071 \\
(0.8-1.8 \mathrm{~mm})\end{array}$ \\
\hline D & 9 & $0.92(23.4 \mathrm{~mm})$ & $0.177 / 18(4.5 \mathrm{~mm})$ & $\begin{array}{c}0.16-3.35 \\
(4.1-85.1 \mathrm{~mm})\end{array}$ & $\begin{array}{c}0.109-0.247 \\
(2.8-6.3 \mathrm{~mm})\end{array}$ \\
\hline $\mathbf{L}$ & 14 & $7.39(187.7 \mathrm{~mm})$ & not measured ${ }^{(a)}$ & $\begin{array}{c}3.6-10 \\
(91.4-254 \mathrm{~mm})\end{array}$ & $\begin{array}{c}0.170-0.440 \\
(4.3-11.2 \mathrm{~mm})\end{array}$ \\
\hline
\end{tabular}

(a) The long cracks were destructively analyzed over only a portion of their length, so the location and value of greatest depth is unknown. However, the data reported is for the greatest depth in the destructively analyzed zones. 
Each specimen inspected by the SAFT-UT field system was first prepared by an MRR official; i.e., each specimen was masked to expose only a specified area to be scanned. There were a total of 47 such areas averaging $254 \mathrm{~mm}(10 \mathrm{in}$.) in length. The areas were divided among three different sizes of pipe; 14 were on 254mm- (10-in.) diameter Schedule 80,29 on $305-\mathrm{mm}$ - (12in.) diameter Schedule 80, and 4 areas on 305-mm- (12in.) diameter Schedule 100 pipe specimens.

\subsubsection{SAFT-UT Inspection Procedure}

Three different transducer configurations were used on each area scanned using SAFT-UT. A single transducer normal to the front surface was used for profiling the pipe inner surface to help identify geometrical conditions that may present crack-like indications. A single transducer using angle beam was used for crack detection and lateral location relative to the weld center, and tandem transducers using angle beam were used for crack detection confirmation.

The normal beam transducer was chosen to provide good penetration through the weldment; for this application, the transducer was $6 \mathrm{~mm}$ (0.25 in.) in diameter and operated at $5 \mathrm{MHz}$. The transducers used for the angle beam inspection and the tandem inspection were $6 \mathrm{~mm}(0.25 \mathrm{in}$.) in diameter but operated at $2.25 \mathrm{MHz}$.

Because the SAFT real-time processor was not operating at this time, the scanning and data analysis took much longer than the time required by the other MRR teams. In this sense, there was not a realistic comparison with the manual or commercially available automated systems. However, the effectiveness of the technique was still validated.

\subsubsection{SAFT-UT Analysis Procedure}

All three scanning configurations were used during the data analysis. The $-6-\mathrm{dB}$ level was chosen to determine the crack length. Each crack was isolated, and the image color scale was adjusted by the maximum value of the isolated crack. This avoided undersizing small cracks or cracks that are not good acoustic reflectors that may be adjacent to a strong acoustic reflection.

At the time this data was collected, the SAFT real-time processor did not exist. Thus, the sizes of the areas scanned were minimized to make three-dimensional SAFT processing practical on the data files. Indications were considered cracks only if they lay in a predetermined "crack zone." This region was approximately one-half the thickness of the pipe specimen plus the weld root width from the weld center. Naturally, indications that lay close to the weld center presented the biggest problem in interpretation because of possible interference from weld root indications in this area. Indications that lay on the outer edge of the crack zone were generally noted but not classified as cracks because the SAFT aperture was limited to make the files small for practical processing.

\subsubsection{SAFT-UT Performance Results and Discussion}

Figures 10.1 through 10.6 show the relative operating characteristic (ROC) curves of the SAFT team, manual teams, and advanced teams under various crack conditions. The ROC curve quantifies the relationship between POD and FCP results based on the decision criteria employed. Five decision criteria were used based on the confidence level of the particular call. These ranged from "definitely not a crack" to "possibly a crack" to "definitely a crack." Each graph shows the ROC curve displayed in the center of the graph; the upper and lower curves describe the upper and lower confidence limits of the ROC fit. The scattered letters are the actual data points used for the ROC curve fit. A performance curve intersecting the area in the upper left-hand corner of these plots is considered acceptable.

Figure 10.1 shows the ROC curves for " $\mathrm{D}$ " and "S" cracks for the SAFT team. The SAFT team performance is better for " $\mathrm{D}$ " cracks because of improved signal-to-noise ratio. Figure 10.2 shows the ROC curves for " $D$ " and "L" cracks. The SAFT team performed equally well for these cracks. Figures 10.3 and 10.4 show the ROC curves for the manual teams' average performance on " $D$," "S," and "L" cracks. When these figures are compared with the previously described plots, they suggest that the SAFT team had better performance in all three crack categories than the average of the manual teams. The final set of ROC curves (Figures 10.5 and 10.6) shows the average performance of the advanced teams on "D," "S," and "L" cracks. A comparison of these plots to Figures 10.1 and 10.2 suggests that the SAFT team's performance 
was better than the average of the advanced teams' performance for these cracks.

Tabular forms of these plots in Tables 10.3, 10.4, and 10.5 show the POD and FCP percentages for various cracks at the crack/no-crack decision threshold for the SAFT, average advanced, and average manual team performance.

Table 10.3. Crack/No-Crack POD and FCP, SAFT-UT

\begin{tabular}{|c|c|c|c|}
\hline & "L" & S" & "D" \\
\hline POD & $95 \%$ & $67 \%$ & $90 \%$ \\
\hline FCP & $18 \%$ & $14 \%$ & $14 \%$ \\
\hline
\end{tabular}

Table 10.4. Crack/No-Crack POD and FCP, Average of Advanced Teams

\begin{tabular}{|c|c|c|c|}
\hline & "L" & "S" & "D" \\
\hline POD & $76 \%$ & $67 \%$ & $87 \%$ \\
\hline FCP & $20 \%$ & $21 \%$ & $21 \%$ \\
\hline
\end{tabular}

Table 10.5. Crack/No-Crack POD and FCP, Average of Manual Teams

\begin{tabular}{|c|c|c|c|}
\hline & "L" & "S" & "D" \\
\hline POD & $72 \%$ & $43 \%$ & $69 \%$ \\
\hline FCP & $33 \%$ & $21 \%$ & $21 \%$ \\
\hline
\end{tabular}

False calls made by the SAFT team proved to be primarily from unusual weld-root zone conditions that presented strong IGSCC-type indications located close to the weld fusion line. Four false calls were made in the short grading units, and two false calls made in the long grading units. Two of the false calls made in the long grading units were on a double weld bounce that produced an IGSCC-type response.
One short and shallow "S" crack, $5 \mathrm{~mm}(0.2 \mathrm{in}$.) long and 5\% through-wall, was not detected. One "D" crack also was not detected because of an error in initial area presentation (the crack was on the far side of the weld). One "L" crack was detected but not classified as a crack because it was located on the edge of the crack zone [approximately $19 \mathrm{~mm}(0.75 \mathrm{in}$.) from the weld center] that had been previously established. The PNL staff had selected the crack zone and SAFT scan area to be slightly larger than one-half the thickness of the pipe specimen plus the weld root width. This was obviously a wrong decision and was corrected for future work.

\subsubsection{Conclusions of the SAFT-UT Perfor- mance on the MRR}

The performance was truly solid and represented a suitable starting point for future work. There were still many things to learn in terms of system performance on various crack conditions, but this work was very encouraging. It should be noted that no sizing was done on these cracks: at that time, the work that had been done was insufficient for PNL to have confidence in the results.

\subsection{Validation of SAFT-UT for IGSCC Sizing}

PNL decided that the technology needed to be demonstrated in a blind test accepted by the industry. One of these tests is the IGSCC Sizing Test conducted at the EPRI NDE Center. Because PNL does not provide qualified inspection services and has no certification program, it was proposed that PNL take the test at the NDE Center and obtain a letter stating that the SAFTUT team performance would have passed or not passed the accepted industry performance demonstration test.

\subsubsection{SAFT-UT Validation Testing Proce- dure}

The SAFT-UT system was sent to the NDE Center. After spending one day in training, the PNL team took the test following all of the normal constraints. The procedure consisted of performing one scan on each specimen using a 6-mm-diameter $\left(0.25\right.$-in.) $45^{\circ}$ shear transducer operating at $2.25 \mathrm{MHz}$. This was done to 
confirm that an IGSCC was present in the specimen. Next, a tandem scan was performed using two transducers identical to that used in pulse-echo. In both cases, the data was then SAFT processed. Data interpretation consisted of confirming the presence of the IGSCC in the pulse-echo scan and then examining the tandem SAFT image. The sizing data was taken from the tandem image; the value measured in this image was reported for the sizing test.

\subsubsection{Discussion of SAFT-UT Sizing Results}

The results from this test are shown in Figure 10.7. As can be seen, the system performed very well. The values obtained were ones that anyone could generate because they were taken directly from the image without any manipulations.

After the test, discussions occurred with other people who have taken this test. PNL discovered that the specimens were constructed using a $30^{\circ}$ weld preparation angle. In retrospect, it would have been advisable to have used a $60^{\circ}$ inspection angle to reflect off the inside surface and strike the crack normally. This would have potentially provided the maximum and best signal, particularly for the deepest cracks. The results in Figure 10.7 indicate a tendency to undersize the deeper cracks. This is believed to be related to the cracks following the weld preparation angle. Use of the $60^{\circ}$ inspection angle would have provided some refinement of the crack sizes for these deeper cracks.

\subsubsection{Conclusions of SAFT-UT from the IGSCC Sizing Test}

The performance demonstrated in these test results shows that a tremendous amount of sizing information contained in the $45^{\circ}$ inspection is normally not utilized. Signal processing techniques like SAFT are needed to effectively use the information contained there.

Researchers concluded also that it is important to understand how the defects propagate so that, as in this case, optimum illumination (i.e., $60^{\circ}$ ) can be employed to provide the highest precision in the estimates generated.
Finally, this test provides a blind demonstration of the effectiveness of the SAFT-UT technique that anyone could duplicate by following the simple rules stated.

\subsection{Validation of SAFT-UT for IGSCC Detection}

After the IGSCC Sizing Test, it was decided to follow a similar procedure and participate in the performance demonstration IGSCC Detection Test also administered at the EPRI NDE Center. Again, this was taken not to become qualified but simply to subject the technology to the same blind test that industry has developed to screen those techniques that are effective as a prerequisite for performing inspections in the field.

\subsubsection{SAFT-UT Detection Test Procedure}

The tests that were performed used a number of transducers that included a contact transducer for normal beam to profile the weldment and counterbore region that was based on the specimen either a $2.25 \mathrm{MHz}$ or 5 $\mathrm{MHz}$ unit. The circumferential scans were made using $1.5 \mathrm{MHz}$ at both $45^{\circ}$ and $60^{\circ}$ shear. There were skew scans taken using the $45^{\circ}$ transducer skewed to $\pm 15^{\circ}$ and $\pm 45^{\circ}$. The normal beam, the $45^{\circ}$ and the $60^{\circ}$ scans were SAFT processed. The skew scans were not processed and were used only to aid in detecting axially oriented cracks.

A set of rules was developed for consistently evaluating the SAFT images. The hierarchy for the rules was as listed below:

1. The weld zone must be mapped with a $0^{\circ}$ inspection to determine weld root and any counterbore conditions.

2. There must be a strong response from the $45^{\circ}$ shear transducer unless there is a wide crown; then, only the $60^{\circ}$ shear response may appear.

3. If there is a response from one or both directions for $\pm 15^{\circ}$ skews, then it is indicative of a crack.

4. If there is a response from one direction or both directions for $\pm 45^{\circ}$ skews, then it is probably a crack. 
5. Responses from skewed scans that are much larger than from the non-skewed scans are candidates for classification as axial cracks.

6. Weld roots tend to occur beyond the specimen back surface.

7. Counterbores tend to have very large responses from non-skewed scans and will have virtually no response from the skewed scans unless they are machined rough. In this latter case, they will tend to have similar properties when skewing in both + and - directions. If the counterbore is tapered and disappears, then the ends will tend to yield a response for the skewed scans.

8. If the response from the $60^{\circ}$ is much less than the response from the $45^{\circ}$, then the indication is most likely a geometric condition.

\subsubsection{SAFT-UT Detection Test Results and Discussions}

The test was first taken using the SAFT-UT system containing the DEC VAX 11/730. This system severely limited data collection, processing, and analysis. Consequently, the spatial sampling was reduced and the temporal zone was limited to the inner one-third of the pipe. A second test was taken when the system had been upgraded to include the MicroVAX III. This upgrade provided a significant increase in speed and improved the amount of information that could be taken.

Since that time, the technology has been implemented on a Sun Microsystems SPARCStation, greatly improving the speed over that of the MicroVAX III. This later system would provide further improvements that would make taking this type of test much easier.

In the IGSCC test taken, seven of the cracks were detected, and false calls were acceptably low. In reviewing the reason for missing the three cracks, it was learned that there were, in two of the cases, good data from the cracks that had been recorded. In one case, there was no data, believed to be a result of the fact that skews to angles of at least $70^{\circ}$ would be required to obtain a suitable response from the crack. In one case, the response from the crack was classified as axial versus the true-state data that classified it as circumfer- ential. The decision for this one can only be conjectured, because it is not permissible to relook at the test data. We believe that the judgement was based in part on the strength of the ultrasonic response from the crack as well as its length in the image. This classification is a subjective decision and does not relate to whether the unit of material is determined to be cracked. We believe that the detection grading scheme should only consider calling a unit of material as either cracked or blank. The classification of the cracks into axial or circumferential is related to a performance parameter connected more closely with the false call performance. We feel that this is a better way to judge acceptable performance. Most IGSCCs have axial components, and for long cracks there is no problem with this. But for short cracks, a very subjective decision has to be made.

The other crack missed in the test was one that we were told was detected and correctly identified on the worksheets but not selected for reporting on the test forms. We do not know why it was not selected for reporting.

One major result of this exercise is that much was learned about classification of signals in this test. The SAFT-UT system provided good signals to be used for analysis, and we were told that the cracks reported correlated with high accuracy with the true state. Our experience with this exercise will result in modification of the data collection and analysis rules. These changes are as follows:

1. Skew to much higher angle for detection of missed axial cracks.

2. Change the classification decision for axial cracks to one based solely on a length measure; ignore signal strength.

3. Refine decision for indication response from the ends to classify those with a planar machined-type response versus that from a more cloud-like or branched crack response.

These changes should lead to correct detection of the IGSCC and proper classification. 


\subsubsection{Conclusions Based on the IGSCC Detection Test}

Although the IGSCC Detection Test was not successfully passed, the SAFT-UT system did provide information on nearly all of the cracks in the test. The rules for systematically evaluating the images have evolved with time, and the testing demonstrated their weaknesses and where improvements were needed. These we believe have been identified and corrected and now should form a complete set that will allow proper identification of all the signals.

\subsection{Validation of the SAFT-UT on the PISC-III Full-Scale Vessel Test}

\subsubsection{Overview of the FSV Test}

Another blind test selected for evaluating the SAFT-UT technology was the PISC-III Full-Scale Vessel (FSV) Test. Located in Stuttgart, Germany, at the Materialpruefungsanstalt (MPA) Laboratory, this test involved the characterization of 12 indications in a full-scale reactor pressure vessel.

During December 1990, three PNL staff traveled to Stuttgart and spent 11 days performing inspections on the FSV. Eight of the inspection zones were examined from both the inside and the outside surfaces of the reactor vessel. In these examinations, more than 650 $\mathrm{MB}$ of data were collected. The inspections included $0^{\circ}, \pm 45^{\circ}, \pm 60^{\circ}, \pm 70^{\circ}$, and tandem SAFT. The data was not analyzed in Germany but was shipped back to PNL for this evaluation.

Field trip notes were developed during the testing at the MPA site. These notes were recorded on a PC to document the activities that occurred during the testing. This turned out to be very useful because a number of confusing points were later clarified with these detailed notes.

A number of things were learned in this exercise. The SAFT-UT system, in general, performed very well; however, several areas were found in which improvements were needed. The most important of these was that the header for the files needed to be changed to accommo- date the sizes of numbers to cover the dimensions of a full-sized reactor pressure vessel.

\subsubsection{FSV SAFT-UT Results}

All $650 \mathrm{MB}$ of data were methodically analyzed. The analysis was performed by first evaluating the data from the clad surface, followed by the data from the unclad surface, and then all the data combined. This approach allowed us to learn more from the data evaluation and to determine optimum inspection combinations.

During the analysis, it became apparent that the amount of data to be analyzed was substantially more than had been analyzed previously for any other inspection. In other examinations, most of the scanned material is blank. However, in this case, every area scanned had a substantial number of indications.

Another result from the data analysis was that the UT responses obtained from the various directions and inspection conditions led to different conclusions about how to interpret the indication. Consequently, a decision was needed as to which signals and interpretation to believe. Every attempt was made to follow the rules that had been established from previous work, but there were many instances of ambiguity associated with the interpretation. In these cases, a decision was reached by looking at the strength of the signals (top tip signal amplitude versus bottom tip amplitude), or recognizing that volumetric indications should be detected during a normal beam scan. The tandem scans were very useful in determining if an indication was surface-connected or if two signal tips were from a single indication or two different indications.

When the analysis was complete, several knowledgeable PNL staff reviewed the results to ensure that nothing was overlooked in the evaluation and to confirm the flaw size estimates. The SAFT-UT inspection results were provided to the JRC at Ispra, Italy. Overall, the results were felt to be good, but there were difficulties in interpreting the data. This was due, in part, to the number of indications in each of the zones examined and the fact that defects have a large range of scattering properties. Some indications were easy to interpret, but others were exceedingly complex and did not fit simple models. 
The data was analyzed by examining all files of a particular zone. The data was reduced by the PNL staff, and those indications that were felt to be of importance were reported; this sometimes included several indications in a particular zone. In several other cases, the indications were all plotted and then judged to all be part of a single larger indication, so were reported as a single large indication. However, we did provide documentation showing the detailed images evaluated to reach that conclusion.
Listed in Table 10.6 is the true-state data obtained by destructive analysis along with the results obtained using SAFT-UT. There was good general agreement for all the defects inspected except for areas 5 and 6.

In reviewing the data, the indications reported for defect zone 5 correlate with the intended defect, but this is not true for defect zone 6 . This latter zone might best be classified as not detected. Because the analysis

Table 10.6. PISC-III Action No. 2 Full-Scale Vessel Test - Phase 1

\begin{tabular}{|c|c|c|c|c|c|c|c|c|c|c|c|c|}
\hline \multirow{2}{*}{$\begin{array}{l}\text { Defect } \\
\text { Numiber }\end{array}$} & \multicolumn{3}{|c|}{1} & \multicolumn{3}{|c|}{ \% } & \multicolumn{3}{|c|}{$\begin{array}{lll}1 & 3\end{array}$} & \multicolumn{3}{|c|}{4} \\
\hline & $\begin{array}{l}\Delta \mathrm{X}, \\
\mathrm{mm}\end{array}$ & $\begin{array}{l}\Delta \mathrm{Y} \\
\text { mm }\end{array}$ & $\begin{array}{l}\Delta \mathrm{Z} ; \\
\mathrm{mm}\end{array}$ & $\begin{array}{l}\Delta \mathrm{X} \\
\mathrm{mm}\end{array}$ & $\begin{array}{l}\Delta \mathrm{Y}, \\
\dot{\mathrm{mm}}\end{array}$ & $\begin{array}{l}\Delta Z_{i} \\
\mathrm{~mm}\end{array}$ & $\begin{array}{l}\Delta \mathrm{X}_{\mathrm{x}} \\
\mathrm{mm}\end{array}$ & $\begin{array}{l}\Delta: Y_{3} \\
\mathrm{~mm}\end{array}$ & $\begin{array}{l}\Delta Z_{\text {, }} \\
\text { mom }\end{array}$ & $\frac{\Delta X_{j}}{m m}$ & $\begin{array}{l}\Delta \mathrm{Y}, \\
\mathrm{mm}\end{array}$ & $\begin{array}{l}\Delta \mathrm{Z}, \\
\mathrm{mm}\end{array}$ \\
\hline True-State & 9 & 80 & 18 & 2 & 24 & 6 & \multicolumn{3}{|c|}{$\mathrm{L}=24, \Delta=6$} & \multicolumn{3}{|c|}{$L=83, \Delta=25$} \\
\hline SAFT Results & 9 & 82 & 17 & $\begin{array}{c}28 \\
5.1 \\
-\end{array}$ & $\begin{array}{c}58 \\
30.7 \\
11.8\end{array}$ & $\begin{array}{c}9^{(a)} \\
5 \\
5\end{array}$ & \multicolumn{3}{|c|}{ not inspected } & \multicolumn{3}{|c|}{ not inspected } \\
\hline
\end{tabular}

\begin{tabular}{|c|c|c|c|c|c|c|c|c|c|c|c|c|}
\hline \multirow{2}{*}{$\begin{array}{l}\text { Defect } \\
\text { Number }\end{array}$} & \multicolumn{3}{|c|}{5} & \multicolumn{3}{|c|}{$6, \cdots$} & \multicolumn{3}{|c|}{$\begin{array}{r}37 \\
\end{array}$} & \multicolumn{3}{|c|}{8} \\
\hline & $\begin{array}{l}\Delta \mathrm{X} \\
\mathrm{mm}\end{array}$ & $\begin{array}{l}\Delta \mathrm{Y} \\
\mathrm{mm}\end{array}$ & $\begin{array}{l}\Delta \mathrm{Z} \\
\mathrm{mm}\end{array}$ & $\begin{array}{l}\Delta \mathrm{X} \\
\mathrm{mm}\end{array}$ & $\begin{array}{l}\Delta \mathrm{Y} \\
\mathrm{mm}\end{array}$ & $\frac{\Delta \mathrm{Z}}{\mathrm{mim}}$ & $\stackrel{\Delta \mathrm{X}}{\mathrm{mm}}$ & $\begin{array}{l}\Delta \mathrm{Y} \\
\mathrm{mm}\end{array}$ & $\frac{\Delta \mathrm{Z},}{\mathrm{mm}}$ & $\Delta x$, & $\begin{array}{l}\Delta Y, \\
\mathrm{~mm}\end{array}$ & $\begin{array}{l}\Delta \mathrm{Z}, \\
\mathrm{mm}\end{array}$ \\
\hline True-State & 13 & 33 & 33 & 15 & 32 & 22 & 2 & 31 & 12 & 28 & 1089 & 110 \\
\hline SAFT Results & $\begin{array}{c}43 \\
17.8 \\
43.1\end{array}$ & $\begin{array}{c}42 \\
20.5 \\
112\end{array}$ & $\begin{array}{c}23 \\
10.1 \\
7.6\end{array}$ & 38 & 168 & 20 & \multicolumn{3}{|c|}{ not inspected } & $\begin{array}{l}23 \\
18 \\
5.1\end{array}$ & 1 & $\begin{array}{c}110 \\
58 \\
33\end{array}$ \\
\hline
\end{tabular}

\begin{tabular}{|c|c|c|c|c|c|c|c|c|c|c|c|c|}
\hline \multirow{2}{*}{$\begin{array}{l}\text { Defect } \\
\text { Number }\end{array}$} & \multicolumn{3}{|c|}{9} & \multicolumn{3}{|c|}{10} & \multicolumn{3}{|c|}{11} & \multicolumn{3}{|c|}{12} \\
\hline & $\begin{array}{l}\Delta X, \\
\mathrm{~mm}\end{array}$ & $\begin{array}{l}\Delta Y, \\
\mathrm{~mm}\end{array}$ & $\begin{array}{l}\Delta \mathrm{Z}, \\
\mathrm{mm}\end{array}$ & $\begin{array}{l}\Delta \mathrm{X} \\
\mathrm{mm}\end{array}$ & $\begin{array}{l}\Delta \mathrm{Y}, \\
\mathrm{mm}\end{array}$ & $\begin{array}{l}\Delta \mathrm{Z} \\
\mathrm{mm}\end{array}$ & $\begin{array}{c}\Delta X \\
\square m\end{array}$ & $\Delta \mathrm{Y}_{\mathrm{m}}$ & $\begin{array}{l}\Delta Z ; \\
\min \end{array}$ & $\underset{\mathrm{m} m}{\Delta \mathrm{X}}$ & $\begin{array}{l}\Delta \mathrm{Y}, \\
\mathrm{mm}\end{array}$ & $\begin{array}{l}\Delta \mathrm{Z} \\
\mathrm{mm}\end{array}$ \\
\hline True-State & 20 & 1089 & 82 & 800 & 4 & 20 & 116 & 16 & 27 & 4 & 77 & 22 \\
\hline SAFT Results & $\begin{array}{c}20 \\
8 \\
8\end{array}$ & $\begin{array}{l}- \\
-\end{array}$ & $\begin{array}{l}71 \\
58 \\
64\end{array}$ & \multicolumn{3}{|c|}{ not inspected } & 109 & 3 & 25 & 9 & 81 & 21 \\
\hline
\end{tabular}

(a) This first one is formed by adding the reported zones together and forming a super large region containing all reported indications. 
is still in progress, the actual outcome and overall assessment will need to await further details.

However, if one takes a simplistic approach to the assessment of the SAFT-UT performance and computes the mean, standard deviation, and variance for the data as a function of the three orthogonal coordinates $X, Y$ and $Z$, then one finds as shown in Table 10.7 that the SAFT-UT technology performed very well. It needs to be noted that most of the error for the $Y$ dimension of the defects was associated with defect 6 . If this defect is eliminated, the greatest effect will be on the Y measurements as shown in Table 10.8. This is more like what is expected because the operating parameters were selected so that the resolution of the system would be similar in both the depth and lateral directions. Because of the degraded performance due to surface roughness and the cladding, it was expected that the resolution would be 2 wavelengths, which would be 2.44 $\mathrm{mm}$ to $3.0 \mathrm{~mm}$ ( $0.1 \mathrm{in}$. to $0.12 \mathrm{in}$.) for the transducer frequencies and modes used versus the theoretical limit of 1 wavelength.

\subsubsection{Conclusions Based on FSV Test}

The SAFT-UT system performed extremely well and much as expected in these tests. This provided some good validation data for thick-section materials. We believe the SAFT technology performed very well in comparison with other techniques used in this test, but a full comparison will have to await the detailed analysis that is in progress by the PISC-III Action 2 Data Analysis Group.

\subsection{Conclusions}

The validation tests reported in this section provide a very good and strong case for the effectiveness of the SAFT technology. This, coupled with the improvements in computer processing speed, RAM capacity, and laser disks, makes for very compact and high performance systems that can process data as it is acquired and provide a high-performance platform for conducting the image analysis. These validation tests of SAFTUT strongly supports using this technology for detecting and characterizing ultrasonic indications in light-water reactor components.
Table 10.7. Statistical Values of SAFT-UT Performance on ALL FSV Flaws

\begin{tabular}{||c|c|c|c||}
\cline { 2 - 4 } \multicolumn{1}{c|}{} &.$\Delta \mathrm{X}$ & $\Delta \mathrm{X}$ & $\Delta \mathrm{Z}$ \\
\hline \hline Mean & $6.1 \mathrm{~mm}$ & $19.5 \mathrm{~mm}$ & $-3.5 \mathrm{~mm}$ \\
\hline $\begin{array}{l}\text { Standard } \\
\text { Deviation }\end{array}$ & $13.3 \mathrm{~mm}$ & $47.2 \mathrm{~mm}$ & $4.4 \mathrm{~mm}$ \\
\hline Variance & $12.4 \mathrm{~mm}^{2}$ & $44.1 \mathrm{~mm}^{2}$ & $4.1 \mathrm{~mm}^{2}$ \\
\hline
\end{tabular}

Table 10.8. Statistical Values of SAFT-UT Performance on All FSV Flaws Except No. 6

\begin{tabular}{|c|c|c|c|}
\hline & rax & $\Delta Y$ & $\Delta \mathbf{Z}$ \\
\hline Mean & $3.7 \mathrm{~mm}$ & $3.0 \mathrm{~mm}$ & $-3.7 \mathrm{~mm}$ \\
\hline $\begin{array}{l}\text { Standard } \\
\text { Deviation }\end{array}$ & $12.3 \mathrm{~mm}$ & $3.8 \mathrm{~mm}$ & $4.7 \mathrm{~mm}$ \\
\hline Variance & $11.4 \mathrm{~mm}^{2}$ & $3.5 \mathrm{~mm}^{2}$ & $4.3 \mathrm{~mm}^{2}$ \\
\hline
\end{tabular}




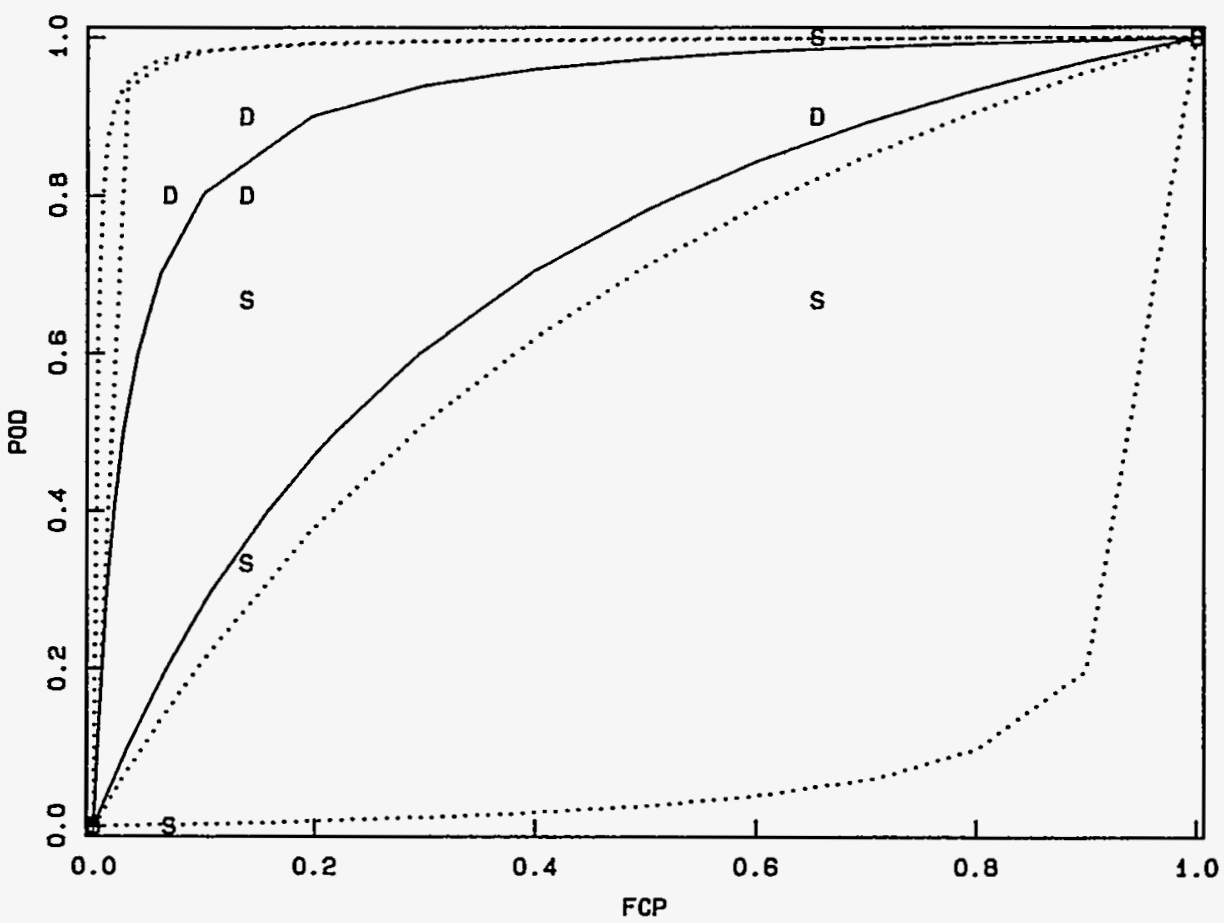

Figure 10.1. SAFT Team ROC Curves for " $D$ " and "S" Cracks

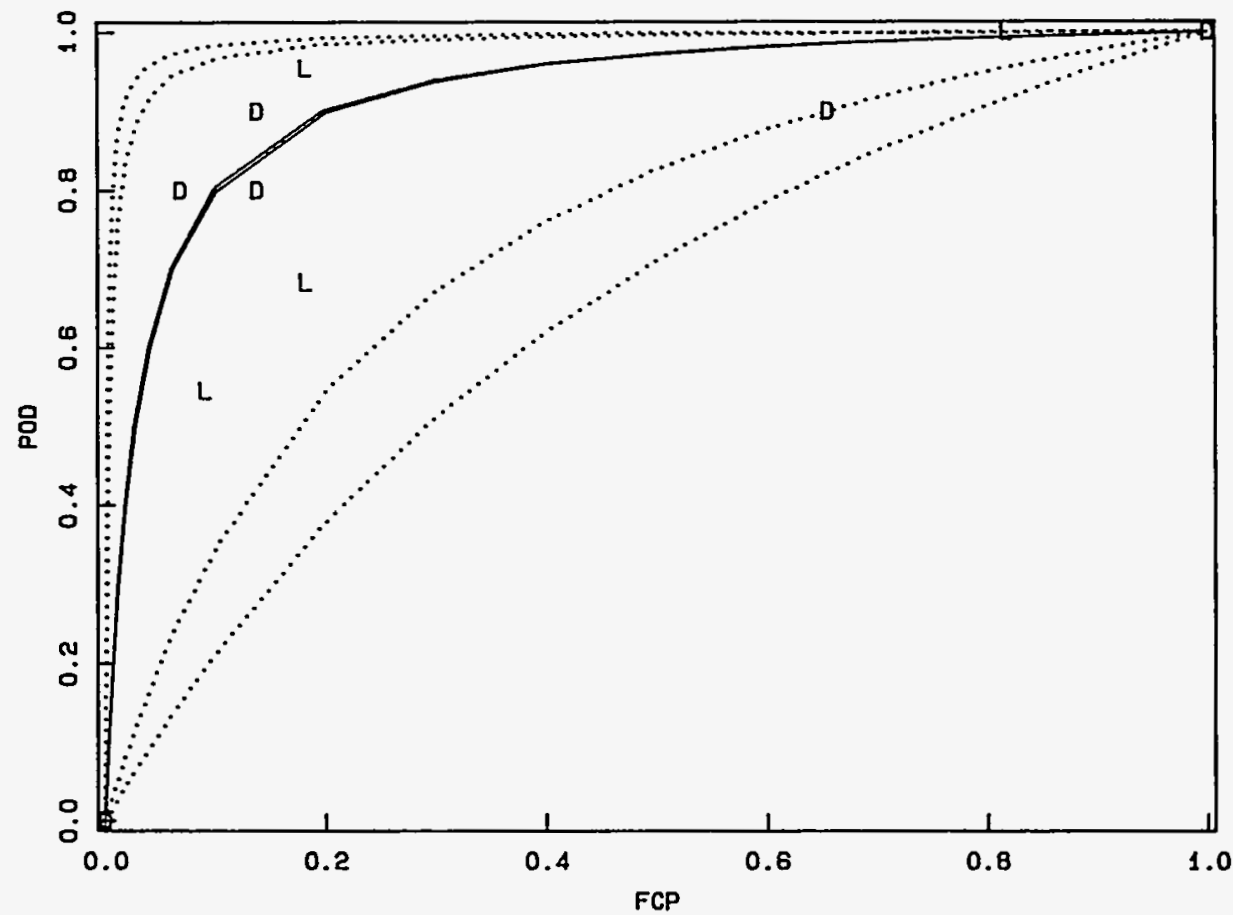

Figure 10.2. SAFT Team ROC Curves for "D" and "L" Cracks 


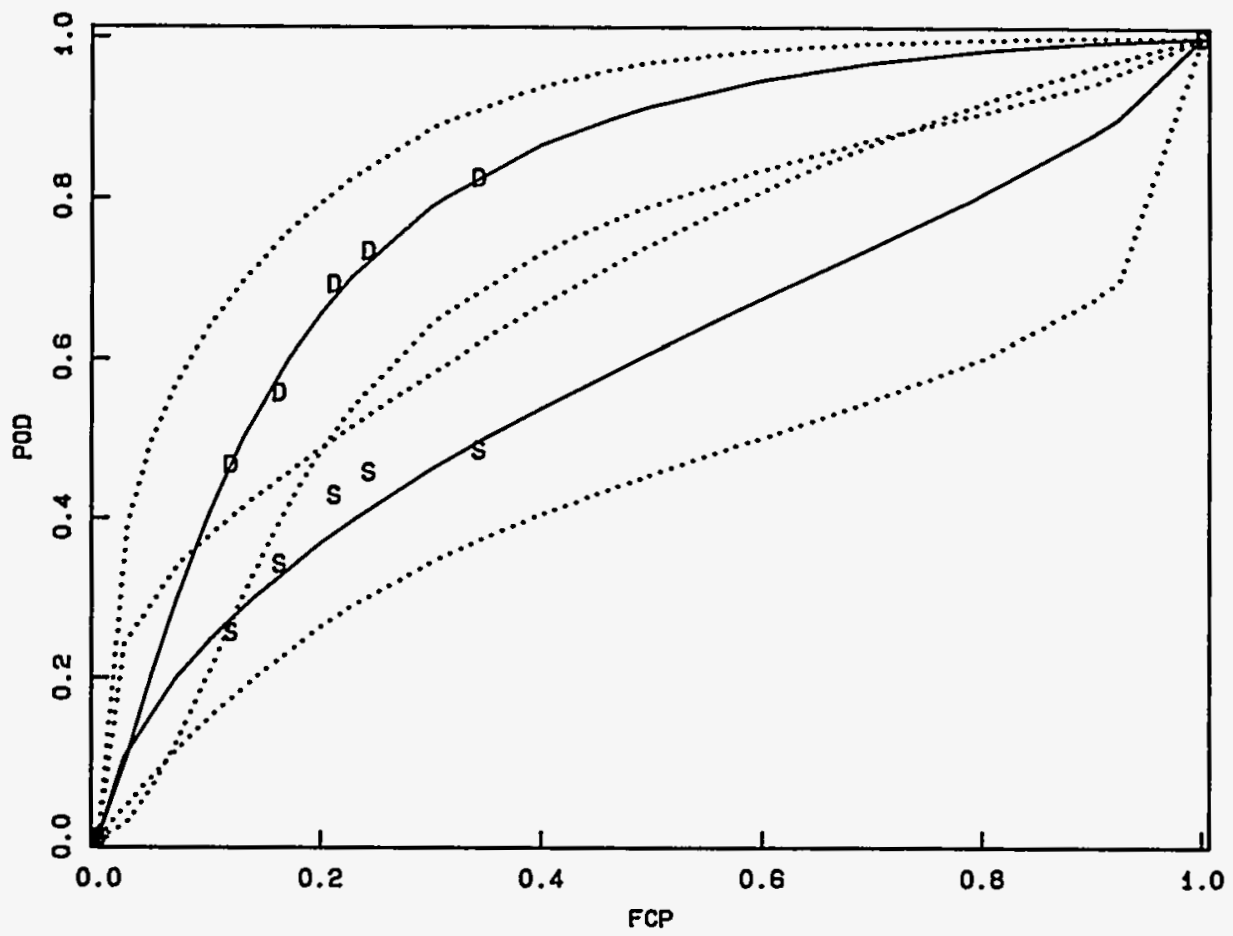

Figure 10.3. Manual Teams' (Average) ROC Curves for " $D$ " and "S" Cracks

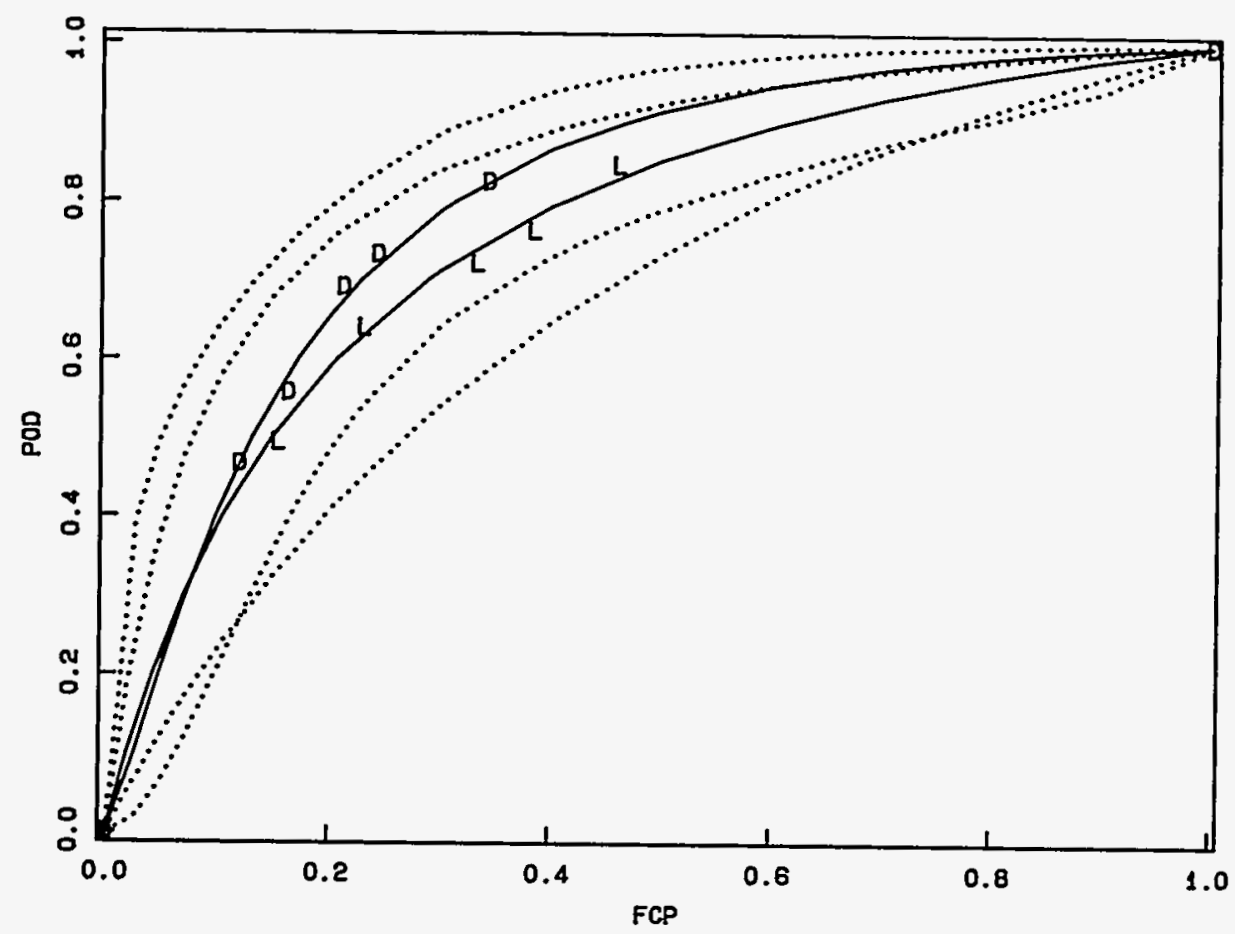

Figure 10.4. Manual Teams' (Average) ROC Curves for "D" and "L" Cracks 


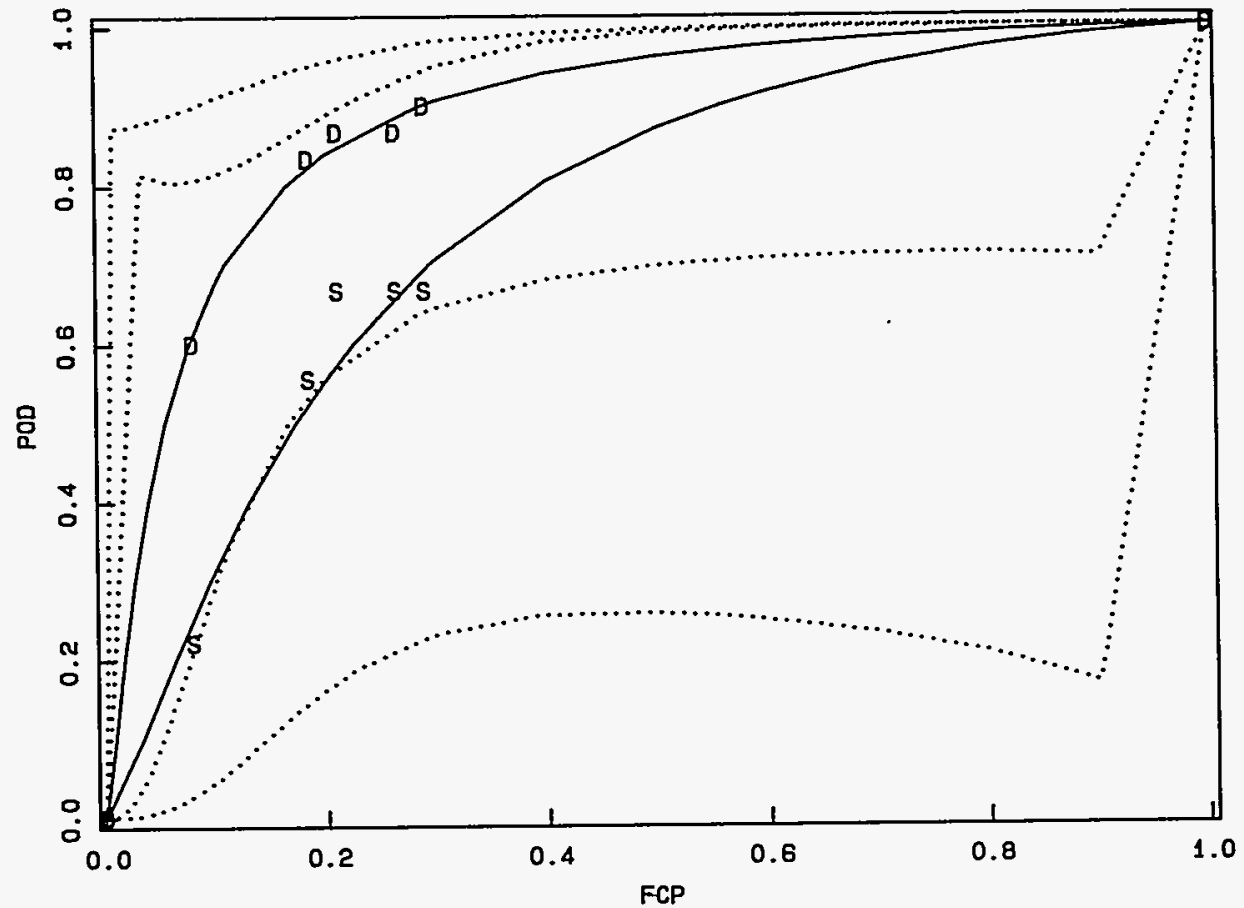

Figure 10.5. Advanced Teams' (Average) ROC Curves for "D" and "S" Cracks

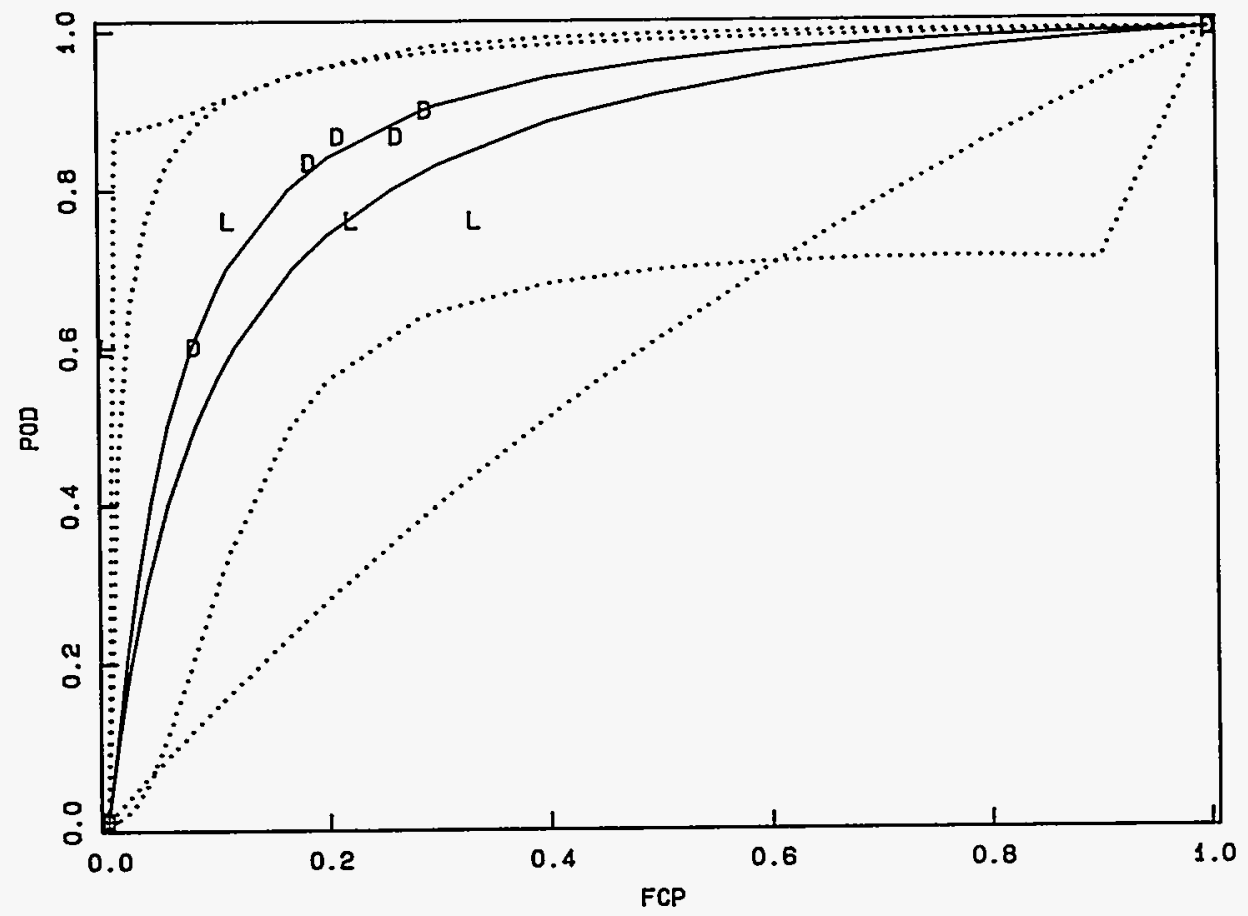

Figure 10.6. Advanced Teams' (Average) ROC Curves for " $D$ " and "L" Cracks 


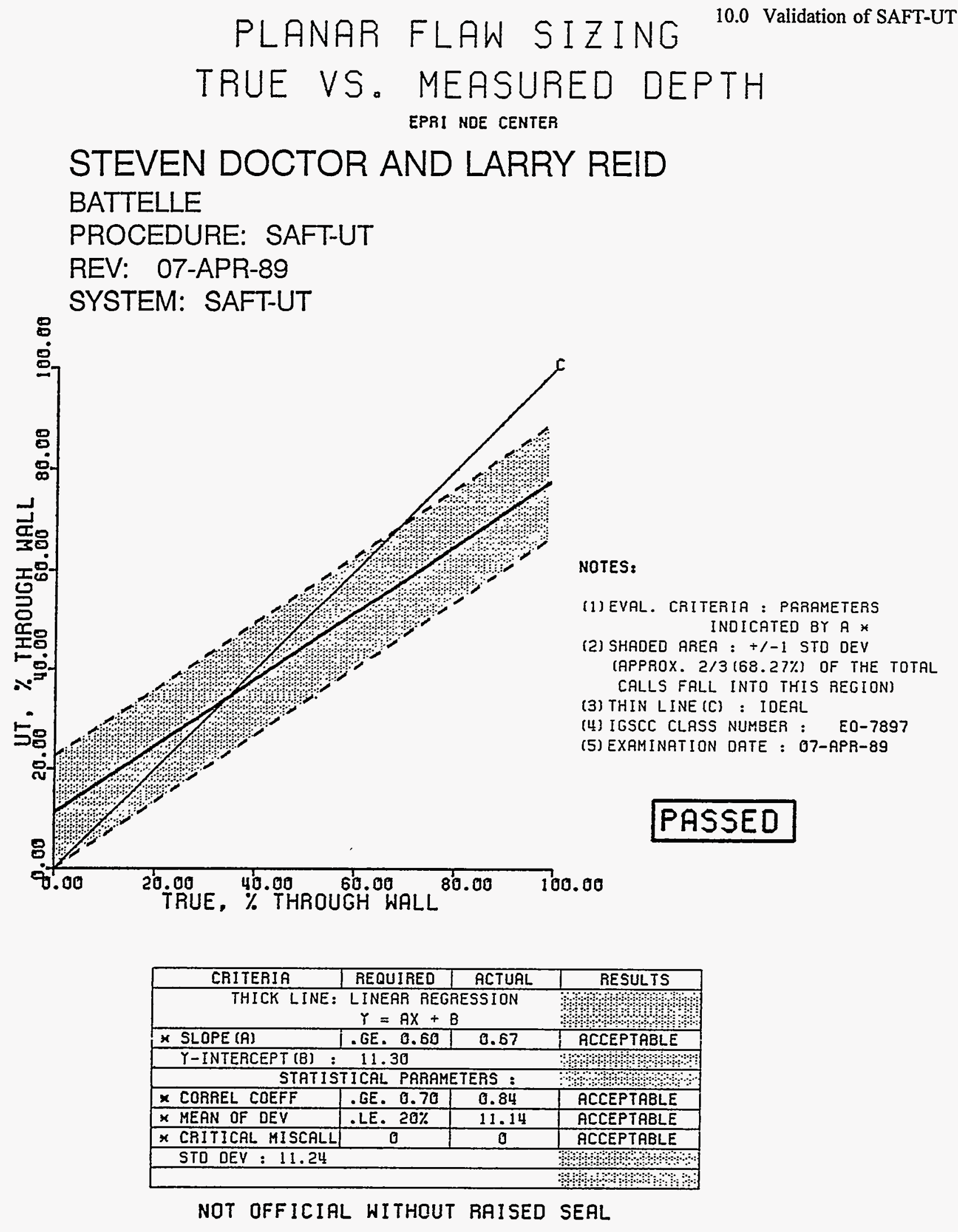

Figure 10.7. Results of SAFT-UT Technique on IGSCC Performance Demonstration Sizing Test Conducted at EPRI NDE Center 


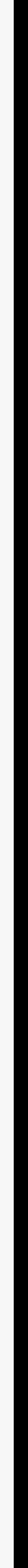




\subsection{Conclusions}

The SAFT technology has evolved into a practical and useful tool for performing inservice inspections on light water reactor components. The SAFT algorithm has been accelerated to process inspection data at the same rate at which the data is collected. The SAFT images are displayed in the true perspective that exists relative to the inspected part and with uniform dimensions, thus making the images easy to interpret. Extensive laboratory testing has shown the viability of the technology. Field trials have shown that SAFT can be applied in the adverse environment of light water reactors and that the resulting images are easy to interpret. A procedure based on the SAFT-UT real-time field system has been written for the SAFT technology.

Over the past 20 years, work has continued to improve SAFT-UT inspection systems for the field and the laboratory. The following list summarizes the highlights of work performed:

- Software from the University of Michigan was modified to reduce processing times.

- Software was developed to run on the host VAX that would communicate with the data acquisition system, graphics processor, and real-time processor.

- The focusing properties of SAFT-UT were numerically analyzed. The point spread function of SAFT was studied as a function of aperture size, pulse attenuation, pulse bandwidth, and horizontal sample intervals.

- Advanced data presentation techniques were analyzed. Perspective contour plotting, grey scale, color plotting, and interactive displays were considered. Finally, a RAMTEK high-resolution graphics imaging system was purchased and a graphics/analysis utility was written.

- Processing needs associated with performing realtime SAFT-UT were analyzed. Commercially available computer systems and array processors were analyzed and found to be either inadequate or too expensive for performing real-time SAFTUT. The concept of a special-purpose, hardware implementation, SAFT processor was proposed and developed. However, this also was inadequate (variations of the SAFT algorithm could not be easily implemented) and a second real-time processor was developed using a parallel processor architecture. This second real-time processor utilized commercially available hardware, and the software could be easily modified to accommodate modified SAFT algorithms.

- Special-purpose scanning head hardware has been developed. Such hardware allows for high-speed scanning of arbitrary surfaces and constant offset of transducers. The scanning hardware was further modified to accommodate multiple transducer configurations such as TSAFT-2 and TSAFT-3.

- Tandem SAFT configurations were developed to overcome the ambiguities inherent in pulse-echo, single-transducer configurations. Further, a special tandem configuration (TSAFT-3) was developed for thick-section materials such as reactor pressure vessels.

- Parametric studies were performed to study the effects of errors in parameters used during SAFT processing. Included in these studies were effects from assumptions made within the SAFT algorithm itself.

- A SAFT-UT field data collection system was developed and employed in the field during inservice inspections of operating reactors (Dresden and Vermont Yankee). Further, SAFT data was acquired using two different commercial data acquisition systems and subsequently processed and analyzed to characterize real defects in a reactor pressure vessel (Indian Point Unit II) and a static-cast stainless steel elbow in the hot-leg of a reactor (Trojan Nuclear Power Plant).

- Advanced preprocessing techniques were developed for anisotropic materials such as centrifugally cast stainless steels. Utilities were written to determine the spectrum characteristics of defects and nondefects in anisotropic materials. A filter utility could subsequently be used on raw data, prior to SAFT processing, to enhance defect responses and diminish nondefect responses. A time-derivative utility was developed to improve the signal-to-noise ratio of wide-band data files. 
- Installation of the SAFT utilities on a VAX 11/750 owned by Combustion Engineering constituted the first step in examining how to transfer the SAFT technology. Also, a real-time processor was fabricated for use by Sandia National Laboratories, and the SAFT utilities were installed on a MicroVAX computer, which was designated as the host computer in the Sandia SAFT-UT system.

- The SAFT technology has been formally integrated into ASME Code Section V, Article 4, Appendix E, which addresses computerized UT imaging systems. This article contains general requirements plus specific requirements covering calibration, SAFT-UT, L-SAFT, broadband holography, and UT-phased arrays.

- Because of the new computer products that have become available during the time that the SAFTUT system was under development, we have seen significant improvement in the system. Tremendous amounts of data generated by SAFT-UT inspections need efficient handling and effective storage. Particularly for the inspection of pressure vessels, SAFT-UT effectively uses advancing computer technology.
- The validation testing provides a very good and strong case for the effectiveness of the SAFT technology. This, coupled with the improvements in computer processing speed, RAM, capacity, and laser disks, makes for very compact and highperformance systems that can process data as it is acquired and provide a high-performance platform for conducting the image analysis. This work provides a powerful case for use of this technology for detecting and characterizing ultrasonic indications in light water reactor components.

- Development, training, and delivery of a compact SAFT-UT field system based on the latest computer advances has been achieved through the system now used by the NRC NDE Mobile Laboratory staff.

To summarize, much developmental research work has been performed over the past 20 years to develop and implement SAFT-UT. Many of the basic questions regarding the ultimate resolution and computational complexity associated with performing SAFT-UT have been answered. The SAFT program has resulted in a realtime SAFT-UT field system and hand-in-hand work with commercial companies performing special inservice inspections of operating reactors. Transfer of the SAFT technology to the nuclear industry has been a goal of the program, and PNL is continuing to work toward achieving that goal. 


\subsection{References}

Bates, D. J., S. R. Doctor, and P. G. Heasler. 1987. Stainless Steel Round Robin Test Centrifugally Cast Stainless Steel Screening Phase, NUREG/CR-4970, U.S. Nuclear Regulatory Commission, Washington, D.C.

Bracewell, R. 1965 The Fourier Transform and Its Applications. McGraw-Hill, NY.

Burckhardt, C. B., P. A. Grandchamp, and H. Hoffmann. 1974. "Methods for Increasing the Lateral Resolution of B-Scan," in Acoustical Imaging, Vol. 5, ed. P. S. Green. Plenum Press, New York, NY.

Busse, L. J., H. D. Collins, and S. R. Doctor. 1984. Review and Discussion of the Development of Synthetic Aperture Focusing Technique for Ultrasonic Testing (SAFT-UT), NUREG/CR-3625. U.S. Nuclear Regulatory Commission, Washington, D.C.

Collins, H. D. and Gribble, R. P. 1986. Siamese Imaging Technique for Quasi-Vertical Type (QVT) Defects in Nuclear Reactor Piping. NUREG/CR-4472. U.S. Nuclear Regulatory Commission, Washington, D.C.

Doctor, S. R., L. J. Busse, S. L. Crawford, T. E. Hall, R. P. Gribble, A. J. Baldwin, and L. P. Van Houten. 1986. Development and Validation of a Real-Time SAFT-UT System for the Inspection of Light Water Reactor Components, Semi-annual Report, April 1984 to September 1984, NUREG/CR-4583, Vol. 1. U.S. Nuclear Regulatory Commission, Washington, D.C.

Doctor, S. R., T. E. Hall, L. D. Reid, S. L. Crawford, R. J. Littlefield, and R. W. Gilbert. 1987a. Development and Validation of a Real-Time SAFT-UT System for the Inspection of Light Water Reactor Components, Annual Report, October 1984 to September 1985, NUREG/CR-4583, Vol. 2. U.S. Nuclear Regulatory Commission, Washington, D.C.

Doctor, S. R., T. E. Hall, L. D. Reid, and G. A. Mart. 1987b. Development and Validation of a Real-Time SAFT-UT System for the Inspection of Light Water Reactor Components, Annual Report, October 1985 to September 1986, NUREG/CR-4583, Vol. 3. U.S. Nuclear Regulatory Commission, Washington, D.C.

Frederick, J. R., C. VandenBroek, S. Ganapathy, M. Elzinga, W. DeVries, D. Papworth, and N. Hamano. 1979. Improved Ultrasonic Nondestructive Testing of
Pressure Vessels, NUREG/CR-0909. U.S. Nuclear Regulatory Commission, Washington, D.C.

Frederick, J. R., R. C. Fairchild, and B. H. Anderson. 1977. Improved Ultrasonic Nondestructive Testing of Pressure Vessels, NUREG-0007-2. U.S. Nuclear Regulatory Commission, Washington, D.C.

Frederick, J. R., C. VandenBroek, R. C. Fairchild, and M. B. Elzinga. 1978. Improved Ultrasonic Nondestructive Testing of Pressure Vessels, NUREG/CR-0135. U.S. Nuclear Regulatory Commission, Washington, D.C.

Frederick, J. R., C. VandenBroek, M. Elzinga, M. Dixon, D. Papworth, N. Hamano, and K. Ganapathy. 1979. Improved Ultrasonic Nondestructive Testing of Pressure Vessels, NUREG/CR-0581. U.S. Nuclear Regulatory Commission, Washington, D.C.

Frederick, J. R. Seydel, and R. C. Fairchild. 1976. Improved Ultrasonic Non-Destructive Testing of Pressure Vessels, NUREG-0007-1. U.S. Nuclear Regulatory Commission, Washington, D.C.

Ganapathy, S., W. S. Wu, and B. Schmult. 1982. "Analysis and Design Considerations for a Real-Time System for Nondestructive Evaluation in the Nuclear Industry." Ultrasonics 20:249.

Ganapathy, S., B. Schmult, W. S. Wu, T. G. Dennehy, N. Moayeri, and P. Kelly. 1985. Design and Development of a Special Purpose SAFT System for Nondestructive Evaluation of Nuclear Reactor Vessels and Piping Components, NUREG/CR-4365. U.S. Nuclear Regulatory Commission, Washington, D.C.

Ganapathy, S. N. Hamano, M. R. Bether, W. S. Wu, T. G. Dennehy, C. Purnaveja, M. M. Murray, M. B. Elzinga, and F. Raam. 1981. Ultrasonic Imaging Techniques for Real-time In-service Inspection of Nuclear Power Reactors, NUREG/CR-2154. U.S. Nuclear Regulatory Commission, Washington, D.C.

Ganapathy, S. and B. Schmult. 1982. "Design of a Real-time Inspection System for NDE of Reactor Vessels and Piping Components." Presented at I MECH E Conference, London, England. I MECH E 1982-9. 
Ganapathy, S., B. Schmult, W. S. Wu, N. Hamano, and D. Bristor. 1983. Investigation of Special Purpose Processors for Real-Time Synthetic Aperture Focusing Techniques for Nondestructive Evaluation of Nuclear Reactor Vessels and Piping Components, NUREG/CR-2703. U.S. Nuclear Regulatory Commission, Washington, D.C.

Hall, T. E., L. D. Reid, and S. R. Doctor. 1988. The SAFT-UT Real-Time Inspection System-Operational Principles and Implementation. NUREG/CR-5075. U.S. Nuclear Regulatory Commission, Washington, D.C.

Hamano, N. 1980. "Deconvolution of Ultrasonic Nondestructive Testing Data." PhD Dissertation, Electrical Engineering Dept., University of Missouri, Columbia, Missouri.

Hamlin, D. R. 1985. Program for Field Validation of the Synthetic Aperture Focusing Technique for Ultrasonic Testing (SAFT-UT), NUREG/CR-4078. U.S. Nuclear Regulatory Commission, Washington, D.C.

Heasler, P. G., T. T. Taylor, J. C. Spanner, S. R. Doctor, and J. D. Deffenbaugh. 1990. Ultrasonic Inspection Reliability for Intergranular Stress Corrosion Cracks - A Round Robin Study of the Effects of Personnel, Procedures, Equipment and Crack Characteristics, NUREG/ CR-4908. U.S. Nuclear Regulatory Commission, Washington, D.C.

Hildebrand, B. P. 1986. Investigation of Advanced Acoustic and Optical Nondestructive Evaluation Techniques. Final Report NP-4897 for Research Project 606-7. Sigma Research, Inc., Redmond, Washington. pp. 3-44 to 3-51.

Jackson, J. L. 1978a. Program for Field Validation of the Synthetic Aperture Focusing Technique for Ultrasonic Testing (SAFT-UT) - Analysis Before Test, NU-

REG/CR-0288. U.S. Nuclear Regulatory Commission, Washington, D.C.

Jackson, J. L. 1978b. Program for Field Validation of the Synthetic Aperture Focusing Technique for Ultrasonic Testing (SAFT-UT) Midyear Progress Report. NUREG/ CR-0290. U.S. Nuclear Regulatory Commission, Washington, D.C.
Jackson, J. L. 1981. Program for Field Validation of the Synthetic Aperture Focusing Technique for Ultrasonic Testing (SAFT-UT), NUREG/CR-1885, Vols. 1, 2, and 3. U.S. Nuclear Regulatory Commission, Washington, D.C.

Johnson, S. A., J. F. Greenleaf, F. A. Duck, A. Chu, W. R. Samayou, and B. K. Gilbert. 1975. "Digital Computer Simulation Study of a Real-Time Collection, PostProcessing Synthetic Focusing Ultrasound Cardiac Camera," in Acoustical Holography, Vol. 6, ed. Newell Booth. Plenum Press, New York, NY.

Norton, S. J. 1976. Theory of Acoustic Imaging, Technical Report No. 4956-2. Stanford University, Stanford, California.

Polky, J. N. 1986. Development of a Real-Time Number Processor for SAFT Inspection, NUREG/CR-4634. U.S. Nuclear Regulatory Commission, Washington, D.C.

Polky, J. N. and D. D. Miller. 1985. An Ultra-High Speed Residue Processor for SAFT Image Enhancement, NUREG/CR-4170. U.S. Nuclear Regulatory Commission, Washington, D.C.

Prine, D. W. 1972. "Synthetic Aperture Ultrasonic Imaging," in Proceedings of the Engineering Applications of Holography Symposium:287. Society of Photo-optical Instrumentation Engineers.

Seydel, J. A. 1978. (Signal Processing) Methods Development, ed. J. R. Frederick, NUREG/CR-0135. U.S. Nuclear Regulatory Commission, Washington, D.C.

Thome, David K. 1985. "Ultrasonic Image Analysis." in Proceedings for The World Conference on Nondestructive Testing, Vol. 2. American Society for Nondestructive Testing, Columbus, $\mathrm{OH}$. 
Appendix A

Detailed Hardware Description of the SAFT-UT Field Inspection System 


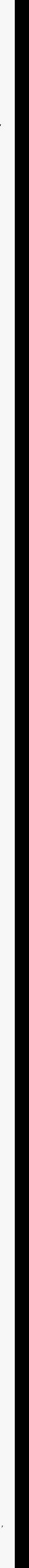




\section{Detailed Description of the SAFT-UT Field Inspection System}

The SAFT-UT field system can be described by four functional subsystems as shown in Figure A.1. The data acquisition subsystem controls the scanning of the part and collects data for subsequent processing. The host computer acts as the system executive and performs the human interface. The Real-Time Processor is a peripheral device to the host computer that performs the computationally intensive SAFT algorithm. Finally, the graphics processor provides the image display to the operator for interpretation.

In the sections that follow, hardware requirements of the complete SAFT-UT field system are discussed, including a detailed hardware description of each subsystem. Specifications, block diagrams, parts lists, and schematics are provided in this Appendix.

\section{A.1 SAFT-UT Data Acquisition Sys- tem}

The SAFT-UT data acquisition system is shown in block diagram form in Figure A.2. It is divided into five sub-systems: mechanical scanner components, mechanical scanner drive/control, the acoustic system, data acquisition computer, and additional miscellaneous components.

Requirements for each of these subsystems will be addressed in this section in the order listed above. The letter superscripts, shown in Figure A.2, help identify the hardware, and are referenced throughout the text in this chapter for clarity. Figure A.3 is a photograph of the SAFT-UT field system with labels indicating the physical location of modules corresponding to the block diagram of Figure A.2.

\section{A.1.1 Mechanical Scanner}

The MECHANICAL SCANNER <DD $>$ is capable of scanning as many as two axes simultaneously and incrementing a third axis perpendicular to the scanned axes. The SAFT-UT scanning convention assumes the $X$ axis to be the scanned axis and the $Y$ axis to be the increment axis. The chosen increment size is dependent on the expected image resolution. The mechanical scanner STEPPING MOTORS <U,Y,T $>$ have a resolution of 1000 steps/inch on the scan axes (X-axes) and 4988 steps/inch on the increment axis (Y-axis). This selection of position resolution allows sufficient flexibility when choosing the increment size.

Usually, positional information is based entirely on the motor pulses; however, there are instances when operating the scanner remotely that absolute position is desired. OPTICAL ENCODERS <K,M,L> with 1024 counts/revolution are used to give absolute position information for reference, and can be optionally employed to provide the pulses used for increment counting. The optical encoders used on the SAFT-UT mechanical scanner provide 2560 counts/inch on the $X$ axes and 865 counts/inch on the $Y$ axis. This provides a sufficient number of counts for increment selection.

Figure A.4 shows a close-up photograph of the pipe scanner in the normal-beam pulse-echo configuration. This shows a side-view of the water column probe used for normal-beam data collection. Figure A.5 shows the same view of a contact probe attached to the SAFT-UT pipe scanner in the 45-degree shear-wave configuration. Finally, Figure A.6 shows a photograph of two contact transducers placed in the dual transducer tandem configuration.

The SAFT-UT mechanical scanner uses magnetic wheels that adhere to a metal track to maintain $\mathrm{Y}$ axis alignment and attach the scanner to the structure being scanned. The scanner originally purchased employed only one $X$ axis; it was modified at PNL to employ two $X$ axes that are independently controllable.

Transducer selection should be considered of utmost importance. When a scan is performed using a plastic wedge, the transducer must be of a small diameter but have a good signal response with the goal of simulating a point source. If a water column is to be used, then a focused probe with the focal point residing on the surface of the structure is needed. Experience on stainless steel piping has shown that a 0.25 -inch-diameter, 2.25MHz unfocussed transducer used with various plastic wedge assortments is sufficient for many field appli- 
cations. A water column is normally used for far-surface profiling only. A 5-MHz, 3-inch focal length, and 0.75 -inch-diameter transducer has been used for farsurface profiling using a water column coupling device.

A number of considerations effected the design of the SAFT-UT pipe scanner. These are itemized as follows:

- It was designed to provide the capability for attaching either a plastic wedge or water column coupling device to the probes involved.

- Two independently controlled scan axes were implemented to accommodate the TSAFT/TSAFT-2 configurations. Also for these configurations the source transducer was aligned directly behind the receive transducer.

- Sufficient tension was required to maintain coupling throughout the scan, overhead or underneath an object such as a pipe.

- When using water columns for normal-beam or angle-beam data collection, the mechanical attachment was designed to be rigid enough to ensure accurate tracking and maintaining the incident angles.

- The mechanical attachment was designed to allow the transducer to be rotated 180-degrees for looking back towards the scanner with the transducer; i.e., reaching across a weld when it is not convenient to mount the scanner on the far side.

- To fully benefit from the ability to straddle a weld, it was necessary to be able to remotely select either transducer to be a source, receiver, or to operate in the pulse-echo or tandem mode.

- Strain reliefs were used on the cables to prevent cable fatigue and to keep the cables out of the pathway of the scanner. Motor and encoder signals utilized shielded pair cabling to ensure electrical isolation and a separate $75-\mathrm{ohm}$ coaxial cable were used for preamplifier signals.

- Decontamination was considered since the scanner was to be used in a contaminated area during inservice inspections. The scanner assembly was designed to be easy to replace, low cost, and simple to disassemble.

- $\quad$ Provision for mounting a remote pulser/preamp on the mechanical scanner was needed. The mounting of the pulser/preamp was studied at length so that the overall size of the scanner was not compromised and interference with the transducer movement was minimized.

\section{A.1.2 Scanner Drive/Control}

Processing data using the SAFT algorithm requires reliable positional information and the ability to accurately read the position of the mechanical scanner during operation. The stepper motor and driver combination was designed to provide sufficient torque to drive the loads that are be encountered, such as the transducers when they are used in conjunction with a water column or when scanning curved surfaces such as horizontal piping. The $Y$ axis stepper motor and driver provides enough torque to transport the scanner mechanism on a vertical surface. The mechanical scanner gear ratio, stepper motor, and driver combination used in the SAFT-UT field system provide adequate torque (53-oz.in.) up to 2 inches per second, which is a sufficient scanning speed for the field application work that has been performed.

Control of the mechanical scanner is accomplished by the Data Acquisition System computer interfacing with a stepper motor controller. The STEPPER MOTOR CONTROLLER $<P, Q, R>$ has the following specifications:

- The number of steps is defined by a number with 23-bit resolution and an additional sign bit for direction.

- Adjustable linear acceleration and deceleration.

- Remote operating mode for connection to a joystick.

- Ability to stop and start a motor during a cycle without losing position information.

- Programmable control of speed and acceleration. 
- Half-step mode for improved torque and resolution.

- Motor pulse and direction outputs.

The motor or encoder pulses are counted as the motor is in motion; when the increment size is reached, data is then collected. Therefore, hardware is needed to count the motor pulses or the optionally selectable encoder pulses. Two counters are used in the SAFT-UT system. The first counter, referred to as the PROGRAMMABLE DIVIDER $<N>$, shown in detail in Figure A.7, was designed and assembled at PNL on a CAMAC prototype card and placed into the system. The counter is loaded with the desired increment count. When the counter overflows, a sync pulse is generated that begins the data acquisition cycle. The counter is then reloaded to count the next scan increment. The counter has the following specifications:

- 12-bit presettable up/down counter.

- Hardware selectable to use either the encoder or motor pulses as the input.

- Software selectable to use the X1, X2, or the $\mathrm{Y}$ axes as the input, latch and load a new increment count, set the pulser drive module to remote, and clear the counter.

The second counter is a four-channel UP/DOWN $<O>$ CAMAC module, and is used to continually count the encoder pulses to provide the system withabsolute position information. This counter has the following specifications:

- Four presettable 16-bit up/down counters.

- Separate up/down inputs.

- Counting rates from dc to $20 \mathrm{MHz}$.

- Capacity of 65,535 counts per channel.

- Overflow and underflow status bits for each channel.

- Separate clear command for each channel.

\section{A.1.3 Acoustic System}

The Acoustic System is a critical component of the SAFT-UT field system. The quality of the data collected is dependent on the quality of the hardware selected. The SAFT-UT field system incorporates a PNL-built SQUARE WAVE PULSER $\angle B>$, which is detailed in a block diagram in Figure A.8, and a commercially available PREAMPLIFIER $\langle B\rangle$, into one package called the remote pulser/preamplifier. This unit mounts directly on the mechanical scanner. The function of the remote pulser/preamplifier is to reduce the signal degradation inherent in having 200 feet of cable linking the mechanical scanner and transducers to the Data Acquisition System main frame. The specifications of the remote pulser/preamplifier are:

- $\quad$ TTL trigger input.

- Output pulse width controllable by trigger input pulse width.

- Separate high voltage input.

- Two transducer hookups, each remotely selectable to be a source or receiver when operated in the tandem mode, or a pulser and receiver when operated in the pulse-echo mode.

- An output pulse amplitude is provided of up to 350 volts with the duration of the pulse adjustable from 0.10 to 1.0 microseconds.

- Pulser output impedance is on the order of 2 to 4 ohms.

- RF output drives 200 feet of 75-ohm coaxial cable with no more than a $-6 \mathrm{~dB}$ loss.

- Preamplifier input impedance is 500 ohms for signal voltages less than the clamp voltage, and not less than $100 \mathrm{ohms}$ for signal voltages greater than the clamp voltage.

- The clamp circuit protects the preamplifier from a positive or negative 350-volt pulse of 1 microsecond duration. 
- Gain of the preamplifier is $20 \mathrm{~dB}$ when loaded into $50 \mathrm{ohms}$.

- $\quad-3 \mathrm{~dB}$ bandwidth is at least 0.5 to $7.5 \mathrm{MHz}$.

At times it is desirable to switch the identity of the transducers that are attached to the remote mechanical scanner. When collecting SAFT-UT data, this feature is desirable because of the tandem and pulse-echo modes that are used. One would like to be able to switch between the two modes without having to physically switch the cables, especially if one has to enter a radiation zone to do the switching. The MODE SELECT <A> module, as detailed in the block diagram in Figure A.9, was developed at PNL to accomplish the switching task and has the following specifications:

- Inhibits pulser high voltage when switching outputs.

- Selects any one of four transducer configurations; pulse-echo mode--front or rear transducer; tandem mode--rear transducer as the source, front transducer as the receiver, or the front transducer as the source and the rear transducer as the receiver.

The PULSER DRIVER <G> module, as shown in Figure A.10, was developed at PNL and provides the system sync pulse, remote pulser trigger, and the remote pulser high voltage. The pulser driver has the following specifications:

- Adjustable sync delay to compensate for the electrical time delay experienced in the 200 feet of cable to the remote pulser/preamplifier.

- High voltage power supply adjustable from 150 to 350 volts.

- Remote pulser trigger output with continuously adjustable pulse widths of 0.1 microsecond to 1 microsecond.

- $\quad$ Remote select input - The pulser driver can provide internal timing or the timing can be provided by an external source. External timing is provided by the programmable divider module on the completion of an increment count. Local timing is adjustable from $0.5 \mathrm{kHz}$ to $2 \mathrm{kHz}$ and is selected from the front panel.

- Pulser inhibit input - Useful when remotely switching transducer sources and is controlled by the Mode Select module.

After the RF signal has passed through the preamplifier, and the 200 feet of cable, it is received and amplified by the TIME VARIABLE GAIN AMPLIFIER $<$ C $>$ (TVG Amplifier) which has the following specifications:

- Total gain of at least $60 \mathrm{~dB}$.

- At least $40 \mathrm{~dB}$ of voltage controlled gain that can change in 20 microseconds without changing the output more than $10 \%$ of full scale with the amplifier input terminated into $500 \mathrm{ohms}$ and no input signal.

- The $-3 \mathrm{~dB}$ bandwidth is 0.5 to $7.5 \mathrm{MHz}$.

- An output swing is 10 volts peak to peak into 50 ohms.

- At least $48 \mathrm{~dB}$ of dynamic range at the output between the noise floor and the saturated output level.

- The output stage is the first stage to saturate under any gain setting.

- Transmit overload recovery is 25 microseconds. It is defined as the time required for the output to settle to the noise level after the leading edge of the transmit pulse at any gain setting and any transmit pulse width from 1 microsecond to 67 nanoseconds. This should be measured with a 50 ohm resistor connected to the transducer connector.

- The overload recovery time, from $100 \%$ signal overload, is less than the time required for one half of a cycle of the input signal after the end of the input signal. It is defined as the time required for the output to settle to the noise level after an input signal twice the amplitude required to saturate the output. The input signal should be a 
bipolar, single-cycle sine wave of any frequency between 0.5 and $7.5 \mathrm{MHz}$.

A block diagram of the TVG RAMP GENERATOR $<D>$ module shown in Figure A.11. It is needed to develop the ramps that control the gain of the TVG amplifier. The ramp generator used in the SAFT-UT field system is a PNL-built unit and has the following specifications:

- Minimum ramp time of 8 microseconds.

- Ramp start adjustable from 0 to 200 microseconds.

- Three slope end point adjustments - each slope end point is settable from 0 to 20 microseconds.

- Five gain adjustments - starting gain, slope 1 gain, slope 2 gain, slope 3 gain, and the final gain.

A dual-trace Tektronix oscilloscope is used as MONITOR \#1 $<E>$ to observe the RF signal out of the TVG amplifier and the TVG ramps out of the TVG ramp generator. It is necessary to ensure that signals of interest are not saturating when collecting data to be processed using either the pulse-echo or tandem SAFT algorithm and set up of the TVG ramps is simplified when the RF signal and the TVG ramps can be viewed at the same time.

The RF waveform is digitized for processing by the SAFT algorithm and provides a data base that can be processed in real time or at a later date. The SAFT algorithm requires four samples per cycle as a temporal sampling minimum. A dual trace Tektronix oscilloscope is used as MONITOR \#2 $<\mathrm{F}>$ to observe the digitized RF signal. The ANALOG-TO-DIGITAL CONVERTER <I> module that is used in the SAFTUT field system has the following specifications:

- $\quad 100-\mathrm{MHz}$ analog bandwidth (-3 dB points) over entire input range.

- 8-bit resolution.

- Programmable internal clock with an external clock input option. The internal clock rates are as follows: $0.5,1,2,4,8,16$, and $32 \mathrm{MHz}$.
- Conversion rates of dc to $32 \mathrm{MHz}$.

- Analog input impedance to be 50 ohms with the DC offset adjustable from the front panel. The input must handle \pm 10 volts.

- $\quad$ Stop sampling input that stops sampling after a selected number of post-trigger samples have been stored.

- Display output and trigger for monitor by an oscilloscope.

- Memory capable of storing at least 1024 samples and memory size programmable and readable by computer.

A PROGRAMMABLE DELAY $<\mathrm{H}>$ module is used to trigger the stop sampling input of the analog-todigital converter and is extremely useful when signals of interest are delayed in time from the initial pulse. The programmable delay module has the following specifications:

- Programmable delays from 100 nanoseconds to 10 seconds.

- Delay readout on the front panel.

- Start delay input is TTL compatible.

An external PROGRAMMABLE CLOCK $<J>$ module is used to provide the timing to the analog-to-digital converter because the analog-to-digital converter internal clock rates did not provide the sampling multiples that were desired. With the option of using either the internal clock or the external clock, the majority of sampling rates can be obtained. The external programmable clock has the following specifications:

- Programmable clock rates of $0.5,1,2,5,10$, and $20 \mathrm{MHz}$.

- Outputs are TTL compatible and will drive 50 ohms. 


\section{A.1.4 Data Acquisition Computer}

The data acquisition computer subsystem provides an operator interface and controls the SAFT-UT data acquisition system. Data can be transferred to the host VAX for processing and graphic display or stored directly on the local disk drives. The ability to store data directly onto disk allows the data acquisition system to perform as a stand-alone unit. In the stand-alone mode processing and analysis of the data is then performed at a later date.

An LSI-11/23 <W $>$ minicomputer is the heart of the data acquisition computer system coupled with an RT11 operating system and a FORTRAN IV compiler.

The following is a list of specifications:

- Extended fixed point instruction set, floating point instruction set.

- $256 \mathrm{~KB}$ of RAM.

- $\quad 2$ RS-232 asynchronous serial interface ports.

- Half-rack card cage with four slots.

To extend the usefulness of the LSI-11/23, a full quad EXPANSION BOX $<\mathrm{X}>$ was purchased that attaches, through a bus expansion cable, to the LSI-11/23. The following is a list of specifications:

- $\quad 120$ volts $\mathrm{AC}$ input.

- Forced air cooling.

- Capable of accepting eight double or four quad size LSI-11 compatible cards.

An LSI-11 BUS ADAPTER $<X>$ plugs into the expansion box and interfaces the LSI-11/23 computer, through a ribbon cable, to the CAMAC CONTROLLER $<S>$ card. The CAMAC controller card supervises the various cards housed in the CAMAC card cage. The two cards will be treated as a set which forms a dedicated interface with the following specifications:

- Supports DMA block transfer mode.
- $\quad$ Fully buffered bus.

Transfer of data to the host VAX requires a high-speed serial or parallel interface. The SAFT-UT field system uses a PRONET $<X>$ high-speed serial interprocessor communications device which consists of two boards that plug into the LSI-11/23 bus expansion box. The PRONET hardware has the following specifications:

- $10 \mathrm{Mbits}$ per second throughput; no saturation.

- Low software interface burden on the host computer.

- Data links up to 300 meters.

- DMA interface to the Q-bus.

- Separate $2 \mathrm{~KB}$ packet buffers, plus control and status registers.

- Token arbitration, variable length data packet, and parity checking.

- Full duplex operation.

One of the RS-232 asynchronous serial lines from the LSI-11/23 connects to a VT-100 compatible TERMINAL $\angle A A>$ and provides an operator interface. The second RS-232 serial interface line is connected to the host VAX computer which provides a virtual terminal interface to allow the operator to directly communicate with the VAX 11/730 host computer via the operator terminal.

Finally the LSI-11/23 system must have a mass storage device. An 8-inch Winchester hard disk and 8-inch floppy combination is used. The DISK DRIVE $<\mathrm{Z}>$ unit used in the SAFT-UT field system has the following specifications:

- 20 MByte capacity Winchester hard disk.

- $1 \mathrm{MB}$ floppy disk capacity, RX02 format.

- Disk controller card interface is LSI-11 compatible. 


\section{A.1.5 Additional Hardware}

Two 24-volt power supplies provide the necessary current for the stepper motors and have the following specifications:

- $\quad$ Adjustable output voltage.

- Output rated at 24 volts and 10 amperes.

A CAMAC CRATE POWER SUPPLY AND MAINFRAME <BB> provides power and a card cage to plug the Camac modules into. The following is a list of the modules that plug into the Camac crate:

\section{- 3 STEPPER MOTOR CONTROLLERS} $<\mathrm{P}, \mathrm{Q}, \mathrm{R}\rangle$

- PROGRAMMABLE DELAY $<\mathrm{H}>$

- ANALOG-TO-DIGITAL CONVERTER <I>

- PROGRAMMABLE CLOCK $<\mathrm{J}>$

- UP/DOWN COUNTER <O>

- PROGRAMMABLE DIVIDER $<\mathrm{N}>$ (PNL designed)

\section{- CAMAC CRATE BUS CONTROLLER $<\mathrm{S}>$}

The following is a list of specifications for the powered Camac crate mainframe and power supply unit:

- Power supply provides \pm 24 volts @ 6 amperes, \pm 12 volts@ 3 amperes, +6 volts @ 42 amperes, and -6 volts@ 25 amperes.

- 25 slots available for Camac modules.

- Metering for voltage and currents of the power supply.

\section{A TEKTRONIX POWER SUPPLY AND MAIN-} FRAME $<$ CC $>$ provides the power for the analog modules. The following is a list of the modules that plug into the Tektronix mainframe:

- MODE SELECT <A>

\section{(PNL designed) \\ - TVG AMPLIFIER <C> \\ - TVG RAMP GENERATOR $<$ D $>$ (PNL designed) \\ - MONITOR SCOPE \#1 <E> \\ - PULSER DRIVER $<G>$ \\ (PNL designed)}

The following is a list of specifications for the Tektronix power supply and mainframe:

- Six powered compartments with compartment number six having a higher power rating than the other five.

- Forced air cooling.

- $\quad \pm 26$ volts DC @ 1 ampere per compartment, +8 volts DC @ 3 amperes per compartment, 25 volts AC @ 1-ampere rms (2.5-ampere rms for the high power compartment) - two per compartment, and 17.5 volts @ 350 milliamperes per compartment.

- One NPN and PNP series pass transistor per compartment, 10-watts (30-watts for the high power compartment) maximum dissipation each.

The data acquisition system includes a PULIZZI POWER CONTROLLER <EE> which provides 120 volts $\mathrm{AC}$ for all the equipment within the Data Acquisition rack. The Pulizzi power controller sequences the computer components on first and then the rest of the equipment all from a single front panel switch. The following is a list of specifications:

- Input power: 120 volts $\mathrm{AC}, 60$ cycle, and $20 \mathrm{am}$ peres.

- Output power: 12 circuits total; four on the switched 1 circuit, four on the switched 2 circuit, and four on the unswitched circuit.

- Remote I/O connection for on/off control.

In field applications, it is desirable to insure that one has a clean AC source. The SOLA POWER CONDITIONER <FF> is used in the SAFT-UT field system to provide both a regulated and filtered AC source. The following is a list of specifications: 
- Input power: $95-130$ volts $\mathrm{AC}, 60$ cycle, and 20 amperes.

- Output power: 120 volts $\mathrm{AC} \pm 3 \%$ for an input variation of $\pm 15 \%, 60$ cycle, and 20 amperes.

- $\quad 85 \%$ efficiency at full load, common mode noise rejection $>120 \mathrm{~dB}$, and transverse noise rejection $>60 \mathrm{~dB}$.

\section{A.2 Host Computer}

A Digital Equipment Corporation VAX-11/730 running under the VMS operating system was selected to be the SAFT-UT field system host computer. It functions as a central controller funneling data to the SAFT-UT RealTime Processor, communicating with the graphics generator and data acquisition system, and doing the general housekeeping tasks that are needed. Although a VAX-11/730 was used for the SAFT-UT field system, any VAX-class computer running the VMS operating system could serve as the host computer; e.g., VAX$11 / 780$, VAX-11/750, or a MicroVAX. The hardware specifications of the SAFT-UT field system host computer are the following:

- VAX-11/730 with 5 MB of Random Access Memory.

- $\quad$ R80 $121 \mathrm{MB}$ hard disk.

- DMF32 communications controller

- LA120 printing terminal to act as the computer console.

- VT100-compatible terminal for user interaction. The SAFT-UT field system uses the Tektronix 4105 for this terminal because of its graphics capabilities. Software has been developed to allow use of this terminal as a backup graphics display unit should one have a need to use it in this capacity.

- VAX/VMS operating system with $C$ and FORTRAN 77 compilers.

- Unibus expansion box with four slot backplane.
- Tri-density tape system complete with unibus interface board. The tape drive provides archival storage for the large data bases that can accrue.

- DR11W parallel interface module that resides on the unibus. This parallel interface provides a data link to the SAFT-UT Real-Time Processor.

- High-speed PRONET serial data link interface card that is unibus compatible with appropriate VMS software driver. The data acquisition system interfaces through this high-speed serial data link to the host computer.

\section{A.3 Graphics Processing and Display}

The SAFT-UT field system uses a RAMTEK 9465 graphics processor for image display and analysis. It has the following specifications:

- MC68000-based display generator with 50 feet of interface cable, $1280 \times 1024 \times 12$ planes of refresh memory, 240 KBytes of user RAM, and a joystick cursor controller.

- Vector throughput rates (under DMA control) of 16,000 vectors per second and images (pixel arrays) transferred from the computer interface to the memory control processor at rates up to 892,000 pixels per second.

- VAX/VMS driver and DEC/VAX interface card.

- 19 inch color RGB, long persistance phosphor monitor.

\section{A.4 Real-Time Processor}

SAFT processing is a highly intensive computational process that can require a considerable amount of time to complete for some data files. The host computer has many tasks to perform (i.e., graphics control, data $\mathrm{I} / \mathrm{O}$, etc.); and if one wishes to process data while the data is being collected, an alternative peripheral device must exist to effectively implement the SAFT algorithm. The SAFT-UT Real-Time Processor was developed as a peripheral to the VAX host to compute the SAFT 
functions. Figure A.12 shows a simplified diagram of the hardware structure within the SAFT Processor module. It performs on the VME bus structure in a parallel processing environment to expedite the computational process. The following hardware specifications pertain to this module:

- 20 slot VME card cage having both J1 and J2 backplanes.

- Each board referenced is a double high/single wide VME-bus compatible board.

- \pm 12 volt power supply @ 6 amperes.

- $\quad+5$ volt power supply @ 73 amperes at temperatures to $60^{\circ} \mathrm{C}$.

- $68000 \mathrm{CPU}$ card having the ROM versions of PROBE and PSOS on board. Two RS232 asynchronous serial ports are required and full arbitration capability of the bus is needed. This CPU card performs the supervisor function.

- System controller board that provides general system utilities necessary for VME bus-compatible boards to operate in a VME bus card cage. It must support all four levels of bus request and allocation; system reset generation and bus arbiter options (fixed priority/round-robin) must also be supported.

- Disk controller card that supports both hard and flexible disk drives with the appropriate on-board ROMS to be compatible with the $\mathrm{CP} / \mathrm{M}$ operating system.

- 10 MByte Winchester hard disk and 48 tracks per inch, double-sided floppy disk drive.

- Two 1-MByte and one 256-KByte DRAM boards capable of 8-, 16-, and 32-bit operations with userselectable addressing.

- $\mathrm{CP} / \mathrm{M}$ operating system with BIOS, C-compiler, 68000 assembler, editor, and linker.

- VME bus high-speed parallel DMA interface board, fully emulating the DEC DR11W.
- Ten 68020 single-board computers that function as slave processors with 1 MByte of local RAM. Each of the slave boards must be capable of 32bit data transfers and come complete with EPROM sockets on board.

- A VT100 compatible terminal is used to communicate with the supervisor CPU board and to boot the Real-Time Processor when processing data files.

\section{A.5 SAFT-UT Field System Per- formance Requirements}

Certain requirements of the SAFT-UT field system should be emphasized. Experiences, primarily in field tests, have contributed to establishing the list that is as follows:

- The host computer hard disk storage size is important. When post analysis is performed, many different data files or processed files are needed for review before any conclusions can be reached. It is convenient if all the necessary files can reside on the hard disk. A disk with a capacity of 100 Mbytes or greater is recommended and should be considered a minimum for nuclear applications.

- The mechanical scanner needs to have two independently controllable scan axes to make use of the tandem SAFT mode. Adequate resolution of each of the axes is defined as "having the capability to set the increment size small enough to achieve a spatial sampling of at least one wave length."

- Temporal sampling is also a critical parameter when collecting data. The analog-to-digital converter that is used must be able to sample the transducer center frequency at least four times per cycle.

- A fully programmable delay gate is necessary in thicker materials to reduce the data file size so that more reasonable data display and manipulation rates can be achieved. This delay gate delays the sync pulse used to stop the digitizer sampling, and allows one to digitize only the area 
of interest; the digitizer then stores each digitized value which constitutes one A-scan.

- The host computer should be a VAX-class computer running the VMS operating system. Because of the larger files, the host computer and disk writing rates become the bottleneck for achieving fast processing speeds. Therefore, the faster VAX-class computer and disk writing specifications should be selected.

- Development of the SAFT-UT Real-Time Processor was a major milestone for the SAFT program and deviation from the hardware listed in Table A.4 may jeopardize the performance of this device. Even subtle hardware differences can be detrimental to the successful performance of the SAFT-UT Real-Time Processor due to the complex nature of a parallel processing environment.

\section{A.6 SAFT-UT Complete Hardware Listing}

Comprehensive hardware listings in Tables A.1 through A.4 are provided to facilitate duplication of the SAFTUT field system. The bracketed letters identify the block on the Data Acquisition System block diagram (Figure A.2), and the bracketed numbers refer to the manufacturer address list in Table A.5.

\section{A.7 Mechanical and Electrical System Drawings}

Figures A.13 through A.18 are electronic schematic diagrams of the PNL custom designed electronic modules. Drawings of the mechanical scanner design are shown in Figures A.19 through A.23. 
Table A.1. Data Acquisition System

\begin{tabular}{|c|c|c|}
\hline Description $\quad:$ & \% & H. Model \\
\hline 1. Mode Select Module <A $>$ & PNL & N/A \\
\hline 2. Remote Preamplifier $<\mathrm{B}>$ & Analog Modules $<1>$ & 5LNVA-7.5 \\
\hline 3. TVG Amplifier $<\mathrm{C}>$ & Analog Modules & VGA-7.5 \\
\hline 4. TVG Ramp Generator $<\mathrm{D}>$ & PNL & $\mathrm{N} / \mathrm{A}$ \\
\hline 5. Monitor Scope \#1<E> & Tektronix $<2>$ & SC-504 \\
\hline 6. Monitor Scope \#2 <F> & Tektronix & $5110 / 5 \mathrm{~A} 15 \mathrm{~N} / 5 \mathrm{~B} 10 \mathrm{~N}$ \\
\hline 7. Pulser Driver $\langle G\rangle$ & PNL & $\mathrm{N} / \mathrm{A}$ \\
\hline 8. Square Wave Pulser $<B>$ & PNL & $\mathrm{N} / \mathrm{A}$ \\
\hline 9. Programable Delay $\langle\mathrm{H}\rangle$ & LeCROY Research <3> & 2323 \\
\hline 10. Analog/Digital Converter $<\mathrm{I}>$ & LeCROY Research & TR8837F \\
\hline 11. Optical Encoder $<K, L, M>$ ( 3 each) & Disc Instruments $<4>$ & 821-1024-OALP-TTL \\
\hline 12. Programable Divider $\langle N\rangle$ & PNL & N/A \\
\hline 13. Programable Clock $<\mathrm{J}\rangle$ & LeCROY Research & 8501 \\
\hline 14. Up/Down Counter $<\mathrm{O}>$ & Kinetic Systems <5> & 3920 \\
\hline 15. Inc. Axis Step Motor $<T>$ & Eastern Air Devices $<6\rangle$ & LD11EAM3C \\
\hline 16. Scan Axes Step Motors $<U, V>$ & Superior Electric $<7>$ & M061-FD08 \\
\hline 17. 24V Power Supply (2 ea.) $<Y>$ & Acopian $<8>$ & 24PT10 \\
\hline 18. Step Motor Control $<P, Q, R>$ (3 ea.) & Joerger Enterprises $<9>$ & SMC-24 \\
\hline 19. Camac Crate Controller $<S>$ & Kinetic Systems & 3920 \\
\hline $\begin{array}{l}\text { 20. Computer (LSI } 11 / 23)<W> \\
\text { RT-11 operating system }\end{array}$ & ADAC Corp. $<10>$ & 2200-CRIX-M-R-611 \\
\hline 21. Q-Buss Expansion Box $<\mathrm{X}>$ & Digital Equipment $<11>$ & BA 11-ME \\
\hline 22. LSI-11 Bus Adapter $<X>$ & Kinetic Systems & $2920-Z 1 D$ \\
\hline 23. Drive (8" Hard/Floppy) $<Z>$ & Qualogy $<12>$ & DSD $880 / 20$ \\
\hline 24. Terminal $<A A>$ & Digital Equipment & $\mathrm{Vt}-100$ \\
\hline 25. ProNET Network $\langle\mathrm{X}\rangle$ & Proteon, Inc. $<13>$ & $\mathrm{P} 1100$ \\
\hline $\begin{array}{l}\text { 26. Camac Crate Card Cage }<B B> \\
25 \text { slot with power supply }\end{array}$ & Kinetic Systems & 1500 PIK \\
\hline
\end{tabular}




\begin{tabular}{||l|l|l||}
\hline \multicolumn{1}{|c|}{ Description } & \multicolumn{1}{|c|}{ Manufacturer } & \multicolumn{1}{|c|}{} \\
\hline 27. Analog Mainframe $<\mathrm{CC}>$ & Tektronix & TM-5006 \\
\hline 28. Mechanical Scanner $<\mathrm{DD}>$ & AMDATA $<14>$ & AMAPS 822511 \\
\hline 29. Power Controller $<\mathrm{EE}>$ & Pulizzi Engineering $<15>$ & PC 230 \\
\hline 30. Power Conditioner 2KVA $<\mathrm{FF}>$ & Sola Electric $<16>$ & $63-13-220$ \\
\hline
\end{tabular}

Table A.2. Host Computer

\begin{tabular}{||l|l|l||}
\hline \multicolumn{1}{|c|}{ Description } & \multicolumn{1}{|c|}{ Manifacturer } & \multicolumn{1}{|c||}{ Model } \\
\hline $\begin{array}{l}\text { 1. Vax 11/730 CPU w/1 Mbyte Memory, } \\
\text { VMS, Fortran, and C }\end{array}$ & Digital Equipment & 11730-ZA-SV-CXWMA-CK \\
\hline 2. Floating Point Accel. & Digital Equipment & FP730 \\
\hline 3. 1200 Baud Printing Terminal & Digital Equipment & LS120-AD \\
\hline 4. 3 MB Expansion Memory & Digital Equipment & MS730-CC \\
\hline 5. Multifunction Add-On Expander & Digital Equipment & DMF32-AB \\
\hline 6. 10.4 MByte Disk & Digital Equipment & RL02-AK \\
\hline 7. 124 MByte Fixed Disk & Digital Equipment & R80-AA \\
\hline 8. UNBUSS Expansion Box & Digital Equipment & BA11-KW \\
\hline 9. 4 Slot UNIBUSS Backplane & Digital Equipment & DD11-CK \\
\hline 10. Pronet Network & Proteon, Inc. & P1000 \\
\hline 11. Parallel DMA Interface & MDB Systems, Inc. <17> & DR11-W \\
\hline 12. Graphics Terminal & Tektronix & 4105 \\
\hline 13. Tri Density Tape Drive & Kennedy <18> & 9400 \\
\hline 14. Unibus Controller & DILOG <19> & DU132 \\
\hline 15. Power Controller & PULIZZI Engineering & PC 230 \\
\hline 16. Power Conditioner 3KVA & Sola Electric & $63-13-230$ \\
\hline 17. Power Conditioner 2KVA & Sola Electric & $63-13-220$ \\
\hline \hline
\end{tabular}


Table A.3. Graphics Processing and Display

\begin{tabular}{|l|l|l||}
\hline \multicolumn{1}{|c|}{$\quad \therefore \quad$ Despcription } & \multicolumn{1}{|c||}{ Manufacturer } & Model \\
\hline 1. DEC/VAX Compatible Display System & RAMTEK <20> & RM-9465 Marquis 7000 \\
\hline 2. Refresh Memory Upgrade & RAMTEK & MQ-256A \\
\hline 3. Memory Board & RAMTEK & $508072-02$ \\
\hline 4. Joystick Controller & RAMTEK & 4401 \\
\hline 5. $19 "$ Graphics Monitor & RAMTEK & GM-859-CLP \\
\hline 6. Polaroid Camera & PECA Products <21> & DS-34 \\
\hline 7. 19" CRT Hood for Camera & PECA Products & DS-3920 \\
\hline
\end{tabular}

Table A.4. Real-Time SAFT Processor

\begin{tabular}{||l|l|l||}
\hline \multicolumn{1}{|c|}{ Description } & \multicolumn{1}{|c|}{ Manufacturer } & Model \\
\hline 1. J1-20 Slot VME Backplane & Scroff $<22>$ & $60800-306$ \\
\hline 2. J2-20 Slot VME Backplane & Scroff & $60800-345$ \\
\hline 3. 20 Slot Card Cage & Scroff & $20817-032$ \\
\hline 4. 300 Watt Power Supply & Scroff & $11008-177$ \\
\hline 5. 10 MByte Hard Disk & Shugart <23> & SA712 \\
\hline 6. 5.25-inch Mini Floppy Disk & Shugartq & SA455 \\
\hline 7. Disk Drive P/S $+5 V$ & ACDC Electronics <24> & ECV5N6-1 \\
\hline 8. Disk Drive P/S +12V & ACDC Electronics & ECV12N68-1 \\
\hline 9. Disk Drive Enclosure & Tracewell Enclosures <25> & CH519 \\
\hline 10. Disk Controller & Signetics <26> & SMVME-4300 \\
\hline 11. System Controller & Signetics & SMVME-1500 \\
\hline 12. Parallel DMA Interface & IKON Corporation <27> & DR11-W \\
\hline 13. CPU Card/Supervisor with \\
$\begin{array}{l}\text { PROBE-68K, PSOS-68K, } \\
\text { CP/M, and C-Compiler }\end{array}$ & Signetics & SMVME-2000A \\
\hline
\end{tabular}


Appendix A

\begin{tabular}{||l|l|l||}
\hline \multicolumn{1}{|c|}{ Description } & \multicolumn{1}{|c|}{ Manufacturer } & \multicolumn{1}{c|}{ Model } \\
\hline 14. 256KB Ram Memory & Signetics & SMVME-3100SD \\
\hline 15. 1MB Ram Memory (2 ea.) & Signetics & SMVME-3110SD \\
\hline 16. 68020 SBC (10 ea.) & Motorola <28> & MVME 133-1 \\
\hline 17. Terminal & Digital Equipment & VT-100 \\
\hline 18. Power Supply $+5 \mathrm{~V}$ & Lambda <29> & LGS-6A-5-OVR \\
\hline
\end{tabular}

Table A.5. Vendor Addresses

Vender addresses are provided to ease the task of purchasing all or part of the hardware used in the SAFT-UT field system.

\begin{tabular}{||l|l|c||}
\hline \multicolumn{1}{|c|}{ Manufacturer } & \multicolumn{1}{|c|}{ Adddress } & Telephone \\
\hline Analog Modules & 126 Baywood Ave, Longwood, FL 32750 & $305-339-4356$ \\
\hline Tektronix, Inc. & P.O. Box 500, Beaverton, OR 97077 & $503-627-9000$ \\
\hline LeCROY Research Systems & 1806 Embarcadero Road, Palo Alto, CA 94303 & $916-425-2000$ \\
\hline Disc Instruments & 102 E.Baker Street, Costa Mesa, CA 92626 & $714-979-5300$ \\
\hline Kinetic Systems Corp. & 11 Maryknoll Drive, Lockport, II 60441 & $815-838-0005$ \\
\hline Eastern Air Devices & Dover, NH 03820 & $603-742-3330$ \\
\hline Superior Electric & 383G Middle St., Bristol, CT 06010 & $203-582-9561$ \\
\hline Acopian & 131G Loomis St., Easton, PA 18044 & $215-258-5441$ \\
\hline Joerger Enterprises, Inc. & 166 Laurel Road, East Northprot, NY 11731 & $516-757-6200$ \\
\hline ADAC Corporation & 70G Tower Office Pk., Woburn, MA 01801 & $617-935-6668$ \\
\hline Digital Equipment Corp. & 200G Baker Ave., Concord, MA 01742 & $916-447-7600$ \\
\hline Qualogy & 2241 Lundy Ave., San Jose, CA 95131 & $408-946-5800$ \\
\hline Proteon Inc. & 4G Tech Circle, Natick, MA 01760 & $617-655-3340$ \\
\hline Amdata, Inc. & 576 Charcot Avenue, San Jose, CA 95131 & $408-435-1090$ \\
\hline PULIZZI Engineering & 3260 S. Susan St., Santa Ana, CA 92704-6865 & \\
\hline Sola Electric & 1717G Busse Rd., Elk Grove Village, IL 60007 & $312-439-2800$ \\
\hline
\end{tabular}




\begin{tabular}{|c|c|c|}
\hline Manufacturer & - & Telephonë \\
\hline MDB Systems Inc. & $\begin{array}{l}1995 \text { North Batavia St., Box 5508, Orange, CA } 92267- \\
0508\end{array}$ & 714-998-1339 \\
\hline Kennedy & 1600 Shamrock Ave., Monrovia, CA 91016 & $818-357-8831$ \\
\hline DILOG & Dept.G, 1555 S. Sinclair St., Anaheim, CA 92806 & $714-937-5700$ \\
\hline Ramtek Corporation & Suite 211, 110 110th Ave. N.E., Bellevue, WA 98004 & $206-451-2559$ \\
\hline PECA Products & 1700 Highway 14 East, Jamesville, WI 53545 & $617-969-4522$ \\
\hline Schroff Inc. & 170G Commerce Dr., Warwick, RI 02886 & $401-732-3770$ \\
\hline Shugart Corp. & Dept.G, 475 Oakmead Pkwy, Sunnyvale, CA 94086 & $408-737-4355$ \\
\hline ACDC Electronics & 401G Jones Rd., Oceanside, CA 92054 & $619-757-1880$ \\
\hline Tracewell Enclosures, Inc. & $\begin{array}{l}\text { 7032G Worthington Galena Road, Columbus, } \mathrm{OH} \\
43085\end{array}$ & $614-846-6175$ \\
\hline Signetics Corp. & $\begin{array}{l}\text { Dept.G, } 811 \text { E. Arques Ave., PO Box 3409, Sunnyvale, } \\
\text { CA } 94088\end{array}$ & $408-991-2000$ \\
\hline IKON Corp. & 2617 Western Ave., Seattle, WA 98121 & $206-624-3410$ \\
\hline Motorola Inc. & $\begin{array}{l}\text { Dept. G, PO Box 20912, M/S Adv 72, Phoenix, AZ } \\
85036\end{array}$ & $602-244-6900$ \\
\hline Lamda Electronics & $121 G$ International Dr., Corpus Christi, TX 78410 & 512-289-0403 \\
\hline
\end{tabular}


Appendix A

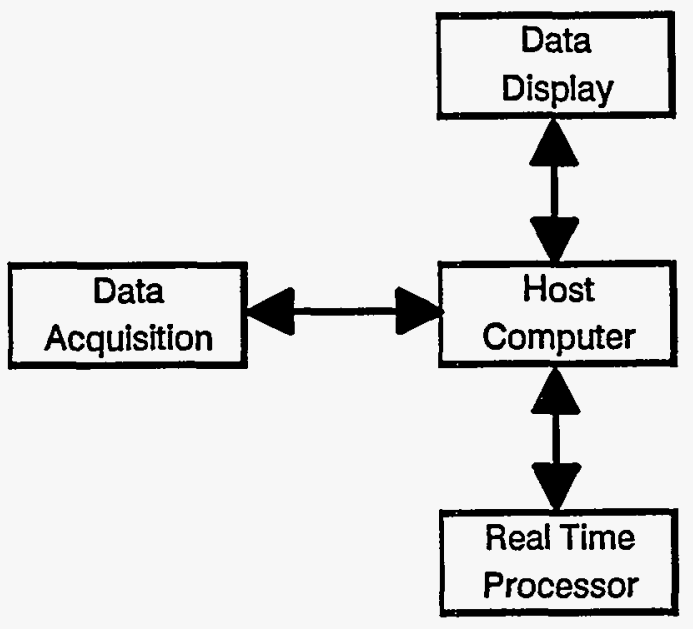

Figure A.1. SAFT-UT Field System Block Diagram 


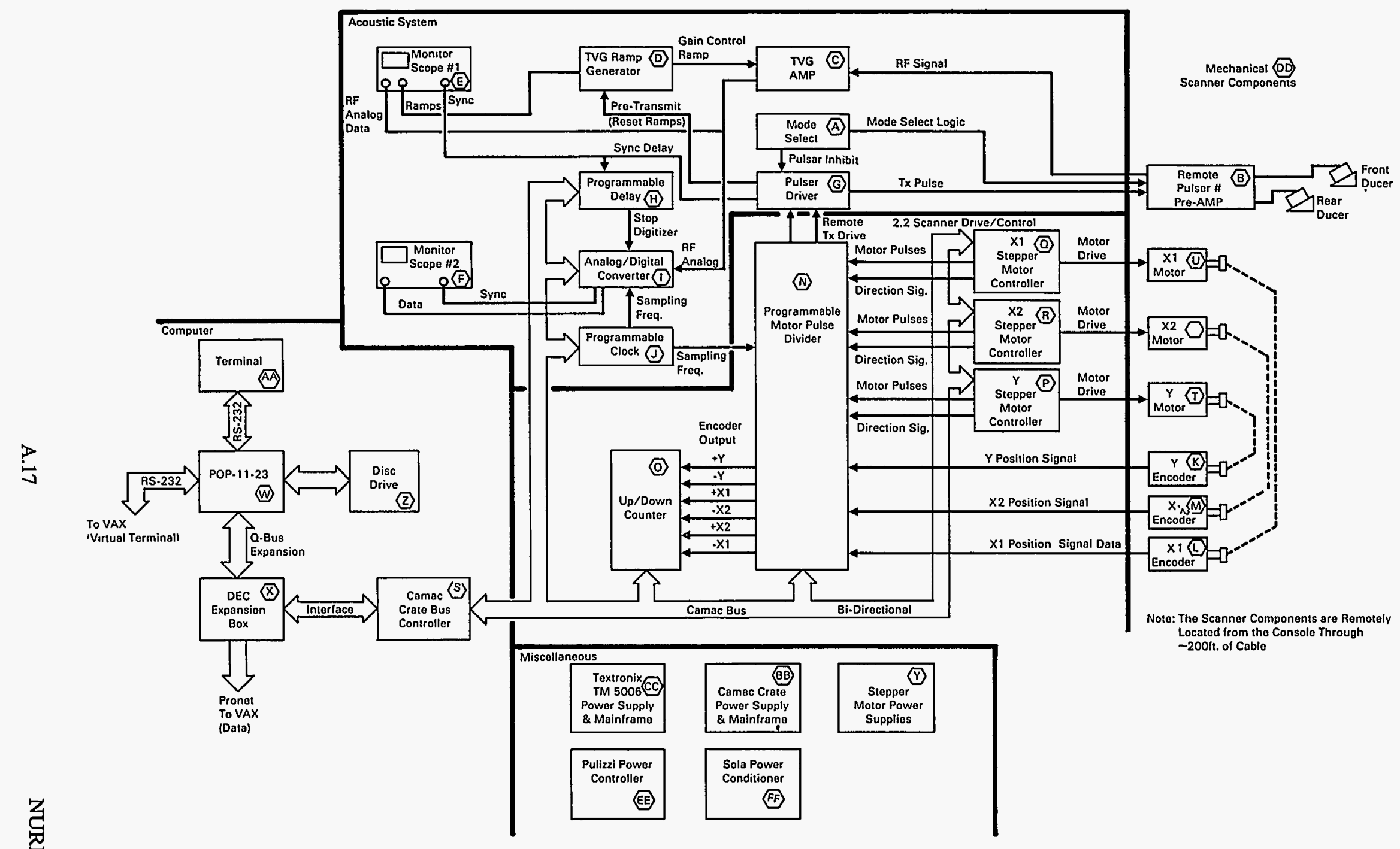

Figure A.2. SAFT-UT Data Acquisition System 
Appendix A

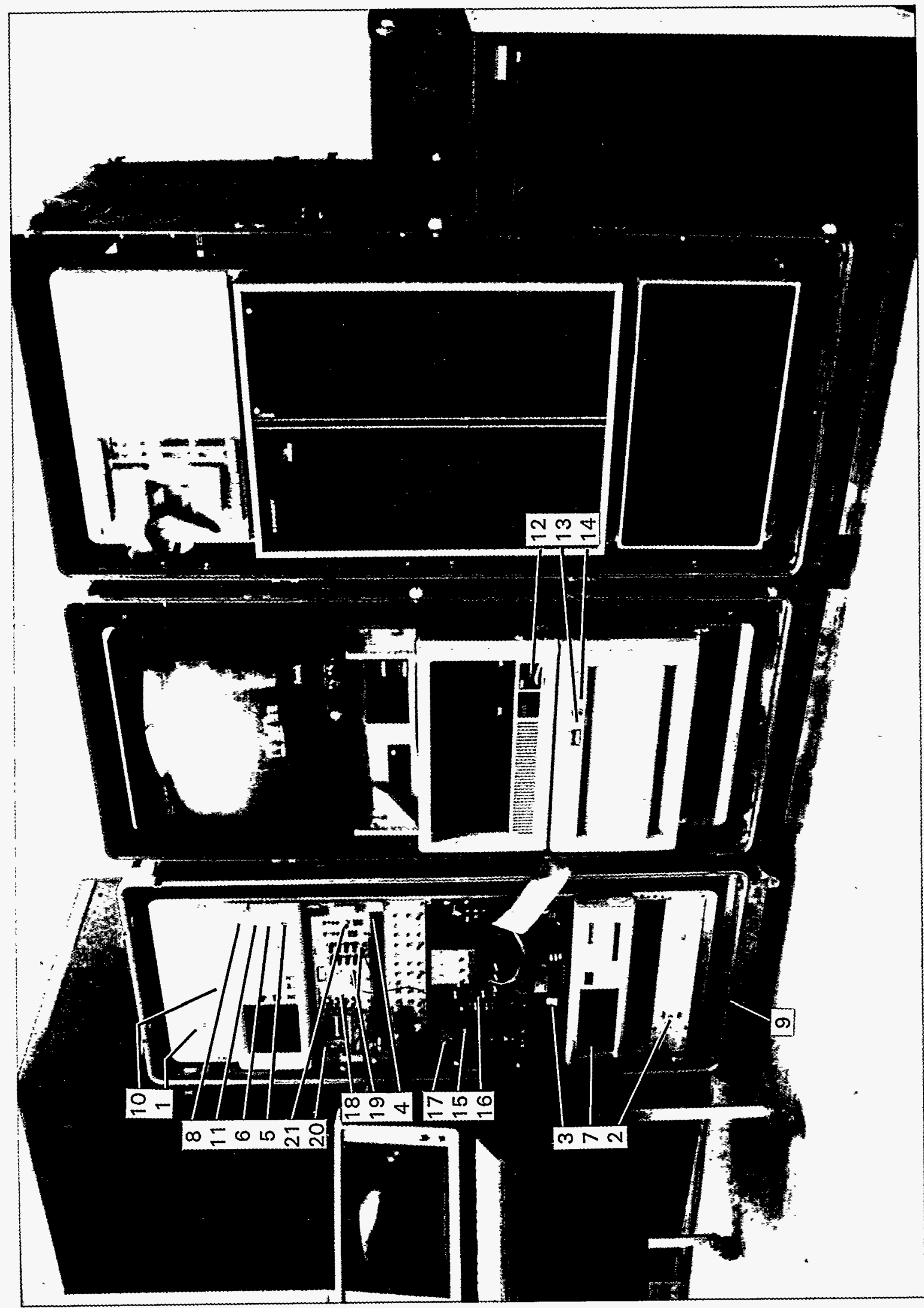

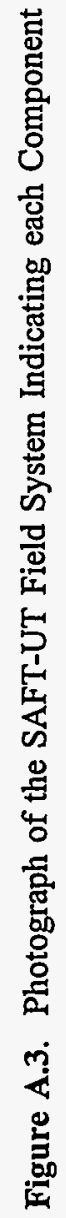




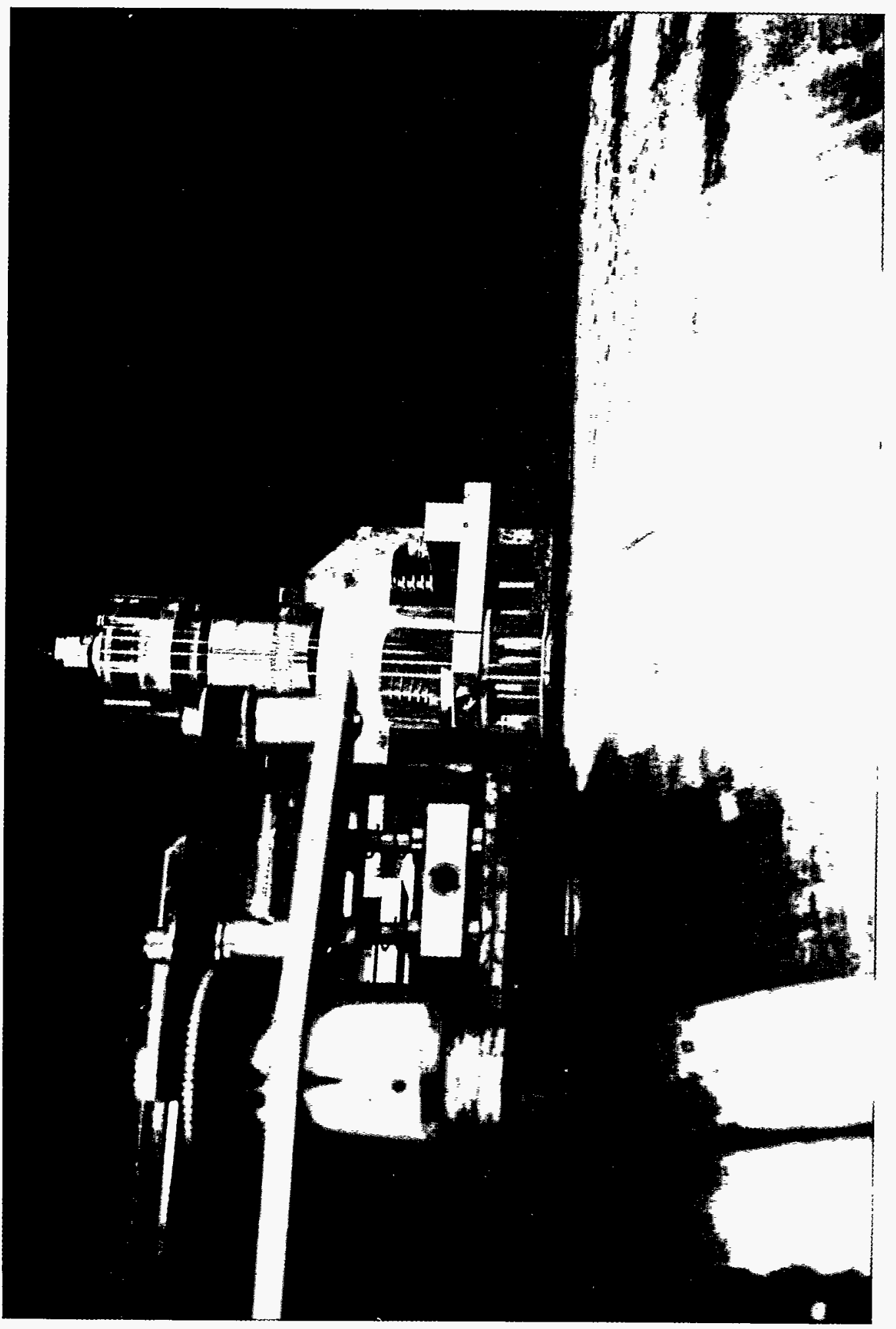

总 


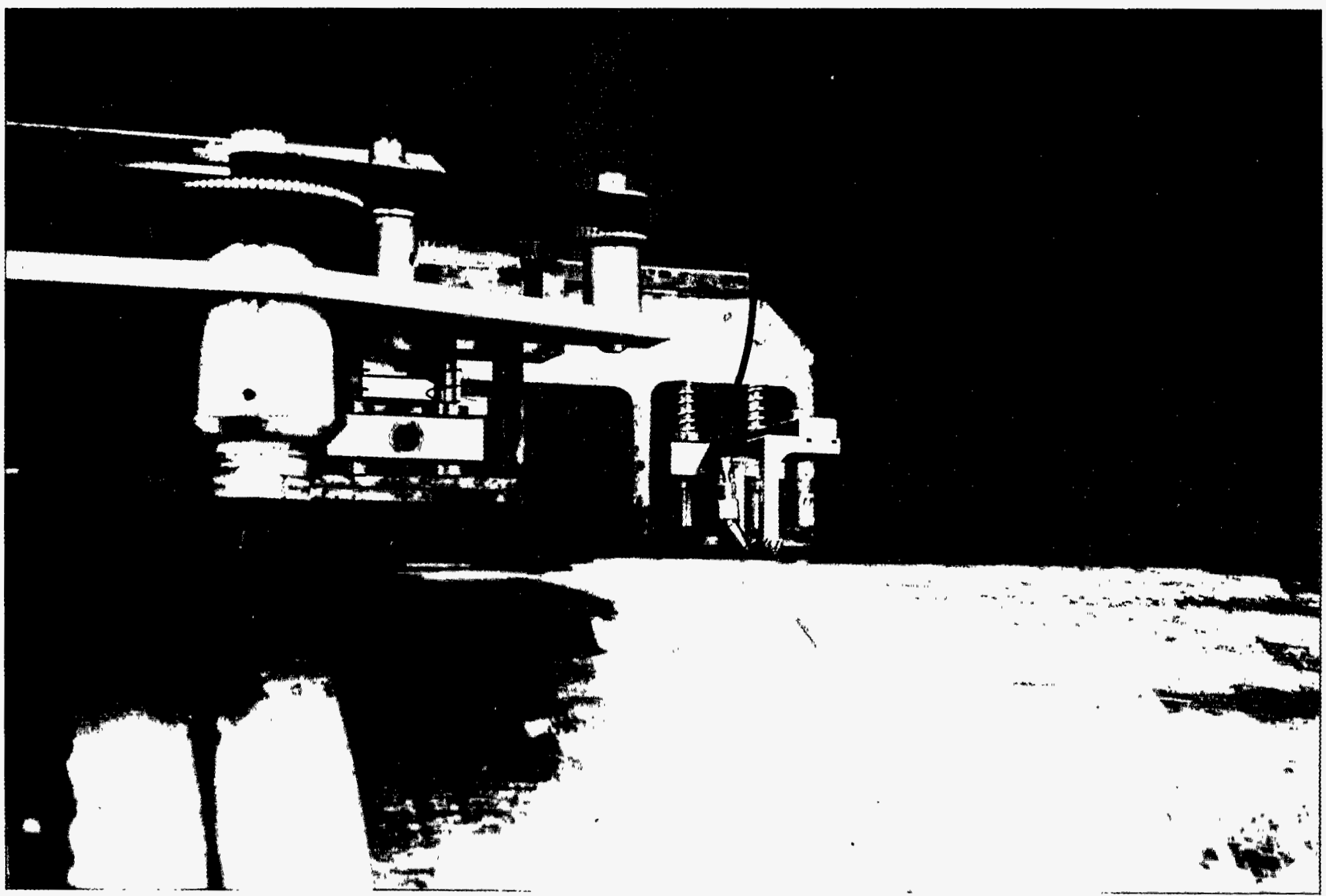


Appendix A

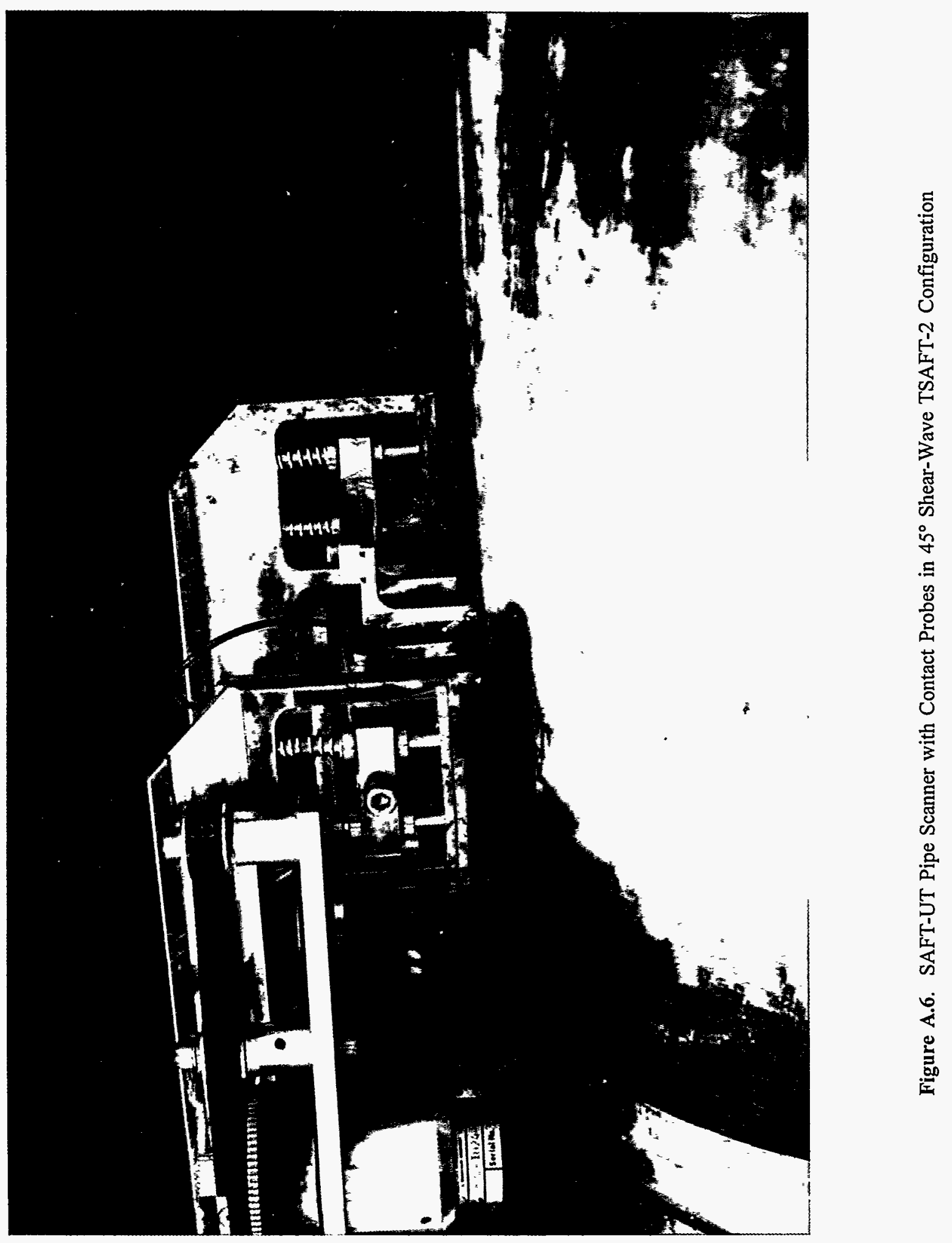


Appendix A

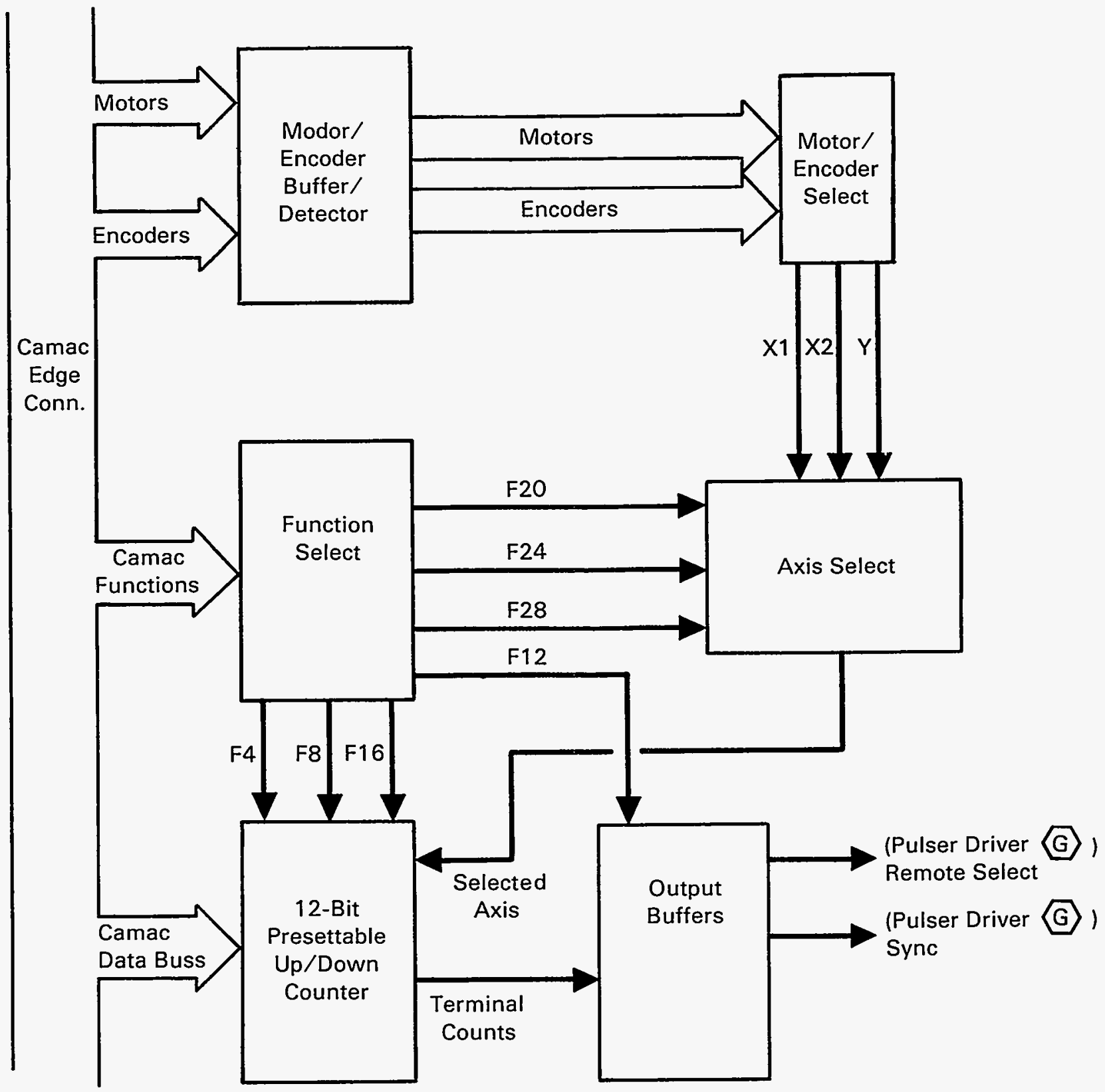

F4 Clears Counter

F8 Loads Counter with New Increment Count

F12 Sets Pulse Driver to Remote

F16 Latches New Increment Count

F20 Select y Axis

F24 Select $x 1$ Axis

F28 Select $\times 2$ Axis

Figure A.7. CAMAC Programmable Divider Block Diagram 
Remote Mode

Selection Control

Line 1

Line 2

(Mode Select A )

w

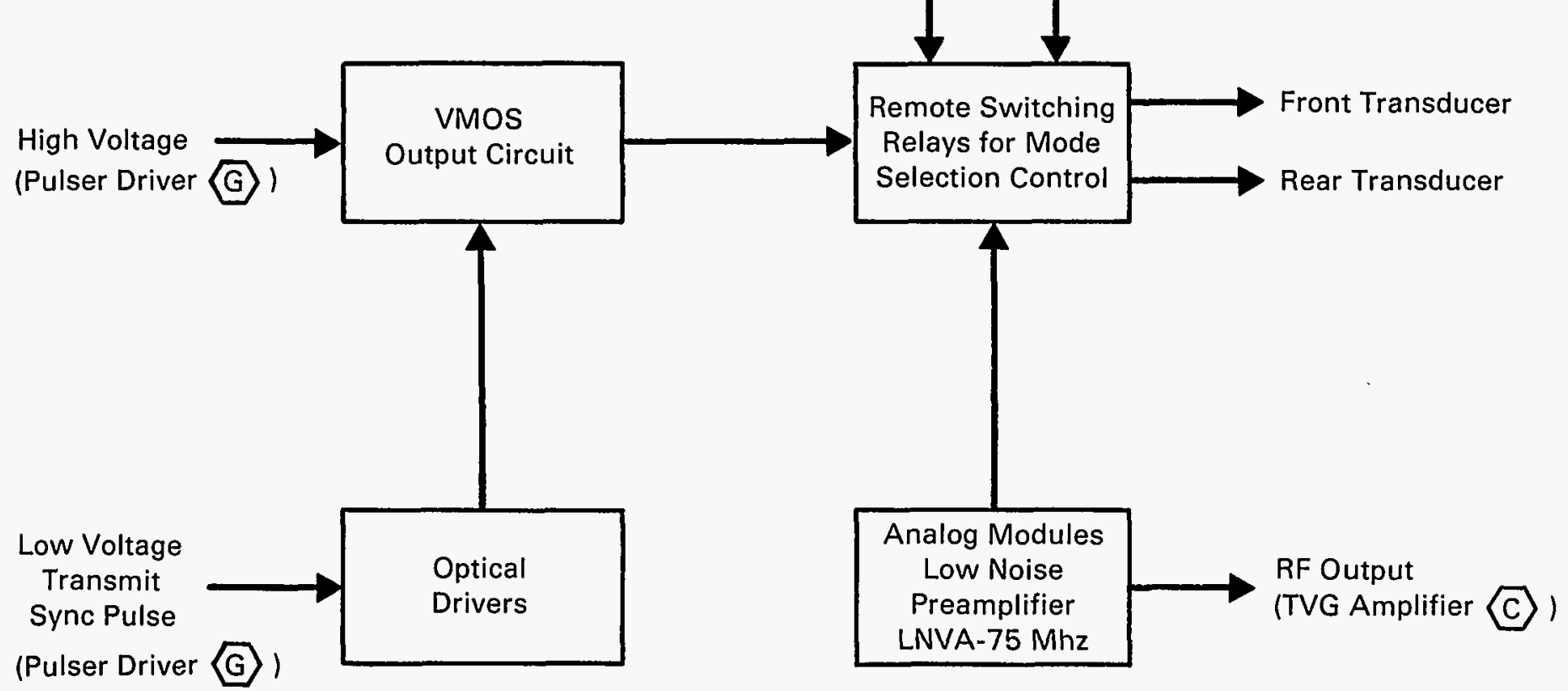

Figure A.8. Block Diagram of Remote Pulser/Preamplifier 


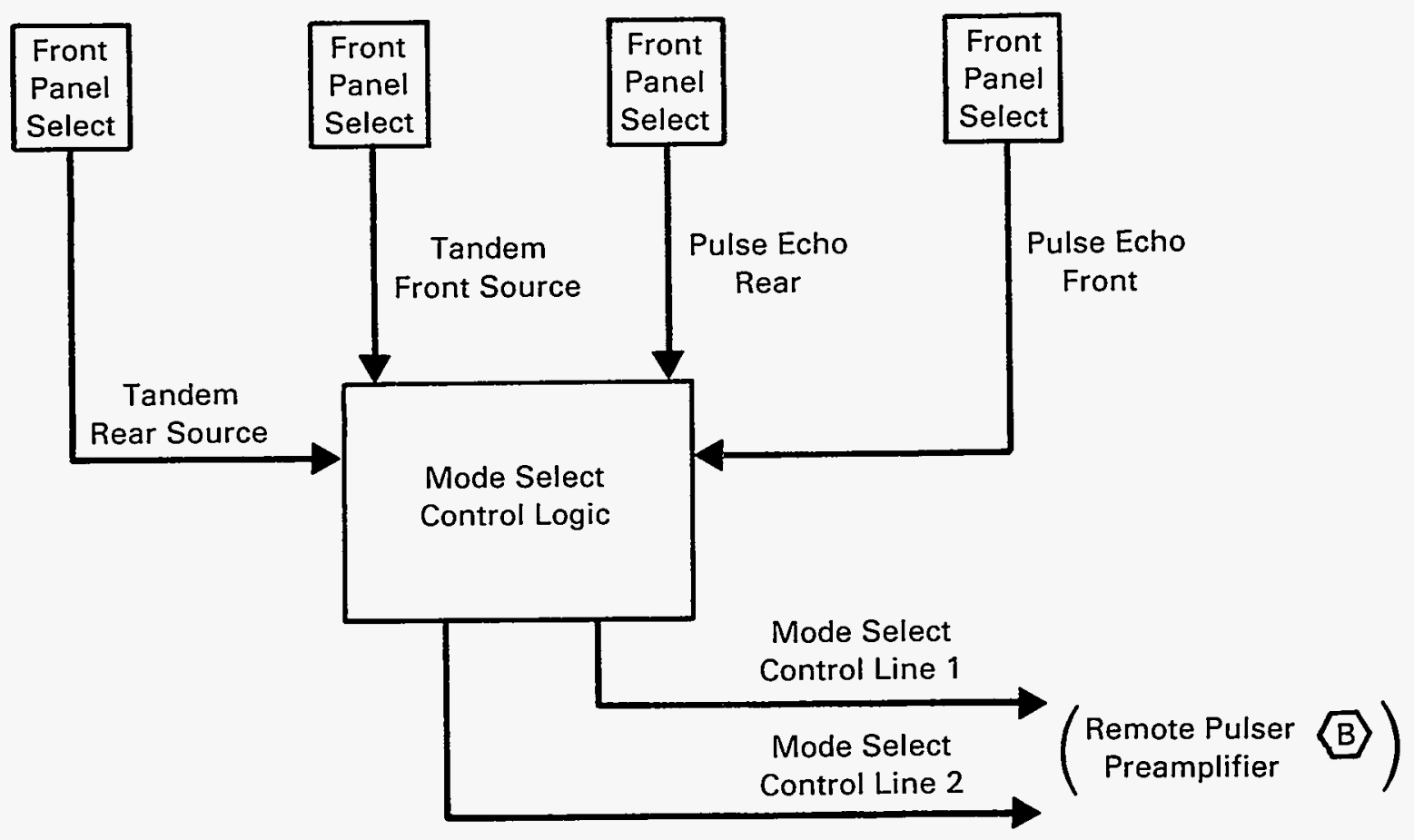

Figure A.9. Block Diagram of SAFT Mode-select Module 


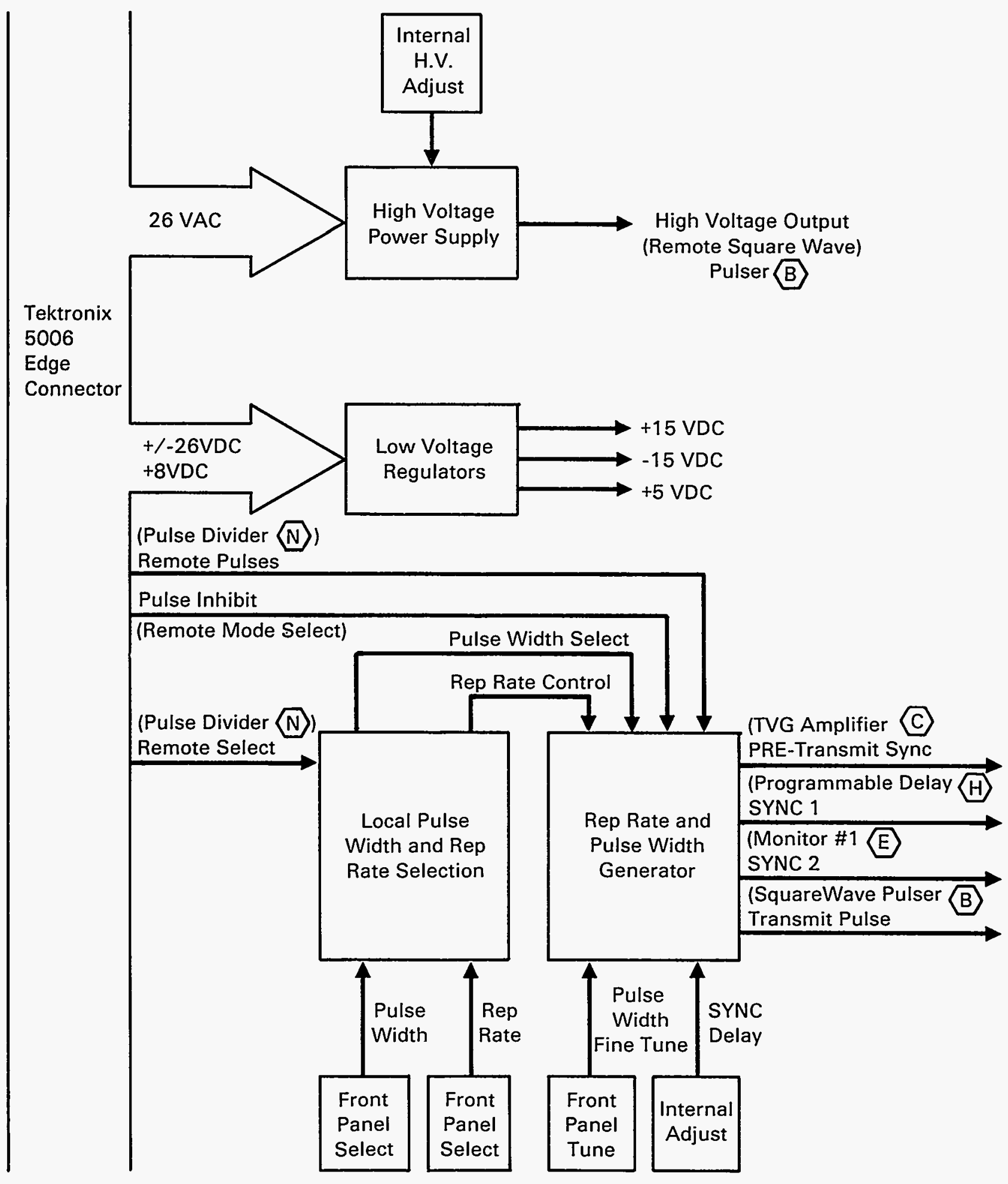

Figure A.10. Square Wave Pulser Drive Module Block Diagram 
Z⿱彐口

胥

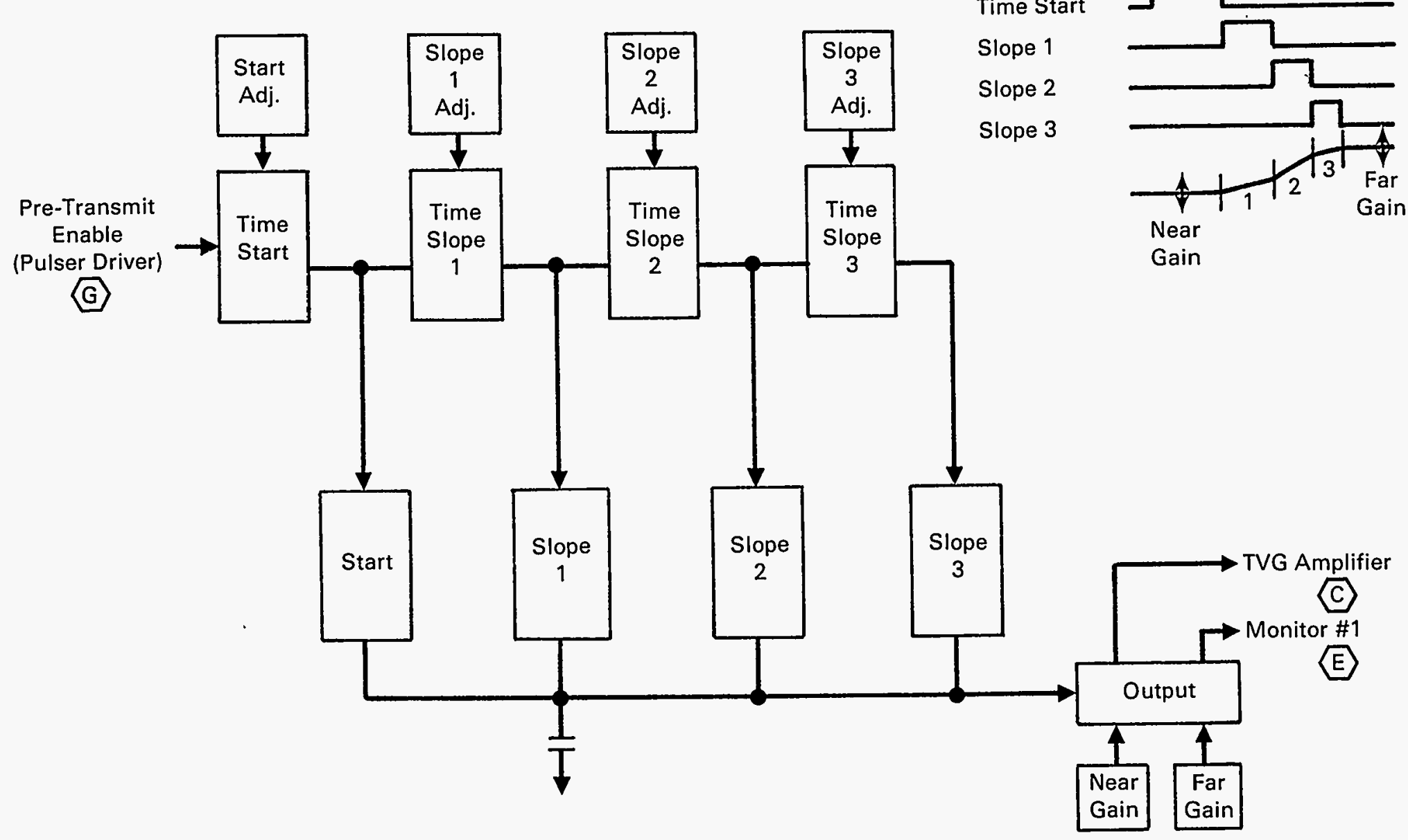

Figure A.11. Time-Variable-Gain (TVG) Ramp Generator Block Diagram 


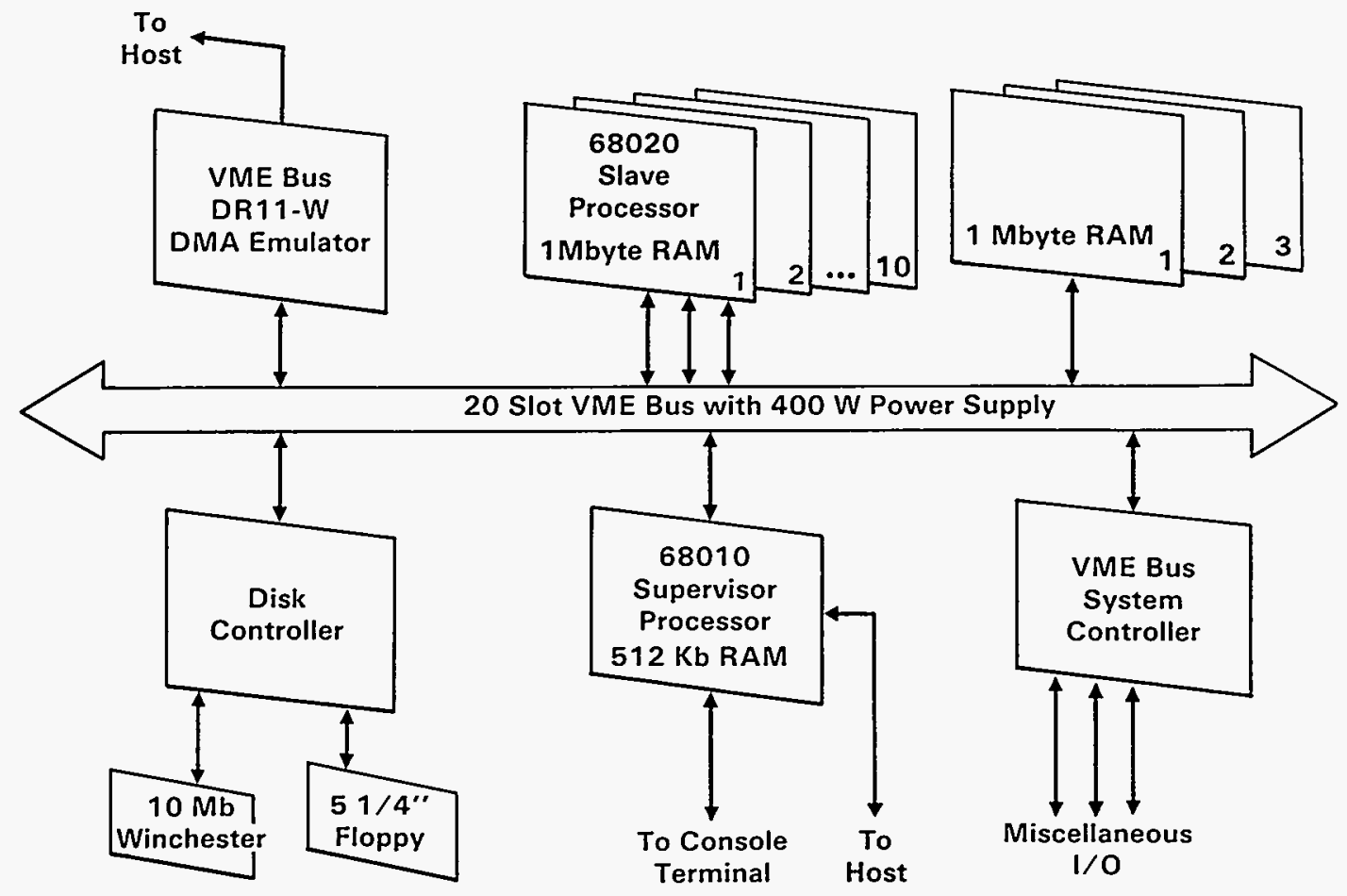

Figure A.12. Block Diagram of the Real-Time SAFT Processor 


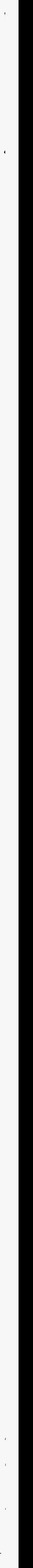




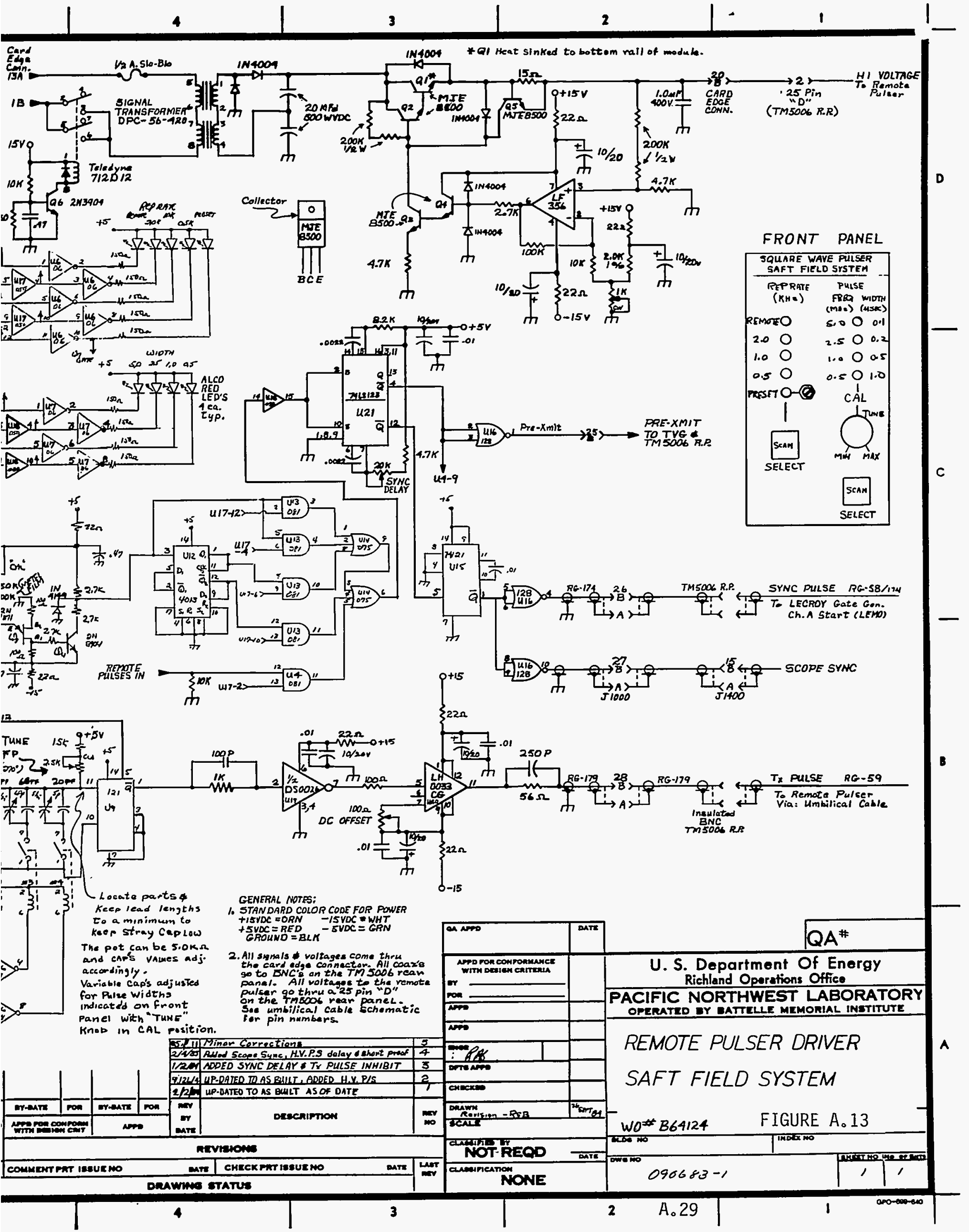




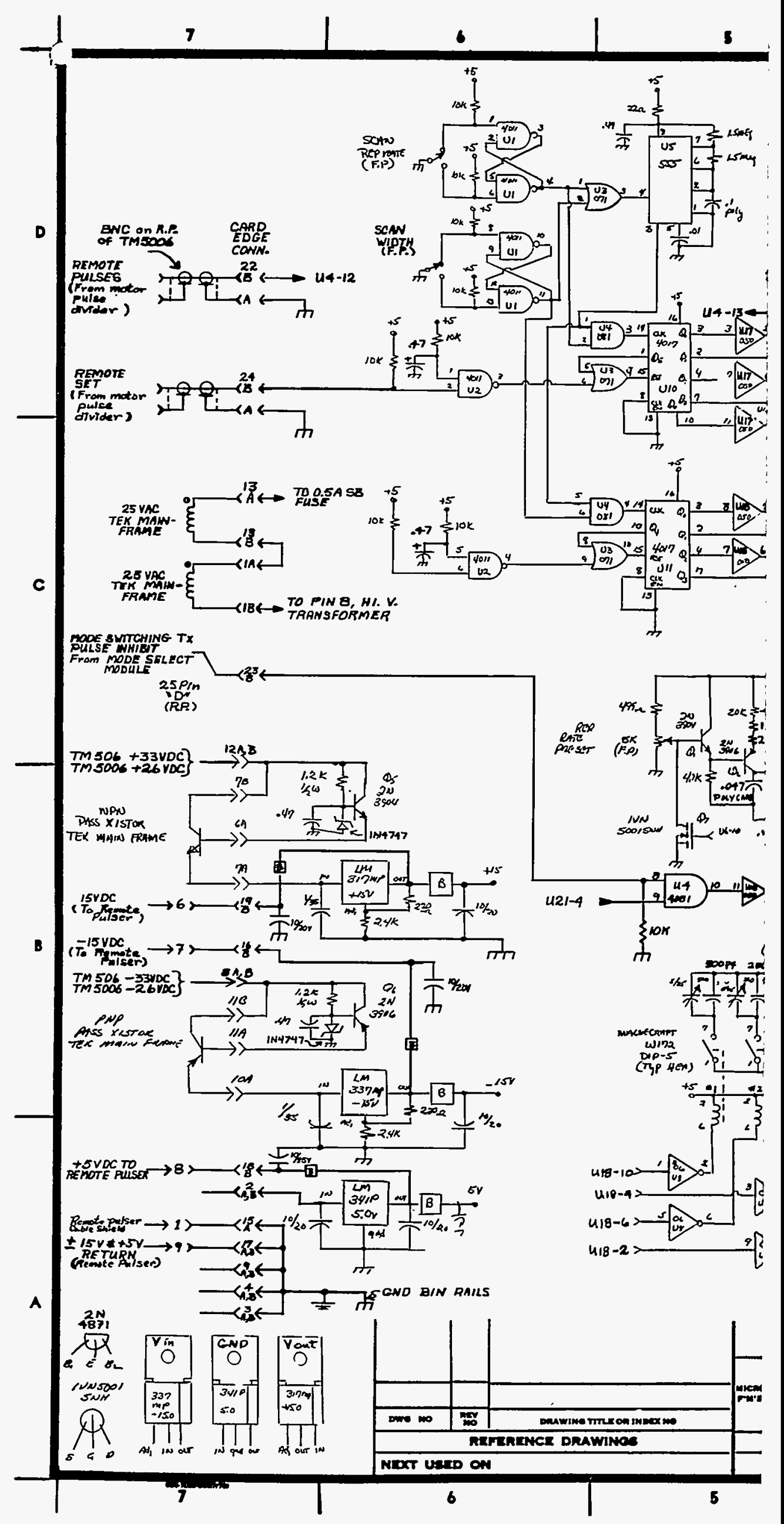




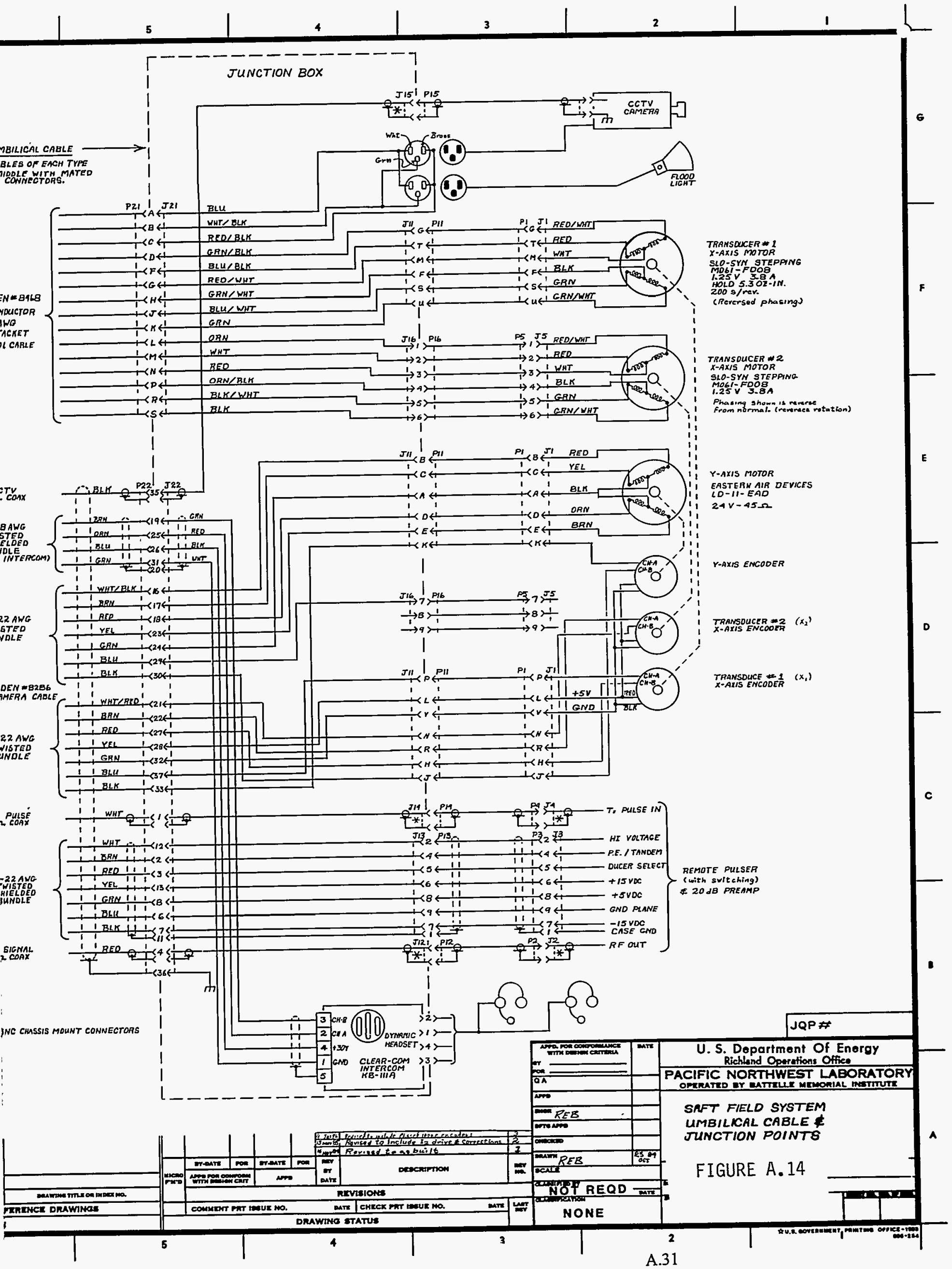




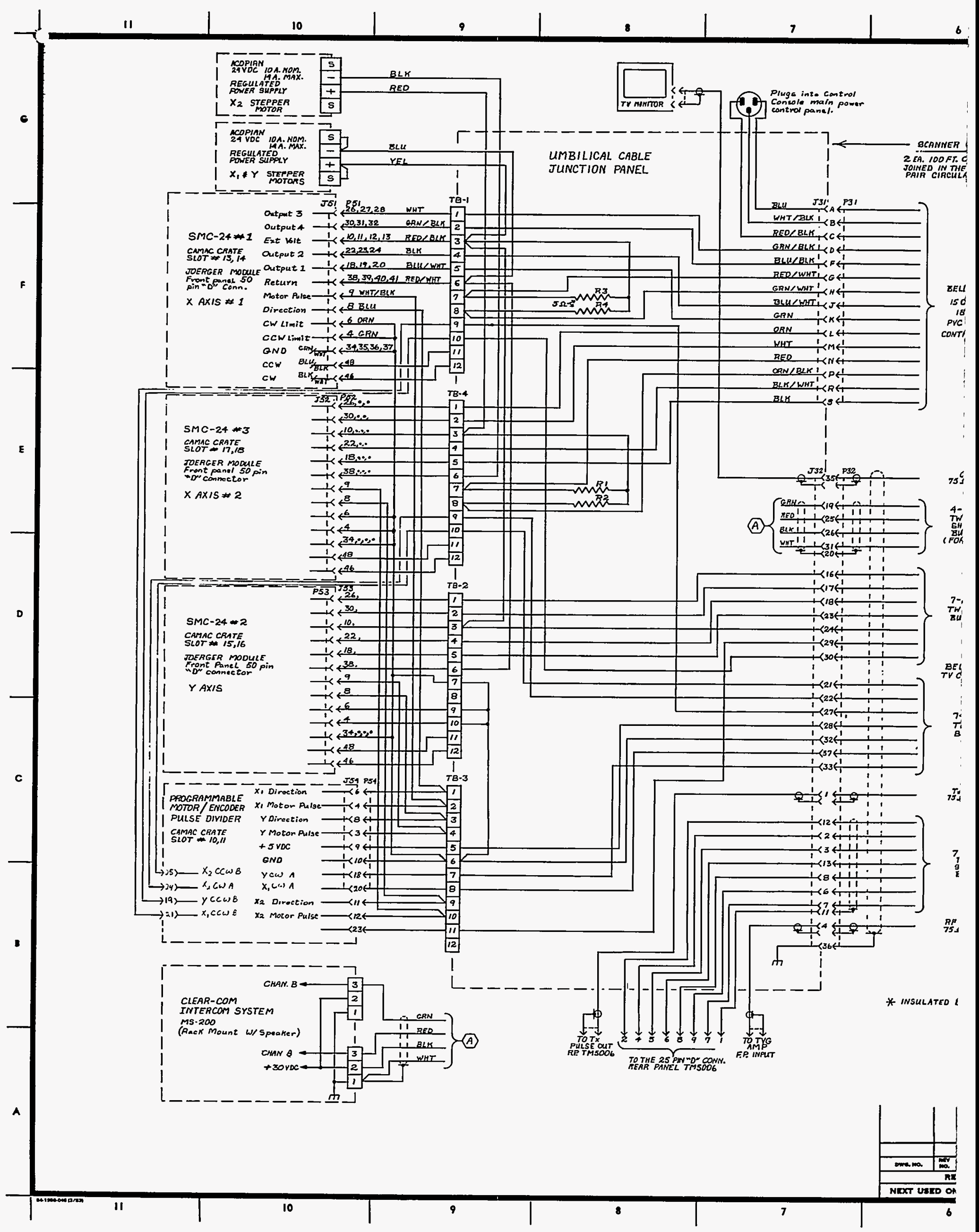



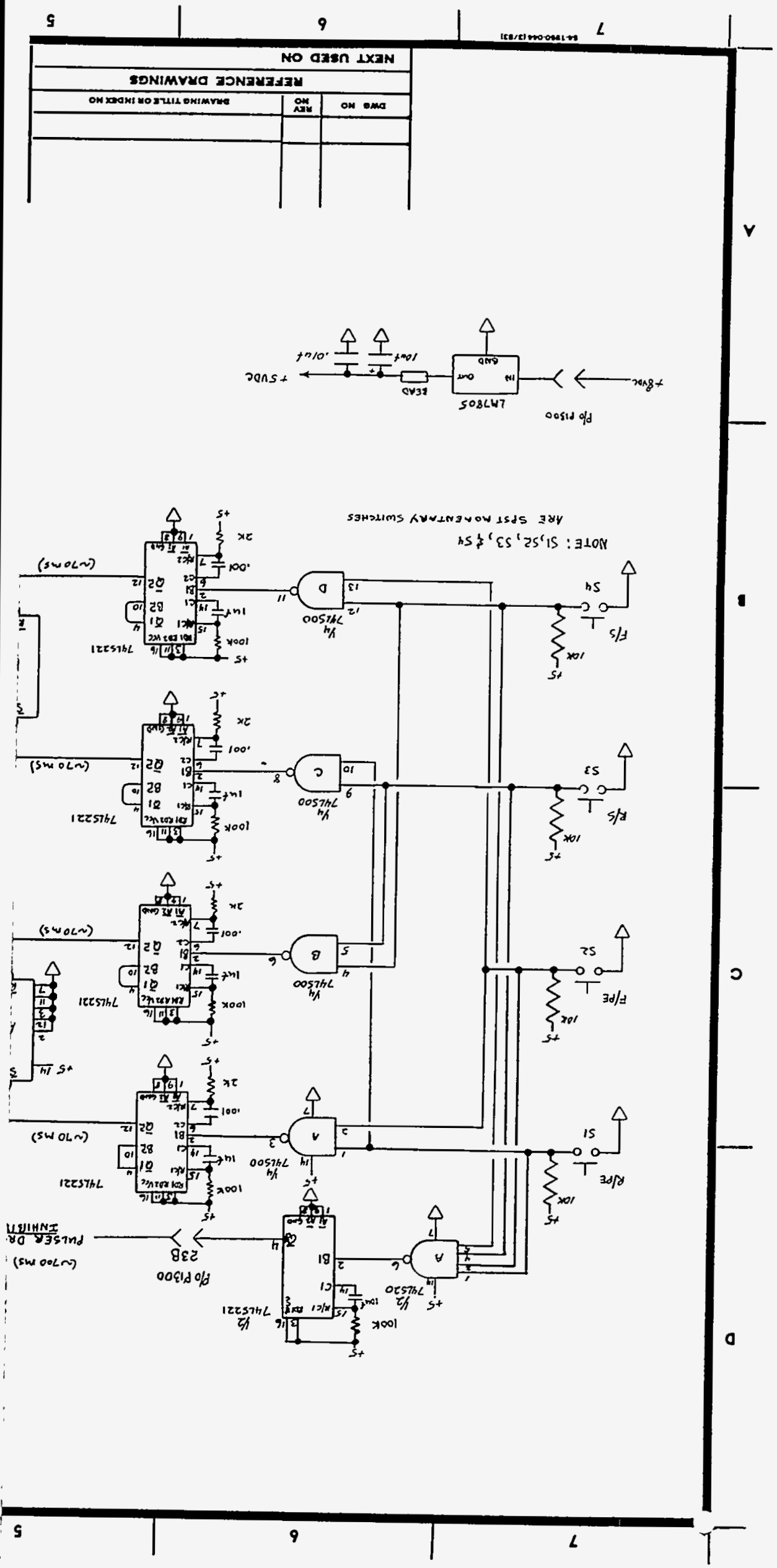


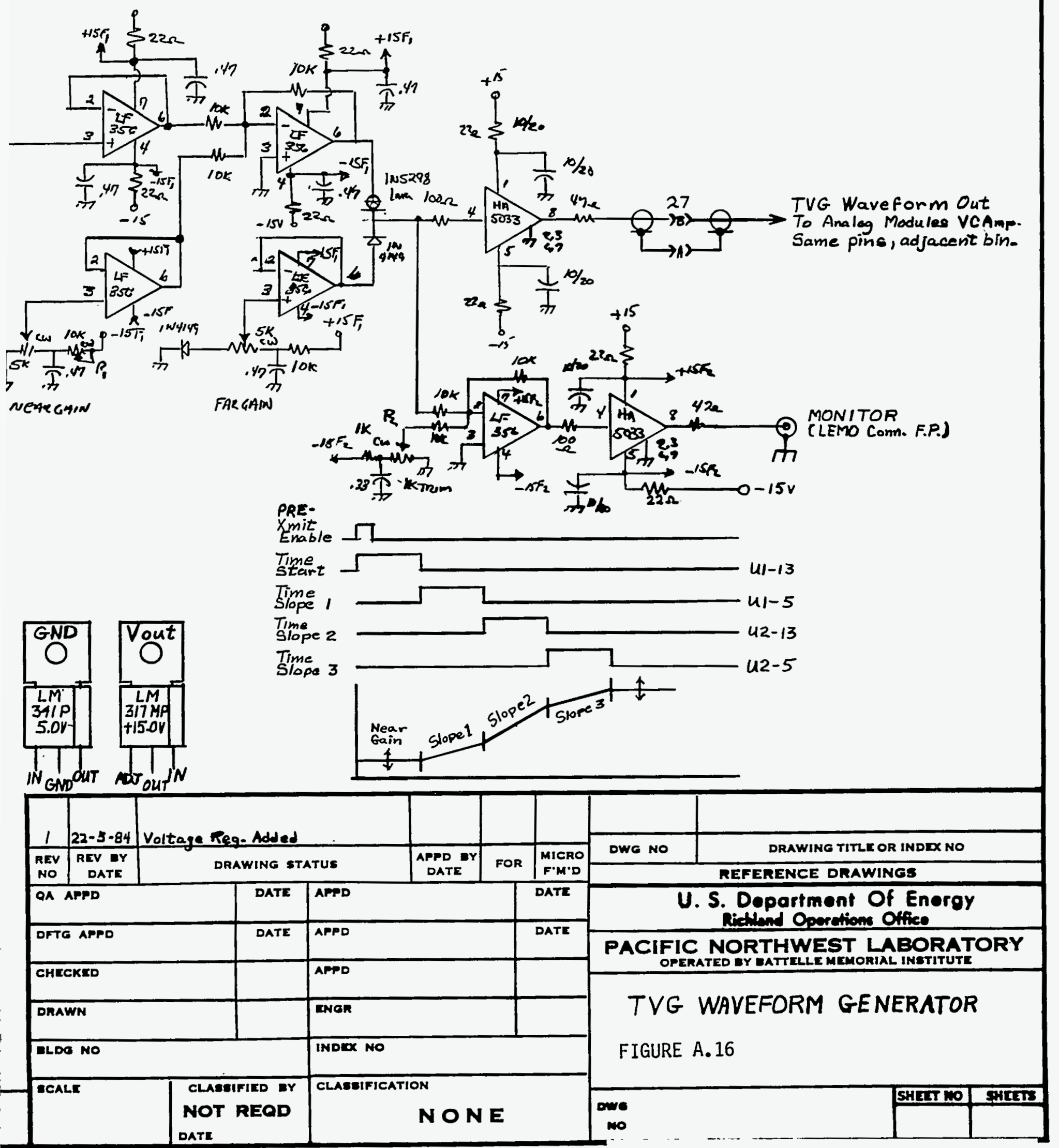




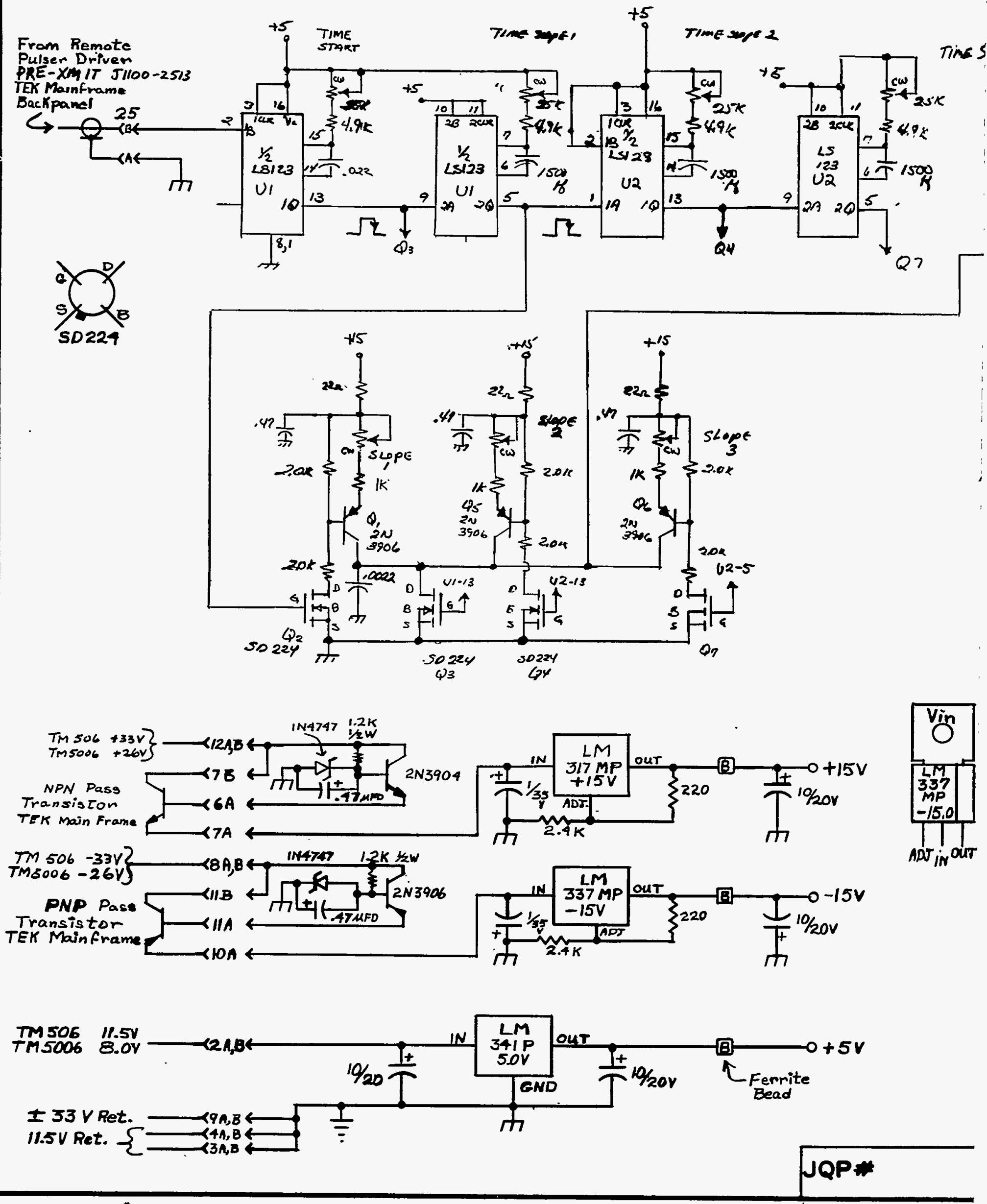




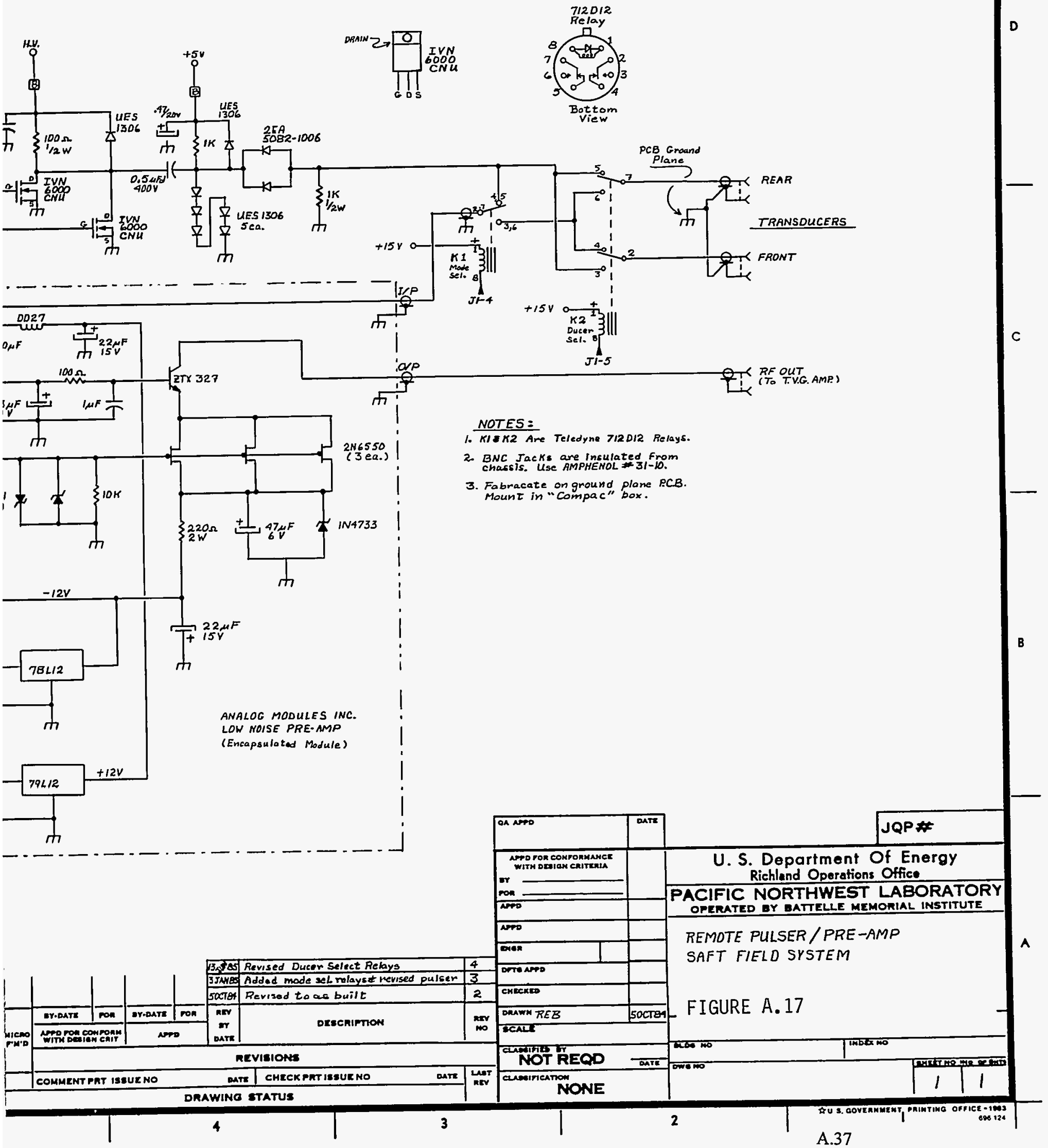




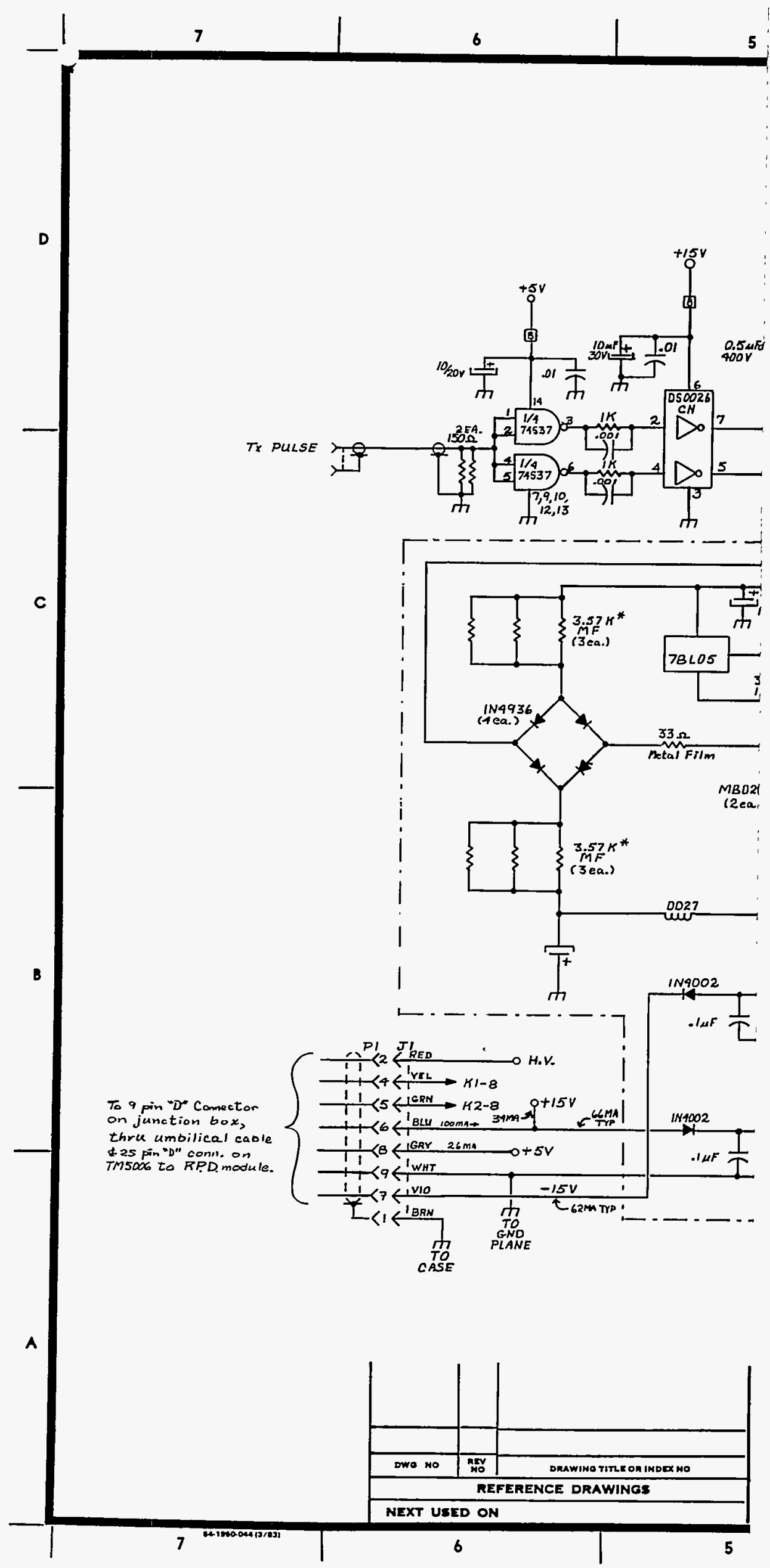




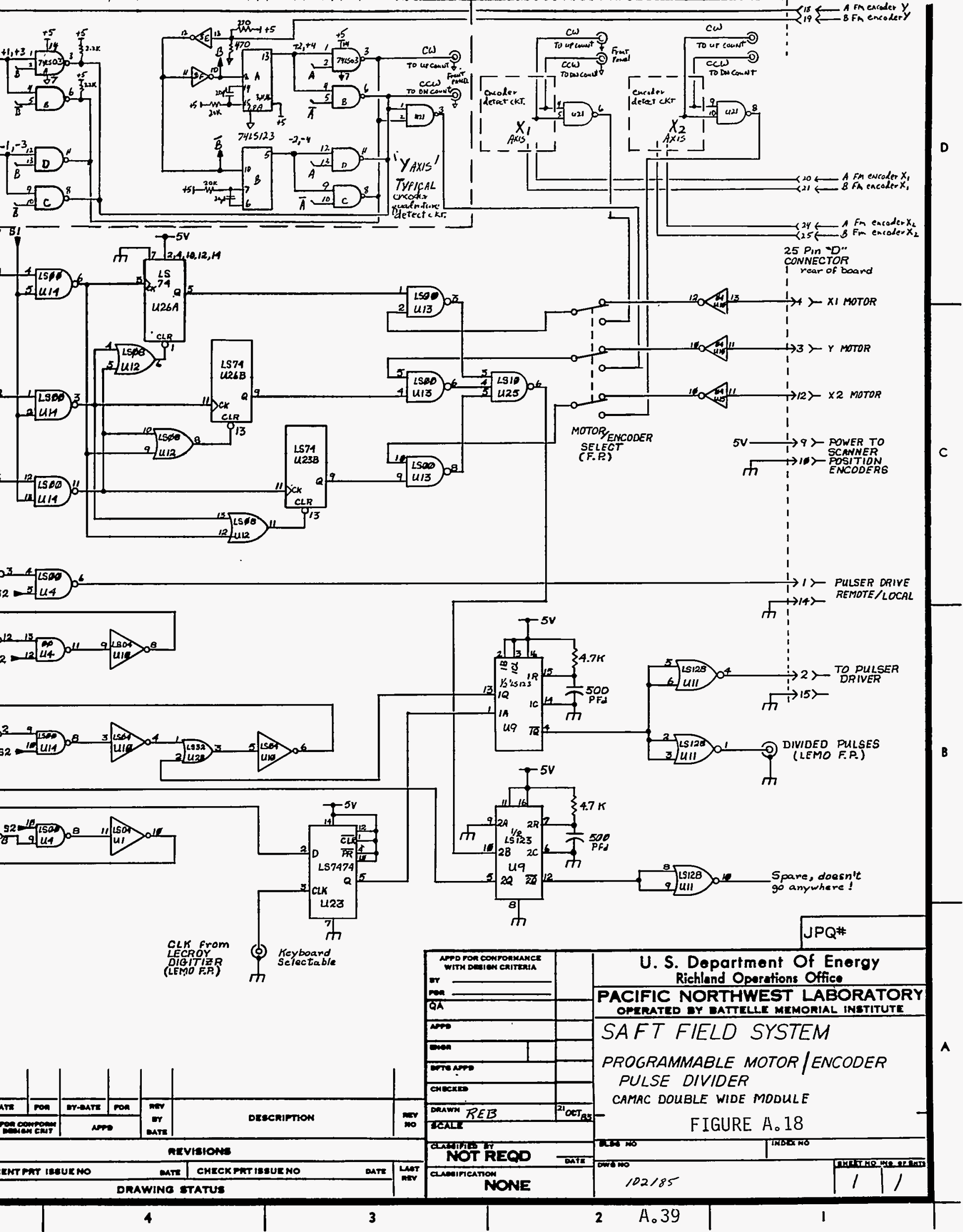




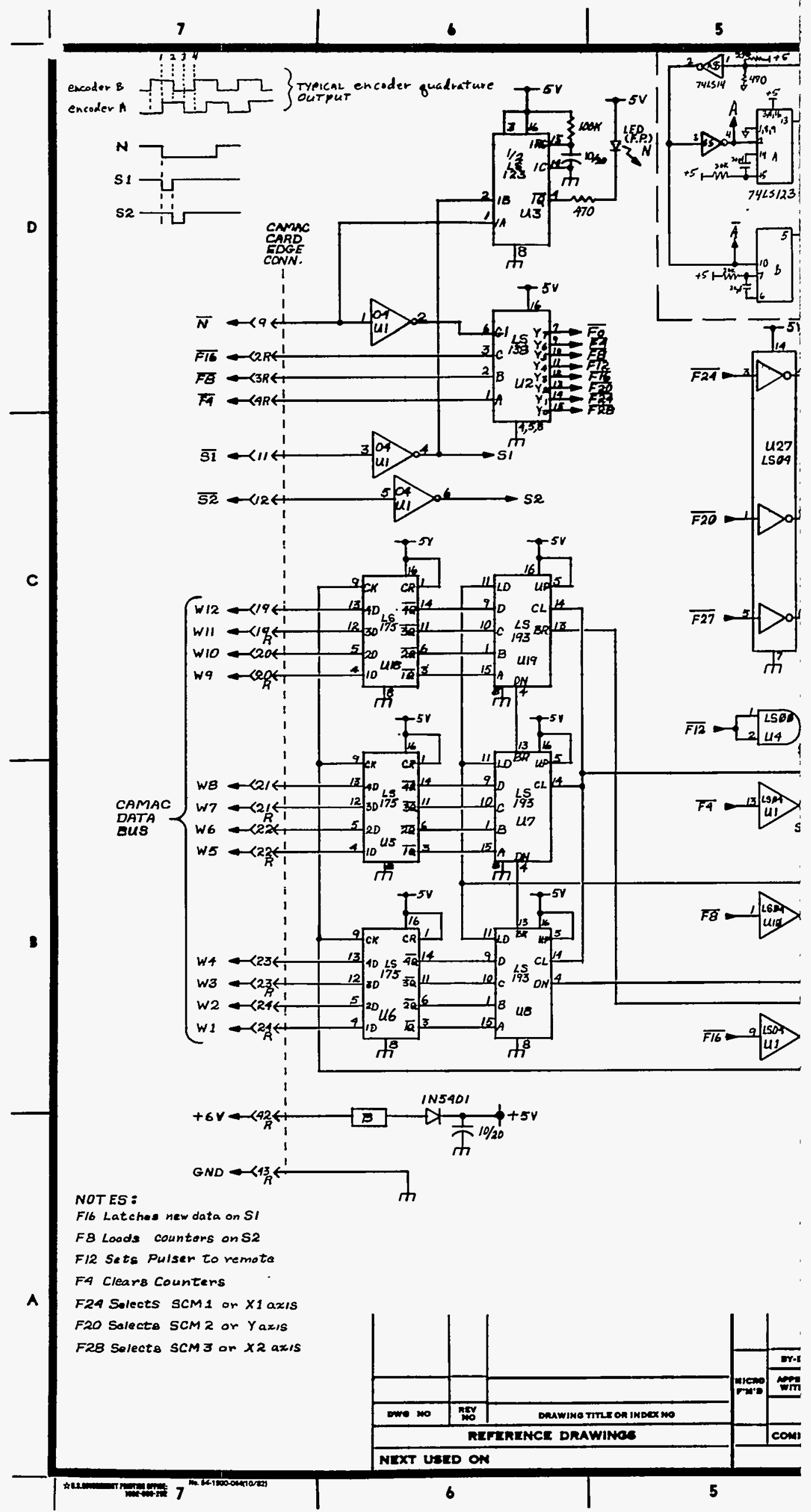




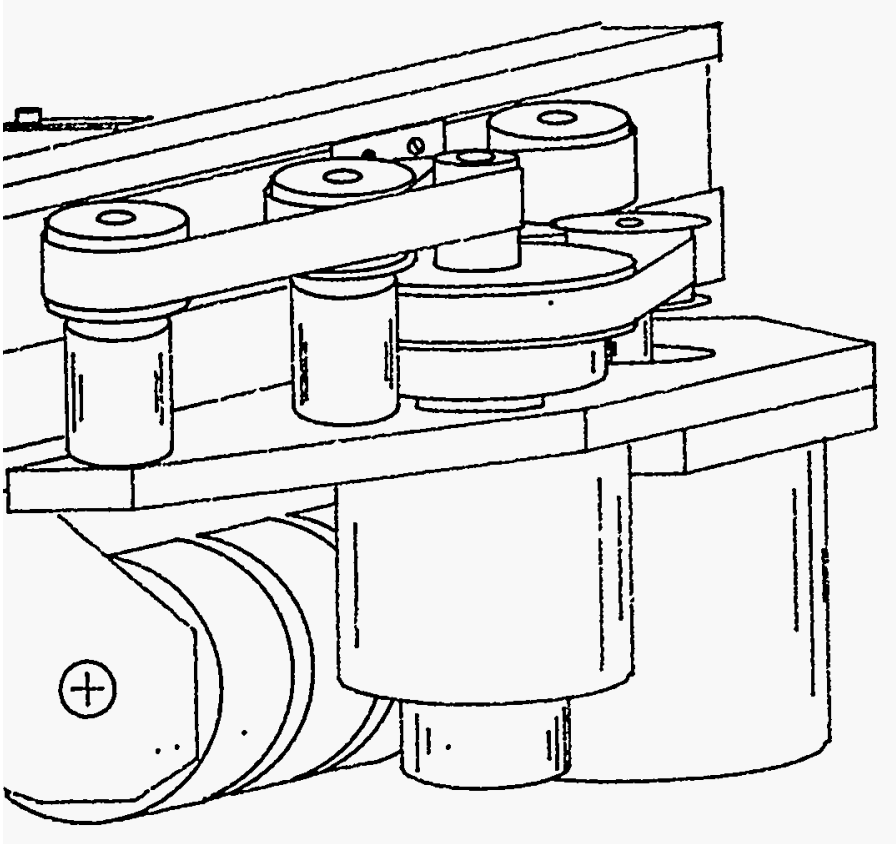

\begin{tabular}{|c|c|c|}
\hline REV. NO. 0 & & SAFT \\
\hline DATE: $10-22-87$ & & \\
\hline $\begin{array}{l}\text { DRMN: JC HARRIS } \\
\text { ENGR: PD SPERUNES }\end{array}$ & & PIPE SCANNER \\
\hline APPD: I DODSOMnfif & 18 & FIGURE A.19 \\
\hline FILE: SAFT-5 & & \\
\hline NO. 5 OF & DRW & NG NO. $8710-5$ \\
\hline
\end{tabular}




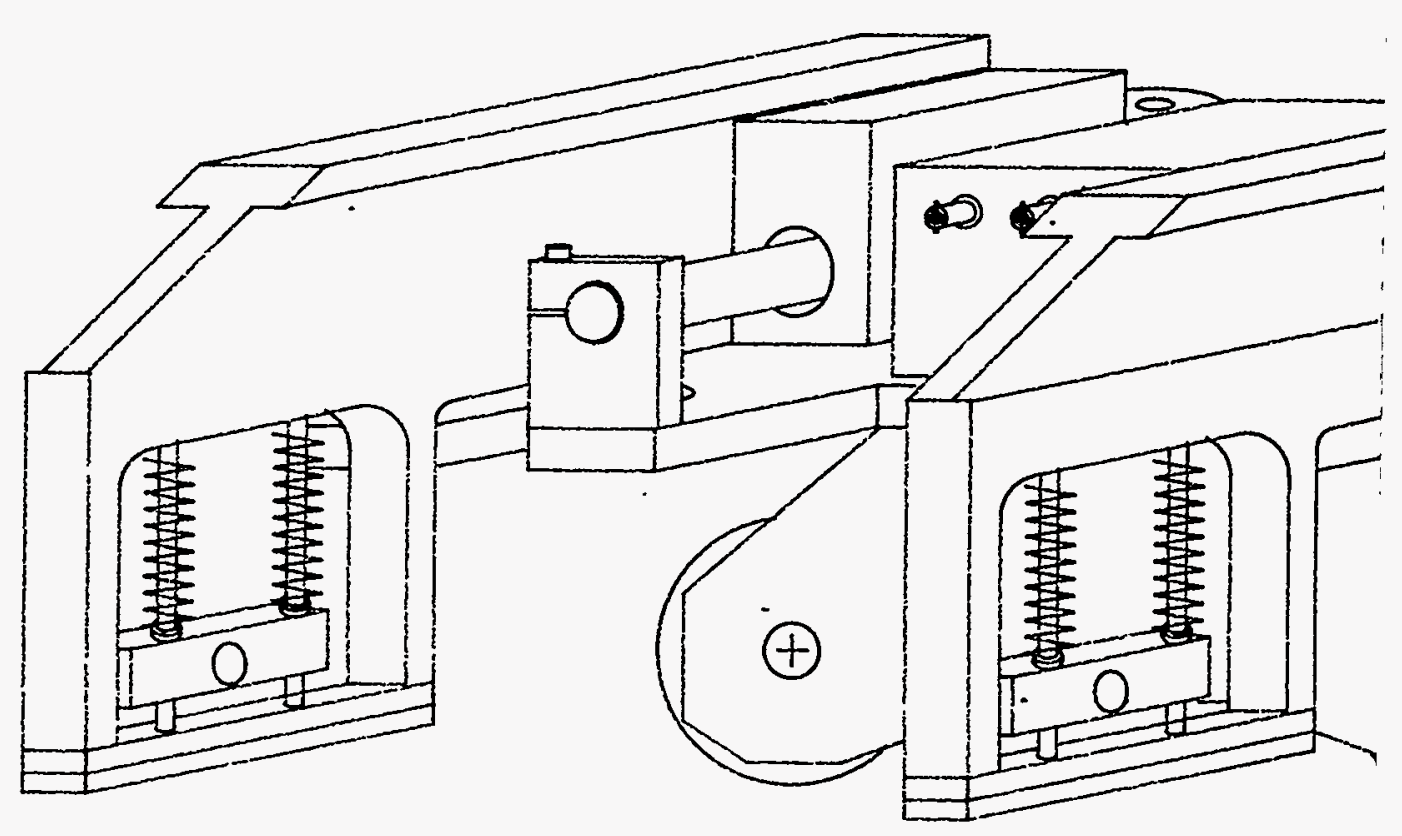




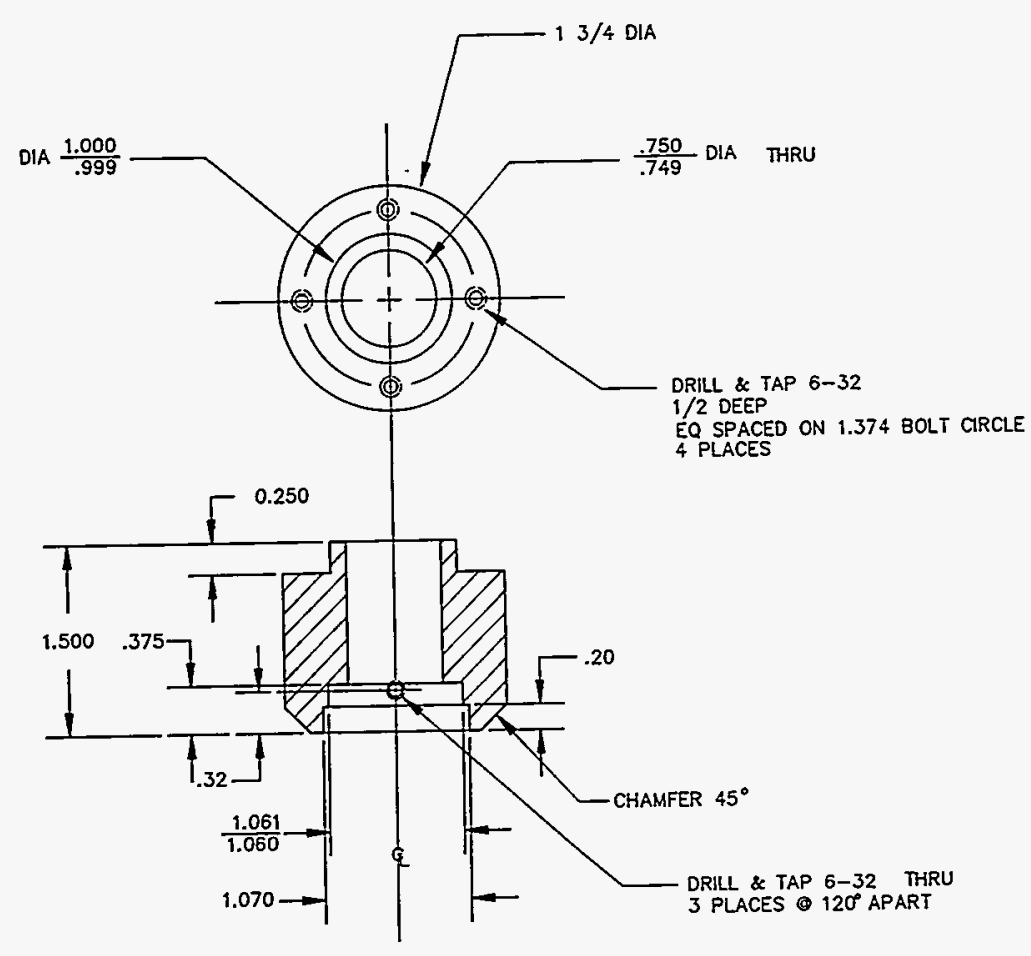

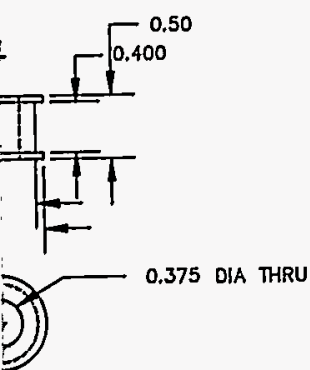

MATL: AL-6061-T6 , MATL:

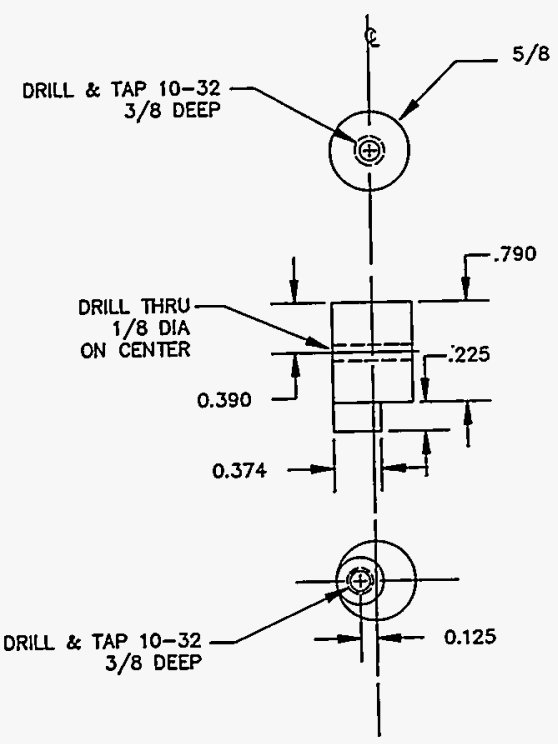

MATL: AL-6061-T6 2 REQ.
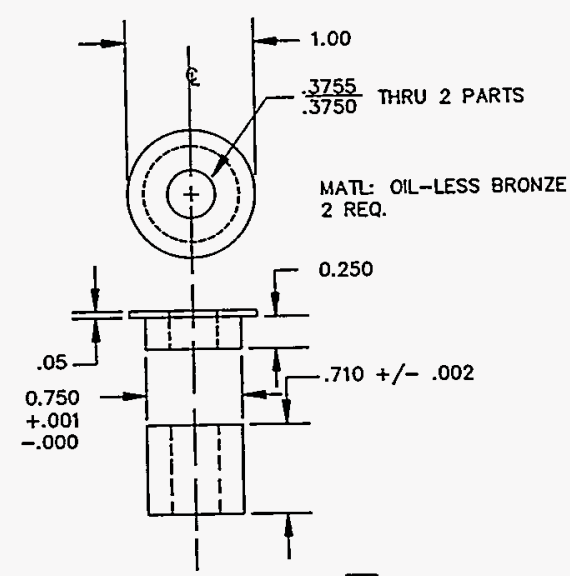

CHAMFER $45^{\circ} 7$ MATL: OLL-LESS BRONZE

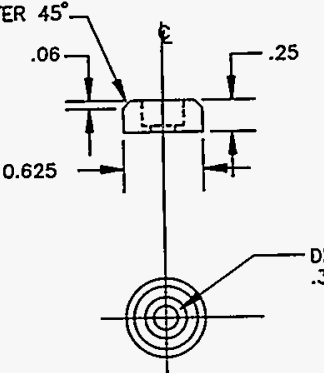

2. REQ. OIL-LESS BRONZE
EACH PART

MATL: AL-6061-T6 REQ.

\begin{tabular}{|c|c|c|}
\hline REV. NO. 0 & & SAFT \\
\hline DATE: $9 / 21 / 87$ & & PIPE SCANNER \\
\hline DRWN: JC HARRIS & & \\
\hline ENGR: M DODSON $N$ & 1/82 & \\
\hline APPO: L REID $A D R$ & $14 \%$ & BELT DRIVE COMPONENTS \\
\hline FLLE: SAFT-2 & & \\
\hline
\end{tabular}

FIGURE A.20 


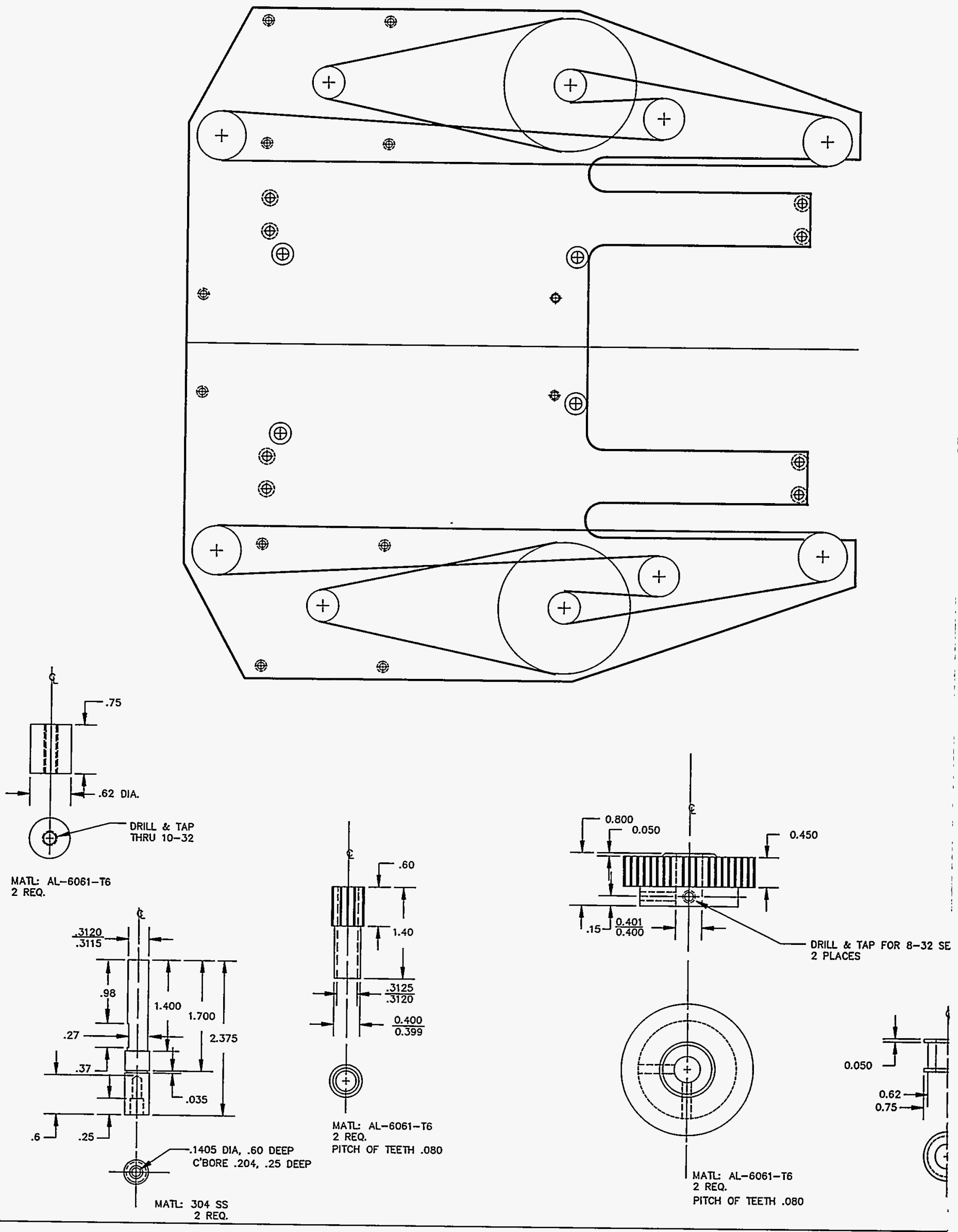



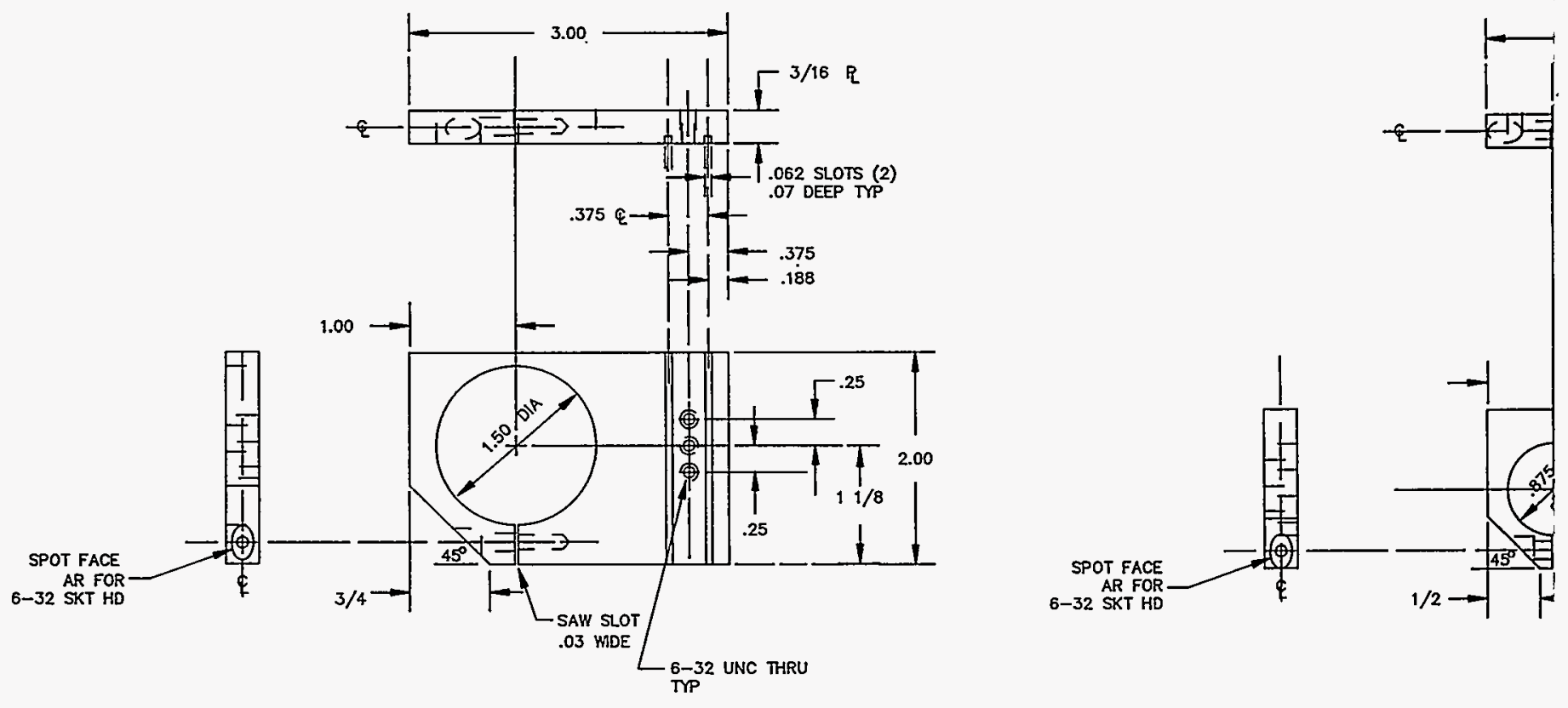

MATL 7075-T6

NO. REQ: 1
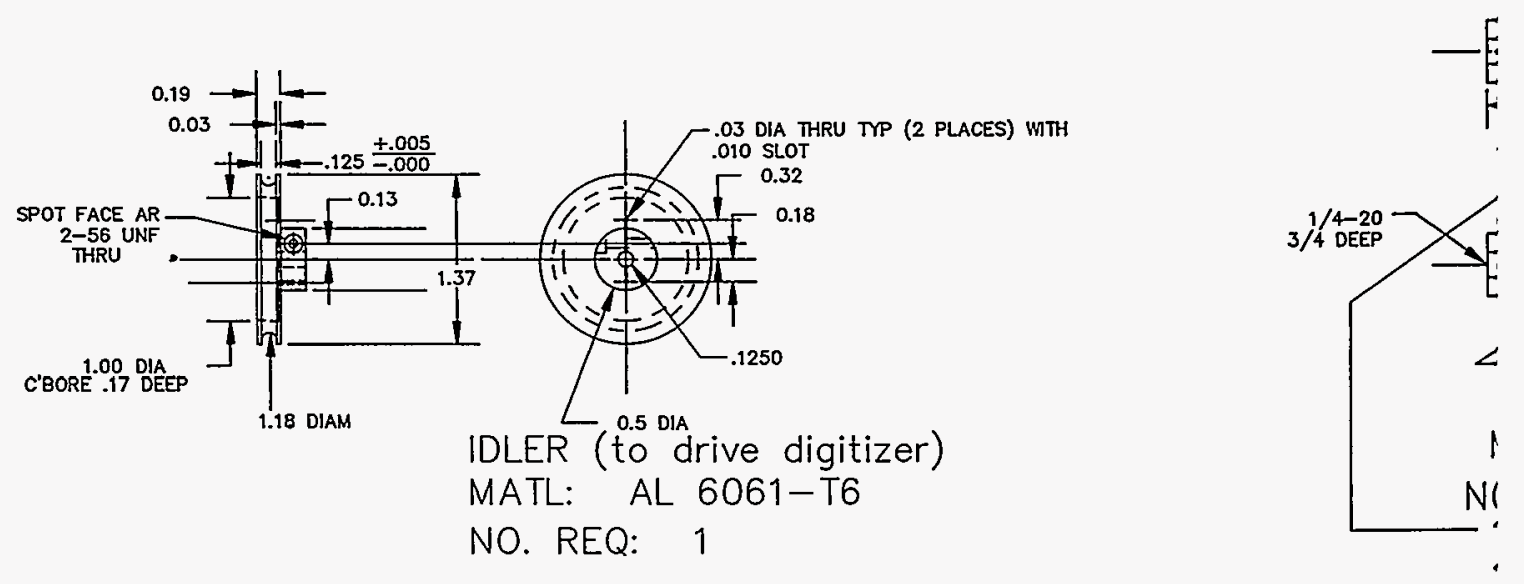

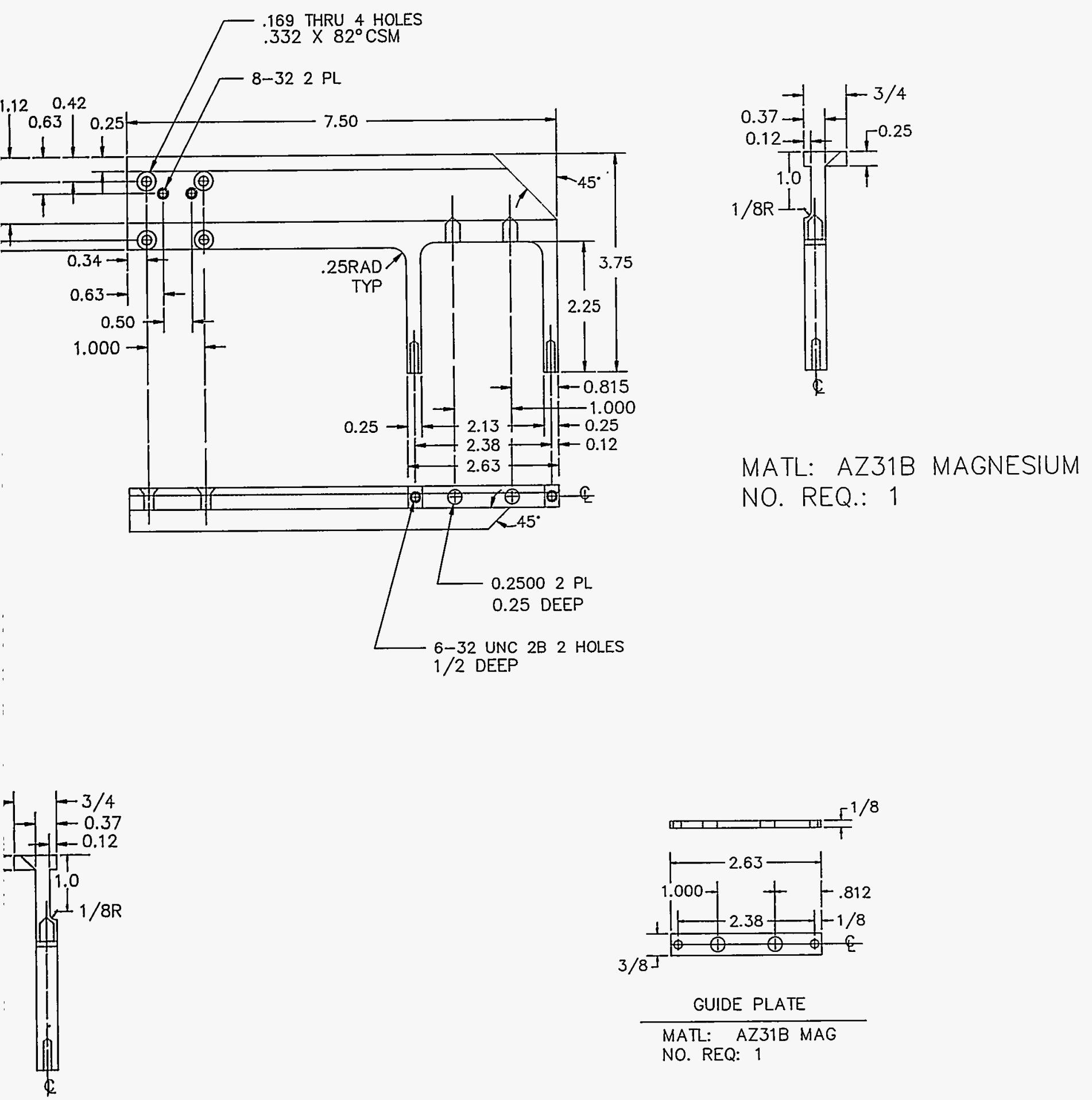

ITL: AZ31B MAGNESIUM

1. REQ.: 1

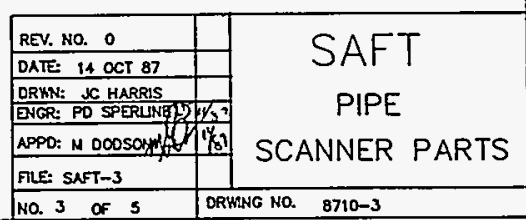



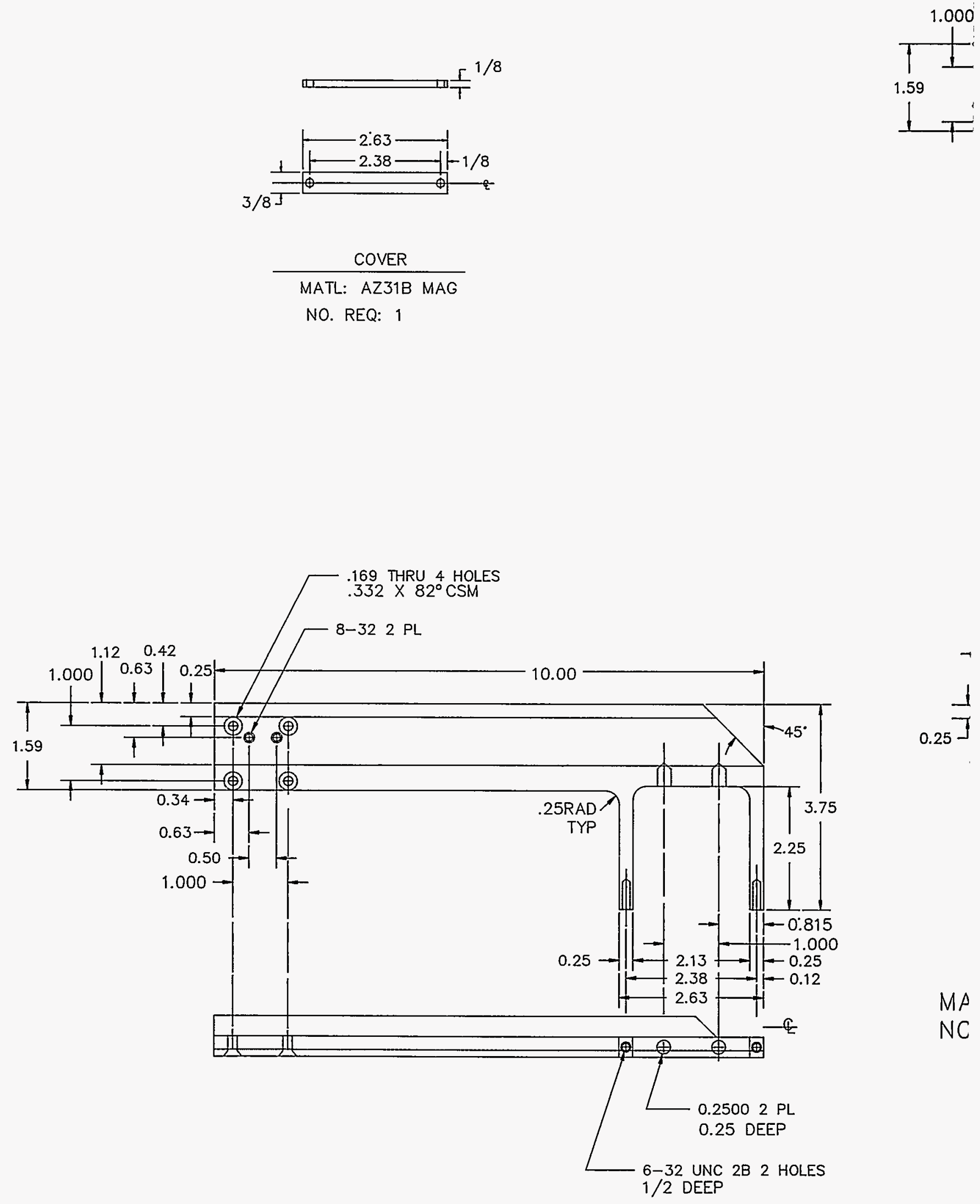

$M A$

$N C$ 

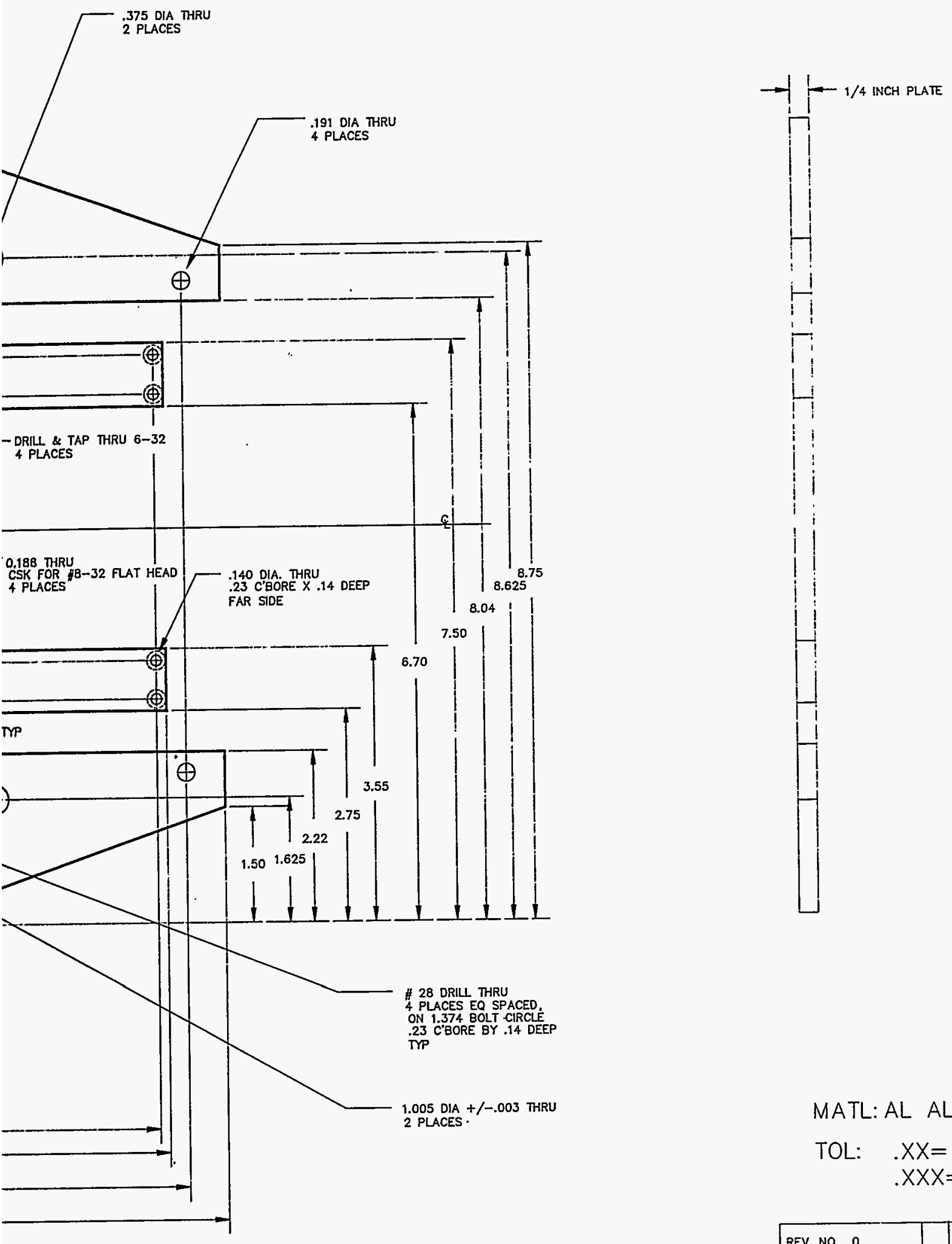

MATL: AL ALLOY 6061-T6

TOL: $. X X=+/-.010$ $. X X X=+/-.005$

\begin{tabular}{|c|c|c|}
\hline \multirow{4}{*}{\multicolumn{2}{|c|}{\begin{tabular}{l|l} 
REV. NO. 0 & \\
DATE: 9/21/87 & \\
DRWN: JC HARRIS & \\
ENGR: M DODSON 2 SG & I"/61 \\
\end{tabular}}} & \multirow{5}{*}{$\begin{array}{l}\text { SAFT } \\
\text { PIPE SCANNER } \\
\text { BASE PLATE }\end{array}$} \\
\hline & & \\
\hline & & \\
\hline & & \\
\hline APPD: L REID $\angle Z R$ & $12 / 82$ & \\
\hline FLE: SAFT-1 & & \\
\hline I. 1 OF & ORI & $8709-1$ \\
\hline
\end{tabular}




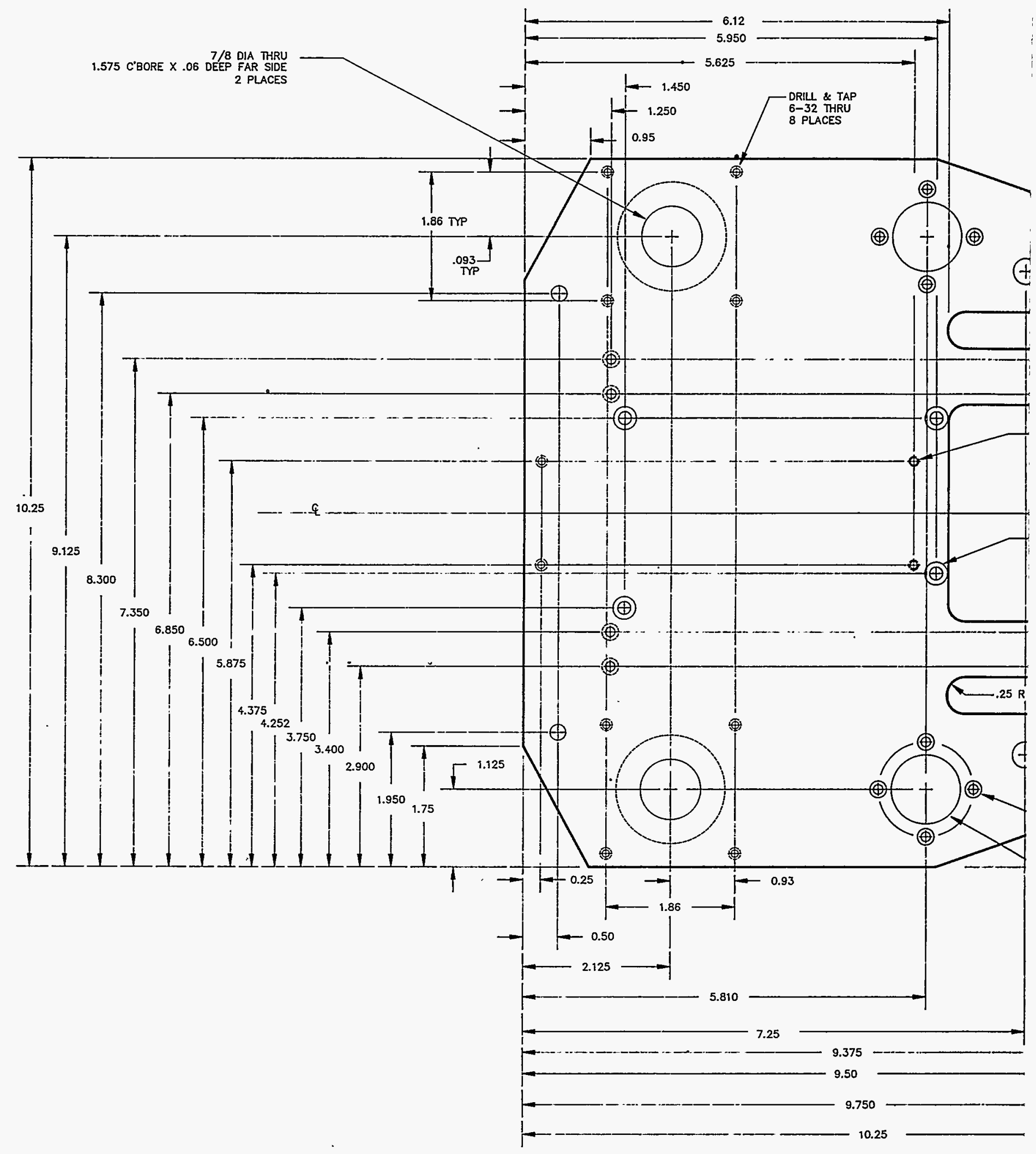




\section{Appendix B}

Tutorial Procedure for Operating the SAFT-UT Robotic Scanner 


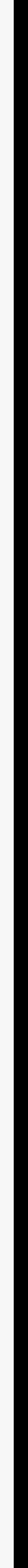




\section{Contents}

B1.0 Performing a Scan of the Vessel Wall . . . . . . . . . . . . . .

B1.1 Invoking the Scanning Software on the Data Acquisition Computer $\ldots \ldots \ldots \ldots \ldots \ldots \ldots \ldots$ B.2

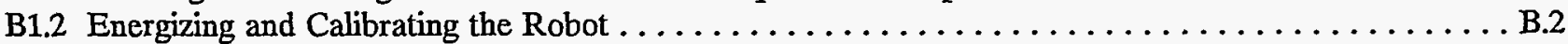

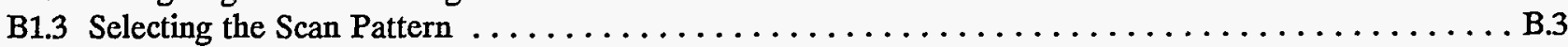

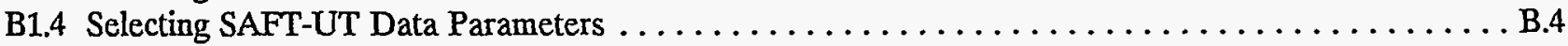

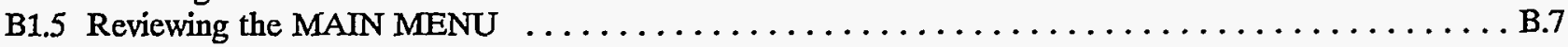

B1.6 Preparing the SAFT-UT Robot for a Scan $\ldots \ldots \ldots \ldots \ldots \ldots \ldots \ldots \ldots \ldots \ldots \ldots \ldots \ldots \ldots \ldots \ldots$

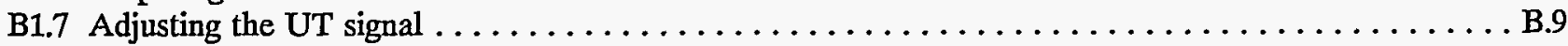

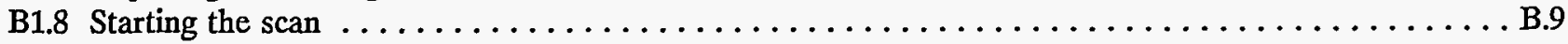

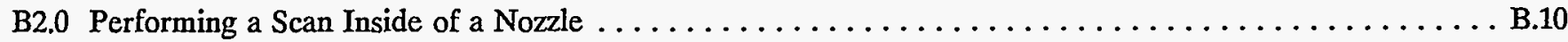

B2.1 Invoking the Scanning Software on the Data Acquisition Computer $\ldots \ldots \ldots \ldots \ldots \ldots \ldots \ldots$ B.10

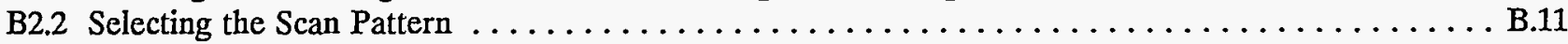

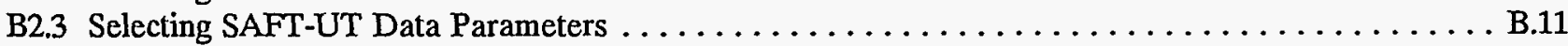

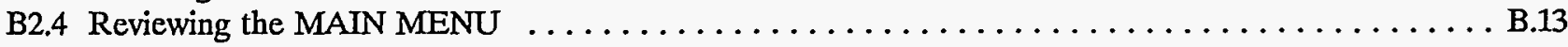

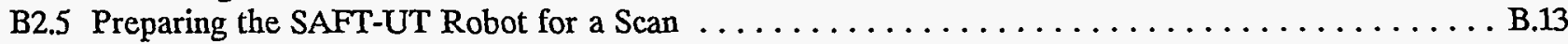

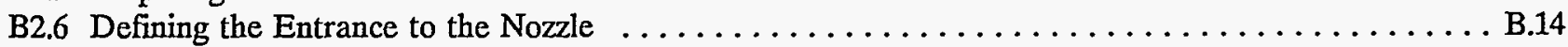

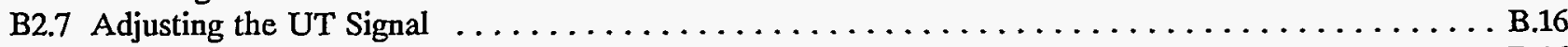

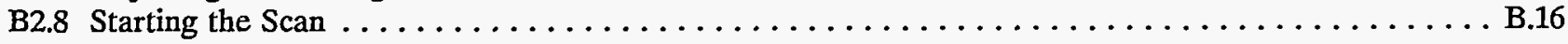

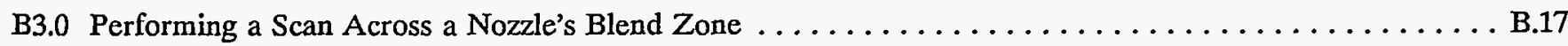

B3.1 Invoking the Scanning Software on the Data Acquisition Computer $\ldots \ldots \ldots \ldots \ldots \ldots \ldots \ldots$ B.17

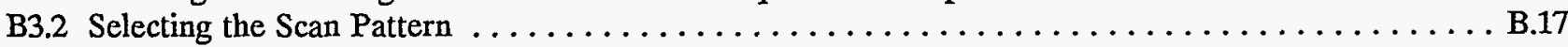

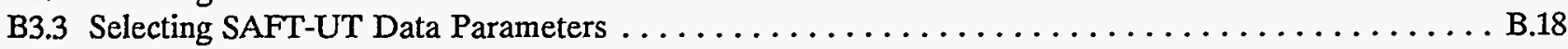

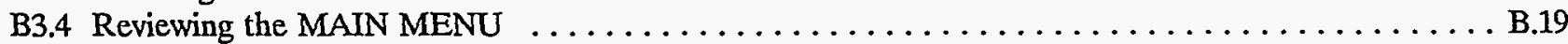

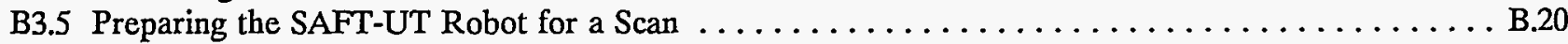

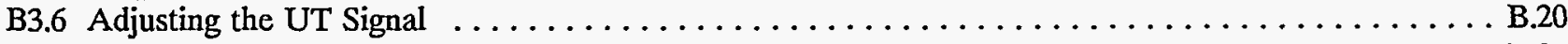

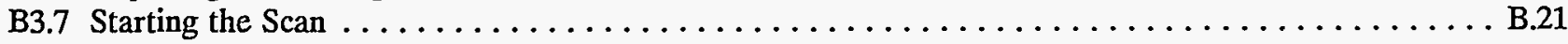


Appendix B

\section{B1.0 Performing a Scan of the Vessel Wall}

The steps are:

B1.1 Invoking the scanning software on the data acquisition computer

B1.2 Energizing and calibrating the robot

B1.3 Selecting the scan pattern

B1.4 Selecting SAFT-UT data parameters

B1.5 Review the MAIN MENU

B1.6 Preparing the SAFT-UT robot for a scan

B1.7 Adjusting the UT signal

B1.8 Starting the scan

\section{B1.1 Invoking the Scanning Software on the Data Acquisition Computer}

After the data acquisition computer boots the DOS operating system, the user should type "scan" at the prompt.

$$
\text { C:1>scan }
$$

The SAFT-UT data acquisition software will start and display the message:

$<S C A N M E N U>$ Has the robot been calibrated $(Y, N)$ :

Assuming that the robot is turned off, answer " $n$ " to this query and proceed to step B1.2 below.

\section{B1.2 Energizing and Calibrating the Robot}

After typing " $\mathrm{n}$ " at the calibration prompt in step B1.1 above, the data acquisition computer will act as a terminal to the robot's controller.

Turn on the robot's controller using the control power key. The screen should show the following messages from the robot controller: 


\section{POWERUP DIAGNOSTIC IN PROGRESS}

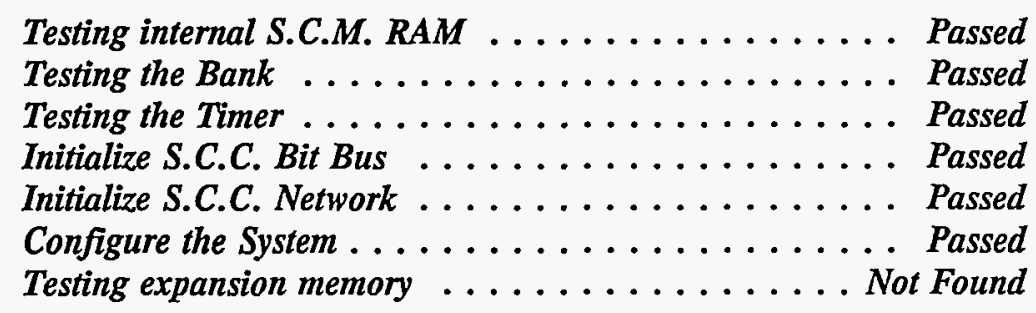

\section{UNIMATION VAL-II Copyright (c) 1986 by Unimation, Inc.}

Initialize $(Y / N)$ ?

Answer "y" to the initialize prompt. The robot controller will prompt with:

Are you sure $(Y / N)$ ?

Answer " $y$ " and the screen show the following messages:

\section{TMS servo gains obtained from EPROM. TP servo gains obtained from $E P R O M$. VAL-II 562.4.1A P/N-4921B17 1-FEB-1990 4.1A $P / N-4919 B 22 \quad 1-F E B-1990 \quad 16 \mathrm{Kw}$}

Note that the robot controller's command prompt is a ".". Turn the "RUN" switch from "HOLD" to "RUN". Press the "ACTIVATE" button. Turn the "ARM POWER" switch from "OFF" to "ON". Press the "ACTIVATE" button.

The robotic arm is now energized. The user should allow 5 minutes for the robot to warm.

After the warm-up period, move the robot free of any obstructions. About 6 inches of clearance will be required for the next step.

Type "cal" at the "." prompt. The robot controller should respond with:

\section{Arm Signature data not applied Are you sure $(Y / N)$ ?}

Type "y" and then watch the robot arm execute a short motion to calibrate its joints. If the calibration completes successfully, then the type "speed 10" at the "." prompt in order to set the speed to reasonable value for setup.

Press the "Esc" key to exit the terminal emulation mode.

\section{B1.3 Selecting the Scan Pattern}

After the "Esc" key is pressed, the data acquisition computer will prompt user for the scan pattern with the following messages: 
Appendix B

Select scan pattern ...

1. XY Scan (vessel wall)

2. Cylindrical scan (inside nozzle)

3. Nozzle scan (nozzle blend zone)

ENTER SELECTION -..>

For this tutorial select "1", XY Scan.

\section{B1.4 Selecting SAFT-UT Data Parameters}

After selecting the scan pattern, the data acquisition computer will prompt the user for SAFT-UT data parameters. These parameters are described in the documentation on the SAFT-UT pipe scanner. An example is shown below for the purpose of comparison to the pipe scanner system.

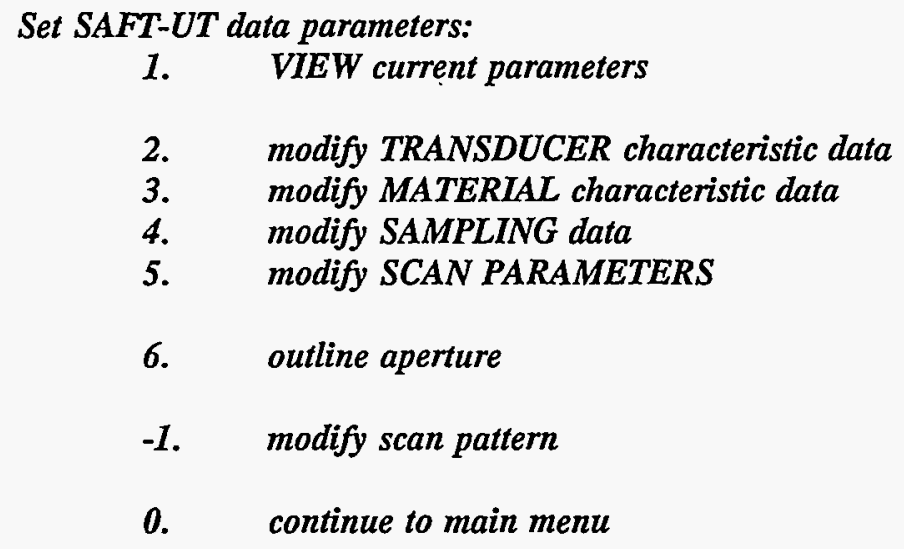

\section{ENTER SELECTION --->}

Menu item 1 displays the settings:

TRANSDUCER: rtd 70 deg, $L$ wave, serial \# 84-23

transducer frequency (MHZ): 2.0

xmit standoff ... hgt $=$ fcc len (in): .29

$X$ incident angle

(deg): -24.9

full beam angle in metal ... (deg): 12.0

mat vel (in/sec): 105000

refracted angle (deg): $-\mathbf{7 0 . 4 4 4}$

beam_entry_diam (in): .125

MATERIAL; man-way dropout, clad vessel material with artificially grown cracks

material velocity (in/sec): $235000 \quad$ thickness ... (in): 4.4

SAMPLING: Beam angle is 70 degrees so 3 inches along sound path is about $I$ inc sample period (ns): 80

depth (along sound path) in material to ...
initial delay
(us): 5.523809
I...
stop sampling (in): 3

number of points: 320

start sampling (in): 0 
SCAN: Scan along secondary axis

$$
\text { step size: } X, Y \text { axis }
$$

scan length: $X, Y$ axis scan dir

$$
(\text { deg, } 0=P, 90=S): 90
$$

(in): .05, $\quad .1$

init. pos.: $P, S$ axis

(in): $3.2, \quad 5.5$

increments: $X, Y$ axis

(in): $101, \quad 51$

final pos.: $P, S$ axis

(in): $-1.8, \quad 10.5$

ENTER RETURN TO CONTINUE

Menu item 2 will set the TRANSDUCER parameters:

\section{$<T R A N S D U C E R$ SETTINGS>}

Enter two lines of comment, up to 40 chars each, or press

[enter] at the first prompt to keep the current comment.

Current: rtd 70 deg, $L$ wave, serial \# 84-23

$1>$

If no change is desired, press enter at that variable.

Current values:

Transducer frequency (mhz)

Standoff, $h g t=$ foc len (in)

$X$ incident angle in metal (deg)

Squint direction

Full beam angle in metal (deg)

Transducer velocity (in/sec)

Beam entry diameter (in)

Refracted angle (deg)

$$
=2.0
$$$$
=.290
$$$$
=-24.9
$$$$
=0
$$

$=12.0$

$=105000$

$=.125$

$=-70.4446$

Menu item 3 will set the MATERIAL parameters:

<MATERIAL SETTINGS>

Enter two lines of comment, up to 40 chars each, or press

[enter] at the first prompt to keep the current comment

Current: man-way dropout, clad vessel material with artificially grown cracks

$1>$

If no change is desired, press enter at the beginning of the line. 
Appendix B

Current values:

Changes:

Material velocity (in/sec)

Thickness (in)

$=23500$

$=4.4$

Refracted angle

$=-70.4446$

Menu item 4 will set the Sampling parameters:

<SAMPLING SETTINGS>

Enter two lines of comment, up to 40 chars each, or press

[enter] at the first prompt to keep the current comment

Current: (no comment)

| ..........................................

$1>$ Beam angle is 70 degrees so 3 inches

$2>$ along sound path is about 1 inch down

If no change is desired, press enter at that variable.

Current values:

Changes:

Sample period (ns)

$=80$

Depth (along sound path)

in material to

start sampling

stop sampling

$$
\begin{aligned}
& =0 \\
& =3
\end{aligned}
$$

Initial delay (us)

Number of points

$$
=5.52381
$$

$=320$

Menu item 5 will set the Scanning parameters:

\section{$<$ SCANNING SETTINGS>}

Enter two lines of comment, up to 40 chars each, or press [enter] at the first prompt to keep the current comment. Current: 270<smiles>[AlH]</smiles>

$1>$ Scan of vessel wall,

$2>$ scan along secondary axis

If no change is desired, press enter at that variable. 
Current values:

step size: $\quad X$ axis $Y$ axis

$X$ scan length (in)

$Y$ scan length (in)

Scan dir. (deg; $0=X, 90=Y$ )

init. pos. primary axis

init. pos. secondary axis
Changes:

$$
\begin{aligned}
& =.05 \\
& =.1 \\
& =5 \\
& =10 \\
& =90 \\
& =100 \\
& =100
\end{aligned}
$$

number of $x$ inc $=101$

number of $y$ inc $=101$

final pos. primary axis $=90$

final pos. secondary axis $=105$

\section{B1.5 Reviewing the MAIN MENU}

After completing the selection of the SAFT-UT data parameters, the user may select the MAIN MENU by entering " 0 " in the menu for the SAFT-UT data parameters.

From the MAIN MENU, the user may return to the previous steps, complete the robotic scanner configuration, adjust the UT signal, start the scan, or quit the program.

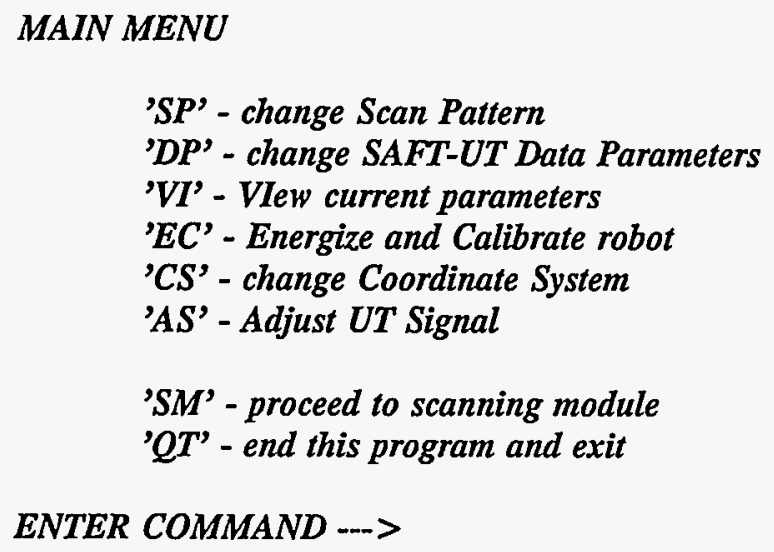

\section{B1.6 Preparing the SAFT-UT Robot for a Scan}

From the MAIN MENU, the user should enter "CS" and the following menu will appear: 
Appendix B

MENU FOR MATERIAL COORDINATE DEFINITION: MODE: XY(1)

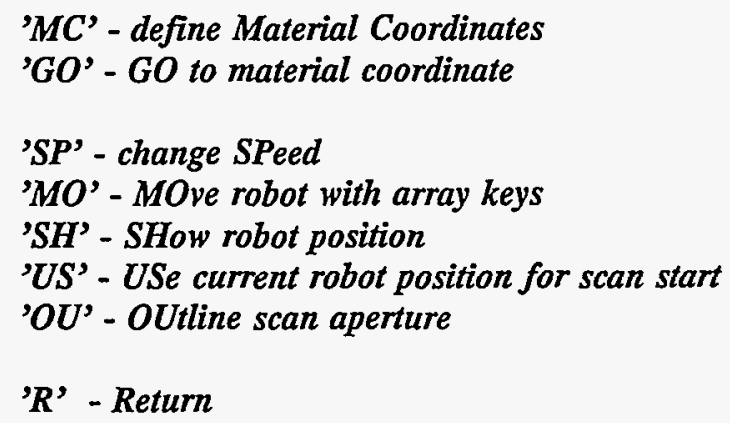

ENTER COMMAND -->

This tutorial assumes that the robot does not know where, in its work space, to find the test material. This happens, for example, whenever the robot is moved. The user can start to teach the robot about the test material by typing "MC". The following prompt should appear:

\section{.Enter the tool length, in inches:}

The tool length is the distance to be maintained between the robots wrist and the test material. When the robot is using the RTD probe holder with a SAFT-UT transducer fixture the distance will be about 7 inches. For this tutorial, run the robot without the probe holder and enter " 2.0 " at the above prompt. Next, the program will ask for a parameter that is not needed for this tutorial:

\section{Enter, in inches, the length of the transition arc for the 90 degree angle:}

Enter "0" and proceed. The user will be prompted as follows:

Coordinates for reference positions must follow the pattern

$(P 1, S 1,0),(P 2, S 1,0),(P 2, S 2,0)$

pt $1->$ pt 2 must be in the positive direction

pt $2->$ pt 3 must be in the positive direction

Use the teach pendant to move robot to reference point 1

Switch to COMP mode and press [enter] when done:

The user must use the teach pendant to move the robotic arm to a position on the test material for which the material coordinates are known. Because a tool length of 2 inches was selected, the robot should be 2 inches above this point. This point will be know as "reference point 1 ". When the robot is positioned in this way, the user may press the "return" key and user will be prompted for the material coordinates of "reference point 1". For the sake of this tutorial, the coordinates are 200.0 (inches) on the primary axis and 100.0 (inches) on the secondary axis. So the user should respond to the next two prompts as follows:

Enter the position on primary axis for reference point $1: 200$

Enter the position on secondary axis $\quad: 100$

This completed, the next prompt is: 
Use the teach pendant to move robot to reference point 2

Switch to COMP mode and press [enter] when done:

The user must use the teach pendant to move to "reference point 2 ". This point must along the positive primary axis from "reference point 1 ". When the robot is positioned 2 inches above "reference point 2 ", the user may press "return" and the prompt for "reference point 3" will appear:

Use the teach pendant to move robot to reference point 3

Switch to COMP mode and press [enter] when done:

The user must use the teach pendant to move to "reference point 3 ". The point must be along the positive secondary axis from "reference point 2". When the robot's tool flange is positioned 2 inches above "reference point 3 " the user may press "return".

The robot now assumes that the surface of the test material lies in the plane of the 3 reference points.

The user should enter "MO" to activate the arrow keys. Move the robot and verify that it will move along the vessel wall. Position the robot for the start of a scan and then select "R" to deactivate the arrow keys. Select "US" to define the start position of a scan.

\section{B1.7 Adjusting the UT signal}

The user should select "AS" from the MAIN MENU in order to adjust the UT signal by changing the parameters of the digitizer and the Time Variable Gain (TVG) module:

$$
\begin{array}{lll}
\text { rate } \ldots \ldots: & 12.5(M h z) \\
\text { length } \ldots \ldots: & 324(25.6 u s e c) \\
\text { delay } \ldots . . & 5.524(\text { usec })
\end{array}
$$

A) range $\ldots . .25$ (volts $p-p)$

B) offset ...: 0.150(volis)

C) coupling ...: (AC coupled)

D) video ...: off $(R F)$

E) reps ....: 1

press ' $R$ " to return:
F) time0 : 4.800

G) slope0 : 0

H) delay : $: 19.873$

I) time1 : 0.835

J) slope1 : 40

K) time2 : 2.839

L) slope2 : 10

\section{B1.8 Starting the scan}

The user may start the scan by entering "sm" at the MAIN MENU prompt. The user will be prompted for a comment that pertains to this data file:

Enter 2 lines of comments, up to 40 characters each, or press

[enter] at the first prompt to keep the current comment

Current: scan of manway, flaw " $C$ "

I> 
Appendix B

The user will be asked to enter the file name:

$$
\text { Enter file name ---> test.dat }
$$

The user may view the A-scan data if "y" is selected to the next prompt:

$$
\text { Display A-scans while taking data? ->y }
$$

Finally, the user must choose a destination for the data file. The programs gives the following choices:

$$
\begin{array}{ll}
\text { select data transfer option: } & \text { a) Transfer to MicroVAX } \\
& \text { b) Save on local disk } \\
\text { c) Both }
\end{array}
$$

Enter choice: $b$

Type "b" and the scan begins.

\section{B2.0 Performing a Scan Inside of a Nozzle}

The steps are:

B2.1 Invoking the scanning software on the data acquisition computer

B2.2 Selecting the scan pattern

B2.3 Selecting SAFT-UT data parameters

B2.4 Review the MAIN MENU

B2.5 Defining the Material Coordinates

B2.6 Defining the Entrance to the Nozzle

B2.7 Adjusting the UT signal

B2.8 Starting the scan

\section{B2.1 Invoking the Scanning Software on the Data Acquisition Computer}

The user should type "scan" at the prompt.

$$
\text { C: } 1>\operatorname{scan}
$$

The SAFT-UT data acquisition software will start and display the message: 
$<S C A N M E N U>$ Has the robot been calibrated $(Y, N)$ :

Assuming that the robot is still on and calibrated, answer " $y$ " to this query and proceed to step B2.2 below. Otherwise the user should perform step B1.2 above.

\section{B2.2 Selecting the Scan Pattern}

The data acquisition computer will prompt user for the scan pattern with the following messages:

Select scan pattern ...

1. XY Scan (vessel wall)

2. Cylindrical scan (inside nozzle)

3. $\quad$ Nozzle scan (nozzle blend zone)

ENTER SELECTION -->

For this tutorial select "2", cylindrical scan.

\section{B2.3 Selecting SAFT-UT Data Parameters}

After selecting the scan pattern, the data acquisition computer will prompt the user for SAFT-UT data parameters. Another example is shown below:

$\begin{array}{cl}\text { Set SAFT-UT data parameters: } \\ \text { 1. } & \text { VIEW current parameters } \\ 2 . & \text { modify TRANSDUCER characteristic data } \\ 3 . & \text { modify MATERIAL characteristic data } \\ \text { 4. } & \text { modify SAMPLING data } \\ 5 . & \text { modify SCAN parameters } \\ 6 . & \text { outline aperture } \\ -1 . & \text { modify scan pattern } \\ 0 . & \text { continue to main menu }\end{array}$

ENTER SELECTION --->

Menu item 1 displays the settings:

TRANSDUCER: rtd 70 deg, $L$ wave, serial \# 84-23

transducer frequency $\quad(M H z): 2.0$

xmit standoff ... hgt $=$ fcc len (in): .29

$X$ incident angle (deg): -24.9

full beam angle in metal ... (deg): 12.0

mat vel (in/sec): 105000

refracted angle (deg): $\mathbf{- 7 0 . 4 4 4}$

beam_entry_diam (in): .125 
Appendix B

MATERIAL; man-way dropout, clad vessel material with artificially grown cracks material velocity

SAMPLING: Beam angle is 70 degrees so 3 inches along sound path is about 1 inc sample period (ns): 80 depth (along sound path) in material to ... initial delay (us): $5.523809 \quad$ /... number of points: 320 start sampling (in): 0 stop sampling (in): 3

SCAN: Scan along secondary axis step size: $\quad X, Y$ axis scan length: $X, Y$ axis scan_tir: 180 init. pos.: $P, S, T$ axis increments: $X, Y$ axis final pos.: $P, S, T$ axis

$\begin{array}{ll}\text { (in): } .05, \quad .1 & \\ \text { (in): } 4, \quad 4 & \\ \text { start angle: } 270 \quad & \text { end angle: } 235.617 \\ \text { (in): } 198.721, & 103.0852, \\ \text { (in): } 81, \quad 41 & \\ \text { (in): } 193.339, & 101.886\end{array}$

\section{ENTER RETURN TO CONTINUE}

Menu item 5 will set the Scanning parameters:

press [enter] at the first prompt to keep the current comment. Current: Scan along secondary axis<smiles></smiles>

If no change is desired, press enter at that variable.

Current values:

step size: $\quad X$ axis $Y$ axis

$X$ scan length (in)

$Y$ scan length (in)

Scan dir. $(0=+T, 180=-T)$

init. pos. primary axis

init. pos. secondary axis

Init. pos. tertiary axis

number of $x$ inc $=81$

number of $y$ inc $=41$

final pos. primary axis $=193.339$

final pos. secondary axis $=101.886$

final pos. tertiary axis $=-6.7537$

Scan type: Cylinder, radius $=9.530353$

\section{Changes:}

$$
\begin{aligned}
& =.05 \\
& =.1 \\
& =4 \\
& =4 \\
& =180 \\
& =198.721 \\
& =103.0853 \\
& =-2.7537
\end{aligned}
$$




\section{B2.4 Reviewing the MAIN MENU}

After completing the selection of the SAFT-UT data parameters, the user may select the MAIN MENU by entering " 0 " in the menu for the SAFT-UT data parameters.

The main menu below is like the one in the previous tutorial except for the addition of item "EN". This item will be needed for scans of the inside of a nozzle.

MAIN MENU

$$
\begin{aligned}
& \text { 'SP' - change Scan Pattern } \\
& \text { 'DP' - change SAFT-UT Data Parameters } \\
& \text { 'VI' - VIew current parameters } \\
& \text { 'EC' - Energize and Calibrate robot } \\
& \text { 'CS' - change Coordinate System } \\
& \text { 'EN' - define entrance to nozzle } \\
& \text { 'AS' - Adjust UT Signal } \\
& \text { 'SM' - proceed to scanning module } \\
& \text { 'QT' - end this program and exit }
\end{aligned}
$$

ENTER COMMAND -->

\section{B2.5 Preparing the SAFT-UT Robot for a Scan}

From the MAIN MENU, the user should enter "CS" and the following menu will appear:

MENU FOR MATERIAL COORDINATE DEFINITION: MODE: IN(2)

$$
\begin{aligned}
& \text { 'MC' - define Material Coordinates } \\
& \text { 'SP' - change SPeed } \\
& \text { 'R' - Return }
\end{aligned}
$$

ENTER COMMAND -->

As in tutorial 1, this tutorial assumes that the robot does not know where, in its work space, to find the test material. This happens, for example, whenever the robot is moved. The user can start to teach the robot about the test material by typing "MC". The following prompt should appear:

.Enter the tool length, in inches:

The tool length is the distance to be maintained between the robots wrist and the test material. When the robot is using the RTD probe holder with a SAFT-UT transducer fixture the distance will be about 7 inches. For this tutorial, run the robot without a transducer and enter " $2.0^{\circ}$ at the above prompt. Next, the program will ask for a nozzle parameter: 


\section{Enter, in inches, the length of the transition arc for the 90 degree angle:}

This is the arc path length of the nozzle's blend zone. The user should measure this distance and enter it (e.g. 4.5). Then user will be prompted as follows:

Coordinates for reference positions must follow the pattern

$(P 1, S 1,0),(P 2, S 1,0),(P 2, S 2,0)$

pt 1 --> pt 2 must be in the positive direction

pt $2 \rightarrow>$ pt 3 must be in the positive direction

Use the teach pendant to move robot to reference point 1

Switch to COMP mode and press [enter] when done:

The user must use the teach pendant to move the robotic arm to a position on the test material for which the material coordinates are known. Because a tool length of 2 inches was selected, the robot should be 2 inches above this point. This point will be know as "reference point 1 ". When the robot is positioned in this way, the user may press the "return" key and user will be prompted for the material coordinates of "reference point 1". For the sake of this tutorial, the coordinates are 200.0 (inches) on the primary axis and 100.0 (inches) on the secondary axis. So the user should respond the next prompts as follows:

\section{Enter the position on primary axis for reference point $1: 200$ Enter the position on secondary axis

This completed, the next prompt is:

Use the teach pendant to move robot to reference point 2

Switch to COMP mode and press [enter] when done:

The user must use the teach pendant to move to "reference point $2 "$. This point must along the positive primary axis from "reference point 1". When the robot is positioned 2 inches above "reference point 2 ", the user may press "return" and the prompt for "reference point 3" will appear:

Use the teach pendant to move robot to reference point 3

Switch to COMP mode and press [enter] when done:

The user must use the teach pendant to move to "reference point 3". The point must be along the positive secondary axis from "reference point 2". When the robot's tool flange is positioned 2 inches above "reference point 3 " the user may press "return".

The robot now assumes that the surface of the test material lies in the plane of the 3 reference points.

\section{B2.6 Defining the Entrance to the Nozzle}

After the material coordinate have been defined in B2.5 above, the user should select " $R$ " to return to the MAIN MENU. The user should select the nozzle definition menu by typing "EN" and the following menu will appear: 
MENU FOR DEFINITION OF NOZZLE OPENING: MODE: IN(2)

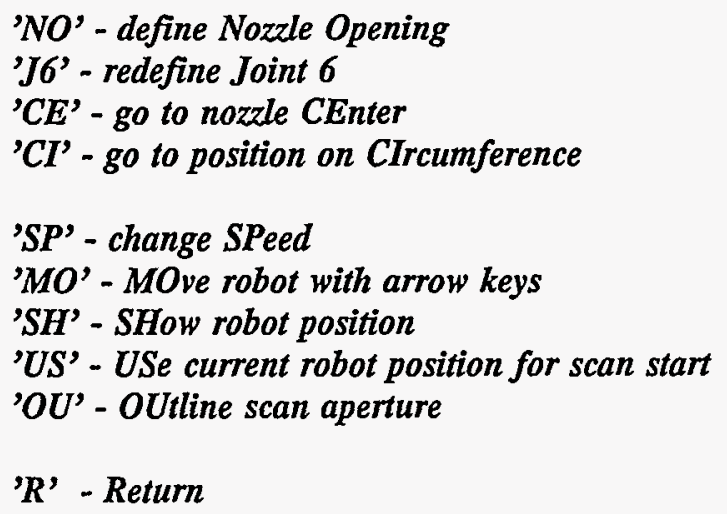

ENTER COMMAND -->

The user should start to teach the robot about the entrance to the nozzle by typing "NO". The following prompt will appear:

Coordinates for circle reference positions are expected to follow the pattern

$(P 1, S 1,0),(P 2, S 2,0),(P 3, S 3,0)$

Use the teach pendant to move robot to circle point 1

Switch to COMP mode and press [enter] when done:

The user must use the teach pendant to move the robotic arm to a position on the circumference of the nozzle. The robot should be positioned just before the blend zone begins on the reactor wall. Because a tool length of 2.0 inches was selected, the robots tool flange should be 2.0 inches above this point. In addition, joint 6 must be positioned so that one of the key-ways on the tool flange points toward the center of the nozzle. Once this is done, the user presses "RETURN" and the following prompt appears:

Enter orientation for joint 6:

(white size toward nozzle: 0, black side: 1) 1

There are 2 key-ways on the tool flange. If the one that is colored white is toward the nozzle center then user should enter " 0 " otherwise enter " 1 ". This completed, the following prompt appears:

Use the teach pendant to move robot to circle point 2

Switch to COMP mode and press [enter] when done:

The user must use the teach pendant to move the robotic arm to a second point on the circumference of the nozzle. Because a tool length of 2.0 inches was selected, the robots tool flange should be 2.0 inches above this point. Press "RETURN" when in position. The user is prompted for the 3rd point:

Use the teach pendant to move robot to circle point 3 Switch to COMP mode and press [enter] when done: 
Appendix B

The user must use the teach pendant to move the robotic arm to a third point on the circumference of the nozzle. Because a tool length of 2.0 inches was selected, the robots tool flange should be 2.0 inches above this point. Press "RETURN" when in position.

The user should enter "MO" to activate the arrow keys. Move the robot and verify that it will move across the bend zone. Position the robot for the start of a scan and then select "R" to deactivate the arrow keys. Select "US" to define the start position of a scan.

\section{B2.7 Adjusting the UT Signal}

The user should select "AS" from the MAIN MENU in order to adjust the UT signal by changing the parameters of the digitizer and the Time Variable Gain (TVG) module:

$$
\begin{array}{lll}
\text { rate } \ldots \ldots: & 12.5(M h z) \\
\text { length } \ldots \ldots: & 324(25.6 u s e c) \\
\text { delay } \ldots \ldots: & 5.524 \text { (usec) }
\end{array}
$$

A) range ...: .25 (volts $p$-p)

B) offset ...: 0.150(volts)

C) coupling ...: (AC coupled)

D) video ...: off $(R F)$

E) reps ....: 1
F) time0 : 4.800

G) slope0 : 0

H) delay : : 19.873

I) time1 : 0.835

J) slope1 : 40

K) time2 : 2.839

L) slope2 : 10

press ' $R$ ' to return:

\section{B2.8 Starting the Scan}

The user may start the scan by entering "sm" at the MAIN MENU prompt. The user will be prompted for a comment that pertains to this data file:

Enter 2 lines of comments, up to 40 characters each, or press

[enter] at the first prompt to keep the current comment

Current: scan of manway, flaw " $C$ "

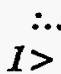

The user will be asked to enter the file name:

$$
\text { Enter file name ---> test.dat }
$$

The user may view the A-scan data if "y" is selected to the next prompt:

$$
\text { Display A-scans while taking data? -->y }
$$

Finally, the user must choose a destination for the data file. The programs gives the following choices: 
select data transfer option:

a) Transfer to MicroVAX

b) Save on local disk

c) Both

Enter choice: $b$

Type "b" and the scan begins.

\section{B3.0 Performing a Scan Across a Nozzle's Blend Zone}

The steps are:

B3.1 Invoking the scanning software on the data acquisition computer

B3.2 Selecting the scan pattern

B3.3 Selecting SAFT-UT data parameters

B3.4 Review the MAIN MENU

B3.5 Setting parameters for a scan of a nozzle's blend zone.

B3.6 Adjusting the UT signal

B3.7 Starting the scan

\section{B3.1 Invoking the Scanning Software on the Data Acquisition Computer}

The user should type "scan" at the prompt.

$$
\text { C:1>scan }
$$

The SAFT-UT data acquisition software will start and display the message:

$<S C A N M E N U>$ Has the robot been calibrated $(Y, N)$ :

Assuming that the robot is still on and calibrated, answer " $y$ " to this query and proceed to step B3.2 below. Otherwise the user should perform step 1.2 above.

\section{B3.2 Selecting the Scan Pattern}

The data acquisition computer will prompt user for the scan pattern with the following messages: 
Appendix B

Select scan pattern ...

1. XY Scan (vessel wall)

2. Cylindrical scan (inside nozzle)

3. Nozzle scan (nozzle blend zone)

ENTER SELECTION -..>

For this tutorial select "3", nozzle scan.

\section{B3.3 Selecting SAFT-UT Data Parameters}

After selecting the scan pattern, the data acquisition computer will prompt the user for SAFT-UT data parameters. Another example is show below:

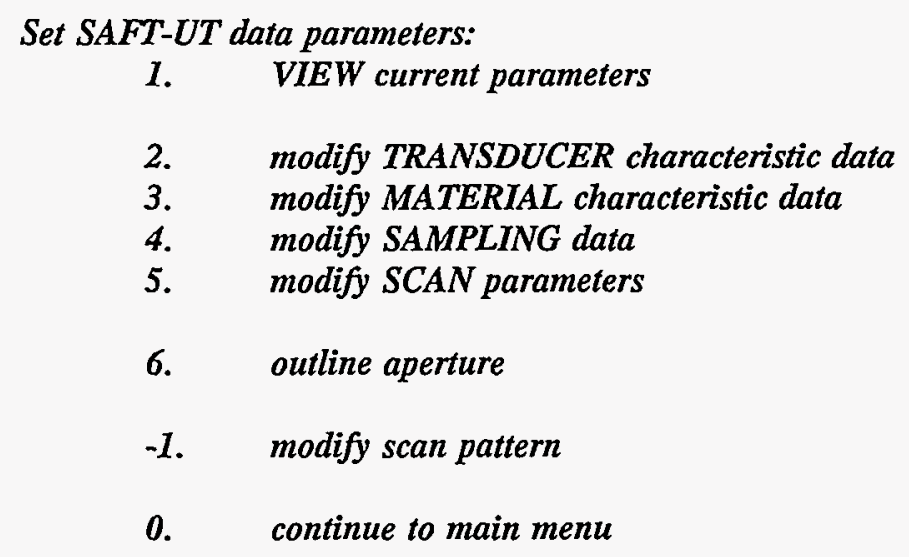

ENTER SELECTION --->

Menu item 1 displays the settings:

TRANSDUCER: rtd $70 \mathrm{deg}, L$ wave, serial \# 84-23

transducer frequency $(M H z): 2.0$

xmit standoff ... hgt $=$ fcc len (in): .29

$X$ incident angle (deg): -24.9

full beam angle in metal ... (deg): 12.0

mat vel (in/sec): 105000

refracted angle (deg): -70.444

beam_entry_diam (in): .125

MATERIAL: WPPSS nozzle mockup

material velocity (in/sec): 235000

thickness ... (in): 4.4

SAMPLING: Beam angle is 70 degrees so 3 inches along sound path is about 1 inc sample period (ns): 80

depth (along sound path) in material to ...

initial delay (us): 5.52380

number of points: 320

start sampling (in): 0

stop sampling (in): 3 
SCAN: Scan of blend zone

step size: $\quad X, Y$ axis

scan length: $X, Y$ axis

scan_dir: 0

235.573 init. pos.: $P, S, T$ axis

increments: $X, Y$ axis

final pos.: $P, S, T$ axis (in): .05, .1

(in): $8, \quad 4$

start angle: 269.957 end angle:

(in): 198.713, 99.21934, 0 (in): $161, \quad 5$

(in): $195.696, \quad 103.509, \quad-2.86479$

$\ldots \ldots \ldots \ldots$

ENTER RETURN TO CONTINUE

Menu item 5 will set the Scanning parameters:

Current: Scan of blend zone

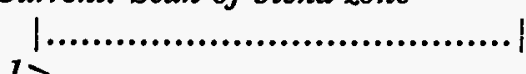

If no change is desired, press enter at that variable.

Current values:

step size: $\quad X$ axis $Y$ axis

$X$ scan length (in)

$Y$ scan length (in)

init. pos. primary axis

init. pos. secondary axis

Init. pos. tertiary axis
Changes:

$$
\begin{aligned}
& =.05 \\
& =1 \\
& =8 \\
& =4 \\
& =198.713 \\
& =99.21934 \\
& =0
\end{aligned}
$$

number of $x$ inc $=161$

number of $y$ inc $=5$

Scan dir. $(0=$ out $->$ in, 180 in- $>$ out $)=0$

final pos. primary axis $=195.696$

final pos. secondary axis $=103.509$

final pos. tertiary axis $=-2.86479$

Scan type: Transition

nozzle radius: 9.530353

break zone radius: 2.86479

\section{B3.4 Reviewing the MAIN MENU}

After completing the selection of the SAFT-UT data parameters, the user may select the MAIN MENU by entering " 0 " in the menu for the SAFT-UT data parameters.

Note that a new item "NS" is available. 
Appendix B

\section{MAIN MENU}

$$
\begin{aligned}
& \text { 'SP' - change Scan Pattern } \\
& \text { 'DP' - change SAFT-UT Data Parameters } \\
& \text { 'VI' - VIew current parameters } \\
& \text { 'EC' - Energize and Calibrate robot } \\
& \text { 'CS' - change Coordinate System } \\
& \text { 'EN' - define entrance to nozzle } \\
& \text { 'NS' - define nozzle scan } \\
& \text { 'AS' - Adjust UT Signal } \\
& \text { 'SM' - proceed to scanning module } \\
& \text { 'QT' - end this program and exit }
\end{aligned}
$$

ENTER COMMAND -->

\section{B3.5 Preparing the SAFT-UT Robot for a Scan}

In this tutorial, it is assumed that the robot has not been moved since the coordinates were defined in tutorial 2 . The user should enter "NS" and the following menu will appear:

MENU FOR NOZZLE BLEND ZONE SCANS: MODE: BZ(3)

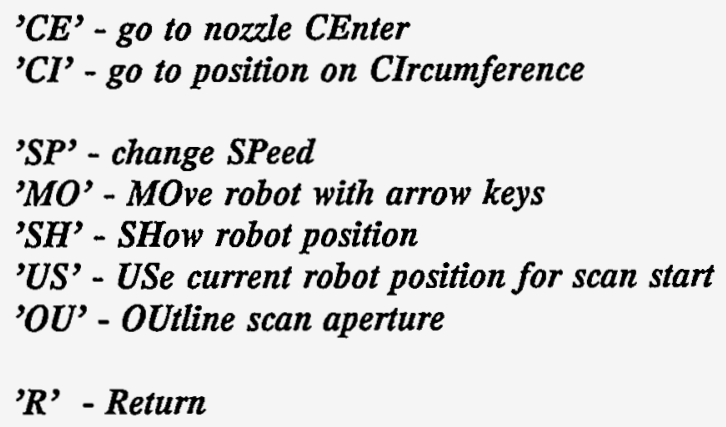

ENTER COMMAND -->

The user should enter "MO" to activate the arrow keys. Move the robot and verify that it will move across the bend zone. When the robot is in position for the start of a scan, select "R" and then select "US" to set the scan start position.

\section{B3.6 Adjusting the UT Signal}

The user should select "AS" from the MAIN MENU in order to adjust the UT signal by changing the parameters of the digitizer and the Time Variable Gain (TVG) module:

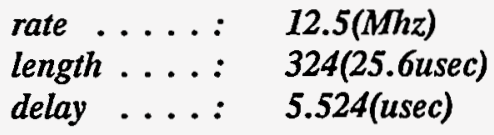

$\begin{array}{lll}\text { F) time0 : } & 4.800 \\ \text { G) } & \text { slope0 : } & 0\end{array}$ 

A) range ...: . .25 (volts p-p)
B) offset ...: 0.150 (volts)
C) coupling ...: (AC coupled)
D) video ...: of off $(R F)$
E) reps ....: 1

press ' $R$ " to return:

\section{B3.7 Starting the Scan}

The user may start the scan by entering "SM" at the MAIN MENU prompt. The user will be prompted for a comment that pertains to this data file:

Enter 2 lines of comments, up to 40 characters each, or press [enter] at the first prompt to keep the current comment Current: scan of manway, flaw " $C$ "

$$
1>
$$

The user will be asked to enter the file name:

\section{Enter file name ---> test.dat}

The user may view the A-scan data if "y" is selected to the next prompt:

Display A-scans while taking data? -->y

Finally, the user must choose a destination for the data file. The programs gives the following choices:

$\begin{array}{ll}\text { select data transfer option: } & \text { a) Transfer to MicroVAX } \\ & \text { b) Save on local disk } \\ \text { c) Both }\end{array}$

Enter choice: $b$

Type "b" and the scan begins. 
Appendix C

Changes to the SAFT Header 


\section{Appendix C}

\section{Changes to the SAFT Header}

1) All fields are now represented as ASCII characters. By way of example, in the old header, 'vel in material' was a floating point number and as such required 4 bytes of header space. In the new header, this is an ASCII character string representation of the floating point number and as such requires 17 bytes. This change will make the SAFT data files portable to any computer.

2) The header size has been increased from 512 bytes to 2048 bytes.

3) All unused fields have been deleted. They are:

$$
\begin{aligned}
& \text { num_sig_sum } \\
& \text { focal_length } \\
& \text { pm_damping } \\
& \text { pm_energy } \\
& \text { pm_attenuation } \\
& \text { pm_gain } \\
& \text { lc_attenuation } \\
& \text { pipe_radius } \\
& \text { sm_flag } \\
& \text { pipe_flag } \\
& \text { sm_depth } \\
& \text { proc_mode } \\
& \text { transy } \\
& \text { bandwidth }
\end{aligned}
$$

4) All header fields have been reorganized into one of the following categories:

\section{FILE PROPERTIES}

TRANNSDUCER PROPERTIES

MATERIAL PROPERTIES

SAMPLING PROPERTIES

SCANNING PROPERTIES

PROCESSING_PROPERTIES

\section{5) Changes to FILE PROPERTIES}

5.1) Added file format_id. This is a flag that indicates what (header) format the file has.
6) Changes to TRANSDUCER PROPERTIES

6.1) Added transducer_comment.

6.2) Renamed hgt_transd to recv_hgt_transd and changed units to inches (from equivalent water path).

6.3) Changed units of xmit hgt transd to inches.

6.4) Renamed beam angle to beam width angle.

6.5) Changed units of squint_dir to $\overline{0}-360 . \overline{0}$ degrees (floating point) from $x=0$; and $y=1$.

6.6) Renamed squint_angle to incident_angle and changed units to shoe degrees (from equivalent water angle).

7) Changes to MATERIAL PROPERTIES

7.1) Added material comment.

7.2) Added refracted_angle.

8) Changes to SAMPLING PROPERTIES

8.1) Added sampling_comment.

8.2) Changed delay inc to floating point number.

8.3) Deleted float_delay increment.

8.4) Changed init_delay to floating point number.

9) Changes to SCANNING PROPERTIES

9.1) Added scanning_comment.

9.2) Renamed scan_axis to scan_direction and changed unit to 0-360.0 degrees (from $x 1, x 2, y$ ).

9.3) Renamed init_x_pos to init_pos_primary_axis, changed to floating point, and changed units to inches.

9.4) Added final_pos_primary_axis.

9.5) Renamed init_y_pos to init_pos_secondary_axis.

9.6) Renamed final_y_pos to final_pos_secondary_axis.

10) Changes to PROCESSING PROPERTIES

10.1) Added processing_comment. 
Appendix D

Definition of SAFT-UT ASCII Header 


\section{Appendix D}

\section{Definition of SAFT-UT ASCII Header}

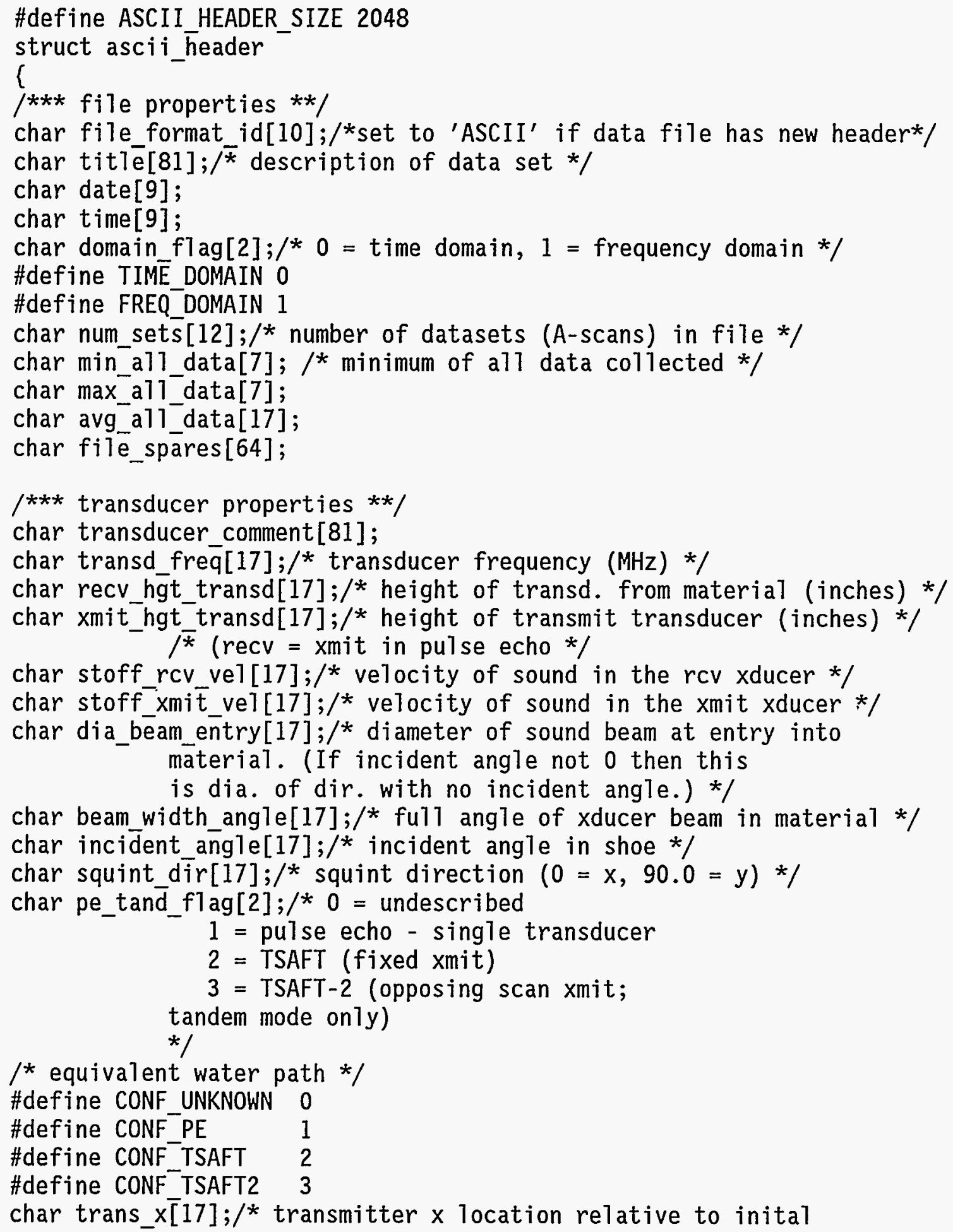


receiver position (inches) */

char transducer_spares[64];

/*** material properties $* * /$

char material_comment[81];

char ve]_in_material[17];/* actual velocity of sound in the material (in/sec) */

char refracted_angle[17];/* refracted angle in material */

char thickness $[17] ; / *$ specimen thickness (inches) */

char material_spares[64];

/*** sampling properites **/

char sampling_comment [81];

char delay_inc [17]; ${ }^{*}$ delay increment, in $10 \mathrm{nS}$ units in time or if domain_flag $==1$ then $10 \mathrm{~Hz}$ units */

char init_delay[17]; /* initial delay, in 10nS units

This value includes standoff delay \& start

delay, and in tandem mode includes

pre bounces of xmit transducer.

*/

char num_pts[7]; $/^{*}$ number of points in each A-scan */

char window_start[17]; /* depth to start of data window */

char window_end[17]; $/ *$ depth to end of data window*/

char sampling_spares[64];/* depth to end of data window */

$/ * * *$ scanning properties **/

char scanning_comment [81];

char scan_direction [17]; ${ }^{*} 0=\operatorname{primary}(\mathrm{x})$ axis of material

$$
90=\operatorname{secondary}(y) * /
$$

char init_pos_primary_axis[17]; $/^{*}$ initial primary material coord of receiver (inches) */

char init_pos_secondary_axis[17];/* secondary coord of receiver (in) */

char finā]pos_primary_axis[17];

char final_pos_secondary_axis[17];

char $x$ inc $[17] \bar{j} / *$ increment (inches) */

char $y_{\text {-inc }}[17] ; / *$ y increment (inches) */

char num_x_inc [7]; $/ *$ total points collected in $x$ direction */

char num_y_inc[7]; $/ *$ total points collected in y direction */

char strattile_weld[2]; $/ * 0=$ scanner not strattling weld

1 = scanner is strattling weld */

char transmit half_vees[12];

char receive_half_vees[12];

char num_pre_half_v[17]; $/^{*}$ number of extimated half $V^{\prime} s$ before signal

encounters object plane (arrow) */

char init_pos_tertiary_axis[17];

char fina $\overline{1} \_$pos_tertiary_axis[17]; 
char scan_pattern[2];

char scanning_spares[28];

$/ * * *$ processing properties $* * /$

char processing_comment[81];

char proc_date $[\overline{9}] ; / *$ date and time when file was processed */

char proc time[9];

char env_det_flag[12];/* non-0 for envdet data */

char deconv $\bar{f} 7 \mathrm{ag}[12] ; / *$ non-0 for deconvoluted data */

char proc f $\mathrm{7}$ ag $[12] ; / *$ non-0 for processed data */

char promode[7];/* process mode chosen in tandem processor

$3=$ CASE $A$
$5=$ CASE B
$7=$ CASE C
$11=$ CASE D

combinations are indicated by multiplying appropriate numbers

*/

\#define TSAFT MODE A 3

\#define TSAFT MODE B 5

\#define TSAFT MODE C 7

\#define TSAFT_MODE D 11

char beyond back $[2] ;{ }^{*} ' y^{\prime}=$ file was processed beyond back surface

$' n^{\prime}=$ file processed only to back surface */

\#define PROC BEYOND BS ' $y$ '

\#define NOT PROC BEYOND BS ' $n$ '

char input_ $\bar{x}$ reduction_factor[7];/* Data reduction that processing */

char input_y_reduction_factor[7];/* performed on data during processing. */

char input_z_reduction_factor[7];/* Data reduction occur during */

char output_z $\bar{z}$ reduction factor [7];/* envelope detection. */

char num_defect_a_scans $\overline{[} 12] ; /^{*}$ count of defect_flags

(see rec struct) */

char num_nondefect_a_scans[12]; $/ *$ initialized in DEDIT */

char proc_clip_level[17]; $/ *$ Tevel in -db of "clipping" performed in processing routine */

char thresh_db[17];/* clipping threshhold level chosen during processing $(\mathrm{db}) * /$

char nbounce proc[7]; ${ }^{*}$ number of transmit bounces chosen in processing */

char nrbounce_proc[7];/* number of receive bounces chosen in processing */

char processing_spares[64];

$/ * * *$ nozzel parameters $* * * /$

char nozzel_radius[17]; $/ *$ inches */

char break_zone_radius[17]; 
Appendix D

char nozzel_center_primary[17];

char nozze1_center_secondary[17];

char ascii_header_spares[2048-1648];

\} ah; 


\section{DISTRIBUTION}

No. of

Copies

\section{OFFSITE}

2 J. Muscara NRC/RES

Mail Stop T-10E10

D. Naujock

NRC/NRR

Mail Stop 7 D4

J. Strosnider NRC/NRR

Mail Stop 7 D4

E. J. Sullivan, Jr. NRC/RES

Mail Stop 7 E23

M. Vagins NRC/RES

Mail Stop NS 217

E. H. Gray NRC/Region I

R. H. Harris NRC/Region I

M. C. Modes NRC/Region I

P. M. Peterson NRC/Region I

A. R. Herdt NRC/Region II

J. J. Blake NRC/Region II
No. of

Copies

J. Jacobson NRC/Region III

L. J. Busse Tetrad Corporation 12741 E. Caley Avenue

Unit 126

Englewood, CO 80111

S. L. Crawford 1308 Brookwood Ave. Richland, WA 99352

R. A. Hewes

General Electric Company Building KW, Room D243

PO Box 8

Schenectady, NY 12301

D. S. Kupperman Materials Science Center Argonne National Laboratory 9700 S. Cass Avenue

Building 212

Argonne, IL 60439

J. LaReau ABB Amdata 1000 Prospect Hill Road Windsor, CT 06095-0500

C. Little Nondestructive Testing Technology Division Sandia National Laboratories Albuquerque, NM 87185

L. E. Mullins

Knolls Atomic Power Laboratory PO Box 1072

Schenectady, NY 12301-1072 
W. W. Shurtleff

Nondestructive Testing

Technology Division

Sandia National Laboratories

Albuquerque, NM 87185

J. H. Tehrune

General Electric Company

175 Curtner Avenue

San Jose, CA 95125

\section{FOREIGN}

S. Crutzen

Joint Research Centre

PO Box 2

1755 ZG Petten

The Netherlands

K. Kussmaul

Materialpruefungsanstalt

Universitat Stuttgart

Pfaffenwaldring 32

7000 Stuttgart 80 (Vaihingen)

West Germany

A. Rogerson

AEA Technology

Risley

Warrington

Cheshire WA3 6AT

United Kingdom

V. Schmitz

Izfp

Universitat, Gerbaude 37

6600 Saarbrucken 11

West Germany

J. R. Tomlinson NDT Application Centre

Nuclear Electric plc

Timpson Road

Wythenshawe

Manchester M23 9LL

United Kingdom

\section{ONSITE}

50 Pacific Northwest Laboratory

R. E. Bowey

D. M. Boyd

S. H. Bush

A. A. Diaz

S. R. Doctor (36)

T. E. Hall

R. V. Harris

L. D. Reid

G. J. Schuster

Publishing Coordination

Technical Report Files (5) 
Real-Time 3-D SAFT-UT System Evaluation and Validation

3. DATE REPORT PUBLISHED \begin{tabular}{l|l} 
MONTH & YEAR \\
\hline
\end{tabular}

September 1996

S. R. Doctor, G.J. Schuster, L.D. Reid, T.E. Hall

8. PERFORMING ORGANIZATION - NAME AND ADDRESS (If NRC, provide Division, Office or Region, U.S. Nuclear Regulatory Commission, and mailing address; if contractor, provide name and mailing addressi

Pacific Northwest National Laboratory

Richland, WA 99352

9. SPONSORING ORGANIZATION - NAME AND ADDRESS II NRC, type "Same as above"; if contractor, provide NRC Division, Office or Region, U.S. Nuclear Regulatory Commission, and malling address.l

Division of Engineering Technology

Office of Nuclear Regulatory Research

U.S. Nuclear Regulatory Commission

Washington, DC 20555-0001

10. SUPPLEMENTARY NOTES

11. ABSTRACT (200 wards or less)

SAFT-UT technology is shown to provide significant enhancements to the inspection of materials used in U.S. nuclear power plants. This report provides guidelines for the implementation of SAFT-UT technology and shows the results from its application. An overview of the development of SAFT-UT is provided so that the reader may become familiar with the technology. Then the basic fundamentals are presented with an extensive 1ist of references. A comprehensive operating procedure, which is used in conjunction with the SAFT-Ut field system developed by Pacific Northwest National Laboratory (PNL), provides the recipe for both SAFT data acquisition and analysis. The specification for the hardware implementation is provided for the SAFT-UT system along with a description of the subsequent developments and improvements. One development of technical interest is the SAFT real-time processor. Performance of the real-time processor is impressive and comparison is made of this dedicated parallel processor to a conventional computer and to the newer high-speed computer architectures designed for image processing. Descriptions of other improvements, including a robotic scanner, are provided. 